\title{
Flavor physics of leptons and dipole moments
}

\section{Journal Article}

\section{Author(s):}

Raidal, Mihkel; Fetscher, Wulf; et al.

Publication date:

2008-09

Permanent link:

https://doi.org/10.3929/ethz-b-000069609

\section{Rights / license:}

Creative Commons Attribution 4.0 International

\section{Originally published in:}

The European Physical Journal C 57(1-2), https://doi.org/10.1140/epjc/s10052-008-0715-2 


\title{
Flavor physics of leptons and dipole moments*
}

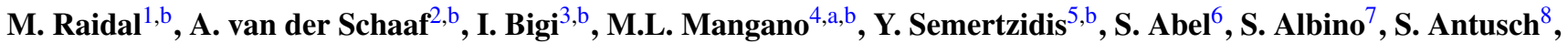

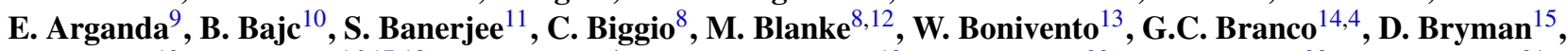 \\ A.J. Buras $^{12}$, L. Calibbi ${ }^{16,17,18}$, A. Ceccucci ${ }^{4}$, P.H. Chankowski ${ }^{19}$, S. Davidson ${ }^{20}$, A. Deandrea ${ }^{20}$, D.P. DeMille ${ }^{21}$, \\ F. Deppisch ${ }^{22}$, M.A. Diaz ${ }^{23}$, B. Duling ${ }^{12}$, M. Felcini ${ }^{4}$, W. Fetscher ${ }^{24}$, F. Forti ${ }^{25}$, D.K. Ghosh ${ }^{26}$, M. Giffels ${ }^{27}$, \\ M.A. Giorgi ${ }^{25}$, G. Giudice ${ }^{4}$, E. Goudzovskij ${ }^{28}$, T. Han $^{29}$, P.G. Harris ${ }^{30}$, M.J. Herrero ${ }^{9}$, J. Hisano ${ }^{31}$, R.J. Holt ${ }^{32}$,

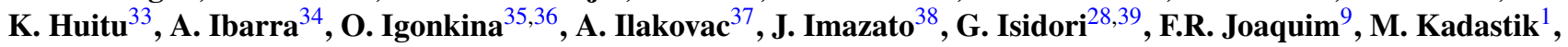

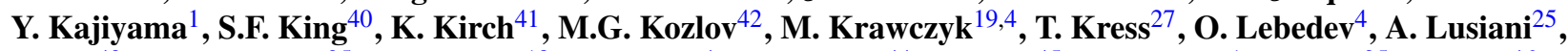

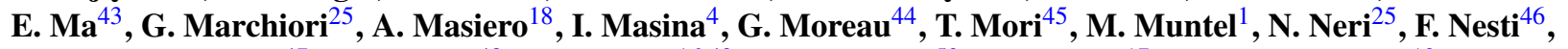 \\ C.J.G. Onderwater ${ }^{47}$, P. Paradisi ${ }^{48}$, S.T. Petcov ${ }^{16,49}$, M. Picariello ${ }^{50}$, V. Porretti ${ }^{17}$, A. Poschenrieder ${ }^{12}$, \\ M. Pospelov ${ }^{51}$, L. Rebane ${ }^{1}$, M.N. Rebelo ${ }^{14,4}$, A. Ritz ${ }^{51}$, L. Roberts ${ }^{52}$, A. Romanino $^{16}$, J.M. Roney ${ }^{11}$, A. Rossi $^{18}$, \\ R. Rückl ${ }^{53}$, G. Senjanovic ${ }^{54}$, N. Serra ${ }^{13}$, T. Shindou ${ }^{34}$, Y. Takanishi ${ }^{16}$, C. Tarantino $^{12}$, A.M. Teixeira ${ }^{44}$, \\ E. Torrente-Lujan ${ }^{55}$, K.J. Turzynski ${ }^{56,19}$, T.E.J. Underwood ${ }^{6}$, S.K. Vempati $^{57}$, O. Vives $^{17}$ \\ ${ }^{1}$ National Institute for Chemical Physics and Biophysics, 10143 Tallinn, Estonia \\ ${ }^{2}$ Physik-Institut der Universität Zürich, 8057 Zürich, Switzerland \\ ${ }^{3}$ Physics Department, University of Notre Dame du Lac, Notre Dame, IN 46556, USA \\ ${ }^{4}$ Physics Department, CERN, 1211 Geneva, Switzerland \\ ${ }^{5}$ Brookhaven National Laboratory, Upton, NY 11973-5000, USA \\ ${ }^{6}$ Institute for Particle Physics Phenomenology, Durham University, Durham DH1 3LE, UK \\ ${ }^{7}$ II. Institute for Theoretical Physics, University of Hamburg, 22761 Hamburg, Germany \\ ${ }^{8}$ Max-Planck-Institut für Physik, 80805 München, Germany \\ ${ }^{9}$ Departamento de Fisica Teorica and IFT/CSIC-UAM, Universidad Autonoma de Madrid, 28049 Madrid, Spain \\ ${ }^{10}$ J. Stefan Institute, 1000 Ljubljana, Slovenia \\ ${ }_{11}^{11}$ Department of Physics, University of Victoria, Victoria, BC V8W 3P6, Canada \\ ${ }^{12}$ Physics Department, TU Munich, 85748 Garching, Germany \\ ${ }^{13}$ Università degli Studi di Cagliari and INFN Cagliari, 09042 Monserrato (CA), Italy \\ ${ }_{14}^{14}$ Departamento de Física and Centro de Física Teórica de Partículas (CFTP), Instituto Superior Técnico (IST), 1049-001 Lisboa, Portugal \\ ${ }^{15}$ Department of Physics and Astronomy, University of British Columbia, TRIUMF, Vancouver, BC, V6T 2A3, Canada \\ ${ }^{16}$ SISSA and INFN, Sezione di Trieste, 34013 Trieste, Italy \\ ${ }^{17}$ Departament de Física Teòrica, Universitat de València-CSIC, 46100 Burjassot, Spain \\ ${ }^{18}$ Dipartimento di Fisica 'G. Galilei’ and INFN, 35131 Padova, Italy \\ ${ }^{19}$ Institute of Theoretical Physics, University of Warsaw, 00-681 Warsaw, Poland \\ ${ }^{20}$ IPNL, CNRS, Université Lyon-1, 69622 Villeurbanne Cedex, France \\ ${ }^{21}$ Physics Department, Yale University, New Haven, CO 06520, USA \\ ${ }^{22}$ School of Physics and Astronomy, University of Manchester, Manchester M13 9PL, UK \\ ${ }^{23}$ Facultad de Fisica, Pontificia Universita Catolica de Chile, Santiago 22, Chile \\ ${ }^{24}$ Department of Physics, ETH Honggerberg, 8093 Zurich, Switzerland \\ ${ }^{25}$ INFN and Dipartimento di Fisica, Universita di Pisa, 56127 Pisa, Italy \\ ${ }^{26}$ Theoretical Physics Division, Physical Research Lab., Navrangpura, Ahmedabad 380 009, India \\ ${ }^{27}$ III. Physikalisches Institut B, RWTH Aachen, 52056 Aachen, Germany \\ ${ }^{28}$ Scuola Normale Superiore, 56100 Pisa, Italy \\ ${ }^{29}$ Department of Physics, High Energy Physics, University of Wisconsin, Madison, WI 53706, USA \\ ${ }^{30}$ Department of Physics and Astronomy, University of Sussex, Falmer, Brighton BN1 9QH, UK \\ ${ }^{31}$ ICRR, University of Tokyo, Tokyo, Japan \\ ${ }^{32}$ Physics Division, Argonne National Laboratory, Argonne, IL 60439-4843, USA \\ ${ }^{33}$ Department of Physics, University of Helsinki, and Helsinki Institute of Physics, 00014 Helsinki, Finland \\ ${ }^{34}$ Theory Group, DESY, 22603 Hamburg, Germany \\ ${ }_{36}^{35}$ Physics Department, University of Oregon, Eugene, OR, USA \\ ${ }^{36}$ NIKHEF, 1098 SJ Amsterdam, The Netherlands \\ ${ }^{37}$ Department of Physics, University of Zagreb, 10002 Zagreb, Croatia \\ ${ }^{38}$ IPNS, KEK, Ibaraki 305-0801, Japan \\ ${ }^{39}$ INFN, Laboratori Nazionali di Frascati, 00044 Frascati, Italy \\ ${ }^{40}$ School of Physics and Astronomy, University of Southampton, SO17 1BJ Southampton, UK \\ ${ }^{41}$ Paul Scherrer Institut, 5232 Villigen, Switzerland \\ ${ }^{42}$ Petersburg Nuclear Physics Institute, Gatchina 188300, Russia \\ ${ }^{43}$ Department of Physics and Astronomy, University of California, Riverside, CA 92521, USA
}




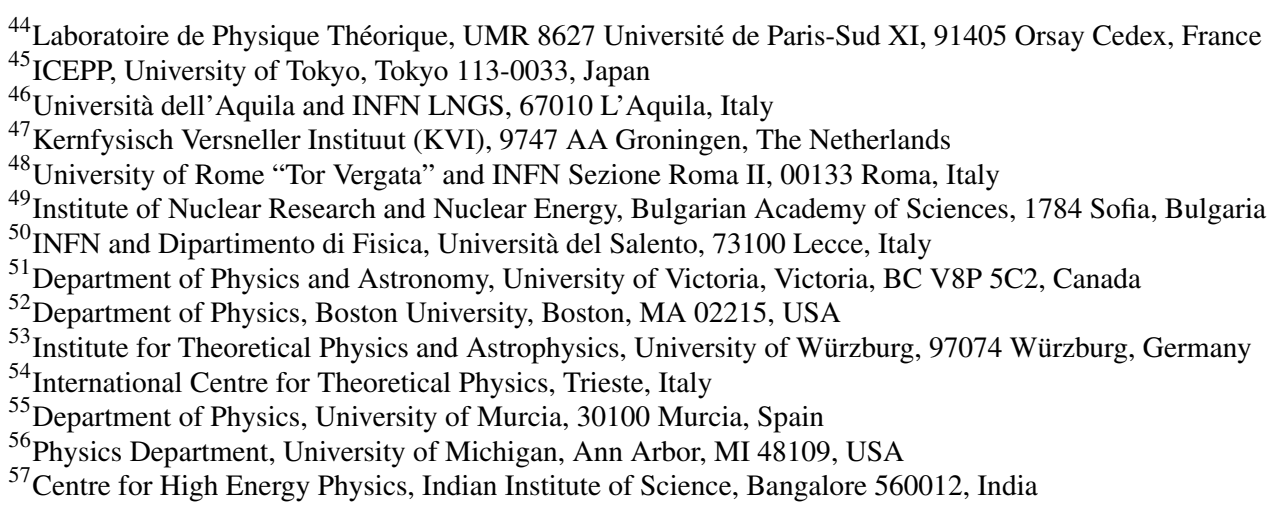

Received: 19 February 2008 / Revised: 12 August 2008 / Published online: 12 November 2008 (C) Springer-Verlag / Società Italiana di Fisica 2008

\begin{abstract}
This chapter of the report of the "Flavor in the era of the LHC" Workshop discusses the theoretical, phenomenological and experimental issues related to flavor phenomena in the charged lepton sector and in flavor conserving $\mathrm{CP}$ violating processes. We review the current experimental limits and the main theoretical models for the flavor structure of fundamental particles. We analyze the phenomenological consequences of the available data, setting constraints on explicit models beyond the standard model, presenting benchmarks for the discovery potential of forthcoming measurements both at the LHC and at low energy, and exploring options for possible future experiments.
\end{abstract}

\section{Contents}

1 Charged leptons and fundamental dipole moments: alternative probes of the origin of flavor and CP

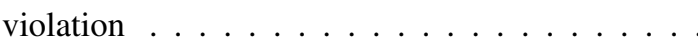

2 Theoretical framework and flavor symmetries . .

2.1 The flavor puzzle . . . . . . . . . . .

2.2 Flavor symmetries . . . . . . . . . .

3 Observables and their parameterization . . . . . .

3.1 Effective operators and low scale observables

3.2 Phenomenological parameterizations of quark and lepton Yukawa couplings . . . . . . . .

3.3 Leptogenesis and cosmological observables . 44

4 Organizing principles for flavor physics . . . . . . 46

4.1 Grand unified theories . . . . . . . . . . . . 46

4.2 Higher dimensional approaches ...... . 50

4.3 Minimal flavor violation in the lepton sector . 53

\footnotetext{
*Report of Working Group 3 of the CERN Workshop "Flavor in the era of the LHC", Geneva, Switzerland, November 2005-March 2007.

a e-mail: michelangelo.mangano@ cern.ch

${ }^{\mathrm{b}}$ Convenors
}

5 Phenomenology of theories beyond the standard model . . . . . . . . . . . 57

5.1 Flavor violation in non-SUSY models directly testable at LHC . . . . . . . . . . .

5.2 Flavor and CP violation in SUSY extensions of the SM ............... 66

5.3 SUSY GUTs . . . . . . . . . . . . . . . . . . 86

5.4 R-parity violation . . . . . . . . . . . . 97

5.5 Higgs-mediated lepton flavor violation in supersymmetry . . . . . . . . . . . . . 101

5.6 Tests of unitarity and universality in the lepton sector . . . . . . . . . . . 109

5.7 EDMs from RGE effects in theories with low energy supersymmetry . . . . . . . 116

6 Experimental tests of charged lepton universality . 122

$6.1 \pi$ decay ................. . . . . . . . . . . . . 124

$6.2 K$ decay ................ 125

$6.3 \tau$ decay . . . . . . . . . . . . . 127

7 CP violation with charged leptons . . . . . . . 128

$7.1 \mu$ decays . . . . . . . . . . . . . 129

7.2 CP violation in $\tau$ decays . . . . . . . . . 130

7.3 Search for T violation in $K^{+} \rightarrow \pi^{0} \mu^{+} v$ decay 131

7.4 Measurement of CP violation in ortho-positronium decay . . . . . . . . 133

8 LFV experiments . . . . . . . . . . . . . . . . 135

8.1 Rare $\mu$ decays . . . . . . . . . . . . . 135

8.2 Searches for lepton flavor violation in $\tau$ decays 141 $8.3 B_{d, s}^{0} \rightarrow e^{ \pm} \mu^{\mp} \ldots \ldots \ldots . \ldots . \ldots 146$

8.4 In flight conversions . . . . . . . . . . 147

9 Experimental studies of electric and magnetic dipole moments . . . . . . . . . . . . . . . . 148

9.1 Electric dipole moments . . . . . . . . . . . 148

9.2 Neutron EDM . . . . . . . . . . . . . . . . . . . . . . . . . . 151

9.3 Deuteron EDM . . . . . . . . . . . . . . 153

9.4 EDM of deformed nuclei: ${ }^{225} \mathrm{Ra} \ldots \ldots . . . .156$

9.5 Electrons bound in atoms and molecules . . . 158

9.6 Muon EDM . . . . . . . . . . . . . . . . 164 
9.7 Muon $g-2 \ldots \ldots \ldots 5$

Acknowledgements ............ . . 167

References .............. 167

\section{Charged leptons and fundamental dipole moments: alternative probes of the origin of flavor and CP violation}

The understanding of the flavor structure and $\mathrm{CP}$ violation (CPV) of fundamental interactions has so far been dominated by the phenomenology of the quark sector of the standard model (SM). More recently, the observation of neutrino masses and mixing has begun extending this phenomenology to the lepton sector. While no experimental data available today link flavor and CP violation in the quark and in the neutrino sectors, theoretical prejudice strongly supports the expectation that a complete understanding should ultimately expose their common origin. Most attempts to identify the common origin, whether through grand unified (GUT) scenarios, supersymmetry (SUSY), or more exotic electroweak symmetry breaking mechanisms predict in addition testable correlations between the flavor and $\mathrm{CP}$ violation observables in the quark and neutrino sector on the one side, and new phenomena involving charged leptons and flavor conserving CP-odd effects on the other. This chapter of the "Flavor in the era of the LHC" report focuses precisely on the phenomenology arising from these ideas, discussing flavor phenomena in the charged lepton sector and flavor conserving CP-violating processes.

Several theoretical arguments make the studies discussed in this chapter particularly interesting.

- The charged lepton sector provides unique opportunities to test scenarios tailored to explain flavor in the quark and neutrino sectors, for example by testing correlations between neutrino mixing and the rate for $\mu \rightarrow e \gamma$ decays, as predicted by specific SUSY/GUT scenarios. Charged leptons are therefore an indispensable element of the flavor puzzle, without which its clarification could be impossible.

- The only observed source of CP violation is so far the Cabibbo-Kobayashi-Maskawa (CKM) mixing matrix. On the other hand, it is by now well established that this is not enough to explain the observed baryon asymmetry of the universe (BAU). The existence of other sources of $\mathrm{CP}$ violation is therefore required. $\mathrm{CP}$-odd phases in neutrino mixing, directly generating the BAU through leptogenesis, are a possibility, directly affecting the charged lepton sector via, e.g., the appearance of electric dipole moments (EDMs). Likewise, EDMs could arise via CP violation in flavor conserving couplings, like phases of the gaugino fields or in extended Higgs sectors. In all cases, the observables discussed in this chapter provide essential experimental input for the understanding of the origin of $\mathrm{CP}$ violation.

- The excellent agreement of all flavor observables in the quark sector with the CKM picture of flavor and CP violation has recently led to the concept of minimal flavor violation (MFV). In scenarios beyond the SM (BSM) with $\mathrm{MFV}$, the smallness of possible deviations from the SM is naturally built into the theory. While these schemes provide a natural setting for the observed lack of new physics (NP) signals, their consequence is often a reduced sensitivity to the underlying flavor dynamics of most observables accessible by the next generation of flavor experiments. Lepton flavor violation (LFV) and EDMs could therefore provide our only probe into this dynamics.

- Last but not least, with the exception of the magnetic dipole moments, where the SM predicts non-zero values and deviations due to new physics compete with the effect of higher order SM corrections, the observation of a non-zero value for any of the observables discussed in this chapter would be unequivocal indication of new physics. In fact, while neutrino masses and mixing can mediate lepton flavor violating transitions, as well as induce CP-odd effects, their size is such that all these effects are by many orders of magnitude smaller than anything measurable in the foreseeable future. This implies that, contrary to many of the observables considered in other chapters of this report, and although the signal interpretation may be plagued by theoretical ambiguities or systematics, there is nevertheless no theoretical systematic uncertainty to claim a discovery once a positive signal is detected.

The observables discussed here are also very interesting from the experimental point of view. They call for a very broad approach, based not only on the most visible tools of high energy physics, namely the high energy colliders, but also on a large set of smaller-scale experiments that draw from a wide variety of techniques. The emphasis of these experiments is by and large on high rates and high precision, a crucial role being played by the control of very large backgrounds and subtle systematics. A new generation of such experiments is ready to start or will start during the first part of the LHC operations. More experiments have been on the drawing board for some time, and could become reality during the LHC era if the necessary resources were made available. The synergy between the techniques and potential results provided by both the large- and small-scale experiments makes this field of research very rich and exciting and gives it a strong potential to play a key role in exploring the physics landscape in the era of the LHC. 
The purpose of this document is to provide a comprehensive overview of the field, from both the theoretical and the experimental perspective. While we cover many model building aspects of neutrino physics that are directly related to the phenomenology of the quark and charged lepton sectors, for the status of the determinations of the mixing parameters and for the review of the future prospects we refer the reader to the vast existing literature, as documented for example in [1-4].

Several of the results presented are already well known, but they are nevertheless documented here to provide a selfcontained review, accessible to physicists whose expertise covers only some of the many diverse aspects of this subject. Many results emerged during the workshop, including ideas on possible new experiments, further enrich this report. We present here a short outline and some highlights of the contents.

Section 2 provides the general theoretical framework that allows us to discuss flavor from a symmetry point of view. It outlines the origin of the flavor puzzles and lists the mathematical settings that have been advocated to justify or predict the hierarchies of the mixing angles in both the quark and neutrino sectors. Section 3 introduces the observables that are sensitive to flavor in the charged lepton sector and to flavor conserving CP violation, providing a unified description in terms of effective operators and effective scales for the new physics that should be responsible for them. The existing data already provide rather stringent limits on the size of these operators, as shown in several tables. We collect here in Table 1 some of the most significant benchmark results (for details, we refer to the discussion in Sect. 3.1.2). We constrain the dimensionless coefficients $\epsilon_{i}$ of effective operators $O_{i}$ describing flavor or CP-violating interactions. Examples of these effective operators include

$\bar{\ell}_{i} \sigma^{\mu \nu} \gamma_{5} \ell_{i} F_{\mu \nu}^{\mathrm{em}}, \quad \bar{\ell}_{i} \sigma^{\mu \nu} \ell_{j} F_{\mu \nu}^{\mathrm{em}}$,

which describe a CP-violating electric dipole moment (EDM) of lepton $\ell_{i}$ or the flavor violating decay $\ell_{i} \rightarrow \ell_{j} \gamma$, or the four-fermion operators:

$\overline{\ell_{i}} \Gamma^{a} \ell_{j} \overline{q_{k}} \Gamma_{a} q_{l}, \quad \overline{\ell_{i}} \Gamma^{a} \ell_{j} \overline{\ell_{k}} \Gamma_{a} \ell_{l}$,

where the $\Gamma_{a}$ represent the various possible Lorentz structures. The overall normalization of the operators is chosen to reproduce the strength of transitions mediated by weak gauge bosons, assuming flavor mixing angles and CPviolating phases of order unity. The smallness of the constraints on $\epsilon$ therefore reflects either the large mass scale of flavor phenomena, or the weakness of the relative interactions.

It is clear from this table that current data are already sensitive to mass scales much larger than the electroweak scale, or to very small couplings. On the other hand, many of these constraints leave room for interesting signals coupled to the new physics at the $\mathrm{TeV}$ scale that can be directly discovered at the LHC. For example, a mixing of order 1 between the supersymmetric scalar partners of the charged leptons and a mass splitting among them of the order of the lepton masses is consistent with the current limits if the scalar lepton masses are just above $100 \mathrm{GeV}$, and it could lead both to their discovery at the LHC, and to observable signals at the next generation of $\ell \rightarrow \ell^{\prime} \gamma$ experiments.

Most of this report will be devoted to the discussion of the phenomenological consequences of limits such as those in Table 1, setting constraints on explicit BSM models, presenting benchmarks for the discovery potential of forthcoming measurements both at the LHC and at low energy, and exploring options for future experiments aimed at increasing the reach even further.

Section 3 also introduces the phenomenological parameterizations of the quark and lepton mixing matrices that are found in the literature, emphasizing with concrete examples the correlations among the neutrino and charged lepton sectors that arise in various proposed models of neutrino masses. The section is completed by a discussion of the possible role played by leptogenesis and cosmological observables in constraining the neutrino sector.

Section 4 reviews the organizing principles for flavor physics. With a favorite dynamical theory of flavor still missing, the extended symmetries of BSM theories can provide some insight in the nature of the flavor structures of quarks and leptons, and give phenomenologically relevant constraints on low energy correlations between them. In GUT theories, for example, leptons and quarks belong to the same irreducible representations of the gauge group, and their mass matrices and mixing angles are consequently tightly related. Extra dimensional theories provide a possible dynamical origin for flavor, linking flavor to the geometry of the extra dimensions. This section also discusses the implications of models adopting for the lepton sector the same concept of MFV already explored in the case of quarks.

Section 5 discusses at length the phenomenological consequences of the many existing models, and represents the main body of this document. We cover models based on

Table 1 Bounds on CP- or flavor violating effective operators, expressed as upper limits on their dimensionless coefficients $\epsilon$, scaled to the strength of weak interactions. For more details, in particular the overall normalization convention for the effective operators, see Sect. 3.1.2

\begin{tabular}{lll}
\hline Observable & Operator & Limit on $\epsilon$ \\
\hline$e \mathrm{EDM}$ & $\overline{e_{L}} \sigma^{\mu \nu} \gamma_{5} e_{R} F_{\mu \nu}$ & $\leq 2.1 \times 10^{-12}$ \\
$B(\mu \rightarrow e \gamma)$ & $\bar{\mu} \sigma^{\mu \nu} e F_{\mu \nu}$ & $\leq 3.4 \times 10^{-12}$ \\
$B(\tau \rightarrow \mu \gamma)$ & $\bar{\tau} \sigma^{\mu \nu} \mu F_{\mu \nu}$ & $\leq 8.4 \times 10^{-8}$ \\
$B\left(K_{L}^{0} \rightarrow \mu^{ \pm} e^{\mp}\right)$ & $\left(\bar{\mu} \gamma^{\mu} P_{L} e\right)\left(\bar{s} \gamma^{\mu} P_{L} d\right)$ & $\leq 2.9 \times 10^{-7}$ \\
\hline
\end{tabular}


SUSY, as well as on alternative descriptions of electroweak symmetry breaking, such as little Higgs or extended Higgs sectors. In this section we discuss the predictions and the detection prospects of standard observables, such as $\ell \rightarrow \ell^{\prime} \gamma$ decays or EDMs, and connect the discovery potential for these observables with the prospects for direct detection of the new massive particles at the LHC or at a future Linear Collider.

This section underlines, as is well known that the exploration of these processes has great discovery potential, since most BSM models anticipate rates that are within the reach of the forthcoming experiments. From the point of view of the synergy with collider physics, the remarkable outcome of these studies is that the sensitivities reached in the searches for rare lepton decays and dipole moments are often quite similar to those reached in direct searches at high energy. We give here some explicit examples. In $S O(10)$ SUSY GUT models, where the charged lepton mixing is induced via renormalization-group evolution of the heavy neutrinos of different generations, the observation of $B(\mu \rightarrow e \gamma)$ at the level of $10^{-13}$, within the range of the just-starting MEG experiment, is suggestive of the existence of squarks and gluinos with a mass of about $1 \mathrm{TeV}$, well within the discovery reach of the LHC. Squarks and gluinos in the range of $2-2.5 \mathrm{TeV}$, at the limit of detectability for the LHC, would push $B(\mu \rightarrow e \gamma)$ down to the level of $10^{-16}$. While this is well beyond the MEG sensitivity, it would well fit the ambitious goals of the next-generation $\mu \rightarrow e$ conversion experiments, strongly endorsing their plans. The decay $\mu \rightarrow e \gamma$ induced by the mixing of the scalar partners of muon and electron, and with a $B(\mu \rightarrow e \gamma)$ at the level of $10^{-13}$, could give a $\chi_{2}^{0} \rightarrow \chi_{1}^{0} \mu^{ \pm} e^{\mp}$ signal at the LHC, with up to 100 events after $300 \mathrm{fb}^{-1}$. Higher statistics and a cleaner signal would arise at a Linear Collider. Models where neutrino masses arise not from a see-saw mechanism at the GUT scale but from triplet Higgs fields at the $\mathrm{TeV}$ scale can be tested at the LHC, where processes like $p p \rightarrow H^{++} H^{--}$can be detected for $m_{H^{++}}$up to $700 \mathrm{GeV}$, using the remarkable signatures due to $B\left(H^{++} \rightarrow \tau^{+} \tau^{+}\right) \approx$ $B\left(H^{++} \rightarrow \mu^{+} \mu^{+}\right) \approx B\left(H^{++} \rightarrow \mu^{+} \tau^{+}\right) \approx 1 / 3$.

Should signals of new physics be observed, alternative interpretations can be tested by exploiting different patterns of correlations that they predict among the various observables. For example, while typical SUSY scenarios predict $B(\mu \rightarrow 3 e) \sim 10^{-2} B(\mu \rightarrow e \gamma)$, these branching ratios are of the same order in the case of little Higgs models with $\mathrm{T}$ parity. Important correlations also exist in see-saw SUSY GUT models between $B(\mu \rightarrow e \gamma)$ and $B(\tau \rightarrow \mu \gamma)$ or $B(\tau \rightarrow e \gamma)$. Furthermore, SUSY models with CP violation in the Higgs or gaugino mass matrix, be they supergravity (SUGRA) inspired or of the split-SUSY type, predict the ratio of electron and neutron EDM to be in the range of
$10^{-2}-10^{-1}$. Furthermore, in SUSY GUT models with seesaw mechanism correlations exist between the values of the neutron and deuteron EDMs and the heavy neutrino masses.

Section 6 discusses studies of lepton universality. The branching ratios $\Gamma(\pi \rightarrow \mu \nu) / \Gamma(\pi \rightarrow e v)$ and $\Gamma(K \rightarrow$ $\mu v) / \Gamma(K \rightarrow e v)$, for example, are very well known theoretically within the SM. Ongoing experiments (at PSI and TRIUMF for the pion, and at CERN and Frascati for the kaon) test the existence of flavor-dependent charged Higgs couplings, by improving the existing accuracies by factors of order 10.

In Sect. 7 we consider CP-violating charged lepton decays, which offer interesting prospects as alternative probes of BSM phenomena. SM-allowed $\tau$ decays, such as $\tau \rightarrow$ $\nu K \pi$, can be sensitive to new CP-violating effects. The decays being allowed by the SM, the CP-odd asymmetries are proportional to the interference of a SM amplitude with the BSM, CP-violating one. As a result, the small CPviolating amplitude contributes linearly to the rate, rather than quadratically, enhancing the sensitivity. In the specific case of $\tau \rightarrow \nu K \pi$, and for some models, a CP asymmetry at the level of $10^{-3}$ would correspond to $B(\tau \rightarrow \mu \gamma)$ around $10^{-8}$. Another example is the CP-odd transverse polarization of the muon, $P_{T}$, in $K \rightarrow \pi \mu \nu$ decays. The current sensitivity of the KEK experiment E246, which resulted in $P_{T}<5 \times 10^{-3}$ at $90 \%$ C.L., can be improved to the level of $10^{-4}$, by TREK proposed at J-PARC, probing models such as multi-Higgs or R-parity-violating SUSY.

Section 8 discusses experimental searches for charged LFV processes. Transitions between $e, \mu$, and $\tau$ might be found in the decay of almost any weakly decaying particle and searches have been performed in $\mu, \tau, \pi, K, B$, $D, W$ and $Z$ decay. Whereas the highest experimental sensitivities were reached in dedicated $\mu$ and $K$ experiments, $\tau$ decay starts to become competitive as well. In Sect. 8 the experimental limitations to the sensitivities for the various decay modes are discussed in some detail, in particular for $\mu$ and $\tau$ decays, and some key experiments are presented. The sensitivities reached in searches for $\mu^{+} \rightarrow e^{+} \gamma$ are limited by accidental $e^{+} \gamma$ coincidences and muon beam intensities have to be reduced now already. Searches for $\mu-e$ conversion, on the other hand, are limited by the available beam intensities, and large improvements in sensitivity may still be achieved. Similarly, in rare $\tau$ decays some decay modes are already background limited at the present $B$-factories and future sensitivities may not scale with the accumulated luminosities. Prospects of LFV decays at the LHC are limited to final states with charged leptons, such as $\tau \rightarrow 3 \mu$ and $B_{d, s}^{0} \rightarrow e^{ \pm} \mu^{\mp}$, which are discussed in detail. This section finishes with the preliminary results of a feasibility study for in-flight $\mu \rightarrow \tau$ conversions using a wide beam of high momentum muons. No working scheme emerged yet. 
Section 9 covers electric and magnetic dipole moments. The muon magnetic moment has been much discussed recently, so we limit ourselves to a short review of the theoretical background and of the current and foreseeable experimental developments. In the case of EDMs, we provide an extensive description of the various theoretical approaches and experimental techniques applied to test electron and quark moments, as well as other possible sources of flavor diagonal CP-violating effects, such as the gluonic $\theta \tilde{F} F$ coupling, or CP-odd four-fermion interactions. While the experimental technique may differ considerably, the various systems provide independent and complementary information. EDMs of paramagnetic atoms such as Tl are sensitive to a combination of the fundamental electron EDM and $\mathrm{CP}$-odd four-fermion interactions between nucleons and electrons. EDMs of diamagnetic atoms such as $\mathrm{Hg}$ are sensitive, in addition, to the intrinsic EDM of quarks, as well as to a non-zero QCD $\theta$ coupling. The neutron EDM more directly probes intrinsic quark EDMs, $\theta$, and possible higher dimension CP-odd quark couplings. EDMs of the electron, without contamination from hadronic EDM contributions, can be tested with heavy diatomic molecules with unpaired electrons, such as $\mathrm{YbF}$. In case of a positive signal the combination of measurements would help to disentangle the various contributions.

The experimental situation looks particularly promising, with several new experiments about to start or under construction. For example, new ultracold-neutron setups at ILL, PSI and Oak Ridge will increase the sensitivity to a neutron EDM by more than two orders of magnitude, to a level of about $10^{-28} e \mathrm{~cm}$ in 5-10 years. This sensitivity probes e.g. CP-violating SUSY phases of the order of $10^{-4}$ or smaller. Similar improvements are expected for the electron EDM. One of the main new ideas developed in the course of the workshop is the use of a storage ring to measure the deuteron EDM. The technical issues related to the design and construction of such an experiment, which could have a statistical sensitivity of about $10^{-29} e \mathrm{~cm}$, are discussed here in some detail.

All the results presented in this document prove the great potential of this area of particle physics to shed light on one of the main puzzles of the standard model, namely the origin and properties of flavor. Low energy experiments are sensitive to scales of new physics that in several cases extend beyond several TeV. The similarity with the scales directly accessible at the LHC supports the expectation of an important synergy with the LHC collider programme, a synergy that clearly extends to future studies of the neutrino and quark sectors. The room for improvement, shown by the projections suggested by the proposed experiments, finally underscores the importance of keeping these lines of research at the forefront of the experimental high energy physics programme, providing the appropriate infrastructure, support and funding.

\section{Theoretical framework and flavor symmetries}

\subsection{The flavor puzzle}

The presence of three fermion families with identical gauge quantum numbers is a puzzle. The very origin of this replication of families constitutes the first element of the SM flavor puzzle. The second element has to do with the Yukawa interactions of those three families of fermions. While the gauge principle allows us to determine all SM gauge interactions in terms of three gauge couplings only (once the SM gauge group and the matter gauge quantum numbers have been specified), we do not have clear evidence of a guiding principle underlying the form of the $3 \times 3$ matrices describing the SM Yukawa interactions. Finally, a third element of the puzzle is represented by the peculiar pattern of fermion masses and mixing originating from those couplings.

The replication of SM fermion families can be rephrased in terms of the symmetries of the gauge part of the SM Lagrangian. The latter is in fact symmetric under a $U(3)^{5}$ symmetry acting on the family indexes of each of the five inequivalent SM representations forming a single SM family $\left(q, u^{c}, d^{c}, l, e^{c}\right.$ in Weyl notation). In other words, the gauge couplings and interactions do not depend on the (canonical) basis we choose in the flavor space of each of the five sets of fields $q_{i}, u_{i}^{c}, d_{i}^{c}, l, e_{i}^{c}, i=1,2,3$.

This $U(3)^{5}$ symmetry is explicitly broken in the Yukawa sector by the fermion Yukawa matrices. It is because of this breaking that the degeneracy of the three families is broken and the fields corresponding to the physical mass eigenstates, as well as their mixing, are defined. An additional source of breaking is provided by neutrino masses. The smallness of neutrino masses is presumably due to the breaking of the accidental lepton symmetry of the SM at a scale much larger than the electroweak, in which case neutrino masses and mixing can be accounted for in the SM effective Lagrangian in terms of a dimension five operator breaking the $U(3)^{5}$ symmetry in the lepton doublet sector.

As mentioned, the special pattern of masses and mixing originating from the $U(3)^{5}$ breaking is an important element of the flavor puzzle. This pattern is quite peculiar. It suffices to mention the smallness of neutrino masses; the hierarchy of charged fermion masses relative to that of the two heavier neutrinos; the smallness of Cabibbo-Kobayashi-Maskawa mixing in the quark sector and the two large mixing angles in Pontecorvo-Maki-Nakagawa-Sakata (PMNS) matrix in the lepton sector; the mass hierarchy in the up quark sector, more pronounced than in the down quark and charged lepton sectors; the presence of a large CP-violating phase in the quark sector and the need of additional $\mathrm{CP}$ violation to account for baryogenesis; the approximate equality of bottom and tau masses at the scale at which the gauge couplings 
unify $^{1}$ and the approximate factor of 3 between the strange and muon masses, both pointing at a grand unified picture at high energy.

The origin of family replication and of the peculiar pattern of fermion masses and mixing are among the most interesting open questions in the SM, which a theory of flavor, discussed in Sect. 2, should address. As seen in Sect. 3, experiment is ahead of theory in this field. All the physical parameters describing the SM flavor structure in the quark sector have been measured with good accuracy. In the lepton sector crucial information on lepton mixing and neutrino masses is being gathered and a rich experimental program is under way to complete the picture.

Several tools are used to attack the flavor problem. Grand unified theories allow one to relate quark and lepton masses at the GUT scale and provide an appealing framework to study neutrino masses, leptogenesis, flavor models, etc. Note that in a grand unified context the $U(3)^{5}$ symmetry of the gauge sector is reduced (to $U(3)$ in the case in which all fermions in a family are unified in a single representation, as in $S O(10))$. Extra dimensions introduce new ways to account for the hierarchy of charged fermion masses (and in some cases for the smallness of neutrino masses) through the mechanism of localization in extra dimensions and by providing a new framework for the study of flavor symmetries. The concept of minimal flavor violation may also provide a framework for addressing flavor. The impact of those organizing principles on flavor physics is discussed in detail in Sect. 4.

From experimental point of view, however, additional handles are needed to gain more insight in the origin of flavor. Essentially this requires a discovery of new physics beyond the SM. New physics at the TeV scale may in fact be associated with an additional flavor structure, whose origin might well be related to the origin of the Yukawa couplings. Some of the present attempts to understand the pattern of fermion masses and mixing do link the flavor structure of the SM and that of the new physics sectors. In which case the search for indirect effects at low energy and for direct effects at colliders may play a primary role in clarifying our understanding of flavor. And conversely, the attempts to understand the pattern of fermion masses and mixing might lead to the prediction of new flavor physics effects. Those issues are addressed in Sect. 5.

Finally, lepton flavor physics is not just related to the lepton flavor violation or $\mathrm{CP}$ violation in the lepton sector but also to understanding the unitarity and universality in the lepton sector. Possible deviations from those are discussed in Sect. 5.6.

\footnotetext{
${ }^{1}$ Needless to say, precise unification requires an extension of the SM, with supersymmetry doing best from this point of view.
}

\subsection{Flavor symmetries}

The SM Lagrangian is $U(3)^{5}$ invariant in the limit in which the Yukawa couplings vanish. This might suggest that the Yukawa couplings, or at least some of them, arise from the spontaneous breaking of a subgroup of $U(3)^{5}$. Needless to say, the use of (spontaneously broken) symmetries as organizing principles to understand physical phenomena has been largely demonstrated in the past (chiral symmetry breaking, electroweak, etc.). In the following, we discuss the possibility of using such an approach to address the origin of the pattern of fermion masses and mixing, the constraints on the flavor structure of new physics, and to put forward expectations for flavor observables.

The spontaneously broken "flavour" or "family" symmetry can be local or global. Many (most) of the consequences of flavor symmetries are independent of this. The flavor breaking scale must be sufficiently high in such a way to suppress potentially dangerous effects associated with the new fields and interactions, in particular with the new gauge interactions (in the local case) or the unavoidable pseudoGoldstone bosons (in the global case). In the context of an analysis in terms of effective operators of higher dimensions, a generic bound of about $10^{3} \mathrm{TeV}$ on the flavor scale from flavor changing neutral currents (FCNC) processes would be obtained. Nevertheless, a certain evidence for $b-\tau$ unification and the appeal of the see-saw mechanism for neutrino masses seem to suggest that these Yukawa couplings are already present near the GUT scale. This is indeed what most flavor models assume, and we shall also assume in the following.

The SM matter fields belong to specific representations of the flavor group, such that in the unbroken limit the Yukawa couplings have a particularly simple form. Typically some or all Yukawa couplings (with the possible exception of third generation ones) are not allowed. The spontaneous symmetry breaking of the flavor symmetry is provided by the vacuum expectation value (VEV) of fields often called "flavons". As the breaking presumably arises at a scale much higher than the electroweak scale, such flavons are SM singlets (or contain a SM singlet in the case of SM extensions) and typically they are only charged under the flavor symmetry. Flavor breaking is communicated dynamically to the SM fields by some interactions (possibly renormalizable, often not specified) living at a scale $\Lambda_{f}$ not smaller than the scale of the flavor symmetry breaking. A typical example for these interactions that communicate the breaking is the exchange of heavy fermions whose mass terms respect the flavor symmetry. In that case the scale $\Lambda_{f}$ would correspond to this fermion mass $M_{f}$. Many consequences of the flavor symmetry are actually independent of the mediation mechanism. It is therefore useful to consider an effective field theory approach below the scale $\Lambda_{f}$ in which the 
Table 2 Transformation of the matter superfields under the family symmetries. The $i$ th generation SM fermion fields are grouped into the representation $\overline{5}_{i}=\left(D^{c}, L\right)_{i}, 10_{i}=\left(Q, U^{c}, E^{c}\right)_{i}, 1_{i}=\left(N^{c}\right)_{i}$

\begin{tabular}{|c|c|c|c|c|c|c|c|c|c|c|}
\hline Field & $10_{3}$ & $10_{2}$ & $10_{1}$ & $\overline{5}_{3}$ & $\overline{5}_{2}$ & $\overline{5}_{1}$ & $1_{3}$ & $1_{2}$ & $1_{1}$ & $\theta$ \\
\hline$U(1)$ & 0 & 2 & 3 & 0 & 0 & 1 & $n_{3}^{c}$ & $n_{2}^{c}$ & $n_{1}^{c}$ & -1 \\
\hline
\end{tabular}

flavor messengers have been integrated out. Once the flavon fields have acquired their VEVs, the structure of the Yukawa matrices (and other flavor parameters) can be obtained from an expansion in non-renormalizable operators involving the flavon fields and respecting the different symmetries (flavor and other symmetries) of the theory.

There are several possibilities for the flavor symmetry, local, global, accidental, continuous or discrete, Abelian or non-Abelian. Many examples are available in the literature for each of those possibilities. Some of them will be discussed in next subsections in relation to the implications considered in this study.

\subsubsection{Continuous flavor symmetries}

In order to provide an explicit example, we shortly discuss here one of the simplest possibilities, which goes back to the pioneering work of Froggatt-Nielsen [5]. In this model we have a $U(1)$ flavor symmetry under which the three generation of SM fields have different charges. In the simplest version we assign positive integer charges to the SM fermionic fields, the Higgs field is neutral, and we have a single flavon field $\theta$ of charge -1 . The VEV of the flavon field is somewhat smaller than the mass of the heavy mediator fields $M_{f}$, so that the ratio $\epsilon=v / M_{f} \ll 1$. In this way the various entries in the Yukawa matrices are determined by epsilon to the power of the sum of the fermion charges with an undetermined order 1 coefficient. This mechanism explains nicely the hierarchy of fermion masses and mixing angles.

This idea is the basis for most flavor symmetries. It can be implemented in a great variety of different models. For the sake of definiteness, we show here how it works using as a concrete example a supersymmetric GUT model. Its superpotential is of the form

$$
\begin{aligned}
W_{\text {Yukawa }}= & c_{i j}^{d} \epsilon^{q_{i}+d_{j}^{c}} Q_{i} D_{j}^{c} H_{1}+c_{i j}^{u} \epsilon^{q_{i}+u_{j}^{c}} Q_{i} U_{j}^{c} H_{2} \\
& +c_{i j}^{e} \epsilon^{l_{i}+e_{j}^{c}} L_{i} E_{j}^{c} H_{1}+c_{i j}^{v} \epsilon^{l_{i}+l_{j}} L_{i} L_{j} \frac{H_{2} H_{2}}{\bar{M}},
\end{aligned}
$$

where the $c$ 's are $O(1)$ coefficients and $\bar{M}$ is the scale associated to $B-L$ breaking. The last term in this equation is an effective operator, giving Majorana masses to neutrinos, which can be generated, e.g., through a see-saw mechanism. Notice that the power of $\epsilon$ in each Yukawa coupling is proportional to the sum of the fermion charges: $Y_{i j}^{u}=c_{i j}^{u} \epsilon^{q_{i}+u_{j}^{c}}$,
$Y_{i j}^{d}=c_{i j}^{d} \epsilon^{q_{i}+d_{j}^{c}}$, etc. Hence, this mechanism explains the hierarchy of fermion masses and mixing angles through a convenient choice of charges. The value of these charges and the expansion parameter $\epsilon$ are constrained by the observed masses and angles. A convenient set of charges for example is given in Table 2. It turns out that this set of charges is the only one compatible with minimal $S U(5)$ unification. By introducing three right handed neutrinos with positive charges it is also possible to successfully realize the see-saw mechanism.

These charges give rise to the following Dirac Yukawa couplings for charged fermions at the GUT scale

$Y_{u}=\left(\begin{array}{ccc}\epsilon^{6} & \epsilon^{5} & \epsilon^{3} \\ \epsilon^{5} & \epsilon^{4} & \epsilon^{2} \\ \epsilon^{3} & \epsilon^{2} & 1\end{array}\right), \quad\left(\begin{array}{ccc}\epsilon^{4} & \epsilon^{3} & \epsilon^{3} \\ \epsilon^{3} & \epsilon^{2} & \epsilon^{2} \\ \epsilon & 1 & 1\end{array}\right)$

where $O(1)$ coefficients in each entry are understood here and in the following. With $\epsilon=O\left(\lambda_{c}\right)$ (the Cabibbo angle), the observed features of charged fermion masses and mixing are qualitatively well reproduced. It is known that the high energy relation $Y_{e}^{T}=Y_{d}$ is not satisfactory for the lighter families and should be relaxed by means of some mechanism [6-8]. The Dirac neutrino Yukawa couplings and the Majorana mass matrix of right handed neutrinos are

$Y_{\nu}=\left(\begin{array}{ccc}\epsilon^{n_{1}^{c}+1} & \epsilon^{n_{2}^{c}+1} & \epsilon^{n_{3}^{c}+1} \\ \epsilon^{n_{1}^{c}} & \epsilon^{n_{2}^{c}} & \epsilon^{n_{3}^{c}} \\ \epsilon^{n_{1}^{c}} & \epsilon^{n_{2}^{c}} & \epsilon^{n_{3}^{c}}\end{array}\right)$,
$M_{R}=\left(\begin{array}{ccc}\epsilon^{2 n_{1}^{c}} & \epsilon^{n_{1}^{c}+n_{2}^{c}} & \epsilon_{1}^{n_{1}^{c}+n_{3}^{c}} \\ \epsilon_{1}^{n_{1}^{c}+n_{2}^{c}} & \epsilon^{2 n_{2}^{c}} & \epsilon_{2}^{n_{2}^{c}+n_{3}^{c}} \\ \epsilon^{n_{1}^{c}+n_{3}^{c}} & \epsilon^{n_{2}^{c}+n_{3}^{c}} & \epsilon^{2 n_{3}^{c}}\end{array}\right) \bar{M}$.

Applying the see-saw mechanism to obtain the effective light neutrino mass matrix $M_{v}$ in the basis of diagonal charged lepton Yukawa couplings, ${ }^{2}$ it is well known $[9,10]$ that if all right handed neutrino masses are positive the dependence on the right handed charges disappears:

$$
U_{\mathrm{PMNS}}^{*} m_{\nu}^{\operatorname{diag}} U_{\mathrm{PMNS}}^{\dagger}=m_{v}=\left(\begin{array}{ccc}
\epsilon^{2} & \epsilon & \epsilon \\
\epsilon & 1 & 1 \\
\epsilon & 1 & 1
\end{array}\right) \frac{v_{2}^{2}}{\bar{M}} .
$$

\footnotetext{
${ }^{2}$ Notice that going to the basis of diagonal charged leptons will only change the $O(1)$ coefficients, but not the power in $\epsilon$ of the different entries.
} 
Experiments require $\bar{M} \sim 5 \times 10^{14} \mathrm{GeV}$. The features of neutrino masses and mixing are quite satisfactorily reproduced-the weak point being the tuning in the 23determinant $[9,10]$ that has to be imposed. For later application, it is useful to introduce the unitary matrices which diagonalize $Y_{v}$ in the basis where both $Y_{e}$ and $M_{R}$ are diagonal: $V_{L} Y_{v} V_{R}=Y_{v}^{\mathrm{diag}} \approx \operatorname{diag}\left(\epsilon^{n_{1}^{c}}, \epsilon^{n_{2}^{c}}, \epsilon^{n_{3}^{c}}\right)$. Notice that, as a consequence of the equal charges of the lepton doublets $L_{2}$ and $L_{3}$, the model predicts that $V_{L}$ has a large mixing, although not necessarily maximal, in the $2-3$ sector as observed in $U_{\text {PMNS }}$.

The literature is very rich of models based on flavor symmetries. Some references are [5, 9-40]; for more recent attempts the interested reader is referred for instance to [41-64].

\subsubsection{Discrete flavor symmetries}

2.2.2.1 Finite groups Discrete flavor symmetries have gained popularity because they seem to be appropriate to address the large mixing angles observed in neutrino oscillations. To obtain a non-Abelian discrete symmetry, a simple heuristic way is to choose two specific non-commuting matrices and form all possible products. As a first example, consider the two $2 \times 2$ matrices

$A=\left(\begin{array}{ll}0 & 1 \\ 1 & 0\end{array}\right), \quad B=\left(\begin{array}{cc}\omega & 0 \\ 0 & \omega^{-1}\end{array}\right)$,

where $\omega^{n}=1$, i.e. $\omega=\exp (2 \pi i / n)$. Since $A^{2}=1$ and $B^{n}=1$, this group contains $Z_{2}$ and $Z_{n}$. For $n=1,2$, we obtain $Z_{2}$ and $Z_{2} \times Z_{2}$ respectively, which are Abelian. For $n=3$, the group generated has six elements and is in fact the smallest non-Abelian finite group $S_{3}$, the permutation group of three objects. This particular representation is not the one found in text books, but it is related to it by a unitary transformation [65], and was first used in 1990 for a model of quark mass matrices [66, 67]. For $n=4$, the group generated has eight elements which are in fact $\pm 1, \pm i \sigma_{1,2,3}$, where $\sigma_{1,2,3}$ are the usual Pauli spin matrices. This is the group of quaternions $Q$, which has also been used [68] for quark and lepton mass matrices. In general, the groups generated by (2.5) have $2 n$ elements and may be denoted as $\Delta(2 n)$.

Consider next the two $3 \times 3$ matrices:

$$
A=\left(\begin{array}{ccc}
0 & 1 & 0 \\
0 & 0 & 1 \\
1 & 0 & 0
\end{array}\right), \quad B=\left(\begin{array}{ccc}
\omega & 0 & 0 \\
0 & \omega^{2} & 0 \\
0 & 0 & \omega^{-3}
\end{array}\right)
$$

Since $A^{3}=1$ and $B^{n}=1$, this group contains $Z_{3}$ and $Z_{n}$. For $n=1$, we obtain $Z_{3}$. For $n=2$, the group generated has 12 elements and is $A_{4}$, the even permutation group of 4 objects, which was first used in 2001 in a model of lepton mass matrices [36, 41]. It is also the symmetry group of the tetrahedron, one of five perfect geometric solids, identified by Plato with the element "fire" [69]. In general, the groups generated by (2.6) have $3 n^{2}$ elements and may be denoted as $\Delta\left(3 n^{2}\right)$ [70]. They are in fact subgroups of $S U(3)$. In particular, $\Delta(27)$ has also been used [57, 71]. Generalizing to $k \times k$ matrices, we then have the series $\Delta\left(k n^{k-1}\right)$. However, since there are presumably only three families, $k>3$ is probably not of much interest.

Going back to $k=2$, but using instead the following two matrices:

$A=\left(\begin{array}{cc}0 & 1 \\ 1 & 0\end{array}\right), \quad B=\left(\begin{array}{cc}\omega & 0 \\ 0 & 1\end{array}\right)$.

Now again $A^{2}=1$ and $B^{n}=1$, but the group generated will have $2 n^{2}$ elements. Call it $\Sigma\left(2 n^{2}\right)$. For $n=1$, it is just $Z_{2}$. For $n=2$, it is $D_{4}$, i.e. the symmetry group of the square, which was first used in 2003 [47, 72]. For $k=3$, consider

$$
A=\left(\begin{array}{ccc}
0 & 1 & 0 \\
0 & 0 & 1 \\
1 & 0 & 0
\end{array}\right), \quad B=\left(\begin{array}{ccc}
\omega & 0 & 0 \\
0 & 1 & 0 \\
0 & 0 & 1
\end{array}\right),
$$

then the groups generated have $3 n^{3}$ elements and may be denoted as $\Sigma\left(3 n^{3}\right)$. They are in fact subgroups of $U(3)$. For $n=1$, it is just $Z_{3}$. For $n=2$, it is $A_{4} \times Z_{2}$. For $n=3$, the group $\Sigma(81)$ has been used [73] to understand the Koide formula [74] as well as lepton mass matrices [75]. In general, we have the series $\Sigma\left(k n^{k}\right)$.

\subsubsection{Model recipe}

1. Choose a group, e.g. $S_{3}$ or $A_{4}$, and write down its possible representations. For example $S_{3}$ has $\underline{1}, \underline{1^{\prime}}, \underline{2} ; A_{4}$ has $\underline{1}$, $\underline{1}^{\prime}, \underline{1}^{\prime \prime}, \underline{3}$. Work out all product decompositions. For example $\underline{2} \times \underline{2}=\underline{1}+\underline{1}^{\prime}+\underline{2}$ in $S_{3}$, and $\underline{3} \times \underline{3}=\underline{1}+\underline{1}^{\prime}+\underline{1}^{\prime \prime}+\underline{3}+\underline{3}$ in $A_{4}$.

2. Assign $(v, l)_{1,2,3}$ and $l_{1,2,3}^{c}$ to the representations of choice. To have only renormalizable interactions, it is necessary to add Higgs doublets (and perhaps also triplets and singlets) and, if so desired, neutrino singlets.

3. The Yukawa structure of the model is restricted by the choice of particle content and their representations. As the Higgs bosons acquire vacuum expectation values (which may be related by some extra or residual symmetry), the lepton mass matrices will have certain particular forms, consistent with the known values of $m_{e}, m_{\mu}, m_{\tau}$, etc. If the number of parameters involved is less than the number of observables, there will be one or more predictions.

4. In models with more than one Higgs doublet, flavor nonconservation will appear at some level. Its phenomenological consequences need to be worked out, to ensure 
the consistency with present experimental constraints. The implications for phenomena at the $\mathrm{TeV}$ scale can then be explored.

5. Insisting on using only the single SM Higgs doublet requires effective non-renormalizable interactions to support the discrete flavor symmetry. In such models, there are no predictions beyond the forms of the mass matrices themselves.

6. Quarks can be considered in the same way. The two quark mass matrices $m_{u}$ and $m_{d}$ must be nearly aligned so that their mixing matrix involves only small angles. In contrast, the mass matrices $m_{v}$ and $m_{e}$ should have different structures so that large angles can be obtained.

Some explicit examples will now be outlined.

2.2.2.3 $\mathrm{S}_{3}$ Being the simplest, the non-Abelian discrete symmetry $S_{3}$ was used already [76] in the early days of strong interactions. There are many recent applications [55, 77-86], some of which are discussed in [87]. Typically, such models often require extra symmetries beyond $S_{3}$ to reduce the number of parameters, or assumptions of how $S_{3}$ is spontaneously and softly broken. For illustration, consider the model of Kubo et al. [77] which has recently been updated by Felix et al. [88]. The symmetry used is actually $S_{3} \times Z_{2}$, with the assignments

$(v, l), l^{c}, N,\left(\phi^{+}, \phi^{0}\right) \sim \underline{1}+\underline{2}$,

and equal vacuum expectation values for the two Higgs doublets transforming as $\underline{2}$ under $S_{3}$. The $Z_{2}$ symmetry serves to eliminate four Yukawa couplings, otherwise allowed by $S_{3}$, resulting in an inverted ordering of neutrino masses with

$\theta_{23} \simeq \pi / 4, \quad \theta_{13} \simeq 0.0034, \quad m_{e e} \simeq 0.05 \mathrm{eV}$,

where $m_{e e}$ is the effective Majorana neutrino mass measured in neutrinoless double beta decay. This model relates $\theta_{13}$ to the ratio $m_{e} / m_{\mu}$.

2.2.2.4 $A_{4}$ To understand why quarks and leptons have very different mixing matrices, $A_{4}$ turns out to be very useful. It allows the two different quark mass matrices to be diagonalized by the same unitary transformations, implying thus no mixing as a first approximation, but because of the assumed Majorana nature of the neutrinos, a large mismatch may occur in the lepton sector, thus offering the possibility of obtaining the so-called tri-bi-maximal mixing matrix $[89,90]$, which is a good approximation to the present data. One way of doing this is to consider the decomposition

$U_{\mathrm{PMNS}}=\left(\begin{array}{ccc}\sqrt{2 / 3} & 1 / \sqrt{3} & 0 \\ -1 / \sqrt{6} & 1 / \sqrt{3} & -1 / \sqrt{2} \\ -1 / \sqrt{6} & 1 / \sqrt{3} & 1 / \sqrt{2}\end{array}\right)$

$$
=\frac{1}{\sqrt{3}}\left(\begin{array}{ccc}
1 & 1 & 1 \\
1 & \omega & \omega^{2} \\
1 & \omega^{2} & \omega
\end{array}\right)\left(\begin{array}{ccc}
0 & 1 & 0 \\
1 / \sqrt{2} & 0 & -i / \sqrt{2} \\
1 / \sqrt{2} & 0 & i / \sqrt{2}
\end{array}\right),
$$

where $U_{\mathrm{PMNS}}$ is the observed neutrino mixing matrix and $\omega=\exp (2 \pi i / 3)=-1 / 2+i \sqrt{3} / 2$. The matrix involving $\omega$ has equal moduli for all its entries and was conjectured already in $1978[91,92]$ to be a possible candidate for the $3 \times 3$ neutrino mixing matrix.

Since $U_{\mathrm{PMNS}}=V_{e}^{\dagger} V_{v}$, where $V_{e}, V_{v}$ diagonalize the matrices $m_{e} m_{e}^{\dagger}, m_{v} m_{v}^{\dagger}$ respectively, (2.11) may be obtained if we have

$V_{e}^{\dagger}=\frac{1}{\sqrt{3}}\left(\begin{array}{ccc}1 & 1 & 1 \\ 1 & \omega & \omega^{2} \\ 1 & \omega^{2} & \omega\end{array}\right)$

and

$$
\begin{aligned}
m_{v}= & \left(\begin{array}{ccc}
a+2 b & 0 & 0 \\
0 & a-b & d \\
0 & d & a-b
\end{array}\right) \\
= & \left(\begin{array}{ccc}
0 & 1 & 0 \\
1 / \sqrt{2} & 0 & -i / \sqrt{2} \\
1 / \sqrt{2} & 0 & i / \sqrt{2}
\end{array}\right) \\
& \times\left(\begin{array}{ccc}
a-b+d & 0 \\
0 & a+2 b & 0 \\
0 & 0 & -a+b+d
\end{array}\right) \\
& \times\left(\begin{array}{ccc}
0 & 1 / \sqrt{2} & 1 / \sqrt{2} \\
1 & 0 & 0 \\
0 & -i / \sqrt{2} & i / \sqrt{2}
\end{array}\right) .
\end{aligned}
$$

It was discovered in Ref. [36] that (2.12) is naturally obtained with $\mathrm{A}_{4}$ if

$$
\begin{aligned}
& (v, l)_{1,2,3} \sim \underline{3}, \quad l_{1,2,3}^{c} \sim \underline{1}+\underline{1}^{\prime}+\underline{1}^{\prime \prime}, \\
& \left(\phi^{+}, \phi^{0}\right)_{1,2,3} \sim \underline{3}
\end{aligned}
$$

for $\left\langle\phi_{1}^{0}\right\rangle=\left\langle\phi_{2}^{0}\right\rangle=\left\langle\phi_{3}^{0}\right\rangle$. This assignment also allows $m_{e}$, $m_{\mu}, m_{\tau}$ to take on arbitrary values, because there are here exactly three independent Yukawa couplings invariant un$\operatorname{der} A_{4}$. If we use this also for quarks [41], then $V_{u}^{\dagger}$ and $V_{d}^{\dagger}$ are also given by (2.12), resulting in $U_{\mathrm{CKM}}=1$, i.e. no mixing. This should be considered as a good first approximation because the observed mixing angles are all small. In the general case without any symmetry, we would have expected $V_{u}$ and $V_{d}$ to be very different.

It was later discovered in Ref. [93] that (2.13) may also be obtained with $A_{4}$, using two further assumptions. Consider 
the most general $3 \times 3$ Majorana mass matrix in the form

$m_{\nu}=\left(\begin{array}{ccc}a+b+c & f & e \\ f & a+b \omega+c \omega^{2} & d \\ e & d & a+b \omega^{2}+c \omega\end{array}\right)$,

where $a$ comes from $\underline{1}, b$ from $\underline{1}^{\prime}, c$ from $\underline{1}^{\prime \prime}$, and $(d, e, f)$ from $\underline{3}$ of $A_{4}$. To get (2.13), we need $e=f=0$, i.e. the effective scalar $A_{4}$ triplet responsible for neutrino masses should have its vacuum expectation value along the $(1,0,0)$ direction, whereas that responsible for charged lepton masses should be $(1,1,1)$ as remarked earlier. This misalignment is a technical challenge to all such models [50, 94-104]. The other requirement is that $b=c$. Since they come from different representations of $A_{4}$, this is rather ad hoc. A very clever solution $[50,94]$ is to eliminate both, i.e. $b=c=0$. This results in a normal ordering of neutrino masses with the prediction [96]

$\left|m_{v_{e}}\right|^{2} \simeq\left|m_{e e}\right|^{2}+\Delta m_{\mathrm{atm}}^{2} / 9$.

Other applications [60, 105-120] of $A_{4}$ have also been considered. A natural (spinorial) extension of $A_{4}$ is the binary tetrahedral group $[30,34]$ which is under active current discussion $[64,121-123]$.

Other recent applications of non-Abelian discrete flavor symmetries include those of $D_{4}$ [47, 72, 124], $Q_{4}$ [68], $D_{5}$ [125, 126], $D_{6}$ [127], $Q_{6}$ [128-130], $D_{7}$ [131], $S_{4}[61,132-$ 135], $\Delta(27)$ [57, 71], $\Delta(75)[15,136], \Sigma(81)[73,75]$, and $B_{3} \times Z_{2}^{3}[137,138]$ which has 384 elements.

\subsubsection{Accidental flavor symmetries}

While flavor symmetries certainly represent one of the leading approaches to understanding the pattern of fermion masses and mixing, it was recently found that the hierarchical structure of charged fermion masses and many other peculiar features of the fermion spectrum in the SM (neutrinos included) do not require a flavor symmetry to be understood, nor any other special "horizontal" dynamics involving the family indices of the SM fermions [63, 139]. Surprisingly enough, those features can in fact be recovered in a model in which the couplings of the three SM families not only are not governed by any symmetry, but are essentially anarchical (uncorrelated $\mathcal{O}(1)$ numbers) at a very high scale.

The idea is based on the hypothesis that the SM Yukawa couplings all arise from the exchange of heavy degrees of freedom (messengers) at a scale not far from the unification scale. Examples of diagrams contributing to the up and down quark Yukawa matrices are shown below, where $\phi$ is a SM singlet field getting a VEV. As discussed in Sects. 2.2 and 2.2.1, the same exchange mechanism is often assumed
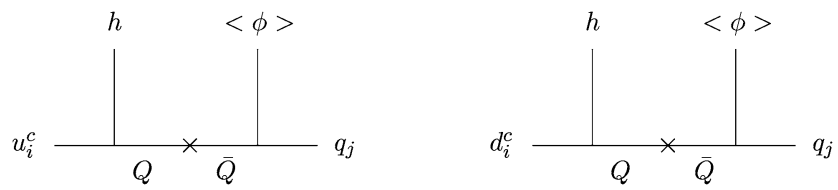

Fig. 1 Contributions to the up- and down-type quark Yukawa mass matrices, from the exchange of heavy messengers

to be at work in models with flavor symmetries. Here, however, the couplings of the heavy messengers to the SM fields are not constrained by any symmetry. ${ }^{3}$ An hierarchy among Yukawa couplings still arises because a single set of left handed messenger fields (heavy quark doublets $Q+\bar{Q}$ in the quark sector and heavy lepton doublets $L+\bar{L}$ in the lepton sector) dominates the exchange at the heavy scale. For example, the diagrams below represents the dominant contribution to the quark Yukawa matrices. As only one field is exchanged, the Yukawa matrices have rank one. Therefore, whatever are the $\mathcal{O}(1)$ couplings in the diagram, the top and bottom Yukawa couplings are generated (at the $\mathcal{O}(1)$ level, giving large $\tan \beta$ ), but the first two families' ones are not, which is a good starting point to obtain a hierarchy of quark masses. This mechanism is similar to a the single right handed neutrino dominance mechanism, used in neutrino model building to obtain a hierarchical spectrum of light neutrinos [140-143]. Note that the diagonalization of the quark Yukawa matrices involves large rotations, as all the couplings are supposed to be $\mathcal{O}(1)$. However, the rotations of the up and down left handed quarks turn out to be the same (because they have same couplings to the left handed doublet messenger). Therefore, the two rotations cancel when combined in the CKM matrix, which ends up vanishing at this level.

The Yukawa couplings of the second family, and a nonvanishing $V_{c b}$ angle, are generated by the subdominant exchange of heavier right handed messengers $D^{c}, U^{c}, E^{c}$, $N^{c}$. Altogether, the messengers form a heavy (vector-like) replica of a SM family, with the left handed fields lighter than the right handed ones. The (inter-family) hierarchy between the masses of the second and the third SM family masses arises from the (intra-family) hierarchy between left and right handed fields in the single family of messengers. In turn, in a Pati-Salam or $S O(10)$ unified model, the hierarchy between right handed and left handed fields can be easily obtained by giving mass to the messengers through a breaking of the gauge group along the $T_{3 R}$ direction. This way, the hierarchy among different families is explained in terms of the breaking of a gauge group acting on single families, with no need of flavor symmetries or other dynamics acting on the family indexes of the SM fermions.

\footnotetext{
${ }^{3}$ A discrete $\mathbf{Z}_{2}$ symmetry, under which all the three SM families (and the field $\phi$ ) are odd, is used for the sole purpose of distinguishing the light SM fields from the heavy messengers.
} 
It is also possible to describe the mechanism outlined above in terms of accidental flavor symmetries. In the effective theory below the scale of the right handed messengers, in fact, the Yukawa couplings of the two lighter families are "protected" by an accidental $U(2)$ symmetry. One can also consider the effective theory below the cut-off of the model, which is supposed to lie one or two orders of magnitude above the mass of the right handed messengers. In the effective theory below the cut-off, the second family gets a nonvanishing Yukawa coupling, but the Yukawa of the lightest family is still "protected" by an accidental $U(1)$ symmetry.

Surprisingly enough, a number of important features of the fermion spectrum can be obtained in this simple and economical model. The relation $\left|V_{c b}\right| \sim m_{s} / m_{b}$ is a direct consequence of the principles of this approach. The stronger mass hierarchy observed in the up quark sector is accounted for without introducing a new scale (besides the left handed and right handed messenger ones) or making the up quark sector somehow different. In spite of the absence of small coefficients, the CKM mixing angles turn out to be small. At the same time, a large atmospheric mixing can be generated in a natural way in the neutrino sector, together with normal hierarchical neutrino masses. In fact, a see-saw mechanism dominated by the single right handed (messenger) neutrino $N^{c}$ is at work. The bottom and tau mass unify at the high scale, while a $B-L$ factor 3 enters the ratios of the muon and strange masses. For a detailed illustration of the model, we refer the reader to [63].

The study of FCNC and CPV effects in a supersymmetric context is still under way. Such effects might represent the distinctive signature of the model, due to the sizable radiative effects one obtains in the (23) block of the "right handed" sfermion mass matrices in both the squark and slepton sector.

\subsubsection{Flavor/CP symmetries and their violation from supersymmetry breaking}

While the vast literature on flavor symmetries covers a number of interesting aspects of the theory and phenomenology of flavor, we are interested here in a (non-exhaustive) review of only those aspects relevant to new physics. The relevance of flavor symmetries to new physics follows from the fact that SM extensions often contain new flavor dependent interactions. In the following we shall consider the case of supersymmetry, in which new flavor violating gaugino or higgsino interactions can be induced by possible new sources of $S U(5)^{5}$ breaking in the soft supersymmetry breaking terms.

While in the SM the Yukawa matrices provide the only source of flavor $\left(U(3)^{5}\right)$ breaking, the supersymmetric extensions of the SM are characterized by a potentially much richer flavor structure associated to the soft supersymmetry breaking Lagrangian. Unfortunately, a generic flavor structure leads to FCNC and CPV processes that can exceed the experimental bounds by up to two orders of magnitudethe so-called supersymmetric flavor and $\mathrm{CP}$ problem. The solution of the latter problem can lie in the supersymmetry breaking and mediation mechanism (this is the case for example of gauge mediated supersymmetry breaking) or in the constraints on the soft terms provided by flavor symmetries.

In turn, the implications of flavor symmetries on the structure of the soft terms depends on the interplay between flavor and supersymmetry breaking. Without entering the details of specific models, we can distinguish two opposite situations.

- The soft terms are flavor universal, or at least symmetric under the flavor symmetry, at the tree level, and

- flavor symmetry breaking enters the soft terms (as for the Yukawa interactions) already at the tree level, through non-renormalizable couplings to the flavon fields.

Let us consider them in greater detail.

The first possibility is that the supersymmetry breaking mechanism takes care of the FCNC and CPV problems. In the simplest case, the new sfermion masses and $A$-terms do not introduce new flavor structure at all. This is the case if

$\mathbf{m}_{i j}^{2}=m_{0}^{2} \delta_{i j}, \quad \mathbf{A}_{i j}=A_{0} \delta_{i j}$,

where $i, j$ are family indexes and the universal values $m_{0}^{2}$, $A_{0}$ can be different in the different sfermion sectors. ${ }^{4}$ The breaking of the flavor symmetry is felt at the tree level only by the Yukawa matrices. Needless to say, the tree level universality of the soft terms will be spoiled by renormalization effects associated to interactions sensitive to Yukawa couplings [144, 145]. These effects can be enhanced by large logarithms if the scale at which the soft terms and the Yukawa interactions appear in the observable sector is sufficiently high. The radiative contributions of Yukawa couplings associated with neutrino masses (or Yukawa couplings occurring in the context of grand unification) are particularly interesting in this context, because they offer new possibilities to test flavor physics by opening a window for physics at very large scales. For example, in the minimal SUSY see-saw model only the off-diagonal elements for left-slepton soft supersymmetry breaking mass terms are generated, while in supersymmetric GUTs also the right handed slepton masses get renormalization induced flavor non-diagonal contributions. In any case, all the flavor effects induced by the soft terms can be traced back to the Yukawa couplings, which remain the only source of flavor breaking.

\footnotetext{
${ }^{4}$ This is the case for example of gauge mediation. In supergravity, supersymmetry breaking can be fully flavor blind in the case of dilaton domination. In this case, we expect the diagonal elements of the soft mass matrices to be exactly universal. However, this is not always the case. Moduli domination is often encountered, in which case fields with different modular weights receive different soft masses.
} 
Such unavoidable effects of flavor breaking on the soft terms will be discussed in Sects. 5.2 and 5.3.

As we have just seen, the radiative contributions to soft masses represent an unavoidable but indirect effect of the physics at the origin of fermion masses and mixing. On the other hand, the mechanism generating the soft terms might not be blind to flavor symmetry breaking, in which case we might also expect flavor breaking to enter the soft terms in a more direct way. If this is the case, the soft term provide a new independent source of flavor violation. Such modeldependent "tree level" effects of flavor breaking on the soft terms add to the radiative effects and will be discussed in Sect. 2.2.4.1. The actual presence in the soft terms of flavor violating effects directly induced by the physics accounting for Yukawa couplings depends on the interplay of the supersymmetry breaking and the flavor generation mechanisms.

Theoretical and phenomenological [146-151] constraints on supersymmetry breaking parameters essentially force supersymmetry breaking to take place in a hidden sector with no renormalizable coupling to observable fields. ${ }^{5}$ The soft terms are therefore often characterized by the scale $\Lambda_{\text {SUSY }}$ at which supersymmetry breaking is communicated to the observable sector by some mediation mechanism. The soft terms arise in fact from non-renormalizable operators in the effective theory below $\Lambda_{\text {SUSY }}$ obtained by integrating out the supersymmetry breaking messenger fields. Analogously, in the context of a theory addressing the origin of flavor, we can define a scale $\Lambda_{f}$ at which the flavor structure arises. Let us consider for definiteness the case of flavor symmetries. The analogy with supersymmetry breaking is in this case even more pronounced. Above $\Lambda_{f}$, the theory is flavor symmetric. By this we mean that we can at least define conserved family numbers, perhaps part of a larger flavor symmetry. The family numbers are then spontaneously broken by the VEV of flavons that couple to observable fields through non-renormalizable interactions suppressed by the scale $\Lambda_{f}$.

We are now in the position to discuss the presence of "tree-level" flavor violating effects in the soft terms. A first possibility is to have $\Lambda_{f} \lesssim \Lambda_{\text {SUSY, as for in- }}$ stance in the case of gravity mediation, in which we expect $\Lambda_{f} \lesssim M_{\text {Planck }}=\Lambda_{\text {SUSY }}$. The soft breaking terms are already present below $M_{\text {Planck }}$. However, the flavor symmetry is still exact at scales larger than $\Lambda_{f}$. Therefore, the soft terms must respect the family symmetries. At the lower scale $\Lambda_{f}$ the effective Yukawa couplings are generated as functions of the flavon VEVs, $\langle\theta\rangle / \Lambda_{f}$, and analogously the soft breaking terms will also be functions of $\langle\theta\rangle / \Lambda_{f}$. In the $\Lambda_{f} \lesssim \Lambda_{\text {SUSY }}$ case, we therefore expect new "tree-level"

\footnotetext{
${ }^{5}$ The fields of the minimal supersymmetric standard model (MSSM) or its relevant extension.
}

sources of flavor breaking in the soft terms on top of the effects radiatively induced by the Yukawa couplings.

On the other hand, if $\Lambda_{\mathrm{SUSY}} \ll \Lambda_{f}$, the soft terms are not present at the scale of flavor breaking. The prototypical example in this case is gauge mediated supersymmetry breaking (GMSB) (see [152] and references therein). At $\Lambda_{f}$ the flavor interactions are integrated and supersymmetry is still unbroken. The only renormalizable remnant of the flavor physics below $\Lambda_{f}$ are the Yukawa couplings. At the scale $\Lambda_{\text {SUSY }}$ soft breaking terms feel flavor breaking only through the Yukawa couplings. Strictly speaking, there could also be non-renormalizable operators involving flavon fields suppressed by the heavier $\Lambda_{f}$. The contributions of these terms to soft masses would be proportional to $\Lambda_{\mathrm{SUSY}} / \Lambda_{f}$ and therefore negligible [152]. We are then only left with the radiatively induced effects of Yukawa couplings. The qualitative arguments above show that flavor physics can provide relevant information on the interplay between the origin of supersymmetry and flavor breaking in the observable sector.

As we just saw, the family symmetry that accounts for the structure of the Yukawa couplings also constrains the structure of sfermion masses. In the limit of exact flavor symmetry, this implies family universal, or at least diagonal, sfermion mass matrices. After the breaking of the flavor symmetry giving rise to the Yukawa couplings, we can have two cases.

- The SUSY breaking mediation mechanism takes place at a scale higher or equal to the flavor symmetry breaking scale and is usually sensitive to flavor. The flavor symmetry breaking accounts for both the structure of the Yukawa couplings and the deviations of the soft breaking terms from universality. This is the general expectation in gravity mediation of the supersymmetry breaking from the hidden sector.

- The supersymmetry breaking mediation mechanism takes place at a scale much smaller than the flavor symmetry breaking scale. In this case the flavor mediation mechanism, which is flavor-blind, guarantees the universality of the soft breaking terms. The flavor symmetry breaking generates the Yukawa couplings but flavor breaking corrections in the soft mass matrices are suppressed by the ratio of the two scales. This is the case of gauge-mediation models of supersymmetry breaking [152].

We begin discussing the first case.

2.2.4.1 "Tree level" effects of flavor symmetries in supersymmetry breaking terms After the breaking of the flavor symmetry responsible for the structure of the Yukawa couplings, we can expect to have non-universal contributions to the soft breaking terms at tree level. Under certain conditions, mainly related to the SUSY-breaking mediation mechanism, these tree-level contributions can be 
sizable and have important phenomenological effects. The main example among these models where the tree level nonuniversality in the soft breaking terms is relevant is provided by models of supergravity mediation [153-157] (for a nice introduction see the appendix in [158]).

The structure of the scalar mass matrices when SUSY breaking is mediated by supergravity interactions is determined by the Kähler potential. We are not going to discuss here the supergravity Lagrangian; we refer the interested reader to Refs. [153-156, 158]. For our purposes, we only need to know that the Kähler potential is a nonrenormalizable, real, and obviously gauge-invariant, function of the chiral superfields with dimensions of mass squared. This non-renormalizable function includes couplings with the hidden sector fields suppressed by different powers of $M_{\text {Planck }}, \phi \phi^{*}\left(1+X X^{*} / M_{\text {Planck }}^{2}+\cdots\right)$ with $\phi$ visible sector fields and $X$ hidden sector fields. This Kähler potential gives rise to SUSY breaking scalar masses once a certain field of the hidden sector gets a non-vanishing Fterm. The important point here is that these couplings with hidden sector fields that will eventually give rise to the soft masses are present in the theory at any scale below $M_{\text {Planck }}$. Below this scale, we can basically consider the hidden sector as frozen and renormalize these couplings only with visible sector interactions.

Therefore, in the following, to simplify the discussion, we concentrate only on the soft masses and treat them as couplings present at all energies below $M_{\text {Planck }}$. The structure of the soft mass matrices is easily understood in terms of the present symmetries. At high energies, our flavor symmetry is still an exact symmetry of the Lagrangian and therefore the soft breaking terms have to respect this symmetry [46]. At some stage, this symmetry is broken generating the Yukawa couplings in the superpotential. In the same way, the scalar masses will also receive new contributions after flavor symmetry breaking from the flavon field VEVs suppressed by mediator masses.

First we must notice that a mass term $\phi_{i}^{\dagger} \phi_{i}$ is clearly invariant under gauge, flavor and global symmetries and hence gives rise to a flavor diagonal contribution to the soft masses even before the family symmetry breaking. ${ }^{6}$ Then, after flavor symmetry breaking, any invariant combination of flavon fields (VEVs) with a pair of sfermion fields, $\phi_{i}^{\dagger} \phi_{j}$, can also contribute to the sfermion mass matrix and will break the universality of the soft masses.

An explicit example with a continuous Abelian $U(1)$ flavor symmetry $[5,11,13,16,19,21,44,48,54]$ was given above in Sect. 2.2.1.

\footnotetext{
${ }^{6}$ As we shall discuss in the following, these allowed contributions may be universal, the same for the different generations, as in the case of non-Abelian flavor symmetries, or they can be different for the three generations in some cases with Abelian flavor symmetries.
}

We turn now to the structure of the scalar mass matrices concentrating mainly on the slepton mass matrix $[13,14,16,43]$. In this case, even before the breaking of the flavor symmetry, we have three different fields with different charges corresponding to each of the three generations. As we have seen, diagonal scalar masses are allowed by the symmetry, but being different fields, there is no reason a priori for these diagonal masses to be the same, and in general we have

$\mathcal{L}_{m^{2}}^{\mathrm{symm}}=m_{1}^{2} \phi_{1}^{*} \phi_{1}+m_{2}^{2} \phi_{2}^{*} \phi_{2}+m_{3}^{2} \phi_{3}^{*} \phi_{3}$.

Notice, however, that this situation is very dangerous, especially in the case of squarks, given that the rotation to the basis of diagonal Yukawa couplings from (2.2) will generate too large off-diagonal entries [43]. In some cases, like dilaton domination, these allowed masses can be equal avoiding this problem. In the following we assume $m_{1}^{2}=m_{2}^{2}=$ $m_{3}^{2}=m_{0}^{2}$. However, even in this case, after the breaking of the flavor symmetry we obtain new contributions proportional to the flavon VEVs that break this universality. All we have to do is to write all possible combinations of two MSSM scalar fields $\phi_{i}$ and an arbitrary number of flavon VEVs invariant under the symmetry:

$$
\begin{aligned}
\mathcal{L}_{m^{2}}= & m_{0}^{2}\left(\phi_{1}^{*} \phi_{1}+\phi_{2}^{*} \phi_{2}+\phi_{3}^{*} \phi_{3}+\left(\frac{\langle\theta\rangle}{M_{\mathrm{fl}}}\right)^{q_{2}-q_{1}} \phi_{1}^{*} \phi_{2}\right. \\
& \left.+\left(\frac{\langle\theta\rangle}{M_{\mathrm{fl}}}\right)^{q_{3}-q_{1}} \phi_{1}^{*} \phi_{3}+\left(\frac{\langle\theta\rangle}{M_{\mathrm{fl}}}\right)^{q_{3}-q_{2}} \phi_{2}^{*} \phi_{3}+\text { h.c. }\right) .
\end{aligned}
$$

Therefore, the structure of the charged slepton mass matrix we would have in this model at the scale of flavor symmetry breaking would be (suppressing $O(1)$ coefficients):

$m_{\tilde{L}}^{2} \simeq\left(\begin{array}{ccc}1 & \epsilon & \epsilon \\ \epsilon & 1 & 1 \\ \epsilon & 1 & 1\end{array}\right) m_{0}^{2}$.

This structure has serious problems with the phenomenological bounds coming from $\mu \rightarrow e \gamma$, etc. There are other $U(1)$ examples that manage to alleviate, in part, these problems [43]. However, large LFV effects are a generic problem of these models due to the required charge assignments to reproduce the observed masses and mixing angles.

These FCNC problems in the sfermion mass matrices of Abelian symmetries were one of the main reasons for the introduction of non-Abelian flavor symmetries [18, 20]. The mechanism used in non-Abelian flavor models to generate the Yukawa couplings is again a variation of the FroggattNielsen mechanism, very similar to the mechanism we have just seen for Abelian symmetries. The main difference is that in this case the left handed fermions are grouped in larger representations of the symmetry group. For instance, 
in a $S U(3)$ symmetry all three generations are unified in a triplet. In a $S O(3)$ flavor symmetry we can assign the three generations to a triplet or to three singlets. In a $U(2)$ flavor symmetry the third generation is a singlet and the two light generations are grouped in a doublet. Then we do not have to assign different charges to the various generations, but in exchange, we need several stages of symmetry breaking by different flavon fields with specially aligned VEVs.

We begin analyzing a non-Abelian $U(2)$ flavor symmetry. As stressed above, if the sfermions mass matrices are only constrained by a $U(1)$ flavor symmetry there is no reason why $m_{1}^{2}$ should be close to $m_{2}^{2}$ in (2.17). Unless an alignment mechanism between fermions and sfermions is available, the family symmetry should then suppress $\left(\tilde{m}_{1}^{2}-\tilde{m}_{2}^{2}\right) / \tilde{m}^{2}$. At the same time, in the fermion sector, the family symmetry must suppress the Yukawa coupling of the first two families, $m_{1}, m_{2} \ll m_{3}$. If the small breaking of a flavor symmetry is responsible for the smallness of $\left(\tilde{m}_{1}^{2}-\tilde{m}_{2}^{2}\right) / \tilde{m}^{2}$ on one hand and of $m_{1} / m_{3}, m_{2} / m_{3}$ on the other, the symmetric limit should correspond to $\tilde{m}_{1}^{2}=\tilde{m}_{2}^{2}$ and to $m_{1}=m_{2}=0$. Interestingly enough, the largest family symmetry compatible with $S O(10)$ unification that forces $m_{1}=m_{2}=0$ automatically also forces $\tilde{m}_{1}^{2}=\tilde{m}_{2}^{2}$. This is a $U(2)$ symmetry under which the first two families transform as a doublet and the third one, as well as the Higgs, as a singlet [16, 18, 20, 24, 26].

$\psi=\psi_{a} \oplus \psi_{3}$.

The same conclusion can be obtained by using discrete subgroups $[30,64]$. In the limit of unbroken $U(2)$, only the third generation of fermions can acquire a mass, whereas the first two generations of scalars are exactly degenerate. While the first property is not a bad approximation of the fermion spectrum, the second one is what is needed to keep FCNC and $\mathrm{CP}$-violating effects under control. This observation can actually be considered as a hint that the flavor structure of the mass matrices of the fermions and of the scalars are related to each other by a symmetry principle. The same physics responsible for the peculiar pattern of fermion masses also accounts for the structure of sfermion masses.

The rank 2 of $U(2)$ allows for a two step breaking pattern:

$$
U(2) \stackrel{\epsilon}{\rightarrow} U(1) \stackrel{\epsilon^{\prime}}{\rightarrow} 0,
$$

controlled by two small parameters $\epsilon$ and $\epsilon^{\prime}<\epsilon$, to be at the origin of the generation mass hierarchies $m_{3} \gg m_{2} \gg m_{1}$ in the fermion spectrum. Although it is natural to view $U(2)$ as a subgroup of $U(3)$, the maximal flavor group in the case of full intra-family gauge unification, $U(3)$ will be anyhow strongly broken to $U(2)$ by the large top Yukawa coupling.

A nice aspect of the $U(2)$ setting is that there is little arbitrariness in the way the symmetry breaking fields couple to the SM fermions. This is unlike what happens e.g. with the choice of fermion charges in the cases of $U(1)$ symmetries. The Yukawa interactions transform as $\left(\psi_{3} \psi_{3}\right)$, $\left(\psi_{3} \psi_{a}\right),\left(\psi_{a} \psi_{b}\right)(a, b, c, \ldots=1,2)$. Hence the only relevant $U(2)$ representations for the fermion mass matrices are $1, \phi^{a}, S^{a b}$ and $A^{a b}$, where $S$ and $A$ are symmetric and antisymmetric tensors, and the upper indices denote a $U(1)$ charge opposite to that of $\psi_{a}$. While $\phi^{a}$ and $A^{a b}$ are both necessary, models with $[20,26]$ or without [24] $S^{a b}$ are both possible.

Let us first consider the case with $S^{a b}$. At leading order, the flavons couple to SM fermions through $D=5$ operators suppressed by a flavor scale $\Lambda$. Normalizing the flavons to $\Lambda$, it is convenient to choose a basis in which $\phi^{2}=\mathcal{O}(\epsilon)$ and $\phi^{1}=0$, while $A^{12}=-A^{21}=\mathcal{O}\left(\epsilon^{\prime}\right)$. If $S$ is present, it turns out to be automatically aligned with $\phi$ [27], in such a way that in the limit $\epsilon^{\prime} \rightarrow 0$ a $U$ (1) subgroup is unbroken. More precisely, $S^{22}=\mathcal{O}(\epsilon)$ and all other components essentially vanish. We are then led to Yukawa matrices of the form

$$
\left(\begin{array}{ccc}
0 & \epsilon^{\prime} & 0 \\
-\epsilon^{\prime} & \epsilon & \epsilon \\
0 & \epsilon & 1
\end{array}\right)
$$

All non-vanishing entries have unknown coefficients of order unity, while still keeping $\lambda_{12}=-\lambda_{21}$. In the context of $S U(5)$ or $S O(10)$ unification, the mass relations $m_{\tau} \approx m_{b}$, $m_{\mu} \approx 3 m_{s}, 3 m_{e} \approx m_{d}$ are accounted for by the choice of the transformations of $A^{a b}, S^{a b}$ under the unified group. The stronger mass hierarchy in the up quark sector, a peculiar feature of the fermion spectrum, is then predicted, due to the interplay of the $U(2)$ and the unified gauge symmetry.

The texture in (2.21) leads to the predictions

$$
\left|\frac{V_{t d}}{V_{t s}}\right|=\sqrt{\frac{m_{d}}{m_{s}}}, \quad\left|\frac{V_{u b}}{V_{c b}}\right|=\sqrt{\frac{m_{u}}{m_{c}}} .
$$

While the experimental determination of $\left|V_{t d} / V_{t s}\right|$ based on one loop observables might be affected by new physics, the tree-level determination of $\left|V_{u b} / V_{c b}\right|$ is less likely to be affected and at present is significantly away from the prediction in (2.22) [29, 39]. A better agreement can be obtained by (i) relaxing the condition $\lambda_{12}=-\lambda_{21}$, (ii) allowing for small contributions to the $11,13,31$ entries in (2.21) or by (iii) allowing for asymmetric textures [39]. The latter possibility is realized in models in which the $S^{a b}$ flavon is not present [20].

While the model building degrees of freedom in the quark and charged lepton sector are limited, a virtue of the $U(2)$ symmetry, the neutrino sector is less constrained. This is due, in the see-saw context, to the several possible choices involved in the modelization of the singlet neutrino mass matrix. This is reflected for example in the possibility to get both small and large mixing angles [25, 28, 31, 34, 35]. 
In the case of an $S U(3)$ flavor symmetry, all three generations are grouped in a single triplet representation, $\psi_{i}$. In addition we have several new scalar fields (flavons) which are either triplets, $\bar{\theta}_{3}, \bar{\theta}_{23}$ and $\bar{\theta}_{2}$, or antitriplets, $\theta_{3}$ and $\theta_{23}$. $S U(3)_{\mathrm{fl}}$ is broken in two steps: the first step occurs when $\theta_{3}$ and $\bar{\theta}_{3}$ get a large VEV breaking $S U(3)$ to $S U(2)$, and defining the direction of the third generation. Subsequently a smaller VEV of $\theta_{23}$ and $\bar{\theta}_{23}$ breaks the remaining symmetry and defines the second generation direction. To reproduce the Yukawa textures the large third generation Yukawa couplings require a $\theta_{3}$ (and $\left.\bar{\theta}_{3}\right) \mathrm{VEV}$ of the order of the mediator scale, $M_{\mathrm{fl}}$, while $\theta_{23} / M_{\mathrm{fl}}$ (and $\bar{\theta}_{23} / M_{\mathrm{fl}}$ ) have small $\mathrm{VEVs}^{7}$ of order $\varepsilon$. After this breaking chain we obtain the effective Yukawa couplings at low energies through the Froggatt-Nielsen mechanism [5] integrating out heavy fields. The resulting superpotential invariant under $S U(3)$ would be

$$
\begin{aligned}
W_{Y}= & H \psi_{i} \psi_{j}^{c}\left[\theta_{3}^{i} \theta_{3}^{j}+\theta_{23}^{i} \theta_{23}^{j}+\epsilon^{i k l} \bar{\theta}_{23, k} \bar{\theta}_{3, l} \theta_{23}^{j}\left(\theta_{23} \overline{\theta_{3}}\right)\right. \\
& +\epsilon^{i j k} \bar{\theta}_{23, k}\left(\theta_{23} \overline{\theta_{3}}\right)^{2}+\epsilon^{i j k} \bar{\theta}_{3, k}\left(\theta_{23} \overline{\theta_{3}}\right)\left(\theta_{23} \overline{\theta_{23}}\right) \\
& +\cdots] .
\end{aligned}
$$

In this equation we can see that each of the $S U(3)$ indices of the external MSSM particles (triplets) are either saturated individually with an antitriplet flavon index (a "meson" in QCD notation) or in an antisymmetric couplings with other two triplet indices (a "baryon"). The presence of other singlets in the different term is due to the presence of additional global symmetries necessaries to ensure the correct hierarchy in the different Yukawa elements $[37,45,46]$. This structure is quite general for the different $S U(3)$ models we can build. Here we are not specially concerned with additional details and we refer to [37, 45, 46] for more complete examples. The Yukawa texture we obtain with this superpotential is the following:

$Y^{f}=\left(\begin{array}{ccc}0 & \alpha \varepsilon^{3} & \beta \varepsilon^{3} \\ \alpha \varepsilon^{3} & \frac{\varepsilon^{2}}{a^{2}} & \gamma \frac{\varepsilon^{2}}{a^{2}} \\ \beta \varepsilon^{3} & \gamma \frac{\varepsilon^{2}}{a^{2}} & 1\end{array}\right) a^{2}$,

with $a=\frac{\left\langle\theta_{3}\right\rangle}{M}$, and $\alpha, \beta, \gamma$ unknown coefficients of or$\operatorname{der} O(1)$.

Let us now analyze the structure of scalar soft masses. In analogy with the Abelian case, in the unbroken limit diagonal soft masses are allowed. However, the three generations belong to the same representation of the flavor symmetry and now this implies the mass is the same for the whole

\footnotetext{
${ }^{7}$ In fact, in realistic models reproducing the CKM mixing matrix, there are two different mediator scales and expansion parameters, $\varepsilon$ in the up quark and $\bar{\varepsilon}$ in the down quark sector $[37,45,46]$.
}

triplet. After the breaking of $S U(3)$ symmetry the scalar soft masses deviate from exact universality [46, 160-162]. Any invariant combination of flavon fields can also contribute to the sfermion masses, although flavor symmetry indices can be contracted with fermion fields. Including these corrections the leading contributions to the sfermion mass matrices are given by

$$
\begin{aligned}
\left(m_{\tilde{f}}^{2}\right)^{i j}= & m_{0}^{2}\left(\delta^{i j}+\frac{1}{M_{f}^{2}}\left[\theta_{3}^{i \dagger} \theta_{3}^{j}+\theta_{23}^{i \dagger} \theta_{23}^{j}\right]\right. \\
& \left.+\frac{1}{M_{f}^{4}}\left(\epsilon^{i k l} \bar{\theta}_{3, k} \bar{\theta}_{23, l}\right)^{\dagger}\left(\epsilon^{j m n} \bar{\theta}_{3, m} \bar{\theta}_{23, n}\right)\right)
\end{aligned}
$$

Notice that each term inside the parentheses is trivially neutral under the symmetry because it contains always a field together with its own complex conjugate field. However, as the flavor indices of the flavon fields are contracted with the external matter fields this gives a non-trivial contribution to the sfermion mass matrices. Therefore in this model, suppressing factors of order 1 we have,

$m_{\tilde{f}}^{2} \simeq\left(\begin{array}{ccc}1 & & \\ & 1 & \\ & & 1\end{array}\right) m_{0}^{2}+\left(\begin{array}{ccc}\varepsilon^{2} & 0 & 0 \\ 0 & \frac{\varepsilon^{2}}{a^{2}} & \frac{\varepsilon^{2}}{a^{2}} \\ 0 & \frac{\varepsilon^{2}}{a^{2}} & 1\end{array}\right) a^{2} m_{0}^{2}$

with $a=\left\langle\theta_{3}\right\rangle / M_{\mathrm{fl}}$ which is still $O(1)$. In the model [37, 45, 46], the expansion parameter for right handed down quarks and charged leptons is $\bar{\varepsilon}=0.15$. Using (2.24) and (2.26) we can obtain the slepton mass matrix in the basis of diagonal charged lepton Yukawa couplings:

$m_{\tilde{e}_{R}}^{2} \simeq\left(\begin{array}{ccc}1+\bar{\varepsilon}^{2} & -\bar{\varepsilon}^{3} & -\bar{\varepsilon}^{3} \\ -\bar{\varepsilon}^{3} & 1+\bar{\varepsilon}^{2} & \bar{\varepsilon}^{2} \\ -\bar{\varepsilon}^{3} & \bar{\varepsilon}^{2} & 1\end{array}\right) m_{0}^{2}$,

where we have used $a_{3} \simeq \mathcal{O}\left(M_{\mathrm{fl}}\right)$. Therefore that generates the order $\bar{\varepsilon}^{3}$ entry in the $(1,2)$ element. The modulo of this entry is order $3 \times 10^{-3}$ at $M_{\mathrm{GUT}}$. These estimates at $M_{\mathrm{GUT}}$ are slightly reduced through renormalization group evolution to the electroweak scale and is order $1 \times 10^{-3}$ at $M_{\mathrm{W}}$. This value implies that supersymmetric contribution to $\mu \rightarrow e \gamma$ is very big and can even exceed the present bounds for light slepton masses and large $\tan \beta$ if we are not in the cancellation region[163-165]. This makes this process perhaps the most promising one to find deviations from universality in flavor models. The presence of the $S U$ (3) flavor symmetry controls the structure of the sfermion mass matrices and the supersymmetric flavor problem can be nicely solved. However, interesting signals of the supersymmetric flavor structure can be found in the near future LFV experiments. 


\section{Observables and their parameterization}

\subsection{Effective operators and low scale observables}

In spite of the clear success of the SM in reproducing all the known phenomenology up to energies of the order of the electroweak scale, nobody would doubt the need of a more complete theory beyond it. There remain many fundamental problems such as the experimental evidence for dark matter (DM) and neutrino masses, as well as the theoretical puzzles posed by the origin of flavor, the three generations, etc., that a complete theory should address. Therefore, we can consider the SM as the low energy effective theory of some more complete model that explains all these puzzles. Furthermore, we have strong reasons (gauge hierarchy problem, unification of couplings, dark matter candidate, etc.) to expect the appearance of new physics close to the electroweak scale. Suppose that these new particles from the more complete theory are to be found at the LHC. Experiments at lower energies $E<m_{\mathrm{NP}}$ are also sensitive [166] to this new physics (NP). Indeed the exchange of new particles can induce:

- corrections to the SM observables (such as S, T and U), and

- the appearance of new observables or new $(d>4)$ operators, (e.g. the flavor violating dipole operators).

Note that both effects can be parameterized by $S U(3) \times$ $S U(2) \times U(1)$-invariant operators of mass dimension $d>4$. We refer to these non-renormalizable operators as effective operators. Any NP proposed to explain new phenomena at the LHC must satisfy the experimental constraints on the effective operators it generates.

\subsubsection{Effective Lagrangian approach: $\mathcal{L}_{\text {eff }}$}

Considering the SM as an effective theory below the scale of NP, $m_{\mathrm{NP}}$, where the heavy fields have been integrated out, we can describe the physics through an effective Lagrangian, $\mathcal{L}_{\text {eff. }}$ This effective Lagrangian contains all possible terms invariant under the SM gauge group and built with the SM fields. Besides the usual SM fields, we could introduce new light singlet fermions with renormalizable Yukawa couplings to the lepton doublets (and possibly small Majorana masses) to accommodate the observed neutrino masses. In this case we would have more operators allowed in the effective Lagrangian of the SM + extra light sterile states. On the assumption that the light sterile particles are weakly interacting, if present, and therefore not relevant to the LHC, we focus on the effective Lagrangian that can be constructed only from the known SM fields. Then, the effective Lagrangian at energies $E \ll m_{\mathrm{NP}}$ can be written as an expansion in $1 / m_{\mathrm{NP}}$ as,

$\mathcal{L}_{\text {eff }}^{\mathrm{SM}}=\mathcal{L}_{0}+\frac{1}{m_{\mathrm{NP}}} \mathcal{L}_{1}+\frac{1}{m_{\mathrm{NP}}^{2}} \mathcal{L}_{2}+\frac{1}{m_{\mathrm{NP}}^{3}} \mathcal{L}_{3}+\cdots$,

where $\mathcal{L}_{0}$ is the renormalizable SM Lagrangian containing the kinetic terms of the $U(1), S U(2)$ and $S U(3)$ gauge bosons $A_{\mu}$, the gauge interactions and kinetic terms of the SM fermions, $\{f\}$, and Higgs, and the Yukawa couplings of the Higgs and SM fermions. In order to fix the notation, we list the SM fermions as

$q_{i}=\left(\begin{array}{c}u_{L i} \\ d_{L i}\end{array}\right), \quad \ell_{i}=\left(\begin{array}{c}v_{L i} \\ e_{L i}\end{array}\right)$,

$u_{R i}, \quad d_{R i}, \quad e_{R i}$,

where $i$ is a flavor/family/generation index. Note that in the following we use always four-component Dirac spinors in the different Lagrangians. Explicit expressions, for $\mathcal{L}_{0}$ in similar notation, can be found in [167].

The different $\mathcal{L}_{n}$ are Lagrangians of dimension $d=4+n$ invariant under $S U(3) \times S U(2) \times U(1)$ and can be schematically written

$\mathcal{L}_{n}=\sum_{a} C_{a} \cdot \mathcal{O}_{a}\left(H,\{f\},\left\{A_{\mu}\right\}\right)+$ h.c.

The local operators $\mathcal{O}_{a}$ are gauge invariant combinations of SM fields of dimension $4+n$. Their coefficient, which in the full Lagrangian has mass dimension $-n$, is unknown in bottom-up effective field theory, but calculable in NP models. We write this coefficient as a dimensionless $C_{a}$ divided by the $n$th power of the mass scale of the NP mediator, $m_{\mathrm{NP}}^{n}$, which for new physics relevant at LHC energies would be $m_{\mathrm{NP}} \sim \sqrt{s_{\mathrm{LHC}}}$. We shall later normalize to $G_{\mathrm{F}}($ see $(3.21))$.

We are mainly interested in dimension five and dimension six operators. We assume that any particles created at the LHC could generate dimension six operators, and then we can neglect higher dimension operators contributing to the same physical processes. Operators of dimension 7 include the lepton number violating operator $\epsilon_{a b} \epsilon_{c d} H^{a} \ell_{[i}^{b} \sigma^{\mu v} H^{c} \ell_{j]}^{d} F_{\mu \nu}$ which gives neutrino transition moments (flavor-changing dipole moments) after electroweak symmetry breaking (EWSB). At dimension 8 are two-Higgs-four-fermion operators, which can give fourfermion operators after EWSB, with a different flavor structure from the dimension six terms. We shall not analyze these operators here, but they are studied in the context of non-standard neutrino interactions [168]. Therefore, in the following, we restrict our analysis to $\mathcal{L}_{1}$ and $\mathcal{L}_{2}$.

The unique operator allowed with the standard model fields and symmetries at dimension five is $\mathcal{O}_{\ell \ell}^{i j}=$ $\epsilon_{a b} \epsilon_{m n} H^{a} \bar{\ell}_{i}^{b} H^{m} \ell_{j}^{n}(a, b, n, m$ are $S U(2)$ indices). Thus we have,

$\mathcal{L}_{1}=\frac{1}{4} \kappa_{\nu \ell \ell}^{i j} \cdot \epsilon_{a b} \epsilon_{m n} H^{a} \bar{\ell}_{i}^{b} H^{m} \ell_{j}^{n}+$ h.c., 
where $\ell^{c}$ is the charge conjugate of the lepton doublet. After electroweak symmetry breaking, this gives rise to a Majorana mass matrix $\frac{1}{4} \kappa_{\ell \ell}^{i j}\left\langle H^{0}\right\rangle^{2}{\overline{v^{c}}}_{i} v_{j}+$ h.c. In the neutrino mass eigenstate basis, the masses are $\kappa_{\ell \ell}^{i i}\left\langle H^{0}\right\rangle^{2} / 2$. The coefficient $\kappa_{\ell \ell}^{i j}=2 Y_{k i} M_{k}^{-1} Y_{k j}$ is generated for instance after integrating out heavy right handed neutrinos of mass $M_{k}$ in a see-saw mechanism with Yukawa coupling $Y$.

$\mathcal{L}_{2}$ is constructed with dimension-six operators built out of SM fields. An exhaustive list is given in [167], including operators with Higgs, $W^{ \pm}$and $Z^{0}$ external legs. Here we list operators which give interactions among leptons and photons, and leptons and quarks. We can classify the possible operators according to the external legs as follows:

- operators with a pair of leptons and an (on-shell) photon:

$$
\begin{aligned}
& \mathcal{O}_{e B}^{i j}=\bar{\ell}_{i} \sigma^{\mu \nu} e_{R j} H B_{\mu \nu}, \\
& \mathcal{O}_{e W}^{i j}=\bar{\ell}_{i} \sigma^{\mu \nu} \tau^{I} e_{R j} H W_{\mu \nu}^{I},
\end{aligned}
$$

- four-lepton operators, with Lorenz structure $\bar{L} L \bar{L} L$, $\bar{R} R \bar{R} R$ or $\bar{L} R \bar{R} L$, singlet or triplet $S U(2)$ gauge contractions (described in the operator subscript), and all possible inequivalent flavor index combinations (see Sect. 3.1.2). The $S U(2) \times U(1)$ invariant operators, with flavor indices in the superscript, are

$$
\begin{aligned}
& \mathcal{O}_{(1) \ell \ell}^{i j k l}=\left(\bar{\ell}_{i} \gamma^{\mu} \ell_{j}\right)\left(\bar{\ell}_{k} \gamma_{\mu} \ell_{l}\right), \\
& \mathcal{O}_{(3) \ell \ell}^{i j k l}=\left(\bar{\ell}_{i} \tau^{I} \gamma^{\mu} \ell_{j}\right)\left(\bar{\ell}_{k} \tau^{I} \gamma_{\mu} \ell_{l}\right), \\
& \mathcal{O}_{e e}^{i j k l}=\left(\bar{e}_{i} \gamma^{\mu} P_{R} e_{j}\right)\left(\bar{e}_{k} \gamma_{\mu} P_{R} e_{l}\right), \\
& \mathcal{O}_{\ell e}^{i j k l}=\left(\bar{\ell}_{i} e_{j}\right)\left(\bar{e}_{k} \ell_{l}\right),
\end{aligned}
$$

- two lepton two-quark operators, with Lorentz structure $\bar{L} L \bar{L} L, \bar{R} R \bar{R} R$ or $\bar{L} R \bar{R} L$, singlet or triplet $S U(2)$ gauge contractions (described in the operator subscript), and all possible inequivalent flavor index combinations (see Sect. 3.1.2). The $S(3) \times S U(2) \times U(1)$ invariant operators, with color indices implicit and flavor indices in the subscript, are

$$
\begin{aligned}
& \mathcal{O}_{(1) \ell q}^{i j k l}=\left(\bar{\ell}_{i} \gamma^{\mu} \ell_{j}\right)\left(\bar{q}_{k} \gamma_{\mu} q_{l}\right), \\
& \mathcal{O}_{(3) \ell q}^{i j k l}=\left(\bar{\ell}_{i} \tau^{I} \gamma^{\mu} \ell_{j}\right)\left(\bar{q}_{k} \tau^{I} \gamma_{\mu} q_{l}\right), \\
& \mathcal{O}_{e d}^{i j k l}=\left(\bar{e}_{i} \gamma^{\mu} P_{R} e_{j}\right)\left(\bar{d}_{k} \gamma_{\mu} P_{R} d_{l}\right), \\
& \mathcal{O}_{e u}^{i j k l}=\left(\bar{e}_{i} \gamma^{\mu} P_{R} e_{j}\right)\left(\bar{u}_{k} \gamma_{\mu} P_{R} u_{l}\right), \\
& \mathcal{O}_{\ell u}^{i j k l}=\left(\bar{\ell}_{i} u_{l}\right)\left(\bar{u}_{k} \ell_{j}\right), \quad \mathcal{O}_{\ell d}^{i j k l}=\left(\bar{\ell}_{i} d_{l}\right)\left(\bar{d}_{k} \ell_{j}\right), \\
& \mathcal{O}_{\ell q S}^{i j k l}=\left(\bar{\ell}_{i} e_{j}\right)\left(\bar{q}_{k} u_{l}\right), \quad \mathcal{O}_{q d e}^{i j k l}=\left(\bar{\ell}_{i} e_{j}\right)\left(\bar{d}_{k} q_{l}\right) .
\end{aligned}
$$


to the right-hand side (RHS) in the above equations. Constraints on the coefficients of the $4 \mathrm{CL}$ operators

$$
\begin{aligned}
\mathcal{O}_{P P}^{i j k l} & =\frac{1}{1+\delta}\left(\bar{e}_{i} \gamma^{\mu} P e_{j}\right)\left(\bar{e}_{k} \gamma_{\mu} P e_{l}\right), \\
\mathcal{O}_{R L}^{i j k l} & =\frac{1}{1+\delta}\left(\bar{e}_{i} \gamma^{\mu} P_{R} e_{j}\right)\left(\bar{e}_{k} \gamma_{\mu} P_{L} e_{l}\right),
\end{aligned}
$$

where $P=P_{R}$ or $P_{L}$, are listed in Tables 4, 5, 6 and 7 .

After electroweak symmetry breaking, the operators $\mathcal{O}_{e B}^{i j}$ and $\mathcal{O}_{e W}^{i j}$ become the chirality-flipping dipole moments as written in (3.9), (3.10) (where we did not include the $Z$ lepton-lepton operators [169]). These dipoles can be flavor conserving or transition dipole moments. The flavor diagonal operators are specially interesting because they correspond to the anomalous magnetic moments and the electric dipole moments of the different fermions. Taking $C_{e \gamma}^{i j}\left(q^{2}\right)=$ $C_{e B}^{i j}\left(q^{2}\right) \cos \theta_{W}-C_{e W}^{i j}\left(q^{2}\right) \sin \theta_{W}$ as the Wilson coefficient with momentum transfer equal to $q^{2}$, we have for $q^{2}=0$,

$$
\begin{gathered}
\frac{C_{e \gamma}^{i i}\left(q^{2}=0\right)}{m_{\mathrm{NP}}^{2}}\langle H\rangle \bar{e}_{i} \sigma^{\mu \nu} P_{R} e_{i} F_{\mu \nu}^{\mathrm{em}}+\text { h.c. } \\
=\frac{\operatorname{Re}\left\{C_{e \gamma}^{i i}\left(q^{2}=0\right)\right\}}{m_{\mathrm{NP}}^{2}}\langle H\rangle \bar{e}_{i} \sigma^{\mu \nu} e_{i} F_{\mu \nu}^{\mathrm{em}} \\
\quad+\frac{\operatorname{Im}\left\{C_{e \gamma}^{i j}\left(q^{2}=0\right)\right\}}{m_{\mathrm{NP}}^{2}}\langle H\rangle i \bar{e}_{i} \sigma^{\mu \nu} \gamma_{5} e_{i} F_{\mu \nu}^{\mathrm{em}} \\
=e \frac{a_{e_{i}}}{4 m_{e_{i}}} \bar{e}_{i} \sigma^{\mu \nu} e_{i} F_{\mu \nu}^{\mathrm{em}}+\frac{i}{2} d_{e_{i}} \bar{e}_{i} \sigma^{\mu \nu} \gamma_{5} e_{i} F_{\mu \nu}^{\mathrm{em}},
\end{gathered}
$$

with $a_{e_{i}}=\left(g_{e_{i}}-2\right) / 2$ the anomalous magnetic moment and $d_{e_{i}}$ the electric dipole moment of the lepton $e_{i}$ that can be found in [170].

In a given model, the coefficients of the effective operators can be obtained by matching the effective theory of (3.1) onto the model, at some matching scale (for instance, the mass scale of new particles). However, in particular models there can appear various pitfalls in constraining the generic coefficients $C_{\ldots}^{i j k l}$. This is illustrated, for example, in the model of [171] which corresponds to adding a singlet slepton $\tilde{E}^{c}$ of flavor $k$, in R-parity violating (RPV) SUSY. In this case, after integrating out the heavy slepton we obtain the following effective operator:

$$
\begin{aligned}
& \frac{\lambda_{[i j]}^{k} \lambda_{[m n]}^{* k}}{M^{2}}\left({\overline{\left(v_{L}\right)^{c}}}_{i} e_{L j}\right)\left({\overline{\left(e_{L}\right)_{n}}}_{n}\left(v_{L}\right)_{m}^{c}\right) \\
& =\frac{\lambda_{[i j]}^{k} \lambda_{[m n]}^{* k}}{2 M^{2}}\left(\bar{e}_{n} \gamma^{\mu} P_{L} e_{j}\right)\left(\bar{v}_{m} \gamma_{\mu} P_{L} v_{i}\right),
\end{aligned}
$$

where $\lambda_{[i j]}^{k}$ is antisymmetric in $i, j$ because the $S U(2)$ contraction of $\ell_{i} \ell_{j}$ is antisymmetric. This is an example of op- erator $\mathcal{O}_{\ell \ell(1)}$, but since it is induced by singlet scalar exchange, there is no four-charged-lepton operator (compare to (3.11)). This illustrates that the bounds obtained here, by assuming that $C_{\ldots}^{i j k l} \neq 0$ for one choice of $i j k l$ at a time, are not generic. Each process receives contributions from a sum of operators, and that sum could contain cancellations in a particular model.

Many models of new physics introduce new $\mathrm{TeV}$-scale particles carrying a conserved quantum number (e.g. Rparity, T-parity...). Such particles appear in pairs at vertices, so they contribute via boxes and penguins to the four-fermion and dipole moment operators considered here. Generic formulae for the one loop contribution to a dipole moment can be found in [172], and for boxes in [173]. Extra Higgses [174, 175] would contribute to the same operators constructed from SM fields, so they are constrained by the experimental limits on the coefficients of such operators.

\subsubsection{Constraints on low scale observables}

In this section we present the low energy constraints on the different Wilson coefficients introduced before. Any NP found at LHC will necessarily respect the bounds presented here.

3.1.2.1 Dipole transitions After electroweak symmetry breaking, the operators of (3.9), (3.10) generate magnetic and electric dipole moments for the charged leptons. Flavordiagonal operators give rise to anomalous magnetic moments and electric dipole moments as shown in (3.15). The anomalous magnetic moment of the electron $a_{e}=$ $(g-2)_{e} / 2$ is used to determine $\alpha_{\mathrm{em}}$. The current measurement of the muon anomalous moment $a_{\mu}=(g-2)_{\mu} / 2$ deviates from the (uncertain) SM expectation by $3.2 \sigma$ using $e^{+} e^{-}$-data [176], and can be taken as a constraint, or indication on the presence of new physics. Currently there is only an upper bound on the magnetic moment of the $\tau$ from the analysis of $e^{+} e^{-} \rightarrow \tau^{+} \tau^{-}[170,177]$. Electric dipole moments have not yet been observed, although we have very constraining bounds specially on the electron dipole moment. In Table 3 we present the bounds of flavor diagonal dipole moments. The EDMs are discussed in detail in Sect. 5.

The bounds on off-diagonal dipole transitions are presented in Table 3. It is convenient to normalize these coefficients, $C_{e \gamma}^{i j}=C_{e B}^{i j} \cos \theta_{W}-C_{e W}^{i j} \sin \theta_{W}$, to the Fermi interactions given our ignorance on the scale of new physics $m_{\mathrm{NP}}$ :

$\frac{C_{e \gamma}^{i j}}{m_{\mathrm{NP}}^{2}}=\frac{4 G_{\mathrm{F}}}{\sqrt{2}} \epsilon_{e \gamma}^{i j}$.

In the literature, it is customary to use the left and right form factors for lepton flavor violating transitions defined by 
Table 3 Bounds on the different dipole coefficients. Flavor diagonal dipole coefficients are given in terms of the corresponding anomalous magnetic moment, $a_{e_{i}}$, and the dipole moment, $d_{e_{i}}$. Bounds on transition moments are given in terms of the dimensionless coefficients $\left|\epsilon_{e \gamma}^{i j}\right|$ (defined in (3.17)) from the bounds on the branching ratios given in the last column. These bounds apply also both to $\left|\epsilon_{e \gamma}^{i j}\right|$ and $\left|\epsilon_{e \gamma}^{j i}\right|$. See Sect. 3.1.2 for details

\begin{tabular}{llll}
\hline$(i j)$ & $a_{i}=\frac{g_{i}-2}{2}$ & $\operatorname{edm}_{i}(e \mathrm{~cm})$ & Ref. \\
\hline $\bar{e} e$ & $0.0011596521859(38)$ & $d_{e} \leq 1.6 \times 10^{-27}$ & PDG [170, 186] \\
$\bar{\mu} \mu$ & $11659208.0(5.4)(3.3) \times 10^{-10}$ & $d_{\mu} \leq 2.8 \times 10^{-19}$ & Muon g-2 Coll. [187, 188] \\
$\bar{\tau} \tau$ & $-0.052<a_{\tau}<0.013$ & $\left(-2.2<d_{\tau}<4.5\right) \times 10^{-17}$ & LEP2 [189], Belle [190] \\
\hline$(i j)$ & $\bar{\ell}_{i} \sigma^{\mu \nu} e_{R j} F_{\mu \nu}^{\mathrm{em}}$ & expt. limit & Ref. \\
\hline $\bar{e} \mu$ & $\leq 3.4 \times 10^{-11}$ & $\leq 1.2 \times 10^{-11}$ & MEGA Coll. [180] \\
$\bar{e} \tau$ & $\leq 1.2 \times 10^{-7}$ & $\leq 1.1 \times 10^{-7}$ & BaBar [182] \\
$\bar{\mu} \tau$ & $\leq 8.4 \times 10^{-8}$ & $\leq 4.5 \times 10^{-8}$ & Belle, BaBar [181, 191] \\
\hline
\end{tabular}

$$
\Delta \mathcal{L}_{2}=e m_{l_{i}} A_{\mu} \bar{f}_{j}\left[i \sigma^{\mu v} q_{v}\left(A_{L}^{i j} P_{L}+A_{R}^{i j} P_{R}\right)\right] f_{i}+\text { h.c. }
$$

where $f$ is a Dirac (4-component) fermion. The radiative decay $f_{i} \rightarrow f_{j}+\gamma$ proceeds at the rate $\Gamma=m_{i}^{5} e^{2} /(16 \pi) \times$ $\left(\left|A_{L}^{i j}\right|^{2}+\left|A_{R}^{i j}\right|^{2}\right)$ [178]. QED corrections to those decays are unusually large and may reach as much as $15 \%$ [179]. Bounds on the dimensionless coefficients $C_{e \gamma}^{i j}$ and $\epsilon_{e \gamma}^{i j}$ can be obtained by translating from $A_{L}^{i j}$ and $A_{R}^{i j}$ :

$$
\frac{C_{e \gamma}^{i j}}{m_{\mathrm{NP}}^{2}}\langle H\rangle=e \frac{m_{i}}{2} A_{R}^{i j}, \quad \frac{C_{e \gamma}^{j i^{*}}}{m_{\mathrm{NP}}^{2}}\langle H\rangle=e \frac{m_{i}}{2} A_{L}^{i j} .
$$

The experimental bounds on radiative lepton decays can be used to set bounds on these off-diagonal Wilson coefficients. The current experimental bounds are $B(\mu \rightarrow e \gamma)<$ $1.2 \times 10^{-11}$ [180], $B(\tau \rightarrow \mu \gamma)<4.5 \times 10^{-8}$ [181], and $B(\tau \rightarrow e \gamma)<1.1 \times 10^{-7}[182]$.

For the off-shell photon, $q^{2} \neq 0$, there exist additional form factors,

$$
\begin{aligned}
\Delta \mathcal{L}= & e m_{l_{i}} A_{\mu} \bar{e}_{j}\left[\left(g_{\mu \nu}-\frac{q_{\mu} q_{\nu}}{q^{2}}\right) \gamma_{\nu}\left(B_{L}^{i j} P_{L}+B_{R}^{i j} P_{R}\right)\right] e_{i} \\
& + \text { h.c. }
\end{aligned}
$$

which induce contributions to the four-fermion operators to be discussed in the next subsections. These form factors may be enhanced by a large factor compared to the on-shell photon form factors [184], $\ln \left(m_{\mathrm{NP}} / m_{l_{i}}\right)$, depending on the nature of new physics. Therefore, those operators become relevant for constraining new physics in R-parity violating SUSY [185] and in low-scale type-II see-saw models [184].

3.1.2.2 Four-charged-lepton operators As before, to present the bounds on the dimensionless four-charged-fermion coefficients in (3.14), we normalize them to the Fermi interactions:

$\frac{C_{(n) \ell \ell}^{i j k l}}{m_{\mathrm{NP}}^{2}}=-\frac{4 G_{\mathrm{F}}}{\sqrt{2}} \epsilon_{(n) \ell \ell}^{i j k l}, \quad \frac{C_{e e}^{i j k l}}{m_{\mathrm{NP}}^{2}}=-\frac{4 G_{\mathrm{F}}}{\sqrt{2}} \epsilon_{e e}^{i j k l}$,

$\frac{C_{\ell e}^{i l k j}}{m_{\mathrm{NP}}^{2}}=\frac{4 G_{\mathrm{F}}}{\sqrt{2}} \epsilon_{\ell e}^{i j k l}$.

The current low energy constraints on the dimensionless $\epsilon$ 's are shown in Tables 4, 5,6 and 7. The rows of the tables are labeled by the flavor combination, and the column by the Lorentz structure. The numbers given in this tables correspond to the best current experimental bound on the coefficient of each operator, assuming it is the only nonzero coefficient present. The last column in the table lists the experiment setting the bound. The compositeness search limits $\Lambda @$ LEP are at $95 \%$ C.L., the decay rate bounds at $90 \%$ C.L.

Regarding the definition of the different coefficients we have to make some comments. First, note the flavor index permutation between $C_{\ell e}$ and $\epsilon_{\ell e}$ :

$C_{\ell e}^{i l k j}\left(\bar{\ell}_{i} e_{l}\right)\left(\bar{e}_{k} \ell_{j}\right)=-\frac{1}{2} \epsilon_{\ell e}^{i j k l}\left(\bar{\ell}_{i} \gamma^{\mu} \ell_{j}\right)\left(\bar{e}_{k} \gamma_{\mu} e_{l}\right)$.

There are relations between the flavor indices of the different operators. For $\mathcal{O}_{L L}=\left(\bar{e} \gamma^{\mu} P_{L} e\right)\left(\bar{e} \gamma_{\mu} P_{L} e\right)$ and $\mathcal{O}_{R R}=$ $\left(\bar{e} \gamma^{\mu} P_{R} e\right)\left(\bar{e} \gamma_{\mu} P_{R} e\right)$ we have

$\mathcal{O}_{P P}^{i j k l}=\mathcal{O}_{P P}^{k l i j}, \quad \mathcal{O}_{P P}^{i j k l}=\mathcal{O}_{P P}^{* j i l k}, \quad \mathcal{O}_{P P}^{i j k l}=\mathcal{O}_{P P}^{i l k j}$,

by symmetry, Hermitian conjugation and Fierz rearrangement, respectively. Therefore, the constraints on $\bar{e} e \bar{\mu} \tau$ in the first two columns of Tables 4 to 7 apply to $\epsilon_{(n) x x}^{e e \mu \tau}, \epsilon_{(n) x x}^{\mu \tau e e}$, $\epsilon_{(n) x x}^{* e e \tau \mu}, \epsilon_{(n) x x}^{* \tau \mu e e}, \epsilon_{(n) x x}^{e \tau \mu e}, \epsilon_{(n) x x}^{\mu e e \tau}, \epsilon_{(n) x x}^{* \tau e e \mu}$, and $\epsilon_{(n) x x}^{* e \mu \tau e}$ with $(n) x x$ 
Table 4 Bounds on coefficients of flavor four-lepton operators, from four-charged-lepton processes. The number is the upper bound on the dimensionless operator coefficient $\epsilon^{i j k l}$ (defined in (3.21)), arising from the measurement in the last column. The bound applies also to $\epsilon^{k l i j}$. The second column is the bounds on $\epsilon_{(3) \ell \ell}^{i j k l}$, and $\epsilon_{(1) \ell \ell}^{i j k l}$ [except in the case of the bracketed limits, which are the upper bound on $\epsilon_{(1) \ell \ell}^{i j k l}$ and $\left.2 \epsilon_{(1) \ell \ell}^{i j k l}\right]$. The third column is the bound on $\epsilon_{(1) e e}^{i j k l}$. The bounds in these two columns apply also when the flavor indices are permuted to jilk and $i l k j$. The fourth column is the bound on $\epsilon_{l e}^{i j k l}$ (which does not apply to the flavor permutation $i l k j$, so this is listed with a line of its own). The constraints in [brackets] apply to the two charged lepton-two neutrino operator of the same flavor structure, and arise from lepton universality in $\tau$ decays. See Sect. 3.1.2 for details

\begin{tabular}{|c|c|c|c|c|c|}
\hline$(i j k l)$ & $\left(\bar{e} \gamma^{\mu} P_{L} e\right)\left(\bar{e} \gamma_{\mu} P_{L} e\right)$ & $\left(\bar{e} \gamma^{\mu} P_{R} e\right)\left(\bar{e} \gamma_{\mu} P_{R} e\right)$ & $\left(\bar{e} \gamma_{\mu} P_{L} e\right)\left(\bar{e} \gamma^{\mu} P_{R} e\right)$ & expt. limit & Ref. \\
\hline $\bar{e} e \bar{e} e$ & $(-1.8-+2.8) \times 10^{-3}$ & $(-1.8-+2.8) \times 10^{-3}$ & $(-2.4-+4.9) \times 10^{-3}$ & $\Lambda @$ LEP2 & [194] \\
\hline $\bar{e} e \bar{\mu} \mu$ & $(-7.2-+5.2) \times 10^{-3}$ & $(-7.8-+5.8) \times 10^{-3}$ & $(-9.0-+9.6) \times 10^{-3}$ & $\Lambda @$ LEP2 & {$[193,195]$} \\
\hline $\bar{e} \mu \bar{\mu} e$ & $(-7.2-+5,2) \times 10^{-3}$ & $(-7.8-+5.8) \times 10^{-3}$ & $1.3 \times 10^{-2}$ & $\Lambda, R P V @$ LEP2 & {$[193,195]$} \\
\hline $\bar{e} e \bar{\tau} \tau$ & $(-7.3-+13) \times 10^{-3}$ & $(-8.0-+15) \times 10^{-3}$ & $(-1.2-+1.8) \times 10^{-2}$ & $\Lambda @$ LEP2 & {$[193,195]$} \\
\hline $\bar{\tau} e \bar{e} \tau$ & $(-7.3-+13) \times 10^{-3}$ & $(-8.0-+15) \times 10^{-3}$ & $1.3 \times 10^{-2}$ & $\Lambda, R P V @ \mathrm{LEP} 2$ & {$[193,195]$} \\
\hline $\bar{\mu} \mu \bar{\mu} \mu$ & $\sim 1$ & $\sim 1$ & $\sim 1$ & $B(Z \rightarrow \mu \bar{\mu})$ & \\
\hline $\bar{\mu} \mu \bar{\tau} \tau$ & $\sim 1[0.0014]$ & $\sim 1$ & $\sim 1[0.01]$ & $B(Z \rightarrow \mu \bar{\mu})$ & \\
\hline $\bar{\mu} \tau \bar{\tau} \mu$ & $\sim 1[0.0014]$ & $\sim 1$ & & $B(Z \rightarrow \mu \bar{\mu})$ & \\
\hline $\bar{\tau} \tau \bar{\tau} \tau$ & $\sim 1$ & $\sim 1$ & $\sim 1$ & $B(Z \rightarrow \tau \bar{\tau})$ & \\
\hline
\end{tabular}

Table 5 Bounds on coefficients of four-lepton operators with $\Delta L_{\alpha}=$ $-\Delta L_{\beta}=1$. They apply also to flavor index permutations $k l i j$ and $i l k j$, except in the case of $\tau \tau e \mu$, where the bound on $\tau \mu e \tau$ in the fourth col- umn is from $\mu$ decay and is listed separately. See the caption of Table 4 and Sect. 3.1.2 for further details

\begin{tabular}{lllll}
\hline$(i j k l)$ & $\left(\bar{e} \gamma^{\mu} P_{L} e\right)\left(\bar{e} \gamma_{\mu} P_{L} e\right)$ & $\left(\bar{e} \gamma^{\mu} P_{R} e\right)\left(\bar{e} \gamma_{\mu} P_{R} e\right)$ & $\left(\bar{e} \gamma_{\mu} P_{L} e\right)\left(\bar{e} \gamma^{\mu} P_{R} e\right)$ & expt. limit \\
\hline $\bar{e} e \bar{e} \mu$ & $7.1 \times 10^{-7}$ & $7.1 \times 10^{-7}$ & $B(\mu \rightarrow e \bar{e} e)<10^{-12}$ \\
$\bar{e} e \bar{e} \tau$ & $7.1 \times 10^{-7}$ & $7.8 \times 10^{-4}$ & $7.8 \times 10^{-4}$ & $B(\tau \rightarrow e \bar{e} e)<2 \times 10^{-7}$ \\
$\bar{e} e \bar{\mu} \tau$ & $7.8 \times 10^{-4}$ & $1.1 \times 10^{-3}$ & $1.1 \times 10^{-3}$ & $B(\tau \rightarrow \bar{e} e \mu)<1.9 \times 10^{-7}$ \\
$\bar{\mu} \mu \bar{e} \mu$ & $1.1 \times 10^{-3}$ & $\sim 1$ & $\sim 1$ & $B(Z \rightarrow e \bar{\mu})<1.7 \times 10^{-6}$ \\
$\bar{\mu} \mu \bar{e} \tau$ & $\sim 1$ & $1.1 \times 10^{-3}$ & $1.1 \times 10^{-3}$ & $B(\tau \rightarrow \bar{\mu} e \mu)<2.0 \times 10^{-7}$ \\
$\bar{\mu} \mu \bar{\mu} \tau$ & $1.1 \times 10^{-3}$ & $7.8 \times 10^{-4}$ & $7.8 \times 10^{-4}$ & $B(\tau \rightarrow 3 \mu)<1.9 \times 10^{-7}$ \\
$\bar{\tau} \tau \bar{e} \mu$ & $7.8 \times 10^{-4}$ & $\sim 1$ & $\sim 1[0.05]$ & $B(Z \rightarrow e \bar{\mu})<1.7 \times 10^{-6}$ \\
$\bar{\tau} \mu \bar{e} \tau$ & $\sim 1[0.05]$ & $\sim 1$ & {$[0.05]$} & $B(Z \rightarrow e \bar{\mu})<1.7 \times 10^{-6}$ \\
$\bar{\tau} \tau \bar{e} \tau$ & $\sim 1[0.05]$ & $\sim 3$ & $\sim 3[0.05]$ & $B(Z \rightarrow e \bar{\tau})<9.8 \times 10^{-6}$ \\
$\bar{\tau} \tau \bar{\tau} \mu$ & $\sim 3[0.05]$ & $\sim 3$ & $\sim 3[0.05]$ & $B(Z \rightarrow \tau \bar{\mu})<1.2 \times 10^{-5}$ \\
\hline
\end{tabular}

Table 6 Bounds on coefficients of four-lepton operators with $\Delta L_{\alpha}=\Delta L_{\beta}=2$. See the caption of Table 4 and Sect. 3.1.2 for details

\begin{tabular}{|c|c|c|c|c|}
\hline$(i j k l)$ & $\left(\bar{e} \gamma^{\mu} P_{L} e\right)\left(\bar{e} \gamma_{\mu} P_{L} e\right)$ & $\left(\bar{e} \gamma^{\mu} P_{R} e\right)\left(\bar{e} \gamma_{\mu} P_{R} e\right)$ & $\left(\bar{e} \gamma_{\mu} P_{L} e\right)\left(\bar{e} \gamma^{\mu} P_{R} e\right)$ & expt. limit \\
\hline $\bar{e} \mu \bar{e} \mu$ & $3.0 \times 10^{-3}$ & $3.0 \times 10^{-3}$ & $2.0 \times 10^{-3}$ & $(\bar{\mu} e) \leftrightarrow(\bar{e} \mu)$ \\
\hline $\bar{e} \tau \bar{e} \tau$ & {$[0.05]$} & & {$[0.05]$} & \\
\hline $\bar{\mu} \tau \bar{\mu} \tau$ & {$[0.05]$} & & {$[0.05]$} & \\
\hline
\end{tabular}

Table 7 Bounds on coefficients of four-lepton operators with $\Delta L_{\alpha}=\Delta L_{\beta}=-\frac{1}{2} \Delta L_{\rho}$. See the caption of Table 4 and Sect. 3.1 .2 for details

\begin{tabular}{lllll}
\hline$(i j k l)$ & $\left(\bar{e} \gamma^{\mu} P_{L} e\right)\left(\bar{e} \gamma_{\mu} P_{L} e\right)$ & $\left(\bar{e} \gamma^{\mu} P_{R} e\right)\left(\bar{e} \gamma_{\mu} P_{R} e\right)$ & $\left(\bar{e} \gamma^{\mu} P_{L} e\right)\left(\bar{e} \gamma^{\mu} P_{R} e\right)$ & expt. limit \\
\hline $\bar{e} \mu \bar{e} \tau$ & $2.3 \times 10^{-4}$ & $2.3 \times 10^{-4}$ & $2.3 \times 10^{-4}$ & $B(\tau \rightarrow \bar{\mu} e e)<1.1 \times 10^{-7}$ \\
$\bar{\mu} e \bar{\mu} \tau$ & $2.6 \times 10^{-4}$ & $2.6 \times 10^{-4}$ & $2.6 \times 10^{-4}$ & $B(\tau \rightarrow \bar{e} \mu \mu)<1.3 \times 10^{-7}$ \\
$\bar{\tau} e \bar{\tau} \mu$ & {$[0.05]$} & & {$[0.05]$} & \\
\hline
\end{tabular}


equal to (3) $\ell \ell,(1) \ell \ell$, or (1)ee. Note, however that it is calculated assuming only one of these $\epsilon$ is non-zero. Similarly, the operator $\mathcal{O}_{L R}^{i j k l}=\left(\bar{e}_{i} \gamma_{\mu} P_{L} e_{j}\right)\left(\bar{e}_{k} \gamma^{\mu} P_{R} e_{l}\right)$, with coefficient $\epsilon_{\ell e}^{i j k l}$, is related by Hermitian conjugation:

$\mathcal{O}_{L R}^{i j k l}=\mathcal{O}_{L R}^{* j i l k}$,

so again the bounds on $\epsilon_{\ell e}^{i j k l}$ apply to $\epsilon_{\ell e}^{* j i l k}$. We can usually apply also these bounds to $\epsilon_{\ell e}^{k l i j}$ because the chirality of the fermion legs does not affect the matrix element squared, but $\epsilon_{\ell e}^{i l k j}$ is bounded separately in the tables.

The bounds from $Z$ decays in Tables 4 and 5 are estimated from the one loop penguin diagram obtained closing two of the legs of the four-fermion operator and coupling it with the $Z$ [192]. These bounds would be more correctly included by renormalization group mixing between the fourfermion operators and the Z-fermion-fermion operators discussed in [169]. They are listed in the tables to indicate the existence of a constraint. The bound can be applied to $\epsilon_{\ell e}^{i i k l}$ and $\epsilon_{\ell e}^{i j k k}$ but it does not apply to $\epsilon_{l e}^{i l k i}$.

Contact interaction bounds are usually quoted on the scale $\Lambda$, where

$\epsilon_{a b}^{i j k l} \frac{4 G_{\mathrm{F}}}{\sqrt{2}}= \pm \frac{1}{1+\delta} \frac{4 \pi}{\Lambda^{2}}$,

and $\delta=1$ for the operators $\mathcal{O}_{L L}^{\text {eeee }}$ and $\mathcal{O}_{R R}^{\text {eeee }}$ of (3.14), 0 otherwise. Since our normalization does not have this factor of 2, we have a Feynman rule $\epsilon 8 G_{\mathrm{F}} / \sqrt{2}$ for these operators, and correspondingly stricter bounds on the $\epsilon$ 's. The bounds are the same for $\epsilon_{\ell e}^{i k k i}$ and $\epsilon_{\ell e}^{k i k}$. However, contact interaction bounds are not quoted on operators of the form $\left(\bar{e}_{i} \gamma^{\mu} P_{L} e_{j}\right)\left(\bar{e}_{j} \gamma_{\mu} P_{R} e_{i}\right)$, corresponding to $\epsilon_{\ell e}^{i i j j}$. Such operators are generated by sneutrino exchange in R-parity violating SUSY, so we estimate the bound $\lambda^{2} / m_{\tilde{v}}^{2}<4 /\left(9 \mathrm{TeV}^{2}\right)$ from the plotted constraints in [193], and impose $4\left|\epsilon_{a b}^{i j k l}\right| G_{\mathrm{F}} /$ $\sqrt{2}<\lambda^{2} /\left(2 m_{\tilde{v}}^{2}\right)$.

Many of the 4CL operators involving two $\tau$ 's are poorly constrained. In some cases, see (3.11), (3.12), new physics that generates 4CL operators also induces $\left(\bar{e}_{i} \gamma^{\lambda} P e_{j}\right) \times$ $\left(\bar{v}_{k} \gamma_{\lambda} L v_{l}\right)$. The coefficients of operators of the form $\left(\bar{\mu} \gamma^{\lambda} P e\right)\left(\bar{v}_{k} \gamma_{\lambda} L v_{l}\right), \quad\left(\bar{\mu} \gamma^{\lambda} P \tau\right)\left(\bar{v}_{k} \gamma_{\lambda} L v_{l}\right)$ or $\left(\bar{e} \gamma^{\lambda} P \tau\right) \times$ $\left(\bar{v}_{k} \gamma_{\lambda} L v_{l}\right)$, are constrained from lepton universality measurements in $\mu$ and $\tau$ decays [196]. The decay rate $\tau \rightarrow$ $e_{i} \nu_{k} \bar{\nu}_{l}$ in the presence of the operators of (3.14), divided by the SM prediction for $\tau \rightarrow e_{i} \nu_{\tau} \bar{\nu}_{i}$, is

$$
\begin{aligned}
& \left(1-2 \delta_{k \tau} \delta_{i l} \operatorname{Re}\left\{\epsilon_{(1) \ell \ell}^{\tau \tau i i}+2 \epsilon_{(3) \ell \ell}^{\tau \tau i i}\right\}+\frac{4 m_{i}}{m_{\tau}} \delta_{k \tau} \delta_{i l} \operatorname{Re}\left\{\epsilon_{\ell e}^{\tau \tau i i}\right\}\right. \\
& \left.+\left|\epsilon_{(1) \ell \ell}^{i \tau k l}\right|^{2}+4\left|\epsilon_{(3) \ell \ell}^{i \tau k l}\right|^{2}+\left|\epsilon_{\ell e}^{i \tau k l}\right|^{2}\right) .
\end{aligned}
$$

Within the experimental accuracy, the weak $\tau$ and $\mu$ decays verify lepton universality and agree with LEP precision measurements of $m_{W}$. Rough bounds on the $\epsilon$ 's can therefore be obtained by requiring the new physics contribution to the decay rates to be less than the errors $\frac{\Delta B}{B}(\tau \rightarrow e v \bar{v})=$ $0.05 / 17.84, \frac{\Delta B}{B}(\tau \rightarrow \mu \nu \bar{v})=0.05 / 17.36$. These are listed in the tables in [brackets]. The bracketed limit in the second column applies to $\epsilon_{(1) \ell \ell}^{i j k l}$; the bound on $\epsilon_{(3) \ell \ell}^{i j k l}$ is $1 / 2$ the quoted number. The limit on $\epsilon_{\ell e}^{\tau e \tau \mu}$ is from its contribution to $\mu \rightarrow e \nu_{\tau} \bar{\nu}_{\tau}$.

Finally, we would like to remind the reader the various caveats to these four-fermion vertex bounds.

- The constraints are calculated "one operator at a time". This is unrealistic; new physics is likely to induce many non-renormalizable operators. In some cases, see (3.16), a symmetry in the new physics can cause cancellations such that it does not contribute to certain observables.

- The coefficients of the 4CL operators, and two $v$-two charged lepton $(2 v 2 \mathrm{CL})$ operators may differ by a factor of few, because they are induced by the exchange of different members of a multiplet, whose masses differ [197].

- The list of operators is incomplete. Perhaps some of the neglected operators give relevant constraints on new physics. For instance, bounds from lepton universality on the $\left(H^{*} \bar{\ell}\right) \gamma^{\mu} \partial_{\mu}(H \ell)$ operator [198] are relevant to extra dimensional scenarios [199].

- Operators of dimension $>6$ are neglected. If the mass scale of the new physics is $\sim \mathrm{TeV}$, then higher dimension operators with Higgs VEVs [200] such as $H H \bar{\psi} \psi \bar{\psi} \psi$ are not significantly suppressed.

3.1.2.3 Two lepton-two quark operators Once more, we normalize the coefficients of the two lepton-two quark operators in (3.6) to the Fermi interactions:

$\frac{C_{(n) \ell q}^{i j k l}}{m_{\mathrm{NP}}^{2}}=-\frac{4 G_{\mathrm{F}}}{\sqrt{2}} \epsilon_{(n) \ell q}^{i j k l}, \quad \frac{C_{e d}^{i j k l}}{m_{\mathrm{NP}}^{2}}=-\frac{4 G_{\mathrm{F}}}{\sqrt{2}} \epsilon_{e d}^{i j k l}$,

$\frac{C_{\ell d}^{i j k l}}{m_{\mathrm{NP}}^{2}}=\frac{4 G_{\mathrm{F}}}{\sqrt{2}} \epsilon_{\ell d}^{i j k l}, \quad \frac{C_{e u}^{i j k l}}{m_{\mathrm{NP}}^{2}}=-\frac{4 G_{\mathrm{F}}}{\sqrt{2}} \epsilon_{e u}^{i j k l}$,

$\frac{C_{\ell u}^{i j k l}}{m_{\mathrm{NP}}^{2}}=-\frac{4 G_{\mathrm{F}}}{\sqrt{2}} \epsilon_{\ell u}^{i j k l}, \quad \frac{C_{\ell q S}^{i j k l}}{m_{\mathrm{NP}}^{2}}=-\frac{4 G_{\mathrm{F}}}{\sqrt{2}} \epsilon_{\ell q S}^{i j k l}$,

$\frac{C_{q d e}^{i j k l}}{m_{\mathrm{NP}}^{2}}=-\frac{4 G_{\mathrm{F}}}{\sqrt{2}} \epsilon_{q d e}^{i j k l}$.

The main bounds on the dimensionless $\epsilon \mathrm{S}$ are given in Tables 8 and 9 . These numbers correspond to the best current experimental bound on the coefficient of each operator, assuming it is the only non-zero coefficient present. The bounds on $\epsilon_{\ell q}$ in Table 8 apply both to $\epsilon_{(1) \ell q}$ and $\epsilon_{(3) \ell q}$. These bounds have been obtained from the corresponding bounds on leptoquark couplings in Refs. [201, 202] that can be checked for further details. 
Table 8 Bounds on coefficients of the left handed two quark-two lepton operators. Bound is the upper bound on the dimensionless operator coefficient $\epsilon^{i j k l}$ (defined in (3.28)), arising from the experimental de- termination of the observable in the next column. Bounds with a * are also valid under the exchange of the lepton indices

\begin{tabular}{|c|c|c|c|c|c|}
\hline \multicolumn{6}{|c|}{$\left(\bar{e} \gamma^{\mu} P_{L} e\right)\left(\bar{q} \gamma_{\mu} P_{L} q\right)$} \\
\hline$(i j k l)$ & Bound on $\epsilon_{\ell q}^{i j k l}$ & Observable & $(i j k l)$ & Bound on $\epsilon_{\ell q}^{i j k l}$ & Observable \\
\hline 1111 & $5.1 \times 10^{-3}$ & $R_{\pi}$ & 2211 & $5.1 \times 10^{-3}$ & $R_{\pi}$ \\
\hline 1211 & $8.5 \times 10^{-7}$ & $\mu-e$ conversion on $\mathrm{Ti}$ & $1212^{*}$ & $2.9 \times 10^{-7}$ & $B\left(K_{L}^{0} \rightarrow \bar{\mu} e\right)$ \\
\hline$i j 12$ & $4.5 \times 10^{-6}$ & $\frac{B\left(K^{+} \rightarrow \pi^{+} \bar{\nu} v\right)}{B\left(K^{+} \rightarrow \pi^{0} e^{+} v_{e}\right)}$ & $i j 22$ & 1.0 & $V_{c s}$ \\
\hline$i j 13$ & $3.6 \times 10^{-3}$ & $V_{u b}$ & $i j 23$ & $4.2 \times 10^{-2}$ & $V_{c b}$ \\
\hline 1123 & $6.6 \times 10^{-5}$ & $B\left(B^{+} \rightarrow e^{+} e^{-} K^{+}\right)$ & 1113 & $9.3 \times 10^{-4}$ & $B\left(B^{+} \rightarrow e^{+} e^{-} \pi^{+}\right)$ \\
\hline 2223 & $5.4 \times 10^{-5}$ & $B\left(B^{+} \rightarrow \mu^{+} \mu^{-} K^{+}\right)$ & 2213 & $1.4 \times 10^{-3}$ & $B\left(B^{+} \rightarrow \mu^{+} \mu^{-} \pi^{+}\right)$ \\
\hline $2123^{*}$ & $4.5 \times 10^{-3}$ & $B\left(B^{+} \rightarrow e^{+} \mu^{-} K^{+}\right)$ & $2113^{*}$ & $3.9 \times 10^{-5}$ & $B\left(B^{+} \rightarrow e^{+} \mu^{-} \pi^{+}\right)$ \\
\hline $1223^{*}$ & $1.2 \times 10^{-2}$ & $B\left(B_{s}^{0} \rightarrow \mu^{+} e^{-}\right)$ & 3312 & $6.6 \times 10^{-2}$ & $K-\bar{K}$ \\
\hline 2222 & $6.0 \times 10^{-2}$ & $\frac{B\left(D_{s}^{+} \rightarrow \mu^{+} v_{\mu}\right)}{B\left(D_{s}^{+} \rightarrow \tau^{+} v_{\tau}\right)}$ & 3322 & $6.0 \times 10^{-2}$ & $\frac{B\left(D_{s}^{+} \rightarrow \mu^{+} v_{\mu}\right)}{B\left(D_{s}^{+} \rightarrow \tau^{+} \nu_{\tau}\right)}$ \\
\hline $3223^{*}$ & $1.2 \times 10^{-3}$ & $B\left(B^{+} \rightarrow \mu^{+} \tau^{-} X^{+}\right)$ & 3323 & $9.3 \times 10^{-3}$ & $B\left(B^{+} \rightarrow \tau^{+} \tau^{-} X^{+}\right)$ \\
\hline
\end{tabular}

Table 9 Bounds on coefficients of the right handed vector and scalar 2 quark-2 lepton operators. Bound is the upper bound on the dimensionless operator coefficient $\epsilon^{i j k l}$ (defined in (3.28)), arising from the ex- perimental determination of the observable in the next column. Bounds with $\mathrm{a}^{*}$ are also valid under the exchange of the lepton indices

\begin{tabular}{|c|c|c|c|c|c|}
\hline \multicolumn{6}{|c|}{$\left(\bar{e} \gamma^{\mu} P_{R} e\right)\left(\bar{q} \gamma_{\mu} P_{R} q\right)$} \\
\hline$\overline{(i j k l)}$ & Bound on $\epsilon_{e u}^{i j k l}$ & Observable & $(i j k l)$ & Bound on $\epsilon_{e u}^{i j k l}$ & Observable \\
\hline 1112 & $1.7 \times 10^{-2}$ & $\frac{B\left(D^{+} \rightarrow \pi^{+} e^{+} e^{-}\right)}{B\left(D^{0} \rightarrow \pi^{-} e^{+} v_{e}\right)}$ & $2112^{*}$ & $1.3 \times 10^{-2}$ & $\frac{B\left(D^{+} \rightarrow \pi^{+} \mu^{-} e^{+}\right)}{B\left(D^{0} \rightarrow \pi^{-} e^{+} v_{e}\right)}$ \\
\hline 2212 & $9.0 \times 10^{-3}$ & $\frac{B\left(D^{+} \rightarrow \pi^{+} \mu^{+} \mu^{-}\right)}{B\left(D^{0} \rightarrow \pi^{-} e^{+} \nu_{e}\right)}$ & 3312 & 0.19 & $B\left(D^{0}-\bar{D}^{0}\right)$ \\
\hline \multicolumn{6}{|c|}{$\left(\bar{\ell} P_{R} e\right)\left(\bar{d} P_{L} q\right)$} \\
\hline$\overline{(i j k l)}$ & Bound on $\epsilon_{q d e}^{i j k l}$ & Observable & $(i j k l)$ & Bound on $\epsilon_{q d e}^{i j k l}$ & Observable \\
\hline 1111 & $1.5 \times 10^{-7}$ & $R_{\pi}$ & 2211 & $3.0 \times 10^{-4}$ & $R_{\pi}$ \\
\hline 1211 & $5.1 \times 10^{-3}$ & $B\left(\pi^{+} \rightarrow \mu^{+} v_{e}\right)$ & $1212^{*}$ & $2.1 \times 10^{-8}$ & $B\left(K_{L}^{0} \rightarrow \mu^{+} e^{-}\right)$ \\
\hline 1112 & $2.7 \times 10^{-8}$ & $B\left(K_{L}^{0} \rightarrow e^{+} e^{-}\right)$ & 2212 & $8.4 \times 10^{-7}$ & $B\left(K_{L}^{0} \rightarrow \mu^{+} \mu^{-}\right)$ \\
\hline 2221 & $1.3 \times 10^{-2}$ & $B\left(D^{+} \rightarrow \mu^{+} v_{\mu}\right)$ & 2222 & $1.2 \times 10^{-2}$ & $\frac{B\left(D_{s}^{+} \rightarrow \mu^{+} v_{\mu}\right)}{B\left(D_{s}^{+} \rightarrow \tau^{+} v_{\tau}\right)}$ \\
\hline 3322 & 0.2 & $\frac{B\left(D_{s}^{+} \rightarrow \mu^{+} v_{\mu}\right)}{B\left(D_{s}^{+} \rightarrow \tau^{+} v_{\tau}\right)}$ & 3313 & $2.5 \times 10^{-3}$ & $B\left(B^{+} \rightarrow \tau^{+} v_{\tau}\right)$ \\
\hline 1113 & $9.0 \times 10^{-5}$ & $B\left(B^{0} \rightarrow e^{+} e^{-}\right)$ & $1213^{*}$ & $1.2 \times 10^{-4}$ & $B\left(B^{0} \rightarrow \mu^{+} e^{-}\right)$ \\
\hline $1313^{*}$ & $2.5 \times 10^{-3}$ & $B\left(B^{0} \rightarrow \tau^{+} e^{-}\right)$ & $2313^{*}$ & $3.3 \times 10^{-3}$ & $B\left(B^{0} \rightarrow \tau^{+} \mu^{-}\right)$ \\
\hline 2213 & $7.5 \times 10^{-5}$ & $B\left(B^{0} \rightarrow \mu^{+} \mu^{-}\right)$ & 1123 & $6.0 \times 10^{-4}$ & $B\left(B_{s}^{0} \rightarrow e^{+} e^{-}\right)$ \\
\hline $1223^{*}$ & $2.1 \times 10^{-4}$ & $B\left(B_{s}^{0} \rightarrow \mu^{+} e^{-}\right)$ & 2223 & $1.2 \times 10^{-4}$ & $B\left(B_{s}^{0} \rightarrow \mu^{+} \mu^{-}\right)$ \\
\hline
\end{tabular}

\subsection{Phenomenological parameterizations of quark and} lepton Yukawa couplings

\subsubsection{Quark sector}

The quark Yukawa sector is described by the following Lagrangian:

$\mathcal{L}_{\text {quark }}=u_{R i}^{c} Y_{i j}^{u} Q_{j} \bar{H}+d_{R i}^{c} Y_{i j}^{d} Q_{j} H+$ h.c., where $i, j=1,2,3$ are generation indices, $Q_{i}=\left(d_{L i}, u_{L i}\right)$ are the left handed quark doublets, $u_{R}^{c}$ and $d_{R}^{c}$ are the right handed up and down quark singlets respectively, and $H$ is the Higgs field. On the other hand, $Y^{u}$ and $Y^{d}$ are complex $3 \times 3$ matrices, which can be cast by means of a singular value decomposition as

$$
\begin{aligned}
& Y^{u}=V_{R}^{u} D_{Y}^{u} V_{L}^{u^{\dagger}}, \\
& Y^{d}=V_{R}^{d} D_{Y}^{d} V_{L}^{d^{\dagger}} .
\end{aligned}
$$


Here, $D_{Y}^{u}=\operatorname{diag}\left(y_{1}^{u}, y_{2}^{u}, y_{3}^{u}\right)$ is a diagonal matrix whose entries can be chosen real and positive with $y_{1}^{u}<y_{2}^{u}<y_{3}^{u}$, and similarly for $D_{Y}^{d}$. $V_{R}^{u, d}$ and $V_{L}^{u, d}$ are $3 \times 3$ unitary matrices that depend on three real parameters and six phases. The unitary matrices $V_{R}^{u, d}$ can be absorbed in the definition of the right handed fields without any physical effect. In neutral currents the left rotations cancel out via the GlashowIliopoulos-Maiani (GIM) mechanism [203]. On the other hand, the redefinition of the left handed fields produces flavor mixing in the charged currents. In the physical basis where both the up and down Yukawa couplings are simultaneously diagonal, the charged current reads

$J_{c c}^{\mu}=u_{L}^{c} \frac{\gamma^{\mu}\left(1-\gamma_{5}\right)}{2}\left(V_{L}^{u \dagger} V_{L}^{d}\right) d_{L}$.

The matrix $V_{L}^{u \dagger} V_{L}^{d}$ can be generically written as $V_{L}^{u \dagger} V_{L}^{d}=$ $\Phi_{1} U_{\mathrm{CKM}} \Phi_{2}$, where $\Phi_{1,2}$ are diagonal unitary matrices (thus, containing only phases) that can be absorbed by appropriate redefinitions of the left handed fields. Finally, $U_{\mathrm{CKM}}$ depends on three angles and one phase that cannot be removed by field redefinitions and accounts for the physical mixing between quark generations and the CP violation [204, 205]. It is usually parameterized thus:

$U_{\mathrm{CKM}}=\left(\begin{array}{ccc}c_{13} c_{12} & c_{13} s_{12} & s_{13} e^{-i \delta} \\ -c_{23} s_{12}-s_{23} s_{13} c_{12} e^{i \delta} & c_{23} c_{12}-s_{23} s_{13} s_{12} e^{i \delta} & s_{23} c_{13} \\ s_{23} s_{12}-c_{23} s_{13} c_{12} e^{i \delta} & -s_{23} c_{12}-c_{23} s_{13} s_{12} e^{i \delta} & c_{23} c_{13}\end{array}\right)$,

where $s_{i j}=\sin \theta_{i j}, c_{i j}=\cos \theta_{i j}$ and $\delta$ is the CP-violating phase. Experiments show a hierarchical structure in the offdiagonal entries of the CKM matrix: $\left|V_{u b}\right| \ll V_{c b} \ll V_{u s}$, that can be well described by the following phenomenological parameterization of the CKM matrix, proposed by Wolfenstein [206]. It reads

$$
\begin{aligned}
U_{\mathrm{CKM}}= & \left(\begin{array}{ccc}
1-\frac{\lambda^{2}}{2} & \lambda & A \lambda^{3}(\rho-i \eta) \\
-\lambda & 1-\frac{\lambda^{2}}{2} & A \lambda^{2} \\
A \lambda^{3}(1-\rho-i \eta) & -A \lambda^{2} & 1
\end{array}\right) \\
& +\mathcal{O}\left(\lambda^{4}\right),
\end{aligned}
$$

where $\lambda$ is determined with a very good precision in semileptonic $K$ decays, giving $\lambda \simeq 0.23$, and $A$ is measured in semileptonic $B$ decays, giving $A \simeq 0.82$. The parameters $\rho$ and $\eta$ are more poorly measured, although a rough estimate is $\rho \simeq 0.1, \eta \simeq 0.3$ [207].

\subsubsection{Leptonic sector with Dirac neutrinos}

A Dirac mass term for the neutrinos requires the existence of three right handed neutrinos, which are singlets under the standard model gauge group. In consequence, the leptonic
Lagrangian would contain in general a Majorana mass term for the right handed neutrinos, which has to be forbidden by imposing exact lepton number conservation. Then the leptonic Lagrangian reads

$\mathcal{L}_{\text {lep }}=e_{R i}^{c} Y_{i j}^{e} L_{j} \bar{H}+v_{R i}^{c} Y_{i j}^{v} L_{j} H+$ h.c.,

where $L_{i}=\left(v_{L i}, e_{L i}\right)$ are the left handed lepton doublets and $e_{R}^{c}$ and $\nu_{R}^{c}$ are respectively the right handed charged lepton and neutrino singlets. Analogously to the quark sector, the Yukawa couplings can be decomposed as

$Y^{e}=V_{R}^{e} D_{Y}^{e} V_{L}^{e^{\dagger}}$,

$Y^{\nu}=V_{R}^{v} D_{Y}^{v} V_{L}^{\nu \dagger}$,

where $V_{R}^{e, v}$ do not have any physical effect, whereas the $V_{L}^{e, v}$ have an effect in the charged current, that in the basis where the charged lepton and neutrino Yukawa couplings are simultaneously diagonal reads

$J_{c c}^{\mu}=e_{L}^{c} \frac{\gamma^{\mu}\left(1-\gamma_{5}\right)}{2}\left(V_{L}^{e^{\dagger}} V_{L}^{\nu}\right) v_{L}$.

As in the case of the quark sector, the matrix $V_{L}^{e \dagger} V_{L}^{v}$ depends on three angles and six phases and can be expressed as $V_{L}^{e \dagger} V_{L}^{v}=\Phi_{1} U_{\mathrm{PMNS}} \Phi_{2}$. The matrices $\Phi_{1}$ and $\Phi_{2}$ can be absorbed by appropriate redefinitions of the left handed fields, yielding a physical mixing matrix $U_{\text {PMNS }}[208,209]$ that depends on three angles and one phase, and that can be parameterized by the same structure as for the quark sector, (3.31). However, the values for the angles differ substantially from the quark sector. The experimental values that result from the global fit are $\sin ^{2} \theta_{12}=0.26-0.36$, $\sin ^{2} \theta_{23}=0.38-0.63$ and $\sin ^{2} \theta_{13} \leq 0.025$ at $2 \sigma[210,211]$. On the other hand, the CP-violating phase $\delta$ is completely unconstrained by present experiments.

In the theory under discussion the total lepton number $L=L_{e}+L_{\mu}+L_{\tau}$ is conserved, but the individual lepton flavors $L_{l}, l=e, \mu, \tau$, are not, and LFV processes like $\mu^{-} \rightarrow e^{-} \gamma$ decay are allowed. For the neutrino masses $m_{v_{j}}$, $j=1,2,3$, satisfying the existing upper limits obtained in ${ }^{3} \mathrm{H} \beta$-decay experiments, $m_{j}<2.3 \mathrm{eV}$, the $\mu^{-} \rightarrow e^{-} \gamma$ decay branching ratio is given by [212]

$B(\mu \rightarrow e \gamma)=\frac{3 \alpha}{32 \pi}\left|\sum_{j} U_{e j}^{\mathrm{PMNS}} U_{\mu j}^{\mathrm{PMNS} *} \frac{m_{v_{j}}^{2}}{M_{W}^{2}}\right|^{2}$,

where $M_{W}$ is the $W^{ \pm}$-boson mass. Thus, the $\mu^{-} \rightarrow e^{-} \gamma$ decay rate is suppressed by the factor $\left(m_{j} / M_{W}\right)^{4}<6.7 \times$ $10^{-43}$, which renders it unobservable. The same conclusion is valid for all other LFV decays and reactions in the minimal extension of the standard theory with light neutrino masses we are considering. The only observable manifestation of the non-conservation of the lepton charges $L_{l}$ in this theory is the oscillations of neutrinos. 


\subsubsection{Leptonic sector with Majorana neutrinos}

Neutrino masses can also be accommodated in the standard model without extending the particle content, just by adding a dimension five operator to the leptonic Lagrangian [213]:

$\mathcal{L}_{\text {lep }}=e_{R i}^{c} Y_{i j}^{e} L_{j} \bar{H}+\frac{1}{4} \kappa_{i j}\left(L_{i} H\right)\left(L_{j} H\right)+$ h.c.

with $\kappa$ a $3 \times 3$ complex symmetric matrix that breaks explicitly lepton number and that has dimensions of mass ${ }^{-1}$. Then, after the electroweak symmetry breaking, a Majorana mass term for neutrinos is generated:

$m_{v}=\frac{1}{2} \kappa\left\langle H^{0}\right\rangle^{2}$

This term can be diagonalized as $m_{v}=V_{L}^{v *} D_{m_{v}} V_{L}^{v^{\dagger}}$, so that the charged current reads as in (3.36), with $V_{L}^{e^{\dagger}} V_{L}^{v}=$ $\Phi_{1} U \Phi_{2}$, where the matrix $U$ has the form of the CKM matrix, (3.31). The matrix $\Phi_{1}$ containing three phases can be removed by a redefinition of the left handed charged lepton fields. However, due to the Majorana nature of the neutrinos, the matrix $\Phi_{2}$ cannot be removed and is physical, yielding a leptonic mixing matrix $U_{\mathrm{PMNS}}=U \Phi_{2}$ that is defined by three angles and three phases [214, 215], one associated to $U$, the "Dirac phase", and two associated to $\Phi_{2}$, the "Majorana phases".

In the leptonic Lagrangian given by (3.38) the origin of the dimension five operator remains open. In the rest of this section, we shall review the heavy Majorana singlet (right handed) neutrino mass mechanism (type I see-saw) [216220] and the triplet Higgs mass mechanism (type II see-saw) [215, 221-224] as the possible origins of this effective operator. The third [225] tree level realization of the operator (3.38) via triplet fermion (type III see-saw) [226] is discussed in Sect. 4.1.

3.2.3.1 Type I see-saw In the presence of singlet right handed neutrinos, the most general Lagrangian compatible with the standard model gauge symmetry reads

$\mathcal{L}_{\text {lep }}=e_{R i}^{c} Y_{i j}^{e} L_{j} \bar{H}+v_{R i}^{c} Y_{i j}^{v} L_{j} H-\frac{1}{2} v_{R i}^{c T} M_{i j} v_{R j}^{c}+$ h.c.,

where lepton number is explicitly broken by the Majorana mass term for the singlet right handed neutrinos. ${ }^{9}$ The seesaw mechanism is implemented when $\operatorname{eig}(M) \gg\left\langle H^{0}\right\rangle$. If this is the case, at low energies the right handed neutrinos are decoupled and the theory can be well described by the

\footnotetext{
${ }^{9}$ Here we explicitly assume three generations of singlet neutrinos. For the phenomenology of a large number of singlets as predicted by string theories, see [227, 228].
}

effective Lagrangian for Majorana neutrinos, (3.38), with [216-220]

$\kappa=2 Y^{\nu T} M^{-1} Y^{\nu}$

Working in the basis where the charged lepton Yukawa matrix and the right handed mass matrix are simultaneously diagonal, it can be checked that the complete Lagrangian, (3.40), contains fifteen independent real parameters and six complex phases [229]. Of these, three correspond to the charged lepton masses, three to the right handed masses, and the remaining nine real parameters and six phases, to the neutrino Yukawa coupling. The independent parameters of the neutrino Yukawa coupling can be expressed in several ways. The most straightforward parameterization uses the singular value decomposition of the neutrino Yukawa matrix:

$Y_{v}=V_{R}^{v} D_{Y}^{v} V_{L}^{v \dagger}$

where $D_{Y}^{v}=\operatorname{diag}\left(y_{1}^{v}, y_{2}^{v}, y_{3}^{v}\right)$, with $y_{i}^{v} \geq 0$ and $y_{1}^{v} \leq y_{2}^{v} \leq$ $y_{3}^{v}$. On the other hand, $V_{L}^{v}$ and $V_{R}^{v}$ are $3 \times 3$ unitary matrices, that depend in general on three real parameters and six phases. Both can be generically written as $\Phi_{1} V \Phi_{2}$, where $V$ has the form of the CKM matrix and $\Phi_{1,2}$ are diagonal unitary matrices (thus, containing only phases). One can check that for $V_{R}^{v}$ the $\Phi_{2}$ matrix can be absorbed into the definition of $V_{L}^{v}$, so that

$$
\begin{aligned}
V_{R}^{v}= & \left(\begin{array}{ccc}
e^{i \alpha_{1}^{R}} & & \\
& e^{i \alpha_{2}^{R}} & \\
& & 1
\end{array}\right) \\
& \times\left(\begin{array}{ccc}
c_{2}^{R} c_{3}^{R} & c_{2}^{R} s_{3}^{R} & s_{2}^{R} e^{-i \delta^{R}} \\
-c_{1}^{R} s_{3}^{R}-s_{1}^{R} s_{2}^{R} c_{3}^{R} e^{i \delta^{R}} & c_{1}^{R} c_{3}^{R}-s_{1}^{R} s_{2}^{R} s_{3}^{R} e^{i \delta^{R}} & s_{1}^{R} c_{2}^{R} \\
s_{1}^{R} s_{3}^{R}-c_{1}^{R} s_{2}^{R} c_{3}^{R} e^{i \delta^{R}} & -s_{1}^{R} c_{3}^{R}-c_{1}^{R} s_{2}^{R} s_{3}^{R} e^{i \delta^{R}} & c_{1}^{R} c_{2}^{R}
\end{array}\right) .
\end{aligned}
$$

Similarly, for $V_{L}$ the $\Phi_{1}$ matrix can be absorbed into the definition of $L$ and $e_{R}$, while keeping $Y_{e}$ diagonal and real. In consequence,

$$
\begin{aligned}
& V_{L}^{v}=\left(\begin{array}{ccc}
c_{2}^{L} c_{3}^{L} & c_{2}^{L} s_{3}^{L} & s_{2}^{L} e^{-i \delta^{L}} \\
-c_{1}^{L} s_{3}^{L}-s_{1}^{L} s_{2}^{L} c_{3}^{L} e^{i \delta^{L}} & c_{1}^{L} c_{3}^{L}-s_{1}^{L} s_{2}^{L} s_{3}^{L} e^{i \delta^{L}} & s_{1}^{L} c_{2}^{L} \\
s_{1}^{L} s_{3}^{L}-c_{1}^{L} s_{2}^{L} c_{3}^{L} e^{i \delta^{L}} & -s_{1}^{L} c_{3}^{L}-c_{1}^{L} s_{2}^{L} s_{3}^{L} e^{i \delta^{L}} & c_{1}^{L} c_{2}^{L}
\end{array}\right) \\
& \times\left(\begin{array}{ccc}
e^{i \alpha_{1}^{L}} & & \\
& e^{i \alpha_{2}^{L}} & \\
& & 1
\end{array}\right)
\end{aligned}
$$

Therefore, in this parameterization the independent parameters in the Yukawa coupling can be identified with the three Yukawa eigenvalues, $y_{i}$, the three angles and three phases in $V_{L}$, and the three angles and three phases in 
$V_{R}$ [229-231]. The requirement that the low energy phenomenology is successfully reproduced imposes constraints among these parameters. To be precise, the low energy leptonic Lagrangian depends just on the three charged lepton masses and the six real parameters and three complex phases of the effective neutrino mass matrix. In consequence, there are still six real parameters and three complex phases that are not determined by low energy neutrino data; this information about the high energy Lagrangian is "lost" in the decoupling of the three right handed neutrinos and cannot be recovered just from neutrino experiments.

The ambiguity in the determination of the high energy parameters can be encoded in the three right handed neutrino masses and an orthogonal complex matrix $R$ defined as [232]

$R=D_{\sqrt{M}}^{-1} \mathbf{Y}_{\nu} U_{\mathrm{PMNS}} D_{\sqrt{m}}^{-1}\left\langle H^{0}\right\rangle$,

so that the most general Yukawa coupling compatible with the low energy data is given by:

$Y^{v}=D_{\sqrt{M}} R D_{\sqrt{m}} U_{\mathrm{PMNS}}^{\dagger}\left\langle H^{0}\right\rangle$.

It is straightforward to check that this equation indeed satisfies the seesaw formula, (3.41). In this expression, $D_{\sqrt{m}}$ and $D_{\sqrt{M}}$ are diagonal matrices whose entries are the square roots of the light neutrino and the right handed neutrino masses, respectively, and $U_{\mathrm{PMNS}}$ is the leptonic mixing matrix. It is customary to parameterize $R$ in terms of three complex angles, $\hat{\theta}_{i}$ :

$R=\left(\begin{array}{ccc}\hat{c}_{2} \hat{c}_{3} & -\hat{c}_{1} \hat{s}_{3}-\hat{s}_{1} \hat{s}_{2} \hat{c}_{3} & \hat{s}_{1} \hat{s}_{3}-\hat{c}_{1} \hat{s}_{2} \hat{c}_{3} \\ \hat{c}_{2} \hat{s}_{3} & \hat{c}_{1} \hat{c}_{3}-\hat{s}_{1} \hat{s}_{2} \hat{s}_{3} & -\hat{s}_{1} \hat{c}_{3}-\hat{c}_{1} \hat{s}_{2} \hat{s}_{3} \\ \hat{s}_{2} & \hat{s}_{1} \hat{c}_{2} & \hat{c}_{1} \hat{c}_{2}\end{array}\right)$,

up to reflections, where $\hat{c}_{i} \equiv \cos \hat{\theta}_{i}, \hat{s}_{i} \equiv \sin \hat{\theta}_{i}$.

Whereas the physical interpretation of the right handed masses is very transparent, the meaning of $R$ is more obscure. $R$ can be interpreted as a dominance matrix in the sense that [233]

- $R$ is an orthogonal transformation from the basis of the left handed leptons mass eigenstates to the one of the right handed neutrino mass eigenstates;

- if and only if an eigenvalue $m_{i}$ of $m_{v}$ is dominated-in the sense already given before - by one right handed neutrino eigenstate $N_{j}$, then $\left|R_{j i}\right| \approx 1$;

- if a light pseudo-Dirac pair is dominated by a heavy pseudo-Dirac pair, then the corresponding $2 \times 2$ sector in $R$ is a boost.

An interesting limit of this dominance behavior is the seesaw model with two right handed neutrinos (2RHN) $[234,235]$. In this limit, the parameterization (3.46) still holds, with the substitutions $D_{\sqrt{M}}=\operatorname{diag}\left(M_{1}^{-1}, M_{2}^{-1}\right)$ and [236-239]

$R=\left(\begin{array}{ccc}0 & \cos \hat{\theta} & \xi \sin \hat{\theta} \\ 0 & -\sin \hat{\theta} & \xi \cos \hat{\theta}\end{array}\right) \quad$ (normal hierarchy),

$R=\left(\begin{array}{ccc}\cos \hat{\theta} & \xi \sin \hat{\theta} & 0 \\ -\sin \hat{\theta} & \xi \cos \hat{\theta} & 0\end{array}\right) \quad$ (inverted hierarchy)

with $\hat{\theta}$ a complex parameter and $\xi= \pm 1$ a discrete parameter that accounts for a discrete indeterminacy in $R$.

A third possible parameterization of the neutrino Yukawa coupling uses the Gram-Schmidt decomposition, in order to cast the Yukawa coupling as a product of a unitary matrix and a lower triangular matrix [240]:

$Y^{v}=U_{\triangle} Y_{\triangle}=U_{\Delta}\left(\begin{array}{ccc}y_{11} & 0 & 0 \\ y_{21} & y_{22} & 0 \\ y_{31} & y_{32} & y_{33}\end{array}\right)$,

where the diagonal elements of $Y_{\triangle}$ are real. Three of the six phases in $U_{\triangle}$ can be absorbed into the definition of the charged leptons. Therefore, the nine real parameters and the six phases of the neutrino Yukawa coupling are identified with the three angles and three phases in $U_{\triangle}$ and the six real parameters and three phases in $Y_{\triangle}$.

In the SM extended with right handed neutrinos, the charged lepton masses and the effective neutrino mass matrix are the only source of information about the leptonic sector. However, if supersymmetry is discovered, the structure of the low energy slepton mass matrices would provide additional information about the leptonic sector, provided the mechanism of supersymmetry breaking is specified. Assuming that the slepton mass matrices are proportional to the identity at the high energy scale, quantum effects induced by the right handed neutrinos would yield at low energies a left handed slepton mass matrix with a complicated structure, whose measurement would provide additional information about the seesaw parameters [144, 145]. To be more specific, in the minimal supersymmetric seesaw model the offdiagonal elements of the low energy left handed and right handed slepton mass matrices and $A$-terms read, in the leading log approximation [178]

$\left(m_{\tilde{L}}^{2}\right)_{i j} \simeq-\frac{1}{8 \pi^{2}}\left(3 m_{0}^{2}+A_{0}^{2}\right) Y_{i k}^{v \dagger} Y_{k j}^{v} \log \frac{M_{X}}{M_{k}}$,

$\left(m_{\tilde{e}_{R}}^{2}\right)_{i j} \simeq 0$,

$\left(A_{e}\right)_{i j} \simeq-\frac{3}{8 \pi^{2}} A_{0} Y_{e} Y_{i k}^{v \dagger} Y_{k j}^{v} \log \frac{M_{X}}{M_{k}}$,

where $m_{0}$ and $A_{0}$ are the universal soft supersymmetry breaking parameters at high scale $M_{X}$. Note that the diagonal elements of those mass matrices include the tree level soft mass matrix, the radiative corrections from gauge and 
charged lepton Yukawa interactions, and the mass contributions from F- and D-terms (which are different for charged sleptons and sneutrinos). Therefore, the measurement at low energies of rare lepton decays, electric dipole moments and slepton mass splittings would provide information about the combination

$C_{i j} \equiv \sum_{k} Y_{i k}^{v \dagger} Y_{k j}^{v} \log \frac{M_{X}}{M_{k}} \equiv\left(Y_{v}^{\dagger} L Y_{v}\right)_{i j}$

where $L_{i j}=\log \frac{M_{X}}{M_{i}} \delta_{i j}$.

Interestingly enough, $C$ encodes precisely the additional information needed to reconstruct the complete seesaw Lagrangian from low energy observations [241, 242] (note in particular that $C$ is a Hermitian matrix that depends on six real parameters and three phases, which together with the nine real parameters and three phases of the neutrino mass matrix sum up to the independent fifteen real parameters and six complex phases in $Y_{v}$ and $M$ ).

To determine $Y_{v}$ and $M$ from the low energy observables $C$ and $m_{v}$, it is convenient to define

$\tilde{Y}^{v}=\operatorname{diag}\left(\sqrt{\log \frac{M_{X}}{M_{1}}}, \sqrt{\log \frac{M_{X}}{M_{2}}}, \sqrt{\log \frac{M_{X}}{M_{3}}}\right) Y^{v}$

$\tilde{M}_{k}=M_{k} \log \frac{M_{X}}{M_{k}}$

so that the effective neutrino mass matrix and $C$ now read

$m_{v}=\tilde{Y}^{v t} \operatorname{diag}\left(\tilde{M}_{1}^{-1}, \tilde{M}_{2}^{-1}, \tilde{M}_{3}^{-1}\right) \tilde{Y}^{v}\left\langle H_{u}^{0}\right\rangle^{2}$,

$C=\tilde{Y}^{\nu \dagger} \tilde{Y}^{v}$,

where $H_{u}^{0}$ is the neutral component of the up-type Higgs doublet. Using the singular value decomposition $\tilde{Y}^{v}=$ $\tilde{V}_{R}^{v} \tilde{D}_{Y}^{v} \tilde{V}_{L}^{v \dagger}$, one finds that $\tilde{V}_{L}^{v \dagger}$ and $\tilde{D}_{Y}^{v}$ could be straightforwardly determined from $C$, since

$C \equiv \tilde{Y}^{\nu \dagger} \tilde{Y}^{v}=\tilde{V}_{L}^{\dagger} \tilde{D}_{Y}^{2} \tilde{V}_{L}$

On the other hand, from $m_{v}=\tilde{Y}^{v t} \tilde{D}_{M}^{-1} \tilde{Y}^{v}\left\langle H_{u}^{0}\right\rangle^{2}$ and the singular value decomposition of $\tilde{Y}^{v}$,

$\tilde{D}_{Y}^{-1} \tilde{V}_{L}^{*} m_{v} \tilde{V}_{L}^{\nu \dagger} \tilde{D}_{Y}^{\nu-1}=\tilde{V}_{R}^{\nu *} \tilde{D}_{M}^{-1} \tilde{V}_{R}^{\nu \dagger}$

where the left hand side of this equation is known $\left(m_{v}\right.$ is one of our inputs, and $\tilde{V}_{L}^{v}$ and $\tilde{D}_{Y}^{v}$ were obtained from (3.57)). Therefore, $\tilde{V}_{R}^{v}$ and $\tilde{D}_{M}$ can also be determined. This simple procedure shows that starting from the low energy observables $m_{v}$ and $C$ it is possible to determine uniquely the matrices $\tilde{D}_{M}$ and $\tilde{Y}^{v}=\tilde{V}_{R}^{v} \tilde{D}_{Y}^{v} \tilde{V}_{L}^{v \dagger}$. Finally, inverting (3.56), the actual parameters of the Lagrangian $M_{k}$ and $Y^{v}$ can be computed.
This procedure is particularly powerful in the case of the two right handed neutrino model, as the number of independent parameters involved (either at high energies or at low energies) is drastically reduced. The matrix $C$ defined in (3.54) depends in general on six moduli and three phases. However, since the Yukawa coupling depends in the 2RHN model on only three unknown moduli and one phase, so does $C$, and consequently it is possible to obtain predictions on the moduli of three $C$-matrix elements and the phases of two $C$-matrix elements. Namely, from (3.46) one obtains

$U^{\dagger} C U=U^{\dagger} \tilde{Y}^{\nu \dagger} \tilde{Y}^{v} U=D_{\sqrt{m}} R^{\dagger} \tilde{D}_{M} R D_{\sqrt{m}} /\left\langle H_{u}^{0}\right\rangle^{2}$,

where we have written $U \equiv U_{\text {PMNS. Since }} m_{1}=0$ in the 2RHN model, ${ }^{10}$ it follows that $\left(U^{\dagger} C U\right)_{1 i}=0$, for $i=$ $1,2,3$, leading to three relations among the elements in $C$. For instance, one could derive the diagonal elements in $C$ in terms of the off-diagonal elements:

$C_{11}=-\frac{C_{12}^{*} U_{21}^{*}+C_{13}^{*} U_{31}^{*}}{U_{11}^{*}}$,

$C_{22}=-\frac{C_{12} U_{11}^{*}+C_{23}^{*} U_{31}^{*}}{U_{21}^{*}}$,

$C_{33}=-\frac{C_{13} U_{11}^{*}+C_{23} U_{21}^{*}}{U_{31}^{*}}$.

The observation of these correlations would be non-trivial tests of the 2 RHN model.

The relations for the phases arise from the hermiticity of $C$, since the diagonal elements in $C$ have to be real. Taking as the independent phase the argument of $C_{12}$, one can derive from (3.60) the arguments of the remaining elements:

$$
\begin{aligned}
e^{i \arg C_{13}}= & {\left[-i \operatorname{Im}\left(C_{12} U_{21} U_{11}^{*}\right)\right.} \\
& \left. \pm \sqrt{\left|C_{13}\right|^{2}\left|U_{11}\right|^{2}\left|U_{31}\right|^{2}-\left[\operatorname{Im}\left(C_{12} U_{21} U_{11}^{*}\right)\right]^{2}}\right] \\
& \times\left[\left|C_{13}\right| U_{31} U_{11}^{*}\right]^{-1}, \\
e^{i \arg C_{23}}= & {\left[i \operatorname{Im}\left(C_{12} U_{21} U_{11}^{*}\right)\right.} \\
& \left. \pm \sqrt{\left|C_{23}\right|^{2}\left|U_{21}\right|^{2}\left|U_{31}\right|^{2}-\left[\operatorname{Im}\left(C_{12} U_{21} U_{11}^{*}\right)\right]^{2}}\right] \\
& \times\left[\left|C_{23}\right| U_{31} U_{21}^{*}\right]^{-1},
\end{aligned}
$$

where the \pm sign has to be chosen so that the eigenvalues of $C$ are positive. We conclude then that the $C$-matrix parameters $C_{12},\left|C_{13}\right|$ and $\left|C_{23}\right|$ can be regarded as independent and can be used as an alternative parameterization of the

\footnotetext{
${ }^{10}$ Here we are assuming a neutrino spectrum with normal hierarchy. In the case with inverted hierarchy, the analysis is similar, using $m_{3}=0$.
} 
2RHN model [243]. Together with the five moduli and the two phases of the neutrino mass matrix, we sum up to the eight moduli and the three phases necessary to reconstruct the high energy Lagrangian of the $2 \mathrm{RHN}$ model.

3.2.3.2 Type II seesaw The type II seesaw mechanism $[215,221-224]$ consists on adding to the SM particle content a Higgs triplet

$T=\left(\begin{array}{cc}T^{0} & -\frac{1}{\sqrt{2}} T^{+} \\ -\frac{1}{\sqrt{2}} T^{+} & -T^{++}\end{array}\right)$.

Then, the leptonic potential compatible with the SM gauge symmetry reads

$\mathcal{L}_{\text {lep }}=e_{R i}^{c} Y_{i j}^{e} L_{j} \bar{H}+Y_{i j}^{T} L_{i} T L_{j}+$ h.c.

From this Lagrangian, it is apparent that the triplet $T$ carries lepton number -2 . If the neutral component of the triplet acquires a VEV and breaks lepton number spontaneously as happens in the Gelmini-Roncadelli model [224], the associated massless majoron rules out the model. Therefore phenomenology suggests to break lepton number explicitly via the triplet coupling to the SM Higgs boson [244]. The most general scalar potential involving one Higgs doublet and one Higgs triplet reads

$$
\begin{aligned}
V= & m_{H}^{2} H^{\dagger} H+\frac{1}{2} \lambda_{1}\left(H^{\dagger} H\right)^{2}+M_{T}^{2} T^{\dagger} T+\frac{1}{2} \lambda_{2}\left(T^{\dagger} T\right)^{2} \\
& +\lambda_{3}\left(H^{\dagger} H\right)\left(T^{\dagger} T\right)+\mu^{\prime} H^{\dagger} T H^{\dagger},
\end{aligned}
$$

where the term proportional to $\mu^{\prime}$ breaks lepton number explicitly. The type II seesaw mechanism is implemented when $M_{T} \gg\left\langle H^{0}\right\rangle$. Then the minimization of the scalar potential yields

$\left\langle H^{0}\right\rangle^{2} \simeq \frac{-m_{H}^{2}}{\lambda_{1}-2 \mu_{\Psi}^{2} / M_{T}^{2}}, \quad\left\langle T^{0}\right\rangle \simeq \frac{-\mu^{\prime}\left\langle H^{0}\right\rangle^{2}}{M_{T}^{2}}$,

which produce Majorana masses for the neutrinos given by

$m_{v}=Y_{T} \frac{-\mu^{\prime}\left\langle H^{0}\right\rangle^{2}}{M_{T}^{2}}$.

The Yukawa matrix $Y^{T}$ has the same flavor structure as the non-renormalizable operator $\kappa$ defined in (3.38) for the effective Lagrangian of Majorana neutrinos. Therefore, the parameterization of the type II seesaw model is completely identical to that case.

Supersymmetric models with low scale triplet Higgses have been extensively considered in studies of collider phenomenology [245-247]. The model [244] was first supersymmetrized in Ref. [248] as a possible scenario for leptogenesis. The requirement of a holomorphic superpotential implies introducing the triplets in a vector-like $S U(2)_{W} \times$ $U(1)_{Y}$ representation, as $T \sim(3,1)$ and $\bar{T} \sim(3,-1)$. The relevant superpotential terms are

$$
\begin{aligned}
& \frac{1}{\sqrt{2}} Y_{T}^{i j} L_{i} T L_{j}+\frac{1}{\sqrt{2}} \lambda_{1} H_{1} T H_{1}+\frac{1}{\sqrt{2}} \lambda_{2} H_{2} \bar{T} H_{2} \\
& \quad+M_{T} T \bar{T}+\mu H_{2} H_{1},
\end{aligned}
$$

where $L_{i}$ are the $S U(2)_{W}$ lepton doublets and $H_{1}\left(H_{2}\right)$ is the Higgs doublet with hypercharge $Y=-1 / 2(1 / 2)$. Decoupling the triplet at high scale at the electroweak scale the Majorana neutrino mass matrix is given by $\left(v_{2}=\left\langle H_{2}\right\rangle\right)$

$m_{v}^{i j}=Y_{T}^{i j} \frac{v_{2}^{2} \lambda_{2}}{M_{T}}$.

Note that in the supersymmetric case there is only one mass parameter, $M_{T}$, while the mass parameter $\mu^{\prime}$ of the nonsupersymmetric version is absent.

The couplings $Y_{T}$ also induce LFV in the slepton mass matrix $m_{\tilde{L}}^{2}$ through renormalization group (RG) running from $M_{X}$ to the decoupling scale $M_{T}$ [249]. In the leadinglogarithm approximation those are given by $(i \neq j)$ :

$$
\begin{aligned}
& \left(m_{\tilde{L}}^{2}\right)_{i j} \approx \frac{-1}{8 \pi^{2}}\left(9 m_{0}^{2}+3 A_{0}^{2}\right)\left(Y_{T}^{\dagger} Y_{T}\right)_{i j} \log \frac{M_{X}}{M_{T}}, \\
& \left(m_{\tilde{e}_{R}}^{2}\right)_{i j} \approx 0, \\
& \left(A_{e}\right)_{i j} \approx \frac{-9}{16 \pi^{2}} A_{0}\left(Y_{e} Y_{T}^{\dagger} Y_{T}\right)_{i j} \log \frac{M_{X}}{M_{T}} .
\end{aligned}
$$

Phenomenological implications of those relations will be presented in Sect. 5.

3.2.3.3 Renormalization of the neutrino mass matrix To make a connection between high scale parameters and low scale observables one needs to consider renormalization effects on neutrino masses and mixing. Below the scale where the dimension five operator is generated, the running of the neutrino mass matrix is governed by the renormalization group (RG) equation of the coupling matrix $\kappa_{v}$, given by [250-253]

$$
(4 \pi)^{2} \frac{d}{d \ln \mu} \kappa_{\nu}=(4 \pi)^{2} A_{g} \kappa_{v}+C_{e}\left(\left(Y_{e}^{\dagger} Y_{e}\right)^{\top} \kappa_{v}+\kappa_{\nu} Y_{e}^{\dagger} Y_{e}\right),
$$

where $C_{e}=-3 / 2$ for the SM and $C_{e}=1$ for the MSSM. The first term does not affect the running of the neutrino mixing angles and $\mathrm{CP}$ violation phases; however, it affects of course the running of the neutrino mass eigenvalues. The 
flavor universal factor $A_{g}$ is given by

$A_{g}=\left\{\begin{array}{l}-3 \alpha_{2}(4 \pi)+\lambda+2 \operatorname{tr}\left(3 Y_{u}^{\dagger} Y_{u}+3 Y_{d}^{\dagger} Y_{d}+Y_{e}^{\dagger} Y_{e}\right) \\ \mathrm{SM}, \\ -2 \alpha_{1}(4 \pi)-6 \alpha_{2}(4 \pi)+\operatorname{tr}\left(Y_{u}^{\dagger} Y_{u}\right) \quad \mathrm{MSSM},\end{array}\right.$

where $\lambda$ denotes the Higgs self-coupling constant and $\alpha_{i}=$ $g_{i}^{2} /(4 \pi)$, where $g_{1}$ and $g_{2}$ are the $U(1)_{Y}$ and $S U(2)$ gauge coupling constants, respectively.

Due to the smallness of the tau-Yukawa coupling in the $\mathrm{SM}$, the mixing angles are not affected significantly by the renormalization group running below the generation scale of the dimension five operator. However, if the neutrino mass matrix $m_{v}=\frac{\langle\phi\rangle^{2}}{2} \kappa_{v}$ is realized in the seesaw scenario (type I), running effects above and between the seesaw scales can also lead to relevant running effects in the $\mathrm{SM}$. Note that in the MSSM case the running of the mixing angles and $\mathrm{CP}$ violation phases can be large even below the seesaw scales due to the possible enhancement of the tauYukawa coupling by the factor $\left(1+\tan \beta^{2}\right)^{1 / 2}$.

In order to understand generic properties of the RG evolution and to estimate the typical size of the RG effects, it is useful to consider RGEs for the leptonic mixing angles, $\mathrm{CP}$ phases and neutrino masses themselves, which can be derived from the RGE in (3.70). For example, below the seesaw scales, up to $\mathcal{O}\left(\theta_{13}\right)$ corrections, the evolution of the mixing angles in the MSSM is given by [254] (see also $[255,256])$

$$
\begin{aligned}
\frac{d \theta_{12}}{d \ln \mu}= & \frac{-y_{\tau}^{2}}{32 \pi^{2}} \sin 2 \theta_{12} s_{23}^{2} \frac{\left|m_{1} e^{i \alpha_{M}}+m_{2}\right|^{2}}{\Delta m_{21}^{2}}, \\
\frac{d \theta_{13}}{d \ln \mu}= & \frac{y_{\tau}^{2}}{32 \pi^{2}} \sin 2 \theta_{12} \sin 2 \theta_{23} \frac{m_{3}}{\Delta m_{31}^{2}(1+\zeta)} \\
& \times I\left(m_{1}, m_{2}, \alpha_{M}, \beta_{M}, \delta\right), \\
\frac{d \theta_{23}}{d \ln \mu}= & \frac{-y_{\tau}^{2}}{32 \pi^{2}} \frac{\sin 2 \theta_{23}}{\Delta m_{31}^{2}}\left[c_{12}^{2}\left|m_{2} e^{i \beta_{M}}+m_{3} e^{i \alpha_{M}}\right|^{2}\right. \\
& \left.+s_{12}^{2} \frac{\left|m_{1} e^{i \beta_{M}}+m_{3}\right|^{2}}{1+\zeta}\right],
\end{aligned}
$$

where $I\left(m_{1}, m_{2}, \alpha_{M}, \beta_{M}, \delta\right) \equiv m_{1} \cos \left(\beta_{M}-\delta\right)-(1+\zeta) \times$ $m_{2} \cos \left(\alpha_{M}-\beta_{M}+\delta\right)-\zeta m_{3} \cos \delta, s_{i j}=\sin \theta_{i j}, c_{i j}=$ $\cos \theta_{i j}$, and $\zeta=\Delta m_{21}^{2} / \Delta m_{31}^{2}$. Here $y_{\tau}$ denotes the tauYukawa coupling, and one can safely neglect the contributions coming from the electron- and muon-Yukawa couplings. For the matrix $P$ containing the Majorana phases, we use the convention $P=\operatorname{diag}\left(1, e^{i \alpha_{M} / 2}, e^{i \beta_{M} / 2}\right)$. In addition to the above formulae, formulae for the running of the $\mathrm{CP}$ phases have been derived [254]. For example, the running of the Dirac CP-violating phase $\delta$, observable in neutrino oscillation experiments, is given by

$$
\frac{d \delta}{d \ln \mu}=\frac{C y_{\tau}^{2}}{32 \pi^{2}} \frac{\delta^{(-1)}}{\theta_{13}}+\frac{C y_{\tau}^{2}}{8 \pi^{2}} \delta^{(0)}+\mathcal{O}\left(\theta_{13}\right) .
$$

The coefficients $\delta^{(-1)}$ and $\delta^{(0)}$ are omitted here and can be found in [254], where also formulae for the running of the Majorana CP phases and for the neutrino mass eigenvalues (mass squared differences) can be found. From (3.75), it can be seen that the Dirac CP phase generically becomes more unstable under RG corrections for smaller $\theta_{13}$.

In the seesaw scenario (type I), the SM or MSSM are extended by heavy right handed neutrinos and their superpartners, which are SM gauge singlets. Integrating them out below their mass scales $M_{R}$ yields the dimension five operator for neutrino masses in the SM or MSSM. Above $M_{R}$, the neutrino Yukawa couplings are active, and the RGEs in the MSSM above the scales $M_{R}$ are

$$
\begin{aligned}
(4 \pi)^{2} \frac{d \kappa_{v}}{d \ln \mu}= & \left\{-\frac{6}{5} \alpha_{1}(4 \pi)-6 \alpha_{2}(4 \pi)+2 \operatorname{tr}\left(Y_{\nu}^{\dagger} Y_{v}\right)\right. \\
& \left.+6 \operatorname{tr}\left(Y_{u}^{\dagger} Y_{u}\right)\right\} \kappa_{v}+\left(Y_{e}^{\dagger} Y_{e}\right)^{\top} \kappa_{v}+\kappa_{v}\left(Y_{e}^{\dagger} Y_{e}\right) \\
& +\left(Y_{v}^{\dagger} Y_{v}\right)^{\top} \kappa_{v}+\kappa_{v}\left(Y_{v}^{\dagger} Y_{v}\right), \\
(4 \pi)^{2} \frac{d M_{R}}{d \ln \mu}= & \frac{1}{8 \pi^{2}}\left[\left(Y_{v} Y_{v}^{\dagger}\right) M_{R}+M_{R}\left(Y_{v} Y_{v}^{\dagger}\right)^{\top}\right], \\
(4 \pi)^{2} \frac{d Y_{v}}{d \ln \mu}= & -Y_{v}\left[\frac{3}{5} \alpha_{1}(4 \pi)+3 \alpha_{2}(4 \pi)\right. \\
& -\operatorname{tr}\left(3 Y_{u}^{\dagger} Y_{u}+Y_{v}^{\dagger} Y_{v}\right) \\
& \left.-3 Y_{v}^{\dagger} Y_{v}-Y_{e}^{\dagger} Y_{e}\right] .
\end{aligned}
$$

For non-degenerate seesaw scales, a method for dealing with the effective theories, where the heavy singlets are partly integrated out, can be found in [257]. Analytical formulae for the running of the neutrino parameters above the seesaw scales are derived in $[258,259]$. The two loop beta functions can be found in Ref. [260].

The running correction to the neutrino mass matrix and its effects on the related issue have been widely analyzed (see e.g. [250-279]). We shall summarize below some of the features of RG running of the neutrino mixing parameters in the MSSM (cf. (3.72)-(3.74)).

- The RG effects are enhanced for relatively large $\tan \beta$, because the tau-Yukawa coupling becomes large.

- The mixing angles are comparatively stable with respect to the RG running in the case of normal hierarchical neutrino mass spectrum, $m_{1} \ll m_{2} \ll m_{3}$ even when $\tan \beta$ is large [261-267]. Nevertheless, the running effects can 
have important implications facing the high precision of future neutrino oscillation experiments.

- For $m_{1} \gtrsim 0.05 \mathrm{eV}$ and the case of $\tan \beta \gtrsim 10$, the RG running effects can be rather large and the leptonic mixing angles can run significantly. Particularly, the RGE effects can be very large for the solar neutrino mixing angle $\theta_{12}$ [261-267, 274, 275].

- The solar neutrino mixing angle $\theta_{12}$ at $M_{R}$ depends strongly on the Majorana phase $\alpha_{M}[254,267,268,275]$, which is the relative phase between $m_{1}$ and $m_{2}$, and plays very important role in the predictions of the effective Majorana mass in $(\beta \beta)_{0 v}$-decay. The effect of RG running for $\theta_{12}$ is smallest for the CP-conserving odd case $\alpha_{M}= \pm \pi$, while it is significant for the CP-conserving even case $\alpha_{M}=0$. For $\alpha_{M}=0$ and $\tan \beta \sim 50$, for instance, we have $\tan ^{2} \theta_{12}\left(M_{R}\right) \lesssim 0.5 \times \tan ^{2} \theta_{12}\left(M_{Z}\right)$ for $m_{1} \gtrsim 0.02 \mathrm{eV}$.

- The RG running effect on $\theta_{12}$ due to the $\tau$-Yukawa coupling always makes $\theta_{12}\left(M_{Z}\right)$ larger than $\theta_{12}\left(M_{R}\right)$ [267]. This constrains the models which predict the value of solar neutrino mixing angle at $M_{R}, \theta_{12}\left(M_{R}\right)>\theta_{12}\left(M_{Z}\right)$. For example, the bi-maximal models are strongly restricted. However, the running effects due to the neutrino Yukawa couplings are free from this feature [257]. Thus, bi-maximal models can predict the correct value of neutrino mixing angles with the neutrino Yukawa contributions [269-272].

- The RG corrections to neutrino mixing angles depend strongly on the deviation of the seesaw parameter matrix $R$ (3.45) from identity [274]. For hierarchical light neutrinos, $m_{1} \lesssim 0.01 \mathrm{eV}, \tan \beta \lesssim 30$ and $R$ non-trivial, the correction to $\theta_{23}$ and $\theta_{13}$ can be beyond their likely future experimental errors while $\theta_{12}$ is quite stable against the RG corrections [274].

- The correction to $\theta_{23}$ can be large when $m_{1}$ and/or $\tan \beta$ are/is relatively large, e.g., (i) when $m_{1} \gtrsim 0.2 \mathrm{eV}$ if $\tan \beta \lesssim 10$, and (ii) for any $m_{1}$ and $\alpha_{M}$ if $\tan \beta \gtrsim 40$ [274, 275].

- The RG corrections to $\sin \theta_{13}$ can be relatively small, even for the large $\tan \beta$ if $m_{1} \lesssim 0.05 \mathrm{eV}$, and for any $m_{1} \gtrsim$ $0.30 \mathrm{eV}$, if $\theta_{13}\left(M_{Z}\right) \cong 0$ and $\alpha_{M} \cong 0$ (with $\beta_{M}=\delta=0$ ). For $\alpha_{M}=\pi$ and $\tan \beta \sim 50$ one can have $\sin \theta_{13}\left(M_{R}\right) \gtrsim$ 0.10 for $m_{1} \gtrsim 0.08 \mathrm{eV}$ even if $\sin \theta_{13}\left(M_{Z}\right)=0$ [274, 275].

- For $\tan \beta \gtrsim 30$, the value of $\Delta m_{21}^{2}\left(M_{R}\right)$ depends strongly on $m_{1}$ in the interval $m_{1} \gtrsim 0.05 \mathrm{eV}$, and on $\alpha_{M}, \beta_{M}, \delta$, and $s_{13}$ for $m_{1} \gtrsim 0.1 \mathrm{eV}$. The dependence of $\Delta m_{31}^{2}\left(M_{R}\right)$ on $m_{1}$ and the CP phases is rather weak, unless $\tan \beta \gtrsim$ $40, m_{1} \gtrsim 0.10 \mathrm{eV}$, and $s_{13} \gg 0.05$ [275].

- Some products of the neutrino mixing parameters, such as $s_{12} c_{12} c_{23}\left(m_{1} / m_{2}-e^{i \alpha_{M}}\right)$ are practically stable with respect to RG running if one neglects the first and second generation charged lepton Yukawa couplings and $s_{13}$ [268, 273, 275].

\subsubsection{Quark-lepton complementarity}

3.2.4.1 Golden complementarity Quark-lepton complementarity [280-282] is based on the observation that $\theta_{12}+$ $\theta_{C}$ is numerically close to $\pi / 4$. Here $\theta_{12}$ is the solar neutrino mixing angle and $\theta_{C}$ is the Cabibbo angle. For hierarchical light neutrino masses this result is relatively stable against the renormalization effects [274]. To illustrate the idea we first review the model of exact golden complementarity.

Consider the following textures [283] for the light neutrino Majorana mass matrix $m_{v}$ and for the charged lepton Yukawa couplings $Y_{e}$ :

$m_{v}=\left(\begin{array}{ccc}0 & m & 0 \\ m & m & 0 \\ 0 & 0 & m_{\mathrm{atm}}\end{array}\right)$

$Y_{e}=\left(\begin{array}{ccc}\lambda_{e} & 0 & 0 \\ 0 & \lambda_{\mu} / \sqrt{2} & \lambda_{\tau} / \sqrt{2} \\ 0 & -\lambda_{\mu} / \sqrt{2} & \lambda_{\tau} / \sqrt{2}\end{array}\right)$

It just assumes some texture zeroes and some strict equalities among different entries. The mass eigenstates of the neutrino mass matrix are given by $m_{1}=-m / \varphi, m_{2}=m \varphi$, $m_{3}=m_{\text {atm }}$, where $\varphi=(1+\sqrt{5}) / 2=1+1 / \varphi \approx 1.62$ is known as the golden ratio [284]. Thanks to its peculiar mathematical properties this constant appears in various natural phenomena, possibly including solar neutrinos. The three neutrino mixing angles obtained from (3.79) are $\theta_{\mathrm{atm}}=\pi / 4$, $\theta_{13}=0$ and, more importantly,

$\tan ^{2} \theta_{12}=1 / \varphi^{2}=0.382, \quad$ i.e. $\quad \sin ^{2} 2 \theta_{12}=4 / 5$,

in terms of the parameter $\sin ^{2} 2 \theta_{12}$ directly measured by vacuum oscillation experiments, such as KamLAND. This prediction for $\theta_{12}$ is $1.4 \sigma$ below the experimental best fit value. A positive measurement of $\theta_{13}$ might imply that the prediction for $\theta_{12}$ suffers an uncertainty up to $\theta_{13}$.

Those properties follow from the $\mathrm{Z}_{2} \otimes \mathrm{Z}_{2}^{\prime}$ symmetry of the neutrino mass matrix. Explicitly $R m_{v} R^{T}=m_{v}$, where

$R=\left(\begin{array}{ccc}-1 / \sqrt{5} & 2 / \sqrt{5} & 0 \\ 2 / \sqrt{5} & 1 / \sqrt{5} & 0 \\ 0 & 0 & 1\end{array}\right), \quad R^{\prime}=\left(\begin{array}{ccc}1 & 0 & 0 \\ 0 & 1 & 0 \\ 0 & 0 & -1\end{array}\right)$

and the rotations satisfy $\operatorname{det} R=-1, R \cdot R^{T}=1$ and $R \cdot R=1$. The first $Z_{2}$ is a reflection along the diagonal of the golden rectangle in the $(1,2)$ plane, see Fig. 2 . The second $Z_{2}^{\prime}$ is the $L_{3} \rightarrow-L_{3}$ symmetry. Those symmetries allow contributions proportional to the identity matrix to be added to $m_{v}$. This property allows one to extend this type symmetries to the quark sector. 
A seesaw model with singlet neutrinos satisfying the $Z_{2} \otimes Z_{2}^{\prime}$ symmetry and giving rise to the mass matrix (3.79) is presented in [283].

Noticing that the golden prediction (3.80) satisfies with high accuracy the quark-lepton complementarity motivates one to give a golden geometric explanation also to the Cabibbo angle. $S U(5)$ unification relates the down-quark Yukawa matrix $Y_{d}$ to $Y_{e}$ and suggests that the up-quark Yukawa matrix $Y_{u}$ is symmetric, like $m_{v}$. One can therefore assume that $Y_{d}$ is diagonal in the two first generations and that $Y_{u}$ is invariant under a $Z_{2}$ reflection described by a matrix analogous to $R$ in (3.81), but with the factors $1 \leftrightarrow 2$ exchanged. Figure 2 illustrates the geometrical meaning of two reflection axis (dashed lines): the up-quark reflection is along the diagonal of the golden rectangle tilted by $\pi / 4$; note also the connection with the decomposition of the golden rectangle as an infinite sum of squares ('golden spiral'). Similarly to the neutrino case, this symmetry allows for two independent terms that can be tuned such that $m_{u} \ll m_{c}$ :

$$
Y_{u}=\lambda\left(\begin{array}{ccc}
1 & 0 & 0 \\
0 & 1 & 0 \\
0 & 0 & 1
\end{array}\right)+\frac{\lambda}{\sqrt{5}}\left(\begin{array}{ccc}
-2 & 1 & 0 \\
1 & 2 & 0 \\
0 & 0 & c
\end{array}\right)
$$

The second term fixes $\cot \theta_{C}=\varphi^{3}$, as can be geometrically seen from Fig. 2. We therefore have

$$
\begin{aligned}
& \sin ^{2} 2 \theta_{C}=1 / 5 \quad \text { i.e. } \theta_{12}+\theta_{C}=\pi / 4 \quad \text { i.e. } \\
& V_{u s}=\sin \theta_{C}=\left(1+\varphi^{6}\right)^{-1 / 2}=0.229 .
\end{aligned}
$$

This prediction is $1.9 \sigma$ above the present best-fit value, $\sin \theta_{C}=0.2258 \pm 0.0021$. However, as the basic elements

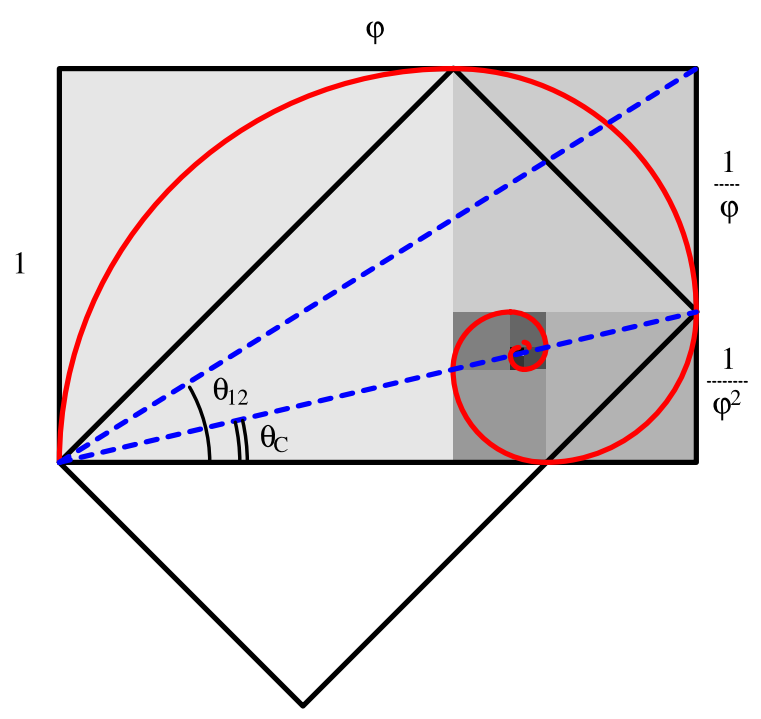

Fig. 2 Geometrical illustration of the connection between the predictions for $\theta_{12}$ and $\theta_{C}$ and the golden rectangle. The two dashed lines are the reflection axis of the $Z_{2}$ symmetry for the neutrino mass matrix and for the up quark mass matrix of flavor presented here follow by construction from the $2 \times 2$ submatrices, one naturally expects that the golden prediction for $V_{u s}$ has an uncertainty at least comparable to $\left|V_{u b}\right| \sim\left|V_{t d}\right| \sim$ few $\times 10^{-3}$. Thus the numerical accuracy is amazing. Should the $1.4 \sigma$ discrepancy between the golden prediction (3.80) and the experimental measurement hold after final SNO and KamLAND results, analogy with the quark sector would allow one to predict the order of magnitude of neutrino mixing angle $\theta_{13}$.

Interestingly, similar predictions on the mixing angles are obtained if some suitably chosen assumptions are made on the properties of neutral currents of quarks and leptons [285].

\subsubsection{Correlation matrix from $S_{3}$ flavor symmetry in GUT} On more general phenomenological ground the quarklepton complementarity $[281,282]$ can be described by the correlation matrix $V^{M}$ between the CKM and the PMNS mixing matrices,

$V^{M}=U^{\mathrm{CKM}} \Omega U^{\mathrm{PMNS}}$,

where $\Omega=\operatorname{diag}\left(e^{i \omega_{i}}\right)$ is a diagonal matrix. In the singlet seesaw mechanism the correlation matrix $V^{M}$ diagonalizes the symmetric matrix

$\mathcal{C}=m_{D}^{\text {diag }} V_{R}^{\nu \dagger} \frac{1}{M} V_{R}^{\nu \star} m_{D}^{\text {diag }}$,

where $M$ is the heavy neutrino Majorana mass matrix and $V_{R}^{v}$ diagonalizes the neutrino Dirac matrix $m_{D}$ from the right. In GUT models such as $S O(10)$ or $E_{6}$ we have intriguing relations between the Yukawa coupling of the quark sector and the one of the lepton sector. For instance, in minimal renormalizable $S O(10)$ with Higgs in the 10, 126, and 120, we have $Y_{e} \approx Y_{d}^{T}$. In fact the flavor symmetry implies the structure of the Yukawa matrices: the equivalent entries of $Y_{e}$ and $Y_{d}$ are usually of the same order of magnitude. In such a case one gets

$U^{\mathrm{PMNS}}=\left(U^{\mathrm{CKM}}\right)^{\dagger} V^{M}$.

As a consequence, a $S_{3}$ flavor permutation symmetry, softly broken into $S_{2}$, gives us the prediction of $V_{13}^{M}=0$ [286] and the correlations between CP-violating phases and the mixing angle $\theta_{12}$ [287].

The six generators of the $S_{3}$ flavor symmetry are the elements of the permutation group of three objects. The action of $S_{3}$ on the fields is to permute the family label of the fields. In the following we shall introduce the $S_{2}$ symmetry with respect the second and third generations. The $S_{2}$ group is an Abelian one and swap the second family $\left\{\mu_{L},\left(v_{\mu}\right)_{L}, s_{L}, c_{L}, \mu_{R},\left(v_{\mu}\right)_{R}, s_{R}, c_{R}\right\}$ with the third one $\left\{\tau_{L},\left(v_{\tau}\right)_{L}, b_{L}, t_{L}, \tau_{R},\left(v_{\tau}\right)_{R}, b_{R}, t_{R}\right\}$ 
Let us assume that there is an $S_{3}$ flavor symmetry at high energy, which is softly broken into $S_{2}$ [84]. In this case, before the $S_{3}$ breaking all the Yukawa matrices have the following structure:

$Y=\left(\begin{array}{lll}a & b & b \\ b & a & b \\ b & b & a\end{array}\right)$,

where $a$ and $b$ independent. The $S_{3}$ symmetry implies that $(1 / \sqrt{3}, 1 / \sqrt{3}, 1 / \sqrt{3})$ is an eigenvector of our matrix in (3.86). Moreover these kind of matrices have two equal eigenvalues. This gives us an undetermined mixing angle in the diagonalizing mixing matrices.

When $S_{3}$ is softly broken into $S_{2}$, one gets

$Y=\left(\begin{array}{lll}a & b & b \\ b & c & d \\ b & d & c\end{array}\right)$,

with $c \approx a$ and $d \approx b$. When $S_{3}$ is broken the degeneracy is removed. In general the $S_{2}$ symmetry implies that $(0,1 / \sqrt{2},-1 / \sqrt{2})$ is an exact eigenvector of our matrix (3.87). The fact that $S_{3}$ is only softly broken into $S_{2}$ allows us to say that $(1 / \sqrt{3}, 1 / \sqrt{3}, 1 / \sqrt{3})$ is still in a good approximation an eigenvector of $Y$ in (3.87). Then the mixing matrix that diagonalize from the right the Yukawa mixing matrix in (3.87) is given in good approximation by the tri-bi-maximal mixing matrix (2.11).

Let us now investigate the $V^{M}$ in this model. The mass matrix $m_{D}$ will have the general structure in (3.87). To be more defined, let us assumed that there is an extra softly broken $Z_{2}$ symmetry under which the 1 st and the 2 nd families are even, while the 3rd family is odd. This extra softly broken $Z_{2}$ symmetry gives us a hierarchy between the offdiagonal and the diagonal elements of $m_{D}$, i.e. $b, d \ll a, c$. In fact if $Z_{2}$ is exact both $b$ and $d$ are zero. For simplicity, we assume also a quasi-degenerate spectrum for the eigenvalues of the Dirac neutrino matrix as in [288].

The right handed neutrino Majorana mass matrix is of the form

$M=\left(\begin{array}{lll}a & b & b^{\prime} \\ b & c & d \\ b^{\prime} & d & e\end{array}\right)$.

Because $S_{3}$ is only softly broken into $S_{2}$ we have that $a \approx$ $c \approx e$, and $b \approx b^{\prime} \approx d$. In this approximation the $M$ matrix is diagonalized by a $U$ of the form in (2.11). In this case we have that $m_{v}$ is near to be $S_{3}$ and $S_{2}$ symmetric, then it is diagonalized by a mixing matrix $U_{v}$ near the tri-bi-maximal one given in (2.11). The $\mathcal{C}$ matrix is diagonalized by the mixing matrix $V_{M}=U_{\nu} U$. We obtain that $V_{M}$ is a rotation in the $(1,2)$ plane, i.e. it contains a zero in the $(1,3)$ entry. As shown in [288], it is possible to fit the CKM and the PMNS mixing matrix within this model.
3.3 Leptogenesis and cosmological observables

\subsubsection{Basic concepts and results}

$\mathrm{CP}$ violation in the leptonic sector can have profound cosmological implications, playing a crucial role in the generation, via leptogenesis, of the observed baryon number asymmetry of the universe [289]:

$\frac{n_{B}}{n_{\gamma}}=\left(6.1_{-0.2}^{+0.3}\right) \times 10^{-10}$.

In the original framework a $\mathrm{CP}$ asymmetry is generated through out-of-equilibrium $L$-violating decays of heavy Majorana neutrinos [290] leading to a lepton asymmetry $L \neq 0$. In the presence of sphaleron processes [291], which are $(B+L)$-violating and $(B-L)$-conserving, the lepton asymmetry is partially transformed to a baryon asymmetry.

The lepton number asymmetry resulting from the decay of heavy Majorana neutrinos, $\varepsilon_{N_{j}}$, was computed by several authors [292-294]. The evaluation of $\varepsilon_{N_{j}}$, involves the computation of the interference between the tree level diagram and one loop diagrams for the decay of the heavy Majorana neutrino $N_{j}$ into charged leptons $l_{\alpha}^{ \pm}(\alpha=e, \mu, \tau)$. Summing the asymmetries $\varepsilon_{N_{j}}^{\alpha}$ over charged lepton flavor, one obtains

$$
\begin{aligned}
\varepsilon_{N_{j}}= & \frac{g^{2}}{M_{W}^{2}} \sum_{\alpha, k \neq j}\left[\operatorname{Im}\left(\left(m_{D}^{\dagger}\right)_{j \alpha}\left(m_{D}\right)_{\alpha k}\left(m_{D}^{\dagger} m_{D}\right)_{j k}\right)\right. \\
& \left.\times \frac{1}{16 \pi}\left(I\left(x_{k}\right)+\frac{\sqrt{x_{k}}}{1-x_{k}}\right)\right] \frac{1}{\left(m_{D}^{\dagger} m_{D}\right)_{j j}}
\end{aligned}
$$

where $M_{k}$ denote the heavy neutrino masses, the variable $x_{k}$ is defined as $x_{k}=\frac{M_{k}^{2}}{M_{j}^{2}}$ and $I\left(x_{k}\right)=\sqrt{x_{k}}(1+(1+$ $\left.\left.x_{k}\right) \log \left(\frac{x_{k}}{1+x_{k}}\right)\right)$. From (3.90) it can be seen that, when one sums over all charged leptons, the lepton number asymmetry is only sensitive to the $\mathrm{CP}$-violating phases appearing in $m_{D}^{\dagger} m_{D}$ in the basis where $M_{R}$ is diagonal. Note that this combination is insensitive to rotations of the left-hand neutrinos.

If the lepton flavors are distinguishable in the final state, it is the flavored asymmetries that are relevant [295-298]. Below $T \sim 10^{12} \mathrm{GeV}$, the $\tau$ Yukawa interactions are fast compared to the Hubble rate, so at least one flavor may be distinguishable. The asymmetry in family $\alpha$, generated from the decay of the $k$ th heavy Majorana neutrino depends on the combination [299] $\operatorname{Im}\left(\left(m_{D}^{\dagger} m_{D}\right)_{k k^{\prime}}\left(m_{D}^{*}\right)_{\alpha k}\left(m_{D}\right)_{\alpha k^{\prime}}\right)$ as well as on $\operatorname{Im}\left(\left(m_{D}^{\dagger} m_{D}\right)_{k^{\prime} k}\left(m_{D}^{*}\right)_{\alpha k}\left(m_{D}\right)_{\alpha k^{\prime}}\right)$. Summing over all leptonic flavors $\alpha$ the second term becomes real so that its imaginary part vanishes and the first term gives rise to the combination $\operatorname{Im}\left(\left(m_{D}^{\dagger} m_{D}\right)_{j k}\left(m_{D}^{\dagger} m_{D}\right)_{j k}\right)$ that appears in (3.90). Clearly, when one works with separate flavors the matrix $U_{\text {PMNS }}$ does not cancel out and one is lead to the 
interesting possibility of having viable leptogenesis even in the case of $R$ being a real matrix [300-303].

The simplest leptogenesis scenario corresponds to the case of heavy hierarchical neutrinos where $M_{1}$ is much smaller than $M_{2}$ and $M_{3}$. In this limit, the asymmetries generated by $N_{2}$ and $N_{3}$ are frequently ignored, because the production of $N_{2}$ and $N_{3}$ can be suppressed by kinematics (for instance, they are not produced thermally, if the re-heat temperature after inflation is $<M_{2}, M_{3}$ ), and the asymmetries from their decays are partially washed out [295, 304, 305]. In this hierarchical limit, the $\varepsilon_{N_{1}}^{\alpha}$ can be simplified into

$\varepsilon_{N_{1}}^{\alpha} \simeq-\frac{3}{16 \pi v^{2}}\left(I_{12}^{\alpha} \frac{M_{1}}{M_{2}}+I_{13}^{\alpha} \frac{M_{1}}{M_{3}}\right)$,

where

$I_{1 i}^{\alpha} \equiv \frac{\operatorname{Im}\left[\left(m_{D}^{\dagger}\right)_{1 \alpha}\left(m_{D}\right)_{\alpha i}\left(m_{D}^{\dagger} m_{D}\right)_{1 i}\right]}{\left(m_{D}^{\dagger} m_{D}\right)_{11}}$.

The flavor-summed CP asymmetry $\varepsilon_{N_{1}}$ can be written in terms of the parameterization equation (3.46) as

$\varepsilon_{N_{1}} \approx-\frac{3}{8 \pi} \frac{M_{1}}{v^{2}} \frac{\sum_{i} m_{i}^{2} \operatorname{Im}\left(R_{1 i}^{2}\right)}{\sum_{i} m_{i}\left|R_{1 i}\right|^{2}}$.

In this case, obviously, leptogenesis demands non-zero imaginary parts in the $R$ matrix. It has an upper bound $\left|\varepsilon_{N_{1}}\right|<\varepsilon_{N_{1}}^{D I}$ where [306]

$\varepsilon_{N_{1}}^{D I}=\frac{3}{8 \pi} \frac{\left(m_{3}-m_{1}\right) M_{1}}{v^{2}}$,

which is proportional to $M_{1}$. So the requirement of generating a sufficient baryon asymmetry gives a lower bound on $M_{1}$ [306, 307]. Depending on the cosmological scenario, the range for minimal $M_{1}$ varies from order $10^{7} \mathrm{GeV}$ to $10^{9} \mathrm{GeV}[308,309]$. This bound does not move much with the inclusion of flavor effects [296, 310, 311]. In a supersymmetric world there is an upper bound $T_{\mathrm{RH}}<10^{8} \mathrm{GeV}$ on the re-heating temperature of the universe from the possible overproduction of gravitinos, the so called gravitino problem [312-315]. Together with the lower bound on $M_{1}$ the gravitino problem puts severe constraints on supersymmetric thermal leptogenesis scenarios.

However, the upper bound (3.94) is based on the (natural) assumption that higher order corrections suppressed by $M_{1} / M_{2}, M_{1} / M_{3}$ in (3.90) are negligible. This may not be true as explicitly demonstrated in [316] in which neutrino mass model is presented realizing $\varepsilon_{N_{1}} \gg \varepsilon_{N_{1}}^{D I}$. In such a case low scale standard thermal leptogenesis consistent with the gravitino bound is possible also for hierarchical heavy neutrinos.

Thermal leptogenesis is a rather involved thermodynamical non-equilibrium process and depends on additional parameters and on the proper treatment of thermal effects [309].
In the simplest case, the $N_{i}$ are hierarchical, and $N_{1}$ decays into a combination of flavors which are indistinguishable. ${ }^{11}$ In this case, the baryon asymmetry only depends on four parameters [306, 308, 318, 319]: the mass $M_{1}$ of the lightest heavy neutrino, together with the corresponding $\mathrm{CP}$ asymmetry $\varepsilon_{N_{1}}$ in its decay, as well as the rescaled $N_{1}$ decay rate, or effective neutrino mass $\tilde{m}_{1}$ defined as

$\tilde{m}_{1}=\sum_{\alpha}\left(m_{D}^{\dagger}\right)_{1 \alpha}\left(m_{D}\right)_{\alpha 1} / M_{1}$

in the weak basis where $M_{R}$ is diagonal, real and positive. Finally, the baryon asymmetry depends also on the sum of all light neutrino masses squared, $\bar{m}^{2}=m_{1}^{2}+m_{2}^{2}+m_{3}^{2}$, since it has been shown that this sum controls an important class of washout processes. If lepton flavors are distinguishable, the final baryon asymmetry depends on partial decay rates $\tilde{m}_{1}^{\alpha}$ and CP asymmetries $\epsilon_{1}^{\alpha}$.

The $N_{1}$ decays in the early universe at temperatures $T \sim M_{1}$, producing asymmetries in the distinguishable final states. A particular asymmetry will survive once washout by inverse decays go out of equilibrium. In the unflavored calculation (where lepton flavors are indistinguishable), the fraction of the asymmetry that survives is of order $\min \{1, H / \Gamma\}$, where the Hubble rate $H$ and the $N_{1}$ total decay rate $\Gamma$ are evaluated at $T=M_{1}$. This is usually written $H / \Gamma=m^{*} / \tilde{m}_{1}$, where [320-322]

$m_{*}=\frac{16 \pi^{5 / 2}}{3 \sqrt{5}} g_{*}^{1 / 2} \frac{v^{2}}{M_{\text {Planck }}} \simeq 10^{-3} \mathrm{eV}$,

and $M_{\text {Planck }}$ is the Planck mass $\left(M_{\text {Planck }}=1.2 \times 10^{19} \mathrm{GeV}\right)$, $v=\left\langle\phi^{0}\right\rangle / \sqrt{2} \simeq 174 \mathrm{GeV}$ is the weak scale and $g_{*}$ is the effective number of relativistic degrees of freedom in the plasma and equals 106.75 in the SM case. In a flavored calculation, the fraction of a flavor asymmetry that survives can be estimated in the same way, replacing $\Gamma$ by the partial decay rate.

\subsubsection{Implications of flavor effects}

For a long time the flavor effects in thermal leptogenesis were known [295] but their phenomenological implications were considered only in specific neutrino flavor models [235]. As discussed, in the single-flavor calculation, the most important parameters for thermal leptogenesis from $N_{1}$ decays are $M_{1}, \tilde{m}_{1}, \epsilon_{N_{1}}$ and the light neutrino mass scale. Including flavor effects gives this parameter space more dimensions $\left(M_{1}, \epsilon^{\alpha}, \tilde{m}_{1}^{\alpha}\right)$, but it can still be projected onto $M_{1}$, $\tilde{m}$ space. For the readers convenience we summarize here

\footnotetext{
${ }^{11}$ This can occur above $\sim 10^{12} \mathrm{GeV}$, before the $\tau$ Yukawa interaction becomes fast compared to the Hubble rate, or in the case where the $N_{1}$ decay rate is faster than the charged lepton Yukawa interactions [317].
} 
some general results on the implications of flavored leptogenesis.

In the unflavored calculation, leptogenesis does not work for degenerate light neutrinos with a mass scale above $\sim 0.1 \mathrm{eV}$ [323-326]. This bound does not survive in the flavored calculation, where models with a neutrino mass scale up to the cosmological bound, $\sum m_{v}<0.68 \mathrm{eV}$ [327], can be tuned to work [296, 317].

Considering the scale of leptogenesis, flavored leptogenesis works for $M_{1}$ a factor of $\sim 3$ smaller in the "interesting" region of $\tilde{m}<m_{\mathrm{atm}}$. But the lower bound on $M_{1}$, in the optimized $\tilde{m}$ region, remains $\sim 10^{9} \mathrm{GeV}[310,311]$. A smaller $M_{1}$ could be possible for very degenerate light neutrinos [296].

An important, but disappointing, observation in singleflavor leptogenesis was the lack of a model-independent connection between $\mathrm{CP}$ violation for leptogenesis and PMNS phases. It was shown [328, 329] that thermal leptogenesis can work with no $\mathrm{CP}$ violation in $U_{\text {PMNS }}$, and conversely, that leptogenesis can fail in spite of phases in

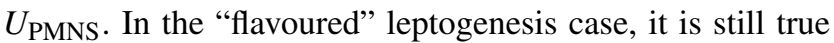
that the baryon asymmetry is not sensitive to PMNS phases [330, 331] (leptogenesis can work for any value of the PMNS phases). However, interesting observations can be made in classes of models [297, 300, 302, 331].

\subsubsection{Other scenarios}

We have presented a brief discussion of minimal thermal leptogenesis in the context of type I seesaw with hierarchical heavy neutrinos. This scenario is the most popular one because it is generic, supported by neutrino mass mechanism and, most importantly, it has predictions for the allowed seesaw parameter space, as described above. There are many other scenarios in which leptogenesis may also be viable.

Resonant leptogenesis [293, 332] may occur when two or more heavy neutrinos are nearly degenerate in mass and in this scenario the scale of the heavy neutrino masses can be lowered whilst still being compatible with thermal leptogenesis [332-335]. Heavy neutrinos of TeV scale or below could in principle be detected at large colliders [336]. In the seesaw context low scale heavy neutrinos may follow from extra symmetry principles [334, 337-339]. Also, the SM extensions with heavy neutrinos at $\mathrm{TeV}$ scale or below include Kaluza-Klein modes in models with extra dimensions or extra matter content of little Higgs models.

Leptogenesis from the out-of-equilibrium decays of a Higgs triplet $[244,340,341]$ is another viable scenario but requires the presence of at least two triplets for non-zero $\mathrm{CP}$ asymmetry. Despite the presence of gauge interactions the washout effects in this scenario are not drastically larger than those in the singlet leptogenesis scenario [341]. Hybrid leptogenesis from type I and type II seesaw can for instance occur in $S O(10)$ models $[340,342,343]$. In that case there are twelve independent CP-violating phases.

"Soft leptogenesis" [344, 345] can work in a one generational SUSY seesaw model because CP violation in this scenario comes from complex supersymmetry breaking terms. If the soft SUSY-breaking terms are of suitable size, there is enough CP violation in $\tilde{N}-\tilde{N}^{*}$ mixing to imply the observed asymmetry. Unlike non-supersymmetric triplet Higgs leptogenesis, soft leptogenesis with a triplet scalar [341, 346] can also work in the minimal supersymmetric model of type II seesaw mechanism.

A very predictive supersymmetric leptogenesis scenario is obtained if the sneutrino is playing the role of inflaton [307, 347-350]. In this scenario the universe is dominated by $\tilde{N}$. Relating $\tilde{N}$ properties to neutrino masses via the seesaw mechanism implies a lower bound $T_{\mathrm{RH}}>10^{6} \mathrm{GeV}$ on the re-heating temperature of the universe [349]. A connection of this scenario with LFV is discussed in Sect. 5.2.

Dirac leptogenesis is another possibility considered in the literature. In this case neutrinos are of Dirac type rather than Majorana. In the original paper [351] two Higgs doublets were required and their decays create the leptonic asymmetry. Recently some authors have studied the connection between leptogenesis and low energy data with two Higgs doublets [352].

Finally, let us mention that right handed neutrinos could have been produced non-thermally in the early universe, by direct couplings to the inflation field. If this is the case, the constraints on neutrino parameters from leptogenesis depend on the details of the inflationary model [353-355].

For a recent overview of the present knowledge of neutrino masses and mixing and what can be learned about physics beyond the standard model from the various proposed neutrino experiments, see [4] and references therein.

\section{Organizing principles for flavor physics}

\subsection{Grand unified theories}

Grand unification is an attempt to unify all known interactions but gravity in a single simple gauge group. It is motivated in part by the arbitrariness of electromagnetic charge in the standard model. One has charge quantization in a purely non-Abelian theory, without an $U(1)$ factor, as in Schwinger's original idea [356] of a $S U(2)$ theory of electroweak interactions. The minimal gauge group which unifies weak and strong interactions, $S U(5)$ [357], automatically implies a quantized $U(1)$ piece too. While Dirac needed a monopole to achieve charge quantization [358], grand unification in turn predicts the existence of magnetic monopoles $[359,360]$. Since it unifies quarks and 
leptons [361], it also predicts another remarkable phenomenon: the decay of the proton. Here we are mostly interested in GUT implications on the flavor structure of Yukawa matrices.

\subsubsection{SU(5): the minimal theory}

The 24 gauge bosons reduce to the 12 ones of the SM plus a $S U$ (2) doublet, color triplet pair $\left(X_{\mu}, Y_{\mu}\right)$ (vector leptoquarks), with $Y=5 / 6$ (charges $+4 / 3,+1 / 3$ ) and their antiparticles. The 15 fermions of a single family in the SM fit in the $\overline{5}_{\mathrm{F}}$ and $10_{\mathrm{F}}$ anomaly-free representations of $S U(5)$, and the new super-weak interactions of leptoquarks with fermions are ( $\alpha, \beta$ and $\gamma$ are color indices):

$$
\begin{aligned}
\mathcal{L}(X, Y)= & \frac{g_{5}}{\sqrt{2}} X_{\mu}^{(-4 / 3) \alpha} \\
& \times\left(\bar{e} \gamma^{\mu} d_{\alpha}^{c}+\bar{d}_{\alpha} \gamma^{\mu} e^{c}-\epsilon_{\alpha \beta \gamma} \bar{u}^{\beta} \beta \gamma^{\mu} u^{\gamma}\right) \\
& -\frac{g_{5}}{\sqrt{2}} Y_{\mu}^{(-1 / 3) \alpha} \\
& \times\left(\bar{v} \gamma^{\mu} d_{\alpha}^{c}+\bar{u}_{\alpha} \gamma^{\mu} e^{c}+\epsilon_{\alpha \beta \gamma} \bar{u}^{\beta} \gamma^{\mu} d^{\gamma}\right) \\
& + \text { h.c. },
\end{aligned}
$$

where all fermions above are explicitly left handed and $\psi^{c} \equiv C \bar{\psi}^{T}$.

The exchange of the heavy gauge bosons leads to the effective interactions suppressed by two powers of their mass $m_{X}\left(m_{X} \simeq m_{Y}\right.$ due to $S U(2)_{L}$ symmetry), which preserves $B-L$, but breaks both $B$ and $L$ symmetries and leads to $(d=6)$ proton decay [213, 362]. From $\tau_{P} \gtrsim 6 \times 10^{33} \mathrm{yr}$ [363], $m_{X} \gtrsim 10^{15.5} \mathrm{GeV}$.

The Higgs sector consists of an adjoint $24_{H}$ and a fundamental $5_{H}$, the first breaks $S U(5) \rightarrow \mathrm{SM}$, the latter completes the symmetry breaking á la Weinberg-Salam. Now, $5_{H}=(T, D)$, where $T$ is a color triplet and $D$ the usual Higgs $S U(2)_{L}$ doublet of the SM and so the Yukawa interactions in the matrix form

$$
\mathcal{L}_{Y}=10_{\mathrm{F}} y_{u} 10_{\mathrm{F}} 5_{H}+\overline{5}_{\mathrm{F}} y_{d} 10_{\mathrm{F}} 5_{H}^{*}
$$

give the quark and lepton mass matrices

$$
m_{u}=y_{u}\langle D\rangle, \quad m_{d}=m_{e}^{T}=y_{d}\langle D\rangle .
$$

Note the correlation between down quarks and charged leptons [364], valid at the GUT scale, and impossible to be true for all three generations. Actually, in the SM it is wrong for all of them. It can be corrected by an extra Higgs, $45_{H}$ [6], or higher dimensional non-renormalizable interaction [7].

From (4.2), one gets also the interactions of the triplet, which lead to proton decay and thus the triplet $T$ must be superheavy, $m_{T} \gtrsim 10^{12} \mathrm{GeV}$. The enormous split between $m_{T}$ and $m_{D} \simeq m_{W}$ can be achieved through the large scale of the breaking of $S U(5)$,

$$
\left\langle 24_{H}\right\rangle=v_{X} \operatorname{diag}(2,2,2,-3,-3),
$$

with $m_{X}^{2}=m_{Y}^{2}=\frac{25}{4} g_{5}^{2} v_{X}^{2}$. This fine-tuning is known as the doublet-triplet problem. Whatever solution one may adopt, the huge hierarchy can be preserved in perturbation theory only by supersymmetry with low scale breaking of order $\mathrm{TeV}$.

The consistency of grand unification requires that the gauge couplings of the SM unify at a single scale, in a tiny window $10^{15.5} \lesssim M_{\mathrm{GUT}} \lesssim 10^{18} \mathrm{GeV}$ (lower limit from proton decay, upper limit from perturbativity, i.e. to stay below $M_{\mathrm{Pl}}$ ). Here the minimal ordinary $S U(5)$ theory described above fails badly, while the version with low energy supersymmetry does great [365-368]. Actually, one needed a heavy top quark [368], with $m_{t} \simeq 200 \mathrm{GeV}$ in order for the theory to work. The same is needed in order to achieve a radiative symmetry breaking of the SM gauge symmetry, where only the Higgs doublet becomes tachyonic $[369,370]$. One can then define the minimal supersymmetric $S U(5)$ GUT with the three families of fermions $10_{\mathrm{F}}$ and $\overline{5}_{\mathrm{F}}$, and with $24_{H}$ and $5_{H}$ and $\overline{5}_{H}$ supermultiplets. It predicts $m_{d}=m_{e}^{T}$ at $M_{\mathrm{GUT}}$, which works well for the third generation; the first two can be corrected by higher dimensional operators. Although this theory typically has a very fast $d=5$ [150,371-374] proton decay [375], the higher dimensional operators can easily make it in accord with experiments [376-378]. The main problem are massless neutrinos, unless one breaks R-parity (whose approximate or exact conservation must be assumed in supersymmetric $S U(5)$, contrary to some supersymmetric $S O(10))$ ). Other ways out include adding singlets, right handed neutrinos (type I seesaw [216-220]), or a $15_{H}$ multiplet (type II see-saw [215, 221-223]). In both cases their Yukawa are not connected to the charged sector, so it is much more appealing to go to $S O(10)$ theory, which unifies all fermions (of a single family) too, besides the interactions.

Before we move to $S O(10)$, what about ordinary nonsupersymmetric $S U(5)$ ? In order to have $m_{v} \neq 0$ and to achieve the unification of gauge couplings one can add either (a) $15_{H}$ Higgs multiplet [379] or (b) $24_{\mathrm{F}}$ fermionic multiplet [380]. The latter one is particularly interesting, since it leads to the mixing of the type I and type III see-saw [225, 226], with the remarkable prediction of a light $S U(2)$ fermionic triplet below $\mathrm{TeV}$ and $M_{\mathrm{GUT}} \leq 10^{16} \mathrm{GeV}$, which offers hope both for the observable see-saw at LHC and detectable proton decay in a future generation of experiments now planned [381].

These fermionic triplets $T_{\mathrm{F}}$ would be produced in pairs through a Drell-Yan process. The production cross section for the sum of all three possible final states, $T_{\mathrm{F}}^{+} T_{\mathrm{F}}^{-}, T_{\mathrm{F}}^{+} T_{\mathrm{F}}^{0}$ 
and $T_{\mathrm{F}}^{-} T_{\mathrm{F}}^{0}$, can be read from Fig. 42 of [382]: it is approximately $20 \mathrm{pb}$ for $100 \mathrm{GeV}$ triplet mass, and around $40 \mathrm{fb}$ for $500 \mathrm{GeV}$ triplets. The triplets then decay into $W$ or $Z$ and a light lepton through the same Yukawa couplings that enter into the seesaw.

The clearest signature would be the three charged lepton decay of the charged triplet, but it has only a $3 \%$ branching ratio. A more promising situation is the decay into two jets with SM gauge boson invariant mass plus a charged lepton: this happens in approximately $23 \%$ of all decays. The signatures in this case is two same charge leptons plus two pairs of jets having the $W$ or $Z$ mass and peaks in the lepton-dijet mass. From the above estimates the cross section for such events is around $1 \mathrm{pb}(2 \mathrm{fb}$ ) for 100 (500) GeV triplet mass. Such signatures were suggested originally in $L-R$ symmetric theories [383] but are quite generic of the seesaw mechanism.

\subsubsection{SO(10): the minimal theory of matter and gauge coupling unification}

There are a number of features that make $S O(10)$ special:

- a family of fermions is unified in a 16 dimensional spinorial representation; this in turn predicts the existence of right handed neutrinos, making the implementation of the see-saw mechanism almost automatic;

- $L-R$ symmetry [361, 384-386] is a finite gauge transformation in the form of charge conjugation. This is a consequence of both left handed fermions $f_{L}$ and its charged conjugated counterparts $\left(f^{c}\right)_{L} \equiv C \bar{f}_{R}^{T}$ residing in the same representation $16_{\mathrm{F}}$;

- in the supersymmetric version, the matter parity $M=$ $(-1)^{3(B-L)}$, equivalent to the R-parity $R=M(-1)^{2 S}$, is a gauge transformation [387-389], a part of the centre $Z_{4}$ of $S O(10)$. In the renormalizable version of the theory it remains exact at all energies [390-392]. The lightest supersymmetric partner (LSP) is then stable and is a natural candidate for the dark matter of the universe;

- its other maximal subgroup, besides $S U(5) \times U(1)$, is $G_{\mathrm{PS}}=S U(2)_{L} \times S U(2)_{R} \times S U(4)_{C}$ quark-lepton symmetry of Pati and Salam, which plays an important role in relating quark and lepton masses and mixings;

- the unification of gauge couplings can be achieved even without supersymmetry (for a recent and complete work and references therein, see [393, 394]).

Fermions belong to the spinor representation $16_{\mathrm{F}}$ (for useful reviews on spinors and $S O(2 N)$ group theory in general see [395-399]). From

$16 \times 16=10+120+126$,

the most general Yukawa sector in general contains $10_{H}$, $120_{H}$ and $\overline{126}_{H}$, respectively the fundamental vector representation, the three-index antisymmetric representation and the five-index antisymmetric and anti-self-dual representation. $\overline{126}_{H}$ is necessarily complex, supersymmetric or not; $10_{H}$ and $\overline{126}_{H}$ Yukawa matrices are symmetric in generation space, while the $120_{H}$ one is antisymmetric.

The decomposition of the relevant representations under $G_{\mathrm{PS}}$ gives

$$
\begin{aligned}
\mathbf{1 6}= & (2,1,4)+(1,2, \overline{4}), \\
\mathbf{1 0}= & (2,2,1)+(1,1,6), \\
\mathbf{1 2 0}= & (2,2,1)+(3,1,6)+(1,3,6)+(2,2,15) \\
& +(1,1,10)+(1,1, \overline{10}), \\
\overline{\mathbf{1 2 6}}= & (3,1, \overline{10})+(1,3,10)+(2,2,15)+(1,1,6) .
\end{aligned}
$$

The see-saw mechanism, whether type I or II, requires $\overline{126}$ : it contains both $(1,3,10)$ whose VEV gives a mass to $v_{R}$ (type I), and $(3,1, \overline{10})$, which contains a color singlet, $B-L=2$ field $\Delta_{L}$, that can give directly a small mass to $v_{L}$ (type II). In $S U(5)$ language this is seen from the decomposition

$\overline{\mathbf{1 2 6}}=1+5+15+\overline{45}+50$.

The 1 of $S U(5)$ belongs to the $(1,3,10)$ of $G_{\mathrm{PS}}$ and gives a mass for $v_{R}$, while 15 corresponds to the $(3,1, \overline{10})$ and gives the direct mass to $v_{L}$.

$\overline{126}$ can be a fundamental field, or a composite of two $\overline{16}_{H}$ fields (for some realistic examples see for example [400-402]), or can even be induced as a two-loop effective representation built out of a $10_{H}$ and two gauge 45 dimensional representations [403-405].

Normally the light Higgs is chosen to be the smallest one, $10_{H}$. Since $\left\langle 10_{H}\right\rangle=\langle(2,2,1)\rangle$ is a $S U(4)_{C}$ singlet, $m_{d}=m_{e}$ follows immediately, independently of the number of $10_{H}$. Thus we must add either $120_{H}$ or $\overline{126}_{H}$ or both in order to correct the bad mass relations. Both of these fields contain $(2,2,15)$, which VEV alone gives the relation $m_{e}=-3 m_{d}^{T}$.

As $\overline{126}_{H}$ is needed anyway for the see-saw, it is natural to take this first. The crucial point here is that in general $(2,2,1)$ and $(2,2,15)$ mix through $\langle(1,3,10)\rangle[222,406]$ and thus the light Higgs is a mixture of the two. In other words, $\langle(2,2,15)\rangle$ in $\overline{126}_{H}$ is in general non-vanishing (in supersymmetry this is not automatic, but depends on the Higgs superfields needed to break $S O(10)$ at $M_{\mathrm{GUT}}$ or on the presence of higher dimensional operators).

If one considers all the operators allowed by $S O(10)$ for the Yukawa couplings, there are too many model parameters, and so no prediction is really possible. One option is to assume that the minimal number of parameters must be employed. It has been shown that 4 ( 3 of them nonrenormalizable) operators are enough in models with 10 and 45 Higgs representations only [8]. Although this is an important piece of information and it has been the starting point of 
a lot of model building, it is difficult to see a reason for some operators (of different dimensions) to be present and other not, without using some sort of flavor symmetry, so these type of models will not be considered in this subsection. On the other hand, a self consistent way of truncating the large number of $S O(10)$ allowed operators without relying on extra symmetries is to consider only the renormalizable ones. This is exactly what we shall assume.

In this case there are just two ways of giving mass to $v_{R}$ : by a nonzero VEV of the Higgs $\overline{126}$, or generate an effective non-renormalizable operator radiatively [403]. We shall consider in turn both of them.

4.1.2.1 Elementary $\overline{126}_{H}$ It is rather appealing that $10_{H}$ and $\overline{126}_{H}$ may be sufficient for all the fermion masses, with only two sets of symmetric Yukawa coupling matrices. The mass matrices at $M_{\mathrm{GUT}}$ are

$$
\begin{aligned}
& m_{d}=v_{10}^{d} Y_{10}+v_{126}^{d} Y_{126}, \\
& m_{u}=v_{10}^{u} Y_{10}+v_{126}^{u} Y_{126}, \\
& m_{e}=v_{10}^{d} Y_{10}-3 v_{126}^{d} Y_{126}, \\
& m_{v}=-m_{D} M_{R}^{-1} m_{D}+m_{v_{L}},
\end{aligned}
$$

where

$$
\begin{aligned}
& m_{D}=v_{10}^{u} Y_{10}-3 v_{126}^{u} Y_{126}, \\
& M_{R}=v_{R} Y_{126}, \\
& m_{v_{L}}=v_{L} Y_{126} .
\end{aligned}
$$

These relations are valid at $M_{\mathrm{GUT}}$, so it is there that their validity must be tested. The analysis done so far used the results of renormalization group running from $M_{Z}$ to $M_{\mathrm{GUT}}$ from [407, 408].

The first attempts in fitting the mass matrices assumed the domination of the type I seesaw. It was pioneered by treating $\mathrm{CP}$ violation perturbatively in a non-supersymmetric framework [406], and later improved with a more detailed treatment of complex parameters and supersymmetric low energy effective theory [409-411]. Nevertheless, these fits had problems to reproduce correctly the PMNS matrix parameters.

A new impetus to the whole program was given by the observation that in case type II seesaw dominates (a way to enforce it is to use a 54 dimensional Higgs representation [412]) the neutrino mass, an interesting relation in these type of models between $b-\tau$ unification and large atmospheric mixing angle can be found [413-415]. The argument is very simple and it can be traced to the relation [416]

$m_{v} \propto m_{d}-m_{e}$ which follows directly from (4.8), (4.10) and (4.14), if only the second term (type II) in (4.11) is considered. Considering only the heaviest two generations as an example and taking the usually good approximation of small second generation masses and small mixing angles, one finds all the elements of the right-hand side small except the 22 element, which is proportional to the difference of two big numbers, $m_{b}-m_{\tau}$. Thus, a large neutrino atmospheric mixing angle is linked to the smallness of this 22 matrix element, and so to $b-\tau$ unification. Note that in these types of models $b-\tau$ unification is no more automatic due to the presence of the $\overline{126}$, which breaks $S U(4)_{C}$. It is, however, quite a good prediction of the RGE running in the case of low energy supersymmetry.

The numerical fitting was able to reproduce also a large solar mixing angle both in case of type II [417, 418] or mixed seesaw [419], predicting also a quite large $\left|U_{e 3}\right| \approx$ 0.16 mixing element, close to the experimental upper bound. The difficulty in fitting the CKM CP-violating phase in the first quadrant was overcome by new solutions found in [420, 421], maintaining the prediction of large $\left|U_{e 3}\right| \geq 0.1$ matrix element.

All these fittings were done assuming no constraints coming from the Higgs sector. Regarding it, it was found that the minimal supersymmetric model [422-424] has only 26 model parameters [425], on top of the usual supersymmetry breaking soft terms, as in the MSSM. When one considers this minimal model, the VEVs in the mass formulae (4.8)(4.14) are not completely arbitrary, but are connected by the restrictions of the Higgs sector. This has been first noticed in [426-428] showing a possible clash with the positive results of the unconstrained Yukawa sector studied in [420, 421]. The issue has been pursued in [429], showing that in the region of parameter space where the fermion mass fitting is successful, there are necessarily intermediate scale thresholds which spoil perturbativity of the RGE evolution of the gauge couplings.

To definitely settle the issue, two further checks should be done. (a) The $\chi^{2}$ analysis used in the fitting procedure should be implemented at $M_{Z}$, not at $M_{\mathrm{GUT}}$. The point is in fact that while the errors at $M_{Z}$ are uncorrelated, they become strongly correlated after running to $M_{\mathrm{GUT}}$, due to the large Yukawa coupling of top and possibly also of bottom, tau and neutrino. (b) Another issue is to consider also the effect of the possible increased gauge couplings on the Yukawas. Only after these two checks will be done, this minimal model could be ruled out.

A further important point is that in the case of VEVs constrained by the Higgs sector one finds from the charged fermion masses that the model predicts large $\tan \beta \simeq 40$, as confirmed by the last fits in [429]. In this regime there may be sizable corrections to the "down" fermion mass matrices from the soft SUSY breaking parameters [430]; this 
brings into the game also the soft SUSY breaking sector, lowering somewhat the predictivity but relaxing the difficulty in fitting the experimental data. In this scenario predictions on masses would become predictions on the soft sector.

Some topics have to be still mentioned in connection with the above: the important calculation of the mass spectrum and Clebsch-Gordan coefficients in SO(10) [399, 431-439], the doublet-triplet splitting problem [440, 441], the Higgs doublet mass matrix [399, 433], the running of the gauge couplings at two loops together with threshold corrections [434], and the study of proton decay [435, 442, 443].

What if this model turns out to be wrong? There are other models on the market. The easiest idea is to add a 120 dimensional Higgs, that may also appear as a natural choice, being the last of the three allowed representations that couple with fermions. There are three different ways of doing it considered in the literature: (a) take 120 as a small, nonleading, contribution, i.e. a perturbation to the previous formulae [444-446]; (b) consider 120 on an equal footing as 10 and $\overline{126}$, but assume some extra discrete symmetry or real parameters in the superpotential, breaking $\mathrm{CP}$ spontaneously [447-450] (and suppressing in the first two references the dangerous $d=5$ proton decay modes); (c) assume small $\overline{126}$ contributions to the charged fermion masses [451-454].

Another limit is to forget the $10_{H}$ altogether, as has been proposed for non-supersymmetric theories [455]. The two generation study predicts a too small ratio $m_{b} / m_{\tau} \approx 0.3$, instead of the value 0.6 that one gets by straight running. The idea is that this could get large corrections due to Dirac neutrino Yukawas [456] and the effect of finite second generation masses, as well as the inclusion of the first generation and CP-violating phases. This is worth pursuing for it provides an alternative minimal version of $S O(10)$, and after all, supersymmetry may not be there.

4.1.2.2 Radiative $\overline{126}_{H}$ The original idea [403] is that there is no $\overline{126}_{H}$ representation in the theory, but the same operator is generated by loop corrections. The representation that breaks the rank of $S O(10)$ is now $16_{H}$, which VEV we call $M_{\Lambda}$. Generically there is a contribution to the righthanded neutrino mass at two loops:

$M_{R} \approx\left(\frac{\alpha}{4 \pi}\right)^{2} \frac{M_{\Lambda}^{2}}{M_{\mathrm{GUT}}} \frac{M_{\mathrm{SUSY}}}{M_{\mathrm{GUT}}} Y_{10}$,

which is too small in low energy supersymmetry (low breaking scale $M_{\text {SUSY }}$ ) as well as non-supersymmetric theories ( $M_{\mathrm{SUSY}}=M_{\mathrm{GUT}}$, but low intermediate scale $M_{\Lambda}$ required by gauge coupling unification). The only exception, proposed in [404], could be split supersymmetry [457, 458].

In the absence of $\overline{126}_{H}$, the charged fermion masses must be given by only $10_{H}$ and $120_{H}$ [404], together with radiative corrections. The simplest analysis of the tree order two generation case gives three interesting predictions-relations [405, 459]: (1) almost exact $b-\tau$ unification; (2) large atmospheric mixing angle related to the small quark $\theta_{b c}$ mixing angle; (3) somewhat degenerate neutrinos. For a serious numerical analysis one needs to use the RGE for the case of split supersymmetry, taking a very small $\tan \beta<1$ to get an approximate $b-\tau$ unification [458]. One needs also some fine-tuning of the parameters to account for the small ratio $M_{\mathrm{SUSY}} / M_{\mathrm{GUT}} \leq 10^{-(3-4)}$ required in realistic models to have gluinos decay fast enough [460].

\subsection{Higher dimensional approaches}

Recently, in the context of theories with extra spatial dimensions, some new approaches toward the question of SM fermion mass hierarchy and flavor structure have arisen [461-468]. For instance, the SM fermion mass spectrum can be generated naturally by permitting the quark/lepton masses to evolve with a power-law dependence on the mass scale $[465,466]$. The most studied and probably most attractive idea for generating a non-trivial flavor structure is the displacement of various SM fermions along extra dimension(s). This approach is totally different from the one discussed in Sect. 2, as it is purely geometrical and thus does not rely on the existence of any novel symmetry in the short distance theory. The displacement idea applies to the scenarios with large flat [467] or small warped [468] extra dimension(s), as we develop in the following subsections.

\subsubsection{Large extra dimensions}

In order to address the gauge hierarchy problem, a scenario with large flat extra dimensions has been proposed by Arkani-Hamed, Dimopoulos and Dvali (ADD) [469-471], based on a reduction of the fundamental gravity scale down to the $\mathrm{TeV}$ scale. In this scenario, gravity propagates in the bulk whereas SM fields live on a 3-brane. One could assume that this 3-brane has a certain thickness $L$ along an extra dimension (as for example in [472]). Then SM fields would feel an extra dimension of size $L$, exactly as in a universal extra dimension (UED) model [473] (where SM fields propagate in the bulk) with one extra dimension of size $L .^{12}$

In such a framework, the SM fermions can be localized at different positions along this extra dimension $L$. Then the relative displacements of quark/lepton wave function peaks produce suppression factors in the effective fourdimensional Yukawa couplings. These suppression factors

\footnotetext{
${ }^{12}$ The constraint from electroweak precision measurements is $R^{-1} \gtrsim$ 2-5 TeV, the one from direct search at LEP collider is $L^{-1} \gtrsim 5 \mathrm{TeV}$ and the expected LHC sensitivity is about $L^{-1} \sim 10 \mathrm{TeV}$.
} 
being determined by the overlaps of fermion wave functions (getting smaller as the distance between wave function peaks increases), they can vary with the fermion flavors and thus induce a mass hierarchy. This mechanism was first suggested in [467] and its variations have been studied in [474-484].

Let us describe this mechanism more precisely. The fermion localization can be achieved through either nonperturbative effects in string/M theory or field-theoretical methods. One field-theoretical possibility is to couple the SM fermion fields $\Psi_{i}\left(x_{\mu}, x_{5}\right)[i=1, \ldots, 3$ being the family index and $\mu=1, \ldots, 4$ the usual coordinate indexes] to five dimensional scalar fields with VEV $\Phi_{i}\left(x_{5}\right)$ depending on the extra dimension (parameterized by $x_{5}$ ) ${ }^{13}$ Indeed, chiral fermions are confined in solitonic backgrounds [485]. If the scalar field profile behaves as a linear function of the form $\Phi_{i}\left(x_{5}\right)=2 \mu^{2} x_{5}-m_{i}$ around its zero-crossing point $x_{i}^{0}=m_{i} / 2 \mu^{2}$, the zero-mode of five dimensional fermion acquires a Gaussian wave function of typical width $\mu^{-1}$ and centered at $x_{i}^{0}$ along the $x_{5}$ direction: $\Psi_{i}^{(0)}\left(x_{\mu}, x_{5}\right)=$ $A e^{-\mu^{2}\left(x_{5}-x_{i}^{0}\right)^{2}} \psi_{i}\left(x_{\mu}\right), \psi_{i}\left(x_{\mu}\right)$ being the four-dimensional fermion field and $A=\left(2 \mu^{2} / \pi\right)^{1 / 4}$ a normalization factor. Then the four-dimensional Yukawa couplings between the five dimensional SM Higgs boson $H$ and zero-mode fermions, obtained by integration on $x_{5}$ over the wall width $L,{ }^{14}$

$$
\begin{aligned}
\mathcal{S}_{\text {Yukawa }} & =\int d^{5} x \sqrt{L} \kappa H\left(x_{\mu}, x_{5}\right) \bar{\Psi}_{i}^{(0)}\left(x_{\mu}, x_{5}\right) \Psi_{j}^{(0)}\left(x_{\mu}, x_{5}\right) \\
& =\int d^{4} x Y_{i j} h\left(x_{\mu}\right) \bar{\psi}_{i}\left(x_{\mu}\right) \psi_{j}\left(x_{\mu}\right),
\end{aligned}
$$

are modulated by the following effective coupling constants,

$$
\begin{aligned}
Y_{i j} & =\int d x_{5} \kappa A^{2} e^{-\mu^{2}\left(x_{5}-x_{i}^{0}\right)^{2}} e^{-\mu^{2}\left(x_{5}-x_{j}^{0}\right)^{2}} \\
& =\kappa e^{-\frac{\mu^{2}}{2}\left(x_{i}^{0}-x_{j}^{0}\right)^{2}} .
\end{aligned}
$$

It can be considered as natural to have a five dimensional Yukawa coupling constant equal to $\sqrt{L} \kappa$, where the dimensionless parameter $\kappa$ is universal (in flavor and nature of fermions) and of order unity, so that the flavor structure is mainly generated by the field localization effect through the exponential suppression factor in (4.18). The remarkable feature is that, due to this exponential factor, large hierarchies can be created among the physical fermion masses, even for all fundamental parameters $m_{i}$ of order of the same energy scale $\mu$.

\footnotetext{
${ }^{13}$ Although we concentrate here on the case with only one extra dimension, for simplicity, the mechanism can be directly extended to more extra dimensions.

${ }^{14}$ Here, the factor $\sqrt{L}$ compensates with the Higgs component along $x_{5}$, since the Higgs boson is not localized.
}

This mechanism can effectively accommodate all the data on quark and charged lepton masses and mixings [486488]. In case that right handed neutrinos are added to the SM so that neutrinos acquire Dirac masses (as those originating from Yukawa couplings (4.17)), neutrino oscillation experiment results can also be reproduced [472]. The fine-tuning, arising there on relative $x_{i}^{0}$ parameters, turns out to be improved when neutrinos get Majorana masses instead [489] (see also [235, 490]).

\subsubsection{Small extra dimensions}

Another type of higher-dimensional scenario solving the gauge hierarchy problem was suggested by Randall and Sundrum (RS) [491, 492]. There, the unique extra dimension is warped and has a size of order $M_{\mathrm{Pl}}^{-1}\left(M_{\mathrm{Pl}}\right.$ being the reduced Planck mass: $M_{\mathrm{Pl}}=2.44 \times 10^{18} \mathrm{GeV}$ ) leading to an effective gravity scale around the $\mathrm{TeV}$. In the initial version, gravity propagates in the bulk and SM particles are all stuck on the TeV-brane. An extension of the original RS model was progressively proposed [493-497], motivated by its interesting features with respect to the gauge coupling unification [498-503] and dark matter problem [504, 505]. This new set-up is characterized by the presence of SM fields, except the Higgs boson (to ensure that the gauge hierarchy problem does not re-emerge), in the bulk.

In this RS scenario with bulk matter, a displacement of SM fermions along the extra dimension is also possible [468]: the effect is that the effective four-dimensional Yukawa couplings are affected by exponential suppression factors, originating from the wave function overlaps between bulk fermions and Higgs boson (confined on our TeVbrane). If the fermion localization depends on the flavor and nature of fermions, then the whole structure in flavor space can be generated by these wave function overlaps. In particular, if the top quark is located closer to the TeV-brane than the up quark, then its overlap with the Higgs boson, and thus its mass after electroweak symmetry breaking, is larger relatively to the up quark (for identical five dimensional Yukawa coupling constants).

More precisely, the fermions can acquire different localizations if each field $\Psi_{i}\left(x_{\mu}, x_{5}\right)$ is coupled to a distinct five dimensional mass $m_{i}: \int d^{4} x \int d x_{5} \sqrt{G} m_{i} \bar{\Psi}_{i} \Psi_{i}, G$ being the determinant of the RS metric. To modify the location of fermions, the masses $m_{i}$ must have a non-trivial dependence on $x_{5}$, like $m_{i}=\operatorname{sign}\left(x_{5}\right) c_{i} k$, where $c_{i}$ are dimensionless parameters and $1 / k$ is the curvature radius of antide Sitter space. Then the fields decompose as, $\Psi_{i}\left(x^{\mu}, x_{5}\right)=$ $\sum_{n=0}^{\infty} \psi_{i}^{(n)}\left(x^{\mu}\right) f_{n}^{i}\left(x_{5}\right) \quad[n$ labeling the tower of KaluzaKlein (KK) excitations], admitting the following solution for the zero-mode wave function, $f_{0}^{i}\left(x_{5}\right)=e^{\left(2-c_{i}\right) k\left|x_{5}\right|} / N_{0}^{i}$, where $N_{0}^{i}$ is a normalization factor. 
The Yukawa interactions with the Higgs boson $H$ read

$$
\begin{aligned}
\mathcal{S}_{\text {Yukawa }} & =\int d^{5} x \sqrt{G}\left(Y_{i j}^{(5)} H \bar{\Psi}_{+i} \Psi_{-j}+\text { h.c. }\right) \\
& =\int d^{4} x M_{i j} \bar{\psi}_{L i}^{(0)} \psi_{R j}^{(0)}+\text { h.c. }+\cdots
\end{aligned}
$$

The $Y_{i j}^{(5)}$ are the five dimensional Yukawa coupling constants and the dots stand for KK mass terms. The fermion mass matrix is obtained after integrating:

$M_{i j}=\int d x_{5} \sqrt{G} Y_{i j}^{(5)} H f_{0}^{i}\left(x_{5}\right) f_{0}^{j}\left(x_{5}\right)$.

The $Y_{i j}^{(5)}$ can be chosen almost universal so that the quark/ lepton mass hierarchies are mainly governed by the overlap mechanism. Large fermion mass hierarchies can be produced for fundamental mass parameters $m_{i}$ all of order of the unique scale of the theory $k \sim M_{\mathrm{Pl}}$.

With this mechanism, the quark masses and CKM mixing angles can be effectively accommodated [506-508], as well as the lepton masses and PMNS mixing angles in both cases where neutrinos acquire Majorana masses (via either dimension five operators [509] or the see-saw mechanism [510]) and Dirac masses (see [511], and [512, 513] for order unity Yukawa couplings leading to mass hierarchies essentially generated by the geometrical mechanism).

\subsubsection{Sources of FCNC in extra dimension scenarios}

GIM-violating FCNC effects in extra dimension scenarios may appear both from tree level and from loop effects.

At tree level FCNC processes can be induced by exchanges of KK excitations of neutral gauge bosons. The neutral current action of the effective four-dimensional coupling, between SM fermions $\psi_{i}^{(0)}\left(x^{\mu}\right)$ and KK excitations of any neutral gauge boson $A_{\mu}^{(n)}\left(x^{\mu}\right)$, reads in the interaction basis

$S_{\mathrm{NC}}=g_{L}^{\mathrm{SM}} \int d^{4} x \sum_{n=1}^{\infty} \bar{\psi}_{L i}^{(0)} \gamma^{\mu} \mathcal{C}_{L i j}^{(n)} \psi_{L j}^{(0)} A_{\mu}^{(n)}+\{L \leftrightarrow R\}$

Therefore, FCNC interactions can be induced by the nonuniversality of the effective coupling constants $g_{L / R}^{\mathrm{SM}} \times C_{0}^{i(n)}$ between KK modes of the gauge fields and the three SM fermion families (which have different locations along $x_{5}$ ).

At the loop level, KK fermion excitations may invalidate the GIM cancellation, as discussed e.g. in [511, 514] for $\ell_{\alpha}^{ \pm} \rightarrow \ell_{\beta}^{ \pm} \gamma$. Indeed, these excitations have KK masses which are not negligible (and thus not quasi-degenerate in family space) compared to $m_{W^{ \pm}}$. The GIM mechanism is also invalidated by the loop contributions of the $\mathrm{KK} W^{ \pm(n)}$ modes which couple (KK level by level), e.g. to leptons in the four-dimensional theory, via an effective mixing matrix of type $V_{\mathrm{MNS}}^{\mathrm{eff}}=U_{L}^{l \dagger} \mathcal{C}_{L}^{(n)} U_{L}^{v}$ being non-unitary due to the non-universality of

$\mathcal{C}_{L}^{(n)} \equiv \operatorname{diag}\left(C_{m}^{1(n)}, C_{m}^{2(n)}, C_{m}^{3(n)}\right)$

In this diagonal matrix, $C_{m}^{i(n)}$ quantifies the wave function overlap along the extra dimension between the $W^{ \pm(n)}$ $[n \geq 1]$ and exchanged ( $m$ th level KK) fermion $f_{m}^{i}\left(x_{5}\right)$ $[i=\{1,2,3\}$ being the generation index] (see below for more details).

The GIM mechanism for leptons can be clearly restored if the three coefficients $C_{m}^{i(n)}$ as well as the three KK fermion masses $m_{\mathrm{KK}}^{i(m)}$ are equal to each other, i.e. are universal with respect to $i=\{1,2,3\}$ (KK level by level) [515]. Within the quark sector, on the other hand, the top quark mass cannot be totally neglected relatively to the KK up-type quark excitation scales, leading to a mass shift of the KK top quark mode from the rest of the KK up-type quark modes and removing the degeneracy among three family masses of the up quark excitations at fixed KK level (with regard to $m_{W^{ \pm(n)}}$ ). Moreover, this means that the Yukawa interaction with the Higgs boson induces a substantial mixing of the top quark KK tower members among themselves $[481,516]$.

For example, the data on $b \rightarrow s \gamma$ (receiving a contribution from the exchange of a $W^{ \pm(n)}[n=0,1, \ldots]$ gauge field and an up quark, or its KK excitations, at one loop-level) can be accommodated in the RS model with $m\left(W^{ \pm(1)}\right) \simeq 1 \mathrm{TeV}$, as shown in [515] using numerical methods for the diagonalization of a large dimensional mass matrix and taking into account the top quark mass effects described previously.

\subsubsection{Mass bounds on Kaluza-Klein excitations}

In this subsection we develop constraints on the $\mathrm{KK}$ gauge boson masses derived from the tree level FCNC effect described above. Our purpose is to determine whether these constraints still allow the KK gauge bosons to be sufficiently light to imply potentially visible signatures at LHC.

4.2.4.1 Large extra dimensions Let us consider the generic framework of a flat extra dimension, with a large size $L$, along which gravity as well as gauge bosons propagate. The SM fermions are located at different points of the fifth dimension, so that their mass hierarchy can be interpreted in term of the geometrical mechanism described in details in Sect. 4.2.1. In such a framework the exchange of the KK excitations of the gluon can bring important contributions to the $K^{0}-\bar{K}^{0}$ mixing $(\Delta F=2)$ at tree level. Indeed, the KK gluon can couple the $d$ quark with the $s$ quark, if these light down-quarks are displaced along the extra dimension. The obtained KK contribution to the mass splitting $\Delta m_{K}$ in 
the kaon system depends on the KK gluon coupling between the $s$ and $d$ quarks (which is fixed by quark locations) and mainly on the mass of the first KK gluon $M_{\mathrm{KK}}^{(1)}$. Assuming that the $s, d$ quark locations are such that the $m_{s}, m_{d}$ mass values are reproduced, the obtained $\Delta m_{K}$ and also $\left|\varepsilon_{K}\right|$ are smaller than the associated experimental values for, respectively,

$M_{\mathrm{KK}}^{(1)} \gtrsim 25 \mathrm{TeV}, \quad$ and $\quad M_{\mathrm{KK}}^{(1)} \gtrsim 300 \mathrm{TeV}$,

as found by the authors of [517]. The same bound coming from the $D^{0}$ meson system is weaker.

In the lepton sector the experimental upper limit on the branching ratio $B(\mu \rightarrow e e e)$ imposes typically the constraint [517]

$M_{\mathrm{KK}}^{(1)} \gtrsim 30 \mathrm{TeV}$,

since the exchange of the KK excitations of the electroweak neutral gauge bosons contributes to the decay $\mu \rightarrow$ eee.

To conclude, we stress that if the extra dimensions treat families in a non-universal way (which could explain the fermion mass hierarchy), the indirect bounds from FCNC physics like the ones in (4.23)-(4.24) force the mass of the KK gauge bosons to be far from the collider reach. As a matter of fact, the LHC will be able to probe the KK excitations of gauge bosons only up to $6-7 \mathrm{TeV}[518-521]$ in the present context.

4.2.4.2 Small extra dimensions In the context of the RS model with SM fields in the bulk, described in Sect. 4.2.2, the exchange of KK excitations of neutral gauge bosons (like e.g. the first $Z^{0}$ excitation: $Z^{(1)}$ ) also contributes to FCNC processes at tree level [468, 507, 522-526] since these KK states possess FC couplings if the different families of fermions are displaced along the warped extra dimension. There exist some configurations of fermion locations, pointed out in [513], which simultaneously reproduce all quark/lepton masses and mixing angles via the wave function effects and lead to amplitudes of FCNC reactions $\left[l_{\alpha} \rightarrow l_{\beta} l_{\gamma} l_{\gamma}\right.$, $Z^{0} \rightarrow l_{\alpha} l_{\beta}, P^{0}-\bar{P}^{0}$ mixing of a generic meson $P, \mu-e$ conversion, $K^{0} \rightarrow l_{\alpha} l_{\beta}$ and $\left.K^{+} \rightarrow \pi^{+} \nu \nu\right]$ compatible with the corresponding experimental constraints even for light neutral KK gauge bosons:

$M_{\mathrm{KK}}^{(1)} \gtrsim 1 \mathrm{TeV}$.

The explanation of this result is the following. If the SM fermions with different locations are localized typically close to the Planck-brane, they have quasi-universal couplings $C_{0}^{i(n)}$ [cf. (4.21)] with the KK gauge bosons which have a wave function almost constant along the fifth dimension near the Planck-brane. Therefore, small FC couplings are generated in the physical basis for these fermions leading to the weak bound (4.25). The fermions from the third family, associated to heavy flavors, cannot be localized extremely close to the Planck-brane since their wave function overlap with the Higgs boson [confined on the TeV-brane] must be large in order to generate high effective Yukawa couplings. Nevertheless, this is compensated by the fact that phenomenological FCNC constraints are usually less severe in the third generation sector.

As a result, the order of lower limits on $M_{\mathrm{KK}}^{(1)}$ coming from the considerations on both fermion mass data and FCNC processes can be as low as TeV. From the purely theoretical point of view, the favored order of magnitude for $M_{\mathrm{KK}}^{(1)}$ is $\mathcal{O}(1) \mathrm{TeV}$ which corresponds to a satisfactory solution for the gauge hierarchy problem. From the model building point of view one has to rely on an appropriate extension of the RS model insuring that, for light KK masses, the deviations of the electroweak precision observables do not conflict with the experimental results. The existing RS extensions, like the scenarios with brane-localized kinetic terms for fermions [527] and gauge bosons [528] (see [529, 530] for the localized gauge boson kinetic terms and [531] for the fermion ones), or the scenarios with an extended gauge symmetry (see [532-534] for different fermion charges under this broken symmetry), allow $M_{\mathrm{KK}}^{(1)}$ to be as low as $\sim 3 \mathrm{TeV}$. In such a case, one can expect a direct detection of the KK excited gauge bosons at LHC.

\subsection{Minimal flavor violation in the lepton sector}

\subsubsection{Motivations and basic idea}

Within the SM the dynamics of flavor-changing transitions is controlled by the structure of fermion mass matrices. In the quark sector, up and down quarks have mass eigenvalues which are up to $10^{5}$ times smaller than the electroweak scale, and mass matrices which are approximately aligned. This results in the effective CKM and GIM suppressions of charged and neutral flavor violating interactions, respectively. Forcing this connection between the low energy fermion mass matrices and the flavor-changing couplings to be valid also beyond the SM, leads to new-physics scenarios with a high level of predictivity (in the flavor sector) and a natural suppression of flavor-changing transitions. The latter achievement is a key ingredient to maintain a good agreement with experiments in models where flavored degrees of freedom are expected around the $\mathrm{TeV}$ scale.

This is precisely the idea behind the minimal flavor violation principle [535-537]. It is a fairly general hypothesis that can be implemented in strongly-interacting theories [535], low energy supersymmetry [536, 537], multi-Higgs [537, 538] and GUT [539] models. In a model independent formulation, the MFV construction consists in identifying the flavor symmetry and symmetry breaking structure of the SM and enforce it in a more general effective theory (written in terms of SM fields and valid above 
the electroweak scale). In the quark sector this procedure is unambiguous: the largest group of flavor changing field transformations commuting with the gauge group is $\mathcal{G}_{q}=$ $S U(3)_{Q_{L}} \times S U(3)_{u_{R}} \times S U(3)_{d_{R}}$, and this group is broken only by the Yukawa couplings. The invariance of the SM Lagrangian under $\mathcal{G}_{q}$ can be formally recovered elevating the Yukawa matrices to spurion fields with appropriate transformation properties under $\mathcal{G}_{q}$. The hypothesis of MFV states that these are the only spurions breaking $\mathcal{G}_{q}$ also beyond the SM. Within the effective theory formulation, this implies that all the higher dimensional operators constructed from SM and Yukawa fields must be (formally) invariant under $\mathcal{G}_{q}$. The consequences of this hypothesis in the quark sector have been extensively analyzed in the literature (see e.g. Refs. [540, 541]). Without entering into the details, we can state that the MFV hypothesis provides a plausible explanation of why no new-physics effects have been observed so far in the quark sector.

Apart from arguments based on the analogy with quarks, and despite the scarce experimental information, the definition of a minimal lepton flavor violation (MLFV) principle [542] is demanded by a severe fine-tuning problem in LFV decays of charged leptons. Within a generic effective theory approach, the radiative decays $l_{i} \rightarrow l_{j} \gamma$ proceed through the following gauge-invariant operator

$\frac{\delta_{i j}^{R L}}{\Lambda_{\mathrm{LFV}}^{2}} H^{\dagger} \bar{e}_{R}^{i} \sigma^{\sigma \rho} L_{L}^{j} F_{\sigma \rho}$,

where $\delta_{i j}^{R L}$ are the generic flavor-changing couplings and $\Lambda_{\mathrm{LFV}}$ denotes the cut-off of the effective theory. In the absence of a specific flavor structure, it is natural to expect $\delta_{i j}^{R L}=\mathcal{O}(1)$. In this case the experimental limit for $\mu \rightarrow e \gamma$ implies $\Lambda_{\mathrm{LFV}}>10^{5} \mathrm{TeV}$, in clear tension with the expectation of new degrees of freedom close to the $\mathrm{TeV}$ scale in order to stabilize the Higgs sector of the SM.

The implementation of a MFV principle in the lepton sector is not as simple as in the quark sector. The problem is that the neutrino mass matrix itself cannot be accommodated within the renormalizable part of the SM Lagrangian. The most natural way to describe neutrino masses, explaining their strong suppression, is to assume they are Majorana mass terms suppressed by the heavy scale of lepton number violation (LNV). In other words, neutrino masses are described by a non-renormalizable interaction of the type equation (3.4) suppressed by the scale $\Lambda_{\mathrm{LNV}} \gg v=|\langle H\rangle|$. This implies that we have to face a two scale problem (presumably with the hierarchy $\Lambda_{\mathrm{LNV}} \gg \Lambda_{\mathrm{LFV}}$ ) and that we need some additional hypothesis to identify the irreducible flavor-symmetry breaking structures. As we shall illustrate in the following, we can choose whether to extend or not the field content of the SM. The construction of the effective theory based on one of these realizations of the MLFV hypothesis can be viewed as a general tool to exploit the observable consequences of a specific (minimalistic) hypothesis about the irreducible sources of lepton-flavor symmetry breaking.

\subsubsection{MLFV with minimal field content}

The lepton field content is the SM one: three left handed doublets $L_{L}^{i}$ and three right handed charged lepton singlets $e_{R}^{i}$. The flavor symmetry group is $\mathcal{G}_{l}=S U(3)_{L_{L}} \times S U(3)_{e_{R}}$ and we assume the following flavor symmetry breaking Lagrangian

$$
\begin{aligned}
\mathcal{L}_{\text {Sym.Br. }}= & -Y_{e}^{i j} \bar{e}_{R}^{i}\left(H^{\dagger} L_{L}^{j}\right) \\
& -\frac{1}{2 \Lambda_{\mathrm{LNV}}} \kappa_{\nu}^{i j}\left(\bar{L}_{L}^{c i} \tau_{2} H\right)\left(H^{T} \tau_{2} L_{L}^{j}\right)+\text { h.c. } \\
\rightarrow & -v Y_{e}^{i j} \bar{e}_{R}^{i} e_{L}^{j}-\frac{v^{2}}{2 \Lambda_{\mathrm{LNV}}} \kappa_{\nu}^{i j} \bar{\nu}_{L}^{c i} v_{L}^{j}+\text { h.c. }
\end{aligned}
$$

Here the two irreducible sources of LFV are the coefficient of dimension five LNV operator $\left(\kappa_{v}^{i j}\right)$ and the charged lepton Yukawa coupling $\left(Y_{e}\right)$, transforming respectively as $(6,1)$ and $(\overline{3}, 3)$ under $\mathcal{G}_{l}$. An explicit realization of this scenario is provided by the so-called triplet see-saw mechanism (or seesaw of type II). This approach has the advantage of being highly predictive, but it differs in an essential way from the MFV hypothesis in the quark sector since one of the basic spurion originates from a non-renormalizable coupling.

Having identified the irreducible sources of flavor symmetry breaking and their transformation properties, we can classify the non-renormalizable operators suppressed by inverse powers of $\Lambda_{\mathrm{LFV}}$ which contribute to flavor violating processes. These operators must be invariant combinations of SM fields and the spurions $Y_{e}$ and $\kappa_{\nu}$. The complete list of the leading operators contributing to LFV decays of charged leptons is given in Refs. [542, 543]. The case of the radiative decays $l_{i} \rightarrow l_{j} \gamma$ is particularly simple since there are only two dimension six operators (operators with a structure as in (3.4), with $F_{\sigma \rho}$ replaced by the stress tensors of the $U(1)_{Y}$ and $S U(2)_{L}$ gauge groups, respectively). The MLFV hypothesis forces the flavor-changing couplings of these operators to be a spurion combination transforming as $(\overline{3}, 3)$ under $\mathcal{G}_{l}$ :

$\left(\delta_{\min }^{R L}\right)_{i j} \propto\left(Y_{e} \kappa_{v}^{\dagger} \kappa_{v}\right)_{i j}+\cdots$

where the dots denote terms with higher powers of $Y_{e}$ or $\kappa_{\nu}$. Up to the overall normalization, this combination can be completely determined in terms of the neutrino mass eigenvalues and the PMNS matrix. In the basis where $Y_{e}$ is diagonal we can write, 


$$
\begin{aligned}
\left(Y_{e} \kappa_{v}^{\dagger} \kappa_{v}\right)_{i \neq j}= & \frac{m_{l_{i}}}{v}\left(\frac{\Lambda_{\mathrm{LNV}}^{2}}{v^{4}} U_{\mathrm{PMNS}} m_{v}^{2} U_{\mathrm{PMNS}}^{\dagger}\right)_{i \neq j} \\
\rightarrow & \frac{m_{l_{i}}}{v} \frac{\Lambda_{\mathrm{LNV}}^{2}}{v^{4}}\left[\left(U_{\mathrm{PMNS}}\right)_{i 2}\left(U_{\mathrm{PMNS}}\right)_{j 2}^{*} \Delta m_{\mathrm{sol}}^{2}\right. \\
& \left. \pm\left(U_{\mathrm{PMNS}}\right)_{i 3}\left(U_{\mathrm{PMNS}}\right)_{j 3}^{*} \Delta m_{\mathrm{atm}}^{2}\right]
\end{aligned}
$$

where $\Delta m_{\text {atm }}^{2}$ and $\Delta m_{\text {sol }}^{2}$ denote the squared mass differences deduced from atmospheric- and solar-neutrino data, and $+/-$ correspond to normal/inverted hierarchy, respectively. The overall factor $\Lambda_{\mathrm{LNV}}^{2} / v^{2}$ implies that the absolute normalization of LFV rates suffers of a large uncertainty. Nonetheless, a few interesting conclusions can still be drawn [542].

- The LFV decay rates are proportional to $\Lambda_{\mathrm{LNV}}^{4} / \Lambda_{\mathrm{LFV}}^{4}$ and could be detected only in presence of a large hierarchy between these two scales. In particular, $\mathcal{B}(\mu \rightarrow e \gamma)>$ $10^{-13}$ only if $\Lambda_{\mathrm{LNV}}>10^{9} \Lambda_{\mathrm{LFV}}$.

- Ratios of similar LFV decay rates, such as $B(\mu \rightarrow$ $e \gamma) / B(\tau \rightarrow \mu \gamma)$, are free from the normalization ambiguity and can be predicted in terms of neutrino masses and PMNS angles: violations of these predictions would unambiguously signal the presence of additional sources of lepton-flavor symmetry breaking. One of these prediction is the $10^{-2}-10^{-3}$ enhancement of $B(\tau \rightarrow \mu \gamma)$ versus $B(\mu \rightarrow e \gamma)$ shown in Fig. 3. Given the present and near-future experimental prospects on these modes, this modest enhancement implies that the $\mu \rightarrow e \gamma$ search is much more promising within this framework.

- Ratios of LFV transitions among the same two families (such as $\mu \rightarrow e \gamma$ versus $\mu \rightarrow 3 e$ or $\tau \rightarrow \mu \gamma$ vs $\tau \rightarrow 3 \mu$ and $\tau \rightarrow \mu e \bar{e}$ ) are determined by known phase space factors and ratios of various Wilson coefficients. As data will become available on different lepton flavor violating processes, if the flavor patter is consistent with the MLFV hypothesis, from these ratios it will be pos-

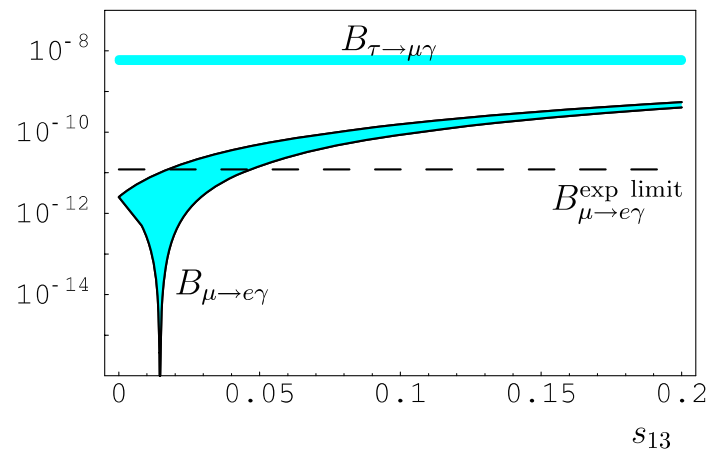

Fig. $3 \quad B_{l_{i} \rightarrow l_{j} \gamma} \equiv \Gamma\left(l_{i} \rightarrow l_{j} \gamma\right) / \Gamma\left(l_{i} \rightarrow l_{j} v_{i} \bar{v}_{j}\right)$ for $\mu \rightarrow e \gamma$ and $\tau \rightarrow \mu \gamma$ as a function of $\sin \theta_{13}$ in the MLFV framework with minimal field content [542]. The normalization of the vertical axis corresponds to $\Lambda_{\mathrm{LNV}} / \Lambda_{\mathrm{LFV}}=10^{10}$. The shading is due to different values of the phase $\delta$ and the normal/inverted spectrum sible to disentangle the contributions of different operators.

- A definite prediction of the MLFV hypothesis is that the rates for decays involving light hadrons $\left(\pi^{0} \rightarrow \mu e\right.$, $\left.K_{L} \rightarrow \mu e, \tau \rightarrow \mu \pi^{0}, \ldots\right)$ are exceedingly small.

\subsubsection{MLFV with extended field content}

In this scenario we assume three heavy right handed Majorana neutrinos in addition to the SM fields. As a consequence, the maximal flavor group becomes $\mathcal{G}_{l} \times S U(3)_{v_{R}}$. In order to minimize the number of free parameters (or to maximize the predictivity of the model), we assume that the Majorana mass term for the right handed neutrinos is proportional to the identity matrix in flavor space: $\left(M_{R}\right)_{i j}=$ $M_{R} \times \delta_{i j}$. This mass term breaks $S U(3)_{v_{R}}$ to $O(3)_{v_{R}}$ and is assumed to be the only source of $\mathrm{LNV}\left(M_{R} \leftrightarrow \Lambda_{\mathrm{LNV}}\right)$.

Once the field content of model is extended, there are in principle many alternative options to define the irreducible sources of lepton flavor symmetry breaking (see e.g. Ref. [544] for an extensive discussion). However, this specific choice has two important advantages: it is predictive and closely resemble the MFV hypothesis in the quark sector. The $v_{R}$ are the counterpart of right handed up quarks and, similarly to the quark sector, the symmetry breaking sources are two Yukawa couplings of (3.40). An explicit example of MLFV with extended field content is the minimal supersymmetric standard model with degenerate right handed neutrinos.

The classification of the higher dimensional operators in the effective theory proceeds as in the minimal field content case. The only difference is that the basic spurions are now $Y_{v}$ and $Y_{e}$, transforming as $(\overline{3}, 1,3)$ and $(\overline{3}, 3,1)$ under $\mathcal{G}_{l} \times O(3)_{v_{R}}$, respectively. The determination of the spurion structures in terms of observable quantities is more involved than in the minimal field content case. In general, inverting the see-saw relation allows us to express $Y_{v}$ in terms of neutrino masses, PMNS angles and an arbitrary complexorthogonal matrix $R$ of (3.45) [232]. Exploiting the $O(3)_{v_{R}}$ symmetry of the MLFV Lagrangian, the real orthogonal part of $R$ can be rotated away. We are then left with a Hermitianorthogonal matrix $H$ [545] which can be parameterized in terms of three real parameters $\left(\phi_{i}\right)$ which control the amount of $\mathrm{CP}$ violation in the right handed sector:

$Y_{v}=\frac{M_{R}^{1 / 2}}{v} H\left(\phi_{i}\right) m_{\mathrm{diag}}^{1 / 2} U_{\mathrm{PMNS}}^{\dagger}$.

With this parameterization for $Y_{v}$ the flavor changing coupling relevant to $l_{i} \rightarrow l_{j} \gamma$ decays reads

$$
\begin{aligned}
\delta_{\mathrm{ext}}^{R L} & \propto Y_{e}\left(Y_{v}^{\dagger} Y_{v}\right) \\
& \rightarrow \frac{m_{e}}{v}\left(\frac{M_{R}}{v^{2}} U_{\mathrm{PMNS}} m_{\mathrm{diag}}^{1 / 2} H^{2} m_{\mathrm{diag}}^{1 / 2} U_{\mathrm{PMNS}}^{\dagger}\right)
\end{aligned}
$$


In the CP-conserving limit $H \rightarrow I$ and the phenomenological predictions turns out to be quite similar to the minimal field content scenario [542]. In particular, all the general observations listed in the previous section remain valid. In the general case, i.e. for $H \neq I$, the predictivity of the model is substantially weakened. However, in principle some information about the matrix $H$ can be extracted by studying baryogenesis through leptogenesis in the MLFV framework [546].

\subsubsection{Leptogenesis}

On general grounds, we expect that the tree-level degeneracy of heavy neutrinos is lifted by radiative corrections. This allows the generation of a lepton asymmetry in the interference between tree-level and one loop decays of right handed neutrinos. Following the standard leptogenesis scenario, we assume that this lepton asymmetry is later communicated to the baryon sector through sphaleron effects and that saturates the observed value of the baryon asymmetry of the universe.

The most general form of the $v_{R}$ mass splittings allowed within the MLFV framework has the following form:

$$
\begin{aligned}
\frac{\Delta M_{R}}{M_{R}}= & c_{v}\left[Y_{v} Y_{v}^{\dagger}+\left(Y_{v} Y_{v}^{\dagger}\right)^{T}\right] \\
& +c_{v v}^{(1)}\left[Y_{v} Y_{v}^{\dagger} Y_{v} Y_{v}^{\dagger}+\left(Y_{v} Y_{v}^{\dagger} Y_{v} Y_{v}^{\dagger}\right)^{T}\right] \\
& +c_{v v}^{(2)}\left[Y_{v} Y_{v}^{\dagger}\left(Y_{v} Y_{v}^{\dagger}\right)^{T}\right]+c_{v v}^{(3)}\left[\left(Y_{v} Y_{v}^{\dagger}\right)^{T} Y_{v} Y_{v}^{\dagger}\right] \\
& +c_{v l}\left[Y_{v} Y_{e}^{\dagger} Y_{e} Y_{v}^{\dagger}+\left(Y_{v} Y_{e}^{\dagger} Y_{e} Y_{v}^{\dagger}\right)^{T}\right]+\cdots .
\end{aligned}
$$

Even without specifying the value of the $c_{i}$, this form allows us to derive a few general conclusions [546].
- The term proportional to $c_{v}$ does not generate a CPV asymmetry, but sets the scale for the mass splittings: these are of the order of magnitude of the decay widths, realizing in a natural way the condition of resonant leptogenesis.

- The right amount of leptogenesis can be generated even with $Y_{e}=0$, if all the $\phi_{i}$ are non-vanishing. However, since $Y_{v} \sim \sqrt{M_{R}}$, for low values of $M_{R}\left(\lesssim 10^{12} \mathrm{GeV}\right)$ the asymmetry generated by the $c_{v l}$ term dominates. In this case $\eta_{B}$ is typically too small to match the observed value and has a flat dependence on $M_{R}$. At $M_{R} \gtrsim 10^{12} \mathrm{GeV}$ the quadratic terms $c_{\nu \nu}^{(i)}$ dominate, determining an approximate linear growth of $\eta_{B}$ with $M_{R}$. These two regimes are illustrated in Fig. 4.

As demonstrated in Ref. [546], baryogenesis through leptogenesis is viable in MLFV models. In particular, assuming a loop hierarchy between the $c_{i}$ (as expected in a perturbative scenario) and neglecting flavor-dependent effects in the Boltzmann equations (one-flavor approximation of Ref. [547]), the right size of $\eta_{B}$ is naturally reached for $M_{R} \gtrsim 10^{12} \mathrm{GeV}$. As discussed in Ref. [301] (see also [303]), this lower bound can be weakened by the inclusion of flavordependent effects in the Boltzmann equations and/or by the $\tan \beta$-enhancement of $Y_{e}$ occurring in two-Higgs doublet models.

From the phenomenological point of view, an important difference with respect to the $\mathrm{CP}$-conserving case is the fact that non-vanishing $\phi_{i}$ change the predictions of the LFV decays, typically producing an enhancement of the $B(\mu \rightarrow$ $e \gamma) / B(\tau \rightarrow \mu \gamma)$ ratio or the both decays separately [545]. For $M_{R} \gg 10^{12} \mathrm{GeV}$ their effect is moderate and the CPconserving predictions are recovered. The other important information following from the leptogenesis analysis is the
Fig. 4 Baryon asymmetry $\left(\eta_{B}\right)$ as a function of the right handed neutrino mass scale $\left(M_{R}\right)$ for $c_{\nu l}=0($ dots $)$ and $c_{\nu l} \neq 0$ (crosses) in the MLFV framework with extended field content [546]

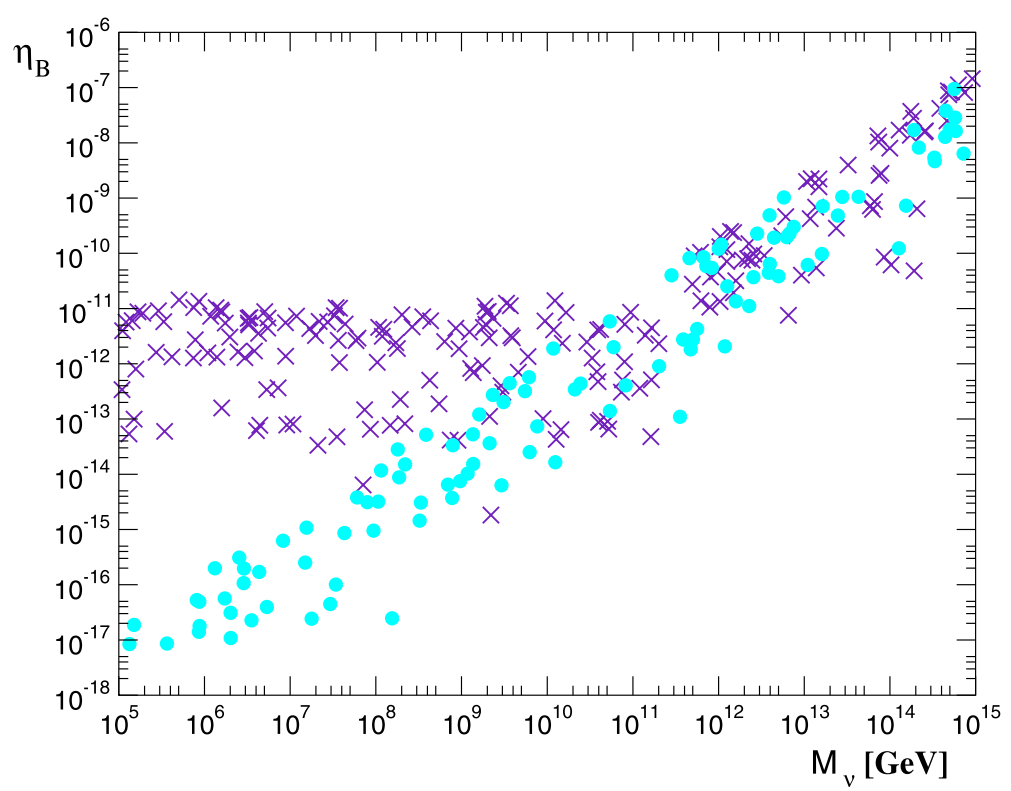


fact that the large $M_{R}$ regime is favored. Assuming $\Lambda_{\mathrm{LFV}}$ to be close to the TeV scale, the $M_{R}$ regime favored by leptogenesis favors a $\mu \rightarrow e \gamma$ rate within the reach of the MEG experiment [548].

\subsubsection{GUT implementation}

Once we accept the idea that flavor dynamics obeys a MFV principle, both in the quark and in the lepton sector, it is interesting to ask if and how this is compatible with a grand unified theory (GUT), where quarks and leptons sit in the same representations of a unified gauge group. This question has recently been addressed in [539], considering the exemplifying case of $S U(5)_{\text {gauge }}$.

Within $S U(5)_{\text {gauge }}$, the down-type singlet quarks $\left(d_{i R}^{c}\right)$ and the lepton doublets $\left(L_{i L}\right)$ belong to the $\overline{\mathbf{5}}$ representation; the quark doublet $\left(Q_{i L}\right)$, the up-type $\left(u_{i R}^{c}\right)$ and lepton singlets $\left(e_{i R}^{c}\right)$ belong to the $\mathbf{1 0}$ representation, and finally the right handed neutrinos $\left(v_{i R}\right)$ are singlet. In this framework the largest group of flavor transformation commuting with the gauge group is $\mathcal{G}_{\mathrm{GUT}}=S U(3)_{5} \times S U(3)_{10} \times S U(3)_{1}$, which is smaller than the direct product of the quark and lepton groups discussed before $\left(\mathcal{G}_{q} \times \mathcal{G}_{l}\right)$. We should therefore expect some violations of the MFV+MLFV predictions either in the quark or in the lepton sector or in both.

A phenomenologically acceptable description of the low energy fermion mass matrices requires the introduction of at least four irreducible sources of $\mathcal{G}_{\mathrm{GUT}}$ breaking. From this point of view the situation is apparently similar to the non-unified case: the four $\mathcal{G}_{\text {GUT }}$ spurions can be put in oneto-one correspondence with the low energy spurions $Y_{u}, Y_{d}$, $Y_{e}$, and $Y_{\nu}$. However, the smaller flavor group does not allow the diagonalization of $Y_{d}$ and $Y_{e}$ (which transform in the same way under $\mathcal{G}_{\mathrm{GUT}}$ ) in the same basis. As a result, two additional mixing matrices can appear in the expressions for flavor changing rates: $C=V_{e_{R}}^{T} V_{d_{L}}$ and $G=V_{e_{L}}^{T} V_{d_{R}}$. The hierarchical texture of the new mixing matrices is known since they reduce to the identity matrix in the limit $Y_{e}^{T}=Y_{d}$. Taking into account this fact, and analyzing the structure of the allowed higher-dimensional operators, a number of reasonably firm phenomenological consequences can be deduced [539]:

- There is a well defined limit in which the standard MFV scenario for the quark sector is fully recovered: $M_{R} \ll$ $10^{12} \mathrm{GeV}$ and small $\tan \beta$ (in a two-Higgs doublet case). For $M_{R} \sim 10^{12} \mathrm{GeV}$ and small $\tan \beta$, deviations from the standard MFV pattern can be expected in rare $K$ decays but not in $B$ physics. Ignoring fine-tuned scenarios, $M_{R} \gg 10^{12} \mathrm{GeV}$ is excluded by the present constraints on quark FCNC transitions. Independently from the value of $M_{R}$, deviations from the standard MFV pattern can appear both in $K$ and in $B$ physics for $\tan \beta \gtrsim m_{t} / m_{b}$.
- Contrary to the non-GUT MFV framework, the rate for $\mu \rightarrow e \gamma$ (and other LFV decays) cannot be arbitrarily suppressed by lowering the average mass $M_{R}$ of the heavy $v_{R}$. This fact can easily be understood by looking at the flavor structure of the relevant effective couplings, which now assume the following form:

$$
\delta_{\mathrm{GUT}}^{\mathrm{RL}}=c_{1} Y_{e} Y_{v}^{\dagger} Y_{v}+c_{2} Y_{u} Y_{u}^{\dagger} Y_{e}+c_{3} Y_{u} Y_{u}^{\dagger} Y_{d}^{T}+\cdots .
$$

In addition to the terms involving $Y_{v} \sim \sqrt{M_{R}}$ already present in the non-unified case, the GUT group allows also $M_{R}$-independent terms involving the quark Yukawa couplings. The latter become competitive for $M_{R} \lesssim 10^{12} \mathrm{GeV}$ and their contribution is such that for $\Lambda_{\mathrm{LFV}} \lesssim 10 \mathrm{TeV}$ the $\mu \rightarrow e \gamma$ rate is above $10^{-13}$ (i.e. within the reach of MEG [548]).

- Improved experimental information on $\tau \rightarrow \mu \gamma$ and $\tau \rightarrow$ $e \gamma$ would be a powerful tool in discriminating the relative size of the standard MFV contributions versus the characteristic GUT-MFV contributions due to the different hierarchy pattern among $\tau \rightarrow \mu, \tau \rightarrow e$, and $\mu \rightarrow e$ transitions.

\section{Phenomenology of theories beyond the standard model}

5.1 Flavor violation in non-SUSY models directly testable at $\mathrm{LHC}$

\subsubsection{Multi-Higgs doublet models}

The arbitrariness of quark masses, mixing and $\mathrm{CP}$ violation in the standard model stems from the fact that gauge invariance does not constrain the flavor structure of Yukawa interactions. In the SM neutrinos are strictly massless. No neutrino Dirac mass term can be introduced, due to the absence of right handed neutrinos and no Majorana mass terms can be generated, due to exact $B-L$ conservation. Since neutrinos are massless, there is no leptonic mixing in the SM, which in turn leads to separate lepton flavor conservation. Therefore, the recent observation of neutrino oscillations is evidence for physics beyond the SM. Fermion masses, mixing and $\mathrm{CP}$ violation are closely related to each other and also to the Higgs sector of the theory.

It has been shown that gauge theories with fermions, but without scalar fields, do not break CP symmetry [549]. A scalar (Higgs) doublet is used in the SM to break both the gauge symmetry and generate gauge boson masses as well as fermion masses through Yukawa interactions. This is known as the Higgs mechanism, which was proposed by several authors [550-553]. It predicts the existence of one 
neutral scalar Higgs particle-the Higgs boson. In the SM where a single Higgs doublet is introduced, it is not possible to have spontaneous $\mathrm{CP}$ violation since any phase in the vacuum expectation value can be eliminated by rephasing the Higgs field. Furthermore, in the SM it is also not possible to violate CP explicitly in the Higgs sector since gauge invariance together with renormalizability restrict the Higgs potential to have only quadratic and quartic terms and hermiticity constrains both of these to be real. Thus, CP violation in the SM requires the introduction of complex Yukawa couplings.

The scenario of spontaneous $\mathrm{CP}$ and $\mathrm{T}$ violation has the nice feature of putting the breakdown of discrete symmetries on the same footing as the breaking of the gauge symmetry, which is also spontaneous in order to preserve renormalizability. A simple extension of the Higgs sector that may give rise to spontaneous $\mathrm{CP}$ violation requires the presence of at least two Higgs doublets, and was introduced by Lee [554].

If one introduces two Higgs doublets, it is possible to have either explicit or spontaneous $\mathrm{CP}$ breaking. Explicit CP violation in the Higgs sector arises due to the fact that in this case there are gauge invariant terms in the Lagrangian which can have complex coefficients. Note however that the presence of complex coefficients does not always lead to explicit $\mathrm{CP}$ breaking.

Extensions of the SM with extra Higgs doublets are very natural since they keep the $\rho$ parameter at tree level equal to one [555]. In multi-Higgs systems there are in general, additional sources of CP violation in the Higgs sector [556]. The most general renormalizable polynomial consistent with the $S U(2) \times U(1) \times S U(3)_{c}$ model with $n_{d}$ Higgs doublets, $\phi_{i}$, may be written as

$\mathcal{L}_{\phi}=Y_{a b} \phi_{a}^{\dagger} \phi_{b}+Z_{a b c d}\left(\phi_{a}^{\dagger} \phi_{b}\right)\left(\phi_{c}^{\dagger} \phi_{d}\right)$,

where repeated indices are summed. Hermiticity of $\mathcal{L}_{\phi}$ implies:

$Y_{a b}^{*}=Y_{b a} ; \quad Z_{a b c d}^{*}=Z_{b a d c}$.

Furthermore, by construction it is obvious that:

$Z_{a b c d}=Z_{c d a b}$.

In models with more than one Higgs doublet, one has the freedom to make Higgs-basis transformations (HBT) that do not change the physical content of the model, but do change both the quadratic and the quartic coefficients. Coefficients that are complex in one Higgs basis may become real in another basis. Furthermore, a given model may have complex quartic coefficients in one Higgs basis, while they may all become real in another basis, with only the quadratic coefficients now complex, thus indicating that in that particular model CP is only softly broken. Such Higgs-basis transformations leave the Higgs kinetic energy term invariant and are of the form:

$\phi_{a} \stackrel{\mathrm{HBT}}{\longrightarrow} \phi_{a}^{\prime}=V_{a i} \phi_{i}, \quad \phi_{a}^{\dagger} \stackrel{\mathrm{HBT}}{\longrightarrow}\left(\phi^{\prime}\right)_{a}^{\dagger}=V_{a i}^{*}\left(\phi^{\prime}\right)_{i}^{\dagger}$,

where $V$ is an $n_{d} \times n_{d}$ unitary matrix acting in the space of Higgs doublets. In [557] conditions for a given Higgs potential to violate $\mathrm{CP}$ at the Lagrangian level, expressed in terms of CP-odd Higgs-basis invariants, were derived. These conditions are expressed in terms of couplings of the unbroken Lagrangian, therefore they are relevant even at high energies, where the $S U(2) \times U(1)$ symmetry is restored. This feature renders them potentially useful for the study of baryogenesis. The derivation of these conditions follows the general method proposed in [558] and already mentioned in previous sections. The method consists of imposing invariance of the Lagrangian under the most general CP transformation of the Higgs doublets, which is a combination of a simple CP transformation for each Higgs field with a Higgsbasis transformation:

$\phi_{a} \stackrel{\mathrm{CP}}{\longrightarrow} W_{a i} \phi_{i}^{*} ; \quad \phi_{a}^{\dagger} \stackrel{\mathrm{CP}}{\longrightarrow} W_{a i}^{*} \phi_{i}^{T}$.

Here $W$ is an $n_{d} \times n_{d}$ unitary matrix operating in Higgs doublets space.

A set of necessary and sufficient conditions for $\mathrm{CP}$ invariance in the case of two Higgs doublets have been derived [557]:

$$
\begin{aligned}
& I_{1} \equiv \operatorname{Tr}\left[Y Z_{Y} \widehat{Z}-\widehat{Z} Z_{Y} Y\right]=0, \\
& I_{2} \equiv \operatorname{Tr}\left[Y Z_{2} \tilde{Z}-\tilde{Z} Z_{2} Y\right]=0 \text {, }
\end{aligned}
$$

where all matrices inside the parenthesis are $2 \times 2$ matrices. In the general case these are $n_{d} \times n_{d}$ matrices, and are defined by:

$$
\begin{array}{ll}
\left(Z_{Y}\right)_{i j} \equiv Z_{i j m n} Y_{m n} ; & \widehat{Z}_{i j} \equiv Z_{i j m m} ; \\
\left(Z_{2}\right)_{i j} \equiv Z_{i p n m} Z_{m n p j} ; & \tilde{Z}_{i j} \equiv Z_{i m m j}
\end{array}
$$

CP-odd HBT invariants are also useful [557] to find out whether, in a given model, there is hard or soft $\mathrm{CP}$ breaking. One may also construct CP-odd weak basis invariants, involving $v_{i} \equiv\left\langle 0\left|\phi_{i}^{0}\right| 0\right\rangle$, i.e., after spontaneous gauge symmetry breaking has occurred $[559,560]$. Further discussions on Higgs-basis independent methods for the two-Higgs-doublet model can be found in [561-564].

So far, we have considered $\mathrm{CP}$ violation at the Lagrangian level in models with multi-Higgs doublets, i.e., explicit CP violation. It is also possible to derive criteria [565] to verify whether $\mathrm{CP}$ and $\mathrm{T}$ in a given model are spontaneously broken. Under T the Higgs fields $\phi_{j}$ transform as

$T \phi_{j} T^{-1}=U_{j k} \phi_{k}$, 
where $U$ is a unitary matrix which may mix the scalar doublets. If no extra symmetries beyond $S U(2) \times U(1)$ are present in the Lagrangian, $U$ reduces to a diagonal matrix possibly with phases. Invariance of the vacuum under $\mathrm{T}$ leads to the following condition:

$$
\left\langle 0\left|\phi_{j}^{0}\right| 0\right\rangle=U_{j k}^{*}\left\langle 0\left|\phi_{k}^{0}\right| 0\right\rangle^{*}
$$

Therefore, a set of vacua lead to spontaneous T, CP violation if there is no unitary matrix $U$ satisfying (5.8) and (5.9) simultaneously.

Most of the previous discussion dealt with the general case of $n$-Higgs doublets. We analyze now the case of two Higgs doublets, where the most general gauge invariant Higgs potential can be explicitly written as

$$
\begin{aligned}
V_{H_{2}}= & m_{1} \phi_{1}^{\dagger} \phi_{1}+p e^{i \varphi} \phi_{1}^{\dagger} \phi_{2}+p e^{-i \varphi} \phi_{2}^{\dagger} \phi_{1}+m_{2} \phi_{2}^{\dagger} \phi_{2} \\
& +a_{1}\left(\phi_{1}^{\dagger} \phi_{1}\right)^{2}+a_{2}\left(\phi_{2}^{\dagger} \phi_{2}\right)^{2}+b\left(\phi_{1}^{\dagger} \phi_{1}\right)\left(\phi_{2}^{\dagger} \phi_{2}\right) \\
& +b^{\prime}\left(\phi_{1}^{\dagger} \phi_{2}\right)\left(\phi_{2}^{\dagger} \phi_{1}\right)+c_{1} e^{i \theta_{1}}\left(\phi_{1}^{\dagger} \phi_{1}\right)\left(\phi_{2}^{\dagger} \phi_{1}\right) \\
& +c_{1} e^{-i \theta_{1}}\left(\phi_{1}^{\dagger} \phi_{1}\right)\left(\phi_{1}^{\dagger} \phi_{2}\right)+c_{2} e^{i \theta_{2}}\left(\phi_{2}^{\dagger} \phi_{2}\right)\left(\phi_{2}^{\dagger} \phi_{1}\right) \\
& +c_{2} e^{-i \theta_{2}}\left(\phi_{2}^{\dagger} \phi_{2}\right)\left(\phi_{1}^{\dagger} \phi_{2}\right)+d e^{i \delta}\left(\phi_{1}^{\dagger} \phi_{2}\right)^{2} \\
& +d e^{-i \delta}\left(\phi_{2}^{\dagger} \phi_{1}\right)^{2}
\end{aligned}
$$

where $m_{i}, p, a_{i}, b, b^{\prime}, c_{i}$, and $d$ are real and all phases are explicitly displayed. It is clear that this potential contains an excess of parameters. With the appropriate choice of Higgs basis some of these may be eliminated, without loss of generality, leaving eleven independent parameters [569-571]. The Higgs sector contains five spinless particles: three neutral and a pair of charged ones, usually denoted by $h, H(\mathrm{CP}$ even), $A$ (CP odd) (or if CP is violated $h_{1,2,3}$ ) and $H^{ \pm}$.

In general, models with two Higgs doublets have tree level Higgs-mediated flavor changing neutral currents (FCNC). This is a problem in view of the present stringent experimental limits on FCNC. In order to solve this problem the concept of natural flavor conservation (NFC) was introduced by imposing extra symmetries on the Lagrangian. These symmetries constrain the Yukawa couplings of the neutral scalars in such a way that the resulting neutral currents are diagonal. Glashow and Weinberg [566] and Paschos [567] have shown that the only way to achieve NFC is to ensure that only one Higgs doublet gives mass to quarks of a given charge.

In the case of two Higgs doublets the simplest solution to avoid FCNC is to require invariance of the Lagrangian under the following transformation of the $Z_{2}$ type:

$$
\begin{array}{ll}
\phi_{1} \longrightarrow \phi_{1}, & \phi_{2} \longrightarrow-\phi_{2}, \\
d_{R} \longrightarrow d_{R}, & u_{R} \longrightarrow-u_{R},
\end{array}
$$

where $d_{R}\left(u_{R}\right)$ denote the right handed down (up) quarks; all other fields remain unchanged.

It is clear from (5.10) that this symmetry eliminates explicit CP violation in the Higgs sector, since the only term of the Higgs potential with a phase that survives is the one with coefficient $d$, moreover a HBT of the form $\phi_{1} \longrightarrow e^{i \delta / 2} \phi_{1}$, $\phi_{2} \longrightarrow \phi_{2}$, eliminates the phase from the Higgs potential. Furthermore, it can be shown that this symmetry also eliminates the possibility of having spontaneous $\mathrm{CP}$ violation.

In conclusion, models with two Higgs doublets and exact NFC cannot give rise to spontaneous CP violation. Explicit CP violation in this case requires complex Yukawa couplings leading to the Kobayashi-Maskawa mechanism with no additional source of $\mathrm{CP}$ violation through neutral scalar Higgs boson exchange. An interesting alternative scenario in the case of two Higgs doublets was considered in [568] with no NFC. Here CP violating Higgs FCNC are naturally suppressed through a permutation symmetry which is softly broken, still allowing for spontaneous CP violation.

Three Higgs doublet models have been considered in an attempt to introduce $\mathrm{CP}$ violation in an extension of the SM with NFC [566] in the Higgs sector. It was shown that indeed, in such models it is possible to violate CP in the Higgs sector either at the Lagrangian level [572] or spontaneously [573-575].

It is also possible to generate spontaneous $\mathrm{CP}$ violation with only one additional Higgs singlet [576], but in this case at least one isosinglet vectorial quark is required in order to generate a non-trivial phase at low energies in the CabibboKobayashi-Maskawa matrix. Such models may provide a solution to the strong CP problem of the type proposed by Nelson [577, 578] and Barr [579] as well as a common origin to all CP violations [580, 581] including the generation of the observed baryon asymmetry of the Universe. The fact that the SM cannot provide the observed baryon asymmetry [582-587], provides yet another reason to study an enlarged Higgs sector.

A lot of work has been done by many authors on possible extensions of the Higgs sector and their implications both for the hadronic and the leptonic sectors at the existing and future colliders, see e.g. [588]. Among the simplest multiHiggs models are the two Higgs Doublet Models (2HDM) which have been analyzed in detail in many different realizations. The need to avoid potentially dangerous tree level Higgs FCNC has led to the consideration of different variants of this model with a certain discrete $Z_{2}$ symmetry imposed.

In the Type-I $2 \mathrm{HDM}$ the $Z_{2}$ discrete symmetry imposed on the Lagrangian is such that only one of the Higgs doublets couples to quarks and leptons. A very well known fermiophobic Higgs boson may arise in such model [589591]. Another example is the Inert Doublet Model, with an unbroken discrete $Z_{2}$ symmetry which forbids one Higgs 
doublet to couple to fermions and to get a non-zero VEV [592, 593]. Physical particles related to such doublets are called "inert" particles, the lightest is stable and contributes to the Dark Matter density. In [594], the naturalness problem has been addressed in the framework of an Inert Doublet Model with a heavy (SM-like) Higgs boson. In this context Dark Matter may be composed of neutral inert Higgs bosons. Predictions are given for multilepton events with missing transverse energy at the LHC, and for the direct detection of dark matter.

The Type-II 2HDM allows one of the Higgs doublet to couple only to the right-handed up quarks while the other Higgs doublet can only couple to right handed down-type quarks and charged leptons. This is achieved by the introduction of an appropriate $Z_{2}$ symmetry, analogous to the one in (5.12). The Higgs sector of the MSSM model can be viewed as a particular realization of Type-II models but with additional constraints required by supersymmetry. Various scenarios are possible for these models-with and without decoupling of heavy Higgs particles [570, 571, 595].

Type-III 2HDM are models where, unlike in models of Type-I and II, NFC is not imposed on the Yukawa interactions. This class of models has in general scalar mediated FCNC at tree level. Various schemes have been proposed to suppress these currents, including the ad-hoc assumption that FCNC couplings are approximately given by the geometric mean of the Yukawa couplings of the two generations [596]. A very interesting alternative [597] is to have an exact symmetry of the Lagrangian which constrains FCNC couplings to be related in an exact way to the elements of the CKM matrix in such a way that FCNC are non-vanishing but naturally suppressed by the smallness of CKM mixing. Another example of Type III 2HDM is the Top Two Higgs Doublet Model which was first proposed in [598], and recently analyzed in detail in [599]. In this framework a discrete symmetry is imposed allowing only the top quark to have Yukawa couplings to one of the doublets while all other quarks and leptons have Yukawa couplings to the other doublet.

Lepton flavor violation is a feature common to many possible extensions of the SM. It can occur both through charged and neutral currents. The possibility of having lepton flavor violation in extensions of the SM, has been considered long before the discovery of neutrino masses [600, 601]. For example, in the case of multi-Higgs doublet models, it has been pointed out that even for massless neutrinos lepton flavor can be violated $[602,603]$. In the context of the minimal extension of the SM, necessary to accommodate neutrino masses, where only right handed neutrinos are included LFV effects are extremely small. It is well known that the effects of LFV can be large in supersymmetry.

CLEO submitted recently a paper [604] where the ratio of the tauonic and muonic branching fractions is examined for the three $\Upsilon(1 S, 2 S, 3 S)$ states. Agreement with expectations from lepton universality is found. The conclusion is that lepton universality is respected within the current experimental accuracy which is roughly $10 \%$. However there is tendency for the tauonic branching fraction to turn out systematically larger than the muonic at a few per cent level.

\subsubsection{Low scale singlet neutrino scenarios}

In the pre-LHC era neutrino oscillations have provided some of the most robust evidence for physics beyond the SM. There are many open questions in this field; why is the absolute mass scale for the neutrinos so small with respect to the other SM particles? what is this mass scale? why is the pattern of mixing so different from the quark sector? If nature has chosen the singlet seesaw scenario [216-220] as an answer to those questions we face the prospect of never being able to produce the heavy neutrinos at a collider. Nevertheless, several extensions of this minimal see-saw scenario contain heavy neutrinos at or around the $\mathrm{TeV}$ scale, these include models based around the group $E_{6}[605,606]$ and also in $S O(10)$ models [403].

Furthermore, even within the usual see-saw scenario, the observed nearly maximal mixing pattern of the light neutrinos requires further explanation. Flavor symmetries are often invoked as possible reasons for the almost tri-bi-maximal structure of the PMNS mixing matrix [607]. It is also possible that the small magnitude of the light neutrino masses is due to an approximate symmetry, allowing the right handed neutrinos to be as light as $\mathcal{O}(200 \mathrm{GeV})$ [337].

$\mathrm{TeV}$ scale right handed neutrinos can also arise in radiative mechanisms of neutrino mass generation. Generically, in these models a tree-level neutrino mass is forbidden or suppressed by a symmetry but small neutrino masses may arise through loops sensitive to symmetry breaking effects [225, 608]. Indeed, several supersymmetric realizations of radiative mechanisms contain $\mathrm{TeV}$ scale right handed neutrinos linked to the scale of supersymmetry breaking [609, 610].

5.1.2.1 Heavy neutrinos accessible to the LHC A low, electroweak-scale mass is not sufficient to imply that heavy neutrinos could be produced and detected at the LHC. They must have a large enough coupling (mixing) with other SM fields so that experiments will be able to distinguish their production and decay from SM background processes. In this review we concentrate on the case where heavy neutrino production and decay occurs through mixing with SM fields only. Quantitatively, we can consider a generalization of the Langacker-London parameters, $\Omega_{l l^{\prime}}$, defined as

$\Omega_{l l^{\prime}}=\delta_{l l^{\prime}}-\sum_{i=1}^{3} B_{l i} B_{l^{\prime} i}^{*}=\sum_{i=4}^{\left(3+n_{R}\right)} B_{l i} B_{l^{\prime} i}^{*}$, 
where $l, l^{\prime}=e, \mu, \tau$ and $B_{l i}$ is the full $3 \times\left(3+n_{R}\right)$ neutrino mixing matrix taking into account all (3 light and $n_{R}$ heavy) neutrinos. The $3 \times 3$ matrix $B_{l i}$ where $i=1, \ldots, 3$ is a good approximation to the usual PMNS matrix and $\Omega_{l l^{\prime}}$ essentially measures the deviation from unitarity of the PMNS matrix.

The $\Omega_{l l^{\prime}}$ are constrained by precision electroweak data [611] and the following upper limits have been set at $90 \%$ C.L.

$\Omega_{e e} \leq 0.012, \quad \Omega_{\mu \mu} \leq 0.0096, \quad \Omega_{\tau \tau} \leq 0.016$.

In addition, the off-diagonal elements of $\Omega_{l l^{\prime}}$ are constrained by limits on lepton flavor violating processes such as $\tau, \mu \rightarrow e \gamma$ and $\tau, \mu \rightarrow e e e$ and $\mu \rightarrow e$ conversion in nuclei $[514,612]$. These limits are rather model dependent but for $M_{R} \gg M_{W}$ and $m_{D} \ll M_{W}$ (where $m_{D}$ is the Dirac component of the neutrino mass matrix), the present upper bounds are [182]

$$
\left|\Omega_{e \mu}\right| \leq 0.0001, \quad\left|\Omega_{e \tau}\right| \leq 0.02, \quad\left|\Omega_{\mu \tau}\right| \leq 0.02 .
$$

It has been pointed out that a heavy Majorana neutrino $(N)$ may be produced via a DY type of mechanism at hadron colliders [608, 613-617], $p p \rightarrow W^{+*} \rightarrow \ell^{+} N$, where $N \rightarrow$ $\ell^{+} W^{-}$, leading to lepton number violation by 2 . Most of the previous studies were concentrated on the $e e$ mode, which would result in a too week signal to be appreciable due to the recent very stringent bound $\left|V_{e N}\right|^{2} / m_{N}<5 \times 10^{-8} \mathrm{GeV}^{-1}$, from the absence of the neutrinoless double beta decay. It has been recently proposed to search for the unique and clean signal, $\mu^{ \pm} \mu^{ \pm}+2$ jets at the LHC [617]. It was concluded that a search at the LHC with an integrated luminosity of $100 \mathrm{fb}^{-1}$ can be sensitive to a mass range of $m_{N} \sim 10$ $400 \mathrm{GeV}$ at a $2 \sigma$ level, and up to $250 \mathrm{GeV}$ at a $5 \sigma$ level. If this type of signal could be established, it would be even feasible to consider the search for $\mathrm{CP}$ violation in the heavy Majorana sector [618].

A recent analysis [619] studied more background processes including some fast detector simulations. In particular, the authors claimed a large background due to the faked leptons $b \bar{b} \rightarrow \mu^{+} \mu^{+}$. The search sensitivity is thus reduced to $175 \mathrm{GeV}$ at a $5 \sigma$ level. However, the background estimate for processes such as $b \bar{b}+n$-jet has large uncertainties due to QCD perturbative calculations and kinematical acceptance. More studies remain to be done for a definitive conclusion.

\subsubsection{Low scale model with successful baryogenesis As} a more detailed example satisfying the constraints of (5.14) we consider a model potentially accessible to colliders, where $M_{R} \simeq 250 \mathrm{GeV}$ which has been shown to successfully explain the baryon asymmetry of the Universe [337].

Leptogenesis has been discussed in Sect. 3.3.1. Low scale leptogenesis scenario would be possible with nearly degenerate heavy neutrinos, where self-energy effects on the leptonic asymmetries become relevant [293, 294]. In this case the $\mathrm{CP}$ asymmetry in the heavy neutrino decays can be resonantly enhanced [332], to the extent that the observed baryon asymmetry can be explained with heavy neutrinos as light as the electroweak scale [335, 337].

We shall consider a model with right handed neutrinos which transform under an $S O$ (3) flavor symmetry. Ignoring effects from the neutrino Yukawa couplings this symmetry is assumed to be exact at some high scale, e.g. the GUT scale $M_{\mathrm{GUT}}$. This restricts the form of the heavy Majorana neutrino mass matrix at $M_{\mathrm{GUT}}$

$M_{R}=\mathbf{1} m_{N}+\delta M_{S}$,

where $\delta M_{S}=0$ at $M_{\mathrm{GUT}}$. This form has also been considered in a class of "minimal flavor violating" models of the lepton sector [542] and naturally provides nearly degenerate heavy neutrinos compatible with resonant leptogenesis.

All other fields are singlets under this $S O(3)$ flavor symmetry and so the neutrino Yukawa couplings will break $S O(3)$ explicitly. We can still choose heavy neutrino Yukawa couplings $Y^{v}$ so that a subgroup of the $S O(3) \times U(1)_{L_{e}} \times$ $U(1)_{L_{\mu}} \times U(1)_{L_{\tau}}$ flavor symmetry present without the neutrino Yukawa couplings remains unbroken. In this case a particular flavor direction can be singled out leaving $S O(2) \simeq U(1)$ unbroken. This residual $U(1)$ symmetry acts to prevent the light Majorana neutrinos from acquiring a mass. The form of the neutrino Yukawa couplings can be written

$Y^{\nu T}=\left(\begin{array}{ccc}0 & a e^{-i \pi / 4} & a e^{i \pi / 4} \\ 0 & b e^{-i \pi / 4} & b e^{i \pi / 4} \\ 0 & c e^{-i \pi / 4} & c e^{i \pi / 4}\end{array}\right)+\delta Y^{\nu}$.

The residual $U(1)$ symmetry is broken both by small $S O(3)$ breaking effects in the heavy Majorana mass matrix, $\delta M_{S}$, and by small effects parameterized by $\delta Y^{v}$ in the Yukawa couplings. Although we shall not consider the specific origin of these effects, $\delta M_{S}$ could arise through renormalization group running for example.

In [337], a specific model was considered where $m_{N}=$ $250 \mathrm{GeV}$ and which successfully explained the baryon asymmetry of the Universe. One of either $a, b$ or $c$ was constrained to be small to allow a single lepton flavor asymmetry (and subsequently a baryon asymmetry) to be generated at $T \sim 250 \mathrm{GeV}$. The other two parameters could be as large as $\mathcal{O}\left(10^{-2}\right)$. This scenario has the features necessary for a model to be visible at the LHC; heavy neutrinos with masses 
around $\mathcal{O}(1 \mathrm{TeV})$ and sufficient mixing between these neutrinos and the light neutrinos to allow them to be produced from a vector boson. Specifically

$\Omega_{e e}=\frac{|a|^{2} v^{2}}{m_{N}^{2}}, \quad \Omega_{\mu \mu}=\frac{|b|^{2} v^{2}}{m_{N}^{2}}, \quad \Omega_{\tau \tau}=\frac{|c|^{2} v^{2}}{m_{N}^{2}}$,

where $v=246 \mathrm{GeV}$ is the vacuum expectation value of the Higgs field.

It should be noted that in this model the heavy neutrinos produced at the LHC would be linked indirectly with the mechanism providing light neutrinos with small masses. The light neutrinos acquire masses directly through the mechanism responsible for breaking the flavor symmetries. However, studying the properties of the heavy neutrinos accessible to the LHC would allow us to better understand the underlying symmetry protecting light neutrinos from large masses and may give us insight into the observed pattern of large mixing. In addition, further knowledge of heavy neutrinos seen at the LHC, for example small couplings with one or more lepton flavors or large, resonantly enhanced $\mathrm{CP}$ violation, would provide us with further information on possible explanations for the baryon asymmetry of the Universe.

\subsubsection{Lepton flavor violation from the mirror leptons in little Higgs models}

Little Higgs models [620-624] offer an alternative route to the solution of the little hierarchy problem. One of the most attractive models of this class is the littlest Higgs model [625] with T-parity (LHT) [626-628], where the discrete symmetry forbids tree-level corrections to electroweak observables, thus weakening the electroweak precision constraints [629]. Under this new symmetry the particles have distinct transformation properties, that is, they are either T-even or T-odd. The model is based on a two-stage spontaneous symmetry breaking occurring at the scale $f$ and the electroweak scale $v$. Here the scale $f$ is taken to be larger than about $500 \mathrm{GeV}$, which allows to expand expressions in the small parameter $v / f$. The additionally introduced gauge bosons, fermions and scalars are sufficiently light to be discovered at LHC and there is a dark matter candidate [630]. Moreover, the flavor structure of the LHT model is richer than the one of the SM, mainly due to the presence of three doublets of mirror quarks and three doublets of mirror leptons and their weak interactions with the ordinary quarks and leptons, as discussed in [631-633].

Now, it is well known that in the SM the FCNC processes in the lepton sector, like $\ell_{i} \rightarrow \ell_{j} \gamma$ and $\mu \rightarrow e e e$, are very strongly suppressed due to tiny neutrino masses. In particular, the branching ratio for $\mu \rightarrow e \gamma$ in the SM amounts to at most $10^{-54}$, to be compared with the present experimental upper bound, $1.2 \times 10^{-11}$ [180], and with the one that will be available within the next two years, $\sim 10^{-13}[634,635]$. Results close to the SM predictions are expected within the LH model without T-parity, where the lepton sector is identical to the one of the SM and the additional $\mathcal{O}\left(v^{2} / f^{2}\right)$ corrections have only minor impact on this result. Similarly the new effects on $(g-2)_{\mu}$ turn out to be small $[636,637]$.

A very different situation is to be expected in the LHT model, where the presence of new flavor violating interactions and of mirror leptons with masses of order $1 \mathrm{TeV}$ can change the SM expectations by up to 45 orders of magnitude, bringing the relevant branching ratios for lepton flavor violating (LFV) processes close to the bounds available presently or in the near future.

5.1.3.1 The model A detailed description of the LHT model can be found in [638], where also a complete set of Feynman rules has been derived. Here we just want to state briefly the ingredients needed for the analysis of LFV decays.

The T-odd gauge boson sector consists of three heavy "partners" of the SM gauge bosons

$W_{H}^{ \pm}, \quad Z_{H}, \quad A_{H}$,

with masses given to lowest order in $v / f$ by

$M_{W_{H}}=g f, \quad M_{Z_{H}}=g f, \quad M_{A_{H}}=\frac{g^{\prime} f}{\sqrt{5}}$.

The T-even fermion sector contains, in addition to the SM fermions, the heavy top partner $T_{+}$. On the other hand, the T-odd fermion sector [631] consists of three generations of mirror quarks and leptons with vectorial couplings under $S U(2)_{L} \times U(1)_{Y}$, that are denoted by

$$
\left(\begin{array}{l}
u_{H}^{i} \\
d_{H}^{i}
\end{array}\right), \quad\left(\begin{array}{l}
v_{H}^{i} \\
\ell_{H}^{i}
\end{array}\right) \quad(i=1,2,3) .
$$

To first order in $v / f$ the masses of up- and down-type mirror fermions are equal. Naturally, their masses are of order $f$. In the analysis of LFV decays, except for $K_{L, S} \rightarrow \mu e$, $K_{L, S} \rightarrow \pi^{0} \mu e, B_{d, s} \rightarrow \ell_{i} \ell_{j}$ and $\tau \rightarrow \ell \pi, \ell \eta, \ell \eta^{\prime}$, only mirror leptons are relevant.

As discussed in detail in [632], one of the important ingredients of the mirror sector is the existence of four CKM-like unitary mixing matrices, two for mirror quarks $\left(V_{H u}, V_{H d}\right)$ and two for mirror leptons $\left(V_{H \nu}, V_{H \ell}\right)$, that are related via

$V_{H u}^{\dagger} V_{H d}=V_{\mathrm{CKM}}, \quad V_{H \nu}^{\dagger} V_{H \ell}=V_{\mathrm{PMNS}}^{\dagger}$.

An explicit parameterization of $V_{H d}$ and $V_{H \ell}$ in terms of three mixing angles and three complex (non-Majorana) phases can be found in [633]. 
The mirror mixing matrices parameterize flavor violating interactions between SM fermions and mirror fermions that are mediated by the heavy gauge bosons $W_{H}^{ \pm}, Z_{H}$ and $A_{H}$. The matrix notation indicates which of the light fermions of a given electric charge participates in the interaction.

In the course of the analysis of charged LFV decays it is useful to introduce the following quantities $(i=1,2,3)$ [639]:

$$
\begin{aligned}
& \chi_{i}^{(\mu e)}=V_{H \ell}^{* i e} V_{H \ell}^{i \mu}, \quad \chi_{i}^{(\tau e)}=V_{H \ell}^{* i e} V_{H \ell}^{i \tau}, \\
& \chi_{i}^{(\tau \mu)}=V_{H \ell}^{* i \mu} V_{H \ell}^{i \tau},
\end{aligned}
$$

that govern $\mu \rightarrow e, \tau \rightarrow e$ and $\tau \rightarrow \mu$ transitions, respectively. Analogous quantities in the mirror quark sector $(i=1,2,3)[638,641]$,

$$
\begin{aligned}
& \xi_{i}^{(K)}=V_{H d}^{* i s} V_{H d}^{i d}, \quad \xi_{i}^{(d)}=V_{H d}^{* i b} V_{H d}^{i d}, \\
& \xi_{i}^{(s)}=V_{H d}^{* i b} V_{H d}^{i s},
\end{aligned}
$$

are needed for the analysis of the decays $K_{L, S} \rightarrow \mu e$, $K_{L, S} \rightarrow \pi^{0} \mu e$ and $B_{d, s} \rightarrow \ell_{i} \ell_{j}$.

As an example, the branching ratio for the $\mu \rightarrow e \gamma$ decay contains the $\chi_{i}^{(\mu e)}$ factors introduced in (5.22) via the short distance function [639]

$$
\begin{aligned}
\bar{D}_{\text {odd }}^{\prime \mu e}= & \frac{1}{4} \frac{v^{2}}{f^{2}} \sum_{i}\left(\chi _ { i } ^ { ( \mu e ) } \left(D_{0}^{\prime}\left(y_{i}\right)-\frac{7}{6} E_{0}^{\prime}\left(y_{i}\right)\right.\right. \\
& \left.\left.-\frac{1}{10} E_{0}^{\prime}\left(y_{i}^{\prime}\right)\right)\right),
\end{aligned}
$$

where $y_{i}=\left(m_{H i}^{\ell} / M_{W_{H}}\right)^{2}, y_{i}^{\prime}=a y_{i}$ with $a=5 / \tan ^{2} \theta_{W}$, and explicit expressions for the functions $D_{0}^{\prime}, E_{0}^{\prime}$ can be found in [642].

The new parameters of the LHT model, relevant for the study of LFV decays, are

$$
\begin{aligned}
& f, \quad m_{H 1}^{\ell}, \quad m_{H 2}^{\ell}, \quad m_{H 3}^{\ell}, \quad \theta_{12}^{\ell}, \quad \theta_{13}^{\ell}, \quad \theta_{23}^{\ell}, \\
& \delta_{12}^{\ell}, \quad \delta_{13}^{\ell}, \quad \delta_{23}^{\ell}
\end{aligned}
$$

and the ones in the mirror quark sector that can be probed by FCNC processes in $K$ and $B$ meson systems, as discussed in detail in [638, 641]. Once the new heavy gauge bosons and mirror fermions will be discovered and their masses measured at the LHC, the only free parameters of the LHT model will be the mixing angles $\theta_{i j}^{\ell}$ and the complex phases $\delta_{i j}^{\ell}$ of the matrix $V_{H \ell}$, that can be determined with the help of LFV processes. Analogous comments apply to the determination of $V_{H d}$ parameters in the quark sector (see [638, 641] for details on $K$ and $B$ physics in the LHT model).

5.1.3.2 Results LFV processes in the LHT model have for the first time been discussed in [643], where the decays $\ell_{i} \rightarrow \ell_{j} \gamma$ have been considered. Further, the new contributions to $(g-2)_{\mu}$ in the LHT model have been calculated by these authors. In [639, 640] the analysis of LFV in the LHT model has been considerably extended, and includes the decays $\ell_{i} \rightarrow \ell_{j} \gamma, \mu \rightarrow e e e$, the six three body leptonic decays $\tau^{-} \rightarrow \ell_{i}^{-} \ell_{j}^{+} \ell_{k}^{-}$, the semileptonic decays $\tau \rightarrow \ell \pi, \ell \eta, \ell \eta^{\prime}$ and the decays $K_{L, S} \rightarrow \mu e, K_{L, S} \rightarrow \pi^{0} \mu e$ and $B_{d, s} \rightarrow \ell_{i} \ell_{j}$ that are flavor violating both in the quark and lepton sector. Moreover, $\mu-e$ conversion in nuclei and the flavor conserving $(g-2)_{\mu}$ have been studied. Furthermore, a detailed phenomenological analysis has been performed in that paper, paying particular attention to various ratios of LFV branching ratios that will be useful for a clear distinction of the LHT model from the MSSM.

In contrast to $K$ and $B$ physics in the LHT model, where the SM contributions constitute a sizable and often the dominant part, the T-even contributions to LFV observables are completely negligible due to the smallness of neutrino masses and the LFV decays considered are entirely governed by mirror fermion contributions.

In order to see how large these contributions can possibly be, it is useful to consider first those decays for which the strongest constraints exist. Therefore Fig. 5 shows $B(\mu \rightarrow e e e)$ as a function of $B(\mu \rightarrow e \gamma)$, obtained from a general scan over the mirror lepton parameter space, with $f=1 \mathrm{TeV}$. It is found that in order to fulfill the present bounds, either the mirror lepton spectrum has to be quasidegenerate or the $V_{H \ell}$ matrix must be very hierarchical. Moreover, as shown in Fig. 6, even after imposing the constraints on $\mu \rightarrow e \gamma$ and $\mu \rightarrow e e e$, the $\mu-e$ conversion rate in $\mathrm{Ti}$ is very likely to be found close to its current bound, and for some regions of the mirror lepton parameter space even violates this bound.

The existing constraints on LFV $\tau$ decays are still relatively weak, so that they presently do not provide a useful constraint on the LHT parameter space. However, as seen in Table 10, most branching ratios in the LHT model can

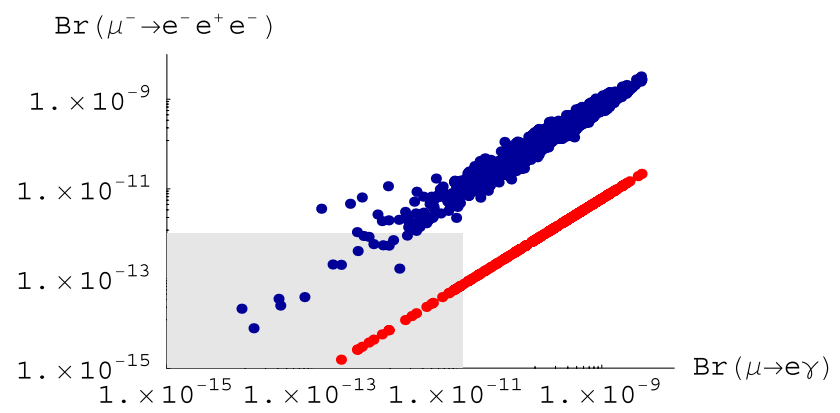

Fig. 5 Correlation between $B(\mu \rightarrow e \gamma)$ and $B(\mu \rightarrow e e e)$ in the LHT model (upper dots) [639]. The lower dots represent the dipole contribution to $\mu \rightarrow$ eee separately, which, unlike in the LHT model, is the dominant contribution in the MSSM. The grey region is allowed by the present experimental bounds 
Fig. $6 \quad R(\mu \mathrm{Ti} \rightarrow e \mathrm{Ti})$ as a function of $B(\mu \rightarrow e \gamma)$, after imposing the existing constraints on $\mu \rightarrow e \gamma$ and $\mu \rightarrow$ eee [639]. The grey region is allowed by the present experimental bounds

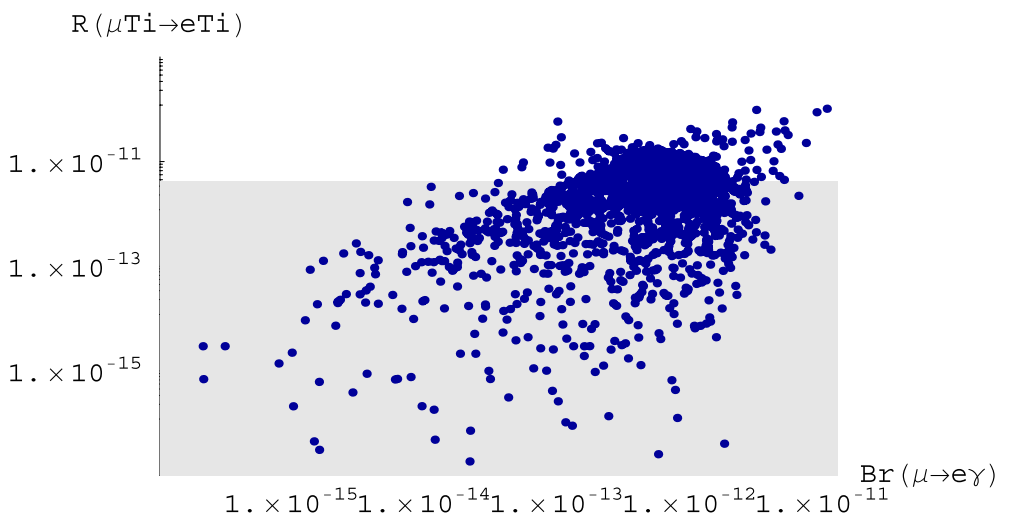

Table 10 Upper bounds on LFV $\tau$ decay branching ratios in the LHT model, for two different values of the scale $f$, after imposing the constraints on $\mu \rightarrow e \gamma$ and $\mu \rightarrow e e e$ [639]. For $f=500 \mathrm{GeV}$, also the bounds on $\tau \rightarrow \mu \pi, e \pi$ have been included. The current experimental upper bounds are also given. The bounds in [183] have been obtained by combining Belle $[646,647]$ and BaBar $[182,648]$ results

\begin{tabular}{lllr}
\hline Decay & $f=1000 \mathrm{GeV}$ & $f=500 \mathrm{GeV}$ & exp. upper bound \\
\hline$\tau \rightarrow e \gamma$ & $8 \times 10^{-10}$ & $1 \times 10^{-8}$ & $9.4 \times 10^{-8}[183]$ \\
$\tau \rightarrow \mu \gamma$ & $8 \times 10^{-10}$ & $2 \times 10^{-8}$ & $1.6 \times 10^{-8}[183]$ \\
$\tau^{-} \rightarrow e^{-} e^{+} e^{-}$ & $7 \times 10^{-10}$ & $2 \times 10^{-8}$ & $2.0 \times 10^{-7}[644]$ \\
$\tau^{-} \rightarrow \mu^{-} \mu^{+} \mu^{-}$ & $7 \times 10^{-10}$ & $3 \times 10^{-8}$ & $1.9 \times 10^{-7}[644]$ \\
$\tau^{-} \rightarrow e^{-} \mu^{+} \mu^{-}$ & $5 \times 10^{-10}$ & $2 \times 10^{-8}$ & $2.0 \times 10^{-7}[645]$ \\
$\tau^{-} \rightarrow \mu^{-} e^{+} e^{-}$ & $5 \times 10^{-10}$ & $2 \times 10^{-8}$ & $1.9 \times 10^{-7}[645]$ \\
$\tau^{-} \rightarrow \mu^{-} e^{+} \mu^{-}$ & $5 \times 10^{-14}$ & $2 \times 10^{-14}$ & $1.3 \times 10^{-7}[644]$ \\
$\tau^{-} \rightarrow e^{-} \mu^{+} e^{-}$ & $5 \times 10^{-14}$ & $2 \times 10^{-14}$ & $1.1 \times 10^{-7}[644]$ \\
$\tau \rightarrow \mu \pi$ & $2 \times 10^{-9}$ & $5.8 \times 10^{-8}$ & $5.8 \times 10^{-8}[183]$ \\
$\tau \rightarrow e \pi$ & $2 \times 10^{-9}$ & $4.4 \times 10^{-8}$ & $4.4 \times 10^{-8}[183]$ \\
$\tau \rightarrow \mu \eta$ & $6 \times 10^{-10}$ & $2 \times 10^{-8}$ & $5.1 \times 10^{-8}[183]$ \\
$\tau \rightarrow e \eta$ & $6 \times 10^{-10}$ & $2 \times 10^{-8}$ & $4.5 \times 10^{-8}[183]$ \\
$\tau \rightarrow \mu \eta^{\prime}$ & $7 \times 10^{-10}$ & $3 \times 10^{-8}$ & $5.3 \times 10^{-8}[183]$ \\
$\tau \rightarrow e \eta^{\prime}$ & $7 \times 10^{-10}$ & $3 \times 10^{-8}$ & $9.0 \times 10^{-8}[183]$ \\
\hline
\end{tabular}

reach the present experimental upper bounds, in particular for low values of $f$, and are very interesting in view of new experiments taking place in this and the coming decade.

The situation is different in the case of $K_{L} \rightarrow \mu e, K_{L} \rightarrow$ $\pi^{0} \mu e$ and $B_{d, s} \rightarrow \ell_{i} \ell_{k}$, due to the double GIM suppression in the quark and lepton sectors. E.g. $B\left(K_{L} \rightarrow \mu e\right)$ can reach values of at most $3 \times 10^{-13}$ which is still one order of magnitude below the current bound, and $K_{L} \rightarrow \pi^{0} \mu e$ is even by two orders of magnitude smaller. Still, measuring the rates for $K_{L} \rightarrow \mu e$ and $K_{L} \rightarrow \pi^{0} \mu e$ would be desirable, as, due to their sensitivity to $\operatorname{Re}\left(\xi_{i}^{(K)}\right)$ and $\operatorname{Im}\left(\xi_{i}^{(K)}\right)$ respectively, these decays can shed light on the complex phases present in the mirror quark sector.

While the possible huge enhancements of LFV branching ratios in the LHT model are clearly interesting, such effects are common to many other NP models, such as the MSSM, and therefore cannot be used to distinguish these models. However, correlations between various branching ratios should allow a clear distinction of the LHT model from the MSSM. While in the MSSM [169, 175, 242, 649, $650]$ the dominant role in decays with three leptons in the final state and in $\mu-e$ conversion in nuclei is typically played by the dipole operator, in [639] it is found that this operator is basically irrelevant in the LHT model, where $Z^{0}$-penguin and box diagram contributions are much more important. As can be seen in Table 11 and also in Fig. 5 this implies a striking difference between various ratios of branching ratios in the MSSM and in the LHT model and should be very useful in distinguishing these two models. Even if for some decays this distinction is less clear when significant Higgs contributions are present $[169,175,650]$, it should be easier than through high energy processes at LHC.

Another possibility to distinguish different NP models through LFV processes is given by the measurement of $\mu \rightarrow e \gamma$ with polarized muons. Measuring the angular distribution of the outgoing electrons, one can determine the 
Table 11 Comparison of various ratios of branching ratios in the LHT model and in the MSSM without and with significant Higgs contributions [639]

\begin{tabular}{llll}
\hline Ratio & LHT & MSSM (dipole) & MSSM (Higgs) \\
\hline$B\left(\mu^{-} \rightarrow e^{-} e^{+} e^{-}\right) / B(\mu \rightarrow e \gamma)$ & $0.4-2.5$ & $\sim 6 \times 10^{-3}$ & $\sim 6 \times 10^{-3}$ \\
$B\left(\tau^{-} \rightarrow e^{-} e^{+} e^{-}\right) / B(\tau \rightarrow e \gamma)$ & $0.4-2.3$ & $\sim 1 \times 10^{-2}$ & $\sim 1 \times 10^{-2}$ \\
$B\left(\tau^{-} \rightarrow \mu^{-} \mu^{+} \mu^{-}\right) / B(\tau \rightarrow \mu \gamma)$ & $0.4-2.3$ & $\sim 2 \times 10^{-3}$ & $0.06-0.1$ \\
$B\left(\tau^{-} \rightarrow e^{-} \mu^{+} \mu^{-}\right) / B(\tau \rightarrow e \gamma)$ & $0.3-1.6$ & $\sim 2 \times 10^{-3}$ & $0.02-0.04$ \\
$B\left(\tau^{-} \rightarrow \mu^{-} e^{+} e^{-}\right) / B(\tau \rightarrow \mu \gamma)$ & $0.3-1.6$ & $\sim 1 \times 10^{-2}$ & $\sim 1 \times 10^{-2}$ \\
$B\left(\tau^{-} \rightarrow e^{-} e^{+} e^{-}\right) / B\left(\tau^{-} \rightarrow e^{-} \mu^{+} \mu^{-}\right)$ & $1.3-1.7$ & $\sim 5$ & $0.3-0.5$ \\
$B\left(\tau^{-} \rightarrow \mu^{-} \mu^{+} \mu^{-}\right) / B\left(\tau^{-} \rightarrow \mu^{-} e^{+} e^{-}\right)$ & $1.2-1.6$ & $\sim 0.2$ & $5-10$ \\
$R(\mu \mathrm{Ti} \rightarrow e \mathrm{Ti}) / B(\mu \rightarrow e \gamma)$ & $0.01-100$ & $\sim 5 \times 10^{-3}$ & $0.08-0.15$ \\
\hline
\end{tabular}

size of left and right handed contributions separately [651]. In addition, detecting also the electron spin would yield information on the relative phase between these two contributions [652]. We recall that the LHT model is peculiar in this respect as it does not involve any right handed contribution.

On the other hand, the contribution of mirror leptons to $(g-2)_{\mu}$, being a flavor conserving observable, is negligible $[639,643]$, so that the possible discrepancy between SM prediction and experimental data [653] cannot be cured. This should also be contrasted with the MSSM with large $\tan \beta$ and not too heavy scalars, where those corrections could be significant, thus allowing to solve the possible discrepancy between SM prediction and experimental data.

\subsubsection{Conclusions We have seen that LFV decays open} up an exciting playground for testing the LHT model. Indeed, they could offer a very clear distinction between this model and supersymmetry. Of particular interest are the ratios $B\left(\ell_{i} \rightarrow e e e\right) / B\left(\ell_{i} \rightarrow e \gamma\right)$ that are $\mathcal{O}(1)$ in the LHT model but strongly suppressed in supersymmetric models even in the presence of significant Higgs contributions. Similarly, finding the $\mu-e$ conversion rate in nuclei at the same level as $B(\mu \rightarrow e \gamma)$ would point into the direction of LHT physics rather than supersymmetry.

\subsubsection{Low scale triplet Higgs neutrino mass scenarios in little Higgs models}

An important open issue to address in the context of little Higgs models is the origin of non-zero neutrino masses [654-658]. The neutrino mass mechanism which naturally occurs in these models is the triplet Higgs mechanism [244] which employs a scalar with the $S U(2)_{L} \times U(1)_{Y}$ quantum numbers $T \sim(3,2)$. The existence of such a multiplet in some versions of the little Higgs models is a direct consequence of global symmetry breaking which makes the SM Higgs light. For example, in the minimal littlest Higgs model [625], the triplet Higgs with non-zero hypercharge occurs from the breaking of global $S U(5)$ down to $S O(5)$ symmetry as one of the Goldstone bosons. Its mass $M_{T} \sim$ $g_{s} f$, where $g_{s}<4 \pi$ is a model dependent coupling constant in the weak coupling regime [659], is therefore predicted to be below the cut-off scale $\Lambda$, and could be within the mass reach of LHC. The present lower bound for the invariant mass of $T$ is set by Tevatron to $M_{T} \geq 136 \mathrm{GeV}[660,661]$.

Although the triplet mass scale is of order $\mathcal{O}(1) \mathrm{TeV}$, the observed neutrino masses can be obtained naturally. Due to the specific quantum numbers the triplet Higgs boson couples only to the left-chiral lepton doublets $L_{i} \sim(2,-1)$, $i=e, \mu, \tau$, via the Yukawa interactions of (3.63) and to the SM Higgs bosons via (3.64). Those interactions induce lepton flavor violating decays of charged leptons which have not been observed. The most stringent constraint on the Yukawa couplings comes from the upper limit on the treelevel decay $\mu \rightarrow$ eee and is ${ }^{15} Y_{T}^{e e} Y_{T}^{e \mu}<3 \times 10^{-5}(M / \mathrm{TeV})^{2}$ [662, 663]. Experimental bounds on the tau Yukawa couplings are much less stringent. The hierarchical light neutrino masses imply $Y_{T}^{e e}, Y_{T}^{e \mu} \ll Y_{T}^{\tau \tau}$ consistently with the direct experimental bounds.

Non-zero neutrino masses and mixing is presently the only experimentally verified signal of new physics beyond the SM. In the triplet neutrino mass mechanism [244] presented in Sect. 3.2.3.2 the neutrino masses are given by

$\left(m_{v}\right)^{i j}=Y_{T}^{i j} v_{T}$,

where $v_{T}$ is the induced triplet VEV of (3.65). It is natural that the smallness of neutrino masses is explained by the smallness of $v_{T}$. In the little Higgs models this can be achieved by requiring the Higgs mixing parameter $\mu \ll M_{T}$, which can be explained, for example, via shining of explicit lepton number violation from extra dimensions as shown in Refs. [664, 665], or if the triplet is related to the Dark Energy of the Universe [666, 667]. Models with additional (approximate) T-parity [626] make the smallness of $v_{T}$ technically

\footnotetext{
${ }^{15}$ In little Higgs models with T-parity there exist additional sources of flavor violation from the mirror fermion sector $[639,643]$ discussed in the previous subsection.
} 
natural (if the T-parity is exact, $v_{T}$ must vanish). In that case $Y_{T} v_{T} \sim \mathcal{O}(0.1) \mathrm{eV}$ while the Yukawa couplings $Y$ can be of order charged lepton Yukawa couplings of the SM. As a result, the branching ratio of the decay $T \rightarrow W W$ is negligible. We also remind that $v_{T}$ contributes to the SM oblique corrections, and the precision data fit $\hat{T}<2 \times 10^{-4}$ [668] sets an upper bound $v_{T} \leq 1.2 \mathrm{GeV}$ on that parameter.

Notice the particularly simple connection between the flavor structure of light neutrinos and the Yukawa couplings of the triplet via (5.26). Therefore, independently of the overall size of the Yukawa couplings, one can predict the leptonic branching ratios of the triplet from neutrino oscillations. For the normally hierarchical light neutrino masses neutrino data implies negligible $T$ branching fractions to electrons and $B\left(T^{++} \rightarrow \mu^{+} \mu^{+}\right) \approx B\left(T^{++} \rightarrow \tau^{+} \tau^{+}\right) \approx$ $B\left(T^{++} \rightarrow \mu^{+} \tau^{+}\right) \approx 1 / 3$. Those are the final state signatures predicted by the triplet neutrino mass mechanism for collider experiments.

At LHC $T^{++}$can be produced singly and in pairs. The cross section of the single $T^{++}$production via the $W W$ fusion process [662] $q q \rightarrow q^{\prime} q^{\prime} T^{++}$scales as $\sim v_{T}^{2}$. In the context of the littlest Higgs model this process, followed by the decays $T^{++} \rightarrow W^{+} W^{+}$, was studied in Refs. [669671]. The detailed ATLAS simulation of this channel shows [671] that in order to observe an $1 \mathrm{TeV} T^{++}$, one must have $v_{T}>29 \mathrm{GeV}$. This is in conflict with the precision physics bound $v_{T} \leq 1.2 \mathrm{GeV}$ as well as with the neutrino data. Therefore the $W W$ fusion channel is not experimentally promising for the discovery of doubly charged Higgs.

On the other hand, the Drell-Yan pair production process [662, 672-678]

$$
p p \rightarrow T^{++} T^{--}
$$

is not suppressed by any small coupling and its cross section is known up to next to leading order [674] (possible additional contributions from new physics such as $Z_{H}$ are strongly suppressed and we neglect those effects here). Followed by the lepton number violating decays $T^{ \pm \pm} \rightarrow \ell^{ \pm} \ell^{ \pm}$, this process allows to reconstruct $T^{ \pm \pm}$invariant mass from the same charged leptons rendering the SM background to be very small in the signal region. If one also assumes that neutrino masses come from the triplet Higgs interactions, one fixes the $T^{ \pm \pm}$leptonic branching ratios. This allows to test the triplet neutrino mass model at LHC. The pure Monte Carlo study of this scenario shows [677] that $T^{++}$ up to the mass $300 \mathrm{GeV}$ is reachable in the first year of LHC
( $L=1 \mathrm{fb}^{-1}$ ) and $T^{++}$up to the mass $800 \mathrm{GeV}$ is reachable for the luminosity $L=30 \mathrm{fb}^{-1}$. Including the Gaussian measurement errors to the Monte Carlo the corresponding mass reaches become [677] $250 \mathrm{GeV}$ and $700 \mathrm{GeV}$, respectively. The errors of those estimates of the required luminosity for discovery depend strongly on the size of statistical Monte Carlo sample of the background processes.

\subsection{Flavor and CP violation in SUSY extensions of the SM}

Supersymmetric models provide the richest spectrum of lepton flavor and CP-violating observables among all models. They are also among the best studied scenarios of new physics beyond the standard model. In this Section we review phenomenologically most interesting aspects of some of the supersymmetric scenarios.

\subsubsection{Mass insertion approximation and phenomenology}

In the low energy supersymmetric extensions of the SM the flavor and CP-violating interactions would originate from the misalignment between fermion and sfermion mass eigenstates. Understanding why all these processes are strongly suppressed is one of the major problems of low energy supersymmetry, the supersymmetric flavor and $C P$ problem. The absence of deviations from the SM predictions in LFV and CPV (and other flavor changing processes in the quark sector) experiments suggests the presence of a quite small amount of fermion-sfermion misalignment. From the phenomenological point of view those effects are most easily described by the mass insertion approximation.

The relevant one loop amplitudes can be exactly written in terms of the general mass matrix of charginos and neutralinos, resulting in quite involved expressions. To obtain simple approximate expressions, it is convenient to use the so-called mass insertion method [145, 679]. This is a particularly convenient method since, in a model independent way, the tolerated deviation from alignment is quantified by the upper limits on the mass insertion $\delta$ 's, defined as the small off-diagonal elements in terms of which sfermion propagators are expanded, normalized with an average sfermion mass, $\delta_{i j}=\Delta_{i j} / m_{\tilde{f}}^{2}$. They are of four types: $\delta^{L L}, \delta^{R R}, \delta^{R L}$ and $\delta^{L R}$, according to the chiralities of the corresponding partner fermions. We shall adopt here the usual convention for the slepton mass matrix in the basis where the lepton mass matrix $m_{\ell}$ is diagonal:

$$
\left(\begin{array}{cc}
\tilde{\ell}_{L}^{\dagger} & \tilde{\ell}_{R}^{\dagger}
\end{array}\right)\left(\begin{array}{cc}
m_{L}^{2}\left(1+\delta^{L L}\right) & \left(A^{*}-\mu \tan \beta\right) m_{\ell}+m_{L} m_{R} \delta^{L R} \\
\left(A-\mu^{*} \tan \beta\right) m_{\ell}+m_{L} m_{R} \delta^{L R^{\dagger}} & m_{R}^{2}\left(1+\delta^{R R}\right)
\end{array}\right)\left(\begin{array}{l}
\tilde{\ell}_{L} \\
\tilde{\ell}_{R}
\end{array}\right)
$$


where $m_{L}, m_{R}$, are respectively the average real masses of the left handed and right handed sleptons and $A$ contains only the diagonal entries the trilinear matrices at the electroweak scale. Notice that these flavor diagonal left-right mixing are always present in any MSSM and play a very important role in LFV processes. In this way, our $\delta^{L R}$ contain only the off-diagonal elements of the trilinear matrices. This definition is then slightly different from the original definition in Refs. [145, 680]. The deviations from universality are then all gathered in the different $\delta$ matrices.

Each element in these $\delta$ matrices can be tested by experiment. Searches for the decay $\ell_{i} \rightarrow \ell_{j} \gamma$ provide bounds on the absolute values of the off-diagonal (flavor violating) $\left|\delta_{i j}^{L L}\right|,\left|\delta_{i j}^{R R}\right|,\left|\delta_{i j}^{L R}\right|$ and $\left|\delta_{i j}^{R L}\right|$, while measurements of the lepton EDM (MDM), parameters and their CP-violating phases, also provide limits on the imaginary (real) part of combinations of flavor violating $\delta$ 's, $\delta_{i j}^{L L} \delta_{j i}^{L R}, \delta_{i j}^{L R} \delta_{j i}^{R R}$, $\delta_{i j}^{L L} \delta_{j i}^{R R}$ and $\delta_{i j}^{L R} \delta_{j i}^{L R}$. Many authors have addressed the issue of the bounds on these misalignment parameters and phases in the sleptonic sector [680]. Following [163] we present the current limits on $\mu \rightarrow e \gamma$ and we analyze the impact of the planned experimental improvements on $\tau \rightarrow \mu \gamma$. In the basis where $Y_{\ell}$ is diagonal, and in the mass insertion approximation, the branching ratio of the process reads

$$
\begin{aligned}
B\left(\ell_{i} \rightarrow \ell_{j} \gamma\right)= & 10^{-5} \times B\left(\ell_{i} \rightarrow \ell_{j} \bar{v}_{j} v_{i}\right) \frac{M_{W}^{4}}{\bar{m}_{L}^{4}} \\
& \times \tan ^{2} \beta\left|\delta_{i j}^{L L}\right|^{2} F_{\text {SUSY }},
\end{aligned}
$$

where $F_{\text {SUSY }}=O(1)$ is a function of supersymmetric masses including both chargino and neutralino exchange (see e.g., [163], and references therein). We focus for definiteness on the mSUGRA scenario, also assuming gaugino and scalar universality at the gauge coupling unification scale and fixing $\mu$ as required by the radiative electroweak symmetry breaking.

As for LFV, Figs. 7 and 8 display the upper bounds on the $|\delta|$ 's in the $\left(M_{1}, m_{R}\right)$ plane, where $M_{1}$ and $m_{R}$ are the bino
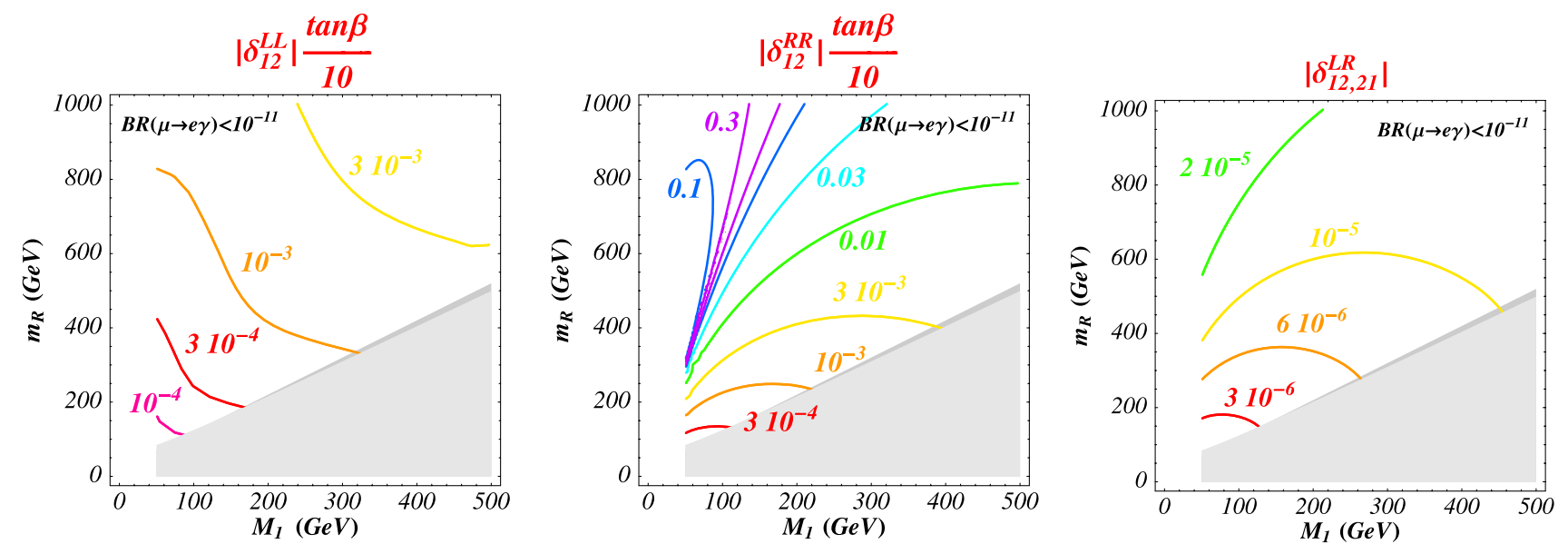

Fig. 7 Upper limits on $\delta_{12}$ 's in mSUGRA. Here $M_{1}$ and $m_{R}$ are the bino and right-slepton masses, respectively
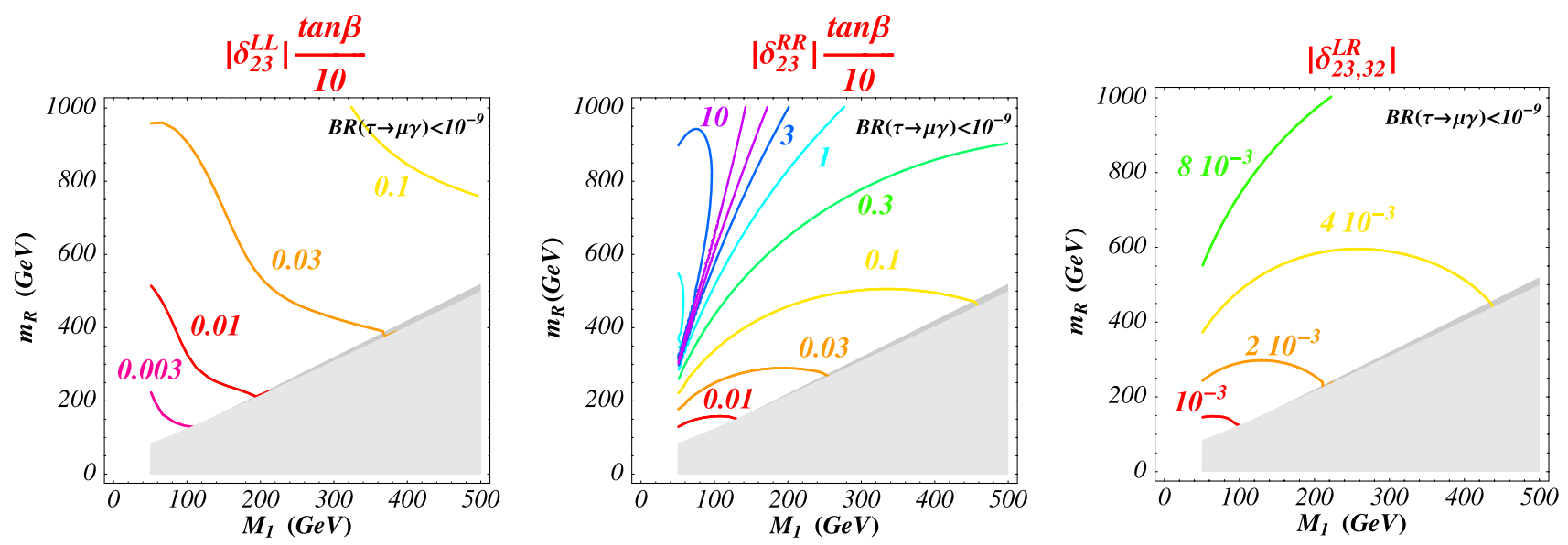

Fig. 8 Upper limits on $\delta_{23}$ 's in mSUGRA. Here $M_{1}$ and $m_{R}$ are the bino and right-slepton masses, respectively 
and right-slepton masses, respectively. Deviations from the mSUGRA assumptions can be estimated by means of relatively simple analytical expressions. In Figs. 7 and 8 we can see that the bounds on $\delta_{j i}^{R R}$ depend strongly and are practically absent for some values of $M_{1}$ and $m_{R}$. This fact is due to a destructive interference between the bino and binohiggsino amplitudes [163]. On the contrary, the limits on $\delta_{j i}^{L L}$ are robust because of a constructive interference between the chargino and bino amplitudes. A weaker bound on $\delta_{12}^{R R}$ on the cancellation regions can be obtained combining the experimental information from the decays $\mu \rightarrow e \gamma$, $\mu \rightarrow e e e$ and $\mu-e$ conversion in nuclei $[165,681]$. The present limits on $\mu \rightarrow e \gamma$ provide interesting constraints on the related $\delta$ 's. As will be discussed in the following, the present sensitivity already allow to test these $\delta$ 's at the level of the radiative effects. Such a sensitivity could hopefully be reached also in future experiments on $\tau \rightarrow \mu \gamma$.

Another issue is the origin of the CP-violating phases in the leptonic EDMs. Unless the sparticle masses are increased above several $\mathrm{TeVs}$, the phases in the flavor diagonal elements of the slepton left-right mass matrices (in the lepton flavor basis), in the parameters $\mu$ and $A_{i}$ of supersymmetric models, have to be quite small, and this constitutes the so-called supersymmetric $\mathrm{CP}$ problem. For the bounds on the sources of CPV also associated to FV, like e.g. $\operatorname{Im}\left(\delta_{i j}^{L L} \delta_{j i}^{R R}\right)_{e e}$ and so on, we refer to the plots in Ref. [163].

\subsubsection{Lepton flavor violation from RGE effects in SUSY seesaw model}

\subsubsection{Predictions from flavor models Consider first the} possibility that flavor and $\mathrm{CP}$ are exact symmetries of the soft supersymmetry breaking sector defined at the appropriate cutoff scale $\Lambda$ (to be identified with the Planck scale for supergravity, the messenger mass for gauge mediation, etc). If below this scale there are flavor and CP-violating Yukawa interactions, it is well-known that in the running down to $m_{\text {SUSY }}$ they will induce a small amount of flavor and CP violation in sparticle masses.

The Yukawa interactions associated to the fermion masses and mixing of the SM clearly violate any flavor and $\mathrm{CP}$ symmetries. However, with the exception of the third generation Yukawa couplings, all the entries in the Yukawa matrices are very small and the radiatively induced misalignment in the sfermion mass matrices turns out to be negligible. The Yukawa interactions of heavy states beyond the SM coupling to the SM fermions induce misalignments proportional to a proper combination of their Yukawa couplings times $\ln m_{\mathrm{F}} / \Lambda$, where $m_{\mathrm{F}}$ represents the heavy state mass scale. This is the case for the seesaw interactions of the right handed neutrinos $[144,145]$ and/or the GUT interactions of the heavy colored triplets $[682,683]$ (those eventually exchanged in diagrams inducing proton decay). Notice that the observation of large mixing in light neutrino masses, may suggest the possibility that also the seesaw interactions could significantly violate flavor- and potentially also $\mathrm{CP}$, in particular in view of the mechanism of leptogenesis. Remarkably, for sparticle masses not exceeding the $\mathrm{TeV}$, the seesaw and colored-triplet induced radiative contributions to the LFV decays and lepton EDM might be close to or even exceed the present or planned experimental limits. Clearly, these processes constitute an important constraint on seesaw and/or GUT models.

For instance, in a type I seesaw model in the low energy basis where charged leptons are diagonal, the $i j$ element of the left handed slepton mass matrix provides the dominant contribution in the decay $\ell_{i} \rightarrow \ell_{j} \gamma$. Assuming, for the sake of simplicity, an mSUGRA spectrum at $\Lambda=M_{\mathrm{Pl}}$, one obtains at the leading $\log [178]$ :

$$
\begin{aligned}
\delta_{i j}^{L L} & =\frac{\left(m_{i j}^{2}\right)_{L L}}{m_{L}^{2}}=-\frac{1}{8 \pi^{2}} \frac{3 m_{0}^{2}+A_{0}^{2}}{m_{L}^{2}} C_{i j}, \\
C_{i j} & \equiv \sum_{k} Y_{\nu k i}^{*} Y_{\nu k j} \ln \frac{M_{\mathrm{Pl}}}{M_{k}},
\end{aligned}
$$

where $m_{0}$ and $A_{0}$ are respectively the universal scalar masses and trilinear couplings at $M_{\mathrm{Pl}}, m_{L}^{2}$ is an average left handed slepton mass and $M_{k}$ the mass of the right handed neutrino with $k=1,2,3$. An experimental limit on $B\left(\ell_{i} \rightarrow \ell_{j} \gamma\right)$ corresponds to an upper bound on $\left|C_{i j}\right|$ $[38,233]$. For $\mu \rightarrow e \gamma$ and $\tau \rightarrow \mu \gamma$ this bound is shown in Fig. 9 as a function of the right handed selectron mass.

The seesaw model dependence resides in $C_{i j}$. Notice that in the fundamental theory at high energy, the size of $C_{i j}$ is determined both by the Yukawa eigenvalues and the largeness of the mixing angles of $V_{R}, V_{L}$, the unitary matrices which diagonalize $Y_{v}$ (in the basis where $M_{R}$ and $Y_{e}$ are diagonal): $V_{R} Y_{\nu} V_{L}=Y_{\nu}^{(\mathrm{diag})}$. The left handed misalignment between neutrino and charged lepton Yukawa's is given by $V_{L}$ and, due to the mild effect of the logarithm in $C_{i j}$, in first approximation $V_{L}$ itself diagonalizes $C_{i j}$. If we consider hierarchical $Y_{v}$ eigenvalues, $Y_{3}>Y_{2}>Y_{1}$, the contributions from $k=1,2$ in (5.28) can in first approximation be neglected with respect to the contribution from the heaviest eigenvalue $(k=3)$ :

$\left|C_{i j}\right| \approx\left|V_{L i 3} V_{L j 3}\right| Y_{3}^{2} \log \left(M_{\mathrm{Pl}} / M_{3}\right)$.

Taking supersymmetric particle masses around the $\mathrm{TeV}$ scale, it has been shown that many seesaw models predict $\left|C_{\mu e}\right|$ and/or $\left|C_{\tau \mu}\right|$ close to the experimentally accessible range. Let us consider the predictions for the seesaw-RGE induced contribution to $\tau \rightarrow \mu \gamma$ and $\mu \rightarrow e \gamma$ in the flavor models discussed previously. 
Fig. 9 Upper limit on $C_{32}$ and $C_{21}$ for the experimental sensitivities displayed [38]

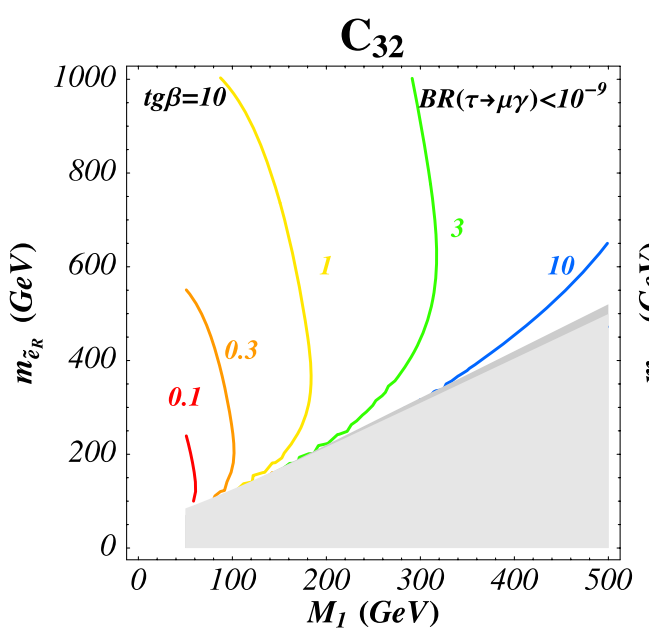

The present experimental bound on $\tau \rightarrow \mu \gamma$ is not very strong but nevertheless promising. In models with "lopsided" $Y_{v}$, one has $V_{L 32} V_{L 32} \approx 1 / 2$, hence $\left|C_{\tau \mu}\right|=$ $O\left(4 \times Y_{3}^{2}\right)$, for $M_{3} \simeq 4 \times 10^{15} \mathrm{GeV}$. This is precisely the case for the $U$ (1) flavor model discussed in Sect. 2.2, where $Y_{3} \approx \epsilon^{n_{3}^{c}}$ with $\epsilon \approx 0.22$ (the Cabibbo angle). For this model, planned $\tau \rightarrow \mu \gamma$ searches could thus be successful if the heaviest right handed neutrino has null charge, $n_{3}^{c}=0$. On the contrary, in models with small $V_{L 23}$ mixing, like in the non-Abelian models discussed previously, the seesaw-RGE induced effect is below the experimental sensitivity.

The present experimental bound on $\mu \rightarrow e \gamma$ is already very severe in constraining $\left|C_{\mu e}\right|$. For instance, if $V_{L} \approx$ $V_{\mathrm{CKM}}$, one obtains $C_{\mu e}=O\left(10^{-3} \times Y_{3}^{2}\right)$. As can be seen from Fig. 9, $V_{L}$ could in future be tested at a CKM-level if $Y_{3}=O$ (1) [164]. The predictions for $\mu \rightarrow e \gamma$ are however very model dependent. For the simple $U(1)$ flavor model of Sect. 2.2, the mixings of $V_{L}$ are of the same order of magnitude as those of $U_{\mathrm{PMNS}}$ and one expects $\left|C_{\mu e}\right|=$ $O\left(8 \times \epsilon^{2 n_{3}^{c}+1}\right)$ : if $n_{3}^{c}=0$ the prediction exceeds the experimental limit, which is respected only with $n_{3}^{c} \geq 1$ [684]. On the contrary, the non-Abelian models discussed previously have $Y_{3} \sim 1$, but the $V_{L}$-mixings are sufficiently small to suppress the seesaw-RGE induced effect below the present experimental level [164].

\subsubsection{Parameter dependence for degenerate heavy neutri-} nos Equation (5.28) indicates that LFV in the minimal supersymmetric seesaw model depends on soft supersymmetry breaking masses as well as on the seesaw parameters. The latter can be parameterized via the heavy and light neutrino masses, the light neutrino mixing matrix and the orthogonal matrix $R$ of (3.45). The three complex mixing angles parameterizing $R$ can be written as $\hat{\theta}_{j}=x_{j}+i y_{j}, j=1,2,3$. For the following numerical examples we use the mSUGRA point SPS1a [685] for SUSY breaking masses.
In the case of degenerate heavy neutrino masses, $M_{i}=$ $M_{R}(i=1,2,3)$, and real $R$, the $R$ dependence in (5.28) and hence also in $B\left(l_{i} \rightarrow l_{j} \gamma\right)$ drops out. However, if $R$ is complex, the LFV observables have more freedom since the dependence on $y_{i}$ can be as significant as the $M_{R}$ dependence, as Fig. 10 shows. For small $\left|y_{i}\right|$, the change in $Y_{v}^{\dagger} Y_{v}$ is approximately

$$
\begin{aligned}
\Delta_{R}\left(Y_{v}^{\dagger} Y_{\nu}\right) \approx & U_{\mathrm{PMNS}} \operatorname{diag}\left(\sqrt{m_{i}}\right)\left(R^{\dagger} R-\mathbf{1}\right) \\
& \times \operatorname{diag}\left(\sqrt{m_{i}}\right) U_{\mathrm{PMNS}}^{\dagger},
\end{aligned}
$$

while the renormalization effects on the soft supersymmetry breaking masses can be estimated via [273]

$m_{L}^{8} \simeq 0.5 m_{0}^{2} M_{1 / 2}^{2}\left(m_{0}^{2}+0.6 M_{1 / 2}^{2}\right)^{2}$,

where $M_{1 / 2}$ is the universal gaugino mass at high scale. In certain cases, the leading logarithmic approximation fails, as pointed out in [238, 273, 275, 686].

Equation (5.30) implies three features seen in Fig. 10:

(i) Compared to the case of degenerate light neutrino masses, the $y$ dependence in the hierarchical case is weaker.

(ii) Observables like (5.27) are larger in the case of complex $R$ than in the case of real $R$. For a given $M_{R}$, even small values of $y$ can enhance a process by orders of magnitude.

(iii) In contrast to the real $R$ case, where $B\left(l_{i} \rightarrow l_{j} \gamma\right)$ for degenerate light neutrinos is always larger than for hierarchical light neutrinos, the relative magnitude can be reversed for complex $R$.

To examine the parameter dependence of rare decays at large $\left|y_{i}\right|>0.1$, we extend the above analysis to the case where the $y_{i}$ are independent of one another. For random values of all parameters in their full ranges, the typical be- 

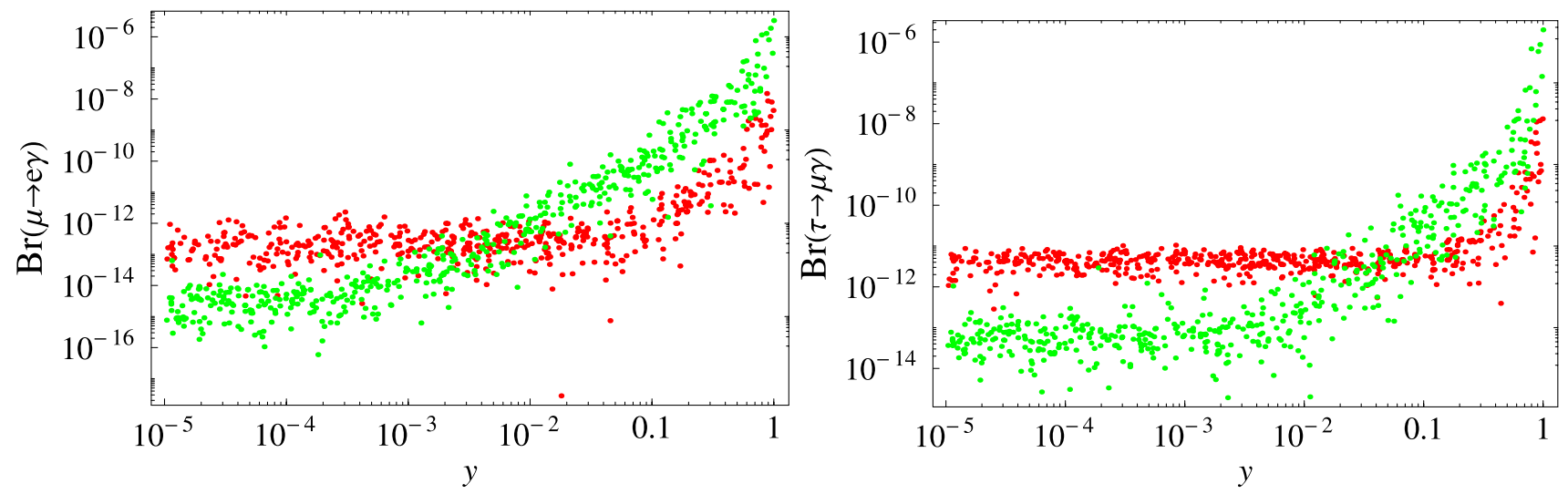

Fig. 10 (Color online) Degenerate heavy neutrinos: LFV branching ratio versus $\left|y_{i}\right|=y$ for fixed $M_{i}=M_{R}=10^{12}$ GeV in mSUGRA scenario SPS1a for hierarchical (dark red) and degenerate (light green) light neutrino masses. The $x_{i}$ are scattered over $0<x_{i}<2 \pi$
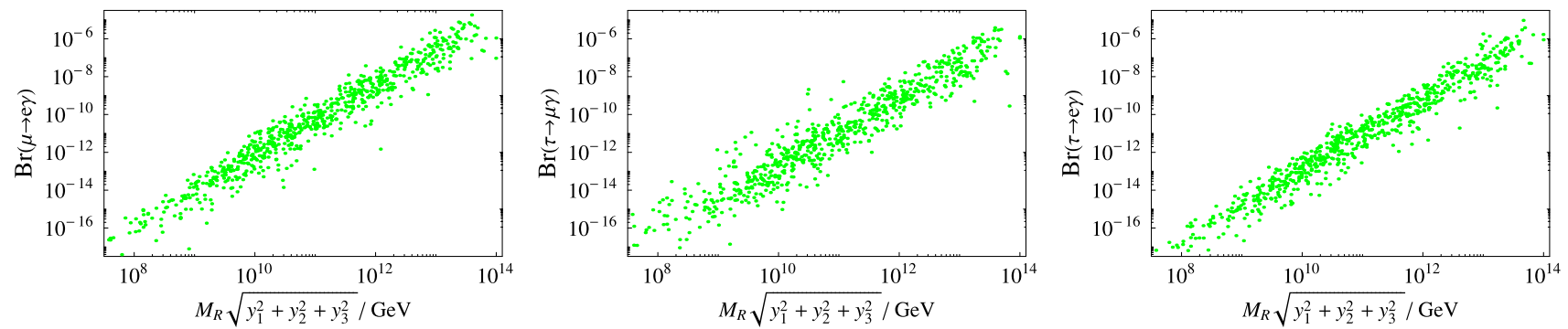

Fig. 11 Degenerate heavy neutrinos: LFV branching ratios versus $M_{R} \sqrt{y_{1}^{2}+y_{2}^{2}+y_{3}^{2}}$, for light neutrinos. The $y_{i}$ are scattered logarithmically in the range $10^{-5}<\left|y_{i}\right|<1$ (independently of one another)

havior

$$
\begin{aligned}
& \left|\left(Y_{v}^{\dagger} L Y_{v}\right)_{j k}\right|^{2} \propto\left\{\begin{array}{lr}
M_{R}^{2}\left(C_{1} y_{1}^{2}+C_{2} y_{2}^{2}+C_{3} y_{3}^{2}\right) & \text { deg. } v_{L} \\
M_{R}^{2} & \text { hier. } v_{L}
\end{array}\right. \\
& (j \neq k),
\end{aligned}
$$

is found, with $C_{i}=O(1)$, slightly dependent on $j, k$. This behavior can be seen in Fig. 11 for degenerate light neutrinos. Thus for large $\left|y_{i}\right|$ all rare decays may be of a similar order of magnitude. For hierarchical light neutrinos, a similar behavior is observed, but versus $M_{R}^{2}$ only.

\subsubsection{Parameter dependence for hierarchical heavy neu-} trinos Hierarchical spectrum of heavy Majorana neutrinos, $M_{1} \ll M_{2} \ll M_{3}$, is well motivated by the arguments of light neutrino mass and mixing generation and leptogenesis. Requiring successful thermal leptogenesis puts additional constraints on the seesaw parameters and constrains the LFV observables [329]. This is the approach we take in this subsection. In particular, the relation (3.93) implies a lower bound on $M_{1}$ [306], e.g., if $\epsilon_{1}>10^{-6}$, then $M_{1}>$ $5 \times 10^{9} \mathrm{GeV}$. Furthermore, to allow for thermal production of right handed neutrinos after inflation, one has to exclude and $M_{R}$ is scattered logarithmically in the range $10^{10}<M_{R}<M_{\mathrm{GUT}}$. The $x_{i}$ are scattered over $0<x_{i}<2 \pi$

$M_{1}>10^{11} \mathrm{GeV}$, at least in simple scenarios. Otherwise a too high re-heating temperature would lead to an overabundance of gravitinos, whose decays into energetic photons can spoil big bang nucleosynthesis. Details of leptogenesis have been described in Sect. 3.3.1.

Assuming hierarchical light neutrinos with $\Delta m_{\text {sol }}^{2}<$ $m_{3}^{2}<\Delta m_{\text {atm }}^{2}$, the condition to reproduce the experimental baryon asymmetry, $\eta_{B}=(6.3 \pm 0.3) \times 10^{-10}$, puts constraints on $M_{1}$ and the $R$ matrix [687]. This is illustrated in Fig. 12 in the $M_{1}-x_{2}$ plane. For $M_{1}<10^{11} \mathrm{GeV}, x_{2}$ has to approach the values $0, \pi, 2 \pi$. A similar behavior is observed in the $M_{1}-x_{3}$ plane.

Taking $M_{1}=10^{10} \mathrm{GeV}$ and $x_{2} \approx x_{3} \approx n \cdot \pi$, experimental bounds on $B(\mu \rightarrow e \gamma)$ can be used to constrain the heavy neutrino scale, here represented by the heaviest right handed neutrino mass $M_{3}$, as shown in the right plot of Fig. 12. Quantitatively, the present bound on $B(\mu \rightarrow$ $e \gamma$ ) already constrains $M_{3}$ to be smaller than $\approx 10^{13} \mathrm{GeV}$, while the MEG experiment at PSI is sensitive to $M_{3} \leq$ $O\left(10^{12}\right) \mathrm{GeV}$. If no signal is observed it will be difficult to test the type I seesaw model considered here at future colliders. 


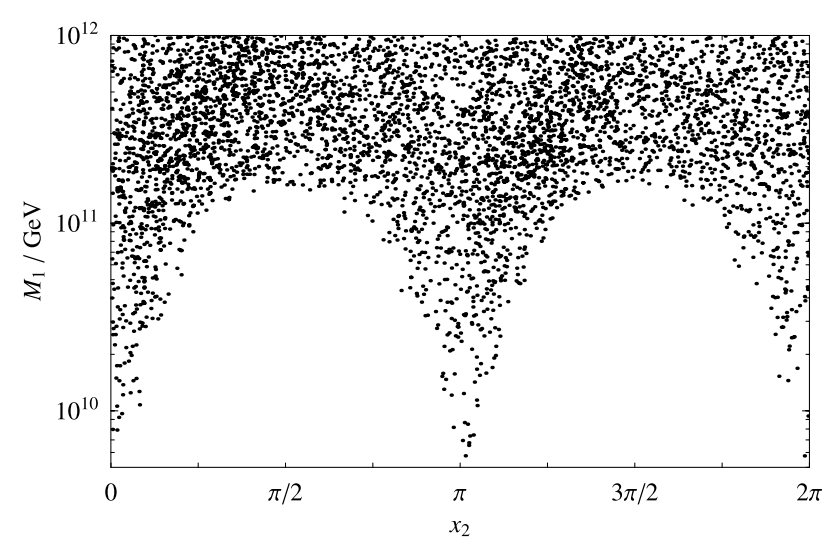

Fig. 12 Hierarchical heavy neutrinos: Region in the plane $\left(x_{2}, M_{1}\right)$ consistent with the generation of the baryon asymmetry $\eta_{B}=(6.3 \pm$ $0.3) \times 10^{-10}$ via leptogenesis (left). [Right] $B(\mu \rightarrow e \gamma)$ versus $M_{3}\left|\cos ^{2} \theta_{2}\right|$ in mSUGRA scenario SPS1a, for $M_{1}=10^{10} \mathrm{GeV}$ and

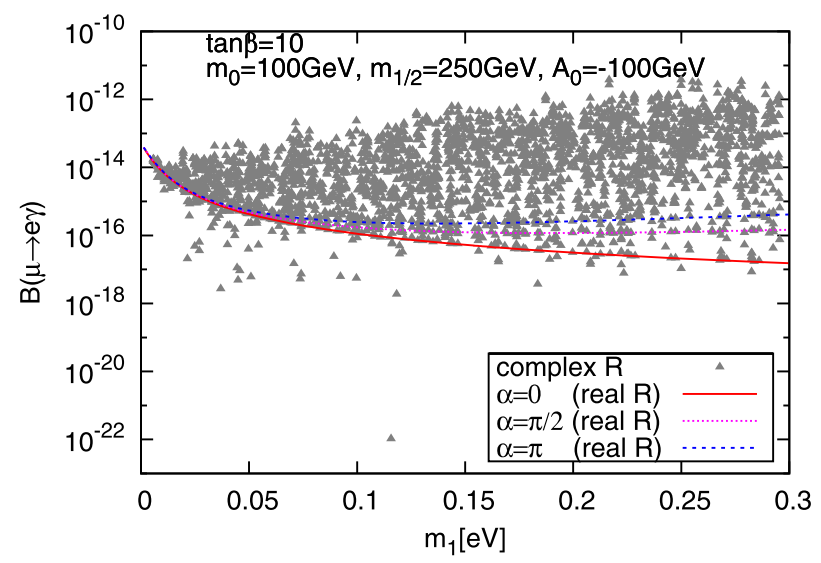

Fig. 13 The branching ratios of the LFV decays $\mu \rightarrow e+\gamma$ and $\tau \rightarrow$ $\mu+\gamma$ versus $m_{1}$ in the cases of complex and real matrix $R$ with $\alpha=$ $0 ; \pi / 2 ; \pi$. The three parameters describing the matrix $R[275,545]$ are generated randomly. The SUSY parameters are $\tan \beta=10, m_{0}=$ $100 \mathrm{GeV}, m_{1 / 2}=250 \mathrm{GeV}, A_{0}=-100 \mathrm{GeV}$, and the neutrino mixing

\subsubsection{Effects of renormalization of light neutrino masses} on $L F V$ The RG running of the neutrino parameters can have an important impact on lepton flavor violating processes in MSSM extended by right handed neutrinos. In this example we assume universal soft SUSY breaking terms at GUT scale and degenerate heavy neutrinos with mass $M_{R}$. The running effects below $M_{R}$ are relatively small when $\tan \beta$ is smaller than 10 and/or $m_{1}$ is much smaller than $0.05 \mathrm{eV}$. Because the combination $s_{12} c_{12} c_{23}\left(m_{1}-m_{2} e^{i \alpha_{M}}\right)$, where we use the notation of Sect. 3.2.3.3, is practically stable against the RG running, and this combination is the dominant term of $\left(Y_{v}^{\dagger} Y_{v}\right)_{21}$ when $\alpha_{M}=0, \theta_{13}=0$ and $R^{*}=R$ are satisfied, the running effect on LFV can be neglected in this case [275]. In general, $\left(Y_{\nu}^{\dagger} Y_{\nu}\right)_{21}$ and $B(\mu \rightarrow e+\gamma)$ can depend strongly on

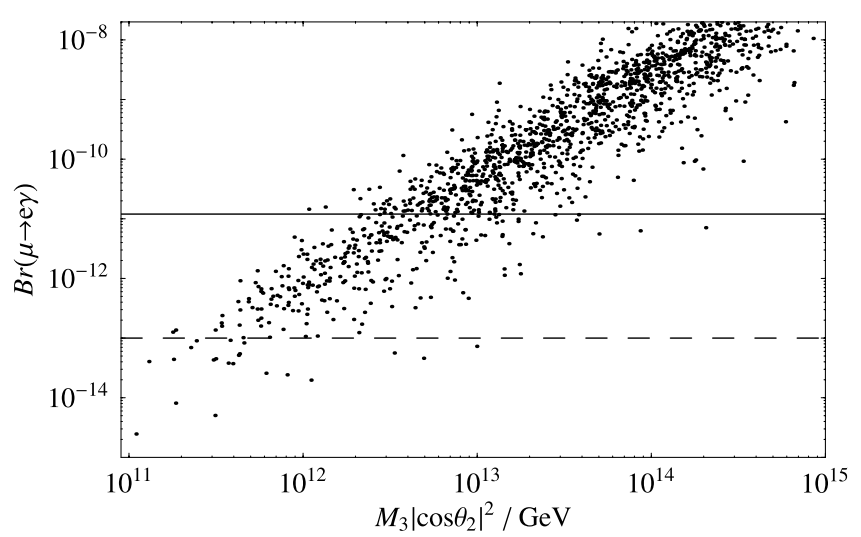

$x_{2} \approx x_{3} \approx n \cdot \pi$. All other seesaw parameters are scattered in their allowed ranges for hierarchical light and heavy neutrinos. The solid (dashed) line indicates the present (expected future) experimental sensitivity

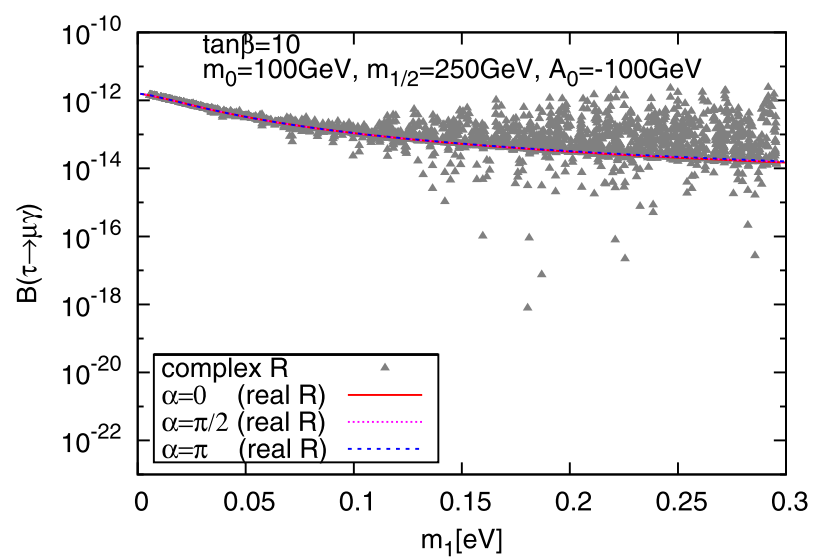

parameters are $\Delta m_{\odot}^{2}=8.0 \times 10^{-5} \mathrm{eV}^{2}, \Delta m_{\mathrm{atm}}^{2}=2.2 \times 10^{-3} \mathrm{eV}^{2}$, $\tan ^{2} \theta_{\odot}=0.4, \tan ^{2} \theta_{\mathrm{atm}}=1$, and $\sin \theta_{13}=0.0$. The neutrino mass spectrum at $M_{Z}$ is assumed to be with normal hierarchy, $m_{1}\left(M_{Z}\right)<$ $m_{2}\left(M_{Z}\right)<m_{3}\left(M_{Z}\right)$. The right handed neutrino mass spectrum is taken to be degenerate as $M_{1}=M_{2}=M_{3}=2 \times 10^{13} \mathrm{GeV}$ [275]

$\theta_{13}$ and RG running has to be taken into account [686, 688]. Note that due to RG running, the value of $\theta_{13}$ at $M_{R}$ differs from 0 , even if $\theta_{13}=0$ is assumed at low energy [275].

In many cases, the running of the neutrino parameters can significantly affect the prediction of the LFV branching ratios. In particular, for $0.05 \lesssim m_{1} \lesssim 0.30 \mathrm{eV}, 30 \lesssim$ $\tan \beta \lesssim 50$, the predicted $\mu \rightarrow e+\gamma$ and $\tau \rightarrow e+\gamma$ decay branching ratios, $B(\mu \rightarrow e+\gamma)$ and $B(\tau \rightarrow e+\gamma)$, can be enhanced by the effects of the RG running of $\theta_{i j}$ and $m_{j}$ by 1 to 3 orders of magnitude if $\pi / 4 \lesssim \alpha_{M} \lesssim \pi$, while $B(\tau \rightarrow \mu+\gamma)$ can be enhanced by up to a factor of 10 [275]. The effects of the running of the neutrino mixing parameters of $B(\mu \rightarrow e+\gamma)$ and $B(\tau \rightarrow e+\gamma)$ are illustrated in Fig. 13. 


\subsubsection{Correlations between LFV observables and collider physics}

5.2.3.1 Correlations of $L F V$ rare decays Equations (5.27) and (5.28) imply correlations between different LFV observables. In addition to the correlations between different classes of LFV observables in the same flavor mixing channels, the assumed LFV mechanism induces also correlations among the $\left|\left(m_{L}\right)_{i j}^{2}\right|^{2}$ and hence among observables of different flavor mixing channels. In this framework, the ratios of the branching ratios are approximately independent of SUSY parameters:

$\frac{B(\tau \rightarrow \mu \gamma)}{B(\mu \rightarrow e \gamma)} \propto \frac{\left|\left(Y_{v}^{\dagger} L Y_{v}\right)_{23}\right|^{2}}{\left|\left(Y_{v}^{\dagger} L Y_{v}\right)_{12}\right|^{2}}$

Thus the measurement of the ratio between the decay rates of the different LFV channels can provide unique information on the flavor structure of the lepton sector. The ratios of interest, such as (5.33), can exhibit, for instance, strong dependence on CP-violating parameters in neutrino Yukawa couplings [691] especially in the case of quasi-degenerate heavy RH neutrinos. As a consequence such correlations have been widely studied (see, e.g., [38, 178, 238, 242, 249, $329,334,649,691,693]$ and the references quoted therein).
Consequently, bounds on one LFV decay channel (process) will limit the parameter space of the LFV mechanism and thus lead to bounds on the other LFV decay channels (processes). In Fig. 14, the correlation induced by the type I seesaw mechanism between $B(\mu \rightarrow e \gamma)$ and $B(\tau \rightarrow \mu \gamma)$ is shown, and the bounds induced by the former on the latter can be easily read off. Interestingly, these bounds do not depend on whether hierarchical or quasi-degenerate heavy and light neutrinos are assumed. The present and future prospective bounds are summarized in Table 12. Note that the present upper bound on $B(\mu \rightarrow e \gamma)$ implies a stronger constraint on $B(\tau \rightarrow \mu \gamma)$ than its expected future bound.

The above results were derived in the simplifying case of a real $R$ matrix. For complex $R$ with $\left|y_{i}\right|<1$ there is no significant change with respect to the results in Table 12 in the case of hierarchical heavy and hierarchical light neutrinos due to the weak $R$ dependence of $B(\mu \rightarrow e \gamma)$ and $B(\tau \rightarrow \mu \gamma)$. However, for quasi-degenerate light neutrinos, $B(\tau \rightarrow \mu \gamma)$ is lowered by roughly one order of magnitude, somewhat spoiling the overlap of all scenarios observed in Fig. 14.

In Fig. 15, we display the correlation between $B(\mu \rightarrow$ $e \gamma)$ and $B(\tau \rightarrow \mu \gamma)$ for complex $R$ and some fixed values of $M_{R}$ in the case of quasi-degenerate RH neutrino masses

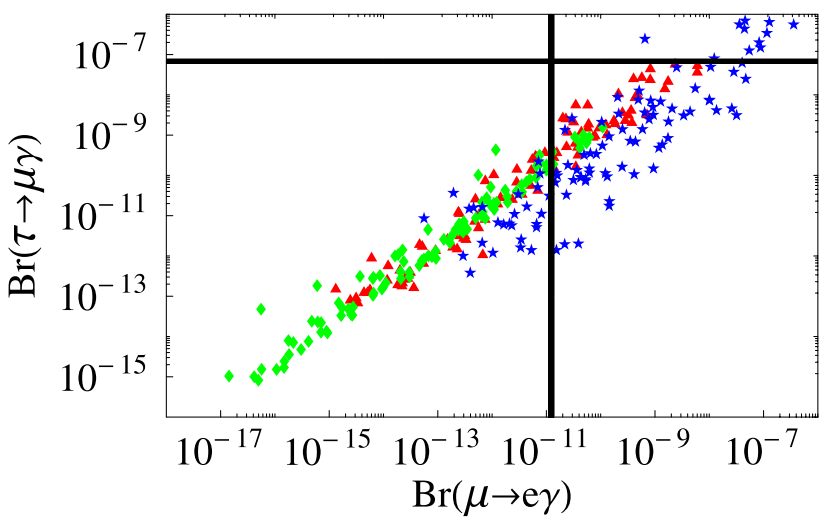

Fig. $14 B(\tau \rightarrow \mu \gamma)$ versus $B(\mu \rightarrow e \gamma)$, in mSUGRA scenario SPS1a with neutrino parameters scattered within their experimentally allowed ranges [689]. For quasi-degenerate heavy neutrino masses, both hierarchical (triangles) and quasi-degenerate (diamonds) light neutrino masses are considered with real $R$ and $10^{11}<M_{R}<10^{14.5} \mathrm{GeV}$.
In the case of hierarchical heavy and light neutrino masses (stars), the $x_{i}$ are scattered over their full ranges $0<x_{i}<2 \pi$ and the $y_{i}$ and $M_{i}$ are scattered within the bounds demanded by leptogenesis and perturbativity. Also indicated are the present experimental bounds $B(\mu \rightarrow e \gamma)<1.2 \times 10^{-11}$ and $B(\tau \rightarrow \mu \gamma)<6.8 \times 10^{-8}[191,690]$

Table 12 Present and expected future bounds on $B(\mu \rightarrow e \gamma)$ from experiment, and bounds on $B(\tau \rightarrow \mu \gamma)$ from (i) experiment (ii) the bound on $B(\mu \rightarrow e \gamma)$ together with correlations from the SUSY type I seesaw mechanism

\begin{tabular}{llll}
\hline & $B(\mu \rightarrow e \gamma)($ exp. $)$ & $B(\tau \rightarrow \mu \gamma)($ exp. $)$ & $B(\tau \rightarrow \mu \gamma)^{\mathrm{a}}$ \\
\hline Present & $1.2 \times 10^{-11}$ & $6.8 \times 10^{-8}$ & $10^{-9}$ \\
Future & $10^{-14}$ & $10^{-9}$ & $10^{-12}$ \\
\hline
\end{tabular}

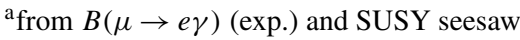



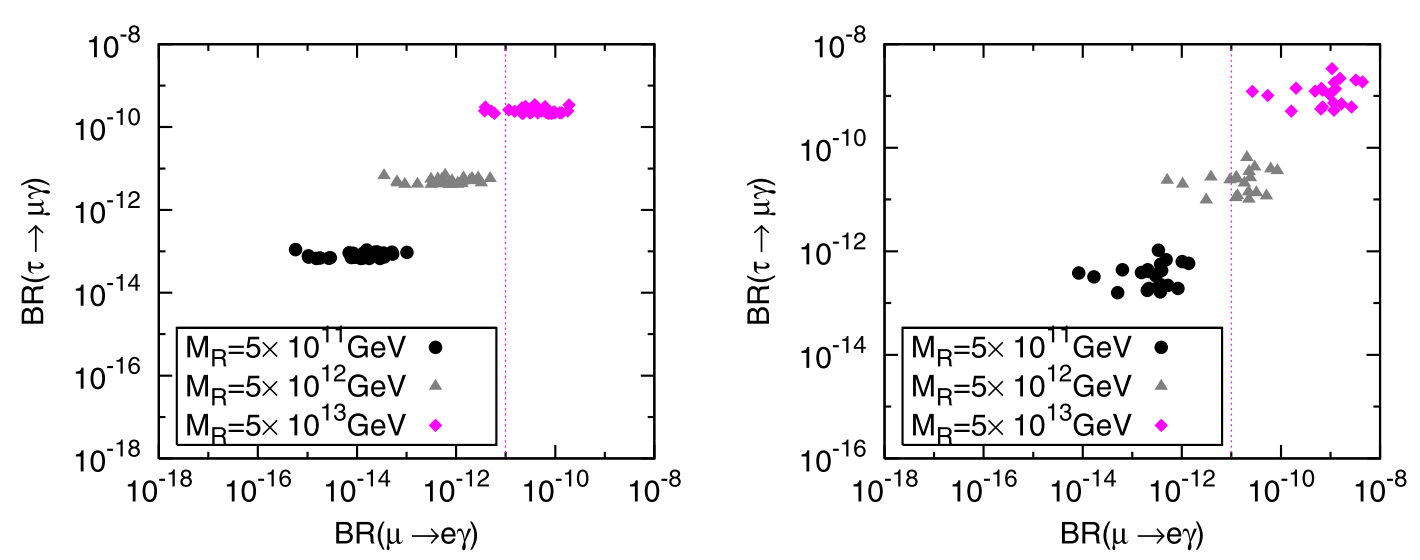

Fig. 15 The correlation between $B(\mu \rightarrow e \gamma)$ and $B(\tau \rightarrow \mu \gamma)$ for quasi-degenerate heavy neutrinos and light neutrino mass spectrum of normal hierarchical (left panel) and inverted hierarchical (right panel) type
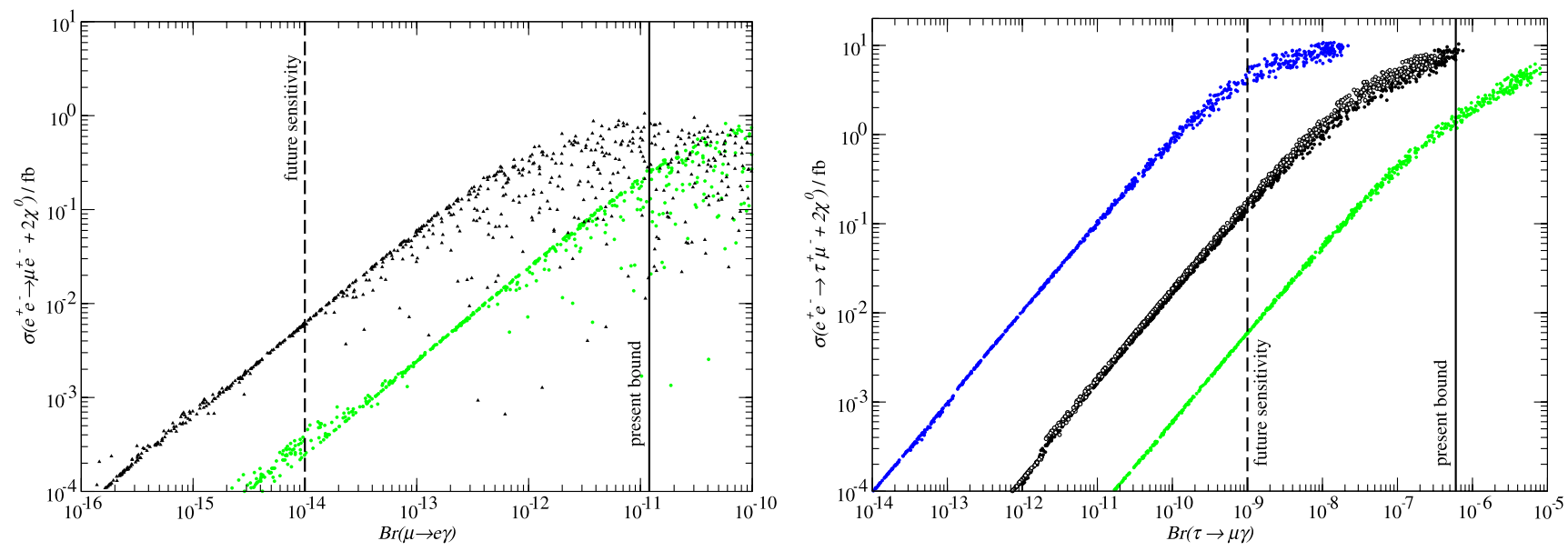

Fig. 16 Correlation of LFV LC processes and rare decays in the $e \mu$-channel $(l e f t)$ and the $\mu \tau$-channel (right). The seesaw parameters are scattered as in Fig. 14. The mSUGRA scenarios used are (from left to right): SPS1a, $\mathrm{G}^{\prime}(e \mu)$ and $\mathrm{C}^{\prime}, \mathrm{B}^{\prime}, \mathrm{SPS} 1 \mathrm{a}, \mathrm{I}^{\prime}(\mu \tau)$

and a normal and inverted hierarchical light neutrino mass spectrum. We note that, as Fig. 15 suggests, $B(\tau \rightarrow \mu \gamma)$ is almost independent of the CP violating parameters and phases respectively in $R$ and $U$, while the dependence of $B(\mu \rightarrow e \gamma)$ on the $\mathrm{CP}$-violating quantities is much stronger. This is reflected, in particular, in the fact that for a fixed $M_{R}$, $B(\tau \rightarrow \mu \gamma)$ is practically constant while $B(\mu \rightarrow \mu \gamma)$ can change by $2-3$ orders of magnitude.

If the $\mu \rightarrow e \gamma$ and $\tau \rightarrow \mu \gamma$ decays will be observed, the ratio of interest can give unique information on the origin of the lepton flavor violation.

5.2.3.2 LFV rare decays and linear collider processes In high energy $e^{+} e^{-}$colliders [692], feasible tests of LFV are provided by the processes $e^{+} e^{-} \rightarrow \tilde{l}_{a}^{-} \tilde{l}_{b}^{+} \rightarrow l_{i}^{-} l_{j}^{+}+2 \tilde{\chi}_{1}^{0}$. Analogously to (5.27), one can derive the approximate expression [695]

$$
\begin{aligned}
& \sigma\left(e^{+} e^{-} \rightarrow l_{i}^{-} l_{j}^{+}+2 \tilde{\chi}_{1}^{0}\right) \\
& \approx \frac{\left|\left(\delta m_{L}\right)_{i j}^{2}\right|^{2}}{m_{\tilde{l}}^{2} \Gamma_{\tilde{l}}^{2}} \sigma\left(e^{+} e^{-} \rightarrow l_{i}^{-} l_{i}^{+}+2 \tilde{\chi}_{1}^{0}\right),
\end{aligned}
$$

for the production cross section in the limit of small slepton mass corrections. By comparing (5.27) with (5.34), it is immediately apparent that the linear collider (LC) processes are flavor-correlated with the rare decays considered previously. These correlations are shown in Fig. 16 for the two most important channels.

This observation implies that once the SUSY parameters are known, a measurement of, e.g., $B(\mu \rightarrow e \gamma)$ will lead to a prediction for $\sigma\left(e^{+} e^{-} \rightarrow \mu e+2 \tilde{\chi}_{1}^{0}\right)$. Quite obviously, this prediction will be independent of the specific LFV mechanism (seesaw or other). Figure 16 also demonstrates that the uncertainties in the neutrino parameters nicely drop out except at large cross sections and branching ratios. 
In the previous results we have assumed a specific choice of the as yet unknown mSUGRA parameters. The results of a more systematic study of the model dependence are visualized in Fig. 17 by contour plots for $\sigma\left(e^{+} e^{-} \rightarrow \mu^{+} e^{-}+2 \tilde{\chi}_{1}^{0}\right)$ and $B(\mu \rightarrow e \gamma)$ in the $m_{0}-m_{1 / 2}$ plane with the remaining mSUGRA parameters fixed.

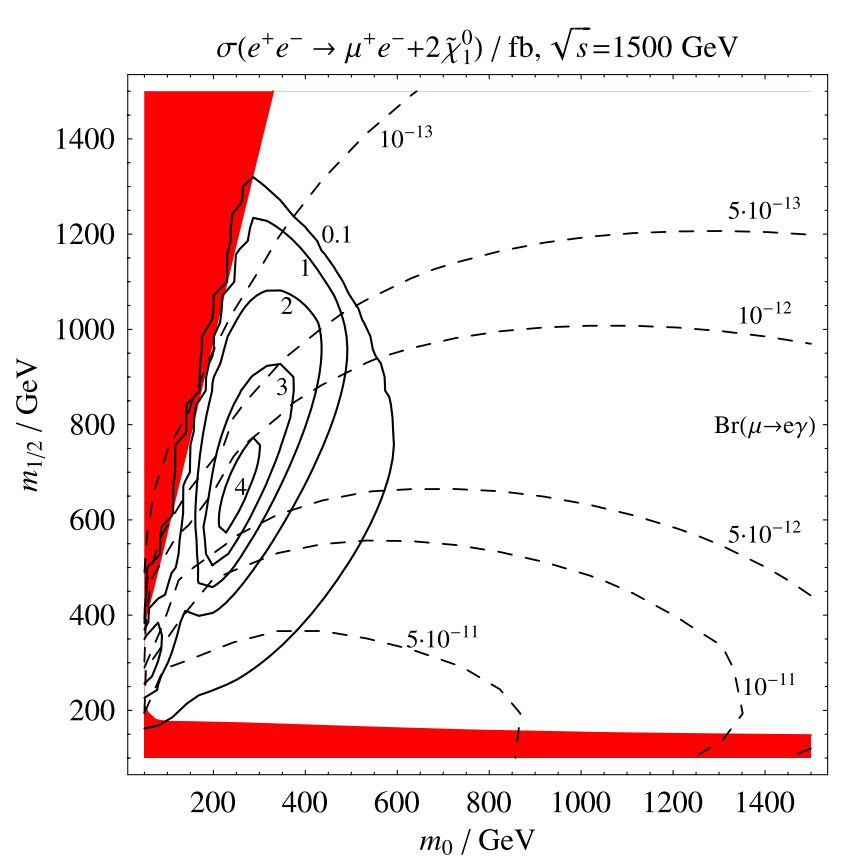

Fig. 17 Contours of the polarized cross section $\sigma\left(e^{+} e^{-} \rightarrow\right.$ $\left.\mu^{+} e^{-}+2 \tilde{\chi}_{1}^{0}\right)($ solid $)$ and $B(\mu \rightarrow e \gamma)$ (dashed) in the $m_{0}-m_{1 / 2}$ plane. The remaining mSUGRA parameters are $A_{0}=0 \mathrm{GeV}, \tan \beta=5$, $\operatorname{sign}(\mu)=+$. The energy and beam polarizations are $\sqrt{s_{e e}}=1.5 \mathrm{TeV}$, $P_{e^{-}}=+0.9, P_{e^{+}}=+0.7$. The neutrino oscillation parameters are fixed at their central values as given in [689], the lightest neutrino mass $m_{1}=0$ and all complex phases are set to zero, and the degenerate right handed neutrino mass scale is $M_{R}=10^{14} \mathrm{GeV}$. The shaded (red) areas are already excluded by mass bounds from various experimental sparticle searches

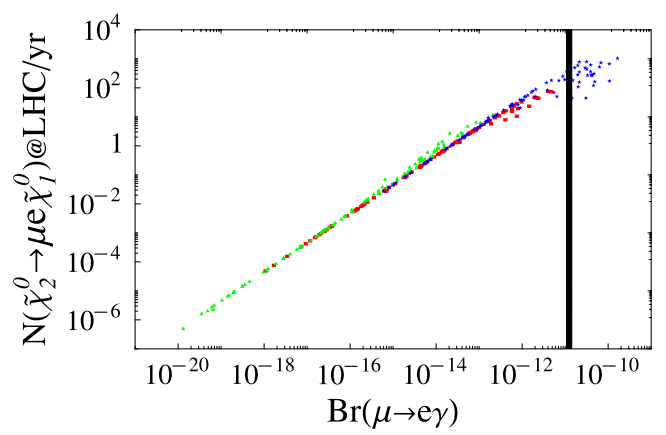

Fig. 18 (Color online) Correlation of the number of $\tilde{\chi}_{2}^{0} \rightarrow \mu^{+} e^{-} \tilde{\chi}_{1}^{0}$ events per year at the LHC and $B(\mu \rightarrow e \gamma)$ in mSUGRA scenario $\mathrm{C}^{\prime}\left(m_{0}=85 \mathrm{GeV}, m_{1 / 2}=400 \mathrm{GeV}, A_{0}=0 \mathrm{GeV}, \tan \beta=10 \mathrm{GeV}\right.$, $\operatorname{sign} \mu=+$ ) for the case of hier. $v_{R / L}$ (blue stars), deg. $v_{R} /$ hier. $v_{L}$
5.2.3.3 LFV rare decays and LHC processes At the LHC, a feasible test of LFV is provided by squark and gluino production, followed by cascade decays of squarks and gluinos via neutralinos and sleptons [696, 697]:

$p p \rightarrow \tilde{q}_{a} \tilde{q}_{b}, \tilde{g} \tilde{q}_{a}, \tilde{g} \tilde{g}$,

$\tilde{q}_{a}(\tilde{g}) \rightarrow \tilde{\chi}_{2}^{0} q_{a}(g)$

$\tilde{\chi}_{2}^{0} \rightarrow \tilde{l}_{\alpha} l_{\beta}$,

$\tilde{l}_{\alpha} \rightarrow \tilde{\chi}_{1}^{0} l_{\beta}$

where $a, b$ run over all squark mass eigenstates, including antiparticles, and $\alpha, \beta$ are slepton (lepton) mass (flavor) eigenstates, including antiparticles. LFV can occur in the decay of the second lightest neutralino and/or the slepton, resulting in different lepton flavors, $\alpha \neq \beta$. The total cross section for the signature $l_{\alpha}^{+} l_{\beta}^{-}+X$ can then be written as

$$
\begin{aligned}
\sigma(p p & \left.\rightarrow l_{\alpha}^{+} l_{\beta}^{-}+X\right) \\
= & {\left[\sum_{a, b} \sigma\left(p p \rightarrow \tilde{q}_{a} \tilde{q}_{b}\right) \times B\left(\tilde{q}_{a} \rightarrow \tilde{\chi}_{2}^{0} q_{a}\right)\right.} \\
& +\sum_{a} \sigma\left(p p \rightarrow \tilde{q}_{a} \tilde{g}\right) \times\left(B\left(\tilde{q}_{a} \rightarrow \tilde{\chi}_{2}^{0} q_{a}\right)\right. \\
& \left.\left.+B\left(\tilde{g} \rightarrow \tilde{\chi}_{2}^{0} g\right)\right)+\sigma(p p \rightarrow \tilde{g} \tilde{g}) \times B\left(\tilde{g} \rightarrow \tilde{\chi}_{2}^{0} g\right)\right] \\
& \times B\left(\tilde{\chi}_{2}^{0} \rightarrow l_{\alpha}^{+} l_{\beta}^{-} \tilde{\chi}_{1}^{0}\right),
\end{aligned}
$$

where $X$ can involve jets, leptons and LSPs produced by lepton flavor conserving decays of squarks and gluinos, as well as low energy proton remnants. The LFV branching ratio $B\left(\tilde{\chi}_{2}^{0} \rightarrow l_{\alpha}^{+} l_{\beta}^{-} \tilde{\chi}_{1}^{0}\right)$ is for example calculated in [698] in the framework of model-independent MSSM slepton mixing. In general, it involves a coherent summation over all intermediate slepton states.

Just as for the linear collider discussed in the previous section, we can correlate the expected LFV event rates at the LHC with LFV rare decays. This is shown in Fig. 18 for

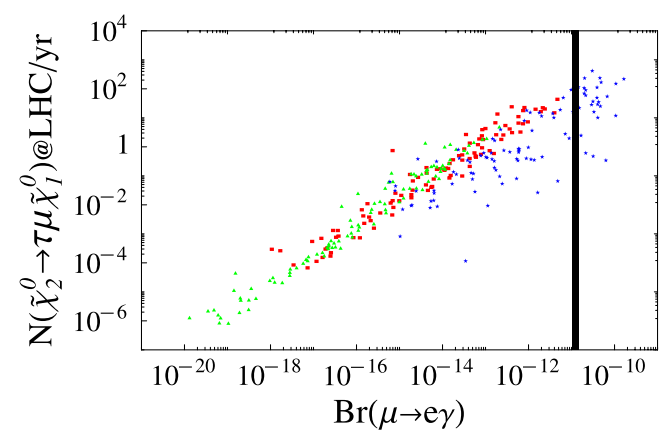

(red boxes) and deg. $v_{R / L}$ (green triangles). The respective neutrino parameter scattering ranges are as in Fig. 14. An integrated LHC luminosity of $100 \mathrm{fb}^{-1}$ is assumed. The current limit on $B(\mu \rightarrow e \gamma)$ is displayed by the vertical line 
the event rates $N\left(\tilde{\chi}_{2}^{0} \rightarrow \mu^{+} e^{-} \tilde{\chi}_{1}^{0}\right)$ and $N\left(\tilde{\chi}_{2}^{0} \rightarrow \tau^{+} \mu^{-} \tilde{\chi}_{1}^{0}\right)$, respectively, originating from the cascade reactions (5.35). Both are correlated with $B(\mu \rightarrow e \gamma)$, yielding maximum rates of around $10^{2-3}$ per year for an integrated luminosity of $\left(100 \mathrm{fb}^{-1}\right)$ in the mSUGRA scenario $\mathrm{C}^{\prime}$, consistent with the current limit on $B(\mu \rightarrow e \gamma)$.

As in the linear collider case, the correlation is approximately independent of the neutrino parameters, but highly dependent on the mSUGRA parameters. This is contemplated further in Fig. 19, comparing the sensitivity of the signature $N\left(\tilde{\chi}_{2}^{0} \rightarrow \mu^{+} e^{-} \tilde{\chi}_{1}^{0}\right)$ at the LHC with $B(\mu \rightarrow e \gamma)$ in the $m_{0}-m_{1 / 2}$ plane. As for the linear collider, LHC searches can be competitive with the rare decay experiments for small $m_{0} \approx 200 \mathrm{GeV}$. Tests in the large- $m_{0}$ region are again severely limited by collider kinematics.

Up to now we have considered LFV in the class of type I SUSY seesaw model described in Sect. 3.2.3.1, which is representative of models of flavor mixing in the left handed slepton sector only. However, it is instructive to analyze general mixing in the left and right handed slepton sector, independent of any underlying model for slepton flavor violation. The easiest way to achieve this is by assuming mixing between two flavors only, which can be parameterized by a mixing angle $\theta_{L / R}$ and a mass difference $(\Delta m)_{L / R}$ between the sleptons, in the case of left/right handed slepton mixing, respectively. ${ }^{16}$ In particular, the left/right handed selectron

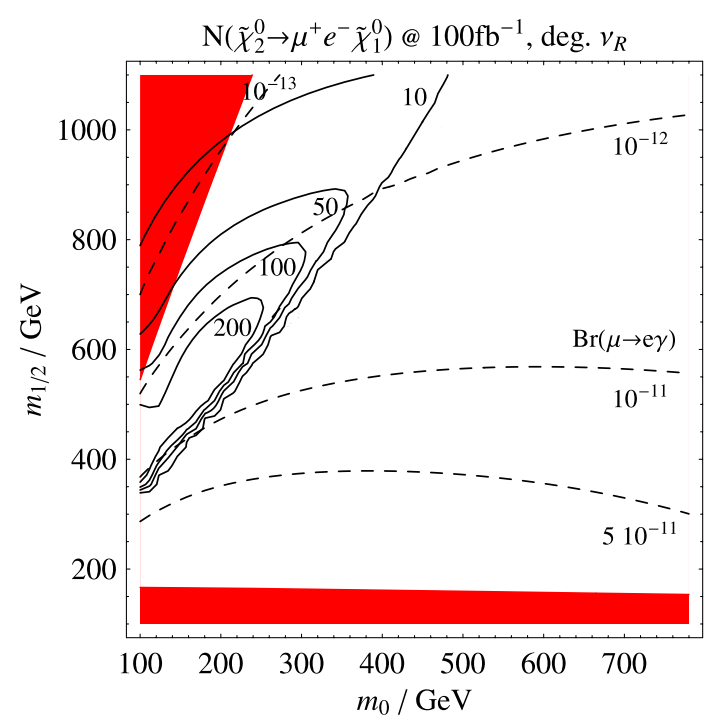

Fig. 19 Contours of the number of $\tilde{\chi}_{2}^{0} \rightarrow \mu^{+} e^{-} \tilde{\chi}_{1}^{0}$ events at the LHC with an integrated luminosity of $100 \mathrm{fb}^{-1}$ (solid) and of $B(\mu \rightarrow e \gamma)$ in the $m_{0}-m_{1 / 2}$ plane. The remaining mSUGRA and neutrino oscillation parameters are as in Fig. 17. The shaded (red) areas are already excluded by mass bounds from various experimental sparticle searches

\footnotetext{
${ }^{16}$ Note that this is different to the approach in [698], where the slepton mass matrix elements are scattered randomly.
}

and smuon sector is then diagonalized by

$$
\begin{aligned}
& \left(\begin{array}{l}
\tilde{l}_{1} \\
\tilde{l}_{2}
\end{array}\right)=U \cdot\left(\begin{array}{c}
\tilde{e}_{L / R} \\
\tilde{\mu}_{L / R}
\end{array}\right), \quad \text { with } \\
& U=\left(\begin{array}{cc}
\cos \theta_{L / R} & \sin \theta_{L / R} \\
-\sin \theta_{L / R} & \cos \theta_{L / R}
\end{array}\right),
\end{aligned}
$$

and a mass difference $m_{\tilde{l}_{2}}-m_{\tilde{l}_{1}}=(\Delta m)_{L / R}$ between the slepton mass eigenvalues. ${ }^{17}$ The LFV branching ratio $B\left(\tilde{\chi}_{2}^{0} \rightarrow \mu^{+} e^{-} \tilde{\chi}_{1}^{0}\right)$ can then be written in terms of the mixing parameters and the flavor conserving branching ratio $B\left(\tilde{\chi}_{2}^{0} \rightarrow e^{+} e^{-} \tilde{\chi}_{1}^{0}\right)$ as

$$
\begin{aligned}
B\left(\tilde{\chi}_{2}^{0}\right. & \left.\rightarrow \mu^{+} e^{-} \tilde{\chi}_{1}^{0}\right) \\
= & 2 \sin ^{2} \theta_{L / R} \cos ^{2} \theta_{L / R} \frac{(\Delta m)_{L / R}^{2}}{(\Delta m)_{L / R}^{2}+\Gamma_{\tilde{l}}^{2}} \\
& \times B\left(\tilde{\chi}_{2}^{0} \rightarrow e^{+} e^{-} \tilde{\chi}_{1}^{0}\right),
\end{aligned}
$$

where $\Gamma_{\tilde{l}}$ is the average width of the two sleptons involved. Maximal LFV is thus achieved by choosing $\theta_{L / R}=\pi / 4$ and $(\Delta m)_{L / R} \gg \Gamma_{\tilde{l}}$. For definiteness, we use $(\Delta m)_{L / R}=$ $0.5 \mathrm{GeV}$. The results of this calculation can be seen in Fig. 20, which shows contour plots of $N\left(\tilde{\chi}_{2}^{0} \rightarrow \mu^{+} e^{-} \tilde{\chi}_{1}^{0}\right)$ in the $m_{0}-m_{1 / 2}$ plane for maximal left and right handed slepton mixing, respectively. Also displayed are the corresponding contours of $B(\mu \rightarrow e \gamma)$. We see that the present bound $B(\mu \rightarrow e \gamma)=10^{-11}$ still permits sizable LFV signal rates at the LHC. However, $B(\mu \rightarrow e \gamma)<10^{-14}$ would exclude the observation of such an LFV signal at the LHC.

\subsubsection{Impact of $\theta_{13}$ on LFV in SUSY seesaw}

In this subsection we present the results of the LFV tau and muon decays within the SUSY singlet-seesaw context. Specifically, we consider the constrained minimal supersymmetric standard model (CMSSM) extended by three right handed neutrinos, $v_{R_{i}}$ and their corresponding SUSY partners, $\tilde{v}_{R_{i}}(i=1,2,3)$, and use the seesaw mechanism for the neutrino mass generation. We include the predictions for the branching ratios (BRs) of two types of LFV channels, $l_{j} \rightarrow l_{i} \gamma$ and $l_{j} \rightarrow 3 l_{i}$, and compare them with the present bounds and future experimental sensitivities. We first analyze the dependence of the BRs with the most relevant SUSY-seesaw parameters, and we then focus on the particular sensitivity to $\theta_{13}$, which we find specially interesting on the light of its potential future measurement. We

\footnotetext{
${ }^{17}$ In case of left handed mixing, the mixing angle $\theta_{L}$ and the mass difference $(\Delta m)_{L}$ are also used to describe the sneutrino sector.
} 


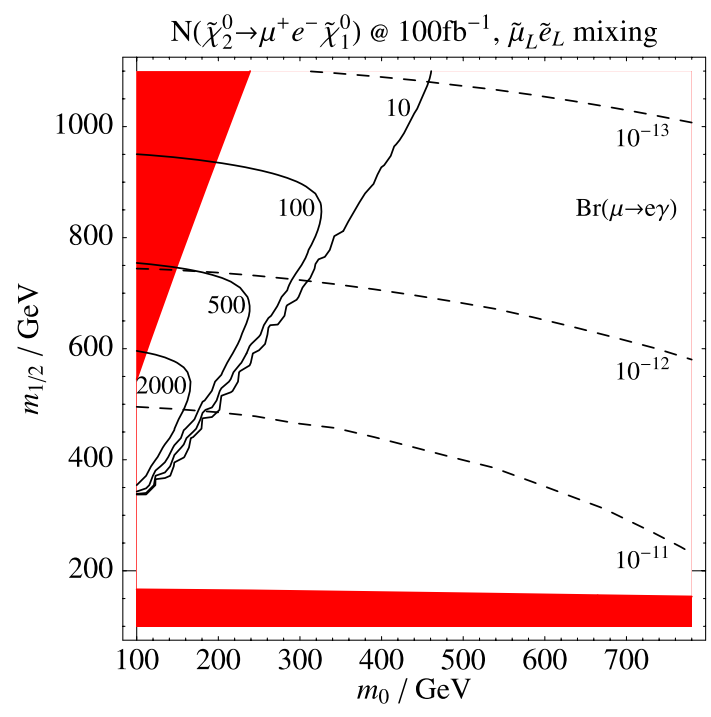

Fig. 20 Contours of the events per year $N\left(\tilde{\chi}_{2}^{0} \rightarrow \mu^{+} e^{-} \tilde{\chi}_{1}^{0}\right)$ at the LHC with an integrated luminosity of $100 \mathrm{fb}^{-1}$ in the $m_{0}-m_{1 / 2}$ plane (solid lines). The remaining mSUGRA parameters are: $A_{0}=$ $-100 \mathrm{GeV}, \tan \beta=10, \operatorname{sign}(\mu)=+$. The left and right panels are

further study the constraints from the requirement of successfully producing the Baryon Asymmetry of the Universe via thermal leptogenesis, which is another appealing feature of the SUSY-seesaw scenario. We conclude with the impact that a potential measurement of the leptonic mixing angle $\theta_{13}$ can have on LFV physics.

Regarding the technical aspects of the computation of the branching ratios, the most relevant points are (for details, see $[649,686]$ :

- It is a full one loop computation of BRs, i.e., we include all contributing one loop diagrams with the SUSY particles flowing in the loops. For the case of $l_{j} \rightarrow l_{i} \gamma$ the analytical formulas can be found in [178, 649]. For the case $l_{j} \rightarrow 3 l_{i}$ the complete set of diagrams (including photon-penguin, Z-penguin, Higgs-penguin and box diagrams) and formulae are given in [649].

- The computation is performed in the physical basis for all SUSY particles entering in the loops. In other words, we do not use the mass insertion approximation (MIA).

- The running of the CMSSM-seesaw parameters from the universal scale $M_{X}$ down to the electroweak scale is performed by numerically solving the full one loop renormalization group equations (RGEs) (including the extended neutrino sector) and by means of the public Fortran Code SPheno2.2.2. [699]. More concretely, we do not use the Leading Log Approximation (LLog).

- The light neutrino sector parameters that are used in $m_{D}=\sqrt{m_{N}^{\text {diag }}} R \sqrt{m_{v}^{\text {diag }}} U_{\text {MNS }}^{\dagger}$ are those evaluated at the seesaw scale $m_{R}$. That is, we start with their low energy

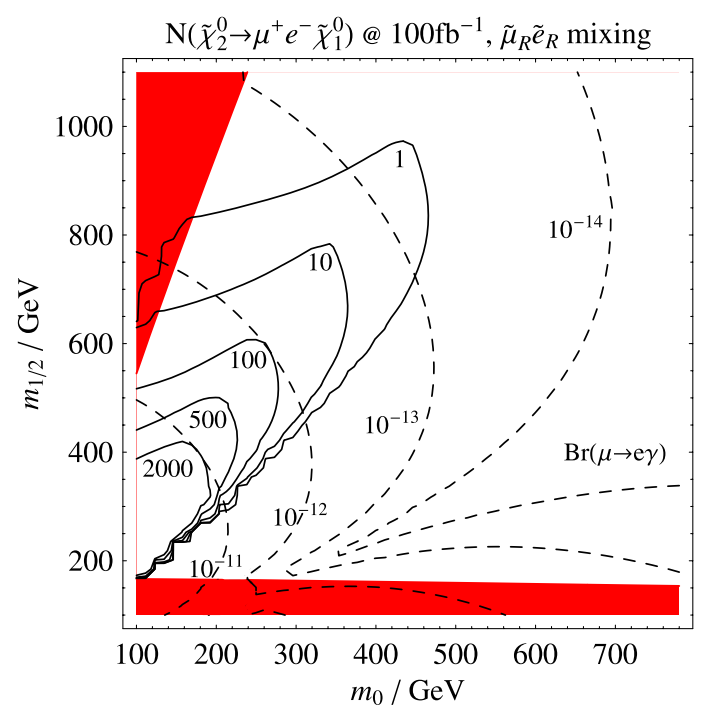

for maximal $\tilde{e}_{L} \tilde{\mu}_{L}$ and $\tilde{e}_{R} \tilde{\mu}_{R}$ mixing $(\theta=\pi / 4, \Delta m=1 \mathrm{GeV})$, respectively. For comparison, $B(\mu \rightarrow e \gamma)$ is shown by dashed lines. The shaded (red) areas are forbidden by mass bounds from various experimental sparticle searches

values (taken from data) and then apply the RGEs to run them up to $m_{R}$.

- We have added to the SPheno code extra subroutines that compute the LFV rates for all the $l_{j} \rightarrow l_{i} \gamma$ and $l_{j} \rightarrow 3 l_{i}$ channels. We have also included additional subroutines to: Implement the requirement of successful baryogenesis (which we define as having $n_{B} / n_{\gamma} \in\left[10^{-10}, 10^{-9}\right]$ ) via thermal leptogenesis in the presence of upper bounds on the reheat temperature; Implement the requirement of compatibility with present bounds on lepton electric dipole moments: $\mathrm{EDM}_{e \mu \tau} \lesssim\left(6.9 \times 10^{-28}, 3.7 \times 10^{-19}\right.$, $\left.4.5 \times 10^{-17}\right) e \mathrm{~cm}$.

In what follows we present the main results for the case of hierarchical heavy neutrinos. We also include a comparison with present bounds on LFV rates [180, 182, 191, 644, 700] and their future sensitivities [694, 701-706]. For hierarchical heavy neutrinos, the BRs are mostly sensitive to the heaviest mass $m_{N_{3}}, \tan \beta, \theta_{1}$ and $\theta_{2}$ (using the $R$ parameterization of [232]). The other input seesaw parameters $m_{N_{1}}, m_{N_{2}}$ and $\theta_{3}$ play a secondary role since the BRs do not strongly depend on them. The dependence on $m_{N_{1}}$ and $\theta_{3}$ appears only indirectly, once the requirement of a successful generation of baryon asymmetry of the universe (BAU) is imposed. We shall comment more on this later.

We display in Fig. 21 the predictions for $B(\mu \rightarrow e \gamma)$ and $B(\tau \rightarrow \mu \gamma)$ as a function of $m_{N_{3}}$, for a specific choice of the other input parameters. This figure clearly shows the strong sensitivity of the BRs to $m_{N_{3}}$. In fact, the BRs vary by as much as six orders of magnitude in the explored range of $5 \times 10^{11} \leq m_{N_{3}} \leq 5 \times 10^{14} \mathrm{GeV}$. Notice also that for 


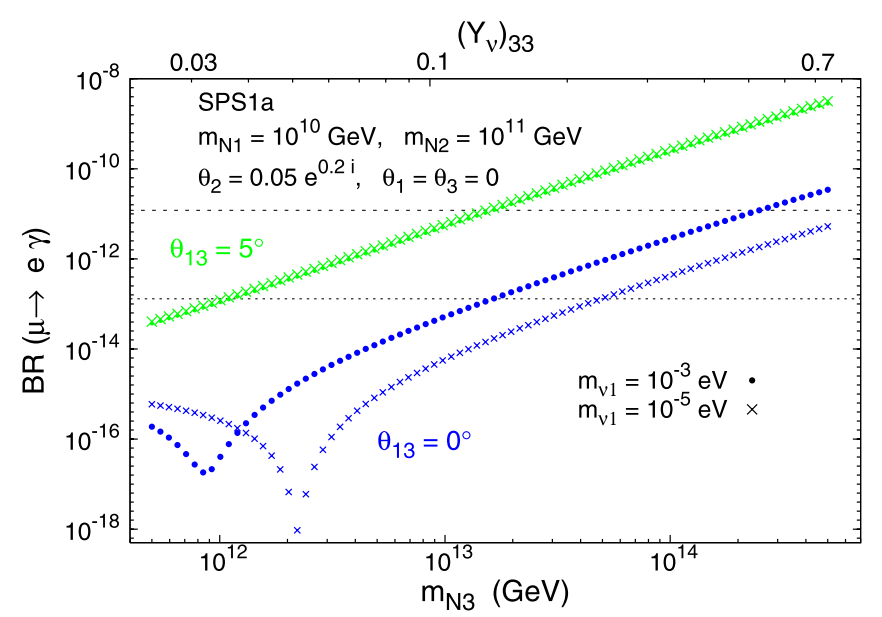

Fig. 21 On the left, $B(\mu \rightarrow e \gamma)$ as a function of $m_{N_{3}}$ for SPS 1a, with $m_{\nu_{1}}=10^{-5} \mathrm{eV}$ and $m_{\nu_{1}}=10^{-3} \mathrm{eV}$ (times, dots, respectively), and $\theta_{13}=0^{\circ}, 5^{\circ}$ (blue/darker, green/lighter lines). Baryogenesis is enabled by the choice $\theta_{2}=0.05 e^{0.2 i}\left(\theta_{1}=\theta_{3}=0\right)$. On the upper horizontal axis we display the associated value of $\left(Y_{v}\right)_{33}$. A dashed (dotted) horizontal line denotes the present experimental bound (future sensi-

the largest values of $m_{N_{3}}$ considered, the predicted rates for $\mu \rightarrow e \gamma$ enter into the present experimental reach and only into the future experimental sensitivity for $\tau \rightarrow \mu \gamma$. It is also worth mentioning that by comparing our full results with the LLog predictions, we find that the LLog approximation dramatically fails in some cases. In particular, for the SPS5 point, the LLog predictions overestimate the BRs by about four orders of magnitude. For the other points SPS4, SPS1a,b and SPS2 the LLog estimate is very similar to the full result, whereas for SPS3 it underestimates the full computation by a factor of three. In general, the divergence of the LLog and the full computation occurs for low $M_{0}$ and large $M_{1 / 2}[238,273]$ and/or large $A_{0}$ values [686]. The failure of the LLog is more dramatic for SUSY scenarios with large $A_{0}$. Figure 21 also shows that while in some cases (as for instance SPS1a) the behavior of the BR with $m_{N_{3}}$ does follow the expected LLog approximation $\left(\mathrm{BR} \sim\left(m_{N_{3}} \log m_{N_{3}}\right)^{2}\right)$, there are other scenarios where this is not the case. A good example is SPS5. It is also worth commenting on the deep minima of $B(\mu \rightarrow e \gamma)$ appearing in Fig. 21 for the lines associated with $\theta_{13}=0^{\circ}$. These minima are induced by the effect of the running of $\theta_{13}$, shifting it from zero to a negative value (or equivalently $\theta_{13}>0$ and $\delta=\pi$ ). In the LLog approximation, they can be understood as a cancellation occurring in the relevant quantity $Y_{v}^{\dagger} L Y_{v}$, with $L_{i j}=\log \left(M_{X} / m_{N_{i}}\right) \delta_{i j}$. Most explicitly, the cancellation occurs between the terms proportional to $m_{N_{3}} L_{33}$ and $m_{N_{2}} L_{22}$ in the limit $\theta_{13}\left(m_{R}\right) \rightarrow 0^{-}$(with $\theta_{1}=\theta_{3}=0$ ). The depth of these minima is larger for smaller $m_{v_{1}}$, as is visible in Fig. 21.

Regarding the $\tan \beta$ dependence of the BRs we obtain that, similar to what was found for the degenerate case, the

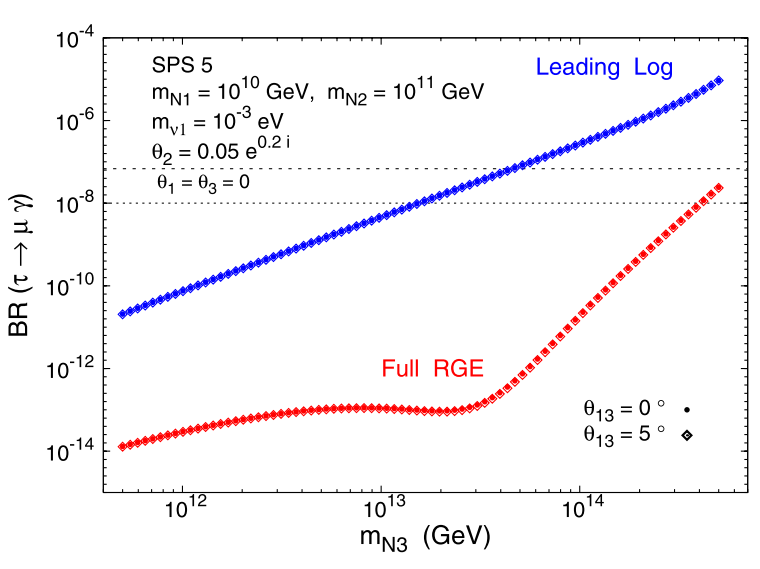

tivity). On the right, $B(\tau \rightarrow \mu \gamma)$ as a function of $m_{N_{3}}$ for SPS5, with $m_{v_{1}}=10^{-3} \mathrm{eV}$ and $\theta_{2}=0.05 e^{0.2 i}\left(\theta_{1}=\theta_{3}=0^{\circ}\right)$. The predictions for $\theta_{13}=0^{\circ}, 5^{\circ}$ are superimposed one on the top of the other. The upper curve is obtained using the LLog approximation and the lower one is the full RGE prediction. The dashed (dotted) horizontal line denotes the present experimental bound (future sensitivity)

BR grow as $\tan ^{2} \beta$. The hierarchy of the BR predictions for the several SPS points is dictated by the corresponding $\tan \beta$ value, with a secondary role being played by the given SUSY spectra. We find again the following generic hierarchy: $B_{\text {SPS } 4}>B_{\text {SPS1b }} \gtrsim B_{\text {SPS1a }}>B_{\text {SPS3 }} \gtrsim B_{\text {SPS2 }}>B_{\text {SPS5 }}$.

In what concerns to the $\theta_{i}$ dependence of the BRs, we have found that they are mostly sensitive to $\theta_{1}$ and $\theta_{2}$. The BRs are nearly constant with $\theta_{3}$. As has been shown in [649], the predictions for $B(\mu \rightarrow e \gamma), B(\mu \rightarrow 3 e), B(\tau \rightarrow \mu \gamma)$ and $B(\tau \rightarrow e \gamma)$ are above their corresponding experimental bound for specific values of $\theta_{1}$. Particularly, the LFV muon decay rates are well above their present experimental bounds for most of the $\theta_{1}$ explored values. Notice also for SPS4 that the predicted $B(\tau \rightarrow \mu \gamma)$ rates are very close to the present experimental reach even at $\theta_{1}=0$ (that is, $R=1$ ). We have also explored the dependence with $\theta_{2}$ and found similar results (not shown here), with the appearance of pronounced dips at particular real values of $\theta_{2}$ with the $B(\mu \rightarrow e \gamma), B(\mu \rightarrow 3 e)$ and $B(\tau \rightarrow \mu \gamma)$ predictions being above the experimental bounds for some $\theta_{2}$ values.

We next address the sensitivity of the LFV BRs to $\theta_{13}$. We first present the results for the simplest $R=1$ case and then discuss how this sensitivity changes when moving from this case towards the more general case of complex $R$, taking into account additional constraints from the requirement of a successful BAU.

For $R=1$, the predictions of the BRs as functions of $\theta_{13}$ in the experimentally allowed range of $\theta_{13}, 0^{\circ} \leq \theta_{13} \leq 10^{\circ}$ are illustrated in Fig. 22. In this figure we also include the present and future experimental sensitivities for all channels. We clearly see that the BRs of $\mu \rightarrow e \gamma, \mu \rightarrow 3 e, \tau \rightarrow e \gamma$ 

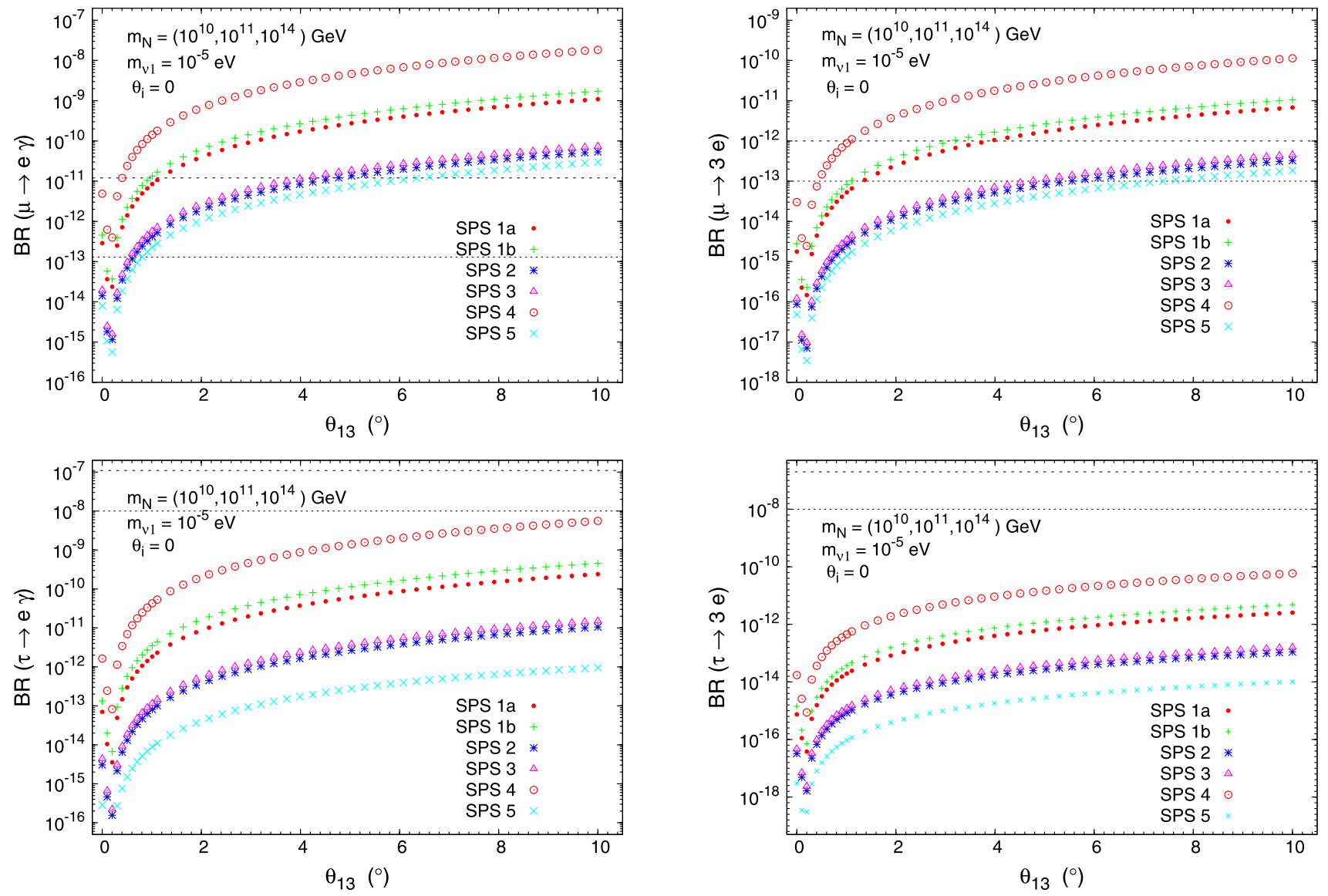

Fig. $22 B(\mu \rightarrow e \gamma)$ and $B(\mu \rightarrow 3 e)$ as a function of $\theta_{13}$ (in degrees), for SPS 1a (dots), 1b (crosses), 2 (asterisks), 3 (triangles), 4 (circles) and 5 (times). A dashed (dotted) horizontal line denotes the present experimental bound (future sensitivity)

and $\tau \rightarrow 3 e$ are extremely sensitive to $\theta_{13}$, with their predicted rates varying many orders of magnitude along the explored $\theta_{13}$ interval. In the case of $\mu \rightarrow e \gamma$ this strong sensitivity was previously pointed out in Ref. [707]. The other LFV channels, $\tau \rightarrow \mu \gamma$ and $\tau \rightarrow 3 \mu$ (not displayed here), are nearly insensitive to this parameter. The most important conclusion from Fig. 22 is that, for this choice of parameters, the predicted BRs for both muon decay channels, $\mu \rightarrow e \gamma$ and $\mu \rightarrow 3 e$, are clearly within the present experimental reach for several of the studied SPS points. The most stringent channel is manifestly $\mu \rightarrow e \gamma$ where the predicted BRs for all the SPS points are clearly above the present experimental bound for $\theta_{13} \gtrsim 5^{\circ}$. With the expected improvement in the experimental sensitivity to this channel, this would happen for $\theta_{13} \gtrsim 1^{\circ}$.

In addition to the small neutrino mass generation, the seesaw mechanism offers the interesting possibility of baryogenesis via leptogenesis [290]. Thermal leptogenesis is an attractive and minimal mechanism to produce a successful BAU with rates which are compatible with present data, $n_{\mathrm{B}} / n_{\gamma} \approx(6.10 \pm 0.21) \times 10^{-10}$ [327]. In the supersymmetric version of the seesaw mechanism, it can be success- fully implemented if provided that the following conditions can be satisfied. Firstly, Big Bang Nucleosynthesis gravitino problems have to be avoided, which is possible, for instance, for sufficiently heavy gravitinos. Since we consider the gravitino mass as a free parameter, this condition can be easily achieved. In any case, further bounds on the reheat temperature $T_{\mathrm{RH}}$ still arise from decays of gravitinos into lightest supersymmetric particles (LSPs). In the case of heavy gravitinos and neutralino LSPs masses into the range $100-150 \mathrm{GeV}$ (which is the case of the present work), one obtains $T_{\mathrm{RH}} \lesssim 2 \times 10^{10} \mathrm{GeV}$. In the presence of these constraints on $T_{\mathrm{RH}}$, the favored region by thermal leptogenesis corresponds to small (but non-vanishing) complex $R$-matrix angles $\theta_{i}$. For vanishing $U_{\text {MNS }} \mathrm{CP}$ phases the constraints on $R$ are basically $\left|\theta_{2}\right|,\left|\theta_{3}\right| \lesssim 1 \operatorname{rad}(\bmod \pi)$. Thermal leptogenesis also constrains $m_{N_{1}}$ to be roughly in the range $\left[10^{9} \mathrm{GeV}, 10 \times T_{\mathrm{RH}}\right]$ (see also [309, 311]). In the present work we have explicitly calculated the produced BAU in the presence of upper bounds on the reheat temperature $T_{\mathrm{RH}}$. We have furthermore set as "favored BAU values" those that are within the interval $\left[10^{-10}, 10^{-9}\right]$, which contains the WMAP value, and choose the value of $m_{N_{1}}=10^{10} \mathrm{GeV}$ 
in some of our plots. Similar studies of the constraints from leptogenesis on LFV rates have been done in [239].

Concerning the EDMs, which are clearly non-vanishing in the presence of complex $\theta_{i}$, we have checked that all the predicted values for the electron, muon and tau EDMs are well below the experimental bounds. In the following we therefore focus on complex but small $\theta_{2}$ values, leading to favorable BAU, and study its effects on the sensitivity to $\theta_{13}$. Similar results are obtained for $\theta_{3}$, but for shortness are not shown here.

Figure 23 shows the dependence of the most sensitive BR to $\theta_{13}, B(\mu \rightarrow e \gamma)$, on $\left|\theta_{2}\right|$. We consider two particular values of $\theta_{13}, \theta_{13}=0^{\circ}, 5^{\circ}$ and choose SPS 1a. Motivated from the thermal leptogenesis favored $\theta_{2}$-regions [686], we take $0 \lesssim\left|\theta_{2}\right| \lesssim \pi / 4$, with $\arg \theta_{2}=\{\pi / 8, \pi / 4,3 \pi / 8\}$. We display the numerical results, considering $m_{\nu_{1}}=10^{-5} \mathrm{eV}$ and $m_{v_{1}}=10^{-3} \mathrm{eV}$, while for the heavy neutrino masses we take $m_{N}=\left(10^{10}, 10^{11}, 10^{14}\right) \mathrm{GeV}$. There are several important conclusions to be drawn from Fig. 23. Let us first discuss the case $m_{v_{1}}=10^{-5} \mathrm{eV}$. We note that one can obtain a baryon asymmetry in the range $10^{-10}$ to $10^{-9}$ for a considerable region of the analyzed $\left|\theta_{2}\right|$ range. Notice also that there is a clear separation between the predictions of $\theta_{13}=0^{\circ}$ and $\theta_{13}=5^{\circ}$, with the latter well above the present experimental bound. This would imply an experimental impact of $\theta_{13}$, in the sense that the BR predictions become potentially detectable for this non-vanishing $\theta_{13}$ value. With the planned MEG sensitivity [701], both cases would be within experimental reach. However, this statement is strongly dependent on the assumed parameters, in particular $m_{v_{1}}$. For instance, a larger value of $m_{v_{1}}=10^{-3} \mathrm{eV}$, illustrated on the right panel of Fig. 23, leads to a very distinct situation regarding the sensitivity to $\theta_{13}$. While for smaller values of $\left|\theta_{2}\right|$ the branching ratio displays a clear sensitivity to having $\theta_{13}$ equal or different from zero (a separation larger than

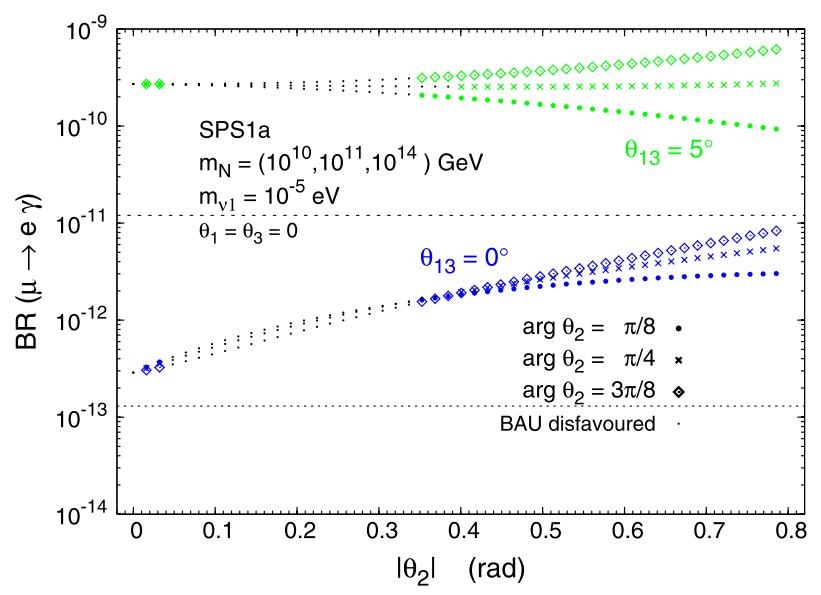

Fig. $23 B(\mu \rightarrow e \gamma)$ as a function of $\left|\theta_{2}\right|$, for $\arg \theta_{2}=$ $\{\pi / 8, \pi / 4,3 \pi / 8\}$ (dots, times, diamonds, respectively) and $\theta_{13}=0^{\circ}$, $5^{\circ}$ (blue/darker, green/lighter lines). We take $m_{\nu_{1}}=10^{-5}\left(10^{-3}\right) \mathrm{eV}$, two orders of magnitude for $\left|\theta_{2}\right| \lesssim 0.05$ ), the effect of $\theta_{13}$ is diluted for increasing values of $\left|\theta_{2}\right|$. For $\left|\theta_{2}\right| \gtrsim 0.3$ the $B(\mu \rightarrow e \gamma)$ associated with $\theta_{13}=5^{\circ}$ can be even smaller than for $\theta_{13}=0^{\circ}$. This implies that in this case, a potential measurement of $B(\mu \rightarrow e \gamma)$ would not be sensitive to $\theta_{13}$. Whether or not a SPS 1a scenario would be disfavored by current experimental data on $B(\mu \rightarrow e \gamma)$ requires a careful weighting of several aspects. Even though Fig. 23 suggests that for this particular choice of parameters only very small values of $\theta_{2}$ and $\theta_{13}$ would be in agreement with current experimental data, a distinct choice of $m_{N_{3}}$ (e.g. $m_{N_{3}}=10^{13} \mathrm{GeV}$ ) would lead to a rescaling of the estimated BRs by a factor of approximately $10^{-2}$. Although we do not display the associated plots here, in the latter case nearly the entire $\left|\theta_{2}\right|$ range would be in agreement with experimental data (in fact the points which are below the present MEGA bound on Fig. 23 would then lie below the projected MEG sensitivity). Regarding the other SPS points, which are not shown here, we find BRs for SPS 1b comparable to those of SPS 1a. Smaller ratios are associated with SPS 2, 3 and 5, while larger (more than one order of magnitude) BRs occur for SPS 4.

Let us now address the question of whether a joint measurement of the BRs and $\theta_{13}$ can shed some light on experimentally unreachable parameters, like $m_{N_{3}}$. The expected improvement in the experimental sensitivity to the LFV ratios supports the possibility that a BR could be measured in the future, thus providing the first experimental evidence for new physics, even before its discovery at the LHC. The prospects are especially encouraging regarding $\mu \rightarrow e \gamma$, where the experimental sensitivity will improve by at least two orders of magnitude. Moreover, and given the impressive effort on experimental neutrino physics, a measurement of $\theta_{13}$ will likely also occur in the future [708-717]. Given

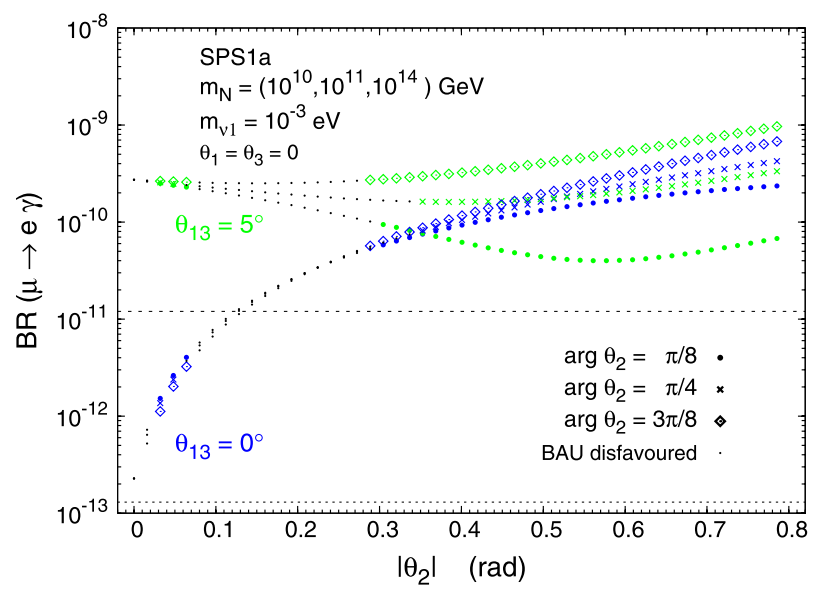

on the left (right) panel. In all cases black dots represent points associated with a disfavored BAU scenario and a dashed (dotted) horizontal line denotes the present experimental bound (future sensitivity) 
that, as previously emphasized, $\mu \rightarrow e \gamma$ is very sensitive to $\theta_{13}$, whereas this is not the case for $B(\tau \rightarrow \mu \gamma)$, and that both BRs display the same approximate behavior with $m_{N_{3}}$ and $\tan \beta$, we now propose to study the correlation between these two observables. This optimizes the impact of a $\theta_{13}$ measurement, since it allows us to minimize the uncertainty introduced from not knowing $\tan \beta$ and $m_{N_{3}}$, and at the same time offers a better illustration of the uncertainty associated with the $R$-matrix angles. In this case, the correlation of the BRs with respect to $m_{N_{3}}$ means that, for a fixed set of parameters, varying $m_{N_{3}}$ implies that the predicted point $(B(\tau \rightarrow \mu \gamma), B(\mu \rightarrow e \gamma))$ moves along a line with approximately constant slope in the $B(\tau \rightarrow \mu \gamma)-$ $B(\mu \rightarrow e \gamma)$ plane. On the other hand, varying $\theta_{13}$ leads to a displacement of the point along the vertical axis.

In Fig. 24, we illustrate this correlation for SPS 1a, choosing distinct values of the heaviest neutrino mass, and we scan over the BAU-enabling $R$-matrix angles (setting $\theta_{3}$ to zero) as

$0 \lesssim\left|\theta_{1}\right| \lesssim \pi / 4, \quad-\pi / 4 \lesssim \arg \theta_{1} \lesssim \pi / 4$

$0 \lesssim\left|\theta_{2}\right| \lesssim \pi / 4, \quad 0 \lesssim \arg \theta_{2} \lesssim \pi / 4$,

$m_{N_{3}}=10^{12}, 10^{13}, 10^{14} \mathrm{GeV}$.

We consider the following values, $\theta_{13}=1^{\circ}, 3^{\circ}, 5^{\circ}$ and $10^{\circ}$, and only include in the plot the BR predictions which allow for a favorable BAU. Other SPS points have also been considered but they are not shown here for brevity (see [686]). We clearly observe in Fig. 24 that for a fixed value of $m_{N_{3}}$, and for a given value of $\theta_{13}$, the dispersion arising from a $\theta_{1}$ and $\theta_{2}$ variation produces a small area rather than a point in the $B(\tau \rightarrow \mu \gamma-B(\mu \rightarrow e \gamma)$ plane. The dispersion along the $B(\tau \rightarrow \mu \gamma)$ axis is of approximately one order of magnitude for all $\theta_{13}$. In contrast, the dispersion along the $B(\mu \rightarrow e \gamma)$ axis increases with decreasing $\theta_{13}$, ranging from an order of magnitude for $\theta_{13}=10^{\circ}$, to over three orders of magnitude for the case of small $\theta_{13}\left(1^{\circ}\right)$. From Fig. 24 we can also infer that other choices of $m_{N_{3}}$ (for $\left.\theta_{13} \in\left[1^{\circ}, 10^{\circ}\right]\right)$ would lead to $\mathrm{BR}$ predictions which would roughly lie within the diagonal lines depicted in the plot. Comparing these predictions for the shaded areas along the expected diagonal "corridor", with the allowed experimental region, allows us to conclude about the impact of a $\theta_{13}$ measurement on the allowed/excluded $m_{N_{3}}$ values. The most important conclusion from Fig. 24 is that for SPS 1a, and for the parameter space defined in (5.39), an hypothetical $\theta_{13}$ measurement larger than $1^{\circ}$, together with the present experimental bound on the $B(\mu \rightarrow e \gamma)$, will have the impact of excluding values of $m_{N_{3}} \gtrsim 10^{14} \mathrm{GeV}$. Moreover, with the planned MEG sensitivity, the same $\theta_{13}$ measurement can further constrain $m_{N_{3}} \lesssim 3 \times 10^{12} \mathrm{GeV}$. The impact of any other $\theta_{13}$ measurement can be analogously extracted from Fig. 24.

As a final comment let us add that, remarkably, within a particular SUSY scenario and scanning over specific $\theta_{1}$ and $\theta_{2}$ BAU-enabling ranges for various values of $\theta_{13}$, the comparison of the theoretical predictions for $B(\mu \rightarrow e \gamma)$ and $B(\tau \rightarrow \mu \gamma)$ with the present experimental bounds allows us to set $\theta_{13}$-dependent upper bounds on $m_{N_{3}}$. Together with the indirect lower bound arising from leptogenesis considerations, this clearly provides interesting hints on the value of the seesaw parameter $m_{N_{3}}$. With the planned future sensitivities, these bounds would further improve by approximately one order of magnitude. Ultimately, a joint measurement of the LFV branching ratios, $\theta_{13}$ and the sparticle spectrum would be a powerful tool for shedding some light on otherwise unreachable SUSY seesaw parameters. It is clear from all this study that the interplay between LFV processes and future improvement in neutrino data is challenging for the searches of new physics.
Fig. 24 (Color online) Correlation between $B(\mu \rightarrow e \gamma)$ and $B(\tau \rightarrow \mu \gamma)$ as a function of $m_{N_{3}}$, for SPS 1a. The areas displayed represent the scan over $\theta_{i}$ as given in (5.39). From bottom to top, the colored regions correspond to $\theta_{13}=1^{\circ}, 3^{\circ}, 5^{\circ}$ and $10^{\circ}$ (red, green, blue and pink, respectively). Horizontal and vertical dashed (dotted) lines denote the experimental bounds (future sensitivities)

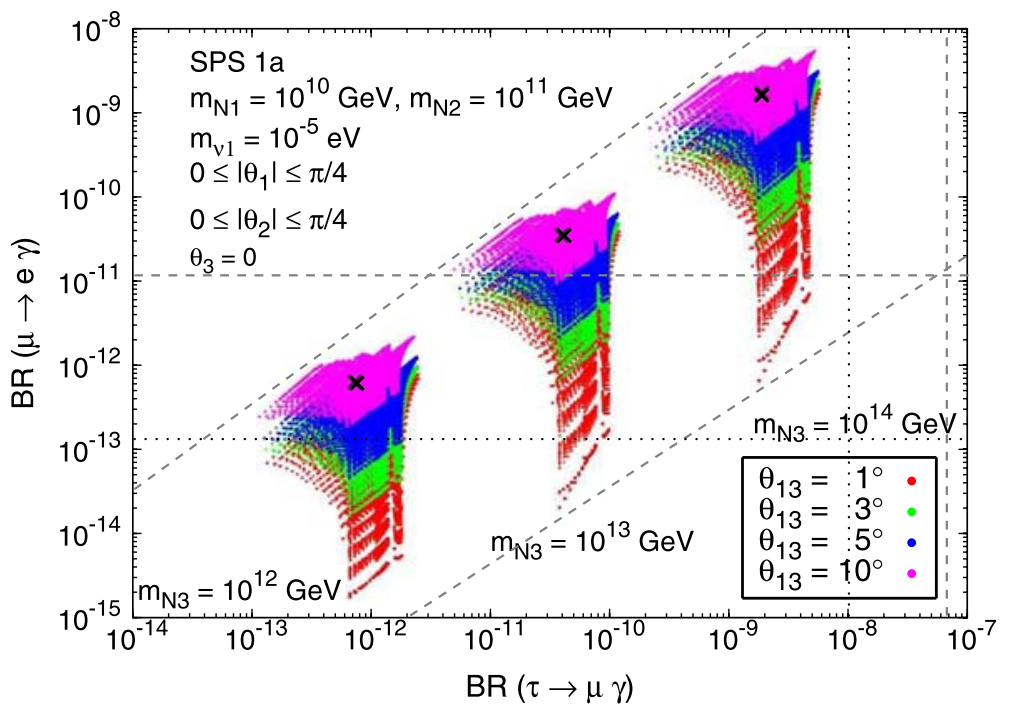




\subsubsection{LFV in the CMSSM with constrained sequential dominance}

Sequential Dominance (SD) [140, 141, 143] represents classes of neutrino models where large lepton mixing angles and small hierarchical neutrino masses can be readily explained within the seesaw mechanism. To understand how Sequential Dominance works, we begin by writing the right handed neutrino Majorana mass matrix $M_{R R}$ in a diagonal basis as $M_{R R}=\operatorname{diag}\left(M_{A}, M_{B}, M_{C}\right)$. We furthermore write the neutrino (Dirac) Yukawa matrix $\lambda_{v}$ in terms of $(1,3)$ column vectors $A_{i}, B_{i}, C_{i}$ as $Y_{v}=(A, B, C)$ using left-right convention. The term for the light neutrino masses in the effective Lagrangian (after electroweak symmetry breaking), resulting from integrating out the massive right handed neutrinos, is

$$
\begin{aligned}
\mathcal{L}_{\text {eff }}^{v}= & \frac{\left(v_{i}^{T} A_{i}\right)\left(A_{j}^{T} v_{j}\right)}{M_{A}}+\frac{\left(v_{i}^{T} B_{i}\right)\left(B_{j}^{T} v_{j}\right)}{M_{B}} \\
& +\frac{\left(v_{i}^{T} C_{i}\right)\left(C_{j}^{T} v_{j}\right)}{M_{C}}
\end{aligned}
$$

where $v_{i}(i=1,2,3)$ are the left handed neutrino fields. Sequential dominance then corresponds to the third term being negligible, the second term subdominant and the first term dominant:

$\frac{A_{i} A_{j}}{M_{A}} \gg \frac{B_{i} B_{j}}{M_{B}} \gg \frac{C_{i} C_{j}}{M_{C}}$.

In addition, we shall shortly see that small $\theta_{13}$ and almost maximal $\theta_{23}$ require that

$\left|A_{1}\right| \ll\left|A_{2}\right| \approx\left|A_{2}\right|$.

Without loss of generality, then, we shall label the dominant right handed neutrino and Yukawa couplings as $A$, the subdominant ones as $B$, and the almost decoupled (subsubdominant) ones as $C$. Note that the mass ordering of right handed neutrinos is not yet specified. Again without loss of generality we shall order the right handed neutrino masses as $M_{1}<M_{2}<M_{3}$, and subsequently identify $M_{A}, M_{B}, M_{C}$ with $M_{1}, M_{2}, M_{3}$ in all possible ways. LFV in some of these classes of SD models has been analyzed in [718]. Tri-bimaximal neutrino mixing corresponds to the choice for example [719], sometimes referred to as constrained sequential dominance (CSD):

$$
Y_{\nu}=\left(\begin{array}{ccc}
0 & b e^{i \beta_{2}} & c_{1} \\
-a e^{i \beta_{3}} & b e^{i \beta_{2}} & c_{2} \\
a e^{i \beta_{3}} & b e^{i \beta_{2}} & c_{3}
\end{array}\right)
$$

When dealing with LFV it is convenient to work in the basis where the charged lepton mass matrix is diagonal. Let us now discuss the consequences of charged lepton corrections with a CKM-like structure, for the neutrino Yukawa matrix with CSD. By CKM-like structure we mean that $V_{e_{\mathrm{L}}}$ is dominated by a $1-2$ mixing $\theta$, i.e. that its elements $\left(V_{e_{\mathrm{L}}}\right)_{13},\left(V_{e_{\mathrm{L}}}\right)_{23},\left(V_{e_{\mathrm{L}}}\right)_{31}$ and $\left(V_{e_{\mathrm{L}}}\right)_{32}$ are very small compared to $\left(V_{e_{\mathrm{L}}}\right)_{i j}(i, j=1,2)$. After re-diagonalizing the charged lepton mass matrix, $Y_{v}$ in (5.43) becomes transformed as $Y_{v} \rightarrow V_{e_{L}} Y_{\nu}$. In the diagonal charged lepton mass basis the neutrino Yukawa matrix therefore becomes

$$
Y_{\nu}=\left(\begin{array}{ccc}
a s_{\theta} e^{-i \lambda} e^{i \beta_{3}} & b\left(c_{\theta}-s_{\theta} e^{-i \lambda}\right) e^{i \beta_{2}} & \left(c_{1} c_{\theta}-c_{2} s_{\theta} e^{-i \lambda}\right) \\
-a c_{\theta} e^{i \beta_{3}} & b\left(c_{\theta}+s_{\theta} e^{i \lambda}\right) e^{i \beta_{2}} & \left(c_{1} s_{\theta} e^{i \lambda}+c_{2} c_{\theta}\right) \\
a e^{i \beta_{3}} & b e^{i \beta_{2}} & c_{3}
\end{array}\right) .
$$

After ordering $M_{A}, M_{B}, M_{C}$ according to their size, there are six possible forms of $Y_{v}$ obtained from permuting the columns, with the convention always being that the dominant one is labeled by $A$, and so on. In particular the third column of the neutrino Yukawa matrix could be $A, B$ or $C$ depending on which of $M_{A}, M_{B}$ or $M_{C}$ is the heaviest. If the heaviest right handed neutrino mass is $M_{A}$ then the third column of the neutrino Yukawa matrix will consist of the (re-ordered) first column of (5.44) and assuming $Y_{33}^{v} \sim 1$ we conclude that all LFV processes will be determined approximately by the first column of (5.44). Similarly if the heaviest right handed neutrino mass is $M_{B}$ then we conclude that all LFV processes will be determined approximately by the second column of (5.44). Note that in both cases the ratios of branching ratios are independent of the unknown Yukawa couplings which cancel, and only depend on the charged lepton angle $\theta$, which in the case of tri-bimaximal neutrino mixing is related to the physical reactor angle by $\theta_{13}=\theta / \sqrt{2}[719,720]$. Also note that $\lambda=\delta-\pi$ where $\delta$ is the standard PDG CP-violating oscillation phase. The results for these two cases are shown in Fig. 25 [721]. The third case $M_{3}=M_{C}$ is less predictive.

\subsubsection{Decoupling of one heavy neutrino and cosmological implications}

The supersymmetric seesaw model involves many free parameters. In order to correlate the model predictions for LFV processes one has to resort to some supplementary hypotheses. Here we discuss the consequences of the assumption that one of the heavy singlet neutrinos (not necessarily the heaviest one) decouples from the see-saw mechanism [238].

If the light neutrino masses are hierarchical, in which case the effects of the renormalization group (RG) running [722] of $\kappa$ are negligible, at least 3 arguments support this assumption. The first one is the naturalness of the see-saw 


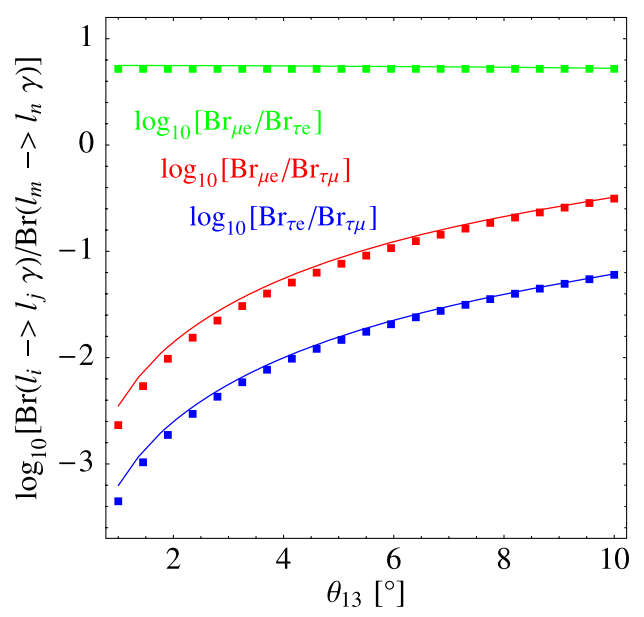

Fig. 25 Ratios of branching ratios of LFV processes $\ell_{i} \rightarrow \ell_{j} \gamma$ in CSD for $M_{3}=M_{A}$ (left panel) and $M_{3}=M_{B}$ (right panel) with right handed neutrino masses $M_{1}=10^{8} \mathrm{GeV}, M_{2}=5 \times 10^{8} \mathrm{GeV}$ and $M_{3}=10^{14} \mathrm{GeV}$. The solid lines show the (naive) prediction, from the MI and LLog approximation and with RG running effects neglected, while the dots show the explicit numerical computation (using SPheno2.2.2. [699] extended by software packages for LFV BRs and

mechanism. Large mixing angles are not generic for hierarchical light neutrino masses (for a review, see [723]). They are natural only for special patterns of the matrix $\kappa$. One is a large hierarchy between one and the remaining two terms in the sum in $(3.41)[38,140,233,724]$. This is what we call decoupling (one term hierarchically smaller) or dominance (hierarchically larger). Seesaw with only two heavy singlet neutrinos $[234,235]$ is the limiting case of the decoupling of $N_{3}$ with $M_{3} \rightarrow \infty$ and $Y_{v}^{3 A} \rightarrow 0$. The immediate consequence of decoupling is $m_{\nu_{1}} \ll m_{\nu_{2}}(\kappa$ has rank 2 if there are only 2 terms in the sum in (3.41)). Similarly, for dominance one has $m_{v_{2}} \ll m_{v_{3}}$.

Secondly, decoupling of the lightest singlet neutrino alleviates the gravitino problem of leptogenesis which in the see-saw models of neutrino masses appears to be the most natural mechanism for producing the observed baryon asymmetry of the Universe ${ }^{18}$ (BAU). As the Universe cools down leptonic asymmetries (subsequently converted into baryon asymmetry through sphaleron transitions) $Y_{\alpha} \equiv$ $\left(n_{\alpha}-\bar{n}_{\alpha}\right) / s \neq 0$ (where $n_{\alpha}$ and $\bar{n}_{\alpha}$ are the flavor $\alpha$ lepton and antilepton number densities, respectively and $s$ is the entropy density) are produced in the decays of $N_{1}$. The final magnitudes of $Y_{\alpha}$ are proportional to the decay asymmetries $\varepsilon_{1 \alpha}$, (which in turn are proportional to the heavy neutrino masses) and crucially depend on the processes which wash out the asymmetries generated by the $N_{1}$ decays. The efficiency of these processes depends on the pa-

\footnotetext{
${ }^{18}$ See [290]; for a review of leptogenesis, see [324]; for a discussion of flavor effects in leptogenesis see, e.g. [296]; for recent analyses of the gravitino problem, see, e.g., [725].
}

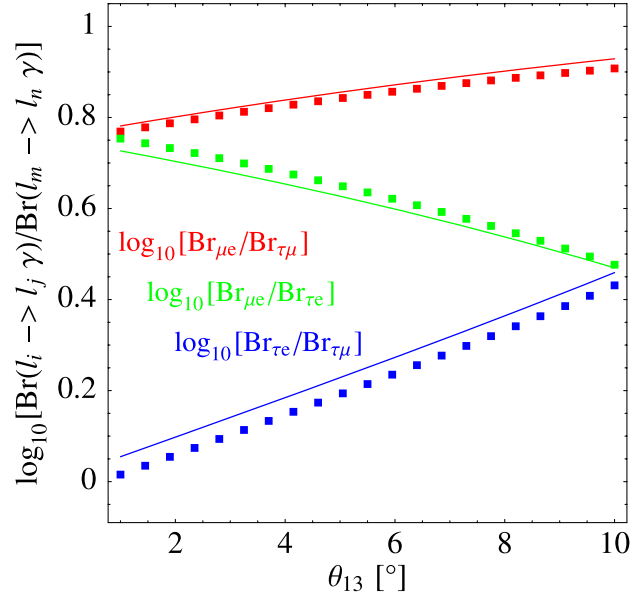

neutrino mass matrix running $[649,686])$ with universal CMSSM parameters chosen as $m_{0}=750 \mathrm{GeV}, m_{1 / 2}=750 \mathrm{GeV}, A_{0}=0 \mathrm{GeV}$, $\tan \beta=10$ and $\operatorname{sign}(\mu)=+1$. While the ratios do not significantly depend on the choice of the SUSY model, since the model-dependence has canceled out, they show a pronounced dependence on $\theta_{13}$ (and $\delta$ ) in the case of $M_{3}=M_{A}\left(\right.$ and $\left.M_{3}=M_{B}\right)$

rameters $\tilde{m}_{1 \alpha}=\sum_{A}\left|R_{1 A} U_{\alpha A}^{*}\right|^{2} m_{v_{A}}$, where $U \equiv U_{\mathrm{PMNS}}$ and it is the smallest (i.e. leptogenesis is most efficient) for $\tilde{m}_{1 \alpha}$ in the meV range (assuming vanishing density of $N_{1}$ after re-heating and strongly hierarchical spectrum of $M_{A}$ ). If it is $N_{1}$ which is decoupled, there are essentially no lower bounds on $\tilde{m}_{1 \alpha}$ and $M_{1}$, hence also the re-heating temperature $T_{\mathrm{RH}}$, already of order $10^{9} \mathrm{GeV}$ are sufficient $[311,726]$ (see, however, e.g., [316]) to reproduce the observed BAU. ${ }^{19}$

Finally, one heavy singlet neutrino $N_{A}$ must be decoupled if its superpartner, $\tilde{N}_{A}$, plays the role of the inflaton field [347]. In such a scenario the (s)neutrino mass $M_{A}$ must be [349] $2 \times 10^{13} \mathrm{GeV}$ and the re-heating temperature following inflaton decay is given by $T_{\mathrm{RH}} \sim \sqrt{\tilde{m}_{A} M_{\mathrm{Pl}}}\left(M_{A} /\langle H\rangle\right)$. Requiring $T_{\mathrm{RH}}<10^{6} \mathrm{GeV}$ (the gravitino problem) then implies $m_{v_{A}} \leq \sum_{\alpha} \tilde{m}_{A \alpha}<10^{-17} \mathrm{eV}$. In this scenario, the leptonic asymmetries must be produced non-thermally in the inflaton decay. Decoupling of $N_{1}$ is favored because if it is $\tilde{N}_{2}$ or $\tilde{N}_{3}$ which is the inflaton the produced asymmetry may be subsequently washed out during the decays of $N_{1}$.

The assumption that $N_{A}$ effectively decouples from the seesaw mechanism or that $N_{A}$ effectively dominates the seesaw mechanism translates into one of the following forms

\footnotetext{
${ }^{19}$ In contrast, for $N_{2}$ or $N_{3}$ decoupled, the washout is much stronger and $M_{1}$ has to be $\gtrsim 10^{10} \mathrm{GeV}$. This requires $T_{\mathrm{RH}}$ leading to a much larger dangerous gravitino production [727]. Lower $T_{\mathrm{RH}}$ is in this case possible only if $N_{1}$ and $N_{2}$ are sufficiently degenerate [728].
} 
of $R$ :

$$
\begin{aligned}
& R_{\mathrm{dec}} \simeq \Pi^{(A)}\left(\begin{array}{ccc}
1 & 0 & 0 \\
0 & z & p \\
0 & \mp p & \pm z
\end{array}\right) \quad \text { or } \\
& R_{\mathrm{dom}} \simeq \Pi^{(A)}\left(\begin{array}{ccc}
\mp p & \pm z & 0 \\
z & p & 0 \\
0 & 0 & 1
\end{array}\right)
\end{aligned}
$$

where $z, p$ are complex numbers satisfying $z^{2}+p^{2}=1$ and $\Pi^{(A)}$ denotes permutation of the rows of $R$. Both conditions can be simultaneously satisfied for $R=\Pi^{(A)} \cdot \mathbf{1}$, known as sequential dominance (for a review, see, e.g. [729]).

In the framework considered, violation of the leptonic flavor is transmitted from the neutrino Yukawa couplings $Y_{v}$ to the slepton mass matrices through the RG corrections. Branching ratios of LFV decays are well described by a single mass-insertion approximation via (5.27) and (5.28). Since decoupling of $N_{1}$ is best motivated we discuss the results for LFV only in this case. ${ }^{20}$

The matrix $R$ has then the first of the patterns displayed in (5.45) with $\Pi^{(1)}=\mathbf{1}$. The discussion simplifies if a technical assumption that $m_{\nu_{3}} M_{2}<m_{v_{2}} M_{3}$ is made. $\left(\tilde{m}_{L}^{2}\right)_{32}$ relevant for $\tau \rightarrow \mu \gamma$ then reads

$$
\begin{aligned}
\left(\tilde{m}_{L}^{2}\right)_{32} \approx & \frac{\kappa m_{\nu_{3}} M_{3} U_{33} U_{23}^{*}}{\langle H\rangle^{2}}\left[\left(|z|^{2}+S|p|^{2}\right)+\rho \frac{U_{22}^{*}}{U_{23}^{*}} x\right. \\
& \left.+\rho \frac{U_{32}}{U_{33}} x^{*}+\rho^{2} \frac{U_{32} U_{22}^{*}}{U_{33} U_{23}^{*}}\left(S|z|^{2}+|p|^{2}\right)\right],
\end{aligned}
$$

where $\rho=\sqrt{m_{v_{2}} / m_{v_{3}}} \sim 0.4, S=M_{2}\left(1+\Delta l_{2} / \Delta t\right) / M_{3} \sim$ $M_{2} / M_{3}$ and $x=S p^{*} z-z^{*} p$. For $\left(\tilde{m}_{L}^{2}\right)_{A 1}$ relevant for $\ell_{A} \rightarrow$ e $\gamma$ we get:

$$
\begin{aligned}
\left(\tilde{m}_{L}^{2}\right)_{A 1} \approx & \frac{\kappa m_{\nu_{3}} M_{3} U_{A 3} U_{12}^{*}}{\langle H\rangle^{2}}\left[\frac{U_{13}^{*}}{U_{12}^{*}}\left(|z|^{2}+S|p|^{2}\right)+\rho x\right. \\
& \left.+\rho^{2} \frac{U_{A 2}}{U_{A 3}}\left(S|z|^{2}+|p|^{2}\right)\right] .
\end{aligned}
$$

Analysis of the expressions (5.46) and (5.47) leads to a number of conclusions [238]. Firstly, the branching ratios of the LFV decays depend (apart from the scales of soft supersymmetry breaking and the value of $\tan \beta$ ) mostly on the

\footnotetext{
${ }^{20}$ Results for $N_{2}$ decoupled are the same as for decoupled $N_{1}$ (including sub-leading effects if $M_{1}$ takes the numerical value of $M_{2}$ ). The same is true also for $N_{3}$ decoupled (including the case with only 2 heavy singlet neutrinos) if $M_{2}$ is numerically the same as $M_{3}$ for decoupled $N_{1}$. However, if $\tilde{N}_{3}$ is the inflaton the LFV decays have the rates too low to be observed. In addition, if $N_{3}$ decouples due to its very large mass its large Yukawa can, for $m_{\nu_{1}} / m_{\nu_{3}}>M_{2} / M_{3}$, still dominate the LFV effects which are then practically unconstrained by the oscillation data; some constraints can then be obtained from the limits on the electron EDM [730].
}

mass of the heaviest of the two un-decoupled singlet neutrinos (in this case $N_{3}$ ). Secondly, for fixed $M_{3}$, they depend strongly on the magnitude and phase of $R_{32}$, mildly on the undetermined element $U_{13}$ of the light neutrino mixing matrix and, in addition, on the Majorana phases of $U$ which cannot be measured in oscillation experiments [545]. The latter dependence is mild for $B(\tau \rightarrow \mu \gamma)$ but can lead to strong destructive interference either in $B(\mu \rightarrow e \gamma)$ or in $B(\tau \rightarrow e \gamma)$ decreasing them by several orders of magnitude. The interference effects are seen in Fig. 26(a), (b) where the predicted ranges (resulting from varying the unknown Majorana phases) of $B(\mu \rightarrow e \gamma)$ are shown as a function of $\left|R_{32}\right|$ for $M_{1}$ appropriate for the sneutrino inflation scenario, three different values of $\arg \left(R_{32}\right)$ and for $m_{0}=100 \mathrm{GeV}, M_{1 / 2}=500 \mathrm{GeV}$ and $\tan \beta=10$, consistent with the dark matter abundance [731]. Results for other values of these parameters can be obtained by appropriate rescalings using (5.27). For comparison, for selected values of $R_{23}$, we also indicate the ranges of $B(\mu \rightarrow e \gamma)$ resulting from generic form of the matrix $R$ (constrained only by the conditions $0<R_{12}, R_{13}<1.5$ and $\operatorname{Re}\left(Y_{v}^{A B}\right)$, $\left.\operatorname{Im}\left(Y_{v}^{A B}\right)<10\right)$.

The bulk of the predicted values of $B(\mu \rightarrow e \gamma)$ shown in Fig. 26(a) and (b) exceed the current experimental limit. Since $M_{1 / 2}=500 \mathrm{GeV}$ leads to masses of the third generation squarks above $1 \mathrm{TeV}$, suppressing $B(\mu \rightarrow e \gamma)$ by increasing the SUSY breaking scale conflicts with the stability of the electroweak scale. Moreover, as discussed in [238] in the scenario considered here generically $B(\tau \rightarrow$ $\mu \gamma) / B(\mu \rightarrow e \gamma) \sim 0.1$. Thus the observation $\tau \rightarrow \mu \gamma$ with $B \gtrsim 10^{-9}$, accessible to future experiments would exclude this scenario.

For completeness, in Fig. 26(c) we also show predictions for $B(\mu \rightarrow e \gamma)$ in the case of $N_{3}$ dominance. $\left(\tilde{m}_{L}^{2}\right)_{21}$ is in this case controlled mainly by $\left|U_{13}\right|$. Moreover, $B(\tau \rightarrow \mu \gamma) / B(\mu \rightarrow e \gamma) \sim \max \left(\left|U_{13}\right|^{2}, \rho^{4} S^{2}\right)$, while $B(\tau \rightarrow e \gamma) / B(\mu \rightarrow e \gamma) \sim 1$ allowing for experimental test of this scenario (cf. [732]). The limits $R_{32} \rightarrow 0$ in panels $a$ and $b$ and or $R_{21} \rightarrow 0$ in panel $c$ correspond to pure sequential dominance.

In conclusion, the well motivated assumption about the decoupling/dominance of one heavy singlet neutrino significantly constrains the predictions for the LFV processes in supersymmetric model. The forthcoming experiments should be able to verify this assumption and, in consequence, to test an interesting class of neutrino mass models.

\subsubsection{Triplet seesaw mechanism and lepton flavor violation}

In this subsection we intend to discuss the aspect of low scale LFV in rare decays arising in the context of the triplet seesaw mechanism of Sect. 3.2.3.2. We consider both nonSUSY and SUSY versions of it. The flavor structure of the 

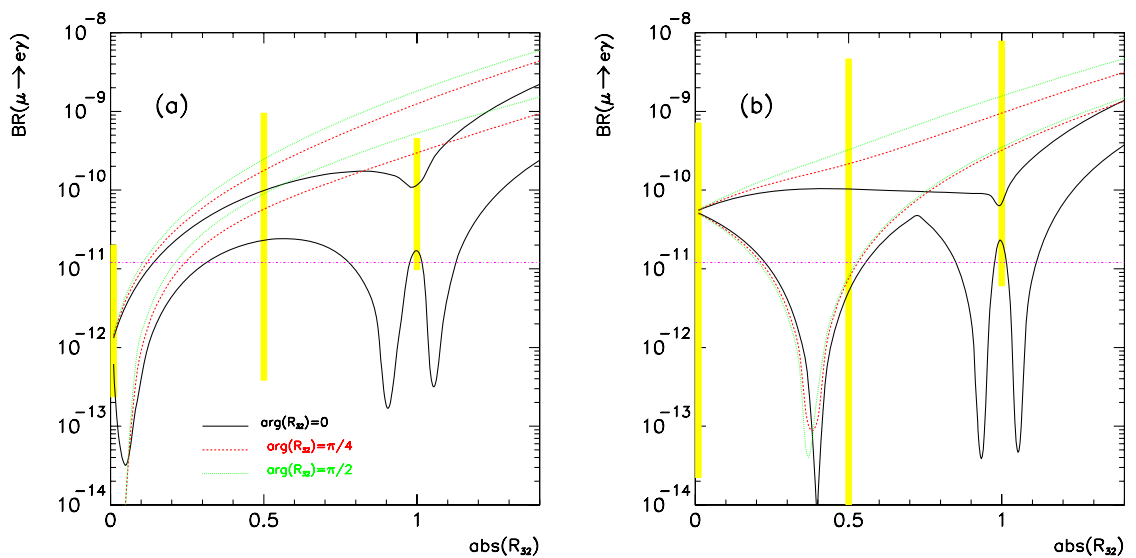

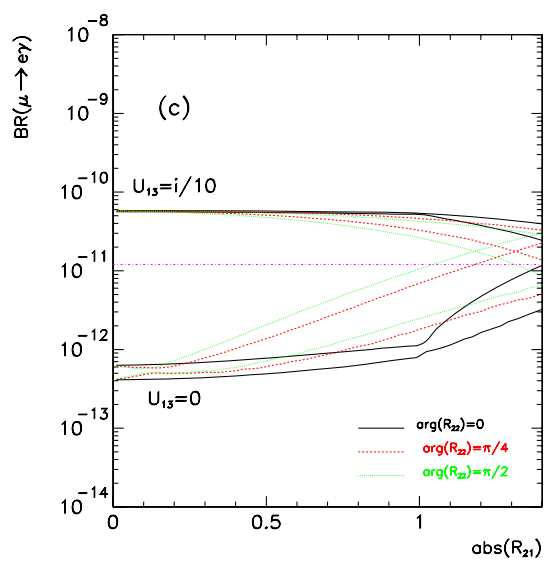

Fig. 26 (Color online) Predicted ranges of $B(\mu \rightarrow e \gamma)$ for $\left(M_{1}, M_{2}, M_{3}\right)=(2,3,50) \times 10^{13} \mathrm{GeV}, m_{0}=100 \mathrm{GeV}, M_{1 / 2}=$ $500 \mathrm{GeV}$ and $\tan \beta=10$, for the decoupling of $N_{1}$ and $U_{13}=0$ (panel a) or $U_{13}=0.1 i$ (panel b). Yellow ranges show the possible

Fig. 27 Diagrams that contribute to the decay $\ell_{j} \rightarrow \ell_{i} \gamma$ through the exchange of the triplet scalars
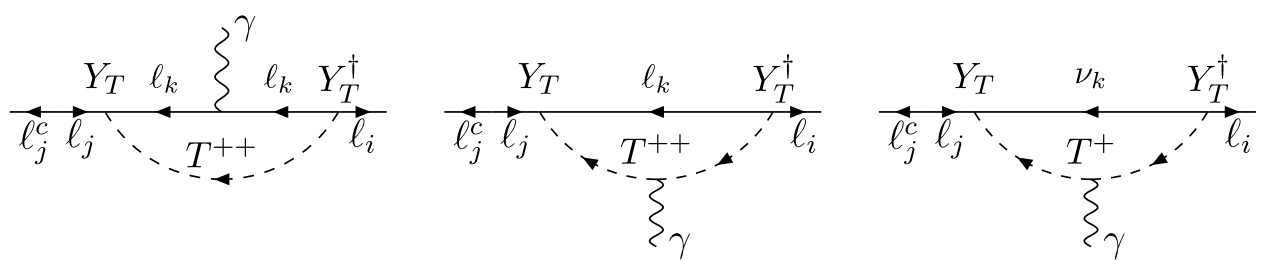

variation for arbitrary form of $R$ with $\arg \left(R_{32}\right)=0$. Lower (upper) pairs of lines in the panel $\mathbf{c}$ show similar ranges for $N_{3}$ dominance for $U_{13}=0(0.1 i)$. The current experimental bound of $1.2 \times 10^{-11}$ [180] is also shown

seesaw case, which represents a concrete and explicit realization of the 'minimal flavor violation' hypothesis [536] in the lepton sector [542]. Indeed, according to the latter, the low energy SM Yukawa couplings are the only source of LFV. This is not generically the case for the seesaw mechanism realized through the exchange of the so-called 'right handed' neutrinos, where the number of independent parameters of the high energy flavor structures is twice more that of the mass matrix $m_{v}$.

Finally, the parametric dependence of the dipole amplitude in Fig. 27 is

$D_{i j} \approx \frac{\left(Y_{T}^{\dagger} Y_{T}\right)_{i j}}{16 \pi^{2} M_{T}^{2}}=\frac{\left(m_{\nu}^{\dagger} m_{v}\right)_{i j}}{16 \pi^{2} v^{4}}\left(\frac{M_{T}}{\mu^{\prime}}\right)^{2}$.

From the present experimental bound on $B(\mu \rightarrow e \gamma)<$ $1.2 \times 10^{-11}$ [180], one infers the bound $\mu^{\prime}>10^{-10} M_{T}$ [comparable limit is obtained from $B(\tau \rightarrow \mu \gamma)$ ]. We can push further our discussion considering the relative size of LFV in different family sectors:

$\frac{\left(Y_{T}^{\dagger} Y_{T}\right)_{\tau \mu}}{\left(Y_{T}^{\dagger} Y_{T}\right)_{\mu e}} \approx \frac{\left[V\left(m_{\nu}^{D}\right)^{2} V^{\dagger}\right]_{\tau \mu}}{\left[V\left(m_{\nu}^{D}\right)^{2} V^{\dagger}\right]_{\mu e}}$

$\frac{\left(Y_{T}^{\dagger} Y_{T}\right)_{\tau e}}{\left(Y_{T}^{\dagger} Y_{T}\right)_{\mu e}} \approx \frac{\left[V\left(m_{v}^{D}\right)^{2} V^{\dagger}\right]_{\tau e}}{\left[V\left(m_{v}^{D}\right)^{2} V^{\dagger}\right]_{\mu e}}$. decays depend only on 7 independent neutrino parameters (there is no dependence on the Majorana phases $\phi_{i}$ ). Notice that this simple flavor structure is peculiar of the triplet where $i, j=e, \mu, \tau$ are family indices. Therefore, the amount of LFV is directly and univocally expressed in terms of the low energy neutrino parameters. In particular, LFV 
These ratios depend only on the neutrino parameters, while do not depend on details of the model, such as the mass scales $M_{T}$, or $\mu^{\prime}$. By taking the present best fit values of the neutrino masses and mixing angles $[211,735]$ provided by the analysis of the experimental data, those ratios can be explicitly expressed as

$\frac{\left(Y_{T}^{\dagger} Y_{T}\right)_{\tau \mu}}{\left(Y_{T}^{\dagger} Y_{T}\right)_{\mu e}} \approx\left(\frac{\Delta m_{A}^{2}}{\Delta m_{S}^{2}}\right) \frac{\sin 2 \theta_{23}}{\sin 2 \theta_{12} \cos \theta_{23}} \sim 40$,

$\frac{\left(Y_{T}^{\dagger} Y_{T}\right)_{\tau e}}{\left(Y_{T}^{\dagger} Y_{T}\right)_{\mu e}} \approx-\tan \theta_{23} \sim-1$,

where $\Delta m_{A}^{2}\left(\Delta m_{S}^{2}\right)$ is the squared-mass difference relevant for the atmospheric (solar) neutrino oscillations. These results hold for $\theta_{13}=0$ and for either hierarchical, quasidegenerate or inverted hierarchical neutrino spectrum (for more details see $[249,736])$. It is immediate to translate the above relations into model-independent predictions for ratios of LFV processes:

$$
\begin{aligned}
& \frac{B(\tau \rightarrow \mu \gamma)}{B(\mu \rightarrow e \gamma)} \approx\left(\frac{\left(Y_{T}^{\dagger} Y_{T}\right)_{\tau \mu}}{\left(Y_{T}^{\dagger} Y_{T}\right)_{\mu e}}\right)^{2} \frac{B\left(\tau \rightarrow \mu \nu_{\tau} \bar{\nu}_{\mu}\right)}{B\left(\mu \rightarrow e v_{\mu} \bar{v}_{e}\right)} \sim 300, \\
& \frac{B(\tau \rightarrow e \gamma)}{B(\mu \rightarrow e \gamma)} \approx\left(\frac{\left(Y_{T}^{\dagger} Y_{T}\right)_{\tau e}}{\left(Y_{T}^{\dagger} Y_{T}\right)_{\mu e}}\right)^{2} \frac{B\left(\tau \rightarrow e v_{\tau} \bar{\nu}_{e}\right)}{B\left(\mu \rightarrow e v_{\mu} \bar{\nu}_{e}\right)} \sim 0.2 .
\end{aligned}
$$

Now we focus upon the supersymmetric version of the triplet seesaw mechanism. (Just recall just that in the supersymmetric case there is only one mass parameter, $M_{T}$, while the mass parameter $\mu^{\prime}$ of the non-supersymmetric version is absent from the superpotential and its role is taken by $\lambda_{2} M_{T}$.) Regarding the aspect of LFV, in this case we have to consider besides the diagrams of Fig. 27 also the related ones with each particle in the loop replaced by its superpart$\operatorname{ner}\left(\ell_{k} \rightarrow \tilde{\ell}_{k}, T \rightarrow \tilde{T}\right)$. Such additional contributions would cancel those in Fig. 27 in the limit of exact supersymmetry. In the presence of soft supersymmetry breaking (SSB) the cancellation is only partial and the overall result for the coefficient of the dipole amplitude behaves like

$D_{i j} \approx \frac{\left(Y_{T}^{\dagger} Y_{T}\right)_{i j}}{16 \pi^{2}} \frac{\tilde{m}^{2}}{M_{T}^{4}} \sim \frac{\left(m_{\nu}^{\dagger} m_{v}\right)_{i j}}{16 \pi^{2}\left(\lambda_{2} v_{2}^{2}\right)^{2}} \frac{\tilde{m}^{2}}{M_{T}^{2}}$,

which is suppressed with respect to the non-supersymmetric result (5.49) for $M_{T}>\tilde{m} \sim \mathcal{O}\left(10^{2} \mathrm{GeV}\right)$ ( $\tilde{m}$ denotes an average soft breaking mass parameter). In the supersymmetric version of the triplet seesaw mechanism flavor violation can also be induced by renormalization effects via (3.69) (the complete set of RGEs of the MSSM with the triplet states have been computed in [249]). Thus in SUSY model the LFV processes can occur also in the case of very heavy triplet. In that case the relevant flavor structure responsible for LFV is again $Y_{T}^{\dagger} Y_{T}$ for which we have already noticed its unambiguous dependence on the neutrino parameters in (5.48). Clearly, we find that analogous ratios as in (5.50) hold also for the LFV entries of the soft breaking parameters, e.g.,

$\frac{\left(m_{\tilde{L}}^{2}\right)_{\tau \mu}}{\left(m_{\tilde{L}}^{2}\right)_{\mu e}} \approx \frac{\left[V\left(m_{v}^{D}\right)^{2} V^{\dagger}\right]_{\tau \mu}}{\left[V\left(m_{\nu}^{D}\right)^{2} V^{\dagger}\right]_{\mu e}}$,

$\frac{\left(m_{\tilde{L}}^{2}\right)_{\tau e}}{\left(m_{\tilde{L}}^{2}\right)_{\mu e}} \approx \frac{\left[V\left(m_{v}^{D}\right)^{2} V^{\dagger}\right]_{\tau e}}{\left[V\left(m_{v}^{D}\right)^{2} V^{\dagger}\right]_{\mu e}}$.

Such SSB flavor violating mass parameters induce extra contributions to the LFV processes. For example, the radiative decays $\ell_{j} \rightarrow \ell_{i} \gamma$ receive also one loop contributions with the exchange of the charged-sleptons/neutralinos and sneutrinos/charginos, where the slepton masses $\left(m_{\tilde{L}}^{2}\right)_{i j}$ are the source of LFV. The relevant dipole terms have a parametric dependence of the form

$$
\begin{aligned}
D_{i j} & \approx \frac{g^{2}}{16 \pi^{2}} \frac{\left(m_{\tilde{L}}^{2}\right)_{i j}}{\tilde{m}^{4}} \tan \beta \\
& \approx \frac{g^{2}}{16 \pi^{2}} \frac{\left(m_{\nu}^{\dagger} m_{v}\right)_{i j}}{\left(\lambda_{2} v_{2}^{2}\right)^{2}} \frac{M_{T}^{2}}{\tilde{m}^{2}} \log \left(\frac{M_{G}}{M_{T}}\right) \tan \beta
\end{aligned}
$$

Notice the inverted dependence on the ratio $\tilde{m} / M_{T}$ with respect to the triplet exchange contribution. Due to this feature, the MSSM sparticle induced contributions (5.55) tends to dominate over the one induced by the triplet exchange. In this case, analogous ratios as in (5.52) can be derived, i.e.,

$$
\begin{aligned}
& \frac{B(\tau \rightarrow \mu \gamma)}{B(\mu \rightarrow e \gamma)} \approx\left(\frac{\left(m_{\tilde{L}}^{2}\right)_{\tau \mu}}{\left(m_{\tilde{L}}^{2}\right)_{\mu e}}\right)^{2} \frac{B\left(\tau \rightarrow \mu v_{\tau} \bar{v}_{\mu}\right)}{B\left(\mu \rightarrow e v_{\mu} \bar{v}_{e}\right)} \sim 300, \\
& \frac{B(\tau \rightarrow e \gamma)}{B(\mu \rightarrow e \gamma)} \approx\left(\frac{\left(m_{\tilde{L}}^{2}\right)_{\tau e}}{\left(m_{\tilde{L}}^{2}\right)_{\mu e}}\right)^{2} \frac{B\left(\tau \rightarrow e v_{\tau} \bar{\nu}_{e}\right)}{B\left(\mu \rightarrow e v_{\mu} \bar{\nu}_{e}\right)} \sim 0.2 .
\end{aligned}
$$

(For more details see [249].)

The presence of extra $S U(2)_{W}$ triplet states at intermediate energy spoils the successful gauge coupling unification of the MSSM. A simple way to recover gauge coupling unification is to introduce more states $X$, to complete a certain representation $R$-such that $R=T+X$-of some unifying gauge group $G, G \supset S U(3) \times S U(2)_{W} \times U(1)_{Y}$. In general the Yukawa couplings of the states $X$ are related to those of the triplet partners $T$. Indeed, this is generally the case in minimal GUT models. In this case RG effects generates not only lepton-flavor violation but also closely correlated flavor violation in the quark sector (due to the $X$-couplings). An explicit scenario with $G=S U(5)$ where both lepton and quark flavor violation arise from RG effects was discussed in Ref. [249]. In Sect. 5.3.2 we review a supersymmetric $S U(5)$ model for the triplet seesaw scenario. 


\subsection{SUSY GUTs}

\subsubsection{Flavor violation in the minimal supersymmetric $S U(5)$ seesaw model}

In this section we review flavor- and/or CP-violating phenomena in the minimal SUSY SU(5) GUT, in which the right handed neutrinos are introduced to generate neutrino masses by the type-I seesaw mechanism [216-220]. Here, it is assumed that the Higgs doublets in this MSSM are embedded in 5- and $\overline{\mathbf{5}}$-dimensional $S U(5)$ multiplets. Rich flavor structure is induced even in those minimal particle contents. The flavor violating SUSY breaking terms for the right handed squarks and sleptons are generated by the GUT interaction, while those are suppressed in the MSSM $\left(+v_{R}\right)$ under the universal scalar mass hypothesis for the SUSY breaking terms.

The Yukawa interactions for quarks and leptons and the Majorana mass terms for the right handed neutrinos in this model are given by the following superpotential,

$$
\begin{aligned}
W= & \frac{1}{4} Y_{i j}^{u} \Psi_{i} \Psi_{j} H+\sqrt{2} Y_{i j}^{d} \Psi_{i} \Phi_{j} \bar{H} \\
& +Y_{i j}^{v} \Phi_{i} \bar{N}_{j} H+\frac{1}{2} M_{N i j} \bar{N}_{i} \bar{N}_{j},
\end{aligned}
$$

where $\Psi$ and $\Phi$ are for $\mathbf{1 0}$ - and $\overline{\mathbf{5}}$-dimensional multiplets, respectively, and $\bar{N}$ is for the right handed neutrinos. $H(\bar{H})$ is 5- (5-) dimensional Higgs multiplets. After removing the unphysical degrees of freedom, the Yukawa coupling constants in (5.57) are given as follows,

$$
\begin{aligned}
Y_{i j}^{u} & =V_{k i} Y_{u_{k}} \mathrm{e}^{i \varphi_{u_{k}} V_{k j},} \\
Y_{i j}^{d} & =Y_{d_{i}} \delta_{i j}, \\
Y_{i j}^{\nu} & =\mathrm{e}^{i \varphi_{d_{i}}} U_{i j}^{\star} Y_{v_{j}} .
\end{aligned}
$$

Here, $Y_{u}, Y_{d}, Y_{v}$ denote diagonal Yukawa couplings, $\varphi_{u_{i}}$ and $\varphi_{d_{i}}(i=1-3)$ are CP-violating phases inherent in the SUSY $S U(5)$ GUT $\left(\sum_{i} \varphi_{u_{i}}=\sum_{i} \varphi_{d_{i}}=0\right)$. The unitary matrix $V$ is the CKM matrix in the extension of the SM to the SUSY $S U(5)$ GUT, and each unitary matrices $U$ and $V$ have only a phase. When the Majorana mass matrix for the right handed neutrinos is real and diagonal in the basis of (5.59), $U$ is the PMNS matrix measured in the neutrino oscillation experiments and the light neutrino mass eigenvalues are given as $m_{v_{i}}=Y_{v_{i}}^{2}\left\langle H_{2}\right\rangle^{2} / M_{N_{i}}$, in which $M_{N_{i}}$ are the diagonal components.

The colored Higgs multiplets $H_{c}$ and $\bar{H}_{c}$ are introduced in $H$ and $\bar{H}$ as $S U(5)$ partners of the Higgs doublets $H_{f}$ and $\bar{H}_{f}$ in the MSSM, respectively, and they have new flavor violating interactions. Equation (5.57) is represented by the fields in the MSSM as follows,

$$
W=W_{\mathrm{MSSM}+\bar{N}}
$$

$$
\begin{aligned}
& +\frac{1}{2} V_{k i} Y_{u_{k}} \mathrm{e}^{i \varphi_{u_{k}}} V_{k j} Q_{i} Q_{j} H_{c}+Y_{u_{i}} V_{i j} \mathrm{e}^{i \varphi_{d_{j}}} \bar{U}_{i} \bar{E}_{j} H_{c} \\
& +Y_{d_{i}} \mathrm{e}^{-i \varphi_{d_{i}}} Q_{i} L_{i} \bar{H}_{c}+\mathrm{e}^{-i \varphi_{u_{i}}} V_{i j}^{\star} Y_{d_{j}} \bar{U}_{i} \bar{D}_{j} \bar{H}_{c} \\
& +\mathrm{e}^{i \varphi_{d_{i}}} U_{i j}^{\star} Y_{v_{j}} \bar{D}_{i} \bar{N}_{j} H_{c} .
\end{aligned}
$$

Here, the superpotential in the MSSM with the right handed neutrinos is

$$
\begin{aligned}
W_{\mathrm{MSSM}+\bar{N}}= & V_{j i} Y_{u_{j}} Q_{i} \bar{U}_{j} H_{f}+Y_{d_{i}} Q_{i} \bar{D}_{i} \bar{H}_{f}+Y_{d_{i}} L_{i} \bar{E}_{i} \bar{H}_{f} \\
& +U_{i j}^{\star} Y_{v_{j}} L_{i} \bar{N}_{j} H_{f}+M_{i j} \bar{N}_{i} \bar{N}_{j} .
\end{aligned}
$$

The flavor violating interactions, which are absent in the MSSM, emerge in the SUSY SU(5) GUT due to existence of the colored Higgs multiplets. The colored Higgs interactions are also baryon-number violating [150, 371], and then proton decay induced by the colored Higgs exchange is a serious problem, especially in the minimal SUSY SU(5) GUT [374]. However, the constraint from the proton decay depends on the detailed structure in the Higgs sector, and it is also loosened by global symmetries, such as the PecceiQuinn symmetry and the $U(1)_{R}$ symmetry. Thus, we may ignore the constraint from the proton decay while we adopt the minimal Yukawa structure in (5.57).

The sfermion mass terms get sizable corrections by the colored Higgs interactions, when the SUSY-breaking terms in the MSSM are generated by dynamics above the colored Higgs masses. In the minimal supergravity scenario the SUSY breaking terms are supposed to be given at the reduced Planck mass scale $\left(M_{G}\right)$. In this case, the flavor violating SUSY breaking mass terms at low energy are induced by the radiative correction, and they are qualitatively given in a flavor basis as

$$
\begin{aligned}
& \left(m_{\tilde{u}_{L}}^{2}\right)_{i j} \simeq-V_{i 3} V_{j 3}^{\star} \frac{Y_{b}^{2}}{(4 \pi)^{2}}\left(3 m_{0}^{2}+A_{0}^{2}\right) \\
& \times\left(2 \log \frac{M_{G}^{2}}{M_{H_{c}}^{2}}+\log \frac{M_{H_{c}}^{2}}{M_{\mathrm{SUSY}^{2}}}\right), \\
& \left(m_{\tilde{u}_{R}}^{2}\right)_{i j} \simeq-\mathrm{e}^{-i \varphi_{u_{i j}}} V_{i 3}^{\star} V_{j 3} \frac{2 Y_{b}^{2}}{(4 \pi)^{2}}\left(3 m_{0}^{2}+A_{0}^{2}\right) \log \frac{M_{G}^{2}}{M_{H_{c}}^{2}}, \\
& \left(m_{\tilde{d}_{L}}^{2}\right)_{i j} \simeq-V_{3 i}^{\star} V_{3 j} \frac{Y_{t}^{2}}{(4 \pi)^{2}}\left(3 m_{0}^{2}+A_{0}^{2}\right) \\
& \times\left(3 \log \frac{M_{G}^{2}}{M_{H_{c}}^{2}}+\log \frac{M_{H_{c}}^{2}}{M_{\mathrm{SUSY}}^{2}}\right), \\
& \left(m_{\tilde{d}_{R}}^{2}\right)_{i j} \simeq-\mathrm{e}^{i \varphi_{d_{i j}}} U_{i k}^{\star} U_{j k} \frac{Y_{v_{k}}^{2}}{(4 \pi)^{2}}\left(3 m_{0}^{2}+A_{0}^{2}\right) \log \frac{M_{G}^{2}}{M_{H_{c}}^{2}}, \\
& \left(m_{\tilde{l}_{L}}^{2}\right)_{i j} \simeq-U_{i k} U_{j k}^{\star} \frac{f_{v_{k}}^{2}}{(4 \pi)^{2}}\left(3 m_{0}^{2}+A_{0}^{2}\right) \log \frac{M_{G}^{2}}{M_{N_{k}}^{2}},
\end{aligned}
$$


$\left(m_{\tilde{e}_{R}}^{2}\right)_{i j} \simeq-\mathrm{e}^{i \varphi_{d_{i j}}} V_{3 i} V_{3 j}^{\star} \frac{3 Y_{t}^{2}}{(4 \pi)^{2}}\left(3 m_{0}^{2}+A_{0}^{2}\right) \log \frac{M_{G}^{2}}{M_{H_{c}}^{2}}$,

with $i \neq j$, where $\varphi_{u_{i j}} \equiv \varphi_{u_{i}}-\varphi_{u_{j}}$ and $\varphi_{d_{i j}} \equiv \varphi_{d_{i}}-\varphi_{d_{i}}$ and $M_{H_{c}}$ is the colored Higgs mass. Here, $M_{\text {SUSY }}$ is the SUSYbreaking scale in the MSSM, $m_{0}$ and $A_{0}$ are the universal scalar mass and trilinear coupling, respectively, in the minimal supergravity scenario. $Y_{t}$ is the top quark Yukawa coupling constant while $Y_{b}$ is for the bottom quark. The offdiagonal components in the right handed squarks and slepton mass matrices are induced by the colored Higgs interactions, and they depend on the CP-violating phases in the SUSY SU(5) GUT with the right handed neutrinos [737].

One of the important features of the SUSY GUTs is the correlation between the leptonic and hadronic flavor violations $[738,739]$. From (5.62), we get a relation

$$
\left(m_{\tilde{d}_{R}}^{2}\right)_{23} \simeq \mathrm{e}^{i \varphi_{d_{23}}}\left(m_{\tilde{l}_{L}}^{2}\right)_{23}^{\star} \times\left(\log \frac{M_{G}^{2}}{M_{H_{c}}^{2}} / \log \frac{M_{G}^{2}}{M_{N_{3}}^{2}}\right) .
$$

The right handed bottom-strange squark mixing may be tested in the $B$ factory experiments since it affects $B_{S}-\bar{B}_{s}$ mixing, $\mathrm{CP}$ asymmetries in the $b-s$ penguin processes such as $B_{d} \rightarrow \phi K_{s}$, and the mixing induced $\mathrm{CP}$ asymmetry in $B_{d} \rightarrow M_{s} \gamma$. (See Chap. 2.) The relation in (5.62) implies that the deviations from the standard model predictions in the $b-s$ transition processes are correlated with $B(\tau \rightarrow \mu \gamma)$ in the SUSY $S U(5)$ GUT. We may test the model in the $B$ factories.

In Fig. 28 we show the CP asymmetry in $B_{d} \rightarrow \phi K_{s}$ $\left(S_{\phi K_{S}}\right)$ and $B(\tau \rightarrow \mu \gamma)$ as an example of the correlation. Here, we assume the minimal supergravity hypothesis for the SUSY breaking terms. See the caption and Ref. [738] for the input parameters and the details of the figure. It is found that $S_{\phi K_{S}}$ and $B(\tau \rightarrow \mu \gamma)$ are correlated and a large deviation from the standard model prediction for $S_{\phi K_{S}}$ is not possible due to the current bound on $B(\tau \rightarrow \mu \gamma)$ in the SUSY $S U(5)$ GUT.

In (5.62), we take the $S U(5)$-symmetric Yukawa interactions given in (5.57), while they fails to explain the fermion mass relations in the first and second generations. We have to extend the minimal model by introducing non-trivial Higgs or matter contents or the higher-dimensional operators including $S U(5)$-breaking Higgs field. These extensions may affect the prediction for the sfermion mass matrices. However, the relation in (5.62) is rather robust when the neutrino Yukawa coupling constant of the third generation is as large as those for the top and bottom quarks and the large mixing in the atmospheric neutrino oscillation comes from the lopside structure of the neutrino Yukawa coupling.

Another important feature of the SUSY GUTs is that both the left and right handed squarks and sleptons have flavor mixing terms. In this case, the hadronic and leptonic electric dipole moments (EDMs) are generated due to the flavor violation, and they may be large enough to be observed in the future EDM measurements [740]. A diagram in Fig. 29(a) generates the electron EDM even at one loop level, when the relative phase between the left and right handed slepton mixing terms is non-vanishing. While this contribution is suppressed by the flavor violation, it is compensated by a heavier fermion mass, that is, $m_{\tau}$. Similar diagrams in Fig. 29(b) contribute to quark EDMs and chromo-electric dipole moments (CEDM), which induce the hadronic EDMs.

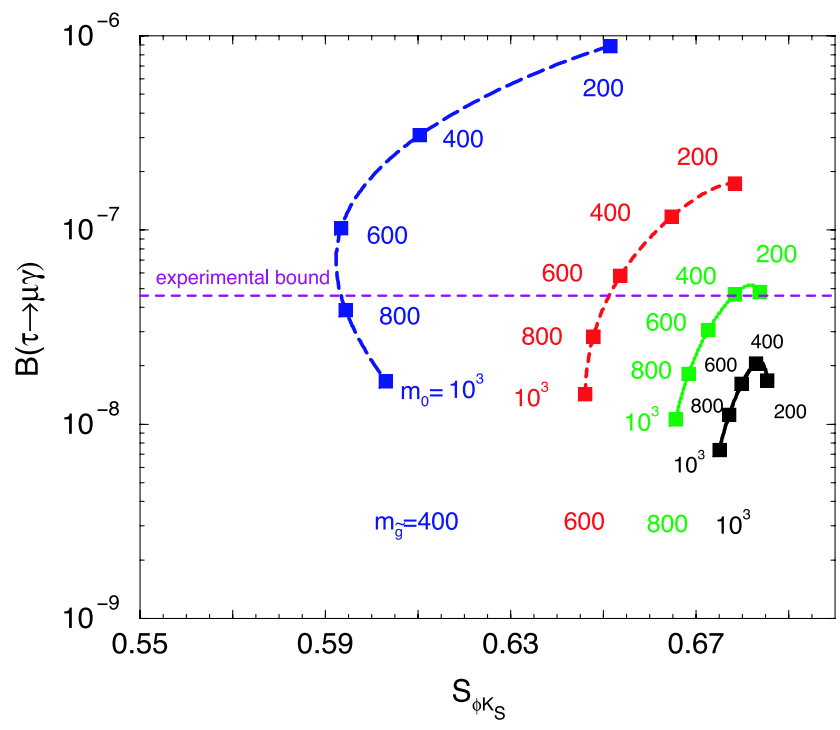

Fig. $28 B(\tau \rightarrow \mu \gamma)$ as a function of the $\mathrm{CP}$ asymmetry in $B_{d} \rightarrow \phi K_{s}$ $\left(S_{\phi K_{S}}\right)$ for fixed gluino masses $m_{\tilde{g}}=400,600,800$, and $1000 \mathrm{GeV}$. Here, $\tan \beta=10,200 \mathrm{GeV}<m_{0}<1 \mathrm{TeV}, A_{0}=0, m_{\nu_{\tau}}=5 \times 10^{-2} \mathrm{eV}$, $M_{N_{3}}=5 \times 10^{14} \mathrm{GeV}$, and $U_{32}=1 / \sqrt{2} . \varphi_{d_{23}}$ is taken for the deviation of $S_{\phi K_{S}}$ from the SM prediction to be maximum. The current experimental bound on $B(\tau \rightarrow \mu \gamma)$ [647] is also shown in the figure
Fig. 29 (a) Diagrams that generate electron EDM and (b) those that generate EDMs and CEDMs of the $i$ th quark due to flavor violation in sfermion mass terms

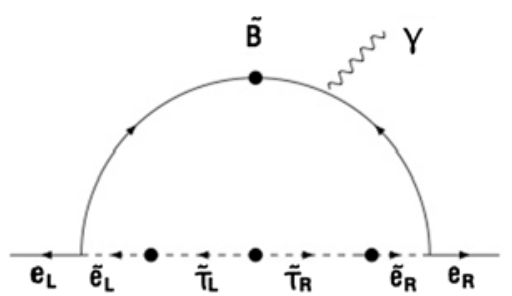

(a)

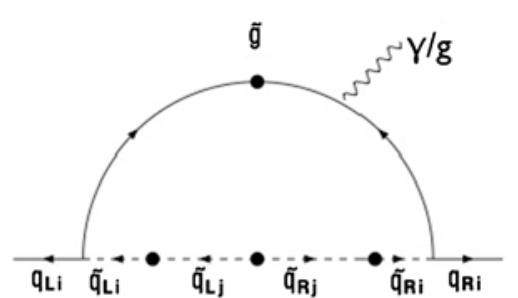

(b) 


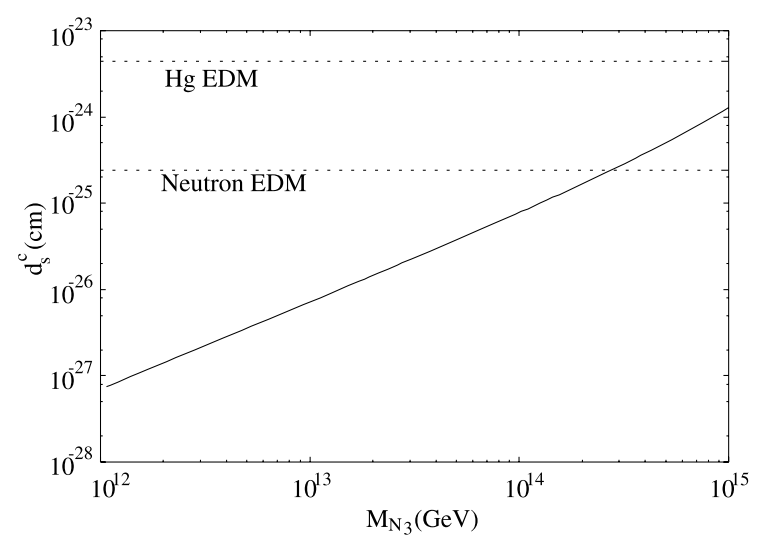

Fig. 30 CEDMs for the strange $\left(d_{s}^{c}\right)$ and down quarks $\left(d_{d}^{c}\right)$ as functions of the right handed tau neutrino mass, $M_{N_{3}}$. Here, $M_{H_{c}}=2 \times$ $10^{16} \mathrm{GeV}, m_{v_{\tau}}=0.05 \mathrm{eV}, U_{23}=1 / \sqrt{2}$, and $U_{13}=0.2,0.02$, and 0.002. For the MSSM parameters, we take $m_{0}=500 \mathrm{GeV}, A_{0}=0$,

The EDM measurements are important to probe the interaction of the SUSY SU(5) GUT.

In Fig. 30 the CEDMs for strange $\left(d_{s}^{c}\right)$ and down quarks $\left(d_{d}^{c}\right)$ are shown as functions of the right handed tau neutrino mass in the SUSY SU(5) GUT with the right handed neutrinos. See the caption and Ref. [741] for the input parameters. The mercury atom EDM, which is a diamagnetic atom, is sensitive to quark CEDMs via the nuclear force, while the neutron EDM depends on them in addition to the quark EDMs. (The evaluation of the hadronic EDMs from the effective operators at the parton level is reviewed in Sect. 9.1 and also Ref. [742].) The strange quark contribution to the mercury atom EDM is suppressed by the strange quark mass. On the other hand, it is argued in Refs. [743, 744] that the strange quark component in nucleon is not negligible and the strange quark CEDM may give a sizable contribution to the neutron EDM. It implies that we may probe the different flavor mixings by measurements of the various hadronic EDMs, though the evaluation of the hadronic EDMs still has large uncertainties.

It is argued that the future measurements of neutron and deuteron EDMs may reach to levels of $\sim 10^{-28} e \mathrm{~cm}$ and $\sim 10^{-29} e \mathrm{~cm}$, respectively. When the sensitivity of deuteron EDM is established, we may probe the new physics to the level of $d_{s}^{c} \sim 10^{-28} e \mathrm{~cm}$ and $d_{d}^{c} \sim d_{u}^{c} \sim 10^{-30} e \mathrm{~cm}$ [849]. The future measurements for the EDMs will give great impacts on the SUSY SU(5) GUT with the right handed neutrinos.

\subsubsection{LFV in the minimal SU(5) GUT with triplet seesaw}

In this section we discuss phenomenology of the minimal $S U(5)$ GUT which incorporates the triplet seesaw mechanism, previously presented in Sects. 3.2.3.2 and 5.2.7. Review of more general class of GUT models also including

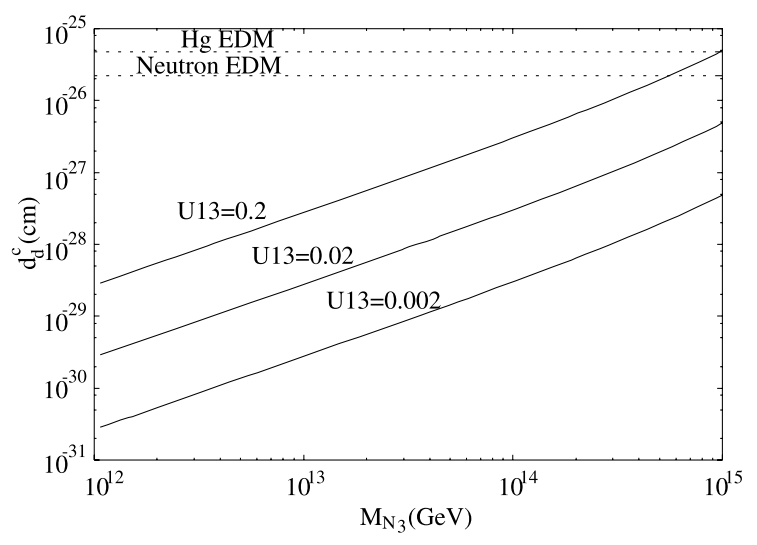

$m_{\tilde{g}}=500 \mathrm{GeV}$ and $\tan \beta=10$. The CP phases $\varphi_{d_{i}}$ are taken for the CEDMs to be maximum. The upper bounds on the strange and down quark CEDMs from the mercury atom and neutron EDMs are shown in the figures

triplet Higgs has been given in Sect. 4.1. In GUTs based on $S U(5)$ there is no natural place for incorporating singlet neutrinos. From this point of view $S U(5)$ presents some advantage for implementing the triplet seesaw mechanism. In particular, a very predictive scenarios can be obtained in the supersymmetric case $[249,736,745]$. The triplet states $T(\bar{T})$ fit into the $15(\overline{15})$ representation, $15=S+T+Z$ with $S$, $T$ and $Z$ transforming as $S \sim\left(6,1,-\frac{2}{3}\right), T \sim(1,3,1), Z \sim$ $\left(3,2, \frac{1}{6}\right)$ under $S U(3) \times S U(2)_{L} \times U(1)_{Y}$ (the $\overline{15}$ decomposition is obvious). We shall briefly show that it is also possible to relate not only neutrino mass parameters and LFV (as shown in Sect. 5.2.7) but also sparticle and Higgs spectra and electroweak symmetry breakdown [736, 745]. For this purpose, consider that the $S U(5)$ model conserves $B-L$, so that the relevant superpotential reads

$$
\begin{aligned}
W_{S U(5)}= & \frac{1}{\sqrt{2}}\left(Y_{15} \overline{5} 15 \overline{5}+\lambda 5_{H} \overline{15} 5_{H}\right)+Y_{5} \overline{5} \overline{5}_{H} 10 \\
& +Y_{10} 10105_{H}+M_{5} 5_{H} \overline{5}_{H}+\xi X 15 \overline{15},
\end{aligned}
$$

where the multiplets are understood as $\overline{5}=\left(d^{c}, L\right), 10=$ $\left(u^{c}, e^{c}, Q\right)$ and the Higgs doublets fit with their colored partners $t$ and $\bar{t}$, like $5_{H}=\left(t, H_{2}\right), \overline{5}_{H}=\left(\bar{t}, H_{1}\right)$ and $X$ is a singlet superfield. The $B-L$ quantum numbers are the combination $Q+\frac{4}{5} Y$ where $Y$ are the hypercharges and $Q_{10}=\frac{1}{5}, Q_{\overline{5}}=-\frac{3}{5}, Q_{5_{H}\left(\overline{5}_{H}\right)}=-\frac{2}{5}\left(\frac{2}{5}\right), Q_{15}=\frac{6}{5}, Q_{\overline{15}}=\frac{4}{5}$, $Q_{X}=-2$. Both the scalar $S_{X}$ and auxiliary $F_{X}$ components of the superfield $X$ are assumed to acquire a VEV through some unspecified dynamics in the hidden sector. Namely, while $\left\langle S_{X}\right\rangle$ only breaks $B-L,\left\langle F_{X}\right\rangle$ breaks both SUSY and $B-L$. These effects are parameterized by the superpotential mass term $M_{15} 15 \overline{15}$, where $M_{15}=\xi\left\langle S_{X}\right\rangle$, and the bilinear SSB term $-B_{15} M_{15} 15 \overline{15}$, with $B_{15} M_{15}=-\xi\left\langle F_{X}\right\rangle$. The 15 and $\overline{15}$ states act, therefore, as messengers of both $B-L$ and SUSY breaking to the MSSM observable sector. Once 
$S U(5)$ is broken to the SM group we find, below the GUT scale $M_{G}$,

$$
\begin{aligned}
& W=W_{0}+W_{T}+W_{S, Z}, \\
& W_{0}=Y_{e} e^{c} H_{1} L+Y_{d} d^{c} H_{1} Q+Y_{u} u^{c} Q H_{2}+\mu H_{2} H_{1}, \\
& W_{T}=\frac{1}{\sqrt{2}}\left(Y_{T} L T L+\lambda H_{2} \bar{T} H_{2}\right)+M_{T} T \bar{T}, \\
& W_{S, Z}=\frac{1}{\sqrt{2}} Y_{S} d^{c} S d^{c}+Y_{Z} d^{c} Z L+M_{Z} Z \bar{Z}+M_{S} S \bar{S} .
\end{aligned}
$$

Here, $W_{0}$ denotes the MSSM superpotential, ${ }^{21}$ the term $W_{T}$ is responsible for neutrino mass generation [cf. (3.67)], while the couplings and masses of the colored fragments $S$ and $Z$ are included in $W_{S, Z}$. It is also understood that $M_{T}=M_{S}=M_{Z} \equiv M_{15}$. At the decoupling of the heavy states $S, T, Z$ we obtain at tree-level the neutrino masses, given by (3.68) and at the quantum level all SSB mass parameters of the MSSM via gauge and Yukawa interactions. At one loop level, only the trilinear scalar couplings, the gaugino masses and the Higgs bilinear mass term $B_{H}$ are generated:

$$
\begin{aligned}
A_{e} & =\frac{3 B_{T}}{16 \pi^{2}} Y_{e}\left(Y_{T}^{\dagger} Y_{T}+Y_{Z}^{\dagger} Y_{Z}\right), \quad A_{u}=\frac{3 B_{T}}{16 \pi^{2}}|\lambda|^{2} Y_{u}, \\
A_{d} & =\frac{2 B_{T}}{16 \pi^{2}}\left(Y_{Z} Y_{Z}^{\dagger}+2 Y_{S} Y_{S}^{\dagger}\right) Y_{d}, \\
M_{a} & =\frac{7 B_{T}}{16 \pi^{2}} g_{a}^{2}, \quad B_{H}=\frac{3 B_{T}}{16 \pi^{2}}|\lambda|^{2} .
\end{aligned}
$$

The scalar mass matrices instead are generated at the twoloop level and receive both gauge-mediated contributions proportional to $C_{a}^{f} g_{a}^{4}\left(C_{a}^{f}\right.$ is the quadratic Casimir of the $f$-particle) and Yukawa-mediated ones of the form $Y_{p}^{\dagger} Y_{p}$ $(p=S, T, Z)$. The former piece is the flavor blind contribution, which is proper of the gauge-mediated scenarios [151, 152, 746-751], while the latter ones constitute the flavor violating contributions transmitted to the SSB terms by the Yukawa's $Y_{S, T, Z}$. These contributions are mostly relevant for the mass matrices $m_{\tilde{L}}^{2}$ and $m_{\tilde{d}^{c}}^{2}$. For example,

$$
\begin{aligned}
m_{\tilde{L}}^{2}= & \left(\frac{B_{T}}{16 \pi^{2}}\right)^{2}\left[\frac{21}{10} g_{1}^{4}+\frac{21}{2} g_{2}^{4}-\left(\frac{27}{5} g_{1}^{2}+21 g_{2}^{2}\right) Y_{T}^{\dagger} Y_{T}\right. \\
& -\left(\frac{21}{15} g_{1}^{2}+9 g_{2}^{2}+16 g_{3}^{2}\right) Y_{Z}^{\dagger} Y_{Z} \\
& +18\left(Y_{T}^{\dagger} Y_{T}\right)^{2}+15\left(Y_{Z}^{\dagger} Y_{Z}\right)^{2}+3 \operatorname{Tr}\left(Y_{T}^{\dagger} Y_{T}\right) Y_{T}^{\dagger} Y_{T} \\
& +12 Y_{Z}^{\dagger} Y_{S} Y_{S}^{\dagger} Y_{Z}+3 \operatorname{Tr}\left(Y_{Z}^{\dagger} Y_{Z}\right) Y_{Z}^{\dagger} Y_{Z}
\end{aligned}
$$

\footnotetext{
${ }^{21}$ This should be regarded as an effective approach where the Yukawa matrices $Y_{d}, Y_{e}, Y_{u}$ include $S U(5)$-breaking effects needed to reproduce a realistic fermion spectrum.
}

$$
\begin{aligned}
& +9 Y_{T}^{\dagger} Y_{Z}^{T} Y_{Z}^{*} Y_{T}+9\left(Y_{T}^{\dagger} Y_{T} Y_{Z}^{\dagger} Y_{Z}+\text { h.c. }\right) \\
& \left.+3 Y_{T}^{\dagger} Y_{e}^{T} Y_{e}^{*} Y_{T}+6 Y_{Z}^{\dagger} Y_{d} Y_{d}^{\dagger} Y_{Z}\right] .
\end{aligned}
$$

Since the flavor structure of $m_{\tilde{L}}^{2}$ is proportional to $Y_{T}$ (and to $Y_{Z}$ which is $S U(5)$-related to $Y_{T}$ ), it can be expressed in terms of the neutrino parameters [cf. (3.68)] and so the relative size of LFV in different leptonic families is predicted according to the results of (5.54).

All the soft masses have the same scaling behavior $\tilde{m} \sim$ $B_{T} /\left(16 \pi^{2}\right)$ which demands $B>10 \mathrm{TeV}$ to fulfill the naturalness principle. This scenario appears very predictive since it contains only three free parameters: the triplet mass $M_{T}$, the effective SUSY breaking scale $B_{T}$ and the coupling constant $\lambda$. The parameter space is then constrained by the experimental bounds on the Higgs boson mass, the $B(\mu \rightarrow$ $e \gamma)$, the sfermion masses, and the requirement of radiative electroweak symmetry breaking. The phenomenological predictions more important and relevant for LHC, the $B$-factories [694] the incoming MEG experiment [548], the Super Flavor factory [752] or the PRISM/PRIME experiment at J-PARC [753], concern the sparticle and Higgs boson spectra and the LFV decays. Regarding the spectrum, the gluino is the heaviest sparticle while, in most of the parameter space, $\tilde{\ell}_{1}$ is the lightest. In the example shown in Fig. 31 the squark and slepton masses lie in the ranges $700-950 \mathrm{GeV}$ and $100-300 \mathrm{GeV}$, respectively. The gluino mass is about $1.3 \mathrm{TeV}$. The chargino masses are $m_{\tilde{\chi}_{1}^{ \pm}} \sim 320 \mathrm{GeV}$ and $m_{\tilde{\chi}_{2}^{ \pm}} \sim 450-550 \mathrm{GeV}$. Moreover, $m_{\tilde{\chi}_{1}^{0}} \sim 190 \mathrm{GeV}, m_{\tilde{\chi}_{2}^{0}} \approx m_{\tilde{\chi}_{1}^{ \pm}}$and $m_{\tilde{\chi}_{3,4}^{0}} \approx m_{\tilde{\chi}_{2}^{ \pm}}$. These mass ranges are within the discovery reach of the LHC.

The Higgs sector is characterized by a decoupling regime with a light SM-like Higgs boson $(h)$ with mass in the range $110-120 \mathrm{GeV}$ which is testable in the near future at LHC (mainly through the decay into 2 photons). The remaining three heavy states $\left(H, A\right.$ and $\left.H^{ \pm}\right)$have mass $m_{H, A, H^{ \pm}} \approx$ $450-550 \mathrm{GeV}$ (again, for $B_{T}=20 \mathrm{TeV}$ ). All the spectra increase almost linearly with $B_{T}$.

Figure 32(b) shows instead several LFV processes: $\mu \rightarrow$ $e X, \mu \rightarrow e$ conversion in nuclei, $\tau \rightarrow e Y$ and $\tau \rightarrow \mu Y(X=$ $\gamma, e e, Y=\gamma, e e, \mu \mu)$. One observe that e.g., the behavior of the radiative-decay branching ratios is in agreement with the estimates given in (5.56) for $\theta_{13}=0$. For $\theta_{13}=0.2$ one obtains instead that $B(\tau \rightarrow \mu \gamma) / B(\mu \rightarrow e \gamma) \sim 2$ and $B(\tau \rightarrow e \gamma) / B(\mu \rightarrow e \gamma) \sim 0.1$ (the full analysis can be found in Ref. [736]). The other LFV processes shown are also correlated to the radiative ones in a model-independent way [736, 745]. The analysis shows that the future experimental sensitivity will allow to measure at most $B(\mu \rightarrow \gamma)$, $B(\mu \rightarrow 3 e), B(\tau \rightarrow \mu \gamma)$ and $\mathrm{CR}\left(\mu \rightarrow e\right.$ Ti) for tiny $\theta_{13}$. In particular, being $B(\tau \rightarrow \mu \gamma) / B(\mu \rightarrow e \gamma) \sim 300, B(\tau \rightarrow$ $\mu \gamma)$ is expected not to exceed $3 \times 10^{-9}$, irrespective of the type of neutrino spectrum. Therefore $\tau \rightarrow \mu \gamma$ falls into 


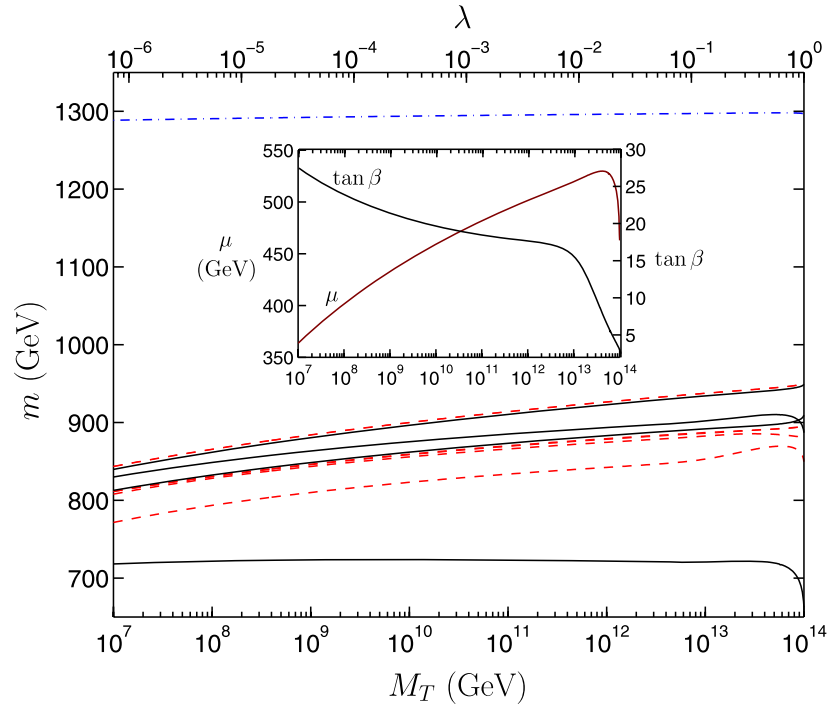

Fig. 31 (Color online) Sparticle and Higgs spectrum for $B_{T}=$ $20 \mathrm{TeV}$. Left panel: squark masses, $m_{\tilde{u}}$ (black solid line), $m_{\tilde{d}}$ (red dashed) and the gluino mass (blue dash-dotted). In the inner plot $\tan \beta$ and $\mu$ are shown as obtained by the electroweak symmetry breaking

the LHC capability. All the decays $\tau \rightarrow \ell_{i} \ell_{k} \ell_{k}$ would have $B<\mathcal{O}\left(10^{-11}\right)$. The predictions for the LFV branching ratios in the present scenario are summarized in Table 13.

Finally, such supersymmetric $S U(5)$ framework with a $15, \overline{15}$ pair may be realized in contexts based on string inspired constructions $[754,755]$.

\subsubsection{LFV from a generic $S O(10)$ framework}

The spinorial representation of the $S O(10)$, given by a 16 dimensional spinor, can accommodate all the SM model particles as well as the right handed neutrino. As discussed in Sect. 4.1, the product of two $\mathbf{1 6}$ matter representations can only couple to 10,120 or 126 representations, which can be formed by either a single Higgs field or a nonrenormalizable product of representations of several Higgs fields. In either case, the Yukawa matrices resulting from the couplings to $\mathbf{1 0}$ and $\mathbf{1 2 6}$ are complex symmetric, whereas they are antisymmetric when the couplings are to the $\mathbf{1 2 0}$. Thus, the most general $S O(10)$ superpotential relevant to fermion masses can be written as

$$
\begin{aligned}
W_{S O(10)=} & Y_{i j}^{10} \mathbf{1 6}_{\mathbf{i}} \mathbf{1 6} \mathbf{j}_{\mathbf{j}} \mathbf{1 0}+Y_{i j}^{126} \mathbf{1 6}_{\mathbf{i}} \mathbf{1 6}_{\mathbf{j}} \mathbf{1 2 6} \\
& +Y_{i j}^{120} \mathbf{1 6}_{\mathbf{i}} \mathbf{1 6} \mathbf{j} \mathbf{j} \mathbf{1 2 0},
\end{aligned}
$$

where $i, j$ refer to the generation indices. In terms of the SM fields, the Yukawa couplings relevant for fermion masses are

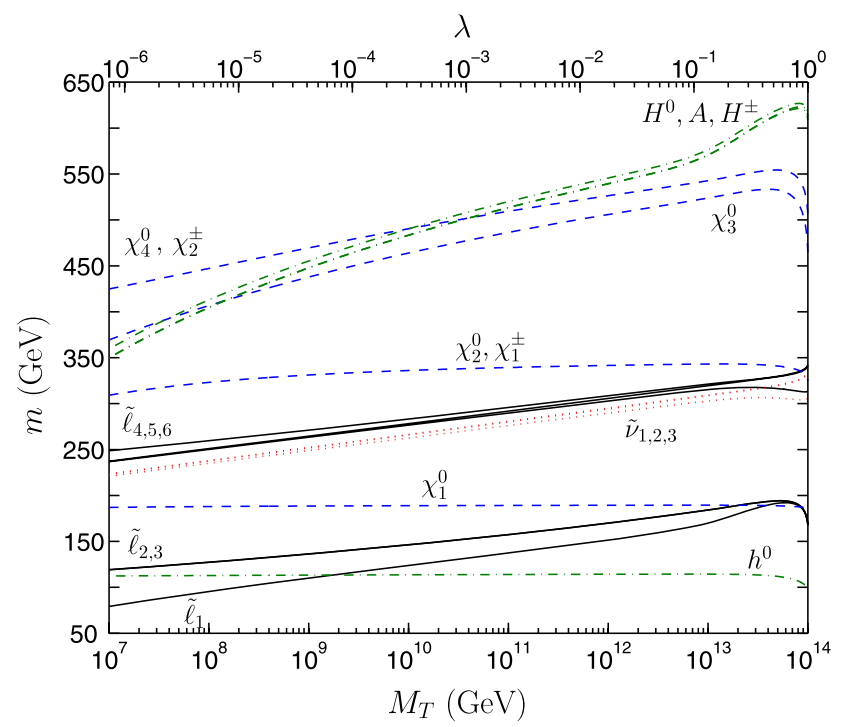

conditions. Right panel: the masses of the charged sleptons, the sneutrinos, the charginos, the neutralinos and the Higgs bosons as the labels indicate

given by $[756,757]$ :

$$
\begin{aligned}
& \mathbf{1 6 1 6 1 0} \supset \mathbf{5}\left(u u^{c}+v v^{c}\right)+\overline{\mathbf{5}}\left(d d^{c}+e e^{c}\right), \\
& \mathbf{1 6 1 6 1 2 6} \supset \mathbf{1} v^{c} v^{c}+15 v v+\mathbf{5}\left(u u^{c}-3 v v^{c}\right)+\overline{\mathbf{4 5}}\left(d d^{c}-3 e e^{c}\right), \\
& \mathbf{1 6 1 6 1 2 0} \supset \mathbf{5} v v^{c}+\mathbf{4 5} u u^{c}+\overline{\mathbf{5}}\left(d d^{c}+e e^{c}\right) \\
&+\overline{\mathbf{4 5}}\left(d d^{c}-3 e e^{c}\right),
\end{aligned}
$$

where we have specified the corresponding $S U(5)$ Higgs representations for each of the couplings and all the fermions are left handed fields. The resulting up-type quarks and neutrinos' Dirac mass matrices can be written as

$m^{u}=M_{10}^{5}+M_{126}^{5}+M_{120}^{45}$,

$m_{D}^{v}=M_{10}^{5}-3 M_{126}^{5}+M_{120}^{5}$.

A simple analysis of the fermion mass matrices in the $S O$ (10) model, as detailed in (5.70) leads us to the following result: At least one of the Yukawa couplings in $Y^{v}=v_{u}^{-1} m_{D}^{v}$ has to be as large as the top Yukawa coupling [164]. This result holds true in general, independently of the choice of the Higgses responsible for the masses in (5.69), (5.70), provided that no accidental fine-tuned cancellations of the different contributions in (5.70) are present. If contributions from the 10's solely dominate, $Y^{v}$ and $Y^{u}$ would be equal. If this occurs for the 126's, then $Y^{v}=-3 Y^{u}$ [395]. In case both of them have dominant entries, barring a rather precisely fine-tuned cancellation between $M_{10}^{5}$ and $M_{126}^{5}$ in (5.70), we expect at least one large entry to be present in $Y^{\nu}$. A dominant antisymmetric contribution to top quark mass 

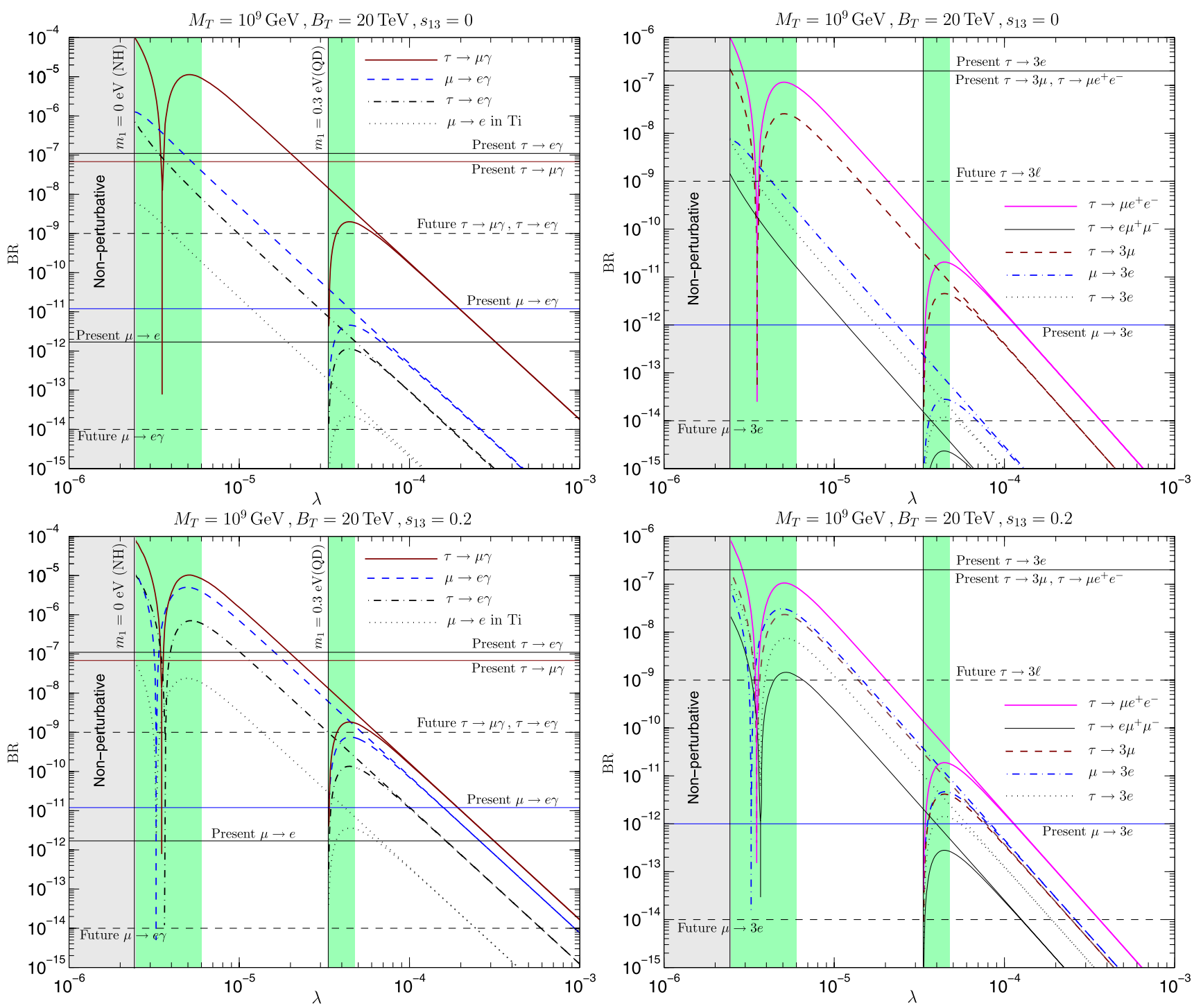

Fig. 32 Branching ratios of several LFV processes as a function of $\lambda$. The left (right) vertical line indicates the lower bound on $\lambda$ imposed by requiring perturbativity of the Yukawa couplings $Y_{T, S, Z}$ when $m_{1}=0$

(0.3) eV [normal-hierarchical (quasi-degenerate) neutrino mass spectrum]. The regions in green (grey) are excluded by the $m_{\tilde{\ell}_{1}}>100 \mathrm{GeV}$ constraint (perturbativity requirement when $m_{1}=0$ )

Table 13 Expectations for the various LFV processes assuming $B(\mu \rightarrow e \gamma)=1.2 \times 10^{-11}$. The results in parenthesis apply to the case of the inverted-hierarchical neutrino spectrum, whenever these are different from those obtained for the normal-hierarchical and quasi-degenerate ones

\begin{tabular}{lll}
\hline Decay mode & Prediction for branching ratio & \\
\cline { 2 - 3 } & $s_{13}=0$ & $s_{13}=0.2$ \\
\hline$\tau^{-} \rightarrow \mu^{-} \gamma$ & $3 \times 10^{-9}$ & $2(3) \times 10^{-11}$ \\
$\tau^{-} \rightarrow e^{-} \gamma$ & $2 \times 10^{-12}$ & $1(3) \times 10^{-12}$ \\
$\mu^{-} \rightarrow e^{-} e^{+} e^{-}$ & $6 \times 10^{-14}$ & $6 \times 10^{-14}$ \\
$\tau^{-} \rightarrow \mu^{-} \mu^{+} \mu^{-}$ & $7 \times 10^{-12}$ & $4(6) \times 10^{-14}$ \\
$\tau^{-} \rightarrow \mu^{-} e^{+} e^{-}$ & $3 \times 10^{-11}$ & $2(3) \times 10^{-13}$ \\
$\tau^{-} \rightarrow e^{-} e^{+} e^{-}$ & $2 \times 10^{-14}$ & $1(3) \times 10^{-14}$ \\
$\tau^{-} \rightarrow e^{-} \mu^{+} \mu^{-}$ & $3 \times 10^{-15}$ & $2(4) \times 10^{-15}$ \\
$\mu \rightarrow e ; \mathrm{Ti}$ & $6 \times 10^{-14}$ & $6 \times 10^{-14}$ \\
\hline
\end{tabular}


due to the $\mathbf{1 2 0}$ Higgs is phenomenologically excluded, since it would lead to at least a pair of heavy degenerate up quarks. Apart from sharing the property that at least one eigenvalue of both $m^{u}$ and $m_{D}^{v}$ has to be large, for the rest it is clear from (5.69) and (5.70) that these two matrices are not aligned in general, and hence we may expect different mixing angles appearing from their diagonalization. This freedom is removed if one sticks to particularly simple choices of the Higgses responsible for up quark and neutrino masses.

Therefore, we see that the $S O(10)$ model with only two ten-plets would inevitably lead to small mixing in $Y^{v}$. In fact, with two Higgs fields in symmetric representations, giving masses to the up sector and the down sector separately, it would be difficult to avoid the small CKM-like mixing in $Y^{v}$. We shall call this case the CKM case. From here, the following mass relations hold between the quark and leptonic mass matrices at the GUT scale: ${ }^{22}$

$Y^{u}=Y^{v} ; \quad Y^{d}=Y^{e}$.

In the basis where charged lepton masses are diagonal, we have

$Y^{v}=V_{\mathrm{CKM}}^{T} Y_{\text {Diag }}^{u} V_{\mathrm{CKM}}$.

The large couplings in $Y^{v} \sim \mathcal{O}\left(Y_{t}\right)$ induce significant offdiagonal entries in $m_{\tilde{L}}^{2}$ through the RG evolution between $M_{\mathrm{GUT}}$ and the scale of the right handed Majorana neutrinos, $M_{R_{i}}$. The induced off-diagonal entries relevant to $l_{j} \rightarrow l_{i}, \gamma$ are of the order of:

$\left(m_{\tilde{L}}^{2}\right)_{i \neq j} \approx-\frac{3 m_{0}^{2}+A_{0}^{2}}{8 \pi^{2}} Y_{t}^{2} V_{t i} V_{t j} \ln \frac{M_{\mathrm{GUT}}}{M_{R_{3}}}+\mathcal{O}\left(Y_{c}^{2}\right)$,

where $V_{i j}$ are elements of $V_{\mathrm{CKM}}$, and $i, j$ flavor indices. In this expression, the CKM angles are small but one would expect the presence of the large top Yukawa coupling to compensate such a suppression. The required right handed neutrino Majorana mass matrix, consistent with both the observed low energy neutrino masses and mixing as well as with CKM-like mixing in $Y^{v}$ is easily determined from the seesaw formula defined at the scale of right handed neutrinos.

The $B\left(l_{i} \rightarrow l_{j} \gamma\right)$ are now predictable in this case. Considering mSUGRA boundary conditions and taking $\tan \beta=$ 40 , we obtain that reaching a sensitivity of $\mathcal{O}\left(10^{-13}\right.$ $\left.10^{-14}\right)$, as planned by the MEG experiment at PSI, for $B(\mu \rightarrow e \gamma)$ would allow us to probe the SUSY spectrum

\footnotetext{
${ }^{22}$ Clearly this relation cannot hold for the first two generations of down quarks and charged leptons. One expects, small corrections due to nonrenormalizable operators or suppressed renormalizable operators [6] to be invoked.
}

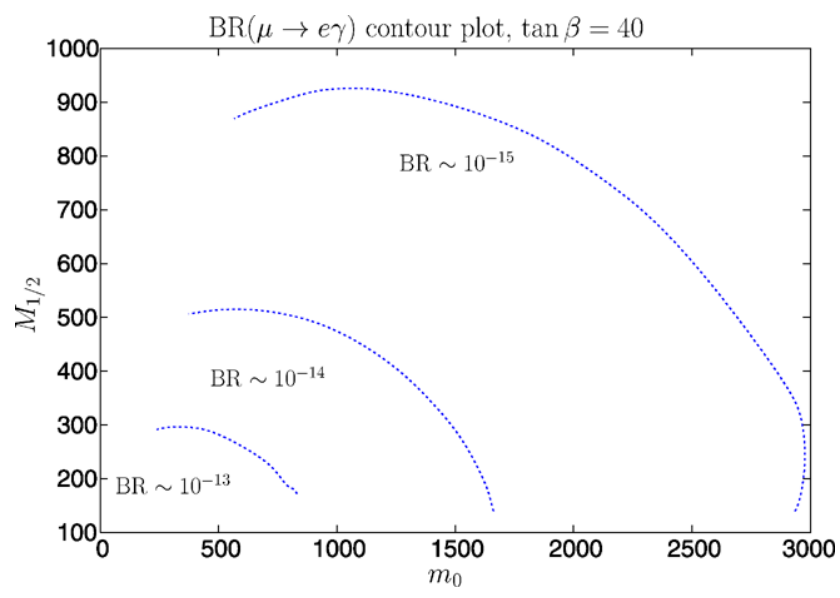

Fig. 33 Contour plot of $B(\mu \rightarrow e, \gamma)$ in the plane of the GUT-scale universal scalar and gaugino masses, $\left(m_{0}, M_{1 / 2}\right)$, at $A_{0}=0$ in the CKM high $\tan \beta$ case. Note that while the plane is presently unconstrained, the planned MEG experiment sensitivity of $\mathcal{O}\left(10^{-13}-10^{-14}\right)$ will be able to probe it in the $\left(m_{0}, m_{\tilde{g}}\right) \lesssim 1 \mathrm{TeV}$ region

completely up to $m_{0}=1200 \mathrm{GeV}, M_{1 / 2}=400 \mathrm{GeV}$ (notice that this corresponds to gluino and squark masses of order $1 \mathrm{TeV})$. This clearly appears from Fig. 33, which shows the $B(\mu \rightarrow e \gamma)$ contour plot in the $\left(m_{0}, M_{1 / 2}\right)$ plane. Thus, in summary, though the present limits on $B(\mu \rightarrow e, \gamma)$ would not induce any significant constraints on the supersymmetry breaking parameter space, an improvement in the limit to $\sim \mathcal{O}\left(10^{-13}-10^{-14}\right)$, as foreseen, would start imposing nontrivial constraints especially for the large $\tan \beta$ region.

To obtain mixing angles larger than CKM angles, asymmetric mass matrices have to be considered. In general, it is sufficient to introduce asymmetric textures either in the up sector or in the down sector. In the present case, we assume that the down sector couples to a combination of Higgs representations (symmetric and antisymmetric) ${ }^{23} \Phi$, leading to an asymmetric mass matrix in the basis where the up sector is diagonal. As we shall see below, this would also require that the right handed Majorana mass matrix be diagonal in this basis. We have:

$$
\begin{aligned}
W_{S O(10)}= & \frac{1}{2} Y_{i i}^{u, v} \mathbf{1 6}_{\mathbf{i}} \mathbf{1 6}_{\mathbf{i}} \mathbf{1 0} \mathbf{1 0}^{\mathbf{u}}+\frac{1}{2} Y_{i j}^{d, e} \mathbf{1 6}_{\mathbf{i}} \mathbf{1 6}_{\mathbf{j}} \Phi \\
& +\frac{1}{2} Y_{i i}^{R} \mathbf{1 6}_{\mathbf{i}} \mathbf{1 6} \mathbf{i} \mathbf{1 2 6},
\end{aligned}
$$

where the 126, as before, generates only the right handed neutrino mass matrix. To study the consequences of these assumptions, we see that at the level of $S U(5)$, we have

\footnotetext{
${ }^{23}$ The couplings of the Higgs fields in the superpotential can be either renormalizable or non-renormalizable. See [758] for a nonrenormalizable example.
} 


$$
\begin{aligned}
W_{S U(5)}= & \frac{1}{2} Y_{i i}^{u} \mathbf{1 0}_{\mathbf{i}} \mathbf{1 0}_{\mathbf{i}} \mathbf{5}_{\mathbf{u}}+Y_{i i}^{v} \overline{\mathbf{5}}_{i} \mathbf{1}_{\mathbf{i}} \mathbf{5}_{\mathbf{u}} \\
& +Y_{i j}^{d} \mathbf{1 0}_{\mathbf{i}} \overline{\mathbf{5}}_{j} \overline{\mathbf{5}}_{d}+\frac{1}{2} M_{i i}^{R} \mathbf{1}_{\mathbf{i}} \mathbf{1}_{\mathbf{i}},
\end{aligned}
$$

where we have decomposed the $\mathbf{1 6}$ into $\mathbf{1 0}+\overline{\mathbf{5}}+\mathbf{1}$ and $\mathbf{5}_{\mathbf{u}}$ and $\overline{\mathbf{5}}_{d}$ are components of $\mathbf{1 0}_{\mathbf{u}}$ and $\Phi$ respectively. To have large mixing $\sim U_{\text {PMNS }}$ in $Y^{v}$ we see that the asymmetric matrix $Y^{d}$ should now give rise to both the CKM mixing as well as PMNS mixing. This is possible if

$V_{\mathrm{CKM}}^{T} Y^{d} U_{\mathrm{PMNS}}^{T}=Y_{\text {Diag }}^{d}$.

Therefore the $\mathbf{1 0}$ that contains the left handed downquarks would be rotated by the CKM matrix whereas the $\overline{5}$ that contains the left handed charged leptons would be rotated by the $U_{\mathrm{PMNS}}$ matrix to go into their respective mass bases [737, 758-760]. Thus we have the following relations in the basis where charged leptons and down quarks are diagonal:

$$
\begin{aligned}
& Y^{u}=V_{\mathrm{CKM}} Y_{\text {Diag }}^{u} V_{\mathrm{CKM}}^{T}, \\
& Y^{v}=U_{\text {PMNS }} Y_{\text {Diag }}^{u} .
\end{aligned}
$$

Using the seesaw formula of (3.41) and (5.76), we have

$M_{R}=\operatorname{Diag}\left\{\frac{m_{u}^{2}}{m_{v_{1}}}, \frac{m_{c}^{2}}{m_{v_{2}}}, \frac{m_{t}^{2}}{m_{v_{3}}}\right\}$.

We now turn our attention to lepton flavor violation in this case. The branching ratio, $B(\mu \rightarrow e, \gamma)$ would now depend on

$$
\left[Y^{v} Y^{\nu T}\right]_{21}=Y_{t}^{2} U_{\mu 3} U_{e 3}+Y_{c}^{2} U_{\mu 2} U_{e 2}+\mathcal{O}\left(h_{u}^{2}\right) .
$$

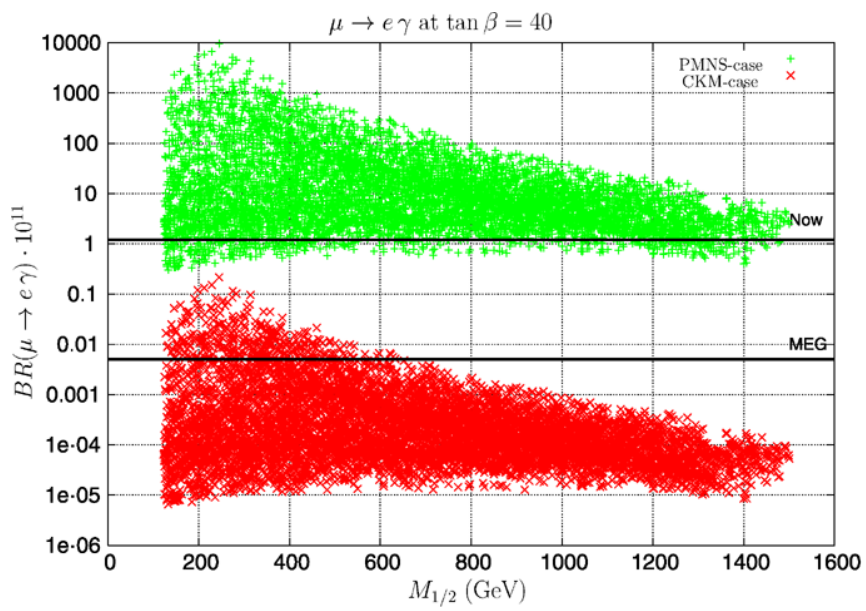

Fig. 34 Scatter plots of $B(\mu \rightarrow e, \gamma)$ (left) and $B(\tau \rightarrow \mu \gamma)$ (right) versus $M_{1 / 2}$ for $\tan \beta=40$, both for the (maximal) PMNS case with $\left|U_{e 3}\right|=0.07$ and the (minimal) CKM case. The plots were obtained by
It is clear from the above that in contrast to the CKM case, the dominant contribution to the off-diagonal entries depends on the unknown magnitude of the element $U_{e 3}$ [761]. If $U_{e 3}$ is close to its present limit $\sim 0.14$ [762] (or at least larger than $\left.\left(Y_{c}^{2} / Y_{t}^{2}\right) U_{e 2} \sim 4 \times 10^{-5}\right)$, the first term on the RHS of the (5.78) would dominate. Moreover, this would lead to large contributions to the off-diagonal entries in the slepton masses with $U_{\mu 3}$ of $\mathcal{O}(1)$. Thus, we have

$$
\left(m_{\tilde{L}}^{2}\right)_{21} \approx-\frac{3 m_{0}^{2}+A_{0}^{2}}{8 \pi^{2}} Y_{t}^{2} U_{e 3} U_{\mu 3} \ln \frac{M_{\mathrm{GUT}}}{M_{R_{3}}}+\mathcal{O}\left(Y_{c}^{2}\right) .
$$

This contribution is larger than the CKM case by a factor of $\left(U_{\mu 3} U_{e 3}\right) /\left(V_{t d} V_{t s}\right) \sim \mathcal{O}\left(10^{2}\right)$. This would mean about a factor $10^{4}$ times larger than the CKM case in $B(\mu \rightarrow e, \gamma)$. Such enhancement with respect to the CKM case is clearly shown in the scatter plots of Fig. 34, where the CKM case is compared with the PMNS case with $U_{e 3}=0.07$. The aim of the figure is to show the capability of MEG to probe the region of mSUGRA parameter space accessible to the LHC. In fact, the plots show the value of $B(\mu \rightarrow e, \gamma)$ obtained by scanning the parameter space in the large region $\left(0<m_{0}<5 \mathrm{TeV}, 0<M_{1 / 2}<1.5 \mathrm{TeV},-3 m_{0}<A_{0}<\right.$ $+3 m_{0}$, $\operatorname{sign}(\mu)$ ), and then keeping the points which give at least one squark lighter than $2.5 \mathrm{TeV}$ (so roughly accessible to the LHC). We see that in this "LHC accessible" region the maximal case (with $U_{e 3}=0.07$ ) is already excluded by the MEGA limit $\left(B(\mu \rightarrow e, \gamma)<1.2 \times 10^{-11}\right)$, and therefore MEG will constrain the parameter space far beyond the LHC sensitivity for this case. If $U_{e 3}$ is very small, i.e. either zero or $\lesssim\left(Y_{c}^{2} / Y_{t}^{2}\right) U_{e 2} \sim 4 \times 10^{-5}$, the second term $\propto Y_{c}^{2}$ in (5.78) would dominate, thus giving a strong suppression to the branching ratio. This could be not true, once RG effects

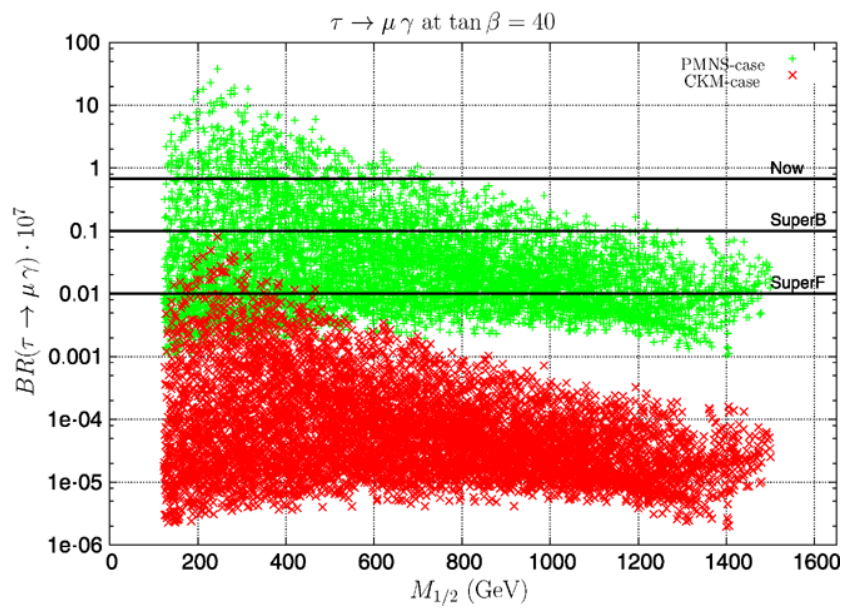

scanning the SUSY parameter space in the LHC accessible region (see the text for details) 
on $U_{e 3}$ itself $[258,722]$ are taken into account. The point is that the PMNS boundary condition (5.76) is valid at high scale. Thus, it is necessary to evolve the neutrino masses and mixing from the low energy scale, where measurements are performed, up to high energy. Such effect turns out to be not negligible in case of low energy $U_{e 3} \lesssim 10^{-3}$, giving a high energy constant enhancement of order $\mathcal{O}\left(10^{-3}\right)$ [688]. The consequence is that the term in (5.78) $\propto Y_{t}^{2}$ always dominates, giving a contribution to the branching ratio larger than the CKM case (which turns out to be really a "minimal" case) and bringing the most of the parameter space in the realm of MEG even for very small low energy values of $U_{e 3}[688,763]$.

The $\tau \rightarrow \mu$ transitions are instead $U_{e 3}$-independent probes of SUSY, whose importance was first pointed out in Ref. [732]. The off-diagonal entry in this case is given by:

$$
\left(m_{\tilde{L}}^{2}\right)_{32} \approx-\frac{3 m_{0}^{2}+A_{0}^{2}}{8 \pi^{2}} Y_{t}^{2} U_{\mu 3} U_{\tau 3} \ln \frac{M_{\mathrm{GUT}}}{M_{R_{3}}}+\mathcal{O}\left(Y_{c}^{2}\right) .
$$

In the $\tau \rightarrow \mu \gamma$ decay the situation is at the moment similarly constrained with respect to $\mu \rightarrow e \gamma$, if $U_{e 3}$ happens to be very small. The main difference is that $B(\tau \rightarrow \mu \gamma)$ does not depend on the value of $U_{e 3}$, so that $\tau \rightarrow \mu \gamma$ will be a promising complementary channel with respect to $\mu \rightarrow e \gamma$. As far as Beauty factories [191, 644, 764] are concerned, we see from Fig. 34, that even with the present bound it is possible to rule out part of LHC accessible region in the PMNS high $\tan \beta$ regime; the planned accuracy of the SuperKEKB [694] machine $\sim \mathcal{O}\left(10^{-8}\right)$ will allow to test much of high $\tan \beta$ region and will start probing the low $\tan \beta$ PMNS case, with a sensitivity to soft masses as high as $\left(m_{0}, m_{\tilde{g}}\right) \lesssim 900 \mathrm{GeV}$. The situation changes dramatically if one takes into account the possibility of a Super Flavor factory: taking the sensitivity of the most promising $\tau \rightarrow \mu \gamma$ process to $\sim \mathcal{O}\left(10^{-9}\right)$, the PMNS case will be nearly ruled out in the high $\tan \beta$ regime and severely constrained in the low $\tan \beta$ one; as for the CKM case we would enter the region of interest.

Let's finish with some remarks. Suppose that the LHC does find signals of low energy supersymmetry, then grand unification becomes a very appealing scenario, because of the successful unification of gauge couplings driven by the SUSY partners. Among SUSY-GUT models, an $S O(10)$ framework is much favored as it is the 'minimal' GUT to host all the fermions in a single representation and it accounts for the smallness of the observed neutrino masses by naturally including the see-saw mechanism. In the above we have addressed the issue by a generic benchmark analysis, within the ansatz that there is no fine-tuning in the neutrino Yukawa sector. We can state that LFV experiments should be able to tell us much about the structure of such a SUSYGUT scenario. If they detect LFV processes, by their rate and exploiting the interplay between different experiments, we would be able to get hints of the structure of the unknown neutrinos' Yukawa's. On the contrary, in the case that both MEG and a future Super Flavor factory happen not to see any LFV process, only two possibilities should be left: (i) the minimal mixing, low $\tan \beta$ scenario; (ii) mSUGRA $S O(10)$ see-saw without fine-tuned $Y_{v}$ couplings is not a viable framework of physics beyond the standard model.

Actually one should remark that LFV experiments will be able to falsify some of above scenarios even in regions of the mSUGRA parameter space that are beyond the reach of LHC experiments. In this sense, the power of LFV experiments of testing/discriminating among different SUSYGUTs models results very interesting and highly complementary to the direct searches at the LHC.

\subsubsection{LFV, QFV and CPV observables in GUTs and their correlations}

In a SUSY grand unified theory (GUT), quarks and leptons sit in same multiplets and are transformed ones into the others through GUT symmetry transformations. If the energy scale where the SUSY breaking terms are transmitted to the visible sector is larger then the GUT scale, as in the case of gravity mediation, such breaking terms, and in particular the sfermion mass matrices, will have to respect the underlying GUT symmetry. Hence, as already discussed in Sect. 5.3.1, the quark-lepton unification seeps also into the SUSY breaking soft sector. If the soft SUSY breaking terms respect boundary conditions which are subject to the GUT symmetry to start with, we generally expect the presence of relations among the (bilinear and trilinear) scalar terms in the hadronic and leptonic sectors [165, 739]. Such relations hold true at the (superlarge) energy scale where the correct symmetry of the theory is the GUT symmetry. After its breaking, the mentioned relations will undergo corrections which are computable through the appropriate RGE's which are related to the specific structure of the theory between the GUT and the electroweak scale (for instance, new Yukawa couplings due to the presence of RH neutrinos acting down to the RH neutrino mass scale, presence of a symmetry breaking chain with the appearance of new symmetries at intermediate scales, etc.). As a result of such a computable running, we can infer the correlations between the softly SUSY breaking hadronic and leptonic MIs at the low scale where FCNC tests are performed. Moreover, given that a common SUSY soft breaking scalar term of $\mathcal{L}_{\text {soft }}$ at scales close to $M_{\text {Planck }}$ can give rise to RG-induced $\delta^{q}$ 's and $\delta^{l}$ 's at the weak scale, one may envisage the possibility to make use of the FCNC constraints on such low energy $\delta$ 's to infer bounds on the soft breaking parameters of the original supergravity Lagrangian $\left(\mathcal{L}_{\text {sugra }}\right)$. Indeed, for each scalar soft 
Table 14 Links between various transitions between up-type, down-type quarks and charged leptons for $S U(5) \cdot m_{\tilde{f}}^{2}$ refers to the average mass for the sfermion $f$,

$m_{\tilde{Q}_{\text {avg }}}^{2}=\sqrt{m_{\tilde{Q}^{2}}^{2} m_{\tilde{d}^{c}}^{2}}$ and

$m_{\tilde{L}_{\mathrm{avg}}}^{2}=\sqrt{m_{\tilde{L}^{2}}^{2} m_{\tilde{e}^{c}}^{2}}$
Boundary conditions at $M_{\mathrm{GUT}}$

$$
\begin{aligned}
& \left(\delta_{i j}^{u}\right)_{R R} \approx\left(m_{\tilde{e}^{c}}^{2} / m_{\tilde{u}^{c}}^{2}\right)\left(\delta_{i j}^{l}\right)_{R R} \\
& \left(\delta_{i j}^{q}\right)_{L L} \approx\left(m_{\tilde{e}^{c}}^{2} / m_{\tilde{Q}^{2}}^{2}\right)\left(\delta_{i j}^{l}\right)_{R R} \\
& \left(\delta_{i j}^{d}\right)_{R R} \approx\left(m_{\tilde{L}_{L}}^{2} / m_{\tilde{d}^{c}}^{2}\left(\delta_{i j}^{l}\right)_{L L}\right. \\
& \left(\delta_{i j}^{d}\right)_{L R} \approx\left(m_{\tilde{L}_{\text {avg }}}^{2} / m_{\tilde{Q}_{\text {avg }}}^{2}\right)\left(m_{b} / m_{\tau}\right)\left(\delta_{i j}^{l}\right)_{L R}^{\star} \\
& \hline
\end{aligned}
$$

parameter of $\mathcal{L}_{\text {sugra }}$ one can ascertain whether the hadronic or the leptonic corresponding bound at the weak scale yields the strongest constraint at the large scale [165].

Let us consider the scalar soft breaking sector of the MSSM:

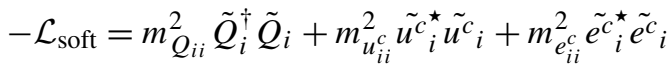

$$
\begin{aligned}
& +m_{d_{i i}^{c}}^{2} \tilde{d}_{i}^{\star} \tilde{d}^{c}{ }_{i}+m_{L_{i i}}^{2} \tilde{L}_{i}^{\dagger} \tilde{L}_{i}+m_{H_{1}}^{2} H_{1}^{\dagger} H_{1} \\
& +m_{H_{2}}^{2} H_{2}^{\dagger} H_{2}+A_{i j}^{u} \tilde{Q}_{i} \tilde{u}^{c}{ }_{j} H_{2}+A_{i j}^{d} \tilde{Q}_{i} \tilde{d}^{c}{ }_{j} H_{1} \\
& +A_{i j}^{e} \tilde{L}_{i} \tilde{e}^{c}{ }_{j} H_{1}+\left(\Delta_{i j}^{l}\right)_{L L} \tilde{L}_{i}^{\dagger} \tilde{L}_{j}+\left(\Delta_{i j}^{e}\right)_{R R} \tilde{e}^{\tilde{e}^{\star}}{ }_{i}^{e^{c}}{ }_{j} \\
& +\left(\Delta_{i j}^{q}\right)_{L L} \tilde{Q}_{i}^{\dagger} \tilde{Q}_{j}+\left(\Delta_{i j}^{u}\right)_{R R}{\tilde{u^{c}}}_{i}^{\star}{\tilde{u^{c}}}_{j} \\
& +\left(\Delta_{i j}^{d}\right)_{R R} \tilde{d}^{c^{\star}}{ }_{i} \tilde{d}^{c}{ }_{j}+\left(\Delta_{i j}^{e}\right)_{L R}{\tilde{L_{L}}}_{i}^{\star} \tilde{e}^{c}{ }_{j} \\
& +\left(\Delta_{i j}^{u}\right)_{L R}{\tilde{u_{L}}}_{i}^{\star} \tilde{u}^{c}{ }_{j}+\left(\Delta_{i j}^{d}\right)_{L R}{\tilde{d_{L}}}_{L_{i}}^{\star} \tilde{d}^{c}{ }_{j}+\cdots,
\end{aligned}
$$

where we have explicitly written down the various offdiagonal entries of the soft SUSY breaking matrices. Consider now that $S U(5)$ is the relevant symmetry at the scale where the above soft terms firstly show up. Then, taking into account that matter is organized into the $S U(5)$ representations $\mathbf{1 0}=\left(q, u^{c}, e^{c}\right)$ and $\overline{\mathbf{5}}=\left(l, d^{c}\right)$, one obtains the following relations

$m_{Q}^{2}=m_{\tilde{e}^{c}}^{2}=m_{\tilde{u}^{c}}^{2}=m_{\mathbf{1 0}}^{2}$,

$m_{\tilde{d}^{c}}^{2}=m_{L}^{2}=m_{\frac{\overline{5}}{2}}^{2}$

$A_{i j}^{e}=A_{j i}^{d}$.

These equations for matrices in flavor space lead to relations between the slepton and squark flavor violating off-diagonal entries $\Delta_{i j}$. These are:

$$
\begin{aligned}
& \left(\Delta_{i j}^{u}\right)_{L L}=\left(\Delta_{i j}^{u}\right)_{R R}=\left(\Delta_{i j}^{d}\right)_{L L}=\left(\Delta_{i j}^{l}\right)_{R R}, \\
& \left(\Delta_{i j}^{d}\right)_{R R}=\left(\Delta_{i j}^{l}\right)_{L L}, \\
& \left(\Delta_{i j}^{d}\right)_{L R}=\left(\Delta_{j i}^{l}\right)_{L R}=\left(\Delta_{i j}^{l}\right)_{R L}^{\star} .
\end{aligned}
$$

These GUT correlations among hadronic and leptonic scalar soft terms are summarized in the second column of Table 14.
Table 15 Links between various transitions between up-type, downtype quarks and charged leptons for PS/SO(10) type models

\begin{tabular}{ll}
\hline Relations at weak-scale & Boundary conditions at $M_{\mathrm{GUT}}$ \\
\hline$\left(\delta_{i j}^{u}\right)_{R R} \approx\left(m_{\tilde{e}^{c}}^{2} / m_{\tilde{u}^{c}}^{2}\right)\left(\delta_{i j}^{l}\right)_{R R}$ & $m_{\tilde{u}^{c}}^{2}(0)=m_{\tilde{e}^{c}}^{2}(0)$ \\
$\left(\delta_{i j}^{q}\right)_{L L} \approx\left(m_{\tilde{L}}^{2} / m_{\tilde{Q}}^{2}\right)\left(\delta_{i j}^{l}\right)_{L L}$ & $m_{\tilde{Q}}^{2}(0)=m_{\tilde{L}^{2}}^{2}(0)$ \\
\hline
\end{tabular}

Assuming that no new sources of flavor structure are present from the $S U(5)$ scale down to the electroweak scale, apart from the usual SM CKM one, one infers the relations in the first column of Table 14 at low scale. Here we have taken into account that due to their different gauge couplings "average" (diagonal) squark and slepton masses acquire different values at the electroweak scale.

Two comments are in order when looking at Table 14. First, the boundary conditions on the sfermion masses at the GUT scale (last column in Table 14) imply that the squark masses are always going to be larger at the weak scale compared to the slepton masses due to the participation of the QCD coupling in the RGEs. As a second remark, notice that the relations between hadronic and leptonic $\delta \mathrm{MI}$ in Table 14 always exhibit opposite "chiralities", i.e. LL insertions are related to RR ones and vice-versa. This stems from the arrangement of the different fermion chiralities in $S U(5)$ five- and ten-plets (as it clearly appears from the final column in Table 14). This restriction can easily be overcome if we move from $S U(5)$ to left-right symmetric unified models like $S O(10)$ or the Pati-Salam (PS) case (we exhibit the corresponding GUT boundary conditions and $\delta \mathrm{MI}$ at the electroweak scale in Table 15).

So far we have confined the discussion within the simple $S U(5)$ model, without the presence of any extra particles like right handed $(\mathrm{RH})$ neutrinos. In the presence of $\mathrm{RH}$ neutrinos, one can envisage of two scenarios [164]: (a) with either very small neutrino Dirac Yukawa couplings and/or very small mixing present in the neutrino Dirac Yukawa matrix, (b) Large Yukawa and large mixing in the neutrino sector. In the latter case, (5.83)-(5.85) are not valid at all scales in general, as large RGE effects can significantly modify the sleptonic flavor structure while keeping the squark sector essentially unmodified; thus essentially breaking the GUT symmetric relations. In the former case where the neutrino Dirac Yukawa couplings are tiny and do not significantly 

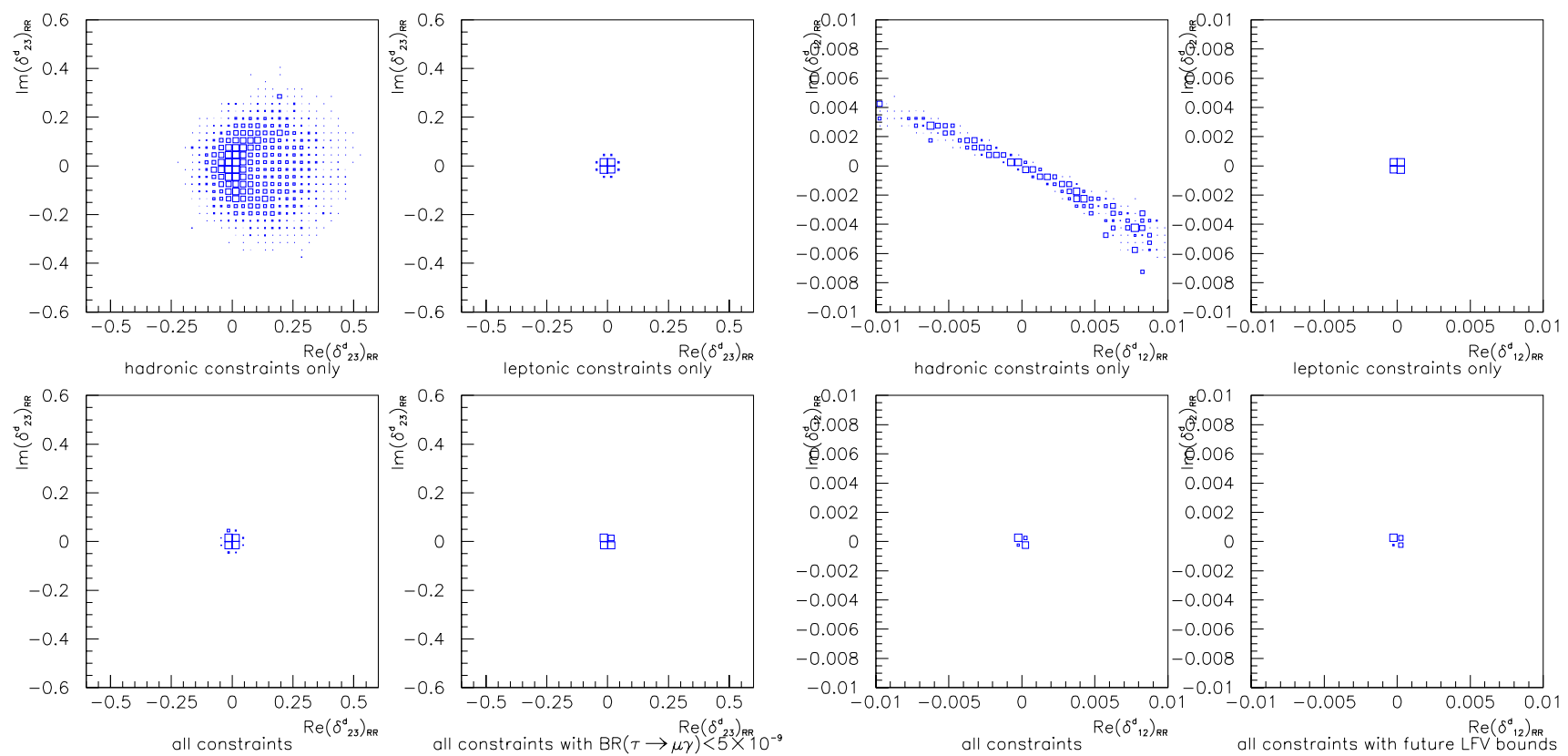

Fig. 35 Left four panels: allowed region for $\left(\delta_{23}^{d}\right)_{R R}$ using constraints as indicated. Right four panels: the same for $\left(\delta_{12}^{d}\right)_{R R}$. For the parameter space considered, please see the text

modify the sleptonic flavor structure, the GUT symmetric relations are expected to be valid at the weak scale. However, in both cases it is possible to say that there exists a bound on the hadronic $\delta$ parameters of the form [739]:

$\left|\left(\delta_{i j}^{d}\right)_{R R}\right| \geq \frac{m_{\tilde{L}^{2}}^{2}}{m_{\tilde{d}^{c}}^{2}}\left|\left(\delta_{i j}^{l}\right)_{L L}\right|$.

The situation is different if we try to translate the bound from quark to lepton MIs. An hadronic MI bound at low energy leads, after RGE evolution, to a bound on the corresponding grand unified $\mathrm{MI}$ at $M_{\mathrm{GUT}}$, applying both to slepton and squark mass matrices. However, if the neutrino Yukawa couplings have sizable off-diagonal entries, the RGE running from $M_{\mathrm{GUT}}$ to $M_{W}$ could still generate a new contribution to the slepton MI that exceeds this GUT bound. Therefore hadronic bounds cannot be translated to leptons unless we make some additional assumptions on the neutrino Yukawa matrices. On general grounds, given that SM contributions in the lepton sector are absent and that the branching ratios of leptonic processes constrain only the modulus of the MIs, it turns out that all the MI bounds arising from the lepton sector are circles in the $\operatorname{Re}\left(\delta_{i j}^{d}\right)_{\mathrm{AB}}-\operatorname{Im}\left(\delta_{i j}^{d}\right)_{\mathrm{AB}}$ plane and are centered at the origin.

In the following the effect of leptonic bounds on the quark mass insertions are reviewed, following the results presented in [165], where constraints on $\delta$ s were studied scanning the mSUGRA parameter space in the ranges: $M_{1 / 2} \leq 160 \mathrm{GeV}, m_{0} \leq 380 \mathrm{GeV},\left|A_{0}\right| \leq 3 m_{0}$ and $5<$ $\tan \beta<15$. For instance, in presence of a $\left(\Delta_{23}^{d}\right)_{L R}$ at the
GUT scale, this would have effects both in the $\tau \rightarrow \mu \gamma$ and $b \rightarrow s \gamma$ decays. Using $\left(\delta_{23}^{d}\right)_{L R} \lesssim\left(m_{b} / m_{\tau}\right)\left(m_{\tilde{l}}^{2} / m_{\tilde{q}}^{2}\right) \times$ $\left(\delta_{23}^{l}\right)_{R L}$, a bound on $\left(\delta_{23}^{l}\right)_{R L}$ from the $\tau \rightarrow \mu \gamma$ decay translates into a bound on $\left(\Delta_{23}^{d}\right)_{L R}$ (neglecting the effects of neutrino Yukawa's the inequality transforms into equality). Thus, leptonic processes set a bound on the SUSY contributions to $B\left(B \rightarrow X_{S} \gamma\right)$. However, it turns out that the present leptonic bounds have no effect on the $\left(\delta_{23}^{d}\right)_{L R}$ couplings. This is due both to the existence of strong hadronic bounds from $b \rightarrow s \gamma$ and CP asymmetries and to the relatively weak leptonic bounds here.

Similarly, in presence of a $\left(\Delta_{23}^{d}\right)_{R R}$ at the GUT scale, the corresponding MIs at the electroweak scale are $\left(\delta_{23}^{d}\right)_{R R}$ and $\left(\delta_{23}^{l}\right)_{L L}$ that contribute to $\Delta M_{B_{s}}$ and $\tau \rightarrow \mu \gamma$ respectively (the impact of $\left(\Delta_{23}^{d}\right)_{R R}$ on $b \rightarrow s \gamma$ and $b \rightarrow s \ell^{+} \ell^{-}$is not relevant because of the absence of interference between SUSY and SM contributions). In Fig. 35 the allowed values of $\operatorname{Re}\left(\delta_{23}^{d}\right)_{R R}$ and $\operatorname{Im}\left(\delta_{23}^{d}\right)_{R R}$ with the different constraints are shown. The leptonic constraints are quite effective as the bound on the $B(\tau \rightarrow \mu \gamma)$ from $B$-factories is already very stringent, while the recent measurement of $\Delta M_{B_{s}}$ is less constraining. The plots correspond to $5<\tan \beta<15$, thus, the absolute bound on $\left(\delta_{23}^{l}\right)_{L L}$ is set by $\tan \beta=5$ and it scales with $\tan \beta$ as $\left(\delta_{23}^{l}\right)_{L L} \sim(5 / \tan \beta) .{ }^{24}$

\footnotetext{
${ }^{24}$ Sizable SUSY contributions to $\Delta M_{B_{S}}$ are still possible from the Higgs sector in the large $\tan \beta$ regime both within [765-767] and also beyond [768] the Minimal Flavor Violating framework. However, for the considered parameter space, the above effects are completely negligible.
} 
As in the LR sector, in the LL one, there is no appreciable improvement from the inclusion of leptonic constraints. In fact, $\tau \rightarrow \mu \gamma$ is not effective to constrain $\left(\delta_{23}^{l}\right)_{R R}$, i.e. the leptonic MI related to $\left(\delta_{23}^{d}\right)_{L L}$ in this SUSY-GUTs scheme, in large portions of the parameter space because of strong cancellations among amplitudes. The analysis of the constraints on the different $\left(\delta_{13}^{d}\right)$ MIs gives similar results to that of the $\left(\delta_{23}^{d}\right)$ MIs. In this case, the hadronic constraints come mainly from $\Delta M_{B_{d}}$ and the different $\mathrm{CP}$ asymmetries measured at B-factories, while the leptonic bounds are due to the decay $\tau \rightarrow e \gamma$.

Coming to the 1-2 sector, let's see, as an example, the allowed values of $\operatorname{Re}\left(\delta_{12}^{d}\right)_{R R}$ and $\operatorname{Im}\left(\delta_{12}^{d}\right)_{R R}$. In this case, as it appears from Fig. 35, leptonic constraints, already using the present limit on $B(\mu \rightarrow e \gamma)$, are competitive and constrain the direction in which the constraint from $\varepsilon_{K}$ is not effective (upper left plot). Similarly in the $L R$ sector, even if the hadronic bounds coming from $\varepsilon^{\prime} / \varepsilon$ are quite stringent, the bounds from $\mu \rightarrow e \gamma$ are even more effective, while the $L L$ sector results less constrained by leptonic processes, as an effect of the cancellations that $\mu \rightarrow e \gamma$ decay suffers in the $R R$ leptonic sector.

\subsection{R-parity violation}

\subsubsection{Introduction}

In supersymmetric extensions of the standard model (SM), baryon and lepton numbers are no longer automatically protected. This is the main reason for introducing R-parity. $\mathrm{R}$-parity is associated with a $Z_{2}$ subgroup of the group of continuous $U(1)$ transformations acting on the gauge superfields and the two chiral doublet Higgs superfields $H_{d}$ and $H_{u}$, with their definition extended to quark and lepton superfields so that quarks and leptons carry $R=0$ and squarks and sleptons $R= \pm 1$. One can express Rparity in terms of spin $S$, baryon $B$ and lepton $L$ number [769]:

R-parity $=(-1)^{2 S}(-1)^{3(B-L)}$.

Taking into account the important phenomenological differences between models with and without R-parity, it is worth studying if and how R-parity can be broken. One of the main reasons to introduce R-parity is avoiding proton decay. However there are in principle other discrete or continuous symmetries that can protect proton decay while allowing for some R-parity violating couplings. In the absence of R-parity, R-parity odd terms allowed by renormalizability and gauge invariance [150] must be included in the superpotential of the minimal supersymmetric standard model,

$$
\begin{aligned}
W_{R p}= & \mu_{i} H_{u} L_{i}+\frac{1}{2} \lambda_{i j k} L_{i} L_{j} E_{k}^{c}+\lambda_{i j k}^{\prime} L_{i} Q_{j} D_{k}^{c} \\
& +\frac{1}{2} \lambda_{i j k}^{\prime \prime} U_{i}^{c} D_{j}^{c} D_{k}^{c},
\end{aligned}
$$

where there is summation over the generation indices $i, j, k=1,2,3$, and summation over gauge indices is understood. One has for example $L_{i} L_{j} E_{k}^{c} \equiv\left(\epsilon_{a b} L_{i}^{a} L_{j}^{b}\right) E_{k}^{c}=$ $\left(N_{i} E_{j}-E_{i} N_{j}\right) E_{k}^{c}$ and $U_{i}^{c} D_{j}^{c} D_{k}^{c} \equiv \epsilon_{\alpha \beta \gamma} U_{i}^{\alpha c} D_{j}^{\beta c} D_{k}^{\gamma c}$, where $a, b=1,2$ are $S U(2)_{L}$ indices, $\alpha, \beta, \gamma=1,2,3$ are $S U(3)_{C}$ indices, and $\epsilon_{a b}$ and $\epsilon_{\alpha \beta \gamma}$ are totally antisymmetric tensors (with $\epsilon_{12}=\epsilon_{123}=+1$ ). Gauge invariance enforces antisymmetry of the $\lambda_{i j k}$ couplings in their first two indices and antisymmetry of the $\lambda_{i j k}^{\prime \prime}$ couplings in their last two indices,

$\lambda_{i j k}=-\lambda_{j i k}$,

$\lambda_{i j k}^{\prime \prime}=-\lambda_{i k j}^{\prime \prime}$.

The bilinear terms $\mu_{i} H_{u} L_{i}$ in (5.88) can be rotated away from the superpotential upon suitably redefining the lepton and Higgs superfields. However, in the presence of generic soft supersymmetry breaking terms of dimension two, bilinear R-parity violation will reappear. The fact that one can make $\mu_{i}=0$ in (5.88) does not mean that the Higgs-lepton mixing associated with bilinear R-parity breaking is unphysical, but rather that there is not a unique way of parameterizing it. If R-parity is violated in the leptonic sector, no quantum numbers differentiate between lepton and Higgs superfields, and they consequently mix with each other [770]. The R-parity violation in the baryonic sector does not imply lepton flavor violation, and we do not consider such option here.

A general consequence of R-parity violation is that unless the relevant couplings are negligibly small, the supersymmetric model does not have a dark matter candidate. Thus experimental studies on dark matter will also shed light on R-parity violation.

\subsubsection{Limits on couplings}

Limits on R-parity violating couplings can be obtained by direct searches at colliders or requiring that the R-parity violating contribution to a given observable does not exceed the limit imposed by the precision of the experimental measurement.

On the collider side R-parity violation implies the possibility of the creation, decay or exchange of single sparticles, thus allowing new decay channels. For example, even for relatively small R-parity violating interactions, the decay of the lightest supersymmetric particle will lead to collider events departing considerably from the characteristic missing momentum signal of R-parity conserving theories. In absence of definite theoretical predictions for the values of the 
45 independent trilinear Yukawa couplings $\Lambda\left(\lambda_{i j k}, \lambda_{i j k}^{\prime}\right.$ and $\left.\lambda_{i j k}^{\prime \prime}\right)$, it is necessary in practice to assume a strong hierarchy among the couplings. A simplifying assumption widely used for the search at colliders is to postulate the existence of a single dominant R-parity violating coupling. When discussing specific bounds, it is necessary to choose a definite basis for quark and lepton superfields. Often it is understood that the single coupling dominance hypothesis applies in the mass eigenstate basis. It can be more natural to apply this hypothesis in the weak eigenstate basis when dealing with models in which the hierarchy among couplings originates from a flavor theory. In this case, a single process allows one to constrain several couplings, provided one has some knowledge of the rotations linking the weak eigenstate and mass eigenstate bases. Indirect bounds from loop processes typically lead to bounds on the products of two most important R-parity violating couplings, or on the sum of products of two couplings. The limits on single dominant couplings, and on products of couplings, as well as a more complete list of references, are collected in [771].

\subsubsection{Spontaneous R-parity breaking}

The spontaneous breaking of R-parity is characterized by an R-parity invariant Lagrangian leading to non-vanishing VEVs for some R-parity odd scalar field, which in turn generates R-parity violating terms. Such a spontaneous breakdown of R-parity generally also entails the breaking of the global $U(1)$ lepton number symmetry $L$ which implies the existence of a massless Nambu-Goldstone real pseudoscalar boson $J$, the majoron. Another light scalar particle, denoted $\rho$, generally accompanies the majoron in the supersymmetric models. If the $U(1)$ symmetry is also explicitly broken by interaction terms in the Lagrangian, both of these particles acquire finite masses. The most severe constraints on the models with a spontaneous R-parity breaking, arise in the cases where the majoron carries electroweak gauge charges and hence is coupled to the $Z$ bosons and to quarks and leptons. The non-singlet components contribute to the $Z$ boson invisible width by an amount of one-half that a single light neutrino, $\delta \Gamma_{\text {inv }}^{Z} / 6 \simeq 83 \mathrm{MeV}$. To suppress the non-singlet components one must allow either for sufficiently small sneutrino VEVs, $v_{L} / M_{Z} \ll 1$, or for some large hierarchy of scales between $v_{L}$ and the VEV parameters associated with additional electroweak singlet scalar fields [772].

However, it is not necessary that models with spontaneous R-parity violation have a majoron. Models without a majoron include a class of models with triplet Higgses, where $B-L$ is a gauge symmetry, which is necessarily spontaneously broken unless effects of non-renormalizable terms or some additional new fields are included [773]. An interesting experimental signal in these models may be a relatively light doubly charged scalar, which decays dominantly to same charge leptons (not necessarily of the same generation) [774]. Another possibility for a model without a majoron is a model where the lepton number is broken by two units explicitly, in which case the spontaneous breaking by one unit (which breaks the R-parity) does not lead to a majoron [775]. The interactions in spontaneously R-parity breaking models through the lepton number violation closely resemble explicitly R-parity breaking models with only bilinear R-parity violation. In the case of spontaneous breaking, the parameters which are free in the model with only bilinear couplings are related to each other via the sneutrino vacuum expectation value (VEV). Thus a constraint from one process affects availability of the other processes. Example bounds for such a model can be found in [776].

It is worth emphasizing that choosing single coupling dominance in the case of spontaneous breaking is not possible and in this sense, the models with spontaneous breaking are more predictive than those without.

\subsubsection{Neutrino sector}

The presence of non-zero couplings $\lambda_{i j k}, \lambda_{i j k}^{\prime}$ or bilinear $\mathrm{R}$-parity violating parameters implies the generation of neutrino masses and mixing [777]. This is an interesting feature of R-parity violating models, but it can also be a problem, since the contribution of R-parity violating couplings may exceed by orders of magnitude the experimental bounds. Two types of contributions can be distinguished: tree-level or loop contributions.

The tree-level contributions are due to bilinear R-parity violation terms which induce a mixing between neutrinos and neutralinos [778]. This gives a massive neutrino state at tree level. When quantum corrections are included, all three neutrinos acquire a mass. The tree-level contribution arising from the neutrino-neutralino mixing can be understood, in the limit of small neutrino-neutralino mixing, as a sort of seesaw mechanism, in which the neutral gauginos and higgsinos play the role of the right handed neutrinos.

The loop contributions are induced by the trilinear R-parity violating couplings $\lambda_{i j k}$ and $\lambda_{i j k}^{\prime}$ and by bilinear $\mathrm{R}$-parity violating parameters [779]. If bilinear R-parity violation is strongly suppressed one can concentrate on the diagrams involving trilinear R-parity violating couplings only. The trilinear couplings $\lambda_{i j k}$ and $\lambda_{i j k}^{\prime}$ contribute to each entry of the neutrino mass matrix through the lepton-slepton and quark-squark loops. The neutrino mass matrix depends therefore on a large number of trilinear R-parity violating couplings. In order to obtain a predictive model, one has to make assumptions on the structure of the trilinear couplings. In general, however, the bilinear R-parity violation contribution cannot be neglected. The presence of bilinear 
terms drastically modifies the calculation of one loop neutrino masses. The neutrino mass matrix receives contributions already at tree level, as discussed above, and moreover in addition to the lepton-slepton and quark-squark loops, one loop diagrams involving insertions of bilinear R-parity violating masses or slepton VEVs must be considered. One should note that the bilinear R-parity violating terms, if not suppressed, give too large loop contributions to neutrino masses.

The scenario known as bilinear R-parity violation $(\mathrm{BRpV})$ corresponds to the explicit introduction of the three mass parameters $\mu_{i}$ in the first term in (5.88), without referring to their origin, and assuming that all the trilinear parameters are zero. The $\mu_{i}$ terms introduce tree-level mixing between the Higgs and lepton superfields. Therefore, they violate R-parity and lepton number, and contribute to the breaking of the $S U(2)$ symmetry by the induction of sneutrino vacuum expectation values $v_{i}$. As it was mentioned before, the mixing between neutralinos and neutrinos leads to an effective tree-level neutrino mass matrix of the form,

$m_{\nu}^{0 i j}=\frac{M_{1} g^{2}+M_{2} g^{2}}{4 \operatorname{det} M_{\chi^{0}}} \Lambda_{i} \Lambda_{j}$,

where the parameters $\Lambda_{i}=\mu v_{i}+\mu_{i} v_{d}$ are proportional to the sneutrino vacuum expectation values in the basis where the $\mu_{i}$ terms are removed from the superpotential. Due to the symmetry of this mass matrix, only one neutrino acquires a mass. Once quantum corrections are included, this symmetry is broken, and the effective neutrino mass matrix takes the form [780],

$m_{v}^{i j}=A \Lambda_{i} \Lambda_{j}+B\left(\Lambda_{i} \epsilon_{j}+\Lambda_{j} \epsilon_{i}\right)+C \epsilon_{i} \epsilon_{j}$.

If the tree-level contribution dominates, as for example in SUGRA models with low values of $\tan \beta$, the atmospheric mass scale is given at tree level, and the solar mass scale is generated at one loop, explaining the hierarchy between them. Most of the time, the dominant loop in SUGRA is the one formed with bottom quarks and squarks, followed in importance by loops with charginos and neutralinos. In the tree-level dominance case the atmospheric mixing angle is well approximated by $\tan ^{2} \theta_{\mathrm{atm}}=\Lambda_{2}^{2} / \Lambda_{3}^{2}$, and the reactor angle by $\tan ^{2} \theta_{13}=\Lambda_{1}^{2} /\left(\Lambda_{2}^{2}+\Lambda_{3}^{2}\right)$. In this case, the smallness of the reactor angle is achieved with a small value of $\Lambda_{1}$, and the maximal mixing in the atmospheric sector with a similar value for $\Lambda_{2}$ and $\Lambda_{3}$. Supergravity scenarios where tree-level contribution does not dominate can also be found [781], in which case the previous approximations for the angles are not valid.

\subsubsection{Lepton flavor violating processes at low energies}

Many processes, which are either rare or forbidden in the R-parity conserving model, become possible when interactions following from the superpotential $W_{R p}$ in (5.88) are available. These interactions include tree-level couplings between different lepton or quark generations, as well as treelevel couplings between leptons and quarks, or leptons and Higgses.

In addition to the trilinear couplings $\lambda$ and $\lambda^{\prime}$, bilinear couplings or spontaneous R-parity breaking contribute to the lepton flavor violating processes mentioned below through mixing.

For references about this section, see Ref. [771].

- $l_{i} \rightarrow l_{j} \gamma, l_{i} \rightarrow l_{j} l_{k} l_{m}$, and $\mu-e$-conversion, and semileptonic decays of $\tau$-leptons. The rare decays of leptons to lighter leptons are excellent probes of new physics, because they do not involve any hadronic uncertainties. Both the lepton flavor violating trilinear $\lambda$ - and $\lambda^{\prime}$ type couplings give rise to LFV decays $l_{i} \rightarrow l_{j} \gamma$ (loop level process with $\tilde{v}-l, v-\tilde{l}$, or $\tilde{q}-q^{\prime}$ in the loop), $l_{i} \rightarrow$ $l_{j} l_{k} l_{m}$ (tree-level process via $\tilde{v}$ or $\tilde{l}$ ), as well as for $\mu-e$ conversion. In these processes, two non-vanishing $\Lambda$ couplings are needed and usual approach is to assume a dominant product of two couplings, when determining bounds on couplings. In the $\mu-e$-conversion, certain pairs of couplings can be probed only in the loop-level process, mediated by virtual $\gamma$ or $Z$, which are logarithmically enhanced compared to $\mu \rightarrow e \gamma$ [185]. The hadronic contributions to the $\mu-e$-conversion in nuclei make the theoretical error larger than in the decays without hadrons. The relatively large mass of $\tau$ allows for new semileptonic decay modes for $\tau$. The bounds from these processes vary between $\Lambda \sim \mathcal{O}\left(10^{-4}-10^{-1}\right)$ for $100 \mathrm{GeV}$ fermion masses, and they scale as mass $^{2}$.

The experimental accuracies of the processes mentioned above are expected to increase considerably in the coming years.

- Leptonic and semileptonic decays of hadrons and top quarks. R-parity violating couplings $\lambda_{i j k}^{\prime}$ allow for lepton flavor violating decays of hadrons, e.g. $K_{L} \rightarrow e^{ \pm} \mu^{\mp}$, $B_{d} \rightarrow \mu^{+} \tau^{-}, K^{+} \rightarrow \pi^{+} v_{i} v_{j}$ [782], as well as semileptonic LFV top decays, e.g. $t \rightarrow \tilde{\tau}^{+} b$, if kinematically allowed. The sensitivity on the couplings is restricted by the theoretical uncertainties in hadronic contributions. For $100 \mathrm{GeV}$ sfermions, the bounds are $\Lambda \sim \mathcal{O}\left(10^{-4}-10^{-1}\right)$.

\subsubsection{Anomalous muon magnetic moment $a_{\mu}$ and electron electric dipole moment}

$\Lambda$ couplings affect leptons also through contributions to dipole moments. The experimental measurement of $a_{\mu}$ is quite precise. The theoretical calculation of the standard model contribution to $a_{\mu}$ contains still uncertainty, which prevents exact comparison with measurement. The contribution of R-parity violation on $a_{\mu}$ is small, and constrained by tiny neutrino masses. 
Contribution from complex $\Lambda$ to electron EDM could be large for large phases. The one loop contribution involving both bilinear and trilinear couplings is sizable for electron EDM, while one loop terms with only trilinear terms are suppressed by neutrino masses.

\subsubsection{Collider signatures}

The main advantage of collider studies compared to the low energy probes is that the particles can be directly produced, and thus their masses and couplings can be experimentally measured.

A major difference between R-parity conserving and breaking models from the detection point of view is the amount of missing energy. If R-parity is violated, the supersymmetric particles decay to the SM particles leaving little or no missing energy. Decays of sparticles through $\lambda$ and $\lambda^{\prime}$-type couplings lead to multi-lepton final states, and $\lambda^{\prime}$ and $\lambda^{\prime \prime}$ to multi-jet final states. Sparticles can decay first via the R-parity conserving couplings to the lightest supersymmetric particle (LSP), which then decays via R-parity violating couplings. If e.g. a neutralino is the LSP, it may be a cascade decay product of a sfermion, chargino, or a heavier neutralino. Thus typically one gets a larger number of jets or leptons in the final state in R-parity violating than in the R-parity conserving decay. The sparticles can also decay directly to the standard model fermions via $\lambda, \lambda^{\prime}$, or $\lambda^{\prime \prime}$ couplings. Assuming all the supersymmetric particles decay inside the detector, a consequence of the decay of the LSP is that the amount of missing energy when R-parity is violated is considerably lower than in the R-parity conserving case, and only neutrinos carry the missing energy. When R-parity is violated, the LSP is not stable and need not be neutral. If then the coupling through which the LSP decays is suppressed, a long lived possibly charged particle appears, leaving a heavily ionizing, easily detectable charged track in the detector.

A simplifying assumption for the search strategy at colliders is to postulate the existence of a single dominant $\mathrm{R}$-parity violating coupling. In case a non-vanishing coupling does exist with a magnitude leading to distinct phenomenology at colliders, a direct sensitivity to a long-lived LSP might be provided by the observation of displaced vertices in an intermediate range of coupling values up to $\mathcal{O}\left(10^{-5}-10^{-4}\right)$. For larger $\Lambda$ values the presence of R-parity violating supersymmetry will become manifest through the decay of short-lived sparticles produced by pair via gauge couplings. A possible search strategy in such cases consists of neglecting R-parity violating contributions at production in non-resonant processes. This is valid provided that the interaction strength remains sufficiently small compared to electromagnetic or weak interaction strengths, for $\Lambda$ values typically below $\mathcal{O}\left(10^{-2}-10^{-1}\right)$. In a similar or larger range of couplings values, R-parity violation could show up at colliders via single resonant or non-resonant production of supersymmetric particles.

For bilinear or spontaneous breaking, the lightest supersymmetric particle decays through mixing with the corresponding $R_{p}=+1$ particle. If the LSP is neutralino or chargino, it decays through mixing with neutrino or charged lepton, and if the LSP is a slepton it decays through mixing with the Higgs bosons, e.g. stau mixes with charged Higgs. Assuming that neutralino is the LSP, the dominant decay mode of stau is to tau and neutralino. Through mixing the charged Higgs has then a branching ratio to tau and neutralino. Thus the detection of R-parity violation includes precise measurement of the branching ratios of particles.

The main signature of $\mathrm{BRpV}$ is the decay of the neutralino, which decays $100 \%$ of the time into R-parity and lepton number violating modes. If squarks and sleptons are heavy and the neutralino is heavier than the gauge bosons, the neutralino decays into on-shell gauge bosons and leptons: $\chi_{1}^{0} \rightarrow W^{\mp} \ell_{i}^{ \pm}, Z v_{i}$. If the gauge bosons are produced off-shell, then the decay modes are $\chi_{1}^{0} \rightarrow$ $q \bar{q}^{\prime} \ell_{i}^{ \pm}, \ell_{j}^{\mp} v_{j} \ell_{i}^{ \pm}, q \bar{q} v_{i}, \ell_{j}^{ \pm} \ell_{j}^{\mp} v_{i}, v_{j} v_{j} v_{i}$. When sfermions cannot be neglected, the decay channels are the same, but squarks and sleptons contribute as intermediate particles [783]. In this model, very useful quantities are formed with ratios of branching ratios, since they can be directly linked to R-parity violating parameters and neutrino observables. We have for example,

$\frac{B\left(\chi_{1}^{0} \rightarrow q \bar{q}^{\prime} \mu\right)}{B\left(\chi_{1}^{0} \rightarrow q \bar{q}^{\prime} \tau\right)} \approx \frac{\Lambda_{2}^{2}}{\Lambda_{3}^{2}} \approx \tan ^{2} \theta_{\mathrm{atm}}$,

where the last approximation is valid in the tree-level dominance scenario. In this way, collider and neutrino measurements, coming from very different experiments, can be contrasted.

Detection possibilities and extraction of limits depend a lot on the specific model and on the collider type and energy. On general grounds a lepton-hadron collider provides both leptonic and baryonic quantum numbers in the initial state and is therefore suited for searches involving $\lambda^{\prime}$. In $e^{+} p$ collisions, the production of $\tilde{u}_{L}^{j}$ squarks of the $j$ th generation via $\lambda_{1 j 1}^{\prime}$ is especially interesting as it involves a valence $d$ quark of the incident proton. In contrast, for $e^{-} p$ collisions where charge conjugate processes are accessible, the $\lambda_{11 k}^{\prime}$ couplings become of special interest as they allow for the production, involving a valence $u$ quark, of $\tilde{d}_{R}^{k}$ squarks of the $k$ th generation.

The excluded regions of the parameter space for R-parity violating scenarios have been worked out from the data at LEP, HERA and Tevatron, see e.g. [784-789]. In the following we shall concentrate on the search possibilities at the LHC. 


\subsubsection{Hadron colliders}

In hadron colliders the $\lambda$ or $\lambda^{\prime}$ couplings can provide a viable signal. In many SUSY scenarios neutralinos and charginos are among the lightest supersymmetric particles. Their pair production or associated production of $\tilde{\chi}_{1}^{ \pm} \tilde{\chi}^{0}$ via gauge couplings and decay via $\lambda$ or $\lambda^{\prime}$ couplings may lead to a trilepton signal from each particle, providing a clean signature. One should notice that if the couplings are small, the vertex may be displaced which makes the analysis more complicated. With small enough couplings the lightest neutralino, if LSP, decays outside the detector.

If kinematically possible, gluinos and squarks are copiously produced at hadron colliders. The NLO cross section has been calculated in [790]. For $m_{\tilde{q}}>m_{\tilde{g}}>m_{\tilde{c}_{L}}$, the production with decay via $\lambda_{121}^{\prime} \neq 0$ was studied at CDF. Also coupling $\lambda_{13 k}^{\prime}$ from $\tilde{t}$ pair production at CDF and $\lambda^{\prime}$ couplings from $\chi_{1}^{0}$ decay at D0 have been investigated.

When R-parity is violated, the supersymmetric particles can be produced singly, and thus they can be produced as resonances through R-parity violating interactions. In a hadron-hadron collider this allows one to probe for resonances in a wide mass range because of the continuous energy distribution of the colliding partons. This production mode requires non-negligible R-parity violating coupling. If a single R-parity violating coupling is dominant, the exchanged supersymmetric particle may decay through the same coupling involved in its production, giving a two fermion final state. It is also possible that the decay of the resonant SUSY particle goes through gauge interactions, giving rise to a cascade decay.

The resonant production of sneutrinos and charged sleptons (via $\lambda^{\prime}$ couplings) has been investigated at hadron colliders [791-795]. The production of a charged lepton with neutralino leads to a like-sign dilepton signature via $\lambda^{\prime}$ couplings. The production of a charged lepton with a chargino in the resonant sneutrino case decay leads to a tri-lepton final state via $\lambda^{\prime}$ couplings. The $\tilde{\chi}_{1}^{0}, \tilde{\chi}_{1}^{ \pm}, \tilde{v}$ masses can be reconstructed using the tri-lepton signal.

Single production is possible also in two-body processes without resonance [796]. Sfermion production with a gauge boson has been studied in either via $\lambda$ or $\lambda^{\prime}$-coupling. (The process $\bar{q}_{i} q_{j} \rightarrow W^{-} \tilde{v}_{k}$ or $\bar{q}_{i} q_{j} \rightarrow W^{+} \tilde{l}_{k L}$ can get contribution also from resonant production, but e.g. in SUGRA $m_{\tilde{l}}-m_{\tilde{\nu}}=\cos 2 \beta m_{W}^{2}$ and resonance production is not kinematically viable.) Similarly via $\lambda^{\prime}$ or $\lambda^{\prime \prime}$ gluino can be produced with a lepton or quark, respectively. Sneutrino production with two associated jets may also provide a detectable signal [797].

Resonant production of squarks can occur via $\lambda^{\prime \prime}$-type couplings, leading eventually to jets in the final states. Although the cross sections can be considerable for these processes, the backgrounds in hadronic colliders are large, and the processes seem difficult to study [798]. In special circumstances the backgrounds can be small, e.g. for stop production in $\bar{d}_{i} \bar{d}_{j} \rightarrow \tilde{t}_{1} \rightarrow b \tilde{\chi}_{1}^{+}$, with $\tilde{\chi}_{i}^{+} \rightarrow \bar{l}_{i} v_{i} \tilde{\chi}_{i}^{0}$ (here it is assumed $\left.m_{\tilde{t}_{1}}>m_{\chi_{1}^{+}}>m_{\chi_{1}^{0}}, m_{\text {top }}>m_{\chi_{1}^{0}}\right)$. Then for $\lambda_{3 j k}^{\prime \prime}, m_{\chi_{1}^{0}}$ is stable $[799,800]$. Also single gluino production, $d_{i} d_{j} \rightarrow \tilde{g} \bar{t}$ via resonant stop production has a good signal to background ratio for $\lambda_{3 j k}^{\prime \prime}=\mathcal{O}(0.1)$ [801].

With the $t \bar{t}$ production cross section of the order of $800 \mathrm{pb}$, the LHC can be considered a top quark factory, with $\sim 10^{8}$ top quarks being produced per year, assuming an integrated luminosity of $100 \mathrm{fb}^{-1}$. This statistics allows for precise studies of top quark physics, in particular, for measurements of rare RpV decays. A simulation of the signal and background using ATLFAST [802], to take into account the experimental conditions prevailing at the ATLAS detector [803], was made for a top quark decaying through a $\lambda_{\ell 31}^{\prime}$ coupling to $t \rightarrow \tilde{\chi}^{0} \ell d$, assuming only one slepton gives the leading contribution as an intermediate state [804].

The importance of treating the top quark production and decay simultaneously $g g \rightarrow t \tilde{\chi}^{0} \ell d$, rather than $\Gamma(g g \rightarrow$ $t \bar{t}) B\left(t \rightarrow \tilde{\chi}^{0} \ell d\right)$, was shown. The latest approach can underestimate the cross section by a factor of a few units, depending on the slepton mass. The reason is that the slepton forces the top quark to be off-shell, becoming the resonance itself, as can be appreciated from $\tilde{\chi}^{0} \ell$ mass invariant distributions.

Two scenarios were chosen for the neutralino decay, $\tilde{\chi}^{0} \rightarrow b d v_{e}$ and $\tilde{\chi}^{0} \rightarrow c d e$, the last one assuming a large stop-scharm mixing. The sensitivity of the LHC is presented as the significance $S / \sqrt{B}$ as a function of $\lambda_{131}^{\prime}$, for slepton masses 150 and $200 \mathrm{GeV}$. The channel $t \rightarrow \tilde{\chi}^{0} e d \rightarrow$ cdeed is more promising with exclusion limits at $2 \sigma$ c.l. for $\lambda^{\prime}>0.03$ and observation at $5 \sigma$ c.l. for $\lambda^{\prime}>0.05$, with these values slightly increasing for heavier sleptons. The $t \rightarrow \tilde{\chi}^{0} e d \rightarrow b d v_{e} e d$ channel is observable only for a $150 \mathrm{GeV}$ slepton mass. The significance is reduced to $\lambda^{\prime}>0.08$ at $2 \sigma$ and $\lambda^{\prime}>0.15$ at $5 \sigma$ level.

Since a $\lambda_{\ell 33}^{\prime} \sim h_{b} \epsilon_{\ell} / \mu$ trilinear term is generated in $\mathrm{BRpV}$ when the $\epsilon_{\ell}$ term is removed from the superpotential, we can see that the above exclusion limits for $\lambda^{\prime}$ are not significant in $\mathrm{BRpV}$, probing only values of $\epsilon_{\ell}$ parameters much larger than what is needed for neutrino oscillations.

\subsection{Higgs-mediated lepton flavor violation in supersymmetry}

If neutrinos are massive, one would expect LFV transitions in the Higgs sector through the decay modes $H^{0} \rightarrow l_{i} l_{j}$ mediated at one loop level by the exchange of the $W$ bosons and neutrinos. However, as for the $\mu \rightarrow e \gamma$ and the $\tau \rightarrow \mu \gamma$ case, also the $H^{0} \rightarrow l_{i} l_{j}$ rates are GIM suppressed. In a supersymmetric (SUSY) framework the situation is completely different. Besides the previous contributions, supersymmetry provides new direct sources of flavor violation, 
namely the possible presence of off-diagonal soft terms in the slepton mass matrices and in the trilinear couplings [144]. In practice, flavor violation would originate from any misalignment between fermion and sfermion mass eigenstates. LFV processes arise at one loop level through the exchange of neutralinos (charginos) and charged sleptons (sneutrinos). The amount of the LFV is regulated by a SuperGIM mechanism that can be much less severe than in the non-supersymmetric case [144]. Another potential source of LFV in models such as the minimal supersymmetric standard model (MSSM) could be the Higgs sector, in fact, extensions of the SM containing more than one Higgs doublet generally allow flavor violating couplings of the neutral Higgs bosons. Such couplings, if unsuppressed, will lead to large flavor-changing neutral currents in direct opposition to experiments. The MSSM avoid these dangerous couplings at the tree level segregating the quark and Higgs fields so that one Higgs $\left(H_{u}\right)$ can couple only to up-type quarks while the other $\left(H_{d}\right)$ couples only to d-type. Within unbroken supersymmetry this division is completely natural, in fact, it is required by the holomorphy of the superpotential. However, after supersymmetry is broken, couplings of the form $Q U_{c} H_{d}$ and $Q D_{c} H_{u}$ are generated at one loop [430]. In particular, the presence of a non-zero $\mu$ term, coupled with SUSY breaking, is enough to induce non-holomorphic Yukawa interactions for quarks and leptons. For large $\tan \beta$ values the contributions to d-quark masses coming from non-holomorphic operator $Q D_{c} H_{u}$ can be equal in size to those coming from the usual holomorphic operator $Q D_{c} H_{d}$ despite the loop suppression suffered by the former. This is because the operator itself gets an additional enhancement of $\tan \beta$.

As shown in Ref. [805] the presence of these loop induced non-holomorphic couplings also leads to the appearance of flavor-changing couplings of the neutral Higgs bosons. These new couplings generate a variety of flavorchanging processes such as $B^{0} \rightarrow \mu^{+} \mu^{-}, \bar{B}^{0}-B^{0}$ etc. [537]. Higgs-mediated FCNC can have sizable effects also in the lepton sector [806, 807]: given a source of non-holomorphic couplings, and LFV among the sleptons, Higgs-mediated LFV is unavoidable. These effects have been widely discussed in the recent literature both in a generic 2HDM $[808,809]$ and in supersymmetry $[807,810]$ frameworks. Through the study of many LFV processes as $\ell_{i} \rightarrow \ell_{j} \ell_{k} \ell_{k}$ [806, 807], $\tau \rightarrow \ell_{j} \eta[169,810], \ell_{i} \rightarrow \ell_{j} \gamma$ [175, 650], $\mu N \rightarrow e N$ [811], $\Phi^{0} \rightarrow \ell_{j} \ell_{k}$ [174] (with $\ell_{i}=\tau, \mu, \ell_{j, k}=$ $\mu, e, \Phi=h^{0}, H^{0}, A^{0}$ ) or the cross section of the $\mu N \rightarrow \tau X$ reaction [812].

\subsubsection{LFV in the Higgs sector}

SM extensions containing more than one Higgs doublet generally allow flavor violating couplings of the neutral Higgs bosons with fermions. Such couplings, if unsuppressed, will lead to large flavor-changing neutral currents in direct opposition to experiments. The possible solution to this problem involves an assumption about the Yukawa structure of the model. A discrete symmetry can be invoked to allow a given fermion type to couple to a single Higgs doublet, and in such case FCNC's are absent at tree level. In particular, when a single Higgs field gives masses to both types of fermions the resulting model is referred as 2HDM-I. On the other hand, when each type of fermion couples to a different Higgs doublet the model is said 2HDM-II.

In the following, we shall assume a scenario where the type-II $2 \mathrm{HDM}$ structure is not protected by any symmetry and is broken by loop effects (this occurs, for instance, in the MSSM).

Let us consider the Yukawa interactions for charged leptons, including the radiatively induced LFV terms [806]:

$-\mathcal{L} \simeq \bar{l}_{R i} Y_{l_{i}} H_{1} \overline{L_{i}}+\bar{l}_{R i}\left(Y_{l_{i}} \Delta_{L}^{i j}+Y_{l_{j}} \Delta_{R}^{i j}\right) H_{2} \overline{L_{j}}+$ h.c.,

where $H_{1}$ and $H_{2}$ are the scalar doublets, $l_{R i}$ are lepton singlet for right handed fermions, $L_{k}$ denote the lepton doublets and $Y_{l_{k}}$ are the Yukawa couplings.

In the mass eigenstate basis for both leptons and Higgs bosons, the effective flavor violating interactions are described by the four dimension operators [806]:

$$
\begin{aligned}
-\mathcal{L} \simeq & \left(2 G_{\mathrm{F}}^{2}\right)^{\frac{1}{4}} \frac{m_{l_{i}}}{c_{\beta}^{2}}\left(\Delta_{L}^{i j} \bar{l}_{R}^{i} l_{L}^{j}+\Delta_{R}^{i j} \bar{l}_{L}^{i} l_{R}^{j}\right) \\
& \times\left(c_{\beta-\alpha} h^{0}-s_{\beta-\alpha} H^{0}-i A^{0}\right) \\
& +\left(8 G_{\mathrm{F}}^{2}\right)^{\frac{1}{4}} \frac{m_{l_{i}}}{c_{\beta}^{2}}\left(\Delta_{L}^{i j} \bar{l}_{R}^{i} v_{L}^{j}+\Delta_{R}^{i j} v_{L}^{i} \bar{l}_{R}^{j}\right) H^{ \pm}+\text {h.c. }
\end{aligned}
$$

where $\alpha$ is the mixing angle between the CP-even Higgs bosons $h_{0}$ and $H_{0}, A_{0}$ is the physical CP-odd boson, $H^{ \pm}$ are the physical charged Higgs-bosons and $t_{\beta}$ is the ratio of the vacuum expectation value for the two Higgs (where we adopt the notation, $c_{x}, s_{x}=\cos x, \sin x$ and $t_{x}=\tan x$ ). Irrespective to the mechanism of the high energy theories generating the LFV, we treat the $\Delta_{L, R}^{i j}$ terms in a model independent way. In order to constrain the $\Delta_{L, R}^{i j}$ parameters, we impose that their contributions to LFV processes do not exceed the experimental bounds [175, 650].

On the other hand, there are several models with a specific ansatz about the flavor-changing couplings. For instance, the famous multi-Higgs-doublet models proposed by Cheng and Sher [596] predict that the LFV couplings of all the neutral Higgs bosons with the fermions have the form $H f_{i} f_{j} \sim \sqrt{m_{i} m_{j}}$.

In supersymmetry, the $\Delta^{i j}$ terms are induced at one loop level by the exchange of gauginos and sleptons, provided 
a source of slepton mixing. In the so mass insertion (MI) approximation, the expressions of $\Delta_{L, R}^{i j}$ are given by

$$
\begin{aligned}
\Delta_{L}^{i j}= & -\frac{\alpha_{1}}{4 \pi} \mu M_{1} \delta_{L L}^{i j} m_{L}^{2} \\
& \times\left[I^{\prime}\left(M_{1}^{2}, m_{R}^{2}, m_{L}^{2}\right)+\frac{1}{2} I^{\prime}\left(M_{1}^{2}, \mu^{2}, m_{L}^{2}\right)\right] \\
& +\frac{3}{2} \frac{\alpha_{2}}{4 \pi} \mu M_{2} \delta_{L L}^{i j} m_{L}^{2} I^{\prime}\left(M_{2}^{2}, \mu^{2}, m_{L}^{2}\right), \\
\Delta_{R}^{i j}= & \frac{\alpha_{1}}{4 \pi} \mu M_{1} m_{R}^{2} \delta_{R R}^{i j}\left[I^{\prime}\left(M_{1}^{2}, \mu^{2}, m_{R}^{2}\right)-\left(\mu \leftrightarrow m_{L}\right)\right],
\end{aligned}
$$

respectively, where $\mu$ is the Higgs mixing parameter, $M_{1,2}$ are the gaugino masses and $m_{L(R)}^{2}$ stands for the left-left (right-right) slepton mass matrix entry. The LFV mass insertions (MIs), i.e. $\delta_{X X}^{3 \ell}=\left(\tilde{m}_{\ell}^{2}\right)_{X X}^{3 \ell} / m_{X}^{2}(X=L, R)$, are the off-diagonal flavor changing entries of the slepton mass matrix. The loop function $I^{\prime}(x, y, z)$ is such that $I^{\prime}(x, y, z)=$ $d I(x, y, z) / d z$, where $I(x, y, z)$ refers to the standard three point one loop integral which has mass dimension-2

$I_{3}(x, y, z)=\frac{x y \log (x / y)+y z \log (y / z)+z x \log (z / x)}{(x-y)(z-y)(z-x)}$.

The above expressions, i.e. (5.96), (5.97), depend only on the ratio of the SUSY mass scales and they do not decouple for large $m_{\text {SUSY }}$. As first shown in Ref. [174], both $\Delta_{R}^{i j}$ and $\Delta_{L}^{i j}$ couplings suffer from strong cancellations in certain regions of the parameter space due to destructive interferences among various contributions. For instance, from (5.97) it is clear that, in the $\Delta_{R}^{i j}$ case, such cancellations happen if $\mu=m_{L}$.

In the SUSY see-saw model, in the mass insertion approximation, one obtains specific values for $\delta_{L L}^{i j}$ depending on the assumptions on the flavor mixing in $Y_{v}[164,707]$. If the latter is of CKM size, $\delta_{L L}^{21(31)} \simeq 3 \times 10^{-5}$ and $\delta_{L L}^{32} \simeq$ $10^{-2}$, while in the case of the observed neutrino mixing, taking $U_{e 3}=0.07$ at about half of the current CHOOZ bound, we get $\delta_{L L}^{21(31)} \simeq 10^{-2}$ and $\delta_{L L}^{32} \simeq 10^{-1}$.

\subsubsection{Phenomenology}

In order to constrain the $\Delta_{L, R}^{i j}$ parameters, we impose that their contributions to LFV processes as $l_{i} \rightarrow l_{j} l_{k} l_{k}$ and $l_{i} \rightarrow$ $l_{j} \gamma$ do not exceed the experimental bounds. At tree level, Higgs exchange contribute only to $\ell_{i} \rightarrow \ell_{j} \ell_{k} \ell_{k}, \tau \rightarrow \ell_{j} \eta$ and $\mu N \rightarrow e N$. On the other hand, a one loop Higgs exchange leads to the LFV radiative decays $\ell_{i} \rightarrow \ell_{j} \gamma$. In the following, we report the expression for the branching ratios of the above processes.

5.5.2.1 $\ell_{i} \rightarrow \ell_{j} \gamma \quad$ The $\ell_{i} \rightarrow \ell_{j} \gamma$ process can be generated by the one loop exchange of Higgs and leptons. How- ever, the dipole transition implies three chirality flips: two in the Yukawa vertices and one in the lepton propagator. This strong suppression can be overcome at higher order level. Going to two loop level, one has to pay the typical price of $g^{2} / 16 \pi^{2}$ but one can replace the light fermion masses from Yukawa vertices with the heavy fermion (boson) masses circulating in the second loop. In this case, the virtual Higgs boson couples only once to the lepton line, inducing the needed chirality flip. As a result, the two loop amplitude can provide the major effects. Naively, the ratio between the two loop fermionic amplitude and the one loop amplitude is

$\frac{A_{l_{i} \rightarrow l_{j} \gamma}^{(2-\text { loop })_{f}}}{A_{l_{i} \rightarrow l_{j} \gamma}^{1-\operatorname{loop}}} \sim \frac{\alpha_{\mathrm{em}}}{4 \pi} \frac{m_{f}^{2}}{m_{l_{i}}^{2}} \log \left(\frac{m_{f}^{2}}{m_{H}^{2}}\right)$

where $m_{f}=m_{b}, m_{\tau}$ is the mass of the heavy fermion circulating in the loop. We remind that in a Model II 2HDM (as SUSY) the Yukawa couplings between neutral Higgs bosons and quarks are $H \bar{t} t \sim m_{t} / \tan \beta$ and $H \bar{b} b \sim m_{b} \tan \beta$. Since the Higgs mediated LFV is relevant only at large $\tan \beta \geq 30$, it is clear that the main contributions arise from the $\tau$ and $b$ fermions and not from the top quark. So, in this framework, $\tau \rightarrow l_{j} \gamma$ does not receive sizable two loop effects by heavy fermionic loops, contrary to the $\mu \rightarrow e \gamma$ case.

However, the situation can drastically change when a $W$ boson circulates in the two loop Barr-Zee diagrams. Bearing in mind that $H W^{+} W^{-} \sim m_{W}$ and that pseudoscalar bosons do not couple to a $W$ pair, it turns out that $A_{l_{i} \rightarrow l_{j} \gamma}^{\left(2-l_{W}\right)} /$ $A_{l_{i} \rightarrow l_{j} \gamma}^{\left(2-l_{f}\right)_{f}} \sim m_{W}^{2} /\left(m_{f}^{2} \tan \beta\right) ;$ thus, two loop $W$ effects are expected to dominate, as it is confirmed numerically [650, 808].

As final result, the following approximate expression holds [175, 650]:

$$
\begin{aligned}
& \frac{B\left(\ell_{i} \rightarrow \ell_{j} \gamma\right)}{B\left(\ell_{i} \rightarrow \ell_{j} \bar{v}_{j} v_{\tau}\right)} \simeq \frac{3 \alpha_{e l}}{2}\left(\frac{m_{\ell_{i}}^{2}}{m_{A}^{2}}\right)^{2} t_{\beta}^{6} \Delta_{i j}^{2}\left\{\frac{\delta m}{m_{A}} \log \frac{m_{\ell_{i}}^{2}}{m_{A}^{2}}+\frac{1}{6}\right. \\
& \quad+\frac{\alpha_{e l}}{\pi}\left[\frac{m_{W}^{2}}{m_{\ell_{i}}^{2}} \frac{F\left(a_{W}\right)}{t_{\beta}}-\sum_{f=b, \tau} N_{f} q_{f}^{2} \frac{m_{f}^{2}}{m_{\ell_{i}}^{2}}\left(\log \frac{m_{f}^{2}}{m_{\ell_{i}}^{2}}+2\right)\right. \\
& \quad-\frac{N_{c}}{4}\left(q_{\tilde{t}}^{2} \frac{m_{t} \mu}{t_{\beta} m_{\ell_{i}}^{2}} s_{2 \theta_{\tilde{t}}} h\left(x_{\tilde{t} H}\right)\right. \\
&\left.\left.\left.\quad-\frac{q_{\tilde{b}}^{2}}{m_{b} A_{b}} s_{2 \theta_{\tilde{b}}} h\left(x_{\tilde{b} H}^{2}\right)\right)\right]\right\}^{2} \\
& \simeq \frac{3 \alpha_{e l}^{3}}{\pi^{3}} \Delta_{21}^{2} t_{\beta}^{4}\left(\frac{m_{W}^{4}}{M_{H}^{4}}\right)\left(F\left(a_{W}\right)\right)^{2},
\end{aligned}
$$

where $\delta m=\left(m_{H}-m_{A}\right) \sim \mathcal{O}\left(m_{Z}^{2} / m_{A^{0}}\right)$. The terms of the first row of (5.99) refer to one loop effects and their 
role is non-negligible only in $\tau$ decays. It turns out that pseudoscalar and scalar one loop amplitudes have opposite signs, so, as we have $m_{A} \simeq m_{H}$, they cancel each other to a very large extent. Since these cancellations occur, two loop effects can become important or even dominant. The two terms of the second row of (5.99) refer to two loop BarrZee effects induced by $W$ and fermionic loops, respectively, while the last row of (5.99) is relative two loop Barr-Zee effects with a squark loop in the second loop. As regards the squark loop effects, it is very easy to realize that they are negligible compared to $W$ effects. In fact, it is well known that Higgs mediated LFV can play a relevant or even a dominant role compared to gaugino mediated LFV provided that slepton masses are not below the $\mathrm{TeV}$ scale while maintaining the Higgs masses at the electroweak scale (and assuming large $t_{\beta}$ values). In this context, it is natural to assume squark masses at least of the same order as the slepton masses (at the TeV scale). So, in the limit where $x_{\tilde{f} H}=m_{\tilde{f}}^{2} / m_{H}^{2} \gg 1$, the loop function $h\left(x_{\tilde{f} H}\right)$ is such that $\left(\log x_{\tilde{f} H}+5 / 3\right) / 6 x_{\tilde{f} H}$ thus, even for maximum squark mixing angles $\theta_{\tilde{t}, \tilde{b}}$, namely for $s_{2 \theta_{\tilde{t}, \tilde{b}}}=\sin 2 \theta_{\tilde{t}, \tilde{b}} \simeq 1$, and large $A_{b}$ and $\mu$ terms, two loop squark effects remain much below the $W$ effects, as it is straightforward to check by (5.99).

As a final result the main two loop effects are provided by the exchange of a $W$ boson, with the loop function $F\left(a_{W}\right) \sim \frac{35}{16}\left(\log a_{W}\right)^{2}$ for $a_{W}=m_{W}^{2} / m_{H}^{2} \ll 1$. It is noteworthy that one and two loop amplitudes have the same signs. In addition, two loops effects dominate in large portions of the parameter space, specially for large $m_{H}$ values, where the mass splitting $\delta m=m_{H}-m_{A}$ decreases to zero.

5.5.2.2 $\ell_{i} \rightarrow \ell_{j} \ell_{k} \ell_{k} \quad$ The $l_{i} \rightarrow l_{j} l_{k} l_{k}$ process can be mediated by a tree level Higgs exchange $[806,807]$. However, up to one loop level, $l_{i} \rightarrow l_{j} l_{k} l_{k}$ gets additional contributions induced by $l_{i} \rightarrow l_{j} \gamma^{*}$ amplitudes [175, 650]. It is worth noting that the Higgs mediated monopole (chirality conserving) and dipole (chirality violating) amplitudes have the same $\tan ^{3} \beta$ dependence. This has to be contrasted to the nonHiggs contributions. For instance, within SUSY, the gaugino mediated dipole amplitude is proportional to $\tan \beta$ while the monopole amplitude is $\tan \beta$ independent. The expression for the Higgs mediated $l_{i} \rightarrow l_{j} l_{k} l_{k}$ can be approximated in the following way $[175,650]$ :

$$
\begin{aligned}
& \left.\frac{B(\tau}{B(\tau} \rightarrow l_{j} l_{k} l_{k}\right) \\
& \simeq \frac{\left.m_{\tau} \bar{v}_{j} m_{\tau}\right)}{32 m_{k}^{4}} \Delta_{\tau j}^{2} \tan ^{6} \beta\left[3+5 \delta_{j k}\right] \\
& \quad+\frac{\alpha_{e l}}{3 \pi}\left(\log \frac{m_{\tau}^{2}}{m_{l_{k}}^{2}}-3\right) \frac{B\left(\tau \rightarrow l_{j} \gamma\right)}{B\left(\tau \rightarrow l_{j} \bar{v}_{j} \nu_{\tau}\right)},
\end{aligned}
$$

where we have disregarded subleading monopole effects.
5.5.2.3 $\mu N \rightarrow e N \quad$ The $\mu \rightarrow e$ conversion in Nuclei process can be generated by a scalar operator through the tree level Higgs exchange [811]. Moreover, at one loop level, additional contributions induced by $l_{i} \rightarrow l_{j} \gamma^{*}$ amplitudes arise [175]; however they are subleading [175]. Finally, the following expression for $B(\mu A l \rightarrow e A l)$ is derived [811]:

$B(\mu A l \rightarrow e A l) \simeq 1.8 \times 10^{-4} \frac{m_{\mu}^{7} m_{p}^{2}}{v^{4} m_{h}^{4} \omega_{\mathrm{capt}}^{A l}} \Delta_{21}^{2} t_{\beta}^{6}$,

where $\omega_{\text {capt }}^{A l} \simeq 0.7054 \times 10^{6} \mathrm{~s}^{-1}$. We observe that $B(\mu \rightarrow$ $3 e)$ is completely dominated by the photonic $\mu \rightarrow e \gamma^{*}$ dipole amplitude so that $B(\mu \rightarrow e e e) \simeq \alpha_{\mathrm{em}} B(\mu \rightarrow e \gamma)$. On the other hand, tree level Higgs mediated contributions are negligible because suppressed by the electron mass through the $H(A) \bar{e} e \sim m_{e}$ coupling. On the contrary, $\mu N \rightarrow e N$ is not suppressed by the light constituent quark $m_{u}$ and $m_{d}$ but only by the nucleon masses, because the Higgs-boson coupling to the nucleon is shown to be characterized by the nucleon mass using the conformal anomaly relation [811]. In particular, the most important contribution turns out to come from the exchange of the scalar Higgs boson $H$ which couples to the strange quark [811].

In fact, the coherent $\mu-e$ conversion process, where the initial and final nuclei are in the ground state, is expected to be enhanced by a factor of $O(Z)$ (where $Z$ is the atomic number) compared to incoherent transition processes. Since the initial and final states are the same, the elements $\langle N|\bar{p} p| N\rangle$ and $\langle N|\bar{n} n| N\rangle$ are nothing but the proton and the neutron densities in a nucleus in the nonrelativistic limit of nucleons. In this limit, the other matrix elements $\left\langle N\left|\bar{p} \gamma_{5} p\right| N\right\rangle$ and $\left\langle N\left|\bar{n} \gamma_{5} n\right| N\right\rangle$ vanish. Therefore, in the coherent $\mu-e$ conversion process, the dominant contributions come from the exchange of $H$, not $A$ [811].

Moreover, we know that $\mu \rightarrow e \gamma^{*}$ (chirality conserving) monopole amplitudes are generally subdominant compared to (chirality flipping) dipole effects [175]. Note also that, the enhancement mechanism induced by Barr-Zee type diagrams is effective only for chirality flipping operators so, in the following, we shall disregard chirality conserving one loop effects.

5.5.2.4 $\tau \rightarrow \mu P\left(P=\pi, \eta, \eta^{\prime}\right) \quad$ Now we consider the implications of virtual Higgs exchange for the decays $\tau \rightarrow$ $\mu P$, where $P$ is a neutral pseudoscalar meson $\left(P=\pi, \eta, \eta^{\prime}\right)$ $[169,810]$. Since we assume CP conservation in the Higgs sector, only the exchange of the $A$ Higgs boson is relevant. Moreover, in the large $\tan \beta$ limit, only the $A$ couplings to down-type quarks are important. These can be written as

$$
\begin{aligned}
& -i\left(\sqrt{2} G_{\mathrm{F}}\right)^{1 / 2} \tan \beta A\left(\xi_{d} m_{d} \bar{d}_{R} d_{L}+\xi_{s} m_{s} \bar{s}_{R} s_{L}\right. \\
& \left.\quad+\xi_{b} m_{b} \bar{b}_{R} b_{L}\right)+ \text { h.c. }
\end{aligned}
$$


The parameters $\xi_{d}, \xi_{s}, \xi_{b}$ are equal to one at tree level, but they can significantly deviate from this value because of higher order corrections proportional to $\tan \beta$ [537, 805], generated by integrating out superpartners. In the limit of quark flavor conservation, each $\xi_{q}(q=d, s, b)$ has the form $\xi_{q}=\left(1+\Delta_{q} \tan \beta\right)^{-1}$, where $\Delta_{q}$ appears in the loopgenerated term $-h_{q} \Delta_{q} H_{2}^{0 *} q^{c} q+$ h.c. [537, 805]. At energies below the bottom mass, the $b$-quark can be integrated out so the bilinear $-i m_{b} b^{c} b+$ h.c. is effectively replaced by the gluon operator $\Omega=\frac{g_{s}^{2}}{64 \pi^{2}} \epsilon^{\mu \nu \rho \sigma} G_{\mu \nu}^{a} G_{\rho \sigma}^{a}$, where $g_{s}$ and $G_{\mu \nu}^{a}$ are the $S U(3)_{C}$ coupling constant and field strength, respectively [169]. In the limit in which the processes $\tau \rightarrow 3 \mu$ and $\tau \rightarrow \mu \eta$ are both dominated by Higgs exchange, these decays are related as [169]:

$$
\begin{aligned}
\frac{B\left(\tau \rightarrow l_{j} \eta\right)}{B\left(\tau \rightarrow l_{j} \bar{v}_{j} \nu_{\tau}\right)} \simeq & 9 \pi^{2}\left(\frac{f_{\eta}^{8} m_{\eta}^{2}}{m_{A}^{2} m_{\tau}}\right)^{2}\left(1-\frac{m_{\eta}^{2}}{m_{\tau}^{2}}\right)^{2} \\
& \times\left[\xi_{s}+\frac{\xi_{b}}{3}\left(1+\sqrt{2} \frac{f_{\eta}^{0}}{f_{\eta}^{8}}\right)\right]^{2} \Delta_{3 j}^{2} \tan ^{6} \beta
\end{aligned}
$$

where $m_{\eta}^{2} / m_{\tau}^{2} \simeq 9.5 \times 10^{-2}$ and the relevant decay constants are $f_{\eta}^{0} \sim 0.2 f_{\pi}, f_{\eta}^{8} \sim 1.2 f_{\pi}$ and $f_{\pi} \sim 92 \mathrm{MeV}$. In the above expression, both the contribution of the (bottom-loop induced) gluon operator $\Omega$ and the factors $\xi_{q}$ were included.

For $\xi_{s} \sim \xi_{b} \sim 1$, it turns out that $B\left(\tau^{-} \rightarrow \mu^{-} \eta\right) / B\left(\tau^{-} \rightarrow\right.$ $\left.\mu^{-} \mu^{+} \mu^{-}\right) \simeq 5$, but it could also be a few times larger or smaller than that, depending on the actual values of $\xi_{s}, \xi_{b}$. Finally, let us compare $\tau \rightarrow \mu \eta^{\prime}$ and $\tau \rightarrow \mu \pi$ with $\tau \rightarrow \mu \eta$ in the limit of Higgs exchange domination. Both ratios are suppressed, although for different reasons. The ratio $B(\tau \rightarrow \mu \pi) / B(\tau \rightarrow \mu \eta)$ is small because it is parametrically suppressed by $m_{\pi}^{4} / m_{\eta}^{4} \sim 10^{-2}$. The ratio $B(\tau \rightarrow$ $\left.\mu \eta^{\prime}\right) / B(\tau \rightarrow \mu \eta)$, which seems to be $\mathcal{O}(1)$, is much smaller because the singlet and octet contributions to $\tau \rightarrow \mu \eta^{\prime}$ tend to cancel against each other [169].

These results, combined with the present bound on $\tau \rightarrow$ $\mu \eta$, imply that the Higgs mediated contribution to $B(\tau \rightarrow$ $\left.\mu \eta^{\prime}\right)$ and $B(\tau \rightarrow \mu \pi)$ can reach $\mathcal{O}\left(10^{-9}\right)$ [169].

5.5.2.5 Higgs $\rightarrow \mu \tau \quad$ The LFV Higgs $\rightarrow \mu \tau$ decays and the related phenomenology have been extensively investigated in [174]. Concerning the Higgs boson decays, we have [174]

$$
B\left(A \rightarrow \mu^{+} \tau^{-}\right)=\tan ^{2} \beta\left(\left|\Delta_{L}\right|^{2}+\left|\Delta_{R}\right|^{2}\right) B\left(A \rightarrow \tau^{+} \tau^{-}\right),
$$

where we have approximated $1 / c_{\beta}^{2} \simeq \tan ^{2} \beta$ since nonnegligible effects can only arise in the large $\tan \beta$ limit. If $A$ is replaced with $H$ [or $h$ ] in (5.103), the r.h.s. should also be multiplied by a factor $\left(c_{\beta-\alpha} / s_{\alpha}\right)^{2}$ [or $\left.\left(s_{\beta-\alpha} / c_{\alpha}\right)^{2}\right]$. We recall that $B(A \rightarrow \mu \tau)$ can reach values of order $10^{-4}$. The same holds for the 'non-standard' CP-even Higgs boson (either $H$ or $h$, depending on $m_{A}$ ).

We now make contact with the physical observable, i.e. the $B\left(\Phi^{0} \rightarrow \mu^{+} \tau^{-}\right)$, and discuss the phenomenological implications. We outline some general features of $B\left(\Phi^{0} \rightarrow\right.$ $\mu^{+} \tau^{-}$) at large $\tan \beta$ and the prospects for these decay channels at the Large Hadron Collider (LHC) and other colliders. Let us discuss the different Higgs bosons, as reported in [174], assuming for definiteness $\tan \beta \sim 50,|50 \Delta|^{2} \sim 10^{-3}$ $\left(\Delta=\Delta_{L}\right.$ or $\left.\Delta_{R}\right)$ and an integrated luminosity of $100 \mathrm{fb}^{-1}$ at $\mathrm{LHC}$.

If $\Phi^{0}$ denotes one of the 'non-standard' Higgs bosons, we have $C_{\Phi} \simeq 1$ and $B\left(\Phi^{0} \rightarrow \tau^{+} \tau^{-}\right) \sim 10^{-1}$, so $B\left(\Phi^{0} \rightarrow\right.$ $\left.\mu^{+} \tau^{-}\right) \sim 10^{-4}$. The main production mechanisms at LHC are bottom-loop mediated gluon fusion and associated production with $b \bar{b}$, which yield cross sections $\sigma \sim\left(10^{3}, 10^{2}\right.$, 20) $\mathrm{pb}$ for $m_{A} \sim(100,200,300) \mathrm{GeV}$, respectively. The corresponding numbers of $\Phi^{0} \rightarrow \mu^{+} \tau^{-}$events are about $\left(10^{4}, 10^{3}, 2 \times 10^{2}\right)$. These estimates do not change much if the bottom Yukawa coupling $Y_{b}$ is enhanced (suppressed) by radiative corrections, since in this case the enhancement (suppression) of $\sigma$ would be roughly compensated by the suppression (enhancement) of $B\left(\Phi^{0} \rightarrow \mu^{+} \tau^{-}\right)$.

If $\Phi^{0}$ denotes the other (more 'standard model-like') Higgs boson, the factor $C_{\Phi} \cdot B\left(\Phi^{0} \rightarrow \tau^{+} \tau^{-}\right)$strongly depends on $m_{A}$, while the production cross section at LHC, which is dominated by top-loop mediated gluon fusion, is $\sigma \sim 30 \mathrm{pb}$. For $m_{A} \sim 100 \mathrm{GeV}$ we may have $C_{\Phi} \cdot B\left(\Phi^{0} \rightarrow\right.$ $\left.\tau^{+} \tau^{-}\right) \sim 10^{-1}$ and $B\left(\Phi^{0} \rightarrow \mu^{+} \tau^{-}\right) \sim 10^{-4}$, which would imply $\sim 300 \mu^{+} \tau^{-}$events. The number of events is generically smaller for large $m_{A}$ since $C_{\Phi}$ scales as $1 / m_{A}^{4}$, consistently with the expected decoupling of LFV effects for such a Higgs boson.

The above discussion suggests that LHC may offer good chances to detect the decays $\Phi^{0} \rightarrow \mu \tau$, especially in the case of non-standard Higgs bosons. This indication should be supported by a detailed study of the background. At Tevatron the sensitivity is lower than at LHC because both the expected luminosity and the Higgs production cross sections are smaller. The number of events would be smaller by a factor $10^{2}-10^{3}$. A few events may be expected also at $e^{+} e^{-}$or $\mu^{+} \mu^{-}$future colliders, assuming integrated luminosities of 500 and $1 \mathrm{fb}^{-1}$, respectively. At a $\mu^{+} \mu^{-}$collider an enhancement may occur for the non-standard Higgs bosons if radiative corrections strongly suppress $Y_{b}$, since in this case both the resonant production cross section $[\sigma \sim$ $\left.\left(4 \pi / m_{A}^{2}\right) B\left(\Phi^{0} \rightarrow \mu^{+} \mu^{-}\right)\right]$and the LFV branching ratios $B\left(\Phi^{0} \rightarrow \mu^{+} \tau^{-}\right)$would be enhanced. As a result, for light $m_{A}$, hundreds of $\mu^{+} \tau^{-}$events could occur.

5.5.2.6 $\mu N \rightarrow \tau X \quad$ Higgs mediated LFV effects can have also relevant impact on the cross section of the $\mu N \rightarrow \tau X$ 
reaction [812]. The contribution of the Higgs boson mediation to the differential cross section $\mu^{-} N \rightarrow \tau^{-} X$ is given by [812]

$$
\begin{aligned}
\frac{d^{2} \sigma}{d x d y}= & \sum_{q} x f_{q}(x)\left\{\left|\mathcal{C}_{L}\right|_{q}^{2}\left(\frac{1-\mathcal{P}_{\mu}}{2}\right)+\left|\mathcal{C}_{R}\right|_{q}^{2}\left(\frac{1+\mathcal{P}_{\mu}}{2}\right)\right\} \\
& \times \frac{s}{8 \pi} y^{2},
\end{aligned}
$$

where the function $f_{q}(x)$ is the PDF for $q$-quarks, $\mathcal{P}_{\mu}$ is the incident muon polarization such that $\mathcal{P}_{\mu}=+1$ and -1 correspond to the right and left handed polarization, respectively, and $s$ is the centre-of-mass (CM) energy. The parameters $x$ and $y$ are defined as $x \equiv Q^{2} / 2 P \cdot q, y \equiv 2 P \cdot q / s$, in the limit of massless tau leptons, where $P$ is the four momentum of the target, $q$ is the momentum transfer, and $Q$ is defined as $Q^{2} \equiv-q^{2}$. As seen in (5.104), experimentally, the form factors of $\mathcal{C}_{L}^{h H}$ and $\mathcal{C}_{L}^{A}\left(\mathcal{C}_{R}^{h H}\right.$ and $\left.\mathcal{C}_{R}^{A}\right)$ can be selectively studied by using purely left handed (right handed) incident muons. In SUSY models such as the MSSM with heavy right handed neutrinos, LFV is radiatively induced due to the left handed slepton mixing, which only affects $\mathcal{C}_{L}^{h H}$ and $\mathcal{C}_{L}^{A}$. Therefore, in the following, we focus only on those $\mathcal{C}_{L}^{h H}$ and $\mathcal{C}_{L}^{A}$ couplings.

The magnitudes of the effective couplings are constrained by the current experimental results of searches for LFV processes of tau decays. Therefore, both couplings are determined by the one that is more constrained, namely the pseudo-scalar coupling. It is constrained by the $\tau \rightarrow \mu \eta$ decay $\left(B(\tau \rightarrow \mu \eta)<3.4 \times 10^{-7}\right)$. Then the constraint is given on the $s$-associated scalar and pseudo-scalar couplings by

$$
\left(\left|\mathcal{C}_{L}^{A}\right|^{2}\right)_{s} \leq 10^{-9}\left[\mathrm{GeV}^{-4}\right] \times B(\tau \rightarrow \mu \eta) .
$$

The largest values of $\mathcal{C}_{L}^{h H}$ and $\mathcal{C}_{L}^{A}$ can be realized with $m_{\mathrm{SUSY}} \sim \mathcal{O}(1) \mathrm{TeV}$ and the higgsino mass $\mu \sim \mathcal{O}(10) \mathrm{TeV}$ $[169,810]$.

The cross sections of the $\mu N \rightarrow \tau X$ reaction in the DIS region is evaluated for the maximally allowed values of the effective couplings as a reference. They are plotted in Fig. 36 for different quark contributions as a function of the muon beam energy in the laboratory frame. For the PDF, CTEQ6L has been used. The target $N$ is assumed to be a proton. For a nucleus target, the cross section would be higher, approximately by the number of nucleons in the target. The cross section sharply increases above $E_{\mu} \sim 50 \mathrm{GeV}$ in Fig. 36 . This enhancement comes from the $b$-quark contribution in addition to the $d$ - and $s$-quark contributions which is enhanced by a factor of $m_{b} / m_{s}$ over the $s$-quark contribution. The cross section is enhanced by one order of magnitude when the muon energy changes from 50 to $100 \mathrm{GeV}$. Typically, for $E_{\mu}=100 \mathrm{GeV}$ and $E_{\mu}=300 \mathrm{GeV}$, the cross section is $10^{-4}$ and $10^{-3} \mathrm{fb}$, respectively. With an intensity of

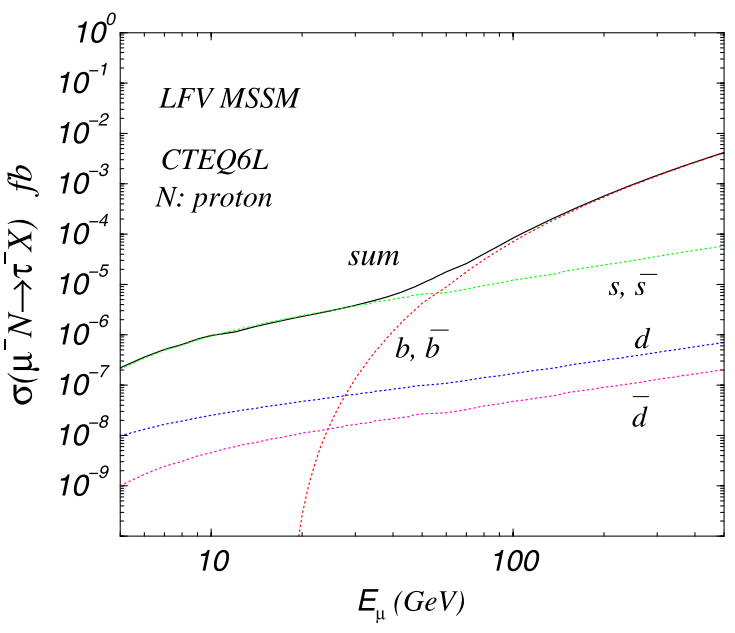

Fig. 36 Cross section of the $\mu^{-} N \rightarrow \tau^{-} X$ DIS process as a function of the muon energy for the Higgs mediated interaction [812]. It is assumed that the initial muons are purely left handed. CTEQ6L is used for the PDF

$10^{20}$ muons per year and a target mass of $100 \mathrm{~g} / \mathrm{cm}^{2}$, about $10^{4}\left(10^{2}\right)$ events could be expected for $\sigma(\mu N \rightarrow \tau X)=$ $10^{-3}\left(10^{-5}\right) \mathrm{fb}$, which corresponds to $E_{\mu}=300$ (50) $\mathrm{GeV}$ from Fig. 36. This would provide good potential to improve the sensitivity by four (two) orders of magnitude from the present limit from $\tau \rightarrow \mu \eta$ decay, respectively. Such a muon intensity could be available at a future muon collider and a neutrino factory.

\subsubsection{Correlations}

The numerical results shown in Figs. 37 and 38 allow us to draw several observations $[175,650]$.

- $\tau \rightarrow l_{j} \gamma$ has the largest branching ratios except for a region around $m_{H} \sim 700 \mathrm{GeV}$ where strong cancellations among two loop effects reduce their size. ${ }^{25}$ The following approximate relations are found:

$$
\begin{aligned}
& \frac{B\left(\tau \rightarrow l_{j} \gamma\right)}{B\left(\tau \rightarrow l_{j} \eta\right)} \\
& \quad \simeq\left(\frac{\delta m}{m_{A}} \log \frac{m_{\tau}^{2}}{m_{A}^{2}}+\frac{1}{6}+\frac{\alpha_{e l}}{\pi}\left(\frac{m_{W}^{2}}{m_{\tau}^{2}}\right) \frac{F\left(a_{W}\right)}{\tan \beta}\right)^{2} \\
& \quad \geq 1,
\end{aligned}
$$

where the last relation is easily obtained by using the approximation for $F(z)$. If two loop effects were disregarded, then we would obtain $B\left(\tau \rightarrow l_{j} \gamma\right) / B(\tau \rightarrow$ $\left.l_{j} \eta\right) \in(1 / 36,1)$ for $\delta m / m_{A} \in(0,10 \%)$. Two loop con-

\footnotetext{
${ }^{25}$ For a detailed discussion about the origin of these cancellations and their connection with non-decoupling properties of two loop $W$ amplitude, see Ref. [808].
} 

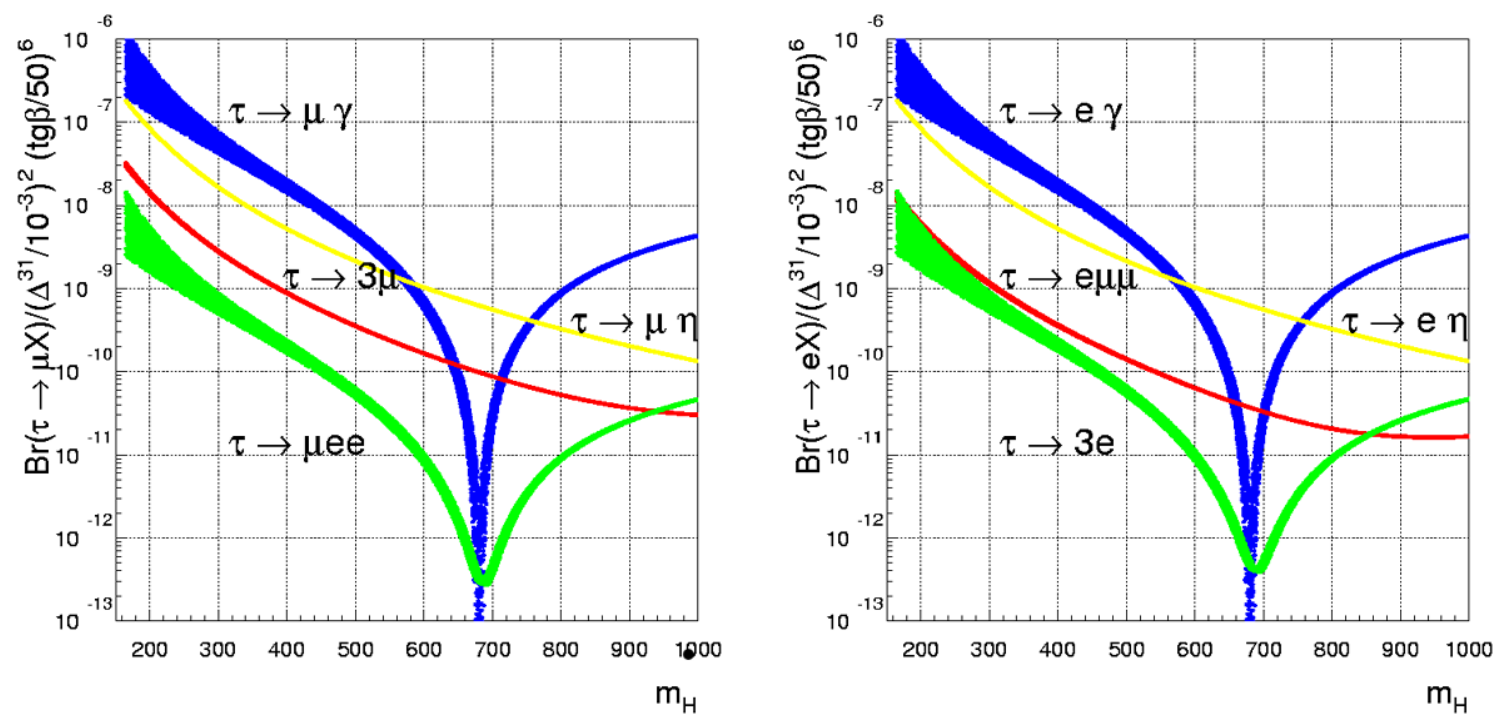

Fig. 37 Branching ratios of various $\tau \rightarrow \mu$ and $\tau \rightarrow e$ LFV processes versus the Higgs boson mass $m_{H}$ in the decoupling limit as reported in [650]. $X=\gamma, \mu \mu, e e, \eta$

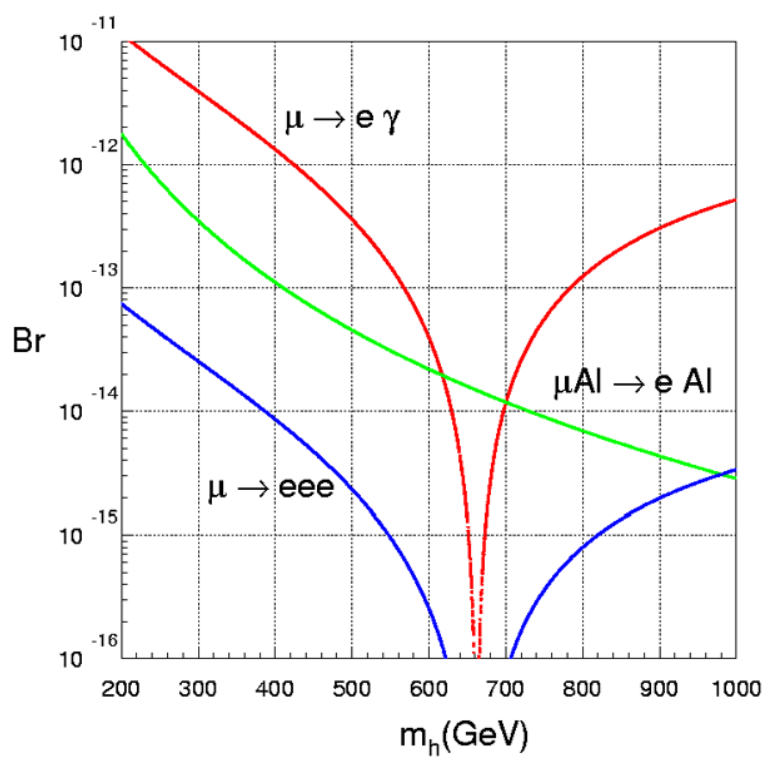

Fig. 38 Left: branching ratios of $\mu \rightarrow e \gamma, \mu \rightarrow e e e$ and $\mu A l \rightarrow e A l$ in the Higgs mediated LFV case versus the Higgs boson mass $m_{h}$ [175]. Right: branching ratios of $\mu \rightarrow e \gamma, \mu \rightarrow e e e$ and $\mu A l \rightarrow e A l$

tributions significantly enhance $B\left(\tau \rightarrow l_{j} \gamma\right)$ specially for $\delta m / m_{A} \rightarrow 0$.

- In Fig. 37 non-negligible mass splitting $\delta m / m_{A}$ effects can be visible at low $m_{H}$ regime through the bands of the $\tau \rightarrow l_{j} \gamma$ and $\tau \rightarrow l_{j} e e$ processes. These effects tend to vanish with increasing $m_{H}$ as is correctly reproduced in Fig. 37. $\tau \rightarrow l_{j} \mu \mu$ does not receive visible effects by $\delta m / m_{A}$ terms being dominated by the tree level Higgs exchange.

- As is shown in Fig. $37 B\left(\tau \rightarrow l_{j} \gamma\right)$ is generally larger than $B\left(\tau \rightarrow l_{j} \mu \mu\right)$; their ratio is regulated by the follow-

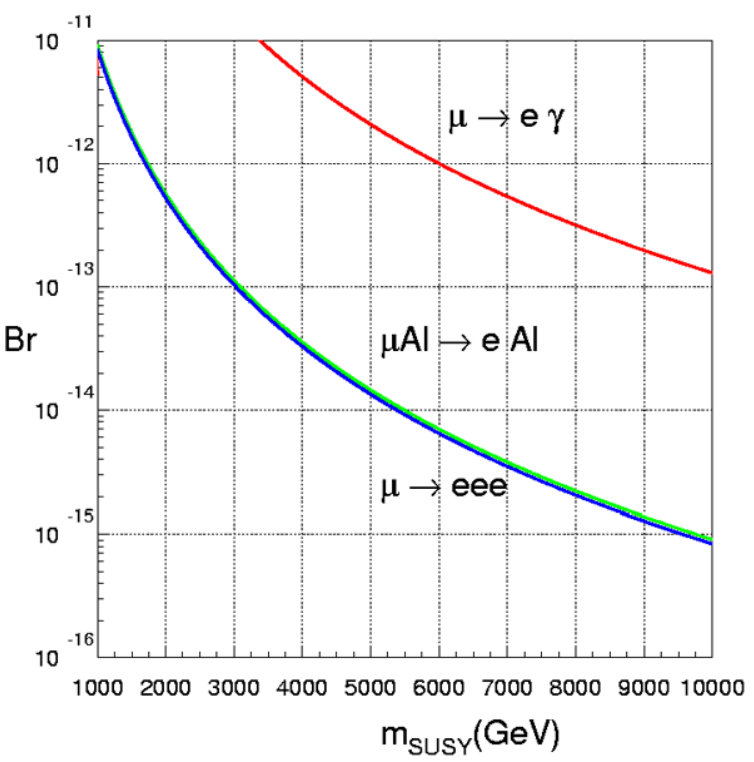

in the gaugino mediated LFV case versus a common SUSY mass $m_{\text {SUSY }}[175]$. In the figure we set $t_{\beta}=50$ and $\delta_{L L}^{21}=10^{-2}$

ing approximate relation:

$$
\frac{B\left(\tau \rightarrow l_{j} \gamma\right)}{B\left(\tau \rightarrow l_{j} \mu \mu\right)} \simeq \frac{36}{3+5 \delta_{j \mu}} \frac{B\left(\tau \rightarrow l_{j} \gamma\right)}{B\left(\tau \rightarrow l_{j} \eta\right)} \geq \frac{36}{3+5 \delta_{j \mu}}
$$

where the last relation is valid only out of the cancellation region. Moreover, from the above relation it turns out that

$$
\frac{B\left(\tau \rightarrow l_{j} \eta\right)}{B\left(\tau \rightarrow l_{j} \mu \mu\right)} \simeq \frac{36}{3+5 \delta_{j \mu}} .
$$


If we relax the condition $\xi_{s, b}=1, B\left(\tau \rightarrow l_{j} \eta\right)$ can get values few times smaller or bigger than those in Fig. 37.

- It is noteworthy that a tree level Higgs exchange predicts that $B\left(\tau \rightarrow l_{j} e e\right) / B\left(\tau \rightarrow l_{j} \mu \mu\right) \sim m_{e}^{2} / m_{\mu}^{2}$ while, at two loop level, we obtain (out of the cancellation region):

$$
\frac{B\left(\tau \rightarrow l_{j} e e\right)}{B\left(\tau \rightarrow l_{j} \mu \mu\right)} \simeq \frac{0.4}{3+5 \delta_{j \mu}} \frac{B\left(\tau \rightarrow l_{j} \gamma\right)}{B\left(\tau \rightarrow l_{j} \eta\right)} \geq \frac{0.4}{3+5 \delta_{j \mu}} .
$$

Let us underline that, in the cancellation region, the lower bound of $B\left(\tau \rightarrow l_{j} e e\right)$ is given by the monopole contributions. So, in this region, $B\left(\tau \rightarrow l_{j} e e\right)$ is much less suppressed than $B\left(\tau \rightarrow l_{j} \gamma\right)$.

- The approximate relations among $\mu A l \rightarrow e A l, \mu \rightarrow e \gamma$ and $\mu \rightarrow e e e$ branching ratios are

$$
\begin{aligned}
& \frac{B(\mu \rightarrow e \gamma)}{B(\mu A l \rightarrow e A l)} \simeq 10^{2}\left(\frac{F\left(a_{W}\right)}{\tan \beta}\right)^{2}, \\
& \frac{B(\mu \rightarrow e e e)}{B(\mu \rightarrow e \gamma)} \simeq \alpha_{e l} .
\end{aligned}
$$

In the above equations we retained only dominant two loop effects arising from $W$ exchange. The exact behavior for the examined processes is reported in Fig. 38 where we can see that $\mu \rightarrow e \gamma$ gets the largest branching ratio except for a region around $m_{H} \sim 700 \mathrm{GeV}$ where strong cancellations among two loop effects sink its size.

The correlations among the rates of the above processes are an important signature of the Higgs-mediated LFV and allow us to discriminate between different SUSY scenarios. In fact, it is well known that, in a supersymmetric framework, besides the Higgs mediated LFV transitions, we have also LFV effects mediated by the gauginos through loops of neutralinos (charginos)-charged sleptons (sneutrinos). On the other hand, the above contributions have different decoupling properties regulated by the mass of the heaviest scalar mass $\left(m_{H}\right)$ or by the heaviest mass in the slepton gaugino loops ( $\left.m_{\mathrm{SUSY}}\right)$. In principle, the $m_{\mathrm{SUSY}}$ and $m_{H}$ masses may be unrelated, so we can always proceed by considering only the Higgs mediated effects (assuming a relatively light $m_{H}$ and an heavy $m_{\text {SUSY }}$ ) or only the gaugino mediated contributions (if $m_{H}$ is heavy). In the following, we are interested to make a comparison between Higgs and gaugino mediated LFV effects. In order to make the comparison as simple as possible, let us consider the simple case where all the SUSY particles are degenerate. In this case, it turns out that

$\Delta_{L}^{21} \sim \frac{\alpha_{2}}{24 \pi} \delta_{L L}^{21}$,

$$
\begin{aligned}
& \left.\frac{B\left(\ell_{i} \rightarrow \ell_{j} \gamma\right)}{B\left(\ell_{i} \rightarrow \ell_{j} \bar{\nu}_{j} v_{i}\right)}\right|_{\text {Gauge }} \\
& =\frac{2 \alpha_{e l}}{75 \pi}\left(1+\frac{5}{4} \tan ^{2} \theta_{W}\right)^{2}\left(\frac{m_{W}^{4}}{m_{\mathrm{SUSY}}^{4}}\right)\left|\delta_{L L}^{i j}\right|^{2} t_{\beta}^{2},
\end{aligned}
$$

$$
\begin{aligned}
& \left.\frac{B\left(\ell_{i} \rightarrow \ell_{j} \gamma\right)}{B\left(\ell_{i} \rightarrow \ell_{j} \bar{v}_{j} v_{i}\right)}\right|_{\text {Higgs }} \\
& \simeq 10 \frac{\alpha_{e l}^{3}}{\pi^{3}}\left(\frac{\alpha_{2}}{24 \pi}\right)^{2}\left(\frac{m_{W}^{4}}{M_{H}^{4}}\right)\left(\log \frac{m_{W}^{2}}{M_{H}^{2}}\right)^{4}\left|\delta_{L L}^{i j}\right|^{2} t_{\beta}^{4} .
\end{aligned}
$$

In Fig. 38 we report the branching ratios of the examined processes as a function of the heaviest Higgs boson mass $m_{H}$ (in the Higgs LFV mediated case) or of the common SUSY mass $m_{\text {SUSY }}$ (in the gaugino LFV mediated case). We set $t_{\beta}=50$ and we consider the PMNS scenario as discussed above so that $\left(\delta_{L L}^{21}\right)_{\mathrm{PMNS}} \simeq 10^{-2}$. Sub-leading contributions proportional to $\left(\delta_{L L(R R)}^{23} \delta_{R R(L L)}^{31}\right)_{\mathrm{PMNS}}$ were neglected since, in the PMNS scenario, it turns out that $\left(\delta_{L L(R R)}^{23} \delta_{R R(L L)}^{31}\right)_{\mathrm{PMNS}} /\left(\delta_{L L}^{21}\right)_{\mathrm{PMNS}} \simeq 10^{-3}$ [707]. As we can see from Fig. 38, Higgs mediated effects start being competitive with the gaugino mediated ones when $m_{\text {SUSY }}$ is roughly one order of magnitude larger than the Higgs mass $m_{H}$. Moreover, we stress that, both in the gaugino and in the Higgs mediated cases, $\mu \rightarrow e \gamma$ gets the largest effects. In particular, within the PMNS scenario, it turns out that Higgs mediated $B(\mu \rightarrow e \gamma) \sim 10^{-11}$ when $m_{H} \sim 200 \mathrm{GeV}$ and $t_{\beta}=50$, that is just closed to the present experimental resolution.

The correlations among different processes predicted in the gaugino mediated case are different from those predicted in the Higgs mediated case. For instance, in the gaugino mediated scenario, $B\left(\tau \rightarrow l_{j} l_{k} l_{k}\right)$ gets the largest contributions by the dipole amplitudes that are $\tan \beta$ enhanced with respect to all other amplitudes resulting in a precise ratio with $B\left(\tau \rightarrow l_{j} \gamma\right)$, namely

$$
\begin{aligned}
& \left.\frac{B\left(\ell_{i} \rightarrow \ell_{j} \ell_{k} \ell_{k}\right)}{B\left(\ell_{i} \rightarrow \ell_{j} \gamma\right)}\right|_{\text {Gauge }} \simeq \frac{\alpha_{e l}}{3 \pi}\left(\log \frac{m_{\tau}^{2}}{m_{l_{k}}^{2}}-3\right) \simeq \alpha_{e l}, \\
& \left.\frac{B\left(\tau \rightarrow \ell_{j} e e\right)}{B\left(\tau \rightarrow \ell_{j} \mu \mu\right)}\right|_{\text {Gauge }} \simeq \frac{\log \frac{m_{\tau}^{2}}{m_{e}^{2}}-3}{\log \frac{m_{\tau}^{2}}{m_{\mu}^{2}}-3} \simeq 5
\end{aligned}
$$

Moreover, in the large $\tan \beta$ regime, one can find the simple theoretical relations

$$
\left.\frac{B(\mu-e \text { in Ti })}{B(\mu \rightarrow e \gamma)}\right|_{\text {Gauge }} \simeq \alpha_{e l} .
$$

If some ratios different from the above were discovered, then this would be clear evidence that some new process is generating the $\ell_{i} \rightarrow l_{j}$ transition, with Higgs mediation being a leading candidate.

\subsubsection{Conclusions}

We have reviewed the allowed rates for Higgs-mediated LFV decays in a SUSY framework. In particular, we have 
analyzed the decay modes of the $\tau, \mu$ lepton, namely $\ell_{i} \rightarrow$ $\ell_{j} \ell_{k} \ell_{k}, \ell_{i} \rightarrow \ell_{j} \gamma, \tau \rightarrow l_{j} \eta$ and $\mu N \rightarrow e N$. We have also discussed the LFV decay modes of the Higgs bosons $\Phi \rightarrow$ $\ell_{i} \ell_{j}\left(\Phi=h^{0}, H^{0}, A^{0}\right)$ so as the impact of Higgs mediated LFV effects on the cross section of the $\mu N \rightarrow \tau X$ reaction. Analytical relations and correlations among the rates of the above processes have been established at the two loop level in the Higgs boson exchange. The correlations among the processes are a precise signature of the theory. In this respect experimental improvements in all the decay channels of the $\tau$ lepton would be very welcome. In conclusion, the Higgsmediated contributions to LFV processes can be within the present or upcoming attained experimental resolutions and provide an important opportunity to detect new physics beyond the standard model.

5.6 Tests of unitarity and universality in the lepton sector

\subsubsection{Deviations from unitarity in the leptonic mixing matrix}

The presence of physics beyond the SM in the leptonic sector can generate deviations from unitarity in the mixing matrix. This is analogous to what happens in the quark sector, where the search for deviations from unitarity of the CKM matrix is considered a sensitive way to look for new physics.

In the leptonic sector a clear example of non-unitarity is given by the see-saw mechanism [216-220]. To generate naturally small neutrino masses, new heavy particles-right handed neutrinos-are added, singlet under the SM gauge group. Thus a Yukawa coupling for neutrinos can be written, as well as Majorana masses for the new heavy fields. The mass matrix of the complete theory is now an enlarged mass matrix ( $5 \times 5$ at least), whose diagonalization leads to small Majorana neutrino masses. The non-unitarity of the $3 \times 3$ leptonic mixing matrix can now be understood simply by observing that it is a sub-matrix of a bigger one which is unitary, since the complete theory must conserve probabilities.

Another way to see this is looking at the effective theory we obtain once the heavy fields are integrated out. The unique dimension five operator is the well-known Weinberg operator [213] which generates neutrino masses when the electroweak symmetry is broken. Masses are naturally small since they are suppressed by the mass $M$ of the heavy particles which have been integrated out: $m_{v} \sim v^{2} / M$, where $v$ is the Higgs VEV. If we go on in the expansion in effective operators, we obtain only one dimension six operator which renormalizes the kinetic energy of neutrinos. Once we perform a field redefinition to go into a mass basis with canonical kinetic terms, a non-unitary mixing matrix is obtained [813]. In minimal models deviations from unitarity gener- ated in this way are very suppressed, since the dimension six operator is proportional to $v^{2} / M^{2}$. However, in more sophisticated versions of this mechanism like double (or inverse) see-saw [814] the suppression can be reduced without affecting the smallness of neutrinos masses and avoiding any fine-tuning of Yukawa couplings. In terms of effective operators, this means that it is possible to "decouple" the dimension five operator from the dimension six, permitting small neutrino masses and not so small unitarity deviations.

Usually the elements of the leptonic mixing matrix are measured using neutrino oscillation experiments assuming unitarity. No information can be extracted from electroweak decays on the individual matrix elements, due to the impossibility of detecting neutrino mass eigenstates. This is quite different from the way of measuring the CKM matrix elements. Here oscillations are important too, but since quark mass eigenstates can be tagged, direct measurements of the matrix elements can be made using electroweak decays.

The situation changes if we relax the hypothesis of unitarity of the leptonic mixing matrix. Electroweak decays acquire now an important meaning, since they can be used to constrain deviations from unitarity. Consider as an example the decay $W \rightarrow l \bar{v}_{l}$. The decay rate is modified as follows: $\Gamma=\Gamma_{\mathrm{SM}}\left(N N^{\dagger}\right)_{l l}$, where $N$ is the non-unitary leptonic mixing matrix and $\Gamma_{\mathrm{SM}}$ is the SM decay rate. This, and other electroweak processes, can therefore be used to obtain information on $\left(N N^{\dagger}\right)_{l l}$. Moreover, lepton flavor violating processes like $\mu \rightarrow 3 e$ or $\mu-e$ conversion in nuclei can occur, while rare lepton decays like $l_{i} \rightarrow l_{j} \gamma$ can be enhanced, permitting to constrain the off-diagonal elements of $\left(N N^{\dagger}\right)$. Finally, universality violation effects are produced, even if the couplings are universal: for example the branching ratio of $\pi$ decay (see Sect. 6) is now proportional to $\left(N N^{\dagger}\right)_{e e} /\left(N N^{\dagger}\right)_{\mu \mu}$.

In Ref. [815] all these processes have been considered, a global fit has been performed and the matrix $\left|\left(N N^{\dagger}\right)\right|$ has been determined (90\% C.L.):

$$
\left|N N^{\dagger}\right| \approx\left(\begin{array}{ccc}
0.994 \pm 0.005 & <7.0 \times 10^{-5} & <1.6 \times 10^{-2} \\
<7.0 \times 10^{-5} & 0.995 \pm 0.005 & <1.0 \times 10^{-2} \\
<1.6 \times 10^{-2} & <1.0 \times 10^{-2} & 0.995 \pm 0.005
\end{array}\right)
$$

Similar bounds can be inferred for $\left|N^{\dagger} N\right|$, leading to the conclusion that deviations from unitarity in the leptonic mixing matrix are experimentally constrained to be smaller than few percent. Notice however that these bounds apply to a $3 \times 3$ mixing matrix, i.e. they constrain deviations from uni- 
tarity induced by higher energy physics which has been integrated out. ${ }^{26}$

However, since on the contrary the quark sector decays can only constrain the elements of $\left|\left(N N^{\dagger}\right)\right|$, to determine the individual elements of the leptonic mixing matrix, oscillation experiments are needed. In Ref. [815] neutrino oscillation physics is reconsidered in the case in which the mixing matrix is not unitary. The main consequence of this is that the flavor basis is no longer orthogonal, which gives rise to two physical effects:

- "zero distance" effect, i.e. flavor conversion in neutrino oscillations at $L=0: P_{v_{\alpha} v_{\beta}}(E, L=0) \hat{A} \cdot \propto\left|\left(N N^{\dagger}\right)_{\beta \alpha}\right|^{2}$; - non-diagonal matter effects.

With the resulting formulas for neutrino oscillations, a fit to present oscillation experiments is performed, in order to determine the individual matrix elements. As in the standard case, no information at all is available on phases (four or six, depending on the nature-Dirac or Majorana-of the neutrinos), since appearance experiments would be needed. However the moduli of matrix elements can be determined, but now they are all independent, so that the free parameters are nine instead of three. The elements of the $e$-row can be constrained using the data from CHOOZ [816], KamLAND [817] and SNO [818], together with the information on $\Delta m_{23}^{2}$ resulting from an analysis of K2K [819]. In contrast, less data are available for the $\mu$-row: only those coming from K2K and Super-Kamiokande [820] on atmospheric neutrinos, and only $\left|N_{\mu 3}\right|$ and the combination $\left|N_{\mu 1}\right|^{2}+\left|N_{\mu 2}\right|^{2}$ can be determined. No information at all is available on the $\tau$-row. The final result is the following ( $3 \sigma$ ranges):

$|N|=\left(\begin{array}{ccc}0.75-0.89 & 0.45-0.66 & <0.34 \\ {\left[\left(\left|N_{\mu 1}\right|^{2}+\left|N_{\mu 2}\right|^{2}\right)^{1 / 2}=\right.} & 0.57-0.86] & 0.57-0.86 \\ ? & ? & ?\end{array}\right)$.

Notice that, without assuming unitarity, only half of the elements can be determined from oscillation experiments alone. Adding the information from near detectors at NOMAD [821], KARMEN [822], BUGEY [823] and MINOS [824], which put bounds on $\left|\left(N N^{\dagger}\right)_{\alpha \beta}\right|^{2}$ by measuring the "zero distance" effect, the degeneracy in the $\mu$-row can be solved, but the $\tau$-row is still unknown.

In order to determine/constrain all the elements of the leptonic mixing matrix without assuming unitarity, data on

\footnotetext{
${ }^{26}$ They do not apply for instance to the case of light sterile neutrinos, where the low energy mixing matrix is larger. Indeed in this case they would be included in the sum over all light mass eigenstates contained inside $\left(N N^{\dagger}\right)_{l l}$ and unitarity would be restored.
}

oscillations must be combined with data from decays. The final result is

$$
|N|=\left(\begin{array}{ccc}
0.75-0.89 & 0.45-0.65 & <0.20 \\
0.19-0.55 & 0.42-0.74 & 0.57-0.82 \\
0.13-0.56 & 0.36-0.75 & 0.54-0.82
\end{array}\right),
$$

which can be compared to the one obtained with standard analysis [825] where similar bounds are found.

It would be good to be able to determine the elements of the mixing matrix with oscillation experiments alone, permitting thus a "direct" test of unitarity. This would be for instance a way to detect light sterile neutrinos [826]. This could be possible exploring the appearance channels for instance at future facilities under discussion, such as SuperBeams [713, 827-829], $\beta$-Beams [830] and Neutrino Factories [831, 832], where the $\tau$-row and phases could be measured. Moreover, near detectors at neutrino factories could also improve the bounds on $\left(N N^{\dagger}\right)_{e \tau}$ and $\left(N N^{\dagger}\right)_{\mu \tau}$ by about one order of magnitude. All this information, coming from both decays and oscillation experiments, will be important not only to detect new physics, but even to discriminate among different scenarios.

\subsubsection{Lepton universality}

High precision electroweak tests (HPET) represent a powerful tool to probe the SM and, hence, to constrain or obtain indirect hints of new physics beyond it. A typical and relevant example of HPET is represented by the Lepton Universality (LU) breaking. Kaon and pion physics are obvious grounds where to perform such tests, for instance in the well studied $\pi_{\ell 2}\left(\pi \rightarrow \ell \nu_{\ell}\right)$ and $K_{\ell 2}\left(K \rightarrow \ell v_{\ell}\right)$ decays, where $l=e$ or $\mu$.

Unfortunately, the relevance of these single decay channels in probing the SM is severely hindered by our theoretical uncertainties, which still remain at the percent level (in particular due to the uncertainties on non-perturbative quantities like $f_{\pi}$ and $f_{K}$ ). This is what prevents us from fully exploiting such decay modes in constraining new physics, in spite of the fact that it is possible to obtain non-SM contributions which exceed the high experimental precision which has been achieved on those modes.

On the other hand, in the ratios $R_{\pi}$ and $R_{K}$ of the electronic and muonic decay modes $R_{\pi}=\Gamma(\pi \rightarrow e v) / \Gamma(\pi \rightarrow$ $\mu \nu)$ and $R_{K}=\Gamma(K \rightarrow e v) / \Gamma(K \rightarrow \mu \nu)$, the hadronic uncertainties cancel to a very large extent. As a result, the SM predictions of $R_{\pi}$ and $R_{K}$ are known with excellent accuracy [833] and this makes it possible to fully exploit the great experimental resolutions on $R_{\pi}$ [834] and $R_{K}[834,835]$ to constrain new physics effects. Given our limited predictive power on $f_{\pi}$ and $f_{K}$, deviations from the $\mu-e$ universality represent the best hope we have at the moment to detect new physics effects in $\pi_{\ell 2}$ and $K_{\ell 2}$. 
The most recent NA48/2 result on $R_{K}$ :

$R_{K}^{\text {exp. }}=\left(2.416 \pm 0.043_{\text {stat. }} \pm 0.024_{\text {syst. }}\right) \times 10^{-5} \mathrm{NA} 48 / 2$,

which will further improve with current analysis, significantly improves on the previous PDG value:

$R_{K}^{\text {exp. }}=(2.44 \pm 0.11) \times 10^{-5}$.

This is to be compared with the SM prediction, which reads

$R_{K}^{\mathrm{SM}}=(2.472 \pm 0.001) \times 10^{-5}$.

The details of the experimental measurement of $R_{K}$ are presented in Sect. 6.2 of this report. Denoting by $\Delta r_{\mathrm{NP}}^{e-\mu}$ the deviation from $\mu-e$ universality in $R_{K}$ due to new physics, i.e.,

$R_{K}=R_{K}^{\mathrm{SM}}\left(1+\Delta r_{\mathrm{NP}}^{e-\mu}\right)$,

the NA48/2 result requires (at the $2 \sigma$ level):

$-0.063 \leq \Delta r_{\mathrm{NP}}^{e-\mu} \leq 0.017 \quad \mathrm{NA} 48 / 2$.

In the following, we consider low energy minimal SUSY extensions of the SM (MSSM) with R parity as the source of new physics to be tested by $R_{K}$ [836]. The question we intend to address is whether SUSY can cause deviations from $\mu-e$ universality in $K_{l 2}$ at a level which can be probed with the present attained experimental sensitivity, namely at the percent level. We shall show that (i) it is indeed possible for regions of the MSSM to obtain $\Delta r_{\mathrm{NP}}^{e-\mu}$ of $\mathcal{O}\left(10^{-2}\right)$ and (ii) such large contributions to $K_{\ell 2}$ do not arise from SUSY lepton flavor conserving (LFC) effects, but, rather, from LFV ones.

At first sight, this latter statement may seem rather puzzling. The $K \rightarrow e v_{e}$ and $K \rightarrow \mu v_{\mu}$ decays are LFC and one could expect that it is through LFC SUSY contributions affecting differently the two decays that one obtains the dominant source of lepton flavor non-universality in SUSY. On the other hand, one can easily guess that, whenever new physics intervenes in $K \rightarrow e v_{e}$ and $K \rightarrow \mu v_{\mu}$ to create a departure from strict SM $\mu-e$ universality, these new contributions will be proportional to the lepton masses; hence, it may happen (and, indeed, this is what occurs in the SUSY case) that LFC contributions are suppressed with respect to the LFV ones by higher powers of the first two generations lepton masses (it turns out that the first contributions to $\Delta r_{\mathrm{NP}}^{e-\mu}$ from LFC terms arise at the cubic order in $m_{\ell}$, with $\ell=e, \mu)$. A second, important reason for such result is that among the LFV contributions to $R_{K}$ one can select those which involve flavor changes from the first two lepton generations to the third one with the possibility of picking up terms proportional to the tau-Yukawa coupling which can be large in the large $\tan \beta$ regime (the parameter $\tan \beta$ denotes the ratio of Higgs vacuum expectation values responsible for the up- and down-quark masses, respectively). Moreover, the relevant one loop induced LFV Yukawa interactions are known [806] to acquire an additional $\tan \beta$ factor with respect to the tree level LFC Yukawa terms. Thus, the loop suppression factor can be (partially) compensated in the large $\tan \beta$ regime.

Finally, given the NA48/2 $R_{K}$ central value below the SM prediction, one may wonder whether SUSY contributions could have the correct sign to account for such an effect. Although the above mentioned LFV terms can only add positive contributions to $R_{K}$ (since their amplitudes cannot interfere with the SM one), it turns out that there exist LFC contributions arising from double LFV mass insertions (MI) in the scalar lepton propagators which can destructively interfere with the SM contribution. We shall show that there exist regions of the SUSY parameter space where the total $R_{K}$ arising from all such SM and SUSY terms is indeed lower than $R_{K}^{\mathrm{SM}}$.

Finally, we also discuss the potentiality of $\tau-\mu(e)$ universality breaking in $\tau$ decays to probe new physics effects.

5.6.2.1 $\mu-e$ universality in $\pi \rightarrow \ell v$ and $K \rightarrow \ell v$ decays Due to the $V-A$ structure of the weak interactions, the SM contributions to $\pi_{\ell 2}$ and $K_{\ell 2}$ are helicity suppressed; hence, these processes are very sensitive to non-SM effects (such as multi-Higgs effects) which might induce an effective pseudoscalar hadronic weak current.

In particular, charged Higgs bosons $\left(H^{ \pm}\right)$appearing in any model with two Higgs doublets (including the SUSY case) can contribute at tree level to the above processes. The relevant four-Fermi interaction for the decay of charged mesons induced by $W^{ \pm}$and $H^{ \pm}$has the following form:

$$
\begin{aligned}
& \frac{4 G_{\mathrm{F}}}{\sqrt{2}} V_{u d}\left[\left(\bar{u} \gamma_{\mu} P_{L} d\right)\left(\bar{l} \gamma^{\mu} P_{L} v\right)\right. \\
& \left.-\tan ^{2} \beta\left(\frac{m_{d} m_{l}}{m_{H^{ \pm}}^{2}}\right)\left(\bar{u} P_{R} d\right)\left(\bar{l} P_{L} v\right)\right],
\end{aligned}
$$

where $P_{R, L}=\left(1 \pm \gamma_{5}\right) / 2$. Here we keep only the $\tan \beta$ enhanced part of the $H^{ \pm} u d$ coupling, namely the $m_{d} \tan \beta$ term. The decays $M \rightarrow l v$ (being $M$ the generic meson) proceed via the axial-vector part of the $W^{ \pm}$coupling and via the pseudoscalar part of the $H^{ \pm}$coupling. Then, once we implement the PCACs

$$
\begin{aligned}
& \left\langle 0\left|\bar{u} \gamma_{\mu} \gamma_{5} d\right| M^{-}\right\rangle=i f_{M} p_{M}^{\mu}, \\
& \left\langle 0\left|\bar{u} \gamma_{5} d\right| M^{-}\right\rangle=-i f_{M} \frac{m_{M}^{2}}{m_{d}+m_{u}},
\end{aligned}
$$


we easily arrive at the amplitude

$$
\begin{aligned}
\mathcal{M}_{M \rightarrow l v}= & \frac{G_{\mathrm{F}}}{\sqrt{2}} V_{u(d, s)} f_{M}\left[m_{l}-m_{l} \tan ^{2} \beta\right. \\
& \left.\times\left(\frac{m_{d}}{m_{d}+m_{u}}\right) \frac{m_{M}^{2}}{m_{H^{ \pm}}^{2}}\right] \bar{l}\left(1-\gamma_{5}\right) v .
\end{aligned}
$$

We observe that the SM term is proportional to $m_{l}$ because of the helicity suppression while the charged Higgs term is proportional to $m_{l}$ because of the Yukawa coupling. The tree level partial width is given by [806]

$\Gamma\left(M^{-} \rightarrow l^{-} \bar{v}\right)=\frac{G_{\mathrm{F}}^{2}}{8 \pi}\left|V_{u(d, s)}\right|^{2} f_{M}^{2} m_{M} m_{l}^{2}\left(1-\frac{m_{l}^{2}}{m_{M}^{2}}\right) \times r_{M}$,

where

$r_{M}=\left[1-\tan ^{2} \beta\left(\frac{m_{d, s}}{m_{u}+m_{d, s}}\right) \frac{m_{M}^{2}}{m_{H^{ \pm}}^{2}}\right]^{2}$,

and where $m_{u}$ is the mass of the up quark while $m_{s, d}$ stands for the down-type quark mass of the $M$ meson $(M=K, \pi)$. From (5.119) it is evident that such tree level contributions do not introduce any lepton flavor dependent correction. The first SUSY contributions violating the $\mu-e$ universality in $\pi \rightarrow \ell v$ and $K \rightarrow \ell v$ decays arise at the one loop level with various diagrams involving exchanges of (charged and neutral) Higgs scalars, charginos, neutralinos and sleptons. For our purpose, it is relevant to divide all such contributions into two classes: (i) LFC contributions where the charged meson $M$ decays without FCNC in the leptonic sector, i.e. $M \rightarrow \ell v_{\ell}$; (ii) LFV contributions $M \rightarrow \ell_{i} v_{k}$, with $i$ and $k$ referring to different generations (in particular, the interesting case will be for $i=e, \mu$, and $k=\tau$ ).

5.6.2.2 The lepton flavor conserving case One-loop corrections to $R_{\pi}$ and $R_{K}$ include box, wave function renormalization and vertex contributions from SUSY particle exchange. The complete calculation of the $\mu$ decay in the MSSM [837] can be easily applied to meson decays.

The dominant diagrams containing one loop corrections to the $l W v_{l}$ vertex have the following suppression factors (compared to the tree level graph):

- $\frac{g_{2}^{2}}{16 \pi^{2}} \frac{m_{l}^{2}}{m_{W}^{2}} \tan \beta \frac{m_{W}^{2}}{m_{h}^{2}}$ for loops with $h W^{ \pm} l$ exchange (with $\left.h=H^{0}, h^{0}\right)$,

$-\frac{g_{2}^{2}}{16 \pi^{2}} \frac{m_{l}^{2}}{m_{W}^{2}} \tan ^{2} \beta \frac{m_{W}^{2}}{m_{h}^{2}}$ for loops with $h H^{ \pm} l$ exchange (with $h=H^{0}, h^{0}$ and $A^{0}$ ),

$-\frac{g_{2}^{2}}{16 \pi^{2}} \frac{m_{W}^{2}}{M_{\text {SUSY }}^{2}}$ for loops generated by charginos/neutralinos and sleptons.
For dominant box contributions we have the following estimates:

$-\frac{g_{2}^{2}}{16 \pi^{2}} \frac{m_{d} m_{l}}{M^{2}} \tan ^{2} \beta$ for boxes with $h W^{ \pm} l$ or $Z^{0} H^{ \pm} l$ exchange (where $M$ is the heavier mass circulating in the loop),

- $\frac{g_{2}^{2}}{16 \pi^{2}}\left(\frac{m_{d} m_{l}}{m_{W} M_{H^{ \pm}}}\right)^{2} \tan ^{4} \beta$ for boxes with $h H^{ \pm} l$,

$-\frac{g_{2}^{2}}{16 \pi^{2}} \frac{m_{W}^{2}}{M_{\text {SUSY }}^{2}}$ for loops generated by charginos/neutralinos and sleptons (where $M_{\text {SUSY }}$ is the heavier mass circulating in the loop).

To get a feeling of the order of magnitude of the above contributions let us show the explicit expression of the dominant Higgs contributions to the $l W v_{l}$ vertex [837]:

$$
\begin{aligned}
\Delta r_{\mathrm{SUSY}}^{e-\mu}= & \frac{\alpha_{2}}{32 \pi} \frac{m_{\mu}^{2}}{M_{W}^{2}} \tan ^{2} \beta\left(-2+I\left(A^{0}, H^{ \pm}\right)\right. \\
& \left.+c_{\alpha}^{2} I\left(H^{0}, H^{ \pm}\right)+s_{\alpha}^{2} I\left(h^{0}, H^{ \pm}\right)\right),
\end{aligned}
$$

where

$I(1,2)=\frac{1}{2} \frac{m_{1}^{2}+m_{2}^{2}}{m_{1}^{2}-m_{2}^{2}} \log \frac{m_{1}^{2}}{m_{2}^{2}}$

and $\alpha$ is the mixing angle in the CP-even Higgs sector. Even if we assume $\tan \beta=50$ and arbitrary relations among the Higgs boson masses we get a value for $\Delta r_{\text {SUSY }}^{e-\mu} \leq 10^{-6}$ much below the actual experimental resolution. In addition, in the large $\tan \beta$ limit, $\alpha \rightarrow 0$ and $m_{A^{0}} \sim m_{H^{0}} \sim m_{H^{ \pm}}$and $\Delta r_{\text {SUSY }}^{e-\mu}$ tends to vanish. The charginos/neutralinos sleptons $\left(\tilde{l}_{e, \mu}\right)$ contributions to $\Delta r_{\text {SUSY }}^{e-\mu}$ are of the form

$\Delta r_{\mathrm{SUSY}}^{e-\mu} \sim \frac{\alpha_{2}}{4 \pi}\left(\frac{\tilde{m}_{\mu}^{2}-\tilde{m}_{e}^{2}}{\tilde{m}_{\mu}^{2}+\tilde{m}_{e}^{2}}\right) \frac{m_{W}^{2}}{M_{\mathrm{SUSY}}^{2}}$.

The degeneracy of slepton masses (in particular those of the first two generations) severely suppresses these contributions. Even if we assume a quite large mass splitting among slepton masses (at the $10 \%$ level for instance) we end up with $\Delta r_{\text {SUSY }}^{e-\mu} \leq 10^{-4}$. For the box-type non-universal contributions, we find similar or even more suppressed effects compared to those we have studied. So, finally, it turns out that all these LFC contributions yield values of $\Delta r_{K \text { SUSY }}^{e-\mu}$ which are much smaller than the percent level required by the achieved experimental sensitivity.

On the other hand, one could wonder whether the quantity $\Delta r_{\text {SUSY }}^{e-\mu}$ can be constrained by the pion physics. In principle, the sensitivity could be even higher: from

$R_{\pi}^{\text {exp. }}=(1.230 \pm 0.004) \times 10^{-4} \quad \mathrm{PDG}$,

and by making a comparison with the SM prediction

$R_{\pi}^{\mathrm{SM}}=(1.2354 \pm 0.0002) \times 10^{-4}$, 
one obtains (at the $2 \sigma$ level)

$-0.0107 \leq \Delta r_{\mathrm{NP}}^{e-\mu} \leq 0.0022$.

Unfortunately, even in the most favorable cases, $\Delta r_{\mathrm{SUSY}}^{e-\mu} \mathrm{re}-$ mains much below its actual experimental upper bound.

In conclusion, SUSY effects with flavor conservation in the leptonic sector can differently contribute to the $K \rightarrow e v_{e}$ and $K \rightarrow \mu v_{\mu}$ decays, hence inducing $\mu-e$ non-universality in $R_{K}$, however such effects are still orders of magnitude below the level of the present experimental sensitivity on $R_{K}$. The same conclusions hold for $R_{\pi}$.

5.6.2.3 The lepton flavor violating case It is well known that models containing at least two Higgs doublets generally allow for flavor violating couplings of the Higgs bosons with the fermions [838]. In the MSSM such LFV couplings are absent at tree level. However, once non-holomorphic terms are generated by loop effects (so called HRS corrections [430]) and given a source of LFV among the sleptons, Higgs-mediated (radiatively induced) $H \ell_{i} \ell_{j}$ LFV couplings are unavoidable $[806,807]$. These effects have been widely discussed through the study of several processes, namely $\tau \rightarrow \ell_{j} \ell_{k} \ell_{k}$ [806, 807], $\tau \rightarrow \mu \eta$ [810], $\mu-e$ conversion in nuclei [811], $B \rightarrow \ell_{j} \tau$ [807], $H \rightarrow \ell_{j} \ell_{k}$ [174] and $\ell_{i} \rightarrow \ell_{j} \gamma$ [650].

Moreover, it has been shown [839] that Higgs-mediated LFV couplings generate a breaking of $\mu-e$ universality in purely leptonic $\pi^{ \pm}$and $K^{ \pm}$decays.

One could naively think that SUSY effects in the LFV channels $M \rightarrow \ell_{i} v_{k}$ are further suppressed with respect to the LFC ones. On the contrary, charged Higgs mediated SUSY LFV contributions, in particular in the kaon decays into an electron or a muon and a tau neutrino, can be strongly enhanced. The quantity which now accounts for the deviation from the $\mu-e$ universality reads

$R_{\pi, K}^{\mathrm{LFV}}=\frac{\sum_{i} \Gamma\left(\pi(K) \rightarrow e \nu_{i}\right)}{\sum_{i} \Gamma\left(\pi(K) \rightarrow \mu \nu_{i}\right)}, \quad i=e, \mu, \tau$,

with the sum extended over all (anti)neutrino flavors (experimentally one determines only the charged lepton flavor in the decay products).

The dominant SUSY contributions to $R_{\pi, K}^{\mathrm{LFV}}$ arise from the charged Higgs exchange. The effective LFV Yukawa couplings we consider are (see Fig. 39)

$\ell H^{ \pm} \nu_{\tau} \rightarrow \frac{g_{2}}{\sqrt{2}} \frac{m_{\tau}}{M_{W}} \Delta_{R}^{3 l} \tan ^{2} \beta, \quad \ell=e, \mu$.

A crucial ingredient for the effects we are going to discuss is the quadratic dependence on $\tan \beta$ in the above coupling: one power of $\tan \beta$ comes from the trilinear scalar coupling in Fig. 39, while the second one is a specific feature of the above HRS mechanism.

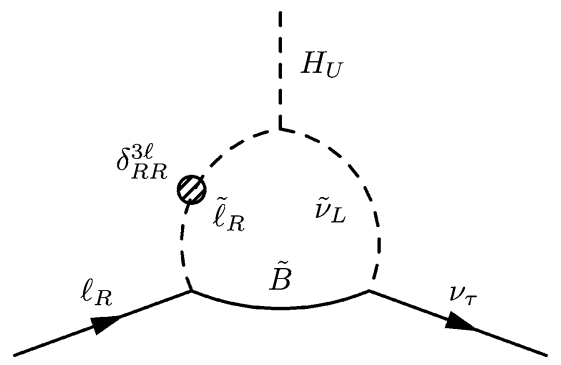

Fig. 39 Contribution to the effective $\bar{v}_{\tau} \ell_{R} H^{+}$coupling

The $\Delta_{R}^{3 \ell}$ terms are induced at one loop level by the exchange of bino (see Fig. 39) or bino-higgsino and sleptons. Since the Yukawa operator is of dimension four, the quantities $\Delta_{R}^{3 \ell}$ depend only on ratios of SUSY masses, hence avoiding SUSY decoupling. In the so called MI approximation the expression of $\Delta_{R}^{3 \ell}$ is given by:

$\Delta_{R}^{3 \ell} \simeq \frac{\alpha_{1}}{4 \pi} \mu M_{1} m_{R}^{2} \delta_{R R}^{3 \ell}\left[I^{\prime}\left(M_{1}^{2}, \mu^{2}, m_{R}^{2}\right)-\left(\mu \leftrightarrow m_{L}\right)\right]$,

where $\mu$ is the Higgs mixing parameter, $M_{1}$ is the bino $(\tilde{B})$ mass and $m_{L(R)}^{2}$ stands for the left-left (right-right) slepton mass matrix entry. The LFV MIs, i.e. $\delta_{X X}^{3 \ell}=\left(\tilde{m}_{\ell}^{2}\right)_{X X}^{3 \ell} / m_{X}^{2}$ $(X=L, R)$, are the off-diagonal flavor changing entries of the slepton mass matrix. The loop function $I^{\prime}(x, y, z)$ is such that $I^{\prime}(x, y, z)=d I(x, y, z) / d z$, where $I(x, y, z)$ refers to the standard three point one loop integral which has mass dimension-2.

Making use of the LFV Yukawa coupling in (5.120), it turns out that the dominant contribution to $\Delta r_{\mathrm{NP}}^{e-\mu}$ reads [839]

$R_{K}^{\mathrm{LFV}} \simeq R_{K}^{\mathrm{SM}}\left[1+\left(\frac{m_{K}^{4}}{M_{H}^{4}}\right)\left(\frac{m_{\tau}^{2}}{m_{e}^{2}}\right)\left|\Delta_{R}^{31}\right|^{2} \tan ^{6} \beta\right]$.

In (5.122) terms proportional to $\Delta_{R}^{32}$ are neglected given that they are suppressed by a factor $m_{e}^{2} / m_{\mu}^{2}$ with respect to the term proportional to $\Delta_{R}^{31}$.

Taking $\Delta_{R}^{31} \simeq 5 \times 10^{-4}$ (by means of a numerical analysis, it turns out that $\Delta_{R}^{3 \ell} \leq 10^{-3}$ [174]), $\tan \beta=40$ and $M_{H}=500 \mathrm{GeV}$ we end up with $R_{K}^{\mathrm{LFV}} \simeq R_{K}^{\mathrm{SM}}(1+0.013)$. We see that in the large (but not extreme) $\tan \beta$ regime and with a relatively heavy $H^{ \pm}$, it is possible to reach contributions to $\Delta r_{K \text { SUSY }}^{e-\mu}$ at the percent level thanks to the possible LFV enhancements arising in SUSY models.

Turning to pion physics, one could wonder whether the analogous quantity $\Delta r_{\pi \text { SUSY }}^{e-\mu}$ is able to constrain SUSY LFV. However, the correlation between $\Delta r_{\pi \text { SUSY }}^{e-\mu}$ and $\Delta r_{K \text { SUSY }}^{e-\mu}$ :

$\Delta r_{\pi \text { SUSY }}^{e-\mu} \simeq\left(\frac{m_{d}}{m_{u}+m_{d}}\right)^{2}\left(\frac{m_{\pi}^{4}}{m_{k}^{4}}\right) \Delta r_{K \text { SUSY }}^{e-\mu}$, 
clearly shows that the constraints on $\Delta r_{K \text { SUSY force }}^{e-\mu}$ $\Delta r_{\pi \text { SUSY }}^{e-\mu}$ to be much below its actual experimental upper bound.

5.6.2.4 On the sign of $\Delta r_{\text {SUSY }}^{e-\mu}$ The above SUSY dominant contribution to $\Delta r_{\mathrm{NP}}^{e-\mu}$ arises from LFV channels in the $K \rightarrow e v$ mode, hence without any interference effect with the SM contribution. Thus, it can only increase the value of $R_{K}$ with respect to the SM expectation. On the other hand, the recent NA48/2 result exhibits a central value lower than $R_{K}^{\mathrm{SM}}$ (and, indeed, also lower than the previous PDG central value). One may wonder whether SUSY could account for such a lower $R_{K}$. Obviously, the only way it can is through terms which, contributing to the LFC $K \rightarrow l \nu_{l}$ channels, can interfere (destructively) with the SM contribution. We already commented that SUSY LFC contributions are subdominant. However, one can envisage the possibility of making use of the large LFV contributions to give rise to LFC ones through double LFV MI that, as a final effect, preserves the flavor.

To see this point explicitly, we report the corrections to the LFC $H^{ \pm} \ell v_{\ell}$ vertices induced by LFV effects

$\ell H^{ \pm} \nu_{\ell} \rightarrow \frac{g_{2}}{\sqrt{2}} \frac{m_{\ell}}{M_{W}} \tan \beta\left(1+\frac{m_{\tau}}{m_{\ell}} \Delta_{R L}^{\ell \ell} \tan \beta\right)$,

where $\Delta_{R L}^{\ell \ell}$ is generated by the same diagram as in Fig. 39 but with an additional $\delta_{L L}^{3 \ell}$ MI in the sneutrino propagator. In the MI approximation, $\Delta_{R L}^{\ell \ell}$ is given by

$\Delta_{R L}^{\ell \ell} \simeq-\frac{\alpha_{1}}{4 \pi} \mu M_{1} m_{L}^{2} m_{R}^{2} \delta_{R R}^{\ell 3} \delta_{L L}^{3 \ell} I^{\prime \prime}\left(M_{1}^{2}, m_{L}^{2}, m_{R}^{2}\right)$,

where $I^{\prime \prime}(x, y, z)=d^{2} I(x, y, z) / d y d z$. In the large slepton mixing case, $\Delta_{R L}^{\ell \ell}$ terms are of the same order of $\Delta_{R}^{3 \ell} \cdot{ }^{27}$ These new effects modify the previous $R_{K}^{\mathrm{LFV}}$ expression in the following way [839]:

$$
\begin{aligned}
R_{K}^{\mathrm{LFV}} \simeq & R_{K}^{\mathrm{SM}}\left[\left|1-\frac{m_{K}^{2}}{M_{H}^{2}} \frac{m_{\tau}}{m_{e}} \Delta_{R L}^{11} t_{\beta}^{3}\right|^{2}\right. \\
& \left.+\left(\frac{m_{K}^{4}}{M_{H}^{4}}\right)\left(\frac{m_{\tau}^{2}}{m_{e}^{2}}\right)\left|\Delta_{R}^{31}\right|^{2} \tan ^{6} \beta\right] .
\end{aligned}
$$

In the above expression, besides the contributions reported in (5.122), we also included the interference between SM and SUSY LFC terms (arising from a double LFV source). Setting the parameters as in the example of the above section and if $\Delta_{R L}^{11}=10^{-4}$ we get $R_{K}^{\mathrm{LFV}} \simeq R_{K}^{\mathrm{SM}}(1-0.032)$, that is

${ }^{27} \operatorname{Im}\left(\delta_{R R}^{13} \delta_{L L}^{31}\right)$ is strongly constrained by the electron electric dipole moment [163]. However, sizable contributions to $R_{K}^{\mathrm{LFV}}$ can still be induced by $\operatorname{Re}\left(\delta_{R R}^{13} \delta_{L L}^{31}\right)$. just within the expected experimental resolution reachable by NA48/2 once all the available data will be analyzed. Finally, we remark that the above effects do not spoil the pion physics constraints.

The extension of the above results to $B \rightarrow \ell v[766]$ is obtained with the replacement $m_{K} \rightarrow m_{B}$, while for the $D \rightarrow \ell v$ case $m_{K}^{2} \rightarrow\left(m_{s} / m_{c}\right) m_{D}^{2}$. In the most favorable scenarios, taking into account the constraints from LFV $\tau$ decays [650], spectacular order-of-magnitude enhancements for $R_{B}^{e / \tau}$ and $\mathcal{O}(50 \%)$ deviations from the $\mathrm{SM}$ in $R_{B}^{\mu / \tau}$ are allowed [766]. There exists a stringent correlation between $R_{B}^{e / \tau}$ and $R_{K}^{e / \mu}$ so that

$R_{B}^{e / \tau} \simeq\left[r_{H}+\frac{m_{B}^{4}}{m_{K}^{4}} \Delta r_{K \text { SUSY }}^{e-\mu}\right] \leq 2 \times 10^{2}$

In particular, it turns out that $\Delta r_{K \text { SUSY }}^{e-\mu}$ is much more effective to constrain $R_{B}^{e / \tau} \Gamma\left(B \rightarrow e \nu_{\tau}\right)$ than LFV tau decay processes.

5.6.2.5 Lepton universality in $M \rightarrow \ell v$ versus $L F V \tau$ decays Obviously, a legitimate worry when witnessing such a huge SUSY contribution through LFV terms is whether the bounds on LFV tau decays, like $\tau \rightarrow e X$ (with $X=$ $\gamma, \eta, \mu \mu)$, are respected [650]. Higgs mediated $B(\tau \rightarrow$ $\left.\ell_{j} X\right)$ and $\Delta r_{K \text { SUSY }}^{e-\mu}$ have exactly the same SUSY dependence; hence, we can compute the upper bounds of the relevant LFV tau decays which are obtained for those values of the SUSY parameters yielding $\Delta r_{K \text { SUSY }}^{e-\mu}$ at the percent level.

The most sensitive processes to Higgs mediated LFV in the $\tau$ lepton decay channels are $\tau \rightarrow \mu(e) \eta, \tau \rightarrow \mu(e) \mu \mu$ and $\tau \rightarrow \mu(e) \gamma$. The related branching ratios are [650]

$$
\begin{aligned}
\frac{B\left(\tau \rightarrow l_{j} \eta\right)}{B\left(\tau \rightarrow l_{j} \bar{\nu}_{j} \nu_{\tau}\right)} \simeq & 18 \pi^{2}\left(\frac{f_{\eta}^{8} m_{\eta}^{2}}{m_{\tau}}\right)^{2}\left(1-\frac{m_{\eta}^{2}}{m_{\tau}^{2}}\right)^{2} \\
& \times\left(\frac{\left|\Delta^{3 j}\right|^{2} \tan ^{6} \beta}{m_{A}^{4}}\right),
\end{aligned}
$$

where $m_{\eta}^{2} / m_{\tau}^{2} \simeq 9.5 \times 10^{-2}$ and the relevant decay constant is $f_{\eta}^{8} \sim 110 \mathrm{MeV}$,

$$
\begin{aligned}
\frac{B\left(\tau \rightarrow l_{j} \gamma\right)}{B\left(\tau \rightarrow l_{j} \bar{v}_{j} \nu_{\tau}\right)} \simeq & 10\left(\frac{\alpha_{e l}}{\pi}\right)^{3} \tan ^{4} \beta\left|\Delta_{\tau j}\right|^{2} \\
& \times\left[\frac{m_{W}}{m_{A}} \log \left(\frac{m_{W}^{2}}{m_{A}^{2}}\right)\right]^{4}, \\
\frac{B\left(\tau \rightarrow l_{j} \mu \mu\right)}{B\left(\tau \rightarrow l_{j} \bar{v}_{j} \nu_{\tau}\right)} \simeq & \frac{m_{\tau}^{2} m_{\mu}^{2}}{32}\left(\frac{\left|\Delta^{3 j}\right|^{2} \tan ^{6} \beta}{m_{A}^{4}}\right) \\
& \times\left(3+5 \delta_{j \mu}\right),
\end{aligned}
$$


where $\left|\Delta^{3 j}\right|^{2}=\left|\Delta_{L}^{3 j}\right|^{2}+\left|\Delta_{R}^{3 j}\right|^{2}$. It is straightforward to check that, in the large $\tan \beta$ regime, $B\left(\tau \rightarrow l_{j} \eta\right)$ and $B\left(\tau \rightarrow l_{j} \gamma\right)$ are of the same order of magnitude [650] and they are dominant compared to $B\left(\tau \rightarrow l_{j} \mu \mu\right) .{ }^{28}$

Given that $\Delta r_{K \text { SUSY }}^{e-\mu}$ and $B\left(\tau \rightarrow l_{j} X\right)$ have the same SUSY dependence, once we saturate the $\Delta r_{K \text { SUSY value (at }}^{e-\mu}$ the $\%$ level), the upper bounds on $B\left(\tau \rightarrow l_{j} X\right)$ (allowed by $\left.\left|\Delta_{R}^{31}\right|^{2}\right)$ are automatically predicted. We find that

$$
\begin{aligned}
B\left(\tau \rightarrow l_{j} \gamma\right) & \sim B\left(\tau \rightarrow l_{j} \eta\right) \simeq 10^{-2}\left(\frac{\left|\Delta^{3 j}\right|^{2} \tan ^{6} \beta}{m_{A}^{4}}\right) \\
& \simeq 10^{-8} \Delta r_{K \text { SUSY }}^{e-\mu} .
\end{aligned}
$$

So, employing the constraints for $\Delta r_{K \text { SUSY }}^{e-\mu}$ at the $\%$ level, we obtain the desired upper bounds: $B(\tau \rightarrow e \eta)$, $B(\tau \rightarrow e \gamma) \leq 10^{-10}$. Given the experimental upper bounds on the LFV $\tau$ lepton decays [764], we conclude that it is possible to saturate the upper bound on $\Delta r_{K \text { SUSY while re- }}^{e-\mu}$ maining much below the present and expected future upper bounds on such LFV decays. There exist other SUSY contributions to LFV $\tau$ decays, like the one loop neutralinocharged slepton exchanges, for instance, where there is a direct dependence on the quantities $\delta_{R R}^{3 j}$. Given that the existing bounds on the leptonic $\delta_{R R}$ involving transitions to the third generation are rather loose [681], it turns out that also these contributions fail to reach the level of experimental sensitivity for LFV $\tau$ decays.

5.6.2.6 $e-\mu$ universality in $\tau$ decays Studying the $\tau-\mu-e$ universality in the leptonic $\tau$ decays is an interesting laboratory for search for physics beyond the SM. In the SM the $\tau$ decay partial width for the leptonic modes is

$$
\begin{aligned}
\Gamma(\tau & \left.\rightarrow l \bar{v}_{l} \nu_{\tau}(\gamma)\right) \\
= & \frac{G_{\mathrm{F}}^{2} m_{\tau}^{5}}{192 \pi^{3}} f\left(m_{l}^{2} / m_{\tau}^{2}\right) \\
& \times\left[1+\frac{3}{5} \frac{m_{\tau}^{2}}{M_{W}^{2}}\right]\left[1+\frac{\alpha\left(m_{\tau}\right)}{\pi}\left(\frac{25}{4}-\pi^{2}\right)\right],
\end{aligned}
$$

where $f(x)=1-8 x+8 x^{3}-x^{4}-12 x^{2} \log x$ is the lepton mass correction and the last two factors are corrections from the nonlocal structure of the intermediate $W^{ \pm}$boson propagator and QED radiative corrections respectively. The Fermi constant $G_{\mathrm{F}}$ is determined by the muon life-time

$$
G_{\mathrm{F}} \equiv G_{\mu}=(1.16637 \pm 0.00002) \times 10^{-5} \mathrm{GeV}^{-2},
$$

\footnotetext{
${ }^{28}$ It is remarkable that $\Delta r_{K}^{e-\mu}$ SUSY $\sim\left|\Delta_{R}^{31}\right|^{2}$ while $B(\tau \rightarrow e X) \sim$ $\left|\Delta_{L}^{31}\right|^{2}+\left|\Delta_{R}^{31}\right|^{2}$ (with $X=\eta, \gamma$ or $\mu \mu$ ). In practice, $\Delta r_{K \text { SUSY }}^{e-\mu}$ is sensitive only to RR-type LFV terms in the slepton mass matrix while $B(\tau \rightarrow e X)$ does not distinguish between left and right sectors.
}

and absorbs all the remaining electroweak radiative (loop) corrections.

The main source of non-universal contributions would be the tree level contribution from the charged Higgs boson (mass dependent couplings) and different slepton masses of the $\tilde{\mu}, \tilde{\tau}$ and $\tilde{e}$ sleptons exchanged in the one loop induced $\ell-W-v_{\ell}$ vertex. On the other hand, as discussed in previous sections, the last contribution can provide a correction that can be at most as large as $10^{-4}$ (in the limiting case of very light sleptons and gauginos $\sim M_{W}$ ), very far for the actual and forthcoming experimental resolutions. However, differently from the $M \rightarrow \ell v$ case, a tree level charged Higgs exchange breaks the lepton universality and it provides a contribution that we are going to discuss.

The deviations from $\tau-\mu-e$ universality can be conveniently discussed by studying the ratios $G_{\tau, e} / G_{\mu, e}$, $G_{\tau, \mu} / G_{\mu, e}$ and $G_{\tau, \mu} / G_{\tau, e}$, given by the ratios of the corresponding branching fractions. With the highly accurate experimental result for the $G_{\mu, e}$, the first two ratios are essentially a direct measure of non-universality in the corresponding tau decays. When the statistical error of future experiments will become negligible, the main problem for achieving maximum precision will be to reduce the systematic errors. One may expect that certain systematic errors will be canceled in the ratio $G_{\tau, \mu} / G_{\tau, e}$.

The 2004 world averaged data for the leptonic $\tau$ decay modes and $\tau$ life-time are [834, 840]

$$
\begin{aligned}
& \left.B^{e}\right|_{\exp }=(17.84 \pm 0.06) \%, \\
& \left.B^{\mu}\right|_{\exp }=(17.37 \pm 0.06) \%, \\
& \tau_{\tau}=(290.6 \pm 1.1) \times 10^{-15} \mathrm{~s} .
\end{aligned}
$$

Note that the relative errors of the above measured quantities are of the $0.34-0.38 \%$, the biggest being for the life-time. One can parameterize a possible beyond the SM contribution by a quantity $\Delta^{l}(l=e, \mu)$, defined as

$B^{l}=\left.B^{l}\right|_{\operatorname{SM}}\left(1+\Delta^{l}\right)$.

Including the $W$-propagator effect and QED radiative corrections, the following results for the branching ratios in the SM are obtained [840]:

$\left.B^{e}\right|_{\mathrm{SM}}=(17.80 \pm 0.07) \%$,

$\left.B^{\mu}\right|_{\mathrm{SM}}=(17.32 \pm 0.07) \%$.

Together with the experimental data this leads to the following $95 \%$ C.L. bounds on $\Delta^{l}$, for the electron and muon decay mode, respectively [840]:

$$
\left(-0.80 \leq \Delta^{e} \leq 1.21\right) \%, \quad\left(-0.76 \leq \Delta^{\mu} \leq 1.27\right) \% .
$$


One can see that the negative contributions are constrained more strongly that the positive ones. A tree level charged Higgs exchange leads to the following contribution [841]:

$$
\begin{aligned}
& \Gamma^{W^{ \pm}+H^{ \pm}} \\
& \quad=\Gamma^{W^{ \pm}}\left[1-2 \frac{m_{l} m_{\tau} \tan ^{2} \beta}{M_{H^{ \pm}}^{2}} \frac{m_{l}}{m_{\tau}} \kappa\left(\frac{m_{l}^{2}}{m_{\tau}^{2}}\right)+\frac{m_{\tau}^{2} m_{l}^{2} \tan ^{4} \beta}{4 M_{H^{ \pm}}^{4}}\right] \\
& \simeq \Gamma^{W^{ \pm}}\left[1-1.15 \times 10^{-3}\left(\frac{200 \mathrm{GeV}}{M_{H^{ \pm}}}\right)^{2}\left(\frac{\tan \beta}{50}\right)^{2}\right],
\end{aligned}
$$

where $\kappa(x)=\frac{g(x)}{f(x)} \simeq 0.94$ with $g(x)=1+9 x-9 x^{2}-x^{3}+$ $6 x(1+x) \ln (x)$. In the above expression, the second term comes from the interference with the SM amplitude and it is much more important than the last one, which is suppressed by a factor $m_{\tau}^{2} \tan ^{2} \beta / 8 M_{H^{ \pm}}^{2}$.

For the future precision of $G_{\tau, \mu}$ and $G_{\tau, e}$ measurements of order $0.1 \%$ ( $G_{\mu, e}$ is known with $0.002 \%$ precision) the only effect that eventually can be observed is the slightly smaller value of $G_{\tau, \mu}$ as compared to $G_{\tau, e}$ and $G_{\mu, e}$. If measured, such effect would mean a rather precise information about MSSM: large $\tan \beta \geq 40$ and small $M_{H^{ \pm}} \sim 200$ $300 \mathrm{GeV}$.

5.6.2.7 Conclusions High precision electroweak tests, such as deviations from the SM expectations of the lepton universality breaking, represent a powerful tool to probe the SM and, hence, to constrain or obtain indirect hints of new physics beyond it. Kaon and pion physics are obvious grounds where to perform such tests, for instance in the well studied $\pi \rightarrow \ell v_{\ell}$ and $K \rightarrow \ell \nu_{\ell}$ decays, where $l=e$ or $\mu$. In particular, a precise measurement of the flavor conserving $K \rightarrow \ell v_{\ell}$ decays may shed light on the size of LFV in new physics. $\mu-e$ non-universality in $K_{\ell 2}$ is quite effective in constraining relevant regions of SUSY models with LFV. A comparison with analogous bounds coming from $\tau$ LFV decays shows the relevance of the measurement of $R_{K}$ to probe LFV in SUSY. Moreover, $\tau-\mu-e$ universality in the leptonic $\tau$ decays is an additional interesting laboratory for searching for physics beyond the SM.

\subsection{EDMs from RGE effects in theories with low energy supersymmetry}

EDMs probe new physics in general and in particular low energy supersymmetry. For definiteness and for simplicity, we focus here on lepton EDMs, as they are free from the theoretical uncertainties associated to the calculation of hadronic matrix elements. After a brief review of the constraints on slepton masses we discuss here a specific kind of sources of $\mathrm{CPV}$, those induced radiatively by the Yukawa interactions of the heavy particles present in see-saw and/or grand unified models. It has been emphasized that these interactions could lead to LFV decays, in particular $\mu \rightarrow e \gamma$, at an observable rate; it is then natural to wonder whether this is also the case for EDMs.

As shown in Sect. 3, LFV decays, EDMs and additional contribution to MDMs all have a common origin, the dimension five dipole operator possibly induced by some new physics beyond the SM:

$\mathcal{L}_{d=5}=\frac{1}{2} \bar{\psi}_{R i} A_{i j} \psi_{L j} \sigma^{\mu v} F_{\mu v}+$ h.c.,

$B\left(\ell_{i} \rightarrow \ell_{j} \gamma\right) \propto\left|A_{i j}\right|^{2}, \quad \delta a_{\ell_{i}}=\frac{2 m_{\ell_{i}}}{e} \operatorname{Re} A_{i i}$,

$d_{\ell_{i}}=\operatorname{Im} A_{i i}$.

If induced at one loop, this amplitude displays a quadratic suppression with respect to the new physics mass scale, $M_{\mathrm{NP}}$, and a linear dependence on the non-dimensional coupling $\Gamma^{\mathrm{NP}}$ encoding the pattern of $\mathrm{F}$ and $\mathrm{CP}$ violations (in the basis where the charged lepton mass matrix is diagonal):

$A_{i j} \approx \frac{e m_{\ell_{i}}}{(4 \pi)^{2}} \frac{\Gamma_{i j}^{\mathrm{NP}}}{M_{\mathrm{NP}}^{2}}$.

For low energy supersymmetry, the loops involve exchange of gauginos and sleptons, so that $\Gamma^{\mathrm{NP}}$ is proportional to the misalignment between leptons and sleptons, conveniently described by the flavor violating $(\mathrm{FV}) \delta$ 's of the mass insertion approximation. It is well known that the flavor conserving (FC) $\mu$ and $a$ terms are potentially a very important source of CPV. In the expansion in powers of the FV $\delta$ 's, they indeed contribute to $d_{\ell_{i}}$ at zero order:

$$
\begin{aligned}
\operatorname{Im}\left(A_{i i}\right)= & f_{\mu} m_{\ell_{i}} \operatorname{Arg}(\mu)+f_{a} m_{\ell_{i}} \operatorname{Im}\left(a_{i}\right) \\
& +f_{L L R R} \operatorname{Im}\left(\delta^{L L} m_{\ell} \delta^{R R}\right)_{i i}+\cdots,
\end{aligned}
$$

where the various $f$ represent supersymmetric loop functions and can be found for instance in [163]. Notice that the contribution arising at second order in the FV $\delta$ 's could be even more important than the FC one, as happens for instance if CPV is always associated to FV.

Assuming no cancellations between the amplitudes, we first review briefly some limits considering for definiteness the mSUGRA scenario with $\tan \beta=10$ and slepton masses in the range suggested by $g_{\mu}$. The strong impact of $\mu \rightarrow e \gamma$ on $\delta^{L L}$ has been emphasized previously, where it was stressed that the impact of $d_{e}$ is also remarkable in constraining the FC sources of CPV: $\arg \mu \leq 2 \times 10^{-3}$, $\operatorname{Im} a_{e} / m_{R} \leq 0.2$. As for the other FV source in (5.142), one obtains $\operatorname{Im}\left(\delta^{L L} m_{\ell} \delta^{R R}\right)_{e e} / m_{\tau} \leq 10^{-5}$. The planned sensitivity $d_{\mu} \leq 10^{-23} e \mathrm{~cm}$ would also give interesting bounds: $\arg \mu \leq 10^{-1}, \operatorname{Im}\left(\delta^{L L} m_{\ell} \delta^{R R}\right)_{\mu \mu} / m_{\tau} \leq 10^{-1}$. Notice that, 
due to the lepton mass scaling law of the $\mu$-term contribution, the present bound on $\arg \mu$ from $d_{e}$ implies that the $\mu$-term contribution to $d_{\mu}$ cannot exceed $2 \times 10^{-25} e \mathrm{~cm}$, below the planned projects. A positive measure of $d_{\mu}$ would thus signal a different source of CPV, i.e. the $a_{\mu}$-term or the FV contribution. In the following we take real $\mu$.

The $a_{i}$-terms and the FV $\delta$ 's at low energy can be thought as the sum of two contributions. The first is already present at the Planck scale where soft masses are defined; we assume that this contribution is absent because of some inhibition mechanism, as could happen in supergravity. The second contribution is induced radiatively running from high to low energies by the Yukawa couplings of heavy particles ${ }^{29}$ that potentially violate F and CP. Since LFV experiments are testing this radiatively induced misalignment, in the following we shall consider what happens for EDMs, beginning with the pure see-saw model and then adding a grand unification scenario, where heavy colored Higgs triplets are present to complete the Higgs doublets representations (in $S U(5)$ for instance they complete the 5 and $\overline{5}$ ). Notice that these triplets are important as in supersymmetric theories proton decays mainly through their exchange.

Consider first the case of degenerate right handed neutrinos with mass $M$. One can solve approximately the RGE by expanding in powers of $\ln (\Lambda / M) /(4 \pi)^{2}$, i.e. the log of the ratio of the two scales between which the neutrino Yukawa couplings $Y_{v}$ are present times the corresponding loop factor suppression. For LFV decays $\delta_{i j}^{L L}$ is induced at 1 st order and is proportional to the combination $\left(Y_{v}^{\dagger} Y_{\nu}\right)_{i j}$. In particular, $\mu \rightarrow e \gamma$ constrains $\left(Y_{\nu}^{\dagger} Y_{v}\right)_{21}$ to be small and this has a strong impact on see-saw models. To obtain an imaginary part for EDMs, one needs a non-hermitian combination of Yukawa couplings, which can be found only at 4th order: $\operatorname{Im}\left(Y_{v}^{\dagger} Y_{v}\left[Y_{v}^{\dagger} Y_{v}, Y_{\ell}^{\dagger} Y_{\ell}\right] Y_{v}^{\dagger} Y_{v}\right)_{i i}$. Such a contribution is negligibly small with respect to the present and planned experimental sensitivities.

Allowing for a non-degenerate spectrum of right handed neutrinos, EDMs get strongly enhanced while LFV decays not. The latter are simply modified by taking into account the different mass thresholds:

$\delta_{i j}^{L L} \propto \sum_{k} C^{k}, \quad C^{k} \equiv Y_{v i k}^{\dagger} \ln \frac{\Lambda}{M_{k}} Y_{v k j}$.

On the contrary for EDMs the see-saw induced FC and FV contributions-coming respectively from $\operatorname{Im} a_{i}$ [229, $242,842,843]$ and $\operatorname{Im}\left(\delta^{R R} m_{\ell} \delta^{L L}\right)_{i i}[843,844]$-arise at 2nd and 3rd order and are proportional to the combinations [684]:

$\operatorname{Im}\left(a_{i}\right) \propto \sum_{k>k^{\prime}} \frac{\ln \left(M_{k} / M_{k^{\prime}}\right)}{\ln \left(\Lambda / M_{k^{\prime}}\right)} \operatorname{Im}\left(C^{k} C^{k^{\prime}}\right)_{i i}$

${ }^{29}$ The SM fermion Yukawa couplings induce negligible effects.
$\operatorname{Im}\left(\delta^{R R} m_{\ell} \delta^{L L}\right)_{i i} \propto \sum_{k>k^{\prime}} \tilde{\ln }_{k^{\prime}}^{k} \operatorname{Im}\left(C^{k} m_{\ell}^{2} C^{k^{\prime}}\right)_{i i}$

where $\tilde{\ln }_{k^{\prime}}^{k}$ is a logarithmic function. The FV contribution generically dominates for $\tan \beta \gtrsim 10$. Without going in the details of this formulae, we just display some representative upper estimates for the see-saw induced EDMs, considering for definiteness the $g_{\mu}$ region with $\tan \beta=20$. The see-saw induced $d_{\mu}$ is below the planned sensitivity, $d_{\mu}^{S S} \lesssim 10^{-25} e \mathrm{~cm}$. On the contrary for $d_{e}$ it could be at hand, $d_{e}^{S S} \lesssim 0.5 \times 10^{-27} e \mathrm{~cm}$; the expectation is however strongly model dependent and usually see-saw models that satisfy the bound from $\mu \rightarrow e \gamma$ predict a much smaller value [684]. The possibility of large $d_{e}$ and its correlation with leptogenesis is discussed in [329].

Perspectives are much more interesting if there is also a stage of grand unification. In minimal supersymmetric $S U(5)$, the Higgs triplet Yukawa couplings contribute to the RGE-running for energies larger than their mass scale $M_{T} \sim M_{\mathrm{GUT}}$. For LFV, $\delta^{R R}$ is generated at 1 st order and is proportional to a combination of the up quark Yukawa couplings [683]:

$\delta_{i j}^{R R} \propto\left(Y_{u}^{T} Y_{u}^{*}\right)_{i j} \ln \frac{\Lambda}{M_{T}}$.

Due to the weaker experimental bounds on $\delta^{R R}$, this contribution is not very significant. On the other hand $\delta^{L L}$ is not changed, as also happens to the FC contribution to EDMs [843]. The FV contribution to EDMs is on the contrary strongly enhanced: it arises at 2nd order (also for degenerate right handed neutrinos) and is proportional to:

$\operatorname{Im}\left(\delta^{R R} m_{\ell} \delta^{L L}\right)_{i i} \propto \operatorname{Im}\left(C m_{\ell} Y_{u}^{T} Y_{u}^{*}\right)_{i i} \ln \frac{\Lambda}{M_{T}}$.

As a result, considering for definiteness the $g_{\mu}$ region with $\tan \beta=20$ and the representative values for triplet and right handed neutrino masses $M_{T}=2 \times 10^{16} \mathrm{GeV}$ and $M_{3}=$ $10^{15} \mathrm{GeV}$, the induced $d_{\mu}$ is still below planned, $d_{\mu}^{S S 5} \lesssim$ $5 \times 10^{-25} e \mathrm{~cm}$, but the induced $d_{e}$ could exceed by much the present limit: $d_{e}^{S S 5} \lesssim 10^{-25} e \mathrm{~cm}$. In turn this means that $\operatorname{Im}\left(e^{-i \beta} C_{13}\right) \lesssim 0.1$ ( $\beta$ being the angle of the unitarity triangle), which has of course an impact on see-saw models. Further details can be found in [843, 845]. Notice however that, in addition to the problems with light fermion masses, minimal supersymmetric $S U(5)$ is generically considered to be ruled out by proton decay induced by Higgs triplet exchange.

More realistic GUTs like $S O(10)$ succeed in suppressing the proton decay rate by introducing more Higgs triplets and enforcing a peculiar structure for their mass matrix. What are the expectation for $d_{e}$ in this case? Consider what happens in a semirealistic $S O(10)$ model [845], where in 


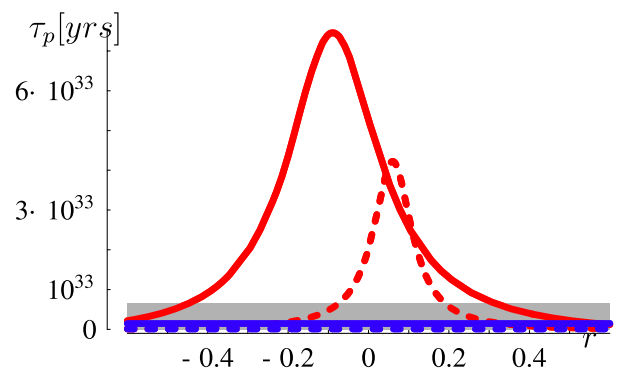

Fig. 40 (Color online) The predictions for $\tau_{p \rightarrow K \bar{v}}$ and $d_{e}$ are shown as a function of $r$ for the degenerate (flat blue) and close to pseudo Dirac (red) cases by taking $m_{T}=10^{17} \mathrm{GeV}, \Lambda=2 \times 10^{18} \mathrm{GeV}$ and

addition to the three 16 fermion representations we introduce a couple of $10_{H}$ 's containing the Higgs doublets and triplets, $10_{H}^{u}=\left(H_{D}^{u}, H_{T}^{u}\right)+\left(\bar{H}_{D}^{u}, \bar{H}_{T}^{u}\right), 10_{H}^{d}=\left(H_{D}^{d}, H_{T}^{d}\right)+$ $\left(\bar{H}_{D}^{d}, \bar{H}_{T}^{d}\right)$. Up and down quark fermion masses arise when the doublets $H_{D}^{u}$ and $\bar{H}_{D}^{d}$ acquire a VEV; in particular $Y_{\nu}=$ $Y_{u}, Y_{\ell}=Y_{d}$, and also the triplet Yukawa couplings are fully determined in terms of $Y_{u}$ and $Y_{d}$. As for the mass matrices of the Higgses, the doublets are diagonal in this basis, while the triplets are a priori undetermined:

$$
\begin{aligned}
& \left(\begin{array}{ll}
\bar{H}_{D}^{d} & \bar{H}_{D}^{u}
\end{array}\right)\left(\begin{array}{cc}
\text { e.w. } & 0 \\
0 & M_{\mathrm{GUT}}
\end{array}\right)\left(\begin{array}{l}
H_{D}^{u} \\
H_{D}^{d}
\end{array}\right), \\
& \left(\begin{array}{ll}
\bar{H}_{T}^{d} & \bar{H}_{T}^{u}
\end{array}\right) M_{T}\left(\begin{array}{l}
H_{T}^{u} \\
H_{T}^{d}
\end{array}\right) .
\end{aligned}
$$

Let consider two limiting cases for the pattern of the triplet mass matrix, diagonal degenerate and close to pseudo-Dirac:

$$
M_{T}^{\mathrm{deg}}=\left(\begin{array}{ll}
1 & 0 \\
0 & 1
\end{array}\right) m_{T}, \quad M_{T}^{c p D}=\left(\begin{array}{cc}
0 & 1 \\
1 & r
\end{array}\right) m_{T},
$$

where $r$ is a small real parameter, $r<1$, and the exact pseudo-Dirac form corresponds to the limit $r \rightarrow 0$. Notice that the close to pseudo-Dirac form is naturally obtained in the Dimopoulos-Wilczek mechanism to solve the doublettriplet splitting problem. The prediction for proton life-time displays a strong dependence on the structure of $M_{T}$, and only the pseudo-Dirac form is allowed, as can be seen in Fig. 40 (there is an intrinsic ambiguity due to GUT phases, so that the prediction is in between the dotted and solid curves). For EDMs on the contrary the Higgs triplets contribution to RGE is cumulative and, due to the log, mildly sensitive to the triplet mass matrix structure. In the case of $O$ (1) CPV phase (a small phase would be unnatural in this context), $d_{e}$ would exceed the present bound for the values of supersymmetric parameters selected in Fig. 40. Planned searches will be a fortiori more constraining. The impact of these results go beyond the essential model described above. Indeed, the week points of the model, like the fermion mass

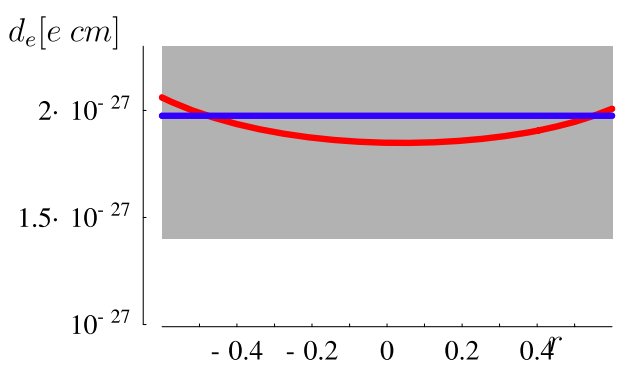

maximal CPV phase for $d_{e}$. The supersymmetric parameters $\tan \beta=3$, $\tilde{M}_{1}=200 \mathrm{GeV}, \bar{m}_{R}=400 \mathrm{GeV}$, have been selected. The shaded (grey) regions are excluded experimentally. See [845] for more details

spectra, could be addressed without changing by much the expectations for $d_{e}$. It is remarkable that EDMs turns out to be complementary to proton decay in constraining supersymmetric GUTs.

In the above model one obtains the relation $d_{\mu} / d_{e} \sim$ $\left|V_{t s} / V_{t d}\right|^{2} \approx 25$, so that the prediction for $d_{\mu}$ is below the planned sensitivity. However, there are GUT models where this is not the case. For instance a significant $d_{\mu}$ is obtained in L-R symmetric guts [846].

\subsubsection{Electron-neutron EDM correlations in SUSY}

One of the questions we would like to address is whether non-zero EDM signals can constitute indirect evidence for supersymmetry. Supersymmetric models contain additional sources of $\mathrm{CP}$ violation compared to the SM, which induce considerable and usually too large EDMs (Fig. 41). In typical (but not all) SUSY models, the same CP-violating source induces both hadronic and leptonic EDMs such that these are correlated. The most important source is usually the CPphase of the $\mu$-term and, in certain non-universal scenarios, the gaugino phases. The $\mathrm{CP}$-phases of the $A$-terms generally lead to smaller contributions.

Typical SUSY models lead to $\left|d_{n}\right| /\left|d_{e}\right| \sim \mathcal{O}(10)_{-}$ $\mathcal{O}(100)$. Thus, if both the neutron and the electron EDMs are observed, and this relation is found, it can be viewed as a clue pointing towards supersymmetry.

Since generic SUSY models suffer from the "SUSY CP problem", EDMs should be analyzed in classes of models which allow for their suppression. These include models with either small CP phases or heavy spectra. $d_{n}-d_{e}$ EDM correlations have been analyzed in mSUGRA, the decoupling scenario with 2 heavy sfermion generations, and split

Fig. 41 One loop EDM contributions

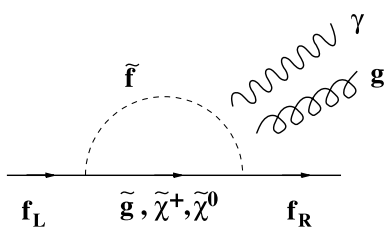




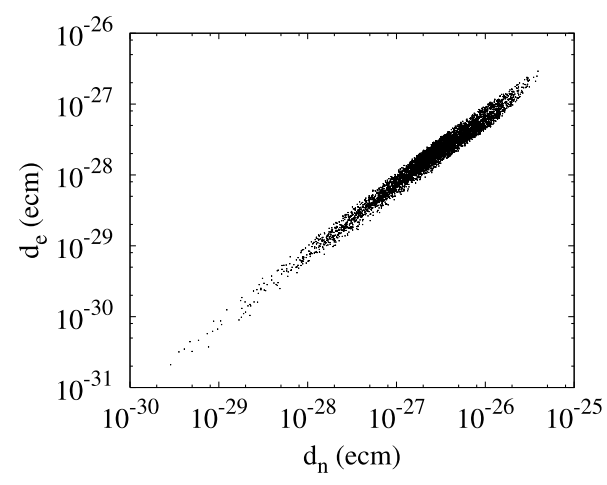

Fig. 42 EDM correlation in mSUGRA

SUSY [847]. Assuming that the SUSY CP phases are all of the same order of magnitude at the GUT scale, one finds

mSUGRA: $\quad d_{e} \sim 10^{-1} d_{n}$,

split SUSY: $\quad d_{e} \sim 10^{-1} d_{n}$,

decoupling: $\quad d_{e} \sim\left(10^{-1}-10^{-2}\right) d_{n}$.

These results are insensitive to $\tan \beta$ and order one variations in the mass parameters. The $d_{e} / d_{n}$ ratio is dominated by the factor $m_{e} / m_{q} \sim 10^{-1}$, although different diagrams contribute to $d_{e}$ and $d_{n}$.

An example of the $d_{n}-d_{e}$ correlation in mSUGRA is presented in Fig. 42. There $m_{0}, m_{1 / 2},|A|$ are varied randomly in the range $[200 \mathrm{GeV}, 1 \mathrm{TeV}], \tan \beta=5$ and the phase of the $\mu$-term $\phi_{\mu}$ is taken to be in the range $[-\pi / 500, \pi / 500]$. The effect of the phase of the $A$-terms, $\phi_{A}$, is negligible as long is it is of the same order of magnitude as $\phi_{\mu}$ at the GUT scale. Clearly, the relation $d_{n} / d_{e} \sim 10$ holds for essentially all parameter values.

As the next step, we would like to see how stable these correlations are. One might expect that breaking universality at the GUT scale would completely invalidate the above results. To answer this question, we study a non-universal MSSM parameterized by

$$
\begin{aligned}
& m_{\text {squark }}, \quad m_{\text {slepton }}, \quad M_{3}, \quad M_{1}=M_{2}, \quad|A|, \\
& \phi_{\mu}, \quad \phi_{A}, \quad \phi_{M_{3}}
\end{aligned}
$$

at the GUT scale. The mass parameters are varied randomly in the range $[200 \mathrm{GeV}, 2 \mathrm{TeV}]$ and the phases in the range $[-\pi / 300, \pi / 300], \tan \beta=5$. We find that although the correlation is not as precise as in the mSUGRA case, about $90 \%$ of the points satisfy the relation $d_{n} / d_{e} \sim 10-100$ (Fig. 43). In most of the remaining $10 \%, 10^{4}>d_{n} / d_{e}>10^{2}$, which arise when the gluino phase dominates. The reason for the correlation is that in most cases $\phi_{\mu}$ is significant and induces both $d_{n}$ and $d_{e}$. Apart from the factor $m_{q} / m_{e}$, the SUSY EDM diagrams are comparable as long as there are no large mass hierarchies in the SUSY spectrum. This means that the EDM correlation survives to a large extent, although it is possible to violate it in certain cases.

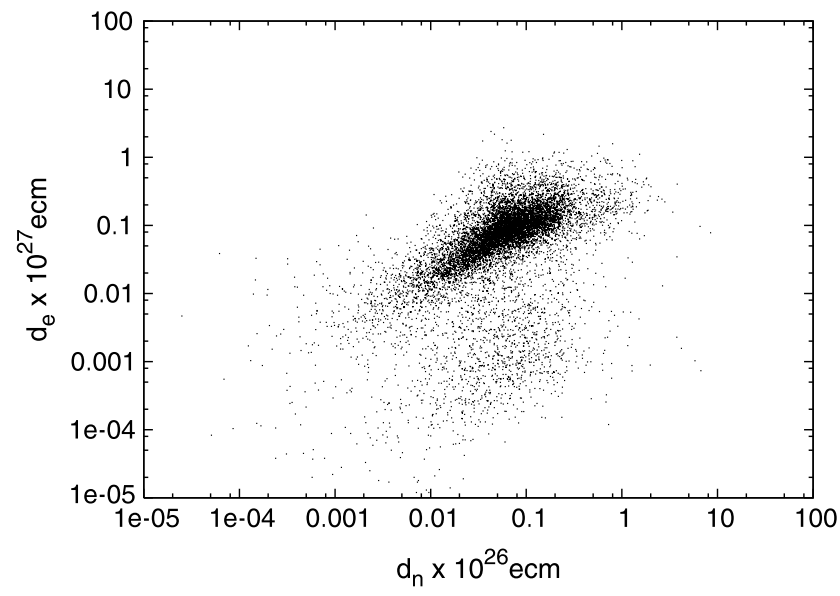

Fig. 43 EDM correlation in non-universal SUSY models

It is instructive to compare the SUSY EDM 'prediction' to those of other models. Start with the standard model. The SM background due to the CKM phase is very small, probably beyond the experimental reach. The neutron EDM can also be induced by the QCD $\theta$-term,

$d_{n} \sim 3 \times 10^{-16} \theta$ e cm,

which does not affect the electron EDM. Thus, one has $d_{n} \gg d_{e}$.

In extra dimensional models, usually there are no extra sources of $\mathrm{CP}$ violation and the EDM predictions are similar to the SM values. Two Higgs doublet models have additional sources of CP violation, however, the leading EDM contributions appear at 2 or 3 loops such that the typical EDM values are significantly smaller than those in SUSY models.

To conclude, we find that typical SUSY models predict $\left|d_{n}\right| /\left|d_{e}\right| \sim \mathcal{O}(10)-\mathcal{O}(100)$. Thus, if

$d_{e}>d_{n}$

or

$d_{e} \ll d_{n}$

is found, common SUSY scenarios would be disfavored, although such relations could still be obtained in baroque SUSY models.

It is interesting to consider SUSY GUT model, where $\mathrm{CP}$ phases in the neutrino Yukawa couplings contributes to hadronic EDMs. For instance, in $S U(5)$ SUSY GUT with right handed neutrinos, not only large mixing but also CPviolating phases in neutrino sector give significant contribution to the mixing and $\mathrm{CP}$ phases in the right handed scalar down sector. Though 1-2 mixing in the neutrino Yukawa coupling is strongly restricted by the $B(\mu \rightarrow e \gamma), 2-3$ mixing in the neutrino Yukawa couplings can be significantly large and this case is interesting in $B$ physics. Large 2-3 
mixing with $\mathrm{CP}$ violation in neutrino sector may give a significant contribution not only to the $B(\tau \rightarrow \mu \gamma)$ but also to color EDM of $s$ quark which may affect [848, 849] neutron and $\mathrm{Hg}$ EDM.

\subsubsection{EDMs in split supersymmetry}

Supersymmetry breaking terms involve many new sources of $\mathrm{CP}$ violation. Particularly worrisome are the phases associated with the invariants $\arg \left(A^{*} M_{\tilde{g}}\right)$ and $\arg \left(A^{*} B\right)$, where $A$ and $B$ represent the usual trilinear and bilinear soft terms and $M_{\tilde{g}}$ the gaugino masses. Such phases survive in the universal limit in which all the flavor structure originates from the SM Yukawa's. If these phases are of order one, the electron and neutron EDMs induced at one loop by gauginosfermion exchange are typically (barring accidental cancellations $[848,850,851])$ a couple of orders of magnitude above the limits [852-855], a difficulty which is known as the supersymmetric $\mathrm{CP}$ problem.

Different remedies are available to this problem making the one loop sfermion contribution to the EDMs small enough, each with its pros and cons. One remedy is to have heavy enough sfermions (say heavier than $50-100 \mathrm{TeV}$ to be on the safe side). Gauginos and higgsinos are not required to be heavy, and can be closer to the electroweak scale, thus preserving the supersymmetric solution to the dark matter problem and gauge coupling unification. This is the "Split" limit of the MSSM [457, 458, 856]. In this limit, the heavy sfermions suppress the dangerous one loop contributions to a negligible level. Nevertheless, some phases survive below the sfermion mass scale and, if they do not vanish for an accidental or a symmetry reason, they give rise to EDMs that are safely below the experimental limits, but sizable enough to be well within the sensitivity of the next generation of experiments [847, 856-858]. Such contributions only arise at the two-loop level, since the new phases appear in the gaugino-higgsino sector, which is not directly coupled to the SM fermions.

Besides the large EDMs, a number of additional unsatisfactory issues, all related to the presence of $\mathrm{TeV}$ scalars, plague the MSSM. The number of parameters exceeds 100; flavor changing neutral current processes are also one or two orders of magnitudes above the experimental limits in most of the wide parameter space; in the context of a grand unified theory, the proton decay rate associated to sfermion-mediated dimension five operators is ruled out by the Super-Kamiokande limit, at least in the minimal version of the supersymmetric $S U(5)$ model; in the supergravity context, another potential problem comes from the gravitino decay, whose rate is slow enough to interfere with primordial nucleosynthesis. While none of those issues is of course deadly - remedies are well known for each of themit should be noted that the split solution of the supersymmetric $\mathrm{CP}$ problem also solves all of those issues at once. At the same time, it gives rise to a predictive framework, characterized by a rich, new phenomenology, mostly determined in terms of only 4 relevant parameters. Of course the price to be paid to make the sfermions heavy is the large fine-tuning (FT) necessary to reproduce the Higgs mass, which exacerbates the FT problem already present in the MSSM. This could be hard to accept, or not, depending on the interpretation of the FT problem, the two extreme attitudes being (i) ignoring the problem, as long as the tuning is not much worse than permille and (ii) accepting a tuning in the Higgs mass as we accept the tuning of the cosmological constant, as in split supersymmetry. The second possibility can in turn be considered as a manifestation of an anthropic selection principle [859-863].

Before moving the quantitative discussion of the effect, let us note that the pure gaugino-higgsino contribution to the EDMs, dominant in split supersymmetry and possibly near the experimental limit, might also be important in the non-split case, depending on the mechanism invoked to push the one loop sfermion contribution below the experimental limit.

5.7.2.1 Sources of CP violation in the split limit Below the heavy sfermion mass scale, denoted generically by $\tilde{m}$, the MSSM gauginos and higgsinos, together with the SM fields constitute the field content of the model. The only interactions of gauginos and higgsinos besides the gauge ones are

$$
\begin{aligned}
-\mathcal{L}= & \sqrt{2}\left(\tilde{g}_{u} H^{\dagger} \tilde{W}^{a} T_{a} \tilde{H}_{u}+\tilde{g}_{u}^{\prime} Y_{H_{u}} H^{\dagger} \tilde{B} \tilde{H}_{u}\right. \\
& \left.+\tilde{g}_{d} H_{c}^{\dagger} \tilde{W}^{a} T_{a} \tilde{H}_{d}+\tilde{g}_{d}^{\prime} Y_{H_{d}} H_{c}^{\dagger} \tilde{B} \tilde{H}_{d}\right)+ \text { h.c. }
\end{aligned}
$$

where the Higgs-higgsino-gaugino couplings $\tilde{g}_{u}, \tilde{g}_{d}, \tilde{g}_{u}^{\prime}$, $\tilde{g}_{d}^{\prime}$ can be expressed in terms of the gauge couplings and $\tan \beta$ through the matching with the supersymmetric gauge interactions at the scale $\tilde{m}, H_{c}=i \sigma_{2} H^{*}, T_{a}$ are the $S U(2)$ generators, and $Y_{H_{u}}=-Y_{H_{d}}=1 / 2$. CP violating phases can enter the effective Lagrangian below the sfermion mass scale $\tilde{m}$ through the $\mu$-parameter, the gaugino masses $M_{i}$, $i=1,2,3$, or the couplings $\tilde{g}_{u}, \tilde{g}_{d}, \tilde{g}_{u}^{\prime}, \tilde{g}_{d}^{\prime}$ (besides of course the Yukawa couplings, not relevant here). Only three combinations of the phases of the above parameters are physical, in a basis in which the Higgs VEV is in its usual form, $\langle H\rangle=$ $(0, v)^{T}$, with $v$ positive. The three combinations are $\phi_{1}=$ $\arg \left(\tilde{g}_{u}^{\prime *} \tilde{g}_{d}^{\prime *} M_{1} \mu\right), \phi_{2}=\arg \left(\tilde{g}_{u}^{*} \tilde{g}_{d}^{*} M_{2} \mu\right), \xi=\arg \left(\tilde{g}_{u} \tilde{g}_{d}^{*} \tilde{g}_{d}^{\prime} \tilde{g}_{u}^{\prime *}\right)$. Actually, the parameters above are not independent themselves. The tree-level matching with the full theory above $\tilde{m}$ gives in fact $\arg \left(\tilde{g}_{u}\right)=\arg \left(\tilde{g}_{u}^{\prime}\right), \arg \left(\tilde{g}_{d}\right)=\arg \left(\tilde{g}_{d}^{\prime}\right)$. As a consequence, the phase $\xi$ vanishes, thus leaving only two independent phases. Moreover, if the phases of $M_{1}$ and $M_{2}$ are equal, as in most models of supersymmetry breaking, there is actually only one CP invariant: $\phi_{2}=\arg \left(\tilde{g}_{u}^{*} \tilde{g}_{d}^{*} M_{2} \mu\right)$. 
Fig. 44 Two loop contributions to the light SM fermion EDMs. The third diagram is for a down-type fermion $f$
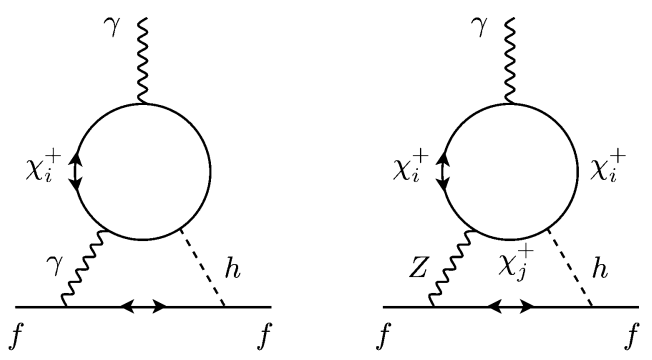

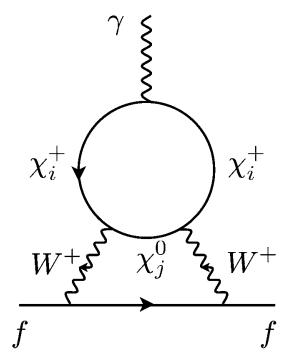

In terms of mass eigenstates, the relevant interactions are

$$
\begin{aligned}
-\mathcal{L}= & \frac{g}{c_{W}} \overline{\chi_{i}^{+}} \gamma^{\mu}\left(G_{i j}^{R} P_{R}+G_{i j}^{L} P_{L}\right) \chi_{j}^{+} Z_{\mu} \\
& +\left[g \overline{\chi_{i}^{+}} \gamma^{\mu}\left(C_{i j}^{R} P_{R}+C_{i j}^{L} P_{L}\right) \chi_{j}^{0} W_{\mu}^{+}\right. \\
& \left.+\frac{g}{\sqrt{2}} \overline{\chi_{i}^{+}}\left(D_{i j}^{R} P_{R}+D_{i j}^{L} P_{L}\right) \chi_{j}^{+} h+\text { h.c. }\right],
\end{aligned}
$$

where

$$
\begin{aligned}
& G_{i j}^{L}=V_{i W^{+}} c_{W^{+}} V_{W^{+} j}^{\dagger}+V_{i h_{u}^{+}} c_{h_{u}^{+}} V_{h_{u}^{+} j}^{\dagger}, \\
& -G_{i j}^{R^{*}}=U_{i W^{-}} c_{W^{-}} U_{W^{-} j}^{\dagger}+U_{i h_{d}^{-}} c_{h_{d}^{-}} U_{h_{d}^{-} j}^{\dagger}, \\
& C_{i j}^{L}=-V_{i W^{+}} N_{j W_{3}}^{*}+\frac{1}{\sqrt{2}} V_{i h_{u}^{+}} N_{j h_{u}^{0}}^{*}, \\
& C_{i j}^{R}=-U_{i W^{-}}^{*} N_{j W_{3}}-\frac{1}{\sqrt{2}} U_{i h_{d}^{-}}^{*} N_{j h_{d}^{0}}, \\
& g D_{i j}^{R}=\tilde{g}_{u}^{*} V_{i h_{u}^{+}} U_{j W^{-}}+\tilde{g}_{d}^{*} V_{i W^{+}} U_{j h_{d}^{-}}, \\
& D^{L}=\left(D^{R}\right)^{\dagger} .
\end{aligned}
$$

In (5.154), $c_{f}=T_{3 f}-s_{W}^{2} Q_{f}\left(s_{W}^{2} \equiv \sin ^{2} \theta_{W}\right)$ is the neutral current coupling coefficient of the fermion $\tilde{f}$ and, accordingly, $c_{W^{ \pm}}= \pm \cos ^{2} \theta_{W}, c_{h_{u}^{+}, h_{d}^{-}}= \pm\left(1 / 2-s_{W}^{2}\right)$. The matrices $U, V, N$ diagonalize the complex chargino and neutralino mass matrices, $M_{+}=U^{T} M_{+}^{D} V, M_{0}=N^{T} N_{0}^{D} N$, where $M_{+}^{D}=\operatorname{Diag}\left(M_{1}^{+}, M_{2}^{+}\right) \geq 0, M_{0}^{D}=\operatorname{Diag}\left(M_{1}^{0}, \ldots\right.$, $\left.M_{4}^{0}\right) \geq 0$ and

$$
\begin{aligned}
M_{+} & =\left(\begin{array}{cc}
M_{2} & \tilde{g}_{u} v \\
\tilde{g}_{d} v & \mu
\end{array}\right), \\
M_{0} & =\left(\begin{array}{cccc}
M_{1} & 0 & -\tilde{g}_{d}^{\prime} v / \sqrt{2} & \tilde{g}_{u}^{\prime} v / \sqrt{2} \\
0 & M_{2} & \tilde{g}_{d} v / \sqrt{2} & -\tilde{g}_{u} v / \sqrt{2} \\
-\tilde{g}_{d}^{\prime} v / \sqrt{2} & \tilde{g}_{d} v / \sqrt{2} & 0 & -\mu \\
\tilde{g}_{u}^{\prime} v / \sqrt{2} & -\tilde{g}_{u} v / \sqrt{2} & -\mu & 0
\end{array}\right) .
\end{aligned}
$$

5.7.2.2 Two loop contributions to EDMs Fermion EDMs are generated only at two loops, since charginos and neutralinos, which carry the information on $\mathrm{CP}$ violation, are only coupled to gauge and Higgs bosons. Three diagrams contribute to the EDM of the light SM fermion $f$ at the twoloop level. They are induced by the effective $\gamma \gamma h, \gamma Z h$, and $\gamma W W$ effective couplings and are shown in Fig. 44. The EDM $d_{f}$ of the fermion $f$ is then given by [857], where

$$
\begin{aligned}
d_{f}= & d_{f}^{\gamma H}+d_{f}^{Z H}+d_{f}^{W W} \\
d_{f}^{\gamma H}= & \frac{e Q_{f} \alpha^{2}}{4 \sqrt{2} \pi^{2} s_{W}^{2}} \operatorname{Im}\left(D_{i i}^{R}\right) \frac{m_{f} M_{i}^{+}}{M_{W} m_{H}^{2}} f_{\gamma H}\left(r_{i H}^{+}\right) \\
d_{f}^{Z H}= & \frac{e\left(T_{3 f_{L}}-2 s_{W}^{2} Q_{f}\right) \alpha^{2}}{16 \sqrt{2} \pi^{2} c_{W}^{2} s_{W}^{4}} \operatorname{Im}\left(D_{i j}^{R} G_{j i}^{R}-D_{i j}^{L} G_{j i}^{L}\right) \\
& \times \frac{m_{f} M_{i}^{+}}{M_{W} m_{H}^{2}} f_{Z H}\left(r_{Z H}, r_{i H}^{+}, r_{j H}^{+}\right) \\
d_{f}^{W W}= & \frac{e T_{3 f_{L} \alpha^{2}}}{8 \pi^{2} s_{W}^{4}} \operatorname{Im}\left(C_{i j}^{L} C_{i j}^{R *}\right) \frac{m_{f} M_{i}^{+} M_{j}^{0}}{M_{W}^{4}} \\
& \times f_{W W}\left(r_{i W}^{+}, r_{j W}^{0}\right) .
\end{aligned}
$$

In (5.159) a sum over indices $i, j$ is understood, $Q_{f}$ is the charge of the fermion $f, T_{3 f_{L}}$ is the third component of the weak isospin of the fermion's left handed component. Also, $r_{Z H}=\left(M_{Z} / m_{H}\right)^{2}, r_{i H}^{+}=\left(M_{i}^{+} / m_{H}\right)^{2}, r_{i W}^{+}=\left(M_{i}^{+} / M_{W}\right)^{2}$, $r_{i W}^{0}=\left(M_{i}^{0} / M_{W}\right)^{2}$, where $m_{H}$ is the Higgs mass, and the loop functions are given by

$$
\begin{aligned}
& f_{\gamma H}(r)=\int_{0}^{1} \frac{d x}{1-x} j\left(0, \frac{r}{x(1-x)}\right) \\
& f_{Z H}\left(r, r_{1}, r_{2}\right)=\frac{1}{2} \int_{0}^{1} \frac{d x}{x(1-x)} j\left(r, \frac{x r_{1}+(1-x) r_{2}}{x(1-x)}\right) \\
& f_{W W}\left(r_{1}, r_{2}\right)=\int_{0}^{1} \frac{d x}{1-x} j\left(0, \frac{x r_{1}+(1-x) r_{2}}{x(1-x)}\right)
\end{aligned}
$$

Their analytic expressions can be found in Ref. [857]. The symmetric loop function $j(r, s)$ is defined recursively by

$j(r)=\frac{r \log r}{r-1}, \quad j(r, s)=\frac{j(r)-j(s)}{r-s}$.

Equation (5.159) hold at the chargino mass scale $\mathrm{M}^{+}$. The neutron EDM is determined as a function of the down 
and up quark dipoles at a much lower scale $\mu$, at which

$d_{q}(\mu)=\eta_{\mathrm{QCD}} d_{q}\left(M^{+}\right), \quad \eta_{\mathrm{QCD}}=\left[\frac{\alpha_{s}\left(M^{+}\right)}{\alpha_{s}(\mu)}\right]^{\gamma / 2 b}$

where the $\beta$-function coefficient is $b=11-2 n_{q} / 3$ and $n_{q}$ is the number of effective light quarks. The anomalousdimension coefficient is $\gamma=8 / 3$. For $\alpha_{s}\left(M_{Z}\right)=0.118 \pm$ 0.004 and $\mu=1 \mathrm{GeV}$ (the scale of the neutron mass), the value of $\eta_{\mathrm{QCD}}$ is 0.75 for $M^{+}=1 \mathrm{TeV}$ and 0.77 for $M^{+}=200 \mathrm{GeV}$. We expect an uncertainty of about $5 \%$ from next-to-leading order effects. This result [857] gives a QCD renormalization coefficient about a factor of 2 smaller than usually considered [864], and it agrees with the recent findings of Ref. [865].

The neutron EDM can be expressed in terms of the quark EDMs using QCD sum rules [866, 867]:

$d_{n}=(1 \pm 0.5) \frac{f_{\pi}^{2} m_{\pi}^{2}}{\left(m_{u}+m_{d}\right)(225 \mathrm{MeV})^{3}}\left(\frac{4}{3} d_{d}-\frac{1}{3} d_{u}\right)$,

where $f_{\pi} \approx 92 \mathrm{MeV}$ and we have neglected the contribution of the quark chromo-electric dipoles, which does not arise at the two-loop level in the heavy-squark mass limit. Since $d_{d}$ and $d_{u}$ are proportional to the corresponding quark masses, $d_{n}$ depends on the light quark masses only through the ratio $m_{u} / m_{d}$, for which we take the value $m_{u} / m_{d}=0.553 \pm 0.043$.

It is instructive to consider the limit $M_{i}, \mu \gg M_{Z}, m_{H}$ which simplifies the EDM dependence on the CP-violating invariants $\left|\tilde{g}_{u} \tilde{g}_{d} / M_{2} \mu\right| \sin \phi_{2}$ and $\left|\tilde{g}_{u}^{\prime} \tilde{g}_{d}^{\prime} / M_{1} \mu\right| \sin \phi_{1}$. The terms depending on the second invariant are actually suppressed, so that both the electron and neutron EDM are mostly characterized by a single invariant even in the case in which the phases of $M_{1}$ and $M_{2}$ are different. The relative importance of the three contributions to $d_{f}$ in (5.158) can be estimated to leading order in $\log \left(M_{2} \mu / m_{H}^{2}\right)$ from

$\frac{d_{f}^{Z H}}{d_{f}^{\gamma H}} \approx \frac{\left(T_{3 f_{L}}-2 s_{W}^{2} Q_{f}\right)\left(3-4 s_{W}^{2}\right)}{8 c_{W}^{2} Q_{f}} ;$

$\frac{d_{f}^{W W}}{d_{f}^{\gamma H}} \approx-\frac{T_{3 f_{L}}}{8 s_{W}^{2} Q_{f}} \quad\left(M_{2}=\mu\right)$.

Numerically, (5.168) gives $d_{e}^{Z H} \approx 0.05 d_{e}^{\gamma H}, d_{e}^{W W} \approx$ $-0.3 d_{e}^{\gamma H}$ and $d_{n}^{Z H} \approx d_{n}^{\gamma H}, d_{n}^{W W} \approx-0.7 d_{n}^{\gamma H}$. These simple estimates show the importance of the $Z H$ contribution to the neutron EDM.
5.7.2.3 Numerical results Let us consider a standard unified framework for the gaugino masses at the GUT scale. Using the RGEs given in Refs. [458, 868], the parameters in (5.159) can be expressed in terms of the (single) phase $\phi \equiv \phi_{2}$ and four positive parameters $M_{2}, \mu$ (evaluated at the low energy scale), $\tan \beta$, and the sfermion mass scale $\tilde{m}$. In first approximation, the dipoles depend on $\beta$ and $\phi$ through an overall factor $\sin 2 \beta \sin \phi$. The overall sfermion scale $\tilde{m}$ enters only logarithmically through the RGE equations for $\tilde{g}_{u, d}, \tilde{g}_{u, d}^{\prime}$. The numerical results for the electron and neutrino EDMs can then conveniently be presented in the $M_{2}-\mu$ plane by setting $\sin 2 \beta \sin \phi=1$ (it is then sufficient to multiply the results by $\sin 2 \beta \sin \phi$ ) and, for example, $\tilde{m}=10^{9} \mathrm{GeV}$. Figure 45 shows the prediction for the electron EDM, the neutron EDM, and their ratio $d_{n} / d_{e}$. The red thick line corresponds to the present experimental limits $d_{e}<1.6 \times 10^{-27} e \mathrm{~cm}$ [186], while the limit $d_{n}<3 \times 10^{-26} e \mathrm{~cm}$ [869] does not impose a constraint on the parameters shown in Fig. 45.

An interesting test of split supersymmetry can be provided by a measurement of both the electron and the neutron EDMs. Indeed, in the ratio $d_{n} / d_{e}$ the dependence on $\sin \phi, \tan \beta$ and $\tilde{m}$ approximately cancels out. Nevertheless, because of the different loop functions associated with the different contributions, the ratio $d_{n} / d_{e}$ varies by $\mathcal{O}(100 \%)$ when $M_{2}$ and $\mu$ are varied in the range spanned in the figures. Still, the variation of $d_{n} / d_{e}$ is comparable to the theoretical uncertainty in (5.167), and is significantly smaller than the variation in the ordinary MSSM prediction, even in the case of universal phases [847]. ${ }^{30}$ On the other hand, the usual tight correlation between the electron and muon EDMs, $d_{\mu} / d_{e}=m_{\mu} / m_{e}$ persists.

\section{Experimental tests of charged lepton universality}

Lepton universality postulates that lepton interactions do not depend explicitly on lepton family number other than through their different masses and mixings. Whereas there is little doubt about the universality of electric charge there are scenarios outside the standard model in which lepton universality is violated in the interactions with $W$ and $Z$ bosons. Violations may also have their origin in non-SM contributions to the transition amplitudes. Such apparent violations of lepton universality can be expected in various particle decays:

- in $W, Z$ and $\pi$ decay resulting from R-parity violating extensions to the MSSM [870, 871],

\footnotetext{
${ }^{30}$ Note that the $Z H$ contribution is missing in the analysis of the split supersymmetry case in Ref. [847], which leads to a somewhat stronger correlation between $d_{e}$ and $d_{n}$.
} 
Fig. 45 Prediction for $d_{n}, d_{e}$, and their ratio $d_{n} / d_{e}$. In the contour plots we have chosen $\tan \beta=1, \sin \phi=1$, and $\tilde{m}=10^{9} \mathrm{GeV}$. The results for $d_{n}$ and $d_{e}$ scale approximately linearly with $\sin 2 \beta \sin \phi$, while the ratio is fairly independent of $\tan \beta, \sin \phi$ and $\tilde{m}$. The red thick line corresponds to the present experimental limit

$d_{e}<1.6 \times 10^{-27} e \mathrm{~cm} \mathrm{[186]}$.

Note that the uncertainty in $d_{n}$ is a factor of a few. The scatter plot shows $d_{n}$ values when $M_{1,3}$ and $\mu$ are varied in the range [200 GeV, $1 \mathrm{TeV}], m_{h}$ in $[100 \mathrm{GeV}, 300 \mathrm{GeV}]$ and the $\mathrm{CP}$ phase in the range $[-\pi, \pi]$
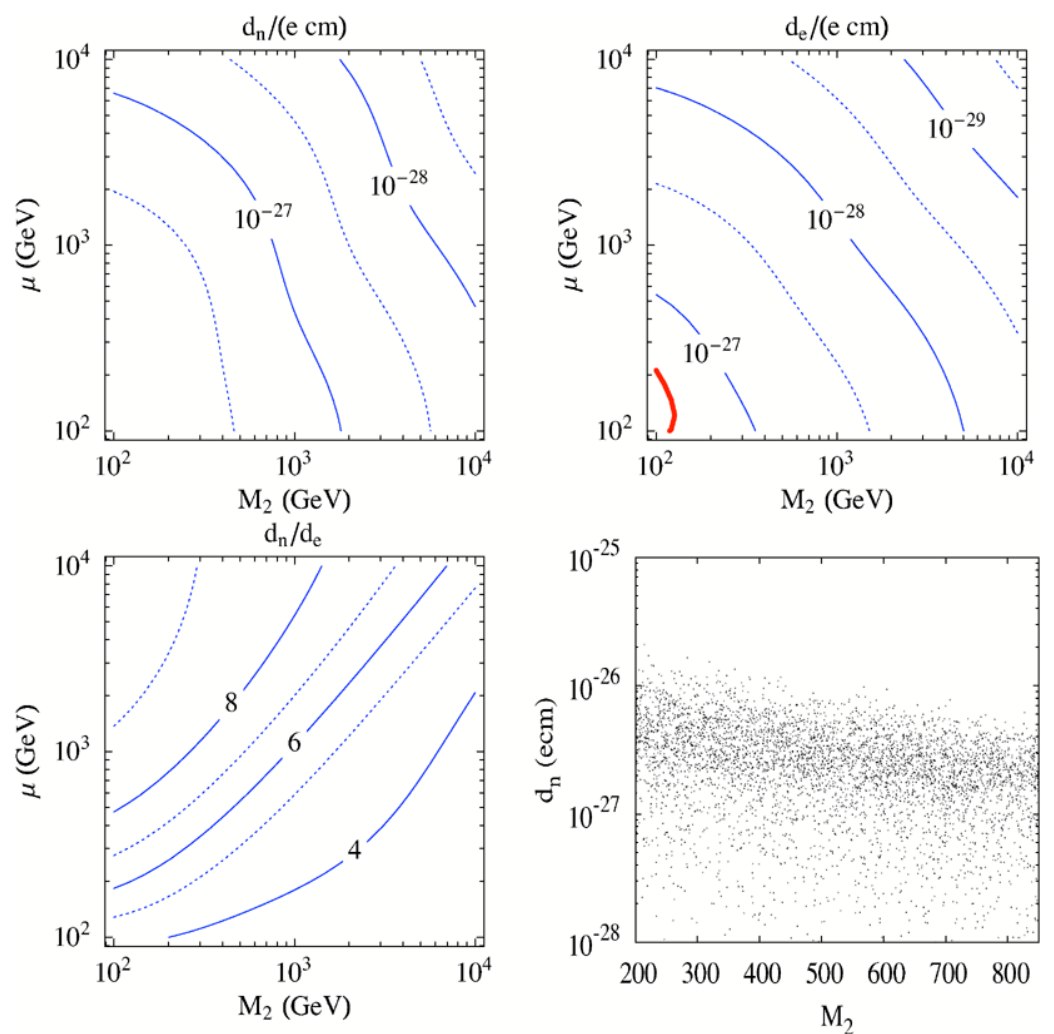

- in $W$ decay resulting from charged Higgs bosons [872, 873],

- in $\pi$ decay resulting from box diagrams involving nondegenerate sleptons [874],

- in $K$ decay resulting from LFV contributions in SUSY [839] (see Sect. 5.6),

- in $\Upsilon$ decay resulting from a light Higgs boson [875],

- in $\pi$ and $K$ decay from scalar interactions [876], enhanced by the strong chiral suppression of the SM amplitude for decays into $e \bar{v}_{e}$. Since these contributions result in interference terms with the SM amplitude the deviations scale with the mass $M$ of the exchange particle like $1 / M^{2}$ rather than $1 / M^{4}$ as may be expected naively.

We assume the $V-A$ Lorentz structure of the charged weak current, and parameterize universality violations by allowing for different strengths of the couplings of the individual lepton flavors: ${ }^{31}$

$\mathcal{L}=\sum_{l=e, \mu, \tau} \frac{g_{l}}{\sqrt{2}} W_{\mu} \bar{\nu}_{l} \gamma^{\mu}\left(\frac{1-\gamma_{5}}{2}\right) l+$ h.c.

Experimental limits have recently been compiled by Loinaz et al. [877]. Results are shown in Table 16.

Following the notation of Ref. [877] one may parameterize the violations by $g_{l} \equiv g\left(1-\epsilon_{l} / 2\right)$. After introducing

${ }^{31}$ Still more general violations lead to deviations from the $1-\gamma_{5}$ structure of the weak interaction.
Table 16 Limits on lepton universality from various processes. One should keep in mind that violations may affect the various tests differently so which constraint is best depends on the mechanism. Hypothetical non- $V-A$ contributions, for example, would lead to larger effects in decay modes with stronger helicity suppression such as $\pi \rightarrow e v$ and $K \rightarrow e v$. Adapted from Ref. [877]. The ratios estimated from tau decays are re-calculated using PDG averages, as described in the text

\begin{tabular}{ll}
\hline Decay mode & Constraint \\
\hline$W \rightarrow e \bar{v}_{e}$ & $\left(g_{\mu} / g_{e}\right)_{W}=0.999 \pm 0.011$ \\
$W \rightarrow \mu \bar{v}_{\mu}$ & $\left(g_{\tau} / g_{e}\right)_{W}=1.029 \pm 0.014$ \\
$W \rightarrow \tau \bar{v}_{\tau}$ & \\
$\mu \rightarrow e \bar{v}_{e} v_{\mu}$ & $\left(g_{\mu} / g_{e}\right)_{\tau}=1.0002 \pm 0.0020$ \\
$\tau \rightarrow e \bar{v}_{e} v_{\tau}$ & $\left(g_{\tau} / g_{e}\right)_{\tau \mu}=1.0012 \pm 0.0023$ \\
$\tau \rightarrow \mu \bar{v}_{\mu} v_{\tau}$ & \\
$\pi \rightarrow e \bar{v}_{e}$ & $\left(g_{\mu} / g_{e}\right)_{\pi}=1.0021 \pm 0.0016$ \\
$\pi \rightarrow \mu \bar{v}_{\mu}$ & $\left(g_{\tau} / g_{e}\right)_{\tau \pi}=1.0030 \pm 0.0034$ \\
$\tau \rightarrow \pi \bar{v}_{\tau}$ & \\
$K \rightarrow e \bar{v}_{e}$ & $\left(g_{\mu} / g_{e}\right)_{K}=1.024 \pm 0.020$ \\
$K \rightarrow \mu \bar{v}_{\mu}$ & $\left(g_{\tau} / g_{\mu}\right)_{K \tau}=0.979 \pm 0.017$ \\
$\tau \rightarrow K \bar{v}_{\tau}$ & \\
\hline
\end{tabular}

$\Delta_{l l^{\prime}} \equiv \epsilon_{l}-\epsilon_{l^{\prime}}$ the various experimental limits on deviations from lepton universality can be compared (see Fig. 46).

It is very fortunate that for most decay modes new dedicated experiments are being prepared. In the following sub- 

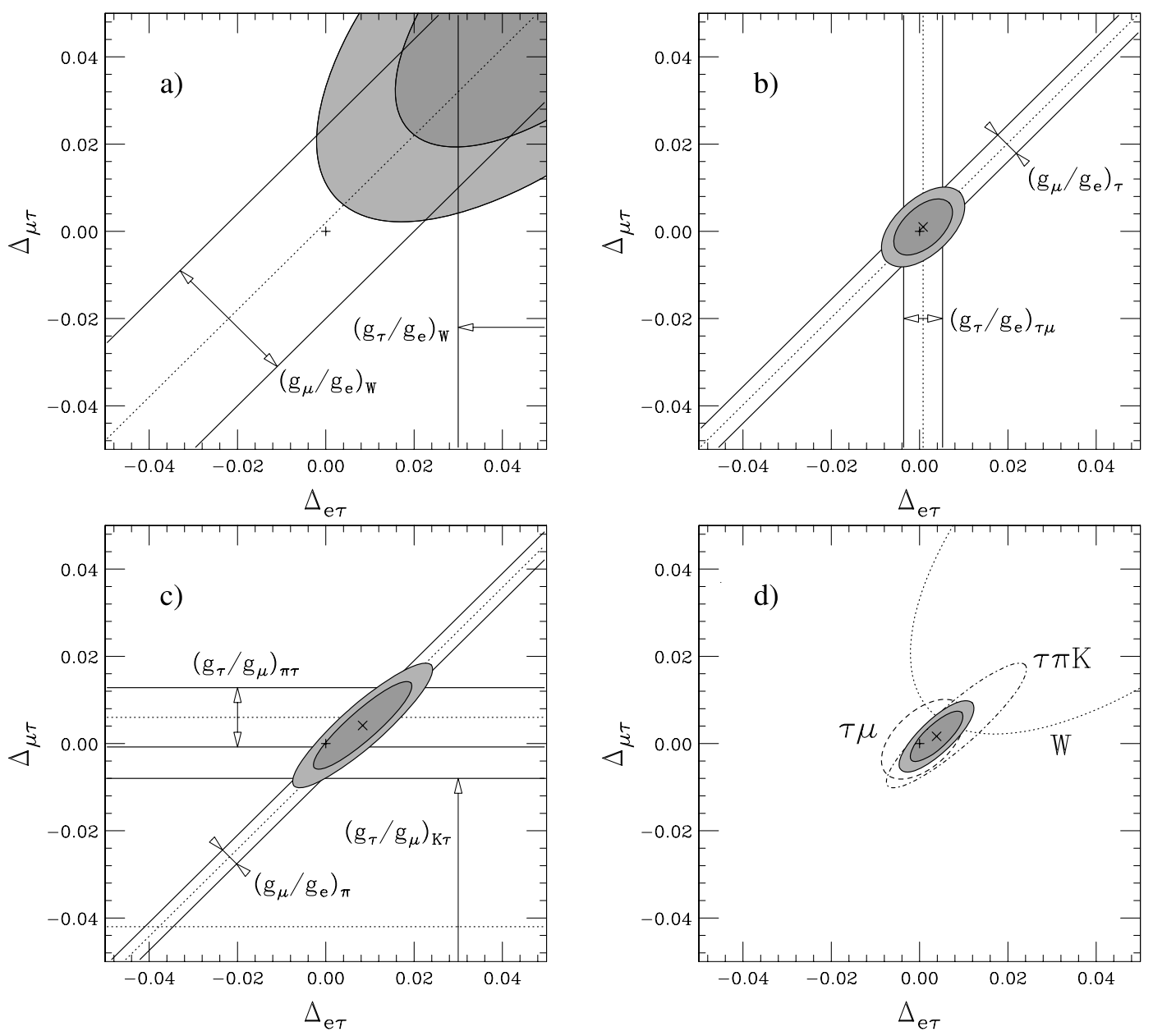

Fig. 46 Experimental constraints on violations of lepton universality from (a) $W$ decay, (b) $\tau$ decay, (c) $\pi$ and $K$ decay and (d) the combination of (a)-(c). Parameters are defined in the text. The $\pm 1 \sigma$

bands are indicated. The shaded areas correspond to $68 \%$ and $90 \%$ confidence levels. Results from the analysis in Ref. [877]

sections the status and prospects of these experimental tests of lepton universality are presented.

\section{$6.1 \pi$ decay}

In lowest order the decay width of $\pi \rightarrow l \bar{\nu}_{l}(l=e, \mu)$ is given by:

$\Gamma_{\pi \rightarrow l \bar{v}_{l}}^{\mathrm{tree}}=\frac{g_{l}^{2} g_{u d}^{2} V_{u d}^{2}}{256 \pi} \frac{f_{\pi}^{2}}{M_{W}^{4}} m_{l}^{2} m_{\pi}\left(1-\frac{m_{l}^{2}}{m_{\pi}^{2}}\right)^{2}$

By taking the branching ratio the factors affected by hadronic uncertainties cancel:

$R_{e / \mu}^{\mathrm{tree}} \equiv \frac{\Gamma_{\pi \rightarrow e \bar{v}}^{\mathrm{tree}}}{\Gamma_{\pi \rightarrow \mu \bar{v}}^{\text {tree }}}=\left(\frac{g_{e}}{g_{\mu}} \times \frac{m_{e}}{m_{\mu}} \times \frac{1-m_{e}^{2} / m_{\pi}^{2}}{1-m_{\mu}^{2} / m_{\pi}^{2}}\right)^{2}$.

Radiative corrections lower this result by 3.74(3)\% [878] when assuming that final states with additional photons are

included. Within the SM (i.e. $g_{e}=g_{\mu}$ ) this leads to:

$R_{e / \mu}^{\mathrm{SM}}=1.2354(2) \times 10^{-4}$.

Two experiments $[879,880]$ contribute to the present world average for the measured value:

$R_{e / \mu}^{\exp }=1.231(4) \times 10^{-4}$.

As a result $\mu e$ universality has been tested at the level: $\left(g_{\mu} / g_{e}\right)_{\pi}=1.0021(16)$.

Measurements of $R_{e / \mu}$ are based on the analysis of $e^{+}$ energy and time delay with respect to the stopping $\pi^{+}$. The decay $\pi \rightarrow e v$ is characterized by $E_{e^{+}}=0.5 m_{\pi} c^{2}=$ $69.3 \mathrm{MeV}$ and an exponential time distribution following the pion life-time $\tau_{\pi}=26 \mathrm{~ns}$. In the case of the $\pi \rightarrow \mu \nu$ decay the $4 \mathrm{MeV}$ muons, which have a range of about $1.4 \mathrm{~mm}$ in plastic scintillator, can be kept inside the target and are monitored by the observation of the subsequent decay $\mu \rightarrow e \nu \bar{\nu}$, which is characterized by $E_{e^{+}}<0.5 m_{\mu} c^{2}=52.3 \mathrm{MeV}$, and 
a time distribution which first grows according to the pion life-time and then falls with the muon life-time. A major systematic error is introduced by uncertainties in the low energy tail of the $\pi \rightarrow e v(\gamma)$ energy spectrum in the region below $0.5 m_{\mu} c^{2}$. This tail fraction typically amounts to $\approx 1 \%$. The low energy tail can be studied by suppressing the $\pi \rightarrow \mu \rightarrow e$ chain by the selection of early decays and by vetoing events in which the muon is observed in the target signal. Suppression factors of typically $10^{-5}$ have been obtained. A study of this region is also interesting, since it might reveal the signal from a heavy sterile neutrino [881].

Although the two experiments contributing to the present world average of $R_{e / \mu}$ reached very similar statistical and systematic errors there were some significant differences. The TRIUMF experiment [880] made use of a single large $\mathrm{NaI}(\mathrm{Tl})$ crystal as main positron detector, with an energy resolution of 5\% (fwhm) and a solid angle acceptance of $2.9 \%$ of $4 \pi$ sr. The PSI experiment [879] used a setup of 132 identical BGO crystals with $99.8 \%$ of $4 \pi$ sr acceptance and an energy resolution of $4.4 \%$ (fwhm). A large solid angle reduces the low energy tail of $\pi \rightarrow e v(\gamma)$ events but may also introduce a high energy tail for $\mu \rightarrow e \nu \bar{\nu} \gamma$.

Two new experiments have been approved recently aiming at a reduction of the experimental uncertainty by an order of magnitude. First results may be expected in the year 2009.

- At PSI [882] the $3 \pi$ sr CsI calorimeter built for a determination of the $\pi^{+} \rightarrow \pi^{0} e^{+} v$ branching ratio will be used. Large samples of $\pi \rightarrow e v$ decays have been recorded parasitically in the past which were used as normalization for $\pi^{+} \rightarrow \pi^{0} e^{+} v$ with an accuracy of $<0.3 \%$, i.e. the level of the present experimental uncertainty of $R_{e / \mu}$. The setup was also used for the most complete studies of the radiative decays $\pi \rightarrow e v \gamma$ [883] and $\mu \rightarrow e v \bar{\nu} \gamma$ [884] done so far. Based on this experience an improvement in precision for $R_{e / \mu}$ by almost an order of magnitude is expected.

- At TRIUMF [885] a single large $\mathrm{NaI}(\mathrm{Tl})$ detector will be used again. The detector is similar in size to the one used in the previous experiment but has significantly better energy resolution. The crystal will be surrounded by CsI detectors to reduce the low energy tail of the $\pi \rightarrow e v$ response function. By reducing the distance between target and positron detector the geometric acceptance will be increased by an order of magnitude.

\section{$6.2 K$ decay}

Despite the poor theoretical control over the meson decay constants, ratios of leptonic decay widths of pseudoscalar mesons such as $R_{K} \equiv \Gamma(K \rightarrow e v) / \Gamma(K \rightarrow \mu \nu)$ can be predicted with high accuracy, and have been traditionally considered as tests of the $V-A$ structure of weak interactions through their helicity suppression and of $\mu-e$ universality. The standard model predicts [878]:

$$
R_{K}(\mathrm{SM})=(2.472 \pm 0.001) \times 10^{-5}
$$

to be compared with the world average [170] of published $R_{K}$ measurements:

$R_{K}(\exp )=(2.44 \pm 0.11) \times 10^{-5}$.

As mentioned above the strong helicity suppression of $\Gamma(K \rightarrow e v)$ makes $R_{K}$ sensitive to physics beyond the SM. As discussed in detail in Sect. 5.6.2.3 lepton flavor violating contributions predicted in SUSY models may lead to a deviation of $R_{K}$ from the SM value in the percent range. Such contributions, arising mainly from charged Higgs exchange with large lepton flavor violating Yukawa couplings, do not decouple if SUSY masses are large and exhibit a strong dependence on $\tan \beta$. For large (but not extreme) values of this parameter, not excluded by other measurements, the interference between the SM amplitude and a double lepton-flavor violating contribution could produce a $-3 \%$ effect. Other experimental constraints such as those from $R_{\pi}$ or lepton flavor violating $\tau$ decays were shown in [839] not to be competitive with those from $R_{K}$ in this scenario.

\subsubsection{Preliminary NA48 results for $R_{K}$}

In the original NA48/2 proposal [886] the measurement of $K$ leptonic decays was not considered interesting enough to be mentioned. Nevertheless, triggers for such decays were implemented during the 2003 run. Since these were not very selective they had to be highly down-scaled. The data still contain about $4000 K_{e 2}$ decays which is more than four times the previous world sample. In the analysis of these data [887] $\sim 15 \%$ background due to misidentified $K_{\mu 2}$ decays was observed (see below). The preliminary result was presented at the HEP2005 Europhysics conference in Lisbon [888]:

$R_{K}(\exp )=\left(2.416 \pm 0.043_{\text {stat }} \pm 0.024_{\text {syst }}\right) \times 10^{-5}$,

marginally consistent with the SM value. While the uncertainty in this result is dominated by the statistical error, the unoptimized $K_{e 2}$ trigger and the lack of a sufficiently large control sample resulted in a $\pm 0.8 \%$ uncertainty.

During 2004 a 56 hours special run with simplified trigger logic at $\sim 1 / 4$ nominal beam intensity was performed, dedicated to the collection of semileptonic $K^{ \pm}$decays for a measurement of $\left|V_{u s}\right|$. About $4000 K_{e 2}$ decays were extracted from these data. The preliminary result for $R_{K}$ is consistent with the 2003 value with similar uncertainty although the trigger efficiencies were better known.

The NA48 apparatus includes the following subsystems relevant for the $R_{K}$ measurement 
- a magnetic spectrometer, composed of four drift chambers and a dipole magnet (MNP33)

- a scintillator hodoscope consisting of two planes segmented into vertical and horizontal strips, providing a fast level-1 (L1) trigger for charged particles

- a liquid krypton electromagnetic calorimeter ( $\mathrm{LKr})$ with an L1 trigger system.

In the analysis of the 2003-04 data $K_{e 2}$ decays were selected using two main criteria:

- $0.95<E / p c<1.05$ where $E$ is the energy deposited in $\mathrm{LKr}$ and $p$ is the momentum measured with the magnetic spectrometer.

- the missing mass $M_{X}$ must be zero within errors, as expected for a neutrino.

The main background resulted from misidentified $K_{\mu 2}$ decays. The $E / p c$ distribution of muons has a tail which extends to $E / p c \sim 1$ and the observed fraction of muons with $0.95<E / p c<1.05$ is $\sim 5 \times 10^{-6} . K_{\mu 2}$ background was present for $p>25 \mathrm{GeV} / c$ where the $M_{X}$ resolution provided by the magnetic spectrometer was insufficient to separate $K_{e 2}$ from $K_{\mu 2}$ decays.

\subsubsection{A new measurement of $\Gamma(\boldsymbol{K} \rightarrow \boldsymbol{e} \nu) / \Gamma(\boldsymbol{K} \rightarrow \mu \nu)$ at the SPS}

During the Summer of 2007 NA62, the evolution of the NA48 experiment, has accumulated more than $100 \mathrm{~K} K_{e 2}$ decays. For this run the spectrometer momentum resolution was improved by increasing the MNP33 momentum kick from 120 to $263 \mathrm{MeV} / c$.

$K_{e 2}$ decays are selected by requiring signals from the two hodoscope planes (denoted by $Q_{1}$ ) and an energy deposition of at least $10 \mathrm{GeV}$ in the $\mathrm{LKr}$ calorimeter. This trigger has an efficiency $>0.99$ for electron momenta $p>15 \mathrm{GeV} / c$. The same down-scaled $Q_{1}$ trigger was used to collect $K_{\mu 2}$ decays. The beam intensity was adjusted to obtain a total trigger rate of $10^{4} \mathrm{~Hz}$, which saturates the data acquisition system.

Figure 47 shows the $M_{X}^{2}$ versus momentum distribution for $K_{e 2}$ and $K_{\mu 2}$ decays for the 2004 data, together with the predicted distributions for the 2004 run and for the 2007 run, as obtained from a Monte Carlo simulation (for $K_{\mu 2}$ decays the electron mass is assigned to the muon). In the 2007 run, for electron momenta up to $35 \mathrm{GeV} / c$ the $K_{\mu 2}$ contamination to the $K_{e 2}$ signal is reduced to a negligible level thanks to the improved spectrometer momentum resolution (see Fig. 48(a)). Using a lower limit of $15 \mathrm{GeV} / c$ for the electron momentum, and taking into account the detector acceptance, this means that $\sim 43 \%$ of the $K_{e 2}$ events will be kinematically background free (see Fig. 48(b)).

The fraction of $K_{\mu 2}$ faking $K_{e 2}$ decays was measured at all momenta in parallel with data taking. For this purpose

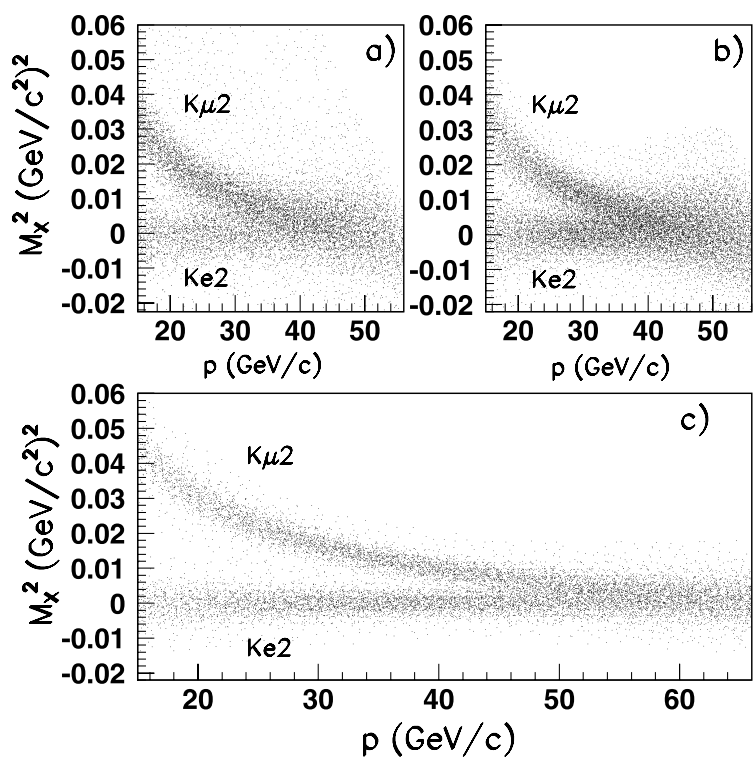

Fig. 47 Distributions of $M_{X}^{2}$ versus $p$ for $K_{e 2}$ and $K_{\mu 2}$ decays. In the $M_{X}$ calculation the electron mass is assumed for both processes: (a) measured data from the 2004 run, (b) Monte Carlo predictions for 2004 conditions, (c) Monte Carlo predictions for the conditions expected in 2007
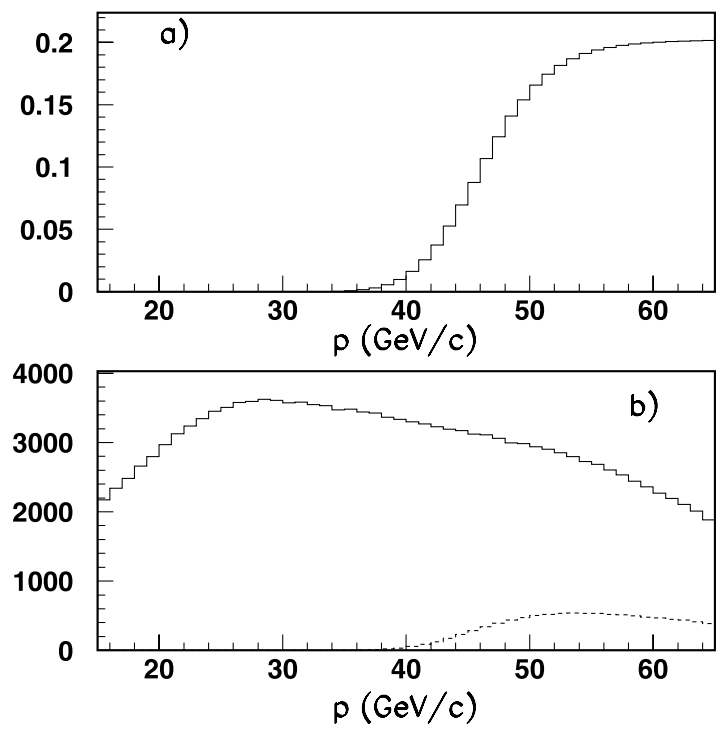

Fig. 48 (a) $K_{\mu 2}$ contamination in the $K_{e 2}$ sample. (b) Simulated momentum distributions of genuine electrons from $K_{e 2}$ decay (full histogram), and of fake electrons from $K_{\mu 2}$ decays (dashed histogram)

a $\sim 5 \mathrm{~cm}$ thick lead plate was inserted between the two hodoscope planes covering six $6.5 \mathrm{~cm}$ wide vertical hodoscope counters. The requirement that charged particles traverse the lead without interacting helps to select a pure sample of $K_{\mu 2}$ decay for which the muon $E / p c$ distribution can be directly measured for the evaluation of the $K_{\mu 2}$ contamination to the $K_{e 2}$ signal. Table 17 lists the relevant parameters describing the running conditions both for the 2004 and 2007 runs. 
Table 17 Comparison of the 2004 and 2007 running conditions

\begin{tabular}{|c|c|c|c|c|c|}
\hline & 2004 & 2007 & & 2004 & 2007 \\
\hline Acceptance $\left(\mathrm{mr}^{2}\right)$ & $0.36 \times 0.36$ & $0.18 \times 0.18$ & SPS duty cycle (s/s) & $4.8 / 16.8$ & $4.8 / 16.8$ \\
\hline$\Delta \Omega(\mathrm{sr})$ & $4 \times 10^{-7}$ & $1 \times 10^{-7}$ & live time (days) & 2.1 & 100 \\
\hline$\Delta p / p$ effective $(\%)$ & \pm 3 & \pm 2.5 & nr. of pulses & $1.08 \times 10^{4}$ & $3 \times 10^{5}$ \\
\hline RMS (\%) & $\sim 3.0$ & $\sim 1.8$ & Protons per pulse & $2.5 \times 10^{11}$ & $1.5 \times 10^{12}$ \\
\hline TRIM3 $x^{\prime}$ (mr) & 0 & \pm 0.3 & beam momentum $(\mathrm{GeV} / c)$ & $\approx 60$ & $\approx 75$ \\
\hline$p_{T}(\mathrm{MeV} / \mathrm{c})$ & 0 & \pm 22.5 & Triggers/pulse & 45,000 & 48,000 \\
\hline MNP33 $x^{\prime}(\mathrm{mr})$ & \pm 2.0 & \pm 3.5 & Good $K_{e 2} /$ pulse & $\sim 0.37$ & $\sim 0.5$ \\
\hline$p_{T}(\mathrm{MeV} / \mathrm{c})$ & \pm 120 & \pm 263 & Good $K_{e 2}($ total $)$ & 4000 & $>100,000$ \\
\hline
\end{tabular}

The overall statistical error, which includes the statistical uncertainty on the background measurement, is expected to be $0.3 \%$. The uncertainty in the trigger efficiency will be reduced to less than $\pm 0.2 \%$. The data collected in 2007 will provide a measurement of $R_{K}$ with a total uncertainty (statistical and systematic errors combined in quadrature) of less than $\pm 0.5 \%$.

\section{$6.3 \tau$ decay}

There are two ways to test lepton universality in charged weak interactions using $\tau$ decays:

- the universality of all three couplings can be tested by comparing the rates of the decays $\tau \rightarrow \mu \nu \bar{\nu}, \tau \rightarrow e \nu \bar{\nu}$ and $\mu \rightarrow e \nu \bar{\nu}$, and

- $g_{\tau} / g_{\mu}$ can be extracted by comparing $\tau \rightarrow \pi \nu$ and $\pi \rightarrow$ $\mu \nu$.

When comparing the experimental constraints one should keep in mind the complementarity of these two tests. Whereas the purely leptonic decay modes are mediated by a transversely polarized $W$, the semileptonic modes involve longitudinal polarization.

\subsubsection{Leptonic $\tau$ decays}

The decay width of $\ell_{i} \rightarrow \ell_{f} v v$ including radiative corrections is given by [889]:

$\Gamma\left(\ell_{i} \rightarrow \ell_{f} v v\right)=\frac{g_{\ell_{i}}^{2} g_{\ell_{f}}^{2}}{32 m_{W}^{2}} \frac{m_{\ell_{i}}^{5}}{192 \pi^{3}}\left(1+C_{\ell_{i} \ell_{f}}\right)$,

where $\left(1+C_{\ell_{i} \ell_{f}}\right)=f(x)\left(1+\frac{3}{5} \frac{m_{\ell_{i}}^{2}}{M_{W}^{2}}\right)\left(1+\frac{\alpha\left(m_{\ell_{i}}\right)}{2 \pi}\left(\frac{25}{4}-\pi^{2}\right)\right)$ combines weak and radiative corrections and $f(x)=1-$ $8 x+8 x^{3}-x^{4}-12 x^{2} \ln x$ with $x \equiv m_{\ell_{f}}^{2} / m_{\ell_{i}}^{2}$.

Electron-muon universality could thus be tested at the $0.2 \%$ level using:

$$
\frac{g_{\mu}}{g_{e}}=\sqrt{\frac{B(\tau \rightarrow \mu \nu \nu)}{B(\tau \rightarrow e \nu \nu)} \frac{\left(1+C_{\tau e}\right)}{\left(1+C_{\tau \mu}\right)}}=1.0002 \pm 0.0020,
$$

where $C_{\tau e}=-0.004$ and $C_{\tau \mu}=-0.0313$ are the corrections from (6.8). The values of the branching ratios of leptonic $\tau$ decays are taken from [170] and are based mostly on measurements from LEP experiments. $e-\tau$ universality has been verified with similar precision:

$$
\begin{aligned}
\frac{g_{\tau}}{g_{e}} & =\sqrt{\frac{\left(1+C_{\mu e}\right)}{\left(1+C_{\tau \mu}\right)} \frac{\tau_{\mu}}{\tau_{\tau}}\left(\frac{m_{\mu}}{m_{\tau}}\right)^{5} B(\tau \rightarrow \mu \nu \nu)} \\
& =1.0012 \pm 0.0023
\end{aligned}
$$

where $\Gamma\left(\ell_{i} \rightarrow \ell_{f} v v\right)=B\left(\ell_{i} \rightarrow \ell_{f} v v\right) / \tau_{\ell_{i}}$ has been used and $C_{\mu e}=-0.0044$. The measurement of $\mu-\tau$ universality can then be derived from $g_{\tau} / g_{e}$ and $g_{\mu} / g_{e}$, giving $g_{\tau} / g_{\mu}=$ $1.0010 \pm 0.0023$.

The measurements used in above formulas are relatively old [170], and no input from BaBar or Belle is used. The measurements of leptonic branching fractions were done by the LEP experiments in the course of the runs at or near the $Z^{0}$ resonance [170]. The $\tau^{+} \tau^{-}$events were selected via their topology, and the $\tau$ decay products were required to pass particle identification, using information from the calorimetry, tracking devices, time projection chambers and muon systems. The largest uncertainty on the measurement of tau branching ratios was statistical, with systematics limitations arising from the simulation and from particle identification.

The measurement of the $\tau$ life-time [170] comes from LEP experiments as well. Due to the large $\sqrt{s}$, each $\tau$ in the event has a large boost and travels $90 \mu \mathrm{m}$ in average. However, as there is nothing but $\tau$ 's produced in each event, their production vertex is unknown and has to be estimated averaging over other events or by minimizing the sum of impact parameters of both $\tau$ 's decay products.

The most accurate published measurement of the $\tau$ mass [890] was done by the BES experiment, through an energy scan of the $\tau^{+} \tau^{-}$production cross section in $e^{+} e^{-}$collisions around the threshold region. The collision energy scale was calibrated with $J / \psi$ and $\psi(2 S)$ resonances, with a precision of $0.25 \mathrm{MeV}$. 
Therefore the major contributions to the uncertainties on the ratios $g_{\tau} / g_{e}$ and $g_{\mu} / g_{e}$ are:

- the $\tau$ leptonic branching fractions $(0.3 \%)$, and

- the $\tau$ life-time $(0.34 \%)$.

In the calculation above, the measurements of leptonic $\tau$ decays are taken as independent. However, there are common sources of systematic uncertainties such as uncertainties on track reconstruction, number of $\tau$ decays registered by an experiment and so on. If one measured the branching ratio $B(\tau \rightarrow e \nu \nu) / B(\tau \rightarrow \mu \nu \nu)$ directly in one experiment, as was done by ARGUS and CLEO and as is done for pion decays as well, most uncertainties would cancel. Taking the PDG average on the branching ratio [170] one obtains $g_{\mu} / g_{e}=1.0028 \pm 0.0055$.

The following improvements can be expected in the future. The KEDR experiment is working, like BES, at the $\tau$-pair production threshold. They plan to measure $m_{\tau}$ with a $0.15 \mathrm{MeV}$ accuracy. A preliminary result, with accuracy comparable to BES's measurement, is available [891]. Both BaBar and Belle have accumulated large statistics of $\tau^{+} \tau^{-}$ events and should be able to perform measurements of leptonic and semileptonic $\tau$ decays, as well as to improve the measurement of the $\tau$ life-time. While the collected $\tau$ sample is much larger than at LEP, there are still significant uncertainties remaining on luminosity, tracking and particle identification. If the ratio of decay fractions is measured, then only the particle identification uncertainties will remain. Currently the electron and muon identification uncertainties for both BaBar and Belle are around 1-2\%. At the $B$-factories the $\tau$ boost in the c.m frame is much smaller than at LEP, and in addition the energies of the $e^{+}$and $e^{-}$ beams are not the same. This leads to significant differences in the technique of the life-time measurement. In particular the 3-dimensional reconstruction of the trajectories of the decay products is poor and only the impact parameter in the plane transverse to the beams, multiplied by the polar angle of the total momentum vector of 3-prong $\tau$ decay products, can be used [892]. While the statistics allows for a very accurate measurement, the work focuses on understanding the alignment of the vertex detector and the systematics in the reconstruction of the impact parameter. The measurement of the $\tau$ mass can also be done at the $B$-factories. Belle has presented a mass measurement analyzing the kinematic limit of the invariant mass of 3-prong $\tau$ decays [893]. This measurement is however less precise than those of BES or KEDR.

If one takes into account recent preliminary measurements of the $\tau$ mass from the KEDR experiment [891] and of the life-time from BaBar [892], the determination of $\tau-e$ universality changes slightly to $g_{\tau} / g_{e}=1.0021 \pm 0.0020$.

\subsubsection{Hadronic $\tau$ decays}

Another way to test $\tau-\mu$ universality is to compare the decay rates for $\tau \rightarrow \pi \nu$ and $\pi \rightarrow \mu \nu$ :

$\frac{g_{\tau}^{2}}{g_{\mu}^{2}}=\frac{B(\tau \rightarrow \pi \nu)}{B(\pi \rightarrow \mu \nu)} \frac{\tau_{\pi}}{\tau_{\tau}} \frac{2 m_{\tau} m_{\mu}^{2}}{m_{\pi}^{3}}\left(\frac{m_{\pi}^{2}-m_{\mu}^{2}}{m_{\tau}^{2}-m_{\pi}^{2}}\right)^{2}\left(1+C_{\tau \pi}\right)$,

where $C_{\tau \pi}=-\left(1.6_{-1.4}^{+0.9}\right) 10^{-3}[833,894]$.

Taking measurements from Ref. [170] one obtains $g_{\tau} / g_{\mu}=0.9996 \pm 0.037$. Here the main uncertainties come from

- $\tau \rightarrow \pi v$ decay (1\%), where the dominant contribution is due to $\tau \rightarrow \pi \pi^{0} v$ contamination and $\pi^{0}$ reconstruction,

- the $\tau$ life-time (0.34\%), and

- the hadronic correction $(0.1 \%)$.

Again, no results from the $B$ factories are available yet, and one should expect that the large $\tau$ samples collected by BaBar and Belle will allow a significant improvement, in case the understanding of particle identification will be improved.

\section{CP violation with charged leptons}

There are two powerful motivations for probing CP symmetry in lepton decays:

- The discovery of CP asymmetries in $B$ decays that are close to $100 \%$ in a sense 'de-mystifies' CP violation. For it established that complex CP phases are not intrinsically small and can be close to 90 degrees even. This demystification would be completed, if $\mathrm{CP}$ violation were found in the decays of leptons as well.

- We know that CKM dynamics, which is so successful in describing quark flavor transitions, is not relevant to baryogenesis. There are actually intriguing arguments for baryogenesis being merely a secondary effect driven by primary leptogenesis [895]. To make the latter less speculative, one has to find $\mathrm{CP}$ violation in dynamics of the leptonic sector.

The strength of these motivations has been well recognized in the community, as can be seen from the planned experiments to measure $\mathrm{CP}$ violation in neutrino oscillations and the ongoing heroic efforts to find an electron EDM. Yet there are other avenues to this goal as well that certainly are at least as difficult, namely to probe CP symmetry in muon and $\tau$ decays. Those two topics are addressed below in Sects. 7.1 and 7.2. There are also less orthodox probes, namely attempts (i) to extract an EDM for $\tau$ leptons from 
$e^{+} e^{-} \rightarrow \tau^{+} \tau^{-}$, (ii) to search for a T-odd correlation in polarized ortho-positronium decays and (iii) to measure the muon transverse polarization in $K^{+} \rightarrow \mu^{+} v \pi^{0}$ decays. It is understood that the standard model does not produce an observable effect in any of these three cases or the other ones listed above (except for $\tau^{ \pm} \rightarrow \nu K_{S} \pi^{ \pm}$, as described below).

Concerning topic (i), one has to understand that one is searching for a CP-odd effect in an electromagnetic production process unlike in $\tau$ decays, which are controlled by weak forces.

In $\left[e^{+} e^{-}\right]_{\mathrm{OP}} \rightarrow 3 \gamma$, topic (ii), one can construct various T-odd correlations or integrated moments between the spin vector $\vec{S}_{\text {OP }}$ of polarized ortho-positronium and the momenta $\vec{k}_{i}$ of two of the photons that define the decay plane:

$$
\begin{aligned}
& A_{T \text { odd }}=\left\langle\vec{S}_{\mathrm{OP}} \cdot\left(\vec{k}_{1} \times \vec{k}_{2}\right)\right\rangle, \\
& A_{\mathrm{CP}}=\left\langle\left(\vec{S}_{\mathrm{OP}} \cdot \vec{k}_{1}\right)\left(\vec{S}_{\mathrm{OP}} \cdot\left(\vec{k}_{1} \times \vec{k}_{2}\right)\right)\right\rangle .
\end{aligned}
$$

- The moment $A_{T \text { odd }}$ is $\mathrm{P}$ and $\mathrm{CP}$ even, yet $\mathrm{T}$ odd. Rather than by $\mathrm{CP}$ or $\mathrm{T}$ violation in the underlying dynamics it is generated by higher order QED processes. It has been conjectured [896] that the leading effect is formally of order $\alpha$ relative to the decay width due to the exchange of a photon between the two initial lepton lines. From it one has to remove the numerically leading contribution, which has to be absorbed into the bound state wave function. The remaining contribution is presumably at the subpermille level. Alternatively $A_{T \text { odd }}$ can be generated at order $\alpha^{2}$-or at roughly the $10^{-5}$ level-through the interference of the lowest order decay amplitude with one where a fermion loop connects two of the photon lines.

- On the other hand the moment $A_{\mathrm{CP}}$ is odd under $\mathrm{T}$ as well as under $\mathrm{P}$ and in particular $\mathrm{CP}$. Final state interactions cannot generate a $\mathrm{CP}$-odd moment with $\mathrm{CP}$ invariant dynamics. Observing $A_{\mathrm{CP}} \neq 0$ thus unambiguously establishes CP violation. The present experimental upper bound is around few percent; it seems feasible, see Sect. 7.4, to improve the sensitivity by more than three orders of magnitude, i.e. down to the $10^{-5}$ level! The caveat arises at the theoretical level: with the 'natural' scale for weak interference effects in positronium given by $G_{\mathrm{F}} m_{e}^{2} \sim 10^{-11}$, one needs a dramatic enhancement to obtain an observable effect.

Discussing topic (iii) — the muon transverse polarization in $K_{\mu 3}$ decays-under the heading of CP violation in the leptonic sector will seem surprising at first. Yet a general, though hand waving argument, suggests that the highly suppressed direct CP violation in nonleptonic $\Delta S=1$-as expressed through $\epsilon^{\prime}$-rules against an observable signal even in the presence of new physics-unless the latter has a special affinity for leptons. The present status of the data and future plans are discussed in Sect. 7.3.

\section{$7.1 \mu$ decays}

The muon decay $\mu^{-} \rightarrow e^{-} \bar{v}_{e} v_{\mu}$ and its 'inverse' $v_{\mu} e^{-} \rightarrow$ $\mu^{-} v_{e}$ are successfully described by the ' $V-A^{\prime}$ ' interaction, which is a particular case of the local, derivative-free, lepton number conserving, four-fermion interaction [897]. The ' $V-A$ ' form and the nature of the neutrinos $\left(\bar{v}_{e}\right.$ and $\left.v_{e}\right)$ have been determined by experiment [898-900].

The observables-energy spectra, polarizations and angular distributions - may be parameterized in terms of the dimensionless coupling constants $g_{\varepsilon \mu}^{\gamma}$ and the Fermi coupling constant $G_{\mathrm{F}}$. The matrix element is

$$
\mathcal{M}=\frac{4 G_{\mathrm{F}}}{\sqrt{2}} \sum_{\substack{\gamma=\mathrm{S}, \mathrm{V}, \mathrm{T} \\ \varepsilon, \mu=\mathrm{R}, \mathrm{L}}} g_{\varepsilon \mu}^{\gamma}\left\langle\bar{e}_{\varepsilon}\left|\Gamma^{\gamma}\right|\left(\nu_{e}\right)_{n}\right)\left\langle\left(\bar{v}_{\mu}\right)_{m}\left|\Gamma_{\gamma}\right| \mu_{\mu}\right\rangle .
$$

We use here the notation of Fetscher et al. $[898,901]$ who in turn use the sign conventions and definitions of Scheck [902]. Here $\gamma=\mathrm{S}, \mathrm{V}, \mathrm{T}$ indicate a (Lorentz) scalar, vector, or tensor interaction, and the chirality of the electron or muon (right or left handed) is labeled by $\varepsilon, \mu=\mathrm{R}, \mathrm{L}$. The chiralities $n$ and $m$ of the $v_{e}$ and the $\bar{v}_{\mu}$ are determined by given values of $\gamma, \varepsilon$ and $\mu$. The 10 complex amplitudes $g_{\varepsilon \mu}^{\gamma}$ and $G_{\mathrm{F}}$ constitute 19 independent parameters to be determined by experiment. The ' $V-A$ ' interaction corresponds to $g_{\mathrm{LL}}^{\mathrm{V}}=1$, with all other amplitudes being 0 .

Experiments show the interaction to be predominantly of the vector type and left handed $\left[g_{\mathrm{LL}}^{\mathrm{V}} \cdot 0.96(90 \%\right.$ C.L. $\left.)\right]$ with no evidence for other couplings. The measurement of the muon life-time yields the most precise determination of the Fermi coupling constant $G_{\mathrm{F}}$, which is presently known with a relative precision of $8 \times 10^{-6}[903,904]$. Continued improvement of this measurement is certainly an important goal [905], since $G_{\mathrm{F}}$ is one of the fundamental parameters of the standard model.

\subsubsection{T invariance in $\mu$ decays}

$P_{\mathrm{T}_{2}}$ - the component of the decay positron polarization which is transverse to the positron momentum and the muon polarization-is T odd and due to the practical absence of a strong or electromagnetic final state interaction it probes $\mathrm{T}$ invariance. A second-generation experiment has been performed at PSI by the ETH Zürich-Cracow-PSI Collaboration [906]. They obtained, for the energy averaged transverse polarization component:

$\left\langle P_{\mathrm{T}_{2}}\right\rangle=\left(-3.7 \pm 7.7_{\text {stat. }} \pm 3.4_{\text {syst. }}\right) \times 10^{-3}$.

\subsubsection{Future prospects}

The precision on the muon life-time can presumably be increased over the ongoing measurements by one order of 
magnitude [903]. Improvement in measurements of the decay parameters seems more difficult. The limits there are not given by the muon rates which usually are high enough already $\left(\approx 3 \times 10^{8} \mathrm{~s}^{-1}\right.$ at the $\mu \mathrm{E} 1$ beam at PSI, for example), but rather by effects like positron depolarization in matter or by the small available polarization $(<7 \%)$ of the electron targets used as analysers. The measurement of the transverse positron polarization might be improved with a smaller phase space (lateral beam dimension of a few millimetres or better). This experiment needs a pulsed beam with high polarization.

\section{$7.2 \mathrm{CP}$ violation in $\tau$ decays}

The betting line is that $\tau$ decays - next to the electron EDM and $v$ oscillations - provide the best stage to search for manifestations of CP breaking in the leptonic sector. There exists a considerable literature on the subject started by discussions on a tau-charm factory more than a decade ago [907-910] and attracting renewed interest recently [911914] stressing the following points:

- There are many more channels than in muon decays making the constraints imposed by CPT symmetry much less restrictive.

- The $\tau$ lepton has sizable rates into multi-body final states. Due to their non-trivial kinematics asymmetries can emerge also in the final state distributions, where they are likely to be significantly larger than in the integrated widths. The channel $K_{L} \rightarrow \pi^{+} \pi^{-} e^{+} e^{-}$can illustrate this point. It commands only the tiny branching ratio of $3 \times 10^{-7}$. The forward-backward asymmetry $\langle A\rangle$ in the angle between the $\pi^{+} \pi^{-}$and $e^{+} e^{-}$planes constitutes a $\mathrm{CP}$ odd observable. It has been measured by $\mathrm{KTeV}$ and NA48 to be truly large, namely about $13 \%$, although it is driven by the small value of $\left|\epsilon_{K}\right| \sim 0.002$. I.e., one can trade branching ratio for the size an $\mathrm{CP}$ asymmetry.

- New physics in the form of multi-Higgs models can contribute on the tree-level like the SM $W$ exchange.

- Some of the channels should exhibit enhanced sensitivity to new physics.

- Having polarized $\tau$ leptons provides a powerful handle on $\mathrm{CP}$ asymmetries and control over systematics.

These features will be explained in more detail below. It seems clear that such measurements can be performed only in $e^{+} e^{-}$annihilation, i.e. at the $B$ factories running now or better still at a Super-Flavor factory, as discussed in the Working Group 2 report. There one has the added advantage that one can realistically obtain highly polarized $\tau$ leptons: This can be achieved directly by having the electron beam longitudinally polarized or more indirectly even with unpolarized beams by using the spin alignment of the produced $\tau$ pair to 'tag' the spin of the $\tau$ under study by the decay of the other $\tau$ like $\tau \rightarrow v \rho$.
7.2.1 $\tau \rightarrow \nu K \pi$

The most promising channels for exhibiting $\mathrm{CP}$ asymmetries are $\tau^{-} \rightarrow \nu K_{S} \pi^{-}, v K^{-} \pi^{0}[910]:$

- Due to the heaviness of the lepton and quark flavors they are most sensitive to non-minimal Higgs dynamics while being Cabibbo suppressed in the SM.

- They can show asymmetries in the final state distributions.

The SM does generate a CP asymmetry in $\tau$ decays that should be observable. Based on known physics one can reliably predict a CP asymmetry [911]:

$$
\begin{aligned}
& \frac{\Gamma\left(\tau^{+} \rightarrow K_{S} \pi^{+} \bar{\nu}\right)-\Gamma\left(\tau^{-} \rightarrow K_{S} \pi^{-} \nu\right)}{\Gamma\left(\tau^{+} \rightarrow K_{S} \pi^{+} \bar{\nu}\right)+\Gamma\left(\tau^{-} \rightarrow K_{S} \pi^{-} \nu\right)} \\
& \quad=(3.27 \pm 0.12) \times 10^{-3}
\end{aligned}
$$

due to $K_{S}$ 's preference for antimatter over matter. Strictly speaking, this prediction is more general than the SM: no matter what produces the $\mathrm{CP}$ impurity in the $K_{S}$ wave function, the effect underlying (7.4) has to be present, while of course not affecting $\tau^{\mp} \rightarrow \nu K^{\mp} \pi^{0}$.

To generate a CP asymmetry, one needs two different amplitudes contribute coherently. This requirement is satisfied, since the $K \pi$ system can be produced from the (QCD) vacuum in a vector and scalar configuration with form factors $F_{V}$ and $F_{S}$, respectively. Both are present in the data, with the vector component (mainly in the form of the $K^{*}$ ) dominant as expected [915]. Within the SM, there does not arise a weak phase between them on an observable level, yet it can readily be provided by a charged Higgs exchange in nonminimal Higgs models, which contributes to $F_{S}$.

A few general remarks on the phenomenology might be helpful to set the stage. For a $\mathrm{CP}$ violation in the underlying weak dynamics to generate an observable asymmetry in partial widths or energy distributions one needs also a relative strong phase between the two amplitudes:

$$
\begin{aligned}
& \Gamma\left(\tau^{-} \rightarrow \nu K^{-} \pi^{0}\right)-\Gamma\left(\tau^{+} \rightarrow \bar{v} K^{+} \pi^{0}\right) \\
& \quad \propto \operatorname{Im}\left(F_{H} F_{V}^{*}\right) \operatorname{Im} g_{H} g_{W}^{*}, \\
& \frac{d}{d E_{K}} \Gamma\left(\tau^{-} \rightarrow \nu K^{-} \pi^{0}\right)-\frac{d}{d E_{K}} \Gamma\left(\tau^{+} \rightarrow \bar{v} K^{+} \pi^{0}\right) \\
& \quad \propto \operatorname{Im}\left(F_{H} F_{V}^{*}\right) \operatorname{Im} g_{H} g_{W}^{*},
\end{aligned}
$$

where $F_{H}$ denotes the Higgs contribution to $F_{S}$ and $g_{H}$ its weak coupling. This should not represent a serious restriction, since the $K \pi$ system is produced in a mass range with several resonances. If on the other hand one is searching for a T-odd correlation like

$$
O_{T} \equiv\left\langle\vec{\sigma}_{\tau} \cdot\left(\vec{p}_{K} \times \vec{p}_{\pi}\right)\right\rangle,
$$


then $\mathrm{CP}$ violation can surface even without a relative strong phase

$O_{T} \propto \operatorname{Re}\left(F_{H} F_{V}^{*}\right) \operatorname{Im} g_{H} g_{W}^{*}$.

Yet there is a caveat: final state interactions can generate T-odd moments even from $\mathrm{T}$ invariant dynamics, when one has

$$
O_{T} \propto \operatorname{Im}\left(F_{H} F_{V}^{*}\right) \operatorname{Re} g_{H} g_{W}^{*} .
$$

Fortunately one can differentiate between the two scenarios of (7.8), (7.9) at a $B$ or a Super-Flavor factory, where one can compare directly the T-odd moments for the $\mathrm{CP}$ conjugate pair $\tau^{+}$and $\tau^{-}$:

$$
O_{T}\left(\tau^{+}\right) \neq O_{T}\left(\tau^{-}\right) \quad \Longrightarrow \quad \mathrm{CP} \text { violation! }
$$

A few numerical scenarios might illuminate the situation: a Higgs amplitude $1 \%$ or $0.1 \%$ the strength of the SM $W$-exchange amplitude-the former [latter] contributing [mainly] to $F_{S}\left[F_{V}\right]$ - is safely in the 'noise' of present measurements of partial widths; yet it could conceivably create a CP asymmetry as large $1 \%$ or $0.1 \%$, respectively. More generally a CP-odd observable in a SM allowed process is merely linear in a new physics amplitude, since the SM provides the other amplitude. On the other hand SM forbidden transitions - say lepton flavor violation as in $\tau \rightarrow \mu \gamma$-have to be quadratic in the new physics amplitude.

$\mathrm{CP}$ odd $\propto\left|T_{\mathrm{SM}}^{*} T_{\mathrm{NP}}\right| \quad$ vs. $\quad \mathrm{LFV} \propto\left|T_{\mathrm{NP}}\right|^{2}$.

Probing CP symmetry at the $0.1 \%$ level in $\tau \rightarrow \nu K \pi$ thus has roughly the same sensitivity for a new physics amplitude as searching for $B(\tau \rightarrow \mu \gamma)$ at the $10^{-8}$ level.

CLEO has undertaken a pioneering search for a CP asymmetry in the angular distribution of $\tau \rightarrow v K_{S} \pi$ placing an upper bound of a few percent [916].

\subsubsection{Other $\tau$ decay modes}

It appears unlikely that analogous asymmetries could be observed in the Cabibbo allowed channel $\tau \rightarrow \nu \pi \pi$, yet detailed studies of $\tau \nu 3 \pi / 4 \pi$ look promising, also because the more complex final state allows us to form T-odd correlations with unpolarized $\tau$ leptons; yet the decays of polarized $\tau$ might exhibit much larger CP asymmetries [912].

Particular attention should be paid to $\tau \rightarrow \nu K 2 \pi$, which has potentially very significant additional advantages:

- One can interfere vector with axial vector $K 2 \pi$ configurations.

- The larger number of kinematical variables and of specific channels should allow more internal cross checks of systematic uncertainties like detection efficiencies for positive vs. negative particles.
7.3 Search for T violation in $K^{+} \rightarrow \pi^{0} \mu^{+} \nu$ decay

The transverse muon polarization in $K^{+} \rightarrow \pi^{0} \mu^{+} v$ decay, $P_{T}$, is an excellent probe of $\mathrm{T}$ violation, and thus of physics beyond the standard model. Most recently the E246 experiment at the KEK proton synchrotron has set an upper bound of $\left|P_{T}\right| \leq 0.0050$ (90\% C.L.). A next generation experiment is now being planned for the high intensity accelerator J-PARC which is aiming at more than one order of magnitude improvement in the sensitivity with $\sigma\left(P_{T}\right) \sim 10^{-4}$.

\subsubsection{Transverse muon polarization}

A non-zero value for the transverse muon polarization $\left(P_{T}\right)$ in the three body decay $K \rightarrow \pi \mu \nu\left(K_{\mu 3}\right)$ violates T conservation with its T-odd correlation [917]. Over the last three decades dedicated experiments have been carried out in search for a non-zero $P_{T}$. Unlike other T-odd channels in e.g. nuclear beta decays, $P_{T}$ in $K_{\mu 3}$ has the advantage that final state interactions (FSI), which may induce a spurious T-odd effect, are very small. With only one charged particle in the final state the FSI contribution originates only in higher loop effects and has been shown to be small. The single photon exchange contribution from twoloop diagrams was estimated more than twenty years ago as $P_{T}^{\mathrm{FSI}} \leq 10^{-6}$ [918]. Quite recently two-photon exchange contributions have been studied [919]. The average value of $P_{T}^{\mathrm{FSI}}$ over the Dalitz plot was calculated to be less than $10^{-5}$.

An important feature of a $P_{T}$ study is the fact that the contribution from the standard model (SM) is practically zero. Since only a single element of the CKM matrix $V_{u s}$ is involved for the semileptonic $K_{\mu 3}$ decay in the SM, no CP violation appears in first order. The lowest order contribution comes from radiative corrections to the $\bar{u} \gamma_{\mu}\left(1-\gamma_{5}\right) s W^{\mu}$ vertex, and this was estimated to be less than $10^{-7}$ [920]. Therefore, non-zero $P_{T}$ in the range of $10^{-3}-10^{-4}$ would unambiguously imply the existence of a new physics contribution [920].

Sizable $P_{T}$ can be accommodated in multi-Higgs doublet models through CP violation in the Higgs sector [921-928]. $P_{T}$ can be induced due to interference between charged Higgs exchange $\left(F_{S}, F_{P}\right)$ and $W$ exchange $\left(F_{V}, F_{A}\right)$ as shown in Fig. 49. It is conceivable that the coupling of charged Higgs fields to leptons is strongly enhanced relative to the coupling to the up-type quarks [929] which would lead to an experimentally detectable $P_{T}$ of $O\left(10^{-3}\right)$. Thus, $P_{T}$ could reveal a source of $\mathrm{CP}$ violation that escapes detection in $K \rightarrow 2 \pi, 3 \pi$ [920].

A number of other models also allow $P_{T}$ at an observable level without conflicting with other experimental constraints, and experimental limits on $P_{T}$ could thus constrain those models. Among them SUSY models with R-parity 
Fig. 49 Two interfering diagrams inducing $P_{T}$ in the multi-Higgs model (from Ref. [920])
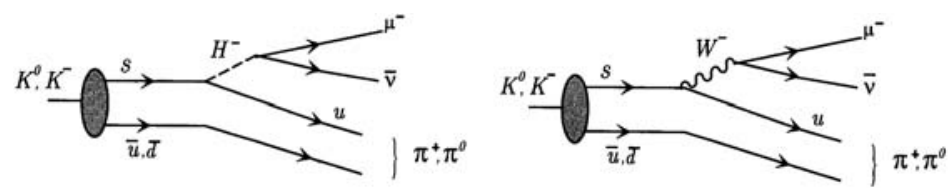

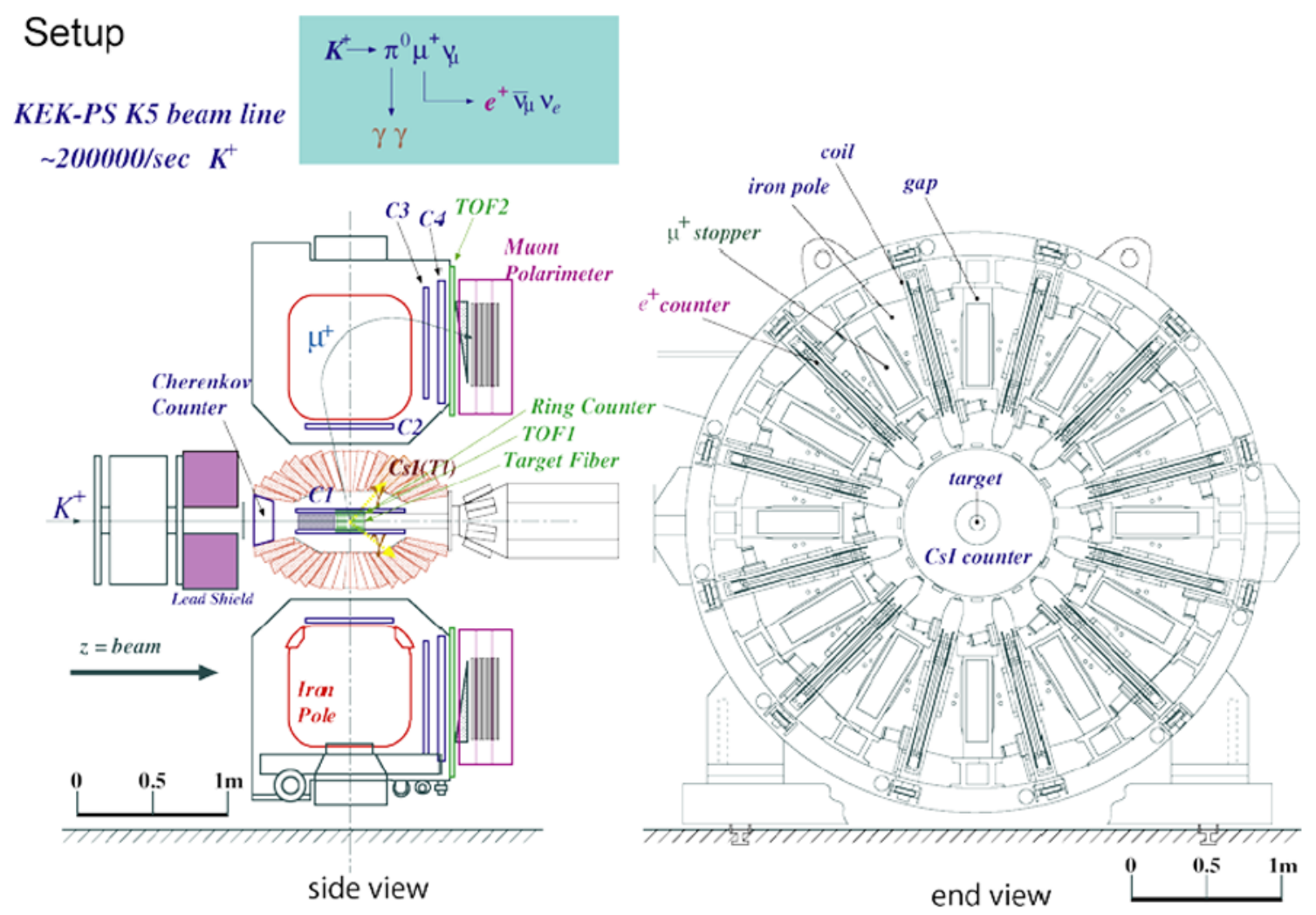

Fig. 50 E246 setup using the superconducting toroidal spectrometer. The elaborate detector system [933] consists of an active target (to monitor stopping $K^{+}$), a large-acceptance $\mathrm{CsI}(\mathrm{Tl})$ barrel (to detect $\pi^{0}$ ), tracking chambers (to track $\mu^{+}$), and muon polarimeters (to measure $P_{T}$ )

breaking [930] and a SUSY model with squark family mixing [931] should be mentioned. A recent paper [932] discusses a generic effective operator leading to a $P_{T}$ expression in terms of a cut-off scale $\Lambda$ and the Wilson coefficients $C_{S}$ and $C_{T}$.

\subsubsection{KEK E246 experiment}

The most recent and highest precision $P_{T}$ experiment was performed at the KEK proton synchrotron. The experiment used a stopped $K^{+}$beam with an intensity of $\sim 10^{5} / \mathrm{s}$ and a setup with a superconducting toroidal spectrometer (Fig. 50). Data were taken between 1996 and 2000 for a total of 5200 hours of beam time. The determination of the muon polarization was based on a measurement of the decay positron azimuthal asymmetry in a longitudinal magnetic field using "passive polarimeters". Thanks to (i) the stopped beam method which enabled total coverage of the decay phase space and hence a forward/backward symmetric measurement with respect to the $\pi^{0}$ direction and (ii) the rotational-symmetric structure of the toroidal system, systematic errors could be substantially suppressed.

The T-odd asymmetry was deduced using a double ratio scheme as

$A_{T}=\left(A_{\mathrm{fwd}}-A_{\mathrm{bwd}}\right) / 2$,

where the fwd(bwd) asymmetry was calculated using the "clockwise" and "counter-clockwise" positron emission rates $N_{\mathrm{cw}}$ and $N_{\mathrm{ccw}}$ as

$A_{\mathrm{fwd}(\mathrm{bwd})}=\frac{N_{\mathrm{fwd}(\mathrm{bwd})}^{\mathrm{cw}}-N_{\mathrm{fwd}(\mathrm{bwd})}^{\mathrm{ccw}}}{N_{\mathrm{fwd}(\mathrm{bwd})}^{\mathrm{cw}}+N_{\mathrm{fwd}(\mathrm{bwd})}^{\mathrm{ccw}}}$

$P_{T}$ was then deduced using

$P_{T}=A_{T} /\left\{\alpha\left\langle\cos \theta_{T}\right\rangle\right\}^{\prime}$

with $\alpha$ the analyzing power and $\left\langle\cos \theta_{T}\right\rangle$ the average kinematic attenuation factor. The final result was [934]

$P_{T}=-0.0017 \pm 0.0023$ (stat) \pm 0.0011 (syst), 
Table 18 Goal of the J-PARC TREK experiment compared with the E246 result

\begin{tabular}{lll}
\hline & E246 @ KEK-PS & TREK @ J-PARC \\
\hline Detector & SC toroidal spectrometer & E246-upgraded \\
Proton beam energy & $12 \mathrm{GeV}$ & $30 \mathrm{GeV}$ \\
Proton intensity & $1.0 \times 10^{12} / \mathrm{s}$ & $6 \times 10^{13} / \mathrm{s}$ \\
$K^{+}$intensity & $1.0 \times 10^{5} / \mathrm{s}$ & $3 \times 10^{6} / \mathrm{s}$ \\
Run time & $\sim 2.0 \times 10^{7} \mathrm{~s}$ & $1.0 \times 10^{7} \mathrm{~s}$ \\
$\sigma\left(P_{T}\right)_{\text {stat }}$ & $2.3 \times 10^{-3}$ & $\sim 1.0 \times 10^{-4}$ \\
$\sigma\left(P_{T}\right)_{\text {syst }}$ & $1.1 \times 10^{-3}$ & $<1.0 \times 10^{-4}$ \\
\hline
\end{tabular}

$\operatorname{Im} \xi=-0.0053 \pm 0.0071$ (stat) \pm 0.0036 (syst),

corresponding to the upper limits of $\left|P_{T}\right|<0.0050$ (90\% C.L.) and $|\operatorname{Im} \xi|<0.016$ (90\% C.L.), respectively. Here $\operatorname{Im} \xi$ is the physics parameter proportional to $P_{T}$ after removal of the kinematic factor. This result constrained the three-Higgs doublet model parameter in the way of $\left|\operatorname{Im}\left(\alpha_{1} \gamma_{1}^{*}\right)\right|<544\left(M_{H_{1}} / \mathrm{GeV}\right)^{2}$, as the most stringent constraint on this parameter. Systematic errors were investigated thoroughly, although the total size was smaller than half of the statistical error. There were two items that could not be canceled out by any of the two cancellation mechanisms of the 12-fold azimuthal rotation and $\pi^{0}$-fwd/bwd: the effect from the decay plane rotation, $\theta_{z}$ and the misalignment of the muon magnetic field, $\delta_{z}$, which should both be eliminated in the next generation J-PARC experiment.

\subsubsection{The proposed J-PARC EO6 (TREK) experiment}

A new possible $P_{T}$ experiment, E06 (TREK), at J-PARC is aiming at a sensitivity of $\sigma\left(P_{T}\right) \sim 10^{-4}$. J-PARC is a high intensity proton accelerator research complex now under construction in Japan with the first beam expected in 2008. In the initial phase of the machine, the main synchrotron will deliver a $9 \mu \mathrm{A}$ proton beam at $30 \mathrm{GeV}$. A low momentum beam of $3 \times 10^{6} K^{+}$per second will be available for stopped $\mathrm{K}^{+}$; this is about 30 times the beam intensity used for E246. Essentially the same detector concept will be adopted; namely the combination of a stopped $K^{+}$beam and the toroidal spectrometer, because this system has the advantage of suppressing systematic errors by means of the double ratio measurement scheme. However, the E246 setup will be upgraded significantly. The E246 detector will be upgraded in several parts so as to accommodate the higher counting rate and to better control the systematics. The major planned upgrades are the following:

- The muon polarimeter will become an active polarimeter, providing the muon-decay vertex and the positron track, leading to an essentially background-free muon decay measurement, with an increased positron acceptance and analyzing power.
- New dipole magnets will be added, improving the field uniformity and the alignment accuracy.

- The electronics and readout of the CsI(Tl) E246 calorimeter will be replaced to maximize the counting rate, fully exploiting the intrinsic crystal speed.

- The tracking system and the active target will be improved for higher resolution and higher decay-in-flight background rejection.

As a result, 20 times higher sensitivity to $P_{T}$ will be obtained after a one year run. The systematic errors will be controlled with sufficient accuracy and a final experimental error of $\sim 10^{-4}$ will be attained (see Table 18). A full description of the experiment can be found in the proposal [935].

It is now proposed to run for net $10^{7} \mathrm{~s}$ corresponding to roughly one year of J-PARC beam-time under the above mentioned beam condition. This would yield $2.4 \times 10^{9}$ good $K_{\mu 3}^{+}$events in the $\pi^{0}$-fwd/bwd regions, providing an estimate of $\sigma\left(P_{T}\right)_{\text {stat }}=1.35 \times 10^{-4}$. The inclusion of other $\pi^{0}$ regions, enabled by the adoption of the active polarimeter, would bring the statistical sensitivity further down to the $10^{-4}$ level. The dominant systematic errors is expected to arise from the misalignment of the polarimeter and the muon magnetic field; this will be determined from data, and Monte Carlo studies indicate a residual systematics at the $10^{-4}$ level.

It is proposed to run TREK in the early stage of J-PARC operation. The experimental group has already started relevant R\&D for the upgrades after obtaining scientific approval, and the exact schedule will be determined after funding is granted.

\subsection{Measurement of $\mathrm{CP}$ violation in ortho-positronium decay}

$\mathrm{CP}$ violation in the o-Ps decay can be detected by an accurate measurement of the angular correlation between the o-Ps spin $\vec{S}_{\text {OP }}$ and the momenta of the photons from the o-Ps decay [936], as shown in (7.1). It is useful then to write the measurable quantity:

$N(\cos \theta)=N_{0}\left(1+C_{\mathrm{CP}} \cos \theta\right)$, 
with the $\mathrm{CP}$ violation amplitude parameter, $C_{\mathrm{CP}}$, different from zero, if $\mathrm{CP}$ violating interactions take part in the o-Ps decay. In this equation, $N(\cos \theta)$ is the number of events with a measured value $\cos \theta \pm|\Delta(\cos (\theta))|$ (hereafter, for the sake of simplicity, it will be referred to as the $\cos \theta$ value, intending that this is measured with an uncertainty, depending on the spatial resolution of the detector). Here $\cos \theta$ is defined as the product of $\cos \theta_{1}$, the cosine of the angle between the $\vec{S}_{\mathrm{OP}}$ and the unit vector in the direction of highest energy photon $\hat{k}_{1}$, and $\cos \theta_{n}$, the cosine of the angle between the $\vec{S}_{\mathrm{OP}}$ and the unit vector in the direction perpendicular to the o-Ps decay plane, $\hat{n}$ [937].

The measured distribution $N(\cos \theta)$ should show an asymmetry given by $N\left(\cos \theta_{+}\right)-N\left(\cos \theta_{-}\right)=2 N_{0} C_{\mathrm{CP}} \times$ $\cos \theta$, for $\cos \theta_{+}=-\cos \theta_{-}=\cos \theta$. The quantity $C_{\mathrm{CP}}$ can be determined by measuring the rate of events $N_{+}$for a given $\cos \theta_{+}=\cos \theta$ and $N_{-}$for $\cos \theta_{-}=-\cos \theta$. In practice, $N_{+}$is the number of events in which $\hat{k}_{2}$ forms an angle with $\hat{k}_{1}$ smaller than $\pi$, and the o-Ps spin forms an angle $\theta_{n}$ smaller than $\pi / 2$ with the perpendicular to the o-Ps decay plane. In the $N_{-}$events, $\hat{k}_{2}$ forms an angle $2 \pi-\theta_{12}$ with $\hat{k}_{1}$ and the $\vec{S}_{\mathrm{OP}}$ forms an angle $\pi-\theta_{n}$ with the normal to the o-Ps decay plane. In other terms, in the $N_{-}$events the perpendicular to the o-Ps decay plane is reversed with respect to the $N_{+}$events, by flipping the direction of $\hat{k}_{2}$ specularly with respect to $\hat{k}_{1}$. Then the measurement of the asymmetry

$A=\frac{\left(N_{+}-N_{-}\right)}{\left(N_{+}+N_{-}\right)}=C_{\mathrm{CP}} \cos \theta$

allows us to derive the experimental value of $C_{\mathrm{CP}}$.

The measurement of the asymmetry $A$ implies that $\cos \theta$ in (7.18) is a well defined quantity in the experiment. In turn, this implies that the o-Ps spin direction is defined. This direction can be selected using an external magnetic field $\vec{B}$, which aligns the o-Ps spin parallel $(m=1)$, perpendicular $(m=0)$ or antiparallel $(m=-1)$ to the field direction. The magnetic field, in addition, perturbs and mixes the two $m=0$ states (one for the para-Ps and the other for the o-Ps). Thus, two new states are possible for the Ps system: the perturbed singlet and the perturbed triplet states, both with $m=0$. Their life-times depend on the $\vec{B}$ field intensity. The perturbed singlet state has a life-time shorter than $1 \mathrm{~ns}$ (as for the unperturbed singlet state of the para-Ps), which is not relevant in the measurement described here, because too short compared to the typical detector time resolution of $1 \mathrm{~ns}$. For values of $|\vec{B}|$ of few kGauss, the perturbed o-Ps life-time can be substantially reduced [938] with respect to the unperturbed value of about $142 \mathrm{~ns}$ [939]. Thanks to this effect, it is possible to separate the $m=0$ from the $m= \pm 1$ states, by measuring the o-Ps decay time. This is the time between the positron emission (by e.g., a ${ }^{22} \mathrm{Na}$ positron source) and the detection of the o-Ps decay photons. The Ps is formed in a target region, where $\mathrm{SiO}_{2}$ powder is used as target material. The value of the $|\vec{B}|$ field that maximizes the decay time separation between $m=0$ and $m= \pm 1$ states is found to be $B=4 \mathrm{kGauss}$, corresponding to a $m=0$ perturbed o-Ps life-time of $30 \mathrm{~ns}$.

The measurement of the asymmetry $A$ is performed in the following way. The direction and intensity of the $\vec{B}$ field are fixed. The $\hat{k}_{1}$ and $\hat{k}_{2}$ detectors are also fixed. In this way $\cos \theta$ has a well defined value. For each event, the Ps decay time and the energies of the three photons from the o-Ps decay are measured. The off-line analysis requires the highest energy photon in the $\hat{k}_{1}$ detector to be within an energy range $\Delta E_{1}=E_{1}^{\mathrm{max}}-E_{1}^{\mathrm{min}}$. The second highest energy photon must be recorded in the $\hat{k}_{2}$ detector within an energy range $\Delta E_{2}=E_{2}^{\max }-E_{2}^{\min }$. Then the $N_{+}$and $N_{-}$ events are counted to determine the asymmetry in (7.18). The measurement of the asymmetry $A$ in both the perturbed states (selected imposing short decay time, e.g., between 10 and $60 \mathrm{~ns}$ ) and unperturbed states (selected imposing long decay time, between 60 and $170 \mathrm{~ns}$ ) allows one to eliminate the time-independent systematics [937]. Other systematics, which are time-dependent, do not cancel out with this method and determine the final uncertainty on the $C_{\mathrm{CP}}$ measurement.

An improved detector with superior spatial and energy resolution, as compared to [937], is sketched in Fig. 51. It consists of a barrel of BGO crystals with the o-Ps forming region at its centre. The crystal signals are read out by avalanche photodiodes (APD), as the detector must work in the magnetic field. Improved spatial and angular resolution is obtained thanks to the smaller size of the crystal face exposed to the photons, $3 \times 3 \mathrm{~cm}^{2}$, and the larger barrel radius, $42 \mathrm{~cm}$. Note that such a detector could also be used efficiently for PET scanning, combined with NMR diagnostic.

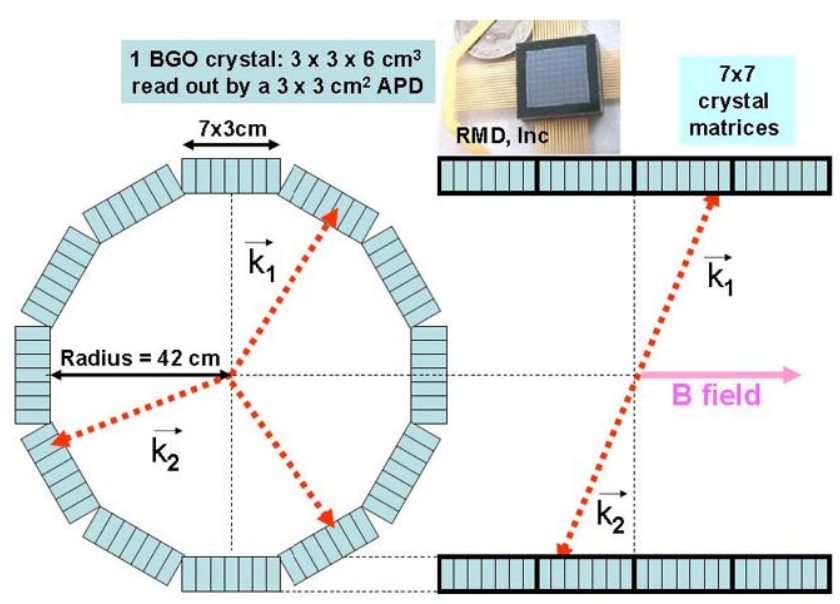

Fig. 51 Schematic view of the BGO crystal barrel calorimeter used to detect the photons from the o-Ps decay: left, detector front view and definition of the $\hat{k}_{1}$ and $\hat{k}_{2}$ vectors; right, detector side view, showing also the direction of the magnetic field $\vec{B}$ 
This possibility makes this detector a valuable investment also for applications in nuclear medical imaging.

With this detector configuration and a simulation of the detector response, the precision to be reached in the measurement of the $C_{\mathrm{CP}}$ parameter has been evaluated. A similar analysis was used for the event selection as described in [938], except that no veto is needed in the present configuration, thanks to the good spatial resolution of the proposed crystals. Various uncertainties affect the $C_{\mathrm{CP}}$ measurement. The time-dependent uncertainties on the asymmetry $A$ are induced mainly by the two-photon background, which affect more strongly the events with shorter decay time, as well as by the inhomogeneity of the o-Ps formation region, which affect the measurement of the o-Ps decay time. For high event statistics (at least $10^{12}$ selected three photon events) the following contributions to the asymmetry measurement were found: $\Delta A_{\text {stat }} \sim 10^{-6}, \Delta A_{\text {syst }}(2 \gamma$ bkgd $) \sim$ $10^{-6}, \Delta A_{\text {syst }}$ (o-Ps formation) $\sim 2 \times 10^{-6}$ resulting into a total uncertainty: $\Delta A_{\text {stat }+ \text { syst }} \sim 2.5 \times 10^{-6}$. Being $\Delta C_{\mathrm{CP}}$ related to the asymmetry total uncertainty by the relation $\Delta C_{\mathrm{CP}}=\Delta A_{\text {stat+syst }} / Q$ [937] with $Q$, the analyzing power, evaluated to be $\sim 0.5$ for this detector configuration, the total uncertainty on the $C_{\mathrm{CP}}$ parameter is $\Delta C_{\mathrm{CP}} \sim 5 \times 10^{-6}$.

Although this precision is not sufficient to measure the expected standard model $C_{\mathrm{CP}}$ value of order of $10^{-9}$, it is suitable to discover CP-violating terms in the order of $10^{-5}$, which if detected would be signal of unexpected new physics beyond the standard model.

\section{LFV experiments}

Mixing of leptonic states with different family number as observed in neutrino oscillations does not necessarily imply measurable branching ratios for LFV processes involving the charged leptons. In the standard model the rates of LFV decays are suppressed relative to the dominant familynumber conserving modes by a factor $\left(\delta m_{v} / m_{W}\right)^{4}$ which results in branching ratios which are out of reach experimentally. Note that a similar family changing quark decay such as $b \rightarrow s \gamma$ does obtain a very significant branching ratio of $O\left(10^{-4}\right)$ due to the large top mass.
As has been discussed in great detail in this report, in almost any further extension to the standard model such as supersymmetry, grand unification or extra dimensions additional sources of LFV appear. For each scenario a large number of model calculations can be found in the literature and have been reviewed in previous sections, with predictions that may well be accessible experimentally. Improved searches for charged LFV thus may either reveal physics beyond the SM or at least lead to a significant reduction in parameter space allowed for such exotic contributions.

Charged LFV processes, i.e. transitions between $e, \mu$, and $\tau$, might be found in the decay of almost any weakly decaying particle. Although theoretical predictions generally depend on numerous unknown parameters these uncertainties tend to cancel in the relative strengths of these modes. Once LFV in the charged lepton sector were found, the combined information from many different experiments would allow us to discriminate between the various interpretations. Searches have been performed in $\mu, \tau, \pi, K, B, D, W$ and $Z$ decay. Whereas highest experimental sensitivities were reached in dedicated $\mu$ and $K$ experiments, $\tau$ decay starts to become competitive as well.

\subsection{Rare $\mu$ decays}

LFV muon decays include the purely leptonic modes $\mu^{+} \rightarrow$ $e^{+} \gamma$ and $\mu^{+} \rightarrow e^{+} e^{+} e^{-}$, as well as the semileptonic $\mu-e$ conversion in muonic atoms and the muonium-antimuonium oscillation. The present experimental limits are listed in Table 19.

Whereas most theoretical models favor $\mu^{+} \rightarrow e^{+} \gamma$, this mode has a disadvantage from an experimental point of view since the sensitivity is limited by accidental $e^{+} \gamma$ coincidences and muon beam intensities have to be reduced now already. Searches for $\mu-e$ conversion, on the other hand, are limited by the available beam intensities and large improvements in sensitivity may still be achieved.

All recent results for $\mu^{+}$decays were obtained with "surface" muon beams containing muons originating in the decay of $\pi^{+}$'s that stopped very close to the surface of the pion production target, or "subsurface" beams from pion decays
Table 19 Present limits on rare $\mu$ decays

\begin{tabular}{lllll}
\hline Mode & Upper limit (90\% C.L.) & Year & Exp./Lab. & Ref. \\
\hline$\mu^{+} \rightarrow e^{+} \gamma$ & $1.2 \times 10^{-11}$ & 2002 & MEGA / LAMPF & {$[180,940]$} \\
$\mu^{+} \rightarrow e^{+} e^{+} e^{-}$ & $1.0 \times 10^{-12}$ & 1988 & SINDRUM I / PSI & {$[700]$} \\
$\mu^{+} e^{-} \leftrightarrow \mu^{-} e^{+}$ & $8.3 \times 10^{-11}$ & 1999 & PSI & {$[941]$} \\
$\mu^{-} \mathrm{Ti} \rightarrow e^{-} \mathrm{Ti}$ & $6.1 \times 10^{-13}$ & 1998 & SINDRUM II / PSI & {$[942]$} \\
$\mu^{-} \mathrm{Ti} \rightarrow e^{+} \mathrm{Ca}{ }^{*}$ & $3.6 \times 10^{-11}$ & 1998 & SINDRUM II / PSI & {$[943]$} \\
$\mu^{-} \mathrm{Pb} \rightarrow e^{-} \mathrm{Pb}$ & $4.6 \times 10^{-11}$ & 1996 & SINDRUM II / PSI & {$[944]$} \\
$\mu^{-} \mathrm{Au} \rightarrow e^{-} \mathrm{Au}$ & $7 \times 10^{-13}$ & 2006 & SINDRUM II / PSI & {$[945]$} \\
\hline
\end{tabular}


just below that region. Such beams are superior to conventional pion decay channels in terms of muon stop density and permit the use of relatively thin (typically $10 \mathrm{mg} / \mathrm{cm}^{2}$ ) foils to stop the beam. Such low-mass stopping targets are required for the ultimate resolution in positron momentum and emission angle, minimal photon yield, or the efficient production of muonium in vacuum.

\section{$8.1 .1 \mu \rightarrow e \gamma$}

Neglecting the positron mass the 2-body decay $\mu^{+} \rightarrow e^{+} \gamma$ of muons at rest is characterized by

$E_{\gamma}=E_{e}=m_{\mu} c^{2} / 2=52.8 \mathrm{MeV}$,

$\Theta_{e \gamma}=180^{\circ}$,

$t_{\gamma}=t_{e}$,

where $t$ is the time of emission from the target, and $\Theta$ the opening angle between positron and photon. All $\mu \rightarrow e \gamma$ searches performed during the past three decades were limited by accidental coincidences between a positron from normal muon decay, $\mu \rightarrow e v \bar{v}$, and a photon produced in the decay of another muon, either by bremsstrahlung or by $e^{+} e^{-}$ annihilation in flight. This background dominates by far the intrinsic background from radiative muon decay $\mu \rightarrow e v \bar{\nu} \gamma$. Accidental $e \gamma$ coincidences can be suppressed by testing the three conditions listed above. The vertex constraint resulting from the ability to trace back positrons and photons to an extended stopping target can further reduce background. Attempts have been made to suppress accidental coincidences by observing the low energy positron associated with the photon, but with minimal success. High muon polarization $\left(P_{\mu}\right)$ could help if one would limit the solid angle to accept only positrons and photons (anti)parallel to the muon spin since their rate is suppressed by the factor $1-P_{\mu}$ for antiparallel emission at $E=m_{\mu} c^{2} / 2$ but the reduced solid angle would have to be compensated by increased beam intensity which would raise the background again.

The most sensitive search to date was performed by the MEGA Collaboration at the Los Alamos Meson Physics Facility (LAMPF) which established an upper limit (90\% C.L.) on $B(\mu \rightarrow e \gamma)$ of $1.2 \times 10^{-11}$ [180, 940]. The MEG experiment [946, 947] at PSI, aims at a single-event sensitivity of $\sim 10^{-13}-10^{-14}$, and began commissioning in early 2007 . A straightforward improvement factor of more than an order of magnitude in suppression of accidental background results from the DC muon beam at PSI, as opposed to the pulsed LAMPF beam which had a macro duty cycle of $7.7 \%$. Another order of magnitude improvement is achieved by superb time resolution $\left(\approx 0.15 \mathrm{~ns}\right.$ FWHM on $\left.t_{\gamma}-t_{e}\right)$.

The MEG setup is shown in Fig. 52. The spectrometer magnet makes use of a novel "COBRA" (COnstant Bending RAdius) design which results in a graded magnetic field varying from $1.27 \mathrm{~T}$ at the centre to $0.49 \mathrm{~T}$ at both ends. This field distribution not only results in a constant projected bending radius for the $52.8 \mathrm{MeV}$ positron, for polar emission angles $\theta$ with $|\cos \theta|<0.35$, but also sweeps away positrons with low longitudinal momenta much faster than a constant field as used by MEGA. This design significantly reduces the instantaneous rates in the drift chambers.

The drift chambers are made of $12.5 \mu \mathrm{m}$ thin foils supported by $\mathrm{C}$-shaped carbon fibre frames which are out of the

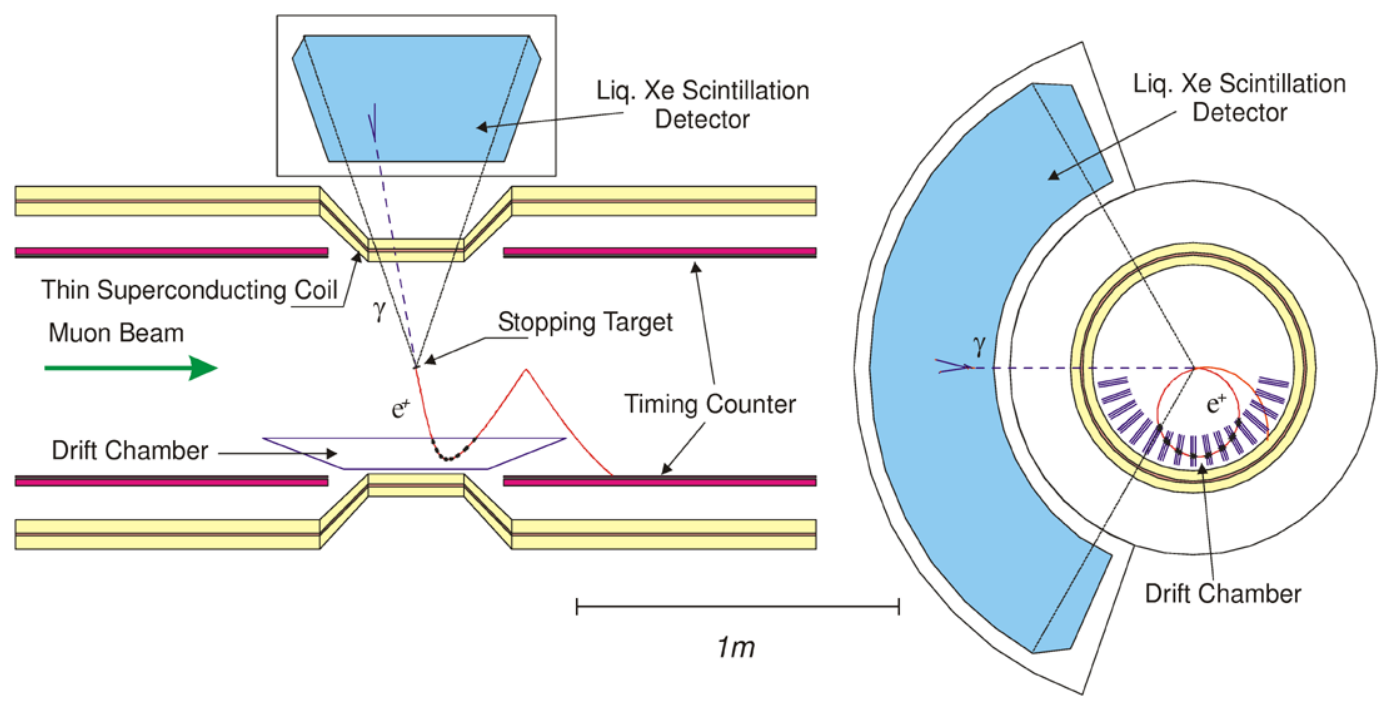

Fig. 52 Side and end views of the MEG setup. The magnetic field is shaped such that positrons are quickly swept out of the tracking region thus minimizing the load on the detectors. The cylindrical $0.8 \mathrm{~m}^{3}$ single-cell LXe detector is viewed from all sides by 846 PMTs im- mersed in the LXe allowing the reconstruction of photon energy, time, conversion point and direction and the efficient rejection of pile-up signals 
Fig. 53 Installing one of the timing counters into the COBRA magnet during the pilot run with the positron spectrometer at the end of 2006. The large ring is one of two Helmholtz coils used to compensate the COBRA stray field at the locations of the photomultipliers of the LXe detector

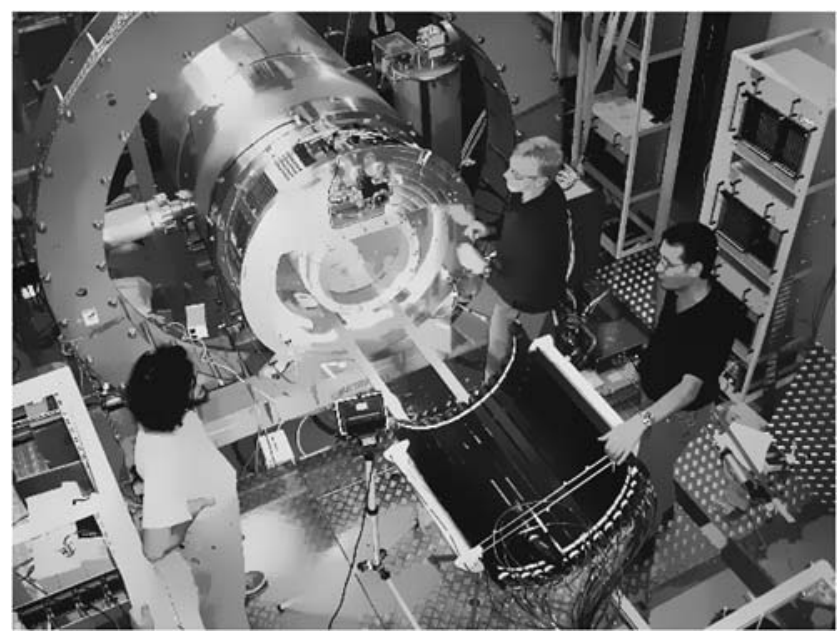

way of the positrons. The foils have "vernier" cathode pads which permit the measurement of the trajectory coordinate along the anode wires with an accuracy of about $500 \mu \mathrm{m}$.

There are two timing counters at both ends of the magnet (see Fig. 53), each of which consists of a layer of plastic scintillator fibers and 15 plastic scintillator bars of dimensions $4 \times 4 \times 90 \mathrm{~cm}^{3}$. The fibers give hit positions along the beam axis and the bars measure positron timings with a precision of $\sigma=40 \mathrm{ps}$. The counters are placed at large radii so only high energy positrons reach them, giving a total rate of a few $10^{4} / \mathrm{s}$ for each bar.

High-strength Al-stabilized conductor for the magnet coil makes the magnet as thin as $0.20 X_{0}$ radially, so that $85 \%$ of $52.8 \mathrm{MeV}$ gamma rays traverse the magnet without interaction before entering the gamma detector placed outside the magnet. Whereas MEGA used rather inefficient pair spectrometers to detect the photon, MEG developed a novel liquid Xe scintillation detector, shown in Fig. 52. By viewing the scintillation light from all sides the electromagnetic shower induced by the photon can be reconstructed which allows for a precise measurement of the photon conversion point [948]. Special PMTs that work at LXe temperature $\left(-110^{\circ} \mathrm{C}\right)$, persist under high pressures and are sensitive to the VUV scintillation light of $\operatorname{LXe}(\lambda \approx 178 \mathrm{~nm})$ have been developed in collaboration with Hamamatsu Photonics. To identify and separate pile-up efficiently, fast waveform digitizing is used for all the PMT outputs.

The performance of the detector was measured with a prototype. The results are shown in Table 20. First data taking with the complete setup took place during the second half of 2007. A sensitivity of $\mathcal{O}\left(10^{-13}\right)$ for the $90 \%$ C.L. upper limit in case no candidates are found should be reached after two years.

8.1.1.1 Beyond MEG Ten times larger surface muon rates than used by MEG can be achieved at PSI today already but the background suppression would have to be improved by
Table 20 Performance of a prototype of the MEG LXe detector at $E_{\gamma}=53 \mathrm{MeV}$

\begin{tabular}{ll}
\hline Observable & Resolution $(\sigma)$ \\
\hline energy & $1.2 \%$ \\
time & $65 \mathrm{ps}$ \\
conversion point & $\approx 4 \mathrm{~mm}$
\end{tabular}

two orders of magnitude. Accidental background $N_{\text {acc }}$ scales with the detector resolutions as

$N_{\text {acc }} \propto \Delta E_{e} \cdot \Delta t \cdot\left(\Delta E_{\gamma} \cdot \Delta \Theta_{e \gamma} \cdot \Delta x_{\gamma}\right)^{2} \cdot A_{T}^{-1}$,

with $x_{\gamma}$ the coordinate of the photon trajectory at the target and $A_{T}$ the target area. Here it is assumed that the photon can be traced back to the target with an uncertainty which is small compared to $A_{T}$. Since the angular resolution is dictated by the positron multiple scattering in the target this can be written:

$N_{\mathrm{acc}} \propto \Delta E_{e} \cdot \Delta t \cdot\left(\Delta E_{\gamma} \cdot \Delta x_{\gamma}\right)^{2} \cdot \frac{d_{T}}{A_{T}}$,

with $d_{T}$ the target thickness. When using a series of $n$ target foils each of them could have a thickness of $d_{T} / n$ and the beam would still be stopped. Since the area would increase like $n \cdot A_{T}$ the background could be reduced in proportion with $1 / n^{2}$ :

$N_{\mathrm{acc}} \propto \Delta E_{e} \cdot \Delta t \cdot\left(\Delta E_{\gamma} \cdot \Delta x_{\gamma}\right)^{2} \cdot \frac{d_{T} / n}{n \cdot A_{T}}$,

so a geometry with ten targets, $1 \mathrm{mg} / \mathrm{cm}^{2}$ each, would lead to the required background suppression.

\section{$8.1 .2 \mu \rightarrow 3 e$}

As has been discussed above the sensitivity of $\mu \rightarrow e \gamma$ searches is limited by background from accidental coinci- 
dences between a positron and a photon originating in the independent decays of two muons. Similarly, searches for the decay $\mu \rightarrow 3 e$ suffer from accidental coincidences between positrons from normal muon decay and $e^{+} e^{-}$pairs originating from photon conversions or scattering of positrons off atomic electrons (Bhabha scattering). For this reason the muon beam should be continuous on the time scale of the muon life-time and longer. In addition to the obvious constraints on relative timing and total energy and momentum, which can be applied in $\mu \rightarrow e \gamma$ searches as well, there are powerful constraints on vertex quality and location to suppress the accidental background. Since the final state contains only charged particles the setup may consist of a magnetic spectrometer without the need for an electromagnetic calorimeter with its limited performance in terms of energy and directional resolution, rate capability, and event definition in general. On the other hand, of major concern are the high rates in the tracking system of a $\mu \rightarrow 3 e$ setup which has to stand the load of the full muon decay spectrum.

8.1.2.1 The SINDRUM I experiment The present experimental limit, $B(\mu \rightarrow 3 e)<1 \times 10^{-12}$ [700], was published way back in 1988. Since no new proposals exist for this decay mode we shall analyse the prospects of an improved experiment with this SINDRUM experiment as a point of reference. A detailed description of the experiment may be found in Ref. [949].

Data were taken during six months using a $25 \mathrm{MeV} / \mathrm{c}$ subsurface beam. The beam was brought to rest with a rate of $6 \times 10^{6} \mu^{+} / \mathrm{s}$ in a hollow double-cone foam target (length $220 \mathrm{~mm}$, diameter $58 \mathrm{~mm}$, total mass $2.4 \mathrm{~g}$ ). SINDRUM I is a solenoidal spectrometer with a relatively low magnetic field of $0.33 \mathrm{~T}$ corresponding to a transverse momentum threshold around $18 \mathrm{MeV} / c$ for particles crossing the tracking system. This system consisted of five cylindrical MWPCs concentric with the beam axis. Three-dimensional space points were found by measuring the charges induced on cathode strips oriented $\pm 45^{\circ}$ relative to the sense wires.
Gating times were typically $50 \mathrm{~ns}$. The spectrometer acceptance for $\mu \rightarrow 3 e$ was $24 \%$ of $4 \pi$ sr (for a constant transition-matrix element) so the only place for a significant improvement in sensitivity would be the beam intensity.

Figure 54 shows the time distribution of the recorded $e^{+} e^{+} e^{-}$triples. Apart from a prompt contribution of correlated triples one notices a dominant contribution from accidental coincidences involving low-invariant-mass $e^{+} e^{-}$ pairs. Most of these are explained by Bhabha scattering of positrons from normal muon decay $\mu \rightarrow e v \bar{\nu}$. The accidental background thus scales with the target mass, but it is not obvious how to reduce this mass significantly below the 11 $\mathrm{mg} / \mathrm{cm}^{2}$ achieved in this search.

Figure 55 shows the vertex distribution of prompt events. One should keep in mind that most of the uncorrelated

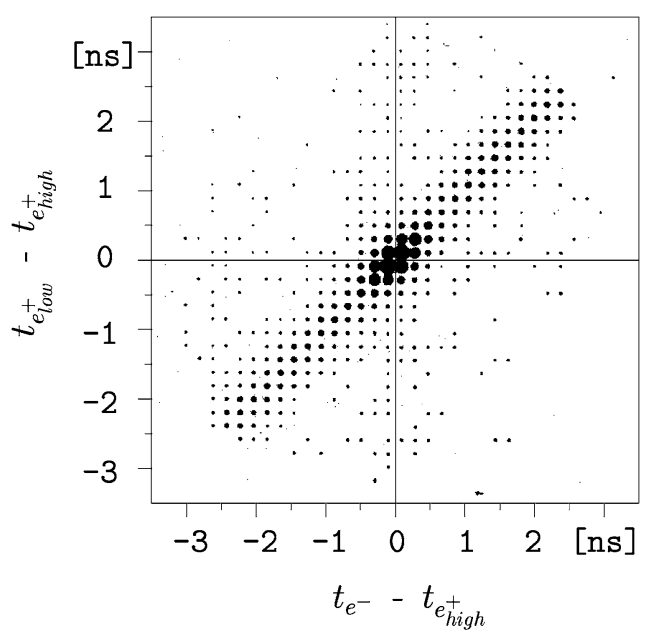

Fig. 54 Relative timing of $e^{+} e^{+} e^{-}$events. The two positrons are labeled low and high according to the invariant mass when combined with the electron. One notices a contribution of correlated triples in the centre of the distribution. These events are mainly $\mu \rightarrow 3 e v \bar{v}$ decays. The concentration of events along the diagonal is due to low-invariant-mass $e^{+} e^{-}$pairs in accidental coincidence with a positron originating in the decay of a second muon. The $e^{+} e^{-}$pairs are predominantly due to Bhabha scattering in the target
Fig. 55 Spatial distribution of the vertex fitted to prompt $e^{+} e^{+} e^{-}$triples. One clearly notices the double-cone target

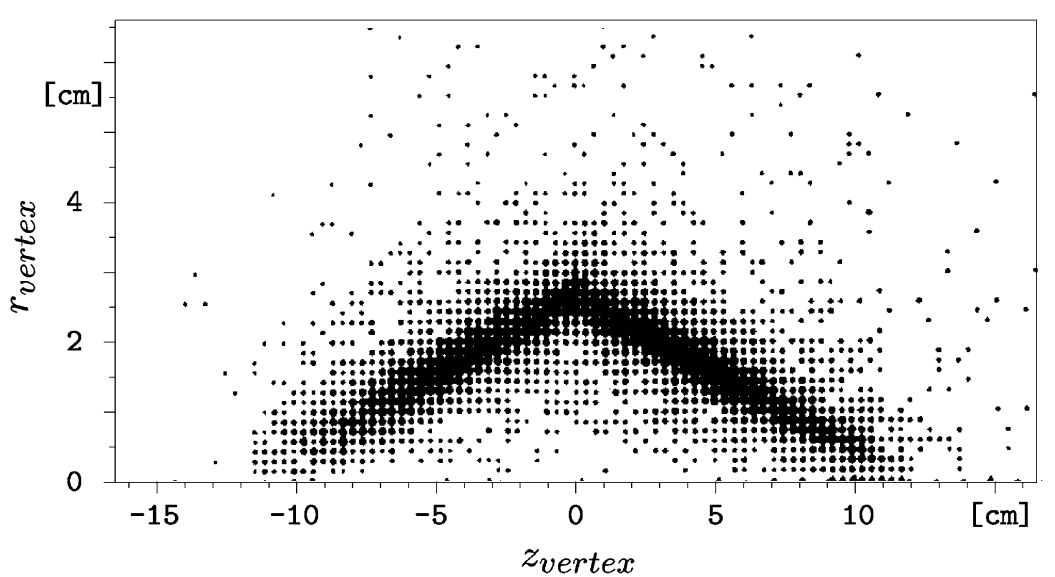


Fig. 56 Total momentum versus total energy for three event classes discussed in the text. The line shows the kinematic limit (within resolution) defined by $\Sigma|\vec{p} c|+|\Sigma \vec{p} c| \leq m_{\mu} c^{2}$ for any muon decay. The enhancement in the distribution of correlated triples below this limit is due to the decay $\mu \rightarrow 3 e v \bar{v}$

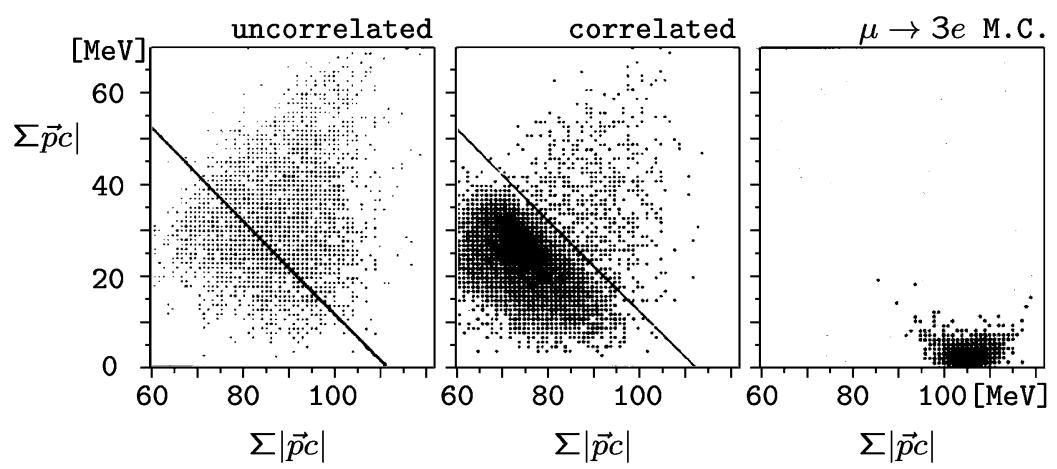

triples contain $e^{+} e^{-}$pairs coming from the target and their vertex distribution will thus follow the target contour as well. This 1-fold accidental background is suppressed by the ratio of the vertex resolution (couple of $\mathrm{mm}^{2}$ ) and the target area. There is no reason, other than the cost of the detection system, not to choose a much larger target. Such an increase might also help to reduce the load on the tracking detectors. Better vertex resolution would help as well. At these low energies tracking errors are dominated by multiple scattering in the first detector layer but it should be possible to gain by bringing it closer to the target.

Finally, Fig. 56 shows the distribution of total momentum versus total energy for three classes of events, (i) uncorrelated $e^{+} e^{+} e^{-}$triples, (ii) correlated $e^{+} e^{+} e^{-}$triples, and (iii) simulated $\mu \rightarrow 3 e$ decays. The distinction between uncorrelated and correlated triples has been made on the basis of relative timing and vertex as discussed above.

8.1.2.2 How to improve? What would a $\mu \rightarrow 3 e$ setup look like that would aim at a single-event sensitivity around $10^{-16}$, i.e., would make use of a beam rate around $10^{10} \mu^{+} / \mathrm{s}$ ? The SINDRUM I measurement was background-free at the level of $10^{-12}$ with a beam of $0.6 \times$ $10^{7} \mu^{+} / \mathrm{s}$. Taking into account that background would have set in at $10^{-13}$, the increased stop rate would raise the background level to $\approx 10^{-10}$, so six orders of magnitude in background reduction would have to be achieved. Increasing the target size and improving the tracking resolution should bring two orders of magnitude from the vertex requirement alone. Since the dominant sources of background are accidental coincidences between two decay positrons (one of which undergoes Bhabha scattering) the background rate scales with the momentum resolution squared. Assuming an improvement by one order of magnitude, i.e., from the $\approx 10 \%$ FWHM obtained by SINDRUM I to $\approx 1 \%$ for a new search, one would gain two orders of magnitude from the constraint on total energy alone. The remaining factor 100 would result from the test on the collinearity of the $e^{+}$and the $e^{+} e^{-}$pair.

As mentioned in Ref. [949] a dramatic suppression of background could be achieved by requiring a minimal open- ing angle (typically $30^{\circ}$ ) for both $e^{+} e^{-}$combinations. Depending on the mechanism for $\mu \rightarrow 3 e$, such a cut might, however, lead to a strong loss in $\mu \rightarrow 3 e$ sensitivity as well.

Whereas background levels may be under control, the question remains whether detector concepts can be developed that work at the high beam rates proposed. A large modularity will be required to solve problems of pattern recognition.

\subsection{3 $\mu-e$ conversion}

When negatively charged muons stop in matter they quickly form muonic atoms which reach their ground states in a time much shorter than the life-time of the atom. Muonic atoms decay mostly through muon decay in orbit (MIO) $\mu^{-}(A, Z) \rightarrow e^{-} v_{\mu} \bar{v}_{e}(A, Z)$ and nuclear muon capture (MC) $\mu^{-}(A, Z) \rightarrow v_{\mu}(A, Z-1)^{*}$ which in lowest order may be interpreted as the incoherent sum of elementary $\mu^{-} p \rightarrow n v_{\mu}$ captures. The MIO rate decreases slightly for increasing values of $Z$ (down to $85 \%$ of the free muon rate in the case of muonic gold) due to the increasing muon binding energy. The $\mathrm{MC}$ rate at the other hand increases roughly proportional to $Z^{4}$. The two processes have about equal rates around $Z=12$.

When the hypothetical $\mu-e$ conversion leaves the nucleus in its ground state the nucleons act coherently, boosting the process relative to the incoherent processes with exited final states. The resulting $Z$ dependence has been studied by several authors [950-953]. For $Z \lesssim 40$ all calculations predict a conversion probability relative to the MC rate which follows the linear rise with $Z$ expected naively. The predictions may, however, deviate by factors $2-3$ at higher $Z$ values.

As a result of the two-body final state the electrons produced in $\mu-e$ conversion are mono-energetic and their energy is given by:

$E_{\mu e}=m_{\mu} c^{2}-B_{\mu}(Z)-R(A)$,

where $B_{\mu}(Z)$ is the atomic binding energy of the muon and $R$ is the atomic recoil energy for a muonic atom with atomic number $Z$ and mass number $A$. In first approximation $B_{\mu}(Z) \propto Z^{2}$ and $R(A) \propto A^{-1}$. 
8.1.3.1 Background Muon decay in orbit (MIO) constitutes an intrinsic background source which can only be suppressed with sufficient electron energy resolution. The process predominantly results in electrons with energy $E_{\mathrm{MIO}}$ below $m_{\mu} c^{2} / 2$, the kinematic endpoint in free muon decay, with a steeply falling high energy component reaching up to $E_{\mu e}$. In the endpoint region the MIO rate varies as $\left(E_{\mu e}-E_{\mathrm{MIO}}\right)^{5}$ and a resolution of $1-2 \mathrm{MeV}$ (FWHM) is sufficient to keep MIO background under control. Since the MIO endpoint rises at lower $Z$ great care has to be taken to avoid low- $Z$ contaminations in and around the target.

Another background source is due to radiative muon capture (RMC) $\mu^{-}(A, Z) \rightarrow \gamma(A, Z-1)^{*} v_{\mu}$ after which the photon creates an $e^{+} e^{-}$pair either internally (Dalitz pair) or through $\gamma \rightarrow e^{+} e^{-}$pair production in the target. The RMC endpoint can be kept below $E_{\mu e}$ for selected isotopes.

Most low energy muon beams have large pion contaminations. Pions may produce background when stopping in the target through radiative pion capture (RPC) which takes place with a probability of $O\left(10^{-2}\right)$. Most RPC photons have energies above $E_{\mu e}$. As in the case of RMC these photons may produce background through $\gamma \rightarrow e^{+} e^{-}$pair production. There are various strategies to cope with RPC background:

- One option is to keep the total number of $\pi^{-}$stopping in the target during the live time of the experiment below $10^{4-5}$. This can be achieved with the help of a moderator in the beam exploiting the range difference between pions and muons of given momentum or with a muon storage ring exploiting the difference in life-time.

- Another option is to exploit the fact that pion capture takes place at a time scale far below a nanosecond. The background can thus be suppressed with a beam counter in front of the experimental target or by using a pulsed beam selecting only delayed events.

Cosmic rays (electrons, muons, photons) are a copious source of electrons with energies around $\approx 100 \mathrm{MeV}$. With the exception of $\gamma \rightarrow e^{+} e^{-}$pair production in the target these events can be recognized by an incoming particle. In addition, passive shielding and veto counters above the detection system help to suppress this background.

8.1.3.2 SINDRUM II The present best limits (see Table 19) have been measured with the SINDRUM II spectrometer at PSI. Most recently a search was performed on a gold target [945]. In this experiment (see Fig. 57) the pion suppression is based on the factor of two shorter range of pions as compared to muons at the selected momentum of $52 \mathrm{MeV} / \mathrm{c}$. A simulation using the measured range distribution shows that about one in $10^{6}$ pions cross an $8 \mathrm{~mm}$ thick $\mathrm{CH}_{2}$ moderator. Since these pions are relatively slow 99.9\% of them decay before reaching the gold target which is situated some $10 \mathrm{~m}$ further downstream. As a result pion stops in the target have been reduced to a negligible level. What remains are radiative pion capture in the degrader and $\pi^{-} \rightarrow e^{-} \bar{v}_{e}$ decay in flight shortly before entering the degrader. The resulting electrons may reach the target where they can scatter into the solid angle acceptance of the spectrometer. $\mathcal{O}(10)$ events are expected with a flat energy distribution between 80 and $100 \mathrm{MeV}$. These events are peaked in forward direction and show a time correlation with the cyclotron rf signal. To cope with this background two event classes have been introduced based on the values of polar angle and rf phase. Fig. 58 shows the corresponding momentum distributions.

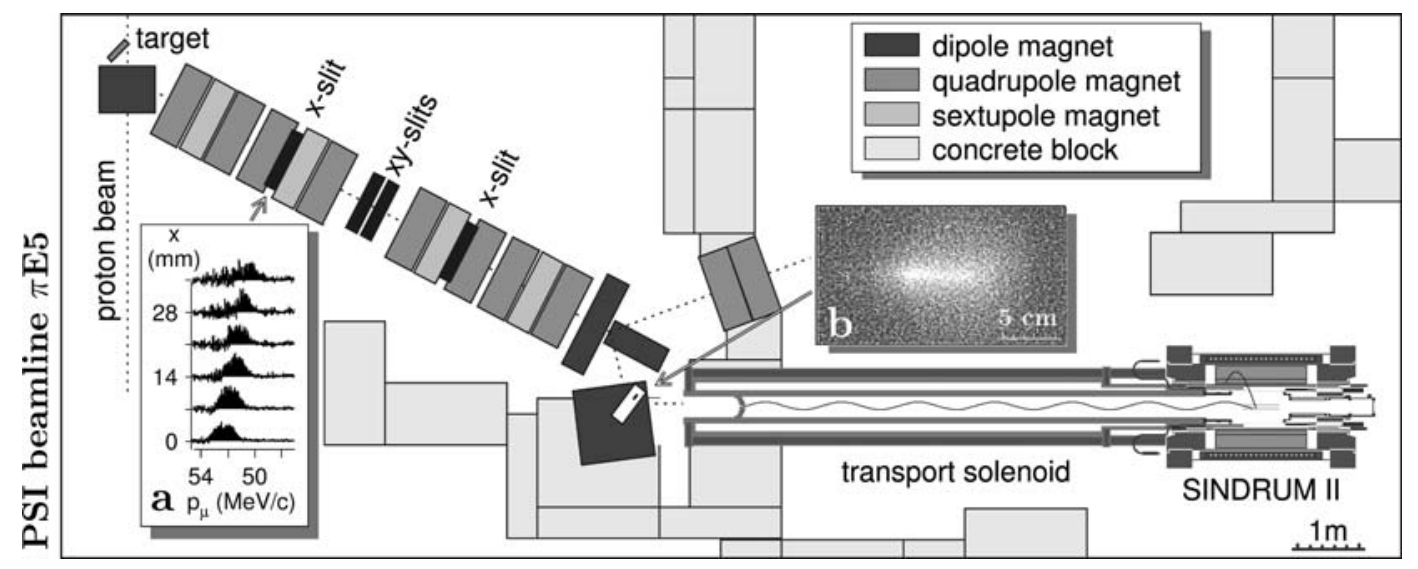

Fig. 57 Plan view of the SINDRUM II experiment. The $1 \mathrm{MW}$ $590 \mathrm{MeV}$ proton beam hits the $40 \mathrm{~mm}$ carbon production target (top left of the figure). The $\pi \mathrm{E} 5$ beam line transports secondary particles $(\pi, \mu, e)$ emitted in the backward direction to a degrader situated at the entrance of a solenoid connected axially to the SINDRUM II spectrometer. Inset $a$ shows the momentum dispersion at the position of the first slit system. Inset $b$ shows a cross section of the beam at the position of the beam focus 

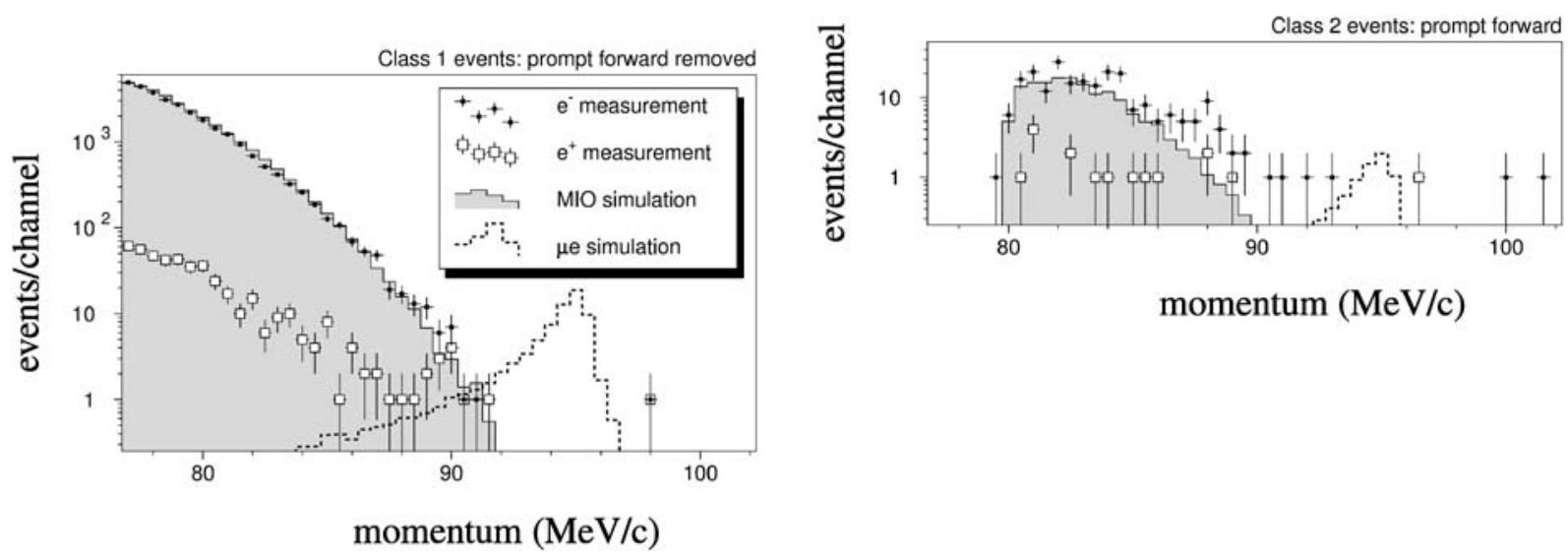

Fig. 58 Momentum distributions of electrons and positrons for two event classes described in the text. Measured distributions are compared with the results of simulations of muon decay in orbit and $\mu-e$ conversion

The spectra show no indication for $\mu-e$ conversion. The corresponding upper limit on

$$
\begin{aligned}
B_{\mu e} & \equiv \Gamma\left(\mu^{-} \mathrm{Au} \rightarrow e^{-} \mathrm{Au}_{\text {g.s. }}\right) / \Gamma_{\text {capture }}\left(\mu^{-} \mathrm{Au}\right) \\
& <7 \times 10^{-13} \quad 90 \% \text { C.L. }
\end{aligned}
$$

has been obtained with the help of a likelihood analysis of the momentum distributions shown in Fig. 58 taking into account muon decay in orbit, $\mu-e$ conversion, a contribution taken from the observed positron distribution describing processes with intermediate photons such as radiative muon capture and a flat component from pion decay in flight or cosmic ray background.

8.1.3.3 New initiatives Based on a scheme originally developed during the eighties for the Moscow Meson Factory [954] $\mu e$-conversion experiments are being considered both in the USA and in Japan. The key elements are:

- A pulsed proton beam allows one to remove pion background by selecting events in a delayed time window. Proton extinction factors below $10^{-9}$ are needed.

- A large acceptance capture solenoid surrounding the pion production target leads to a major increase in muon flux.

- A bent solenoid transporting the muons to the experimental target results in a significant increase in momentum transmission compared to a conventional quadrupole channel. A bent solenoid not only removes neutral particles and photons but also separates electric charges.

Unfortunately, the MECO proposal at BNL [955] designed along these lines was stopped because of the high costs. Presently the possibilities are studied to perform a MECO-type of experiment at Fermilab (mu2e). There is good hope that a proton beam with the required characteristics can be produced with minor modifications to the existing accelerator complex which will become available after the Tevatron stops operation in 2009. A letter of intent is in preparation.

Further improvements are being considered for an experiment at J-PARC. To fully exploit the life-time difference to suppress pion induced background the separation has to occur in the beam line rather than after the muon has stopped since the life-time of the muonic atom may be significantly shorter than the $2.2 \mu$ s of the free muon. For this purpose a muon storage ring PRISM (Phase Rotated Intense Slow Muon source, see Fig. 59) is being considered [956] which makes use of large-acceptance fixed-field alternatinggradient (FFAG) magnets. A portion of the PRISM-FFAG ring is presently under construction as $r \& d$ project. As the name suggests the ring is also used to reduce the momentum spread of the beam (from $\approx 30 \%$ to $\approx 3 \%$ ) which is achieved by accelerating late muons and decelerating early muons in $\mathrm{RF}$ electric fields. The scheme requires the construction of a pulsed proton beam [957] a decision about which has not been made yet. The low momentum spread of the muons allows for the use of a relatively thin target which is an essential ingredient for high resolution in the momentum measurement with the PRIME detector [704, 705].

Table 21 lists the $\mu^{-}$stop rates and single-event sensitivities for the various projects discussed above.

\subsection{Searches for lepton flavor violation in $\tau$ decays}

Highest sensitivities to date are achieved at the $B$-factories and further improvements are to be expected. At the LHC the modes with three charged leptons in the final state such as $\tau \rightarrow 3 \mu$ could be sufficiently clean to reach even higher sensitivity. Studies have been performed for LHCb [159] and CMS (see below).

\subsubsection{B-factories}

Present generation $B$-factories operating around the $\Upsilon(4 \mathrm{~S})$ resonance also serve as $\tau$-factories, because the production 
Fig. 59 Layout of PRISM/PRIME. The experimental target is situated at the entrance of the $180^{\circ}$ bent solenoid that transports decay electrons to the detection system. See text for further explanations

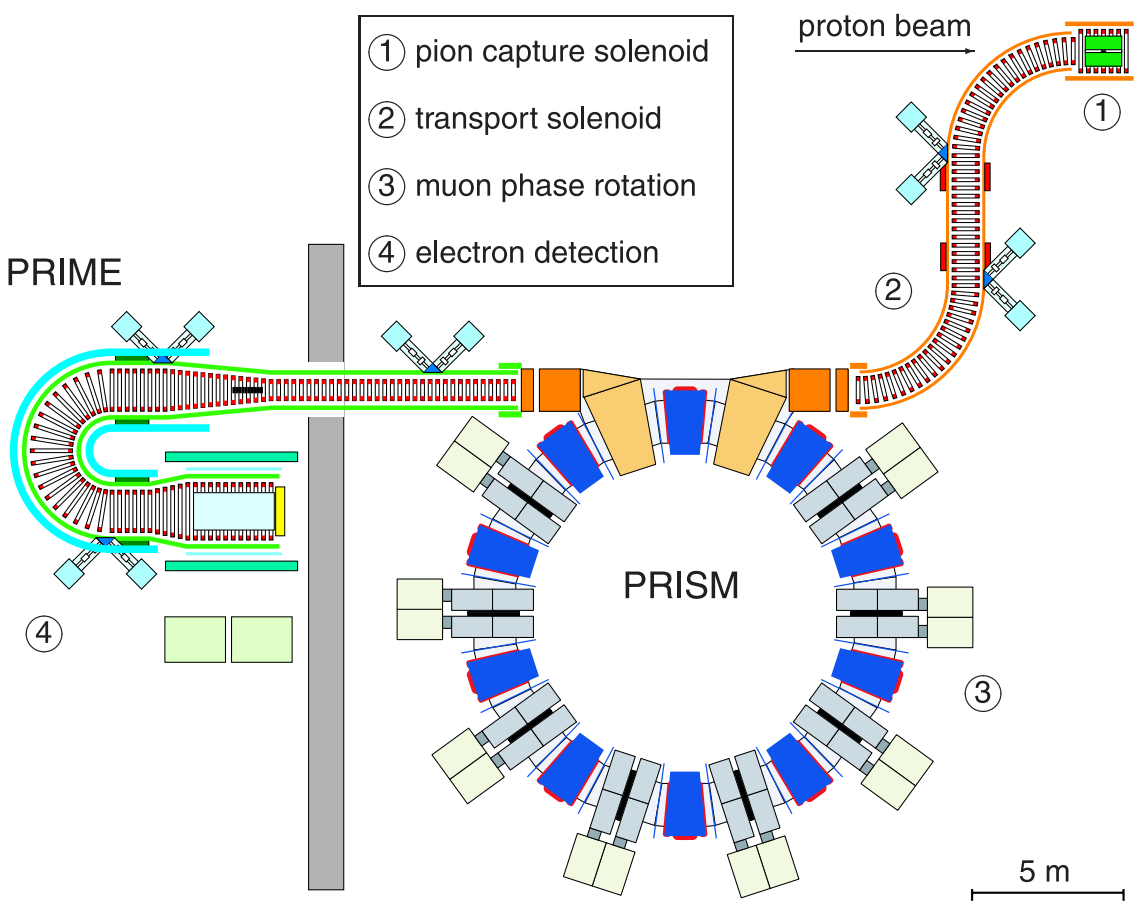

Table $21 \mu-e$ conversion searches

\begin{tabular}{lllllrr}
\hline Project & Lab & Status & $E_{p}[\mathrm{GeV}]$ & $p_{\mu}[\mathrm{MeV} / \mathrm{c}]$ & $\left.\mu^{-} \mathrm{stops}^{-1}\right]$ & $S^{\mathrm{a}}$ \\
\hline SINDRUM II & PSI & finished & 0.6 & $52 \pm 1$ & $10^{7}$ & $2 \times 10^{-13}$ \\
MECO & BNL & canceled & 8 & $45 \pm 25$ & $10^{11}$ & $2 \times 10^{-17}$ \\
mu2e & FNAL & under study & 8 & $45 \pm 25$ & $0.6 \times 10^{10}$ & $4 \times 10^{-17}$ \\
PRISM/PRIME & J-PARC & LOI & 40 & $68 \pm 3$ & $10^{12}$ & $5 \times 10^{-19}$ \\
\hline
\end{tabular}

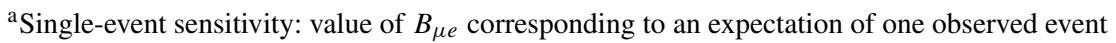

cross sections $\sigma_{b \bar{b}}=1.1 \mathrm{nb}$ and $\sigma_{\tau^{+} \tau^{-}}=0.9 \mathrm{nb}$ are quite similar at centre-of-mass energy near $10.58 \mathrm{GeV}$. BaBar and Belle have thus been able to reach the highest sensitivity to lepton flavor violating tau decays.

Many theories beyond the standard model allow for $\tau^{ \pm} \rightarrow \ell^{ \pm} \gamma$ and $\tau^{ \pm} \rightarrow \ell^{ \pm} \ell^{\mp} \ell^{ \pm}$decays, where $\ell^{-}=e^{-}, \mu^{-}$, at the level of $\sim \mathcal{O}\left(10^{-10}-10^{-7}\right)$. Examples are

- SM with additional heavy right handed Majorana neutrinos or with left handed and right handed neutral isosinglets [958];

- mSUGRA models with right handed neutrinos introduced via the see-saw mechanism [242, 959];

- supersymmetric models with Higgs exchange [174, 807] or $S O(10)$ symmetry $[164,960]$;

- technicolour models with non-universal $Z^{\prime}$ exchange [961].

Large neutrino mixing could induce large mixing between the supersymmetric partners of the leptons. While some scenarios predict higher rates for $\tau^{ \pm} \rightarrow \mu^{ \pm} \gamma$ de- cays, others, for example with inverted mass hierarchy for the sleptons [242], predict higher rates for $\tau^{ \pm} \rightarrow e^{ \pm} \gamma$ decays.

Semi-leptonic neutrino-less decays involving pseudoscalar mesons like $\tau^{ \pm} \rightarrow \ell^{ \pm} P^{0}$, where $P^{0}=\pi^{0}, \eta, \eta^{\prime}$ may be enhanced over $\tau^{ \pm} \rightarrow \ell^{ \pm} \ell^{\mp} \ell^{ \pm}$decays in supersymmetric models, for example, arising out of exchange of CP-odd pseudo-scalar neutral Higgs boson, which are further enhanced by color factors associated with these decays. The large coupling of Higgs at the $s \bar{s}$ vertex enhances final states containing the $\eta$ meson, giving a prediction of $B\left(\tau^{ \pm} \rightarrow\right.$ $\left.\mu^{ \pm} \eta\right): B\left(\tau^{ \pm} \rightarrow \mu^{ \pm} \mu^{\mp} \mu^{ \pm}\right): B\left(\tau^{ \pm} \rightarrow \mu^{ \pm} \gamma\right)=8.4: 1: 1.5$ [810]. Some models with heavy Dirac neutrinos [198, 962], two Higgs doublet models, R-parity violating supersymmetric models, and flavor changing $Z^{\prime}$ models with nonuniversal couplings [963] allow for observable parameter space of new physics [964], while respecting the existing experimental bounds at the level of $\sim \mathcal{O}\left(10^{-7}\right)$. 

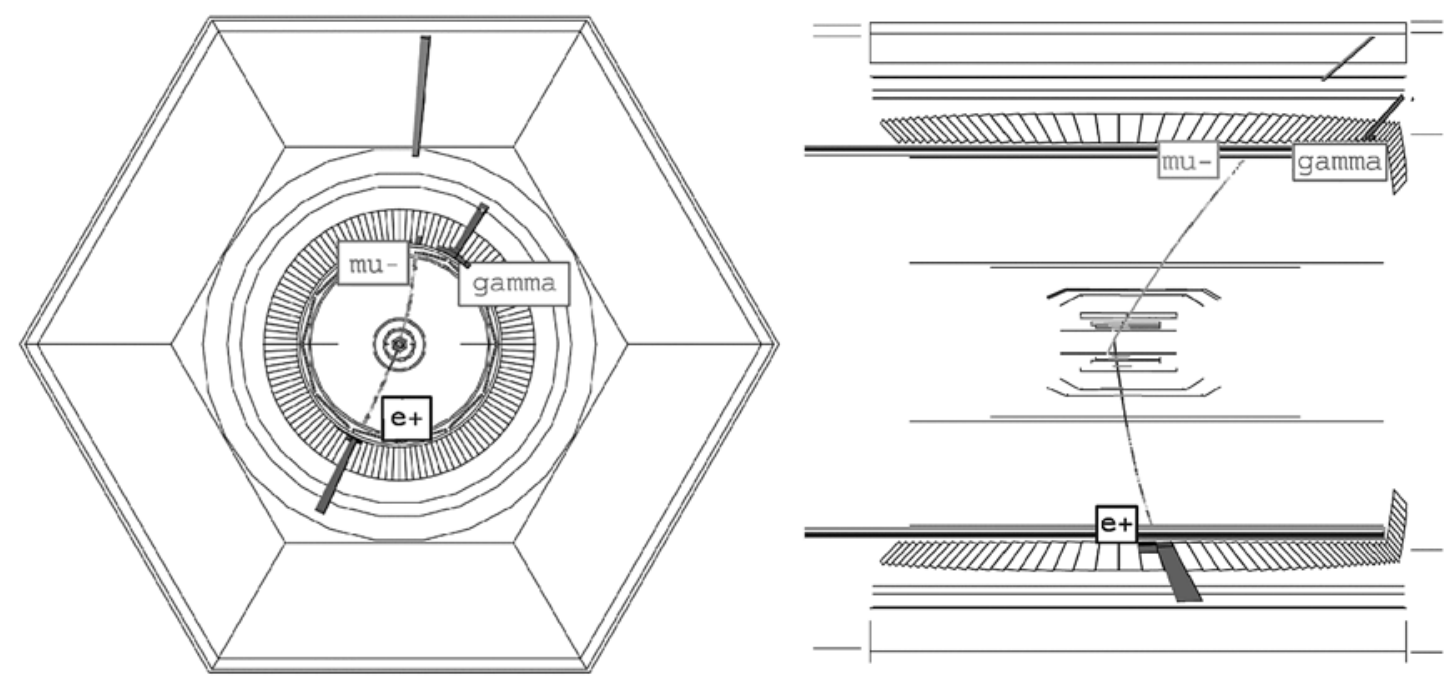

Fig. 60 Transverse and longitudinal views of a simulated $\tau \rightarrow \mu \gamma$ event in the BaBar detector. The second tau decays to $e \nu \bar{v}$

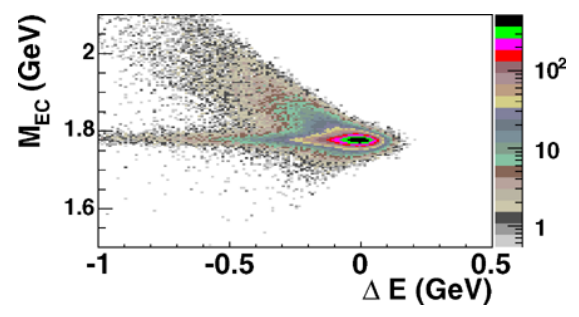

Fig. $61 m_{\mathrm{EC}}$ vs. $\Delta E$ for simulated $\tau \rightarrow \mu \gamma$ events as reconstructed in the BaBar detector. The tails of distributions are due to initial state radiation and photon energy reconstruction effects. Latter causes also the shift in $\langle\Delta E\rangle$

8.2.1.1 Search strategy In the clean $e^{+} e^{-}$annihilation environment, the decay products of two taus produced are well separated in space as illustrated in Fig. 60.

As shown in Fig. 61 neutrino-less $\tau$-decays have two characteristic features:

- the measured energy of $\tau$ daughters is close to half the centre-of-mass energy,

- the total invariant mass of the daughters is centered around the mass of the $\tau$ lepton.

While for $\ell \ell \ell$ modes the achieved mass resolution is excellent, the resolution $(\sigma)$ of the $\ell \gamma$ final state improves from $\sim 20$ to $9 \mathrm{MeV}$ by assigning the point of closest approach of the muon trajectory to the $e^{+} e^{-}$collision axis as the decay vertex and by using a kinematic fit with the $\mu \gamma \mathrm{CM}$ energy constrained to $\sqrt{s} / 2$ [191]. The energy resolution is typically $45 \mathrm{MeV}$ with a long tail due to radiation.

The principal sources of background are radiative QED (di-muon or Bhabha) and continuum $(q \bar{q})$ events as well as $\tau^{+} \tau^{-}$events with a mis-identified standard model decay mode. There is also some irreducible contribution from $\tau^{+} \tau^{-}$events with hard initial state radiation in which one of the $\tau$ 's decays into a mode with the same charged particle as the signal. For example, $\tau \rightarrow \mu \nu \bar{v}$ decays accompanied by a hard $\gamma$ is an irreducible background in the $\tau \rightarrow \mu \gamma$ search.

The general strategy to search for the neutrino-less decays is to define a signal region, typically of size $\sim 2 \sigma$, in the energy-mass plane of the $\tau$ daughters and to reduce the background expectation from well-known CM processes inside the signal region by optimizing a set of selection criteria:

- the missing momentum is consistent with the zero-mass hypothesis

- the missing momentum points inside the acceptance of the detector

- the second tau is found with the correct invariant mass

- minimal opening angle between two tau decay products

- minimal value for the highest momentum of any reconstructed track

- particle identification

The analyses are performed in a blind fashion by excluding events in the region of the signal box until all optimizations and systematic studies of the selection criteria have been completed. The cut values are optimized using control samples, data sidebands and Monte Carlo extrapolation to the signal region to yield the lowest expected upper limit under the no-signal hypothesis. The measured $m_{\mathrm{EC}}$ vs. $\Delta E$ distribution for the $\tau \rightarrow \mu \gamma$ search after applying the constraints listed above is shown in Fig. 62.

For the $\tau^{ \pm} \rightarrow \ell^{ \pm} P^{0}$ searches, the pseudo-scalar mesons $\left(P^{0}\right)$ are reconstructed in the following decay modes: $\pi^{0} \rightarrow$ $\gamma \gamma$ for $\tau^{ \pm} \rightarrow \ell^{ \pm} \pi^{0}, \eta \rightarrow \gamma \gamma$ and $\eta \rightarrow \pi^{+} \pi^{-} \pi^{0}\left(\pi^{0} \rightarrow\right.$ $\gamma \gamma)$ for $\tau^{ \pm} \rightarrow \ell^{ \pm} \eta$, and $\eta^{\prime} \rightarrow \pi^{+} \pi^{-} \eta(\eta \rightarrow \gamma \gamma)$ and $\eta^{\prime} \rightarrow$ $\rho^{0} \gamma$ for $\tau^{ \pm} \rightarrow \ell^{ \pm} \eta^{\prime}$. 
8.2.1.2 Experimental results from BaBar and Belle By the beginning of $2007 \mathrm{BaBar}$ and Belle had recorded integrated luminosities of $\mathcal{L} \sim 400$ and $700 \mathrm{fb}^{-1}$, respectively, which corresponds to a total of $\sim 10^{9} \tau$-decays. Analysis of these data samples is still ongoing and published results include only part of the data analysed. No signal has yet been observed in any of the probed channels and some limits and the corresponding integrated luminosities are summarized in Table 22. Frequentest upper limits have been calculated for the combination of the two experiments [183] using the technique of Cousins and Highland [965] following the implementation of Barlow [966].

\subsubsection{Projection of limits to higher luminosities $B\left(\tau^{ \pm} \rightarrow\right.$} $\left.\mu^{ \pm} \gamma\right)$ and $B\left(\tau^{ \pm} \rightarrow \mu^{ \pm} \mu^{\mp} \mu^{ \pm}\right)$have been lowered by five orders of magnitude over the past twenty-five years. Further significant improvements in sensitivity are expected during the next five years. Depending upon the nature of backgrounds contributing to a given search, two extreme scenarios can be envisioned in extrapolating to higher luminosities:

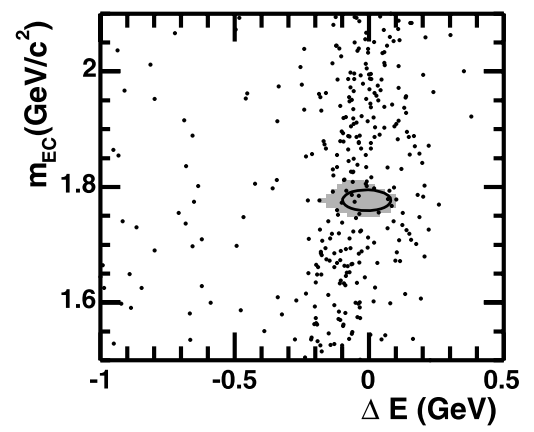

Fig. 62 Measured distribution of $m_{\mathrm{EC}}$ vs. $\Delta E$ for $\tau \rightarrow \mu \gamma$ reconstructed by BaBar [191]. The shaded region taken from Fig. 61 contains $68 \%$ of the hypothetical signal events
- If the expected background is kept below $\mathcal{O}(1)$ events, while maintaining the same efficiency $\mathcal{B}_{U L}^{90} \propto 1 / \mathcal{L}$ if no signal events would be observed. In $\tau^{ \pm} \rightarrow \mu^{ \pm} \mu^{\mp} \mu^{ \pm}$ searches, for example, the backgrounds are still quite low and the irreducible backgrounds are negligible even for projected Super $B$-factories.

- If there is background now already and no reduction could be achieved in the future measurements $\mathcal{B}_{U L}^{90} \propto 1 / \sqrt{\mathcal{L}}$.

The $\sqrt{\mathcal{L}}$ scaling is, however, unduly pessimistic since the analyses improve steadily. Better understanding of the nature of the backgrounds will lead to a more effective separation of signal and background.

The $\tau^{ \pm} \rightarrow \mu^{ \pm} \gamma$ searches suffer from significant background from both $\mu^{+} \mu^{-}$and $\tau^{+} \tau^{-}$events and to a lesser extend from $q \bar{q}$ production. While one can expect to reduce these backgrounds with continued optimization with more luminosity at the present day $B$-factories, much of the background from $\tau^{+} \tau^{-}$events is irreducible coming from $\tau \rightarrow \mu \nu \bar{\nu}$ decays accompanied by initial state radiation. This source represents about $20 \%$ of the total background in the searches performed by the BaBar experiment [191] and it is conceivable that an analysis can be developed that reduces all but this background with minimal impact on the efficiency. One could also include new selection criteria such as a cut on the polar angle of the photon which could reduce the radiative "irreducible" background by $85 \%$ with a $40 \%$ loss of signal efficiency. Table 23 summarizes the future sensitivities for various LFV decay modes.

In order to further reduce the impact of irreducible backgrounds at a future Super $B$-factory experiment, one can consider what is necessary to improve the mass resolution of the, e.g., $\mu-\gamma$ system. Currently, this resolution is limited by the $\gamma$ angular resolution. Therefore improvements might be expected if the granularity of the electromagnetic calorimeter is increased.

Table 22 Integrated luminosities and observed upper limits on the branching fractions at 90\% C.L. for selected LFV tau decays by BaBar and Belle

\begin{tabular}{|c|c|c|c|c|c|c|}
\hline \multirow[t]{2}{*}{ Channel } & \multicolumn{3}{|l|}{ BaBar } & \multicolumn{3}{|l|}{ Belle } \\
\hline & $\overline{\mathcal{L}}\left(\mathrm{fb}^{-1}\right)$ & $\mathcal{B}_{U L}\left(10^{-8}\right)$ & Ref. & $\overline{\mathcal{L}\left(\mathrm{fb}^{-1}\right)}$ & $\mathcal{B}_{U L}\left(10^{-8}\right)$ & Ref. \\
\hline$\tau^{ \pm} \rightarrow e^{ \pm} \gamma$ & 232 & 11 & [182] & 535 & 12 & [181] \\
\hline$\tau^{ \pm} \rightarrow \mu^{ \pm} \gamma$ & 232 & 6.8 & [191] & 535 & 4.5 & [181] \\
\hline$\tau^{ \pm} \rightarrow \ell^{ \pm} \ell^{\mp} \ell^{ \pm}$ & 92 & $11-33$ & [644] & 535 & $2-4$ & {$[967]$} \\
\hline$\tau^{ \pm} \rightarrow e^{ \pm} \pi^{0}$ & 339 & 13 & [648] & 401 & 8.0 & [968] \\
\hline$\tau^{ \pm} \rightarrow \mu^{ \pm} \pi^{0}$ & 339 & 11 & [648] & 401 & 12 & {$[968]$} \\
\hline$\tau^{ \pm} \rightarrow e^{ \pm} \eta$ & 339 & 16 & [648] & 401 & 9.2 & {$[968]$} \\
\hline$\tau^{ \pm} \rightarrow \mu^{ \pm} \eta$ & 339 & 15 & [648] & 401 & 6.5 & {$[968]$} \\
\hline$\tau^{ \pm} \rightarrow e^{ \pm} \eta^{\prime}$ & 339 & 24 & [648] & 401 & 16 & {$[968]$} \\
\hline$\tau^{ \pm} \rightarrow \mu^{ \pm} \eta^{\prime}$ & 339 & 14 & [648] & 401 & 13 & {$[968]$} \\
\hline
\end{tabular}




\subsubsection{CMS}

So far, only $\tau \rightarrow \mu$ transitions have been studied since muons are more easily identified and the CMS trigger thresholds for muons are generally lower than for electrons. The $\tau \rightarrow \mu \gamma$ channel was studied in the past [969] both for CMS and for ATLAS but found not to be competitive with the prospects at the $B$-factories. The $\tau \rightarrow 3 \mu$ channel looks more promising and will be discussed below.

8.2.2.1 $\tau$ production at the $L H C$ It is planned to operate the LHC in three different phases. After a commissioning phase the LHC will be ramped up to an initial luminosity of $\mathcal{L}=10^{32} \mathrm{~cm}^{-2} \mathrm{~s}^{-1}$ followed by a low luminosity phase $\left(\mathcal{L}=2 \times 10^{33} \mathrm{~cm}^{-2} \mathrm{~s}^{-1}\right)$. A high luminosity phase with $\mathcal{L}=10^{34} \mathrm{~cm}^{-2} \mathrm{~s}^{-1}$ will start in 2010 and last for a period of several years. The integrated luminosity per year will be $10-30$ and $100-300 \mathrm{fb}^{-1}$ for the low and high luminosity phases, respectively [970].

The rate of $\tau$ leptons produced was estimated with the help of PYTHIA 6.227 using the parton distribution function CTEQ5L. The results are shown in Table 24. During the low luminosity phase assuming an integrated luminosity of only $10 \mathrm{fb}^{-1}$ per year about $10^{12} \tau$ leptons will be produced within the CMS detector. The dominant production sources of $\tau$ leptons at the LHC are the $D_{s}$ and various $B$ mesons. The $W$ and the $Z$ production sources will provide considerably less $\tau$ leptons per year, but at higher energies which is an advantage for the efficient detection of their decay products (see below).

8.2.2.2 $\tau \rightarrow 3 \mu$ detection A key feature of CMS is a $4 \mathrm{~T}$ magnetic field, which ensures the measurement of chargedparticle momenta with a resolution of $\sigma_{p_{\mathrm{T}}} / p_{\mathrm{T}}=1.5 \%$ for

Table 23 Expected 90\% C.L. upper limits on LFV $\tau$ decays with $75 \mathrm{ab}^{-1}$ assuming no signal is found and reducible backgrounds are small $(\sim \mathcal{O}(1)$ events) and the irreducible backgrounds scale as $1 / \mathcal{L}$

\begin{tabular}{ll}
\hline Decay mode & Sensitivity \\
\hline$\tau \rightarrow \mu \gamma$ & $2 \times 10^{-9}$ \\
$\tau \rightarrow e \gamma$ & $2 \times 10^{-9}$ \\
$\tau \rightarrow \mu \mu \mu$ & $2 \times 10^{-10}$ \\
$\tau \rightarrow e e e$ & $2 \times 10^{-10}$ \\
$\tau \rightarrow \mu \eta$ & $4 \times 10^{-10}$ \\
$\tau \rightarrow e \eta$ & $7 \times 10^{-10}$ \\
\hline
\end{tabular}

$10 \mathrm{GeV}$ muons [970] using a four-station muon system. A silicon pixel detector and tracker allow to reconstruct secondary vertices with a resolution of about $50 \mu \mathrm{m}$ [971] and help to improve the muon reconstruction. Furthermore, CMS has an electromagnetic calorimeter (ECAL) composed of $\mathrm{PbWO}_{4}$ and a copper scintillator hadronic calorimeter (HCAL). As a result of the high magnetic field and the amount of material that has to be crossed only muons with $p_{\mathrm{T}}>3 \mathrm{GeV} / c$ are accepted. The reconstruction efficiency varies between $\approx 70 \%$ at $5 \mathrm{GeV}[972]$ and $\approx 98 \%$ at $100 \mathrm{GeV} / c$ [970].

The two levels of the CMS trigger system are called "level 1" (L1) and "high level" (HLT). The triggers relevant for this analysis are the dedicated single and di-muon triggers. For the low luminosity phase it is planned to use as $p_{\mathrm{T}}$ thresholds for single muons $14 \mathrm{GeV} / c$ at L1 and $19 \mathrm{GeV} / c$ for the HLT. The thresholds for the di-muon trigger will be $3 \mathrm{GeV} / c$ at $\mathrm{L} 1$ and $7 \mathrm{GeV} / c$ for the HLT.

Most $\tau \rightarrow 3 \mu$ events produced via $W$ and $Z$ decays will be accepted by the present triggers. Unfortunately, the low $p_{\mathrm{T}}$ of the muons from the decays of $\tau$ 's originating in $D_{s}$ or $B$ decay result in a very low trigger efficiency (Fig. 63). Dedicated trigger algorithms with improved efficiency are presently being studied.

To improve the identification of low $p_{\mathrm{T}}$ muons a new method is currently under development combining the energy deposit in the ECAL, HCAL and the number of reconstructed muon track segments in the muon systems. The in-

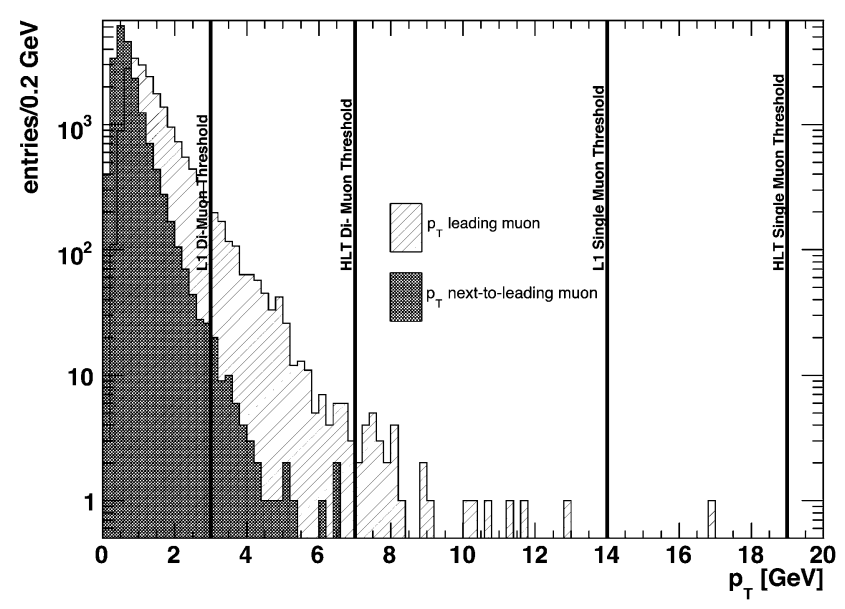

Fig. $63 p_{\mathrm{T}}$ distributions of the leading and next-to-leading muon from the decay $\tau \rightarrow 3 \mu$ at CMS. The indicated trigger thresholds for the low luminosity phase are clearly too high for the efficient detection of these events

Table 24 Number of $\tau$ leptons per year produced during the low-luminosity phase of the LHC

\begin{tabular}{lllllll}
\hline Production channel & $W \rightarrow \tau \nu_{\tau}$ & $\gamma / Z \rightarrow \tau \tau$ & $B^{0} \rightarrow \tau X$ & $B^{ \pm} \rightarrow \tau X$ & $B_{s} \rightarrow \tau X$ & $D_{s} \rightarrow \tau X$ \\
\hline$N_{\tau} / 10 \mathrm{fb}^{-1}$ & $1.7 \times 10^{8}$ & $3.2 \times 10^{7}$ & $4.0 \times 10^{11}$ & $3.8 \times 10^{11}$ & $7.9 \times 10^{10}$ & $1.5 \times 10^{12}$ \\
\hline
\end{tabular}


Fig. 64 Invariant mass distribution from the simulation of $\tau \rightarrow 3 \mu$ events

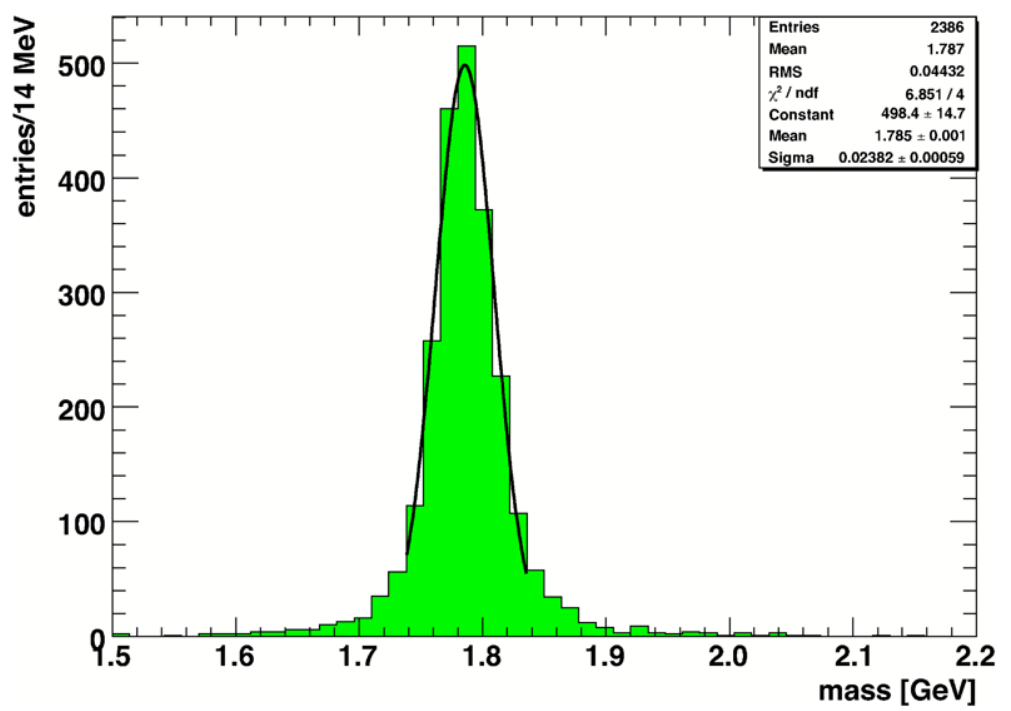

variant mass distribution of reconstructed $\tau \rightarrow 3 \mu$ events is shown in Fig. 64. The resolution is about $24 \mathrm{MeV} / c^{2}$, which ensures a good capability to reduce background events.

8.2.2.3 Background and expected sensitivity The main sources of muons are decays of $D$ and $B$ mesons which are copiously produced at LHC energies. A previous study [973] suggested that these background events can be suppressed by appropriate selection criteria. The probability to misidentify an event from pile-up is small and cosmic rays can be rejected by timing. Due to the high momentum of the muons from direct $W$ and $Z$ decays, the contribution to the background is negligible [974].

One rare decay that can mimic the signal is $D_{s} \rightarrow \phi \mu \nu_{\mu}$ followed by a decay $\phi \rightarrow \mu \mu$. This background can be reduced by an invariant mass cut around the $\phi$ mass. Radiative $\phi$ decay $\phi \rightarrow \mu \mu \gamma$ survives this cut since the photon usually remains undetected. These radiative decays and any other heavy meson decays may be suppressed using secondary vertex properties and isolation criteria and by exploring the three-muon angular distributions. These studies are in progress.

Predictions of the achievable sensitivity are available in an older CMS Note [973]. In case no signal is observed the expected upper limit on the $\tau \rightarrow 3 \mu$ branching ratio at $95 \%$ C.L. for the $W$ source is $7.0 \times 10^{-8}\left(3.8 \times 10^{-8}\right)$ for $10 \mathrm{fb}^{-1}$ $\left(30 \mathrm{fb}^{-1}\right)$ of collected data. For the $Z$ source a limit of $3.4 \times$ $10^{-7}$ and for the $B$ meson source a limit of $2.1 \times 10^{-7}$ was derived assuming an integrated luminosity of $30 \mathrm{fb}^{-1}$. The $D_{s}$ source was not studied in this early paper.

Potentially including the muons from $D$ and $B$ meson decays may lead to significant improvements of the sensitivity. Further studies are necessary to make firm predictions.
$8.3 B_{d, s}^{0} \rightarrow e^{ \pm} \mu^{\mp}$

The present limits $B\left(B_{d}^{0} \rightarrow e \mu\right)<1.7 \times 10^{-7}$ [975] determined by Belle and $B\left(B_{s}^{0} \rightarrow e \mu\right)<6.1 \times 10^{-6}$ [976] from $\mathrm{CDF}$ are of interest since they place bounds on the masses of two Pati-Salam leptoquarks [361] (see below). Both measurements are almost background free so significant improvements should be expected from these experiments. These decay modes have similarities with the $K_{L}^{0} \rightarrow \mu e$ decay for which an upper limit of $4.7 \times 10^{-12}$ exists [977].

The prospects of a more sensitive search have been studied for the LHCb experiment [978]. Although background levels are higher, this is more than compensated by the improved single-event sensitivity. The event selection closely follows that of the $B_{s}^{0} \rightarrow \mu^{+} \mu^{-}$decay. The dominant backgrounds come from (i) events in which two $b$ hadrons decay into leptons combining to a fake vertex and (ii) from two-body charmless hadronic decays when the two hadrons are misidentified as leptons. Signal and background are separated on the basis of particle identification, invariant mass $\left(\sigma\left(m_{B}\right)=50 \mathrm{MeV} / c^{2}\right)$, transverse momenta, proper distance and the isolation of the $B^{0}$ candidate from the other decay products. See Ref. [978] for details. Simulation shows that for an integrated luminosity of $2 \mathrm{fb}^{-1}$ the total background can be reduced to $\approx 80$ events with a selection efficiency of $1.4 \%$. Assuming no signal would be found the $90 \%$ C.L. upper limits would be $1.6 \times 10^{-8}$ and $6.5 \times 10^{-8}$ for $B\left(B_{d}^{0} \rightarrow e^{ \pm} \mu^{\mp}\right)$ and $B\left(B_{s}^{0} \rightarrow e^{ \pm} \mu^{\mp}\right)$, respectively. These values correspond to $90 \%$ C.L. lower limits on the leptoquark mass and mixings of $90 \times F_{\text {mix }}^{d} \mathrm{TeV}$ and $65 \times F_{\text {mix }}^{s} \mathrm{TeV}$, where $F_{\text {mix }}^{d, s}$ are factors taking into account generation mixing within the model. Present limits are 50 and $21 \mathrm{TeV}$, respectively (see Fig. 65). 
Fig. $6590 \%$ C.L. limits on $B\left(B_{d}^{0} \rightarrow e \mu\right)$ (left panel) and $B\left(B_{s}^{0} \rightarrow e \mu\right)($ right panel $)$ and the corresponding lower limits on the products of Pati-Salam leptoquark mass and mixing. Present results are compared with results projected for $\mathrm{LHCb}$ for an integrated luminosity of $2 \mathrm{fb}^{-1}$ in case no signal would be observed. Dashed regions indicate the theoretical uncertainties in the relation between the variables
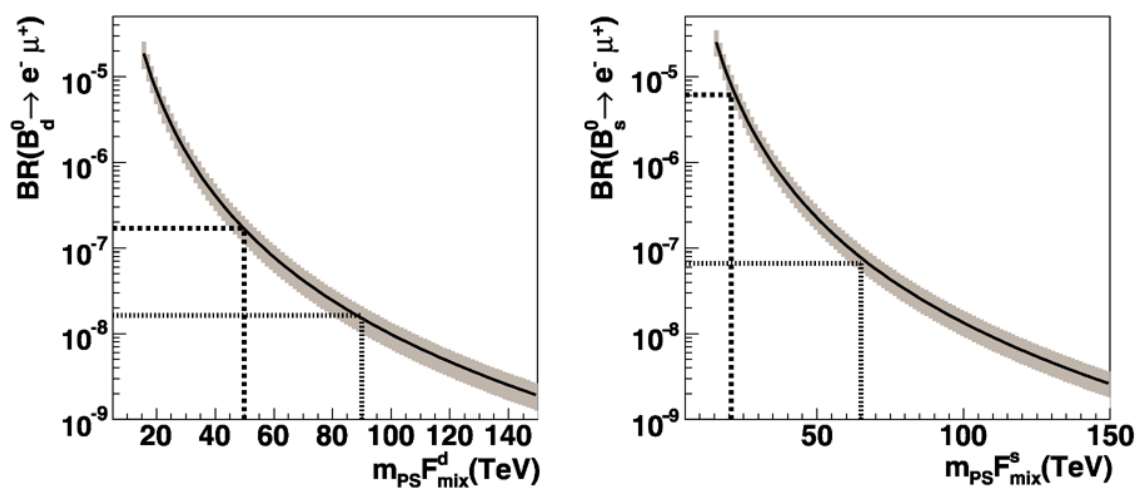

\subsection{In flight conversions}

Lepton Flavor Violation could manifest itself in the conversion of high energy muons into tau leptons when scattering on nucleons in a fixed target configuration [979]. Muons can be produced much more copiously than tau leptons so $\mu \rightarrow \tau$ conversions could be more sensitive than neutrinoless $\tau \rightarrow \mu X$ decays. When considering the effective lepton flavor violating four-fermion couplings, tau decays mainly involve light quarks, so heavy quark couplings are only loosely constrained [980]. In SUSY models, muon to tau conversion could be greatly enhanced by Higgs mediation at energies where heavy quarks contribute [812].

Within the context of this workshop the experimental feasibility of such experiment has been investigated. The cross section for mu to tau lepton conversion on target has been estimated to be at most 550 ab [980] for $50 \mathrm{GeV}$ muons, using an effective model independent interpretation of the tau decay LFV constraints [964] based on the 2000 data [981]. By rescaling the upper limit on $B\left(\tau \rightarrow \mu \pi^{+} \pi^{-}\right)$to the current value [982, 983], one obtains an upper limit at 90\% C.L. on the cross section of $4.7 \mathrm{ab}$. This value scales roughly linearly with the muon energy. In the context of the MSSM, the experimental data available in 2004 constrained the cross section in the range from $0.1 \mathrm{ab}$ to $1 \mathrm{ab}$ for muon energies from 100 to $300 \mathrm{GeV}$ [812].

The following assumptions were made to assess the experimental feasibility:

- the goal is a sensitivity to the cross section corresponding to $1 / 10$ of the present limits from tau decay, collecting at least thousand events per year;

- the active target consists of 330 planes of $300 \mu \mathrm{m}$ thick silicon, with either strip or pixel readout;

- the target has transverse dimensions corresponding to an area of $1 \mathrm{~m}^{2}$ and the beam is distributed homogeneously over the target.

As a consequence, $3.75 \times 10^{19}$ muons/yr are needed which, assuming a $10 \%$ duty cycle and an effective data taking year of $10^{7} \mathrm{~s}$, corresponds to $3.75 \times 10^{13}$ muons/s (peak) and $3.75 \times 10^{12}$ muons/s (average).
Using the LEPTO 6.5.1 generator [984] deep inelastic muon scattering off nucleons was studied. The amount of power dissipated in the target is sustainable, and the interaction rate is 0.6 interactions per $25 \mathrm{~ns}$, which is comparable to LHC experiments. Radiation levels and occupancy in the silicon active target appear to be tractable, provided pixel readout is used.

When requiring momentum transfer above $2 \mathrm{GeV}$ and invariant mass of the hadronic final state above $3 \mathrm{GeV}$ an effective interaction cross section of $47 \mathrm{nb}$ was found. This value reduces to $15 \mathrm{nb}$ when applying the level 0 trigger requirement of at least $60 \mathrm{GeV}$ of hadronic energy which results in a rate of $7.7 \mathrm{MHz}$. The amount of data that needs to be extracted from the tracker for further event selection can probably be handled at such rate.

Unfortunately it appears that the required muon flux is incompatible with the operation of calorimeters as triggering and detecting devices. Assuming an LHCb-like electromagnetic calorimeter with a $2.6 \mathrm{~cm}$ thick lead absorber and an integration time of $25 \mathrm{~ns}$, and assuming that electrons from muon decay travel unscreened for $4 \mathrm{~m}$ before encountering the electro-magnetic calorimeter, three high energy electrons per $25 \mathrm{~ns}$ integration time reach the calorimeter, preventing any effective way of triggering on electrons. Assuming an LHCb-like hadronic calorimeter structured in towers consisting of 75 layers including $13 \times 13 \mathrm{~cm}^{2}$ scintillating pads and $16 \mathrm{~mm}$ of iron each, each tower will detect $25 \mathrm{TeV}$ of equivalent hadronic energy for each $25 \mathrm{~ns}$ of integration time just because of the muon flux energy loss. The Poisson fluctuation of the number of muons will induce a fluctuation in the detected hadronic energy per tower of about $200 \mathrm{GeV}$, preventing the use of the hadronic calorimeter as a trigger for $\mu N \rightarrow \tau X$.

In conclusion, the idea of using an intense but transversely spread muon flux to produce and detect LFV muon conversions to tau leptons does not appear feasible in this preliminary study, mainly because it does not appear possible to operate calorimeters at these rates. 


\section{Experimental studies of electric and magnetic dipole moments}

\subsection{Electric dipole moments}

We review here the current status and prospects of the searches for fundamental EDMs, a flavor diagonal signal of $\mathrm{CP}$ violation. At the non-relativistic level, the EDM $d$ determines a coupling of the spin to an external electric field, $\mathcal{H} \sim d \vec{E} \cdot \vec{S}$. Searches for intrinsic EDMs have a long history, stretching back to the prescient work of Purcell and Ramsey who used the neutron EDM as a test of parity in nuclear physics. At the present time, there are two primary motivations for anticipating a nonzero EDM at or near current sensitivity levels. Firstly, a viable mechanism for baryogenesis requires a new $\mathrm{CP}$-odd source, which if tied to the electroweak scale necessarily has important implications for EDMs. The second is that CP-odd phases appear quite generically in models of new physics introduced for other reasons, e.g. in supersymmetric models. Indeed, it is only the limited field content of the SM which limits the appearance of $\mathrm{CP}$-violation to the CKM phase and $\theta_{\mathrm{QCD}}$. The lack of any observation of a nonzero EDM has, on the flipside, provided an impressive source of constraints on new physics, and there is now a lengthy body of literature on the constraints imposed, for example, on the soft breaking sector of the MSSM. Generically, the EDMs ensure that new CPodd phases in this sector are at most of $\mathcal{O}\left(10^{-1}-10^{-2}\right)$, a tuning that appears rather unwarranted given the $\mathcal{O}(1)$ value of the CKM phase.

The strongest current EDM constraints are shown for three characteristic classes of observables in Table 25, and will be discussed in detail in the following.

We summarize first the details of the EDM constraints, and the induced bounds on a generic class of $\mathrm{CP}$-odd operators normalized at $1 \mathrm{GeV}$, commenting on how the next generation of experiments will impact significantly on the level of sensitivity in all sectors. We then turn to a brief discussion of some of the constraints on new physics that ensue from these bounds. More detailed discussions of phenomenology of EDMs is given in the first half of this report (see e.g. Sect. 5.7).

\subsubsection{CP-odd operators and electric dipole moments}

We shall briefly review the relevant formulae for the observable EDMs in terms of CP-odd operators normalized at
$1 \mathrm{GeV}$. Including the most significant flavor diagonal $\mathrm{CP}$ odd operators (see e.g. [742]) up to dimension six, the corresponding effective Lagrangian takes the form,

$$
\begin{aligned}
\mathcal{L}_{\mathrm{eff}}^{1 \mathrm{GeV}}= & \frac{g_{s}^{2}}{32 \pi^{2}} \bar{\theta} G_{\mu \nu}^{a} \tilde{G}^{\mu \nu, a}-\frac{i}{2} \sum_{i=e, u, d, s} d_{i} \bar{\psi}_{i}(F \sigma) \gamma_{5} \psi_{i} \\
& -\frac{i}{2} \sum_{i=u, d, s} \tilde{d}_{i} \bar{\psi}_{i} g_{s}(G \sigma) \gamma_{5} \psi_{i} \\
& +\frac{1}{3} w f^{a b c} G_{\mu \nu}^{a} \tilde{G}^{\nu \beta, b} G_{\beta}^{\mu, c} \\
& +\sum_{i, j=e, q} C_{i j}\left(\bar{\psi}_{i} \psi_{i}\right)\left(\psi_{j} i \gamma_{5} \psi_{j}\right)+\cdots
\end{aligned}
$$

The $\theta$-term, as it has a dimensionless coefficient, is particularly dangerous leading to the strong $\mathrm{CP}$ problem and in what follows we shall invoke the axion mechanism [986] to remove this term.

The physical observables can be conveniently separated into three main categories, depending on the physical mechanisms via which an EDM can be generated: EDMs of paramagnetic atoms and molecules; EDMs of diamagnetic atoms; and the neutron EDM. The inheritance pattern for these three classes is represented schematically in Fig. 66 and, while the experimental constraints on the three classes of EDMs differ by several orders of magnitude, it is important that the actual sensitivity to the operators in (9.1) turns out to be quite comparable in all cases. This is due to various enhancements or suppression factors which are relevant in each case, primarily associated with various violations of "Schiff shielding" - the non-relativistic statement that an electric field applied to a neutral atom must necessarily be screened and thus remove any sensitivity to the EDM.

\subsubsection{EDMs of paramagnetic atoms}

For paramagnetic atoms, Schiff shielding is violated by relativistic effects which can in fact be very large. One has roughly [987],

$d_{\mathrm{para}}\left(d_{e}\right) \sim 10 \alpha^{2} Z^{3} d_{e}$,

which for large atoms such as Thallium amounts to a huge enhancement of the field seen by the electron EDM (see e.g.
Table 25 Current constraints within three representative classes of EDMs

\begin{tabular}{llll}
\hline Class & EDM & Current bound & Ref. \\
\hline Paramagnetic & ${ }^{205} \mathrm{Tl}$ & $\left|d_{\mathrm{Tl}}\right|<9 \times 10^{-25} e \mathrm{~cm}$ & [186] \\
Diamagnetic & ${ }^{199} \mathrm{Hg}$ & $\left|d_{\mathrm{Hg}}\right|<2 \times 10^{-28} e \mathrm{~cm}$ & {$[985]$} \\
Nucleon & $n$ & $\left|d_{n}\right|<3 \times 10^{-26} e \mathrm{~cm}$ & {$[869]$} \\
\hline
\end{tabular}




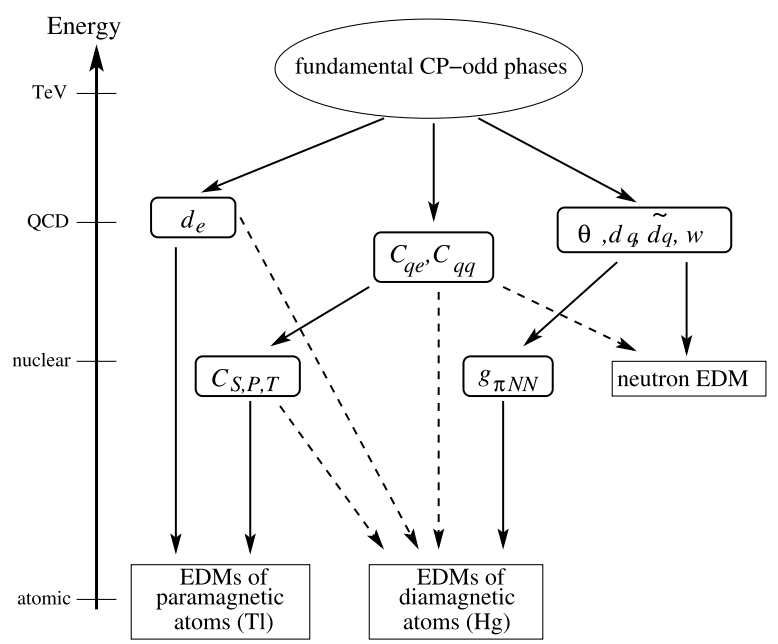

Fig. 66 A schematic plot of the hierarchy of scales between the leptonic and hadronic $\mathrm{CP}$-odd sources and three generic classes of observable EDMs. The dashed lines indicate generically weaker depen-

[987, 988]), which counteracts the apparently lower sensitivity of the Tl EDM bound,

$d_{\mathrm{Tl}}=-585 d_{e}-43 \mathrm{GeV} \times e C_{S}^{\text {singlet }}$.

We have also included here the most relevant $\mathrm{CP}$-odd electron-nucleon interaction, namely $C_{S} \bar{e} i \gamma_{5} e \bar{N} N$, which in turn is related to the semileptonic four-fermion operators in (9.1).

\subsubsection{EDMs of diamagnetic atoms}

For diamagnetic atoms, Schiff shielding is instead violated by the finite size of the nucleus and differences in the distribution of the charge and the EDM. However, this is a rather subtle effect,

$d_{\mathrm{dia}} \sim 10 Z^{2}\left(R_{N} / R_{A}\right)^{2} \tilde{d}_{q}$,

and the suppression by the ratio of nuclear to atomic radii, $R_{N} / R_{A}$, generally leads to a suppression of the sensitivity to the nuclear EDM, parameterized to leading order by the Schiff moment $S$, by a factor of $10^{3}$ (see e.g. [987, 988]). Thus, although the apparent sensitivity to the Hg EDM is orders of magnitude stronger than for the Tl EDM, both experiments currently have comparable sensitivity to various CP-odd operators and thus play a very complementary role. Combining the atomic $d_{\mathrm{Hg}}(S)$, nuclear $S\left(\bar{g}_{\pi N N}\right)$, and $\mathrm{QCD} \bar{g}_{\pi N N}^{(1)}\left(\tilde{d}_{q}\right)$, components of the calculation [742, 988], we have

$d_{\mathrm{Hg}}=7 \times 10^{-3} e\left(\tilde{d}_{u}-\tilde{d}_{d}\right)+10^{-2} d_{e}+\mathcal{O}\left(C_{S}, C_{q q}\right)$,

where the overall uncertainty is rather large, a factor of 2-3, due to significant cancellations between various contribu-

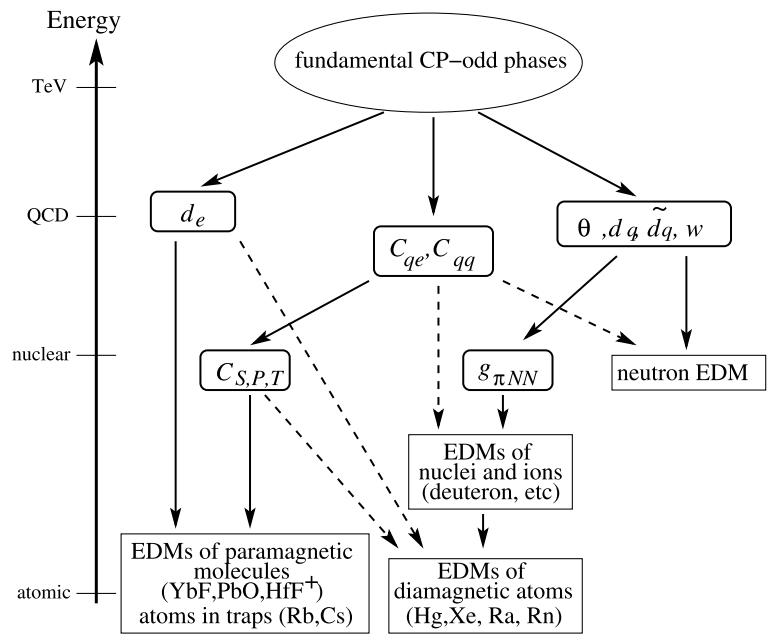

dencies in SUSY models. The current situation is given on the left, while on the right we show the dependencies of several classes of nextgeneration experiments

tions. A valuable feature of $d_{\mathrm{Hg}}$ is its sensitivity to the triplet combination of color EDM operators $\tilde{d}_{q}$.

\subsubsection{Neutron EDM}

The neutron EDM measurement is of course not sensitive to the above atomic enhancement/suppression factors and, using the results obtained using QCD sum rule techniques [742] (see also [849, 989, 990] for alternative chiral approaches), wherein under Peccei-Quinn relaxation of the axion the contribution of sea-quarks is also suppressed at leading order:

$$
\begin{aligned}
d_{n}= & (1.4 \pm 0.6)\left(d_{d}-0.25 d_{u}\right) \\
& +(1.1 \pm 0.5) e\left(\tilde{d}_{d}+0.5 \tilde{d}_{u}\right) \\
& +20 \mathrm{MeV} \times e w+\mathcal{O}\left(C_{q q}\right) .
\end{aligned}
$$

Note that the proportionality to $d_{q}\langle\bar{q} q\rangle \sim m_{q}\langle\bar{q} q\rangle \sim f_{\pi}^{2} m_{\pi}^{2}$ removes any sensitivity to the poorly known absolute value of the light quark masses.

\subsubsection{Future developments}

The experimental situation is currently very active, and a number of new EDM experiments, as detailed in this report, promise to improve the level of sensitivity in all three classes by one-to-two orders of magnitude in the coming years. These include: new searches for EDMs of polarizable paramagnetic molecules, which aim to exploit additional polarization effects enhancing the effective field seen by the unpaired electron by a remarkable factor of up to $10^{5}$, and are therefore primarily sensitive to the electron EDM; new searches for the EDM of the neutron in cryogenic systems; 
and also proposed searches for EDMs of charged nuclei and ions using storage rings. This latter technique clearly aims to avoid the effect of Schiff shielding and enhance sensitivity to the nuclear EDM and its hadronic constituents. A schematic summary of how a number of these new experiments will be sensitive to the set of CP-odd operators is exhibited in Fig. 66.

\subsubsection{Constraints on new physics}

Taking the existing bounds, and the formulae above, we obtain the following set of constraints on the CP-odd sources at $1 \mathrm{GeV}$ (assuming an axion removes the dependence on $\bar{\theta}$ ),

$$
\begin{aligned}
& \left|d_{e}+e(26 \mathrm{MeV})^{2}\left(3 \frac{C_{e d}}{m_{d}}+11 \frac{C_{e s}}{m_{s}}+5 \frac{C_{e b}}{m_{b}}\right)\right| \\
& <1.6 \times 10^{-27} e \mathrm{~cm} \text { from } d_{\mathrm{Tl}} \text {, } \\
& \left|\left(\tilde{d}_{d}-\tilde{d}_{u}\right)+\mathcal{O}\left(\tilde{d}_{s}, d_{e}, C_{q q}, C_{q e}\right)\right|<2 \times 10^{-26} e \mathrm{~cm} \\
& \text { from } d_{\mathrm{Hg}} \text {, } \\
& \left|e\left(\tilde{d}_{d}+0.56 \tilde{d}_{u}\right)+1.3\left(d_{d}-0.25 d_{u}\right)+\mathcal{O}\left(\tilde{d}_{s}, w, C_{q q}\right)\right| \\
& <2 \times 10^{-26} e \mathrm{~cm} \text { from } d_{n},
\end{aligned}
$$

where the additional $\mathcal{O}(\cdots)$ dependencies are known less precisely, but may not always be sub-leading in particular models. The precision of these results varies from $10-15 \%$ for the $\mathrm{Tl}$ bound, to around $50 \%$ for the neutron bound, and to a factor of a few for Hg. It is remarkable to note that, accounting for the naive mass-dependence $d_{f} \propto m_{f}$, all these constraints are of essentially the same order of magnitude and thus highly complementary. Constraints obtained in the hadronic sector using other calculational techniques differ somewhat but generally give results consistent with these within the quoted precision.

The application of these constraints to models of new physics has many facets and is discussed in several specific cases elsewhere in this report. We shall limit our attention here to just a few simple examples relevant to the motivations noted above.

\subsubsection{The SUSY CP-problem}

It is now rather well-known that a generic spectrum of soft SUSY-breaking parameters in the MSSM will generate EDMs via one loop diagrams [852] that violate the existing bounds by one-to-two orders of magnitude leading to the SUSY CP problem. The situation is summarized rather schematically in Fig. 67.

In many respects the situation is better described by the amount of fine tuning of the MSSM spectrum that is required to avoid these leading order contributions, and by how much the ability to avoid the EDM constraints is limited by secondary constraints from numerous, and more robust, two

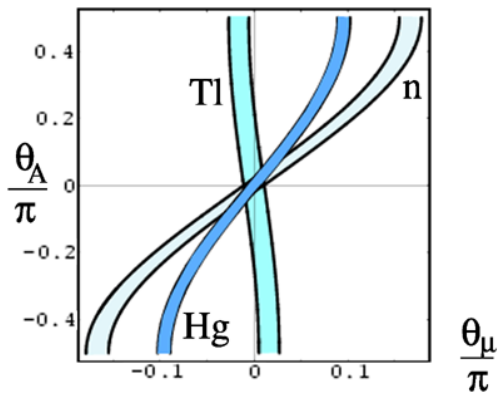

Fig. 67 Constraints on the CMSSM phases $\theta_{A}$ and $\theta_{\mu}$ from a combination of the three most sensitive EDM constraints, $d_{n}, d_{\mathrm{Tl}}$ and $d_{\mathrm{Hg}}$, for $M_{\mathrm{SUSY}}=500 \mathrm{GeV}$, and $\tan \beta=3$ (from [742]). The region allowed by EDM constraints is at the intersection of all three bands around $\theta_{A}=\theta_{\mu}=0$

loop contributions [991] and four-fermion sources [992]. Indeed, if we consider two extreme cases: (i) the 2HDM, where all SUSY fermions and sfermions are very heavy; and (ii) split SUSY, where all SUSY scalars are very heavy, one finds that while one loop EDMs are suppressed, two loop contributions are already very close to the current bounds [857, 858, 992]. This bodes well for the ability of nextgeneration experiments to provide a comprehensive test of large SUSY phases at the electroweak scale, regardless of the detailed form of the SUSY spectrum.

\subsubsection{Constraints on new SUSY thresholds}

If SUSY is indeed discovered at the LHC, but with no sign of phases in the soft sector, one may instead consider the ability of EDMs to detect new supersymmetric CP-odd thresholds. At dimension five there are several R-parity conserving operators, besides those well-known examples associated with neutrino masses and baryon and lepton number violation [150, 371]. Writing the relevant dimension five superpotential as [993, 994]

$$
\begin{aligned}
\Delta \mathcal{W}= & \frac{y_{h}}{\Lambda_{h}} H_{d} H_{u} H_{d} H_{u}+\frac{Y_{i j k l}^{q e}}{\Lambda_{q e}}\left(U_{i} Q_{j}\right) E_{k} L_{l} \\
& +\frac{Y_{i j k l}^{q q}}{\Lambda_{q q}}\left(U_{i} Q_{j}\right)\left(D_{k} Q_{l}\right) \\
& +\frac{\tilde{Y}_{i j k l}^{q q}}{\Lambda_{q q}}\left(U_{i} t^{A} Q_{j}\right)\left(D_{k} t^{A} Q_{l}\right)
\end{aligned}
$$

one finds that order-one CP-odd coefficients with a generic flavor structure, particularly for the semileptonic operators, are probed by the sensitivity of $d_{\mathrm{Tl}}$ and $d_{\mathrm{Hg}}$ at the remarkable level of $\Lambda \sim 10^{8} \mathrm{GeV}$ [993, 994]. This is comparable to, or better than, the corresponding sensitivity of lepton-flavor violating observables. 


\subsubsection{Constraints on minimal electroweak baryogenesis}

As noted above, one of the primary motivations for anticipating nonzero EDMs at or near the current level of sensitivity is through the need for a viable mechanism of baryogenesis. This is clear in essentially all baryogenesis mechanisms that are tied to the electroweak scale. As a simple illustration, one can consider a minimal extension of the SM Higgs sector [995-997],

$$
\begin{aligned}
\mathcal{L}_{\operatorname{dim} 6}= & \frac{1}{\Lambda^{2}}\left(H^{\dagger} H\right)^{3}+\frac{Z_{i j}^{u}}{\Lambda_{\mathrm{CP}}^{2}}\left(H^{\dagger} H\right) U_{i}^{c} H Q_{j} \\
& +\frac{Z_{i j}^{d}}{\Lambda_{\mathrm{CP}}^{2}}\left(H^{\dagger} H\right) D_{i}^{c} H^{\dagger} Q_{j} \\
& +\frac{Z_{i j}^{e}}{\Lambda_{\mathrm{CP}}^{2}}\left(H^{\dagger} H\right) E_{i}^{c} H^{\dagger} L_{j} .
\end{aligned}
$$

The first term is required to induce a sufficiently strong first order electroweak phase transition, while the remaining operators provide the additional source (or sources) of $\mathrm{CP}$ violation, where we have assumed consistency with the principle of minimal flavor violation. Modified Higgs couplings of this kind, including CP-violating effects, are currently the subject of significant research within collider physics, relevant to the LHC in particular [588], making EDM probes of models of this kind quite complementary.

As discussed in [997], such a scenario can reproduce the required baryon-to-entropy ratio, $\eta_{b}=8.9 \times 10^{-11}$, while remaining consistent with the EDM bounds, provided the thresholds and the Higgs mass are quite low, e.g. $400 \mathrm{GeV}<\Lambda, \Lambda_{\mathrm{CP}}<800 \mathrm{GeV}$. The EDMs in this case are generated at the two loop level, and it is clear that an improvement in EDM sensitivity by an order of magnitude would provide a conclusive test of minimal mechanisms of this form.

\subsection{Neutron EDM}

The neutron electric dipole moment is sensitive to many sources of CP violation. Most famously, it constrains QCD (the "strong CP problem"), but it also puts tight constraints on supersymmetry and other physics models beyond the standard model. The standard model prediction of $\sim 10^{-32} e \mathrm{~cm}$ is a factor of $10^{6}$ below existing limits, so any convincing signal within current or foreseen sensitivity ranges will be a clear indication of physics beyond the SM.

All current nEDM experiments use NMR techniques to search for electric-field induced changes in the Larmor precession frequency of bottled ultracold neutrons. Recent results from a room-temperature apparatus at ILL yielded a new limit of $\left|d_{n}\right|<2.9 \times 10^{-26} e \mathrm{~cm}(90 \%$ C.L.) which rules out many "natural" varieties of SUSY. Several new experiments hope to improve on this limit: two of these involve new cryogenic techniques that promise an eventual increase in sensitivity by two orders of magnitude. First results, at the level of $\sim 10^{-27} e \mathrm{~cm}$, are to be expected within about four years.

\subsubsection{ILL}

A measurement of the neutron EDM was carried out at the ILL between 1996 and 2002, by a collaboration from the University of Sussex, the Rutherford Appleton Laboratory, and the ILL itself. The final published result provided a limit of $\left|d_{n}\right|<2.9 \times 10^{-26} e \mathrm{~cm}(90 \%$ C.L.) [869]. This represents a factor of two improvement beyond the intermediate result [998] and almost a factor of four beyond the results existing prior to this experiment [999, 1000]. The collaboration, which has now expanded to include Oxford University and the University of Kure, has designed and developed "CryoEDM", a cryogenic version of the experiment that is expected to achieve two orders of magnitude improvement in sensitivity. Construction and initial testing are underway at the time of writing.

Experimental technique The room-temperature measurement was carried out using stored ultracold neutrons (i.e. having energies $\lesssim 200 \mathrm{neV}$ ) from the ILL reactor. The Ramsey technique of separated oscillatory fields was used to determine the Larmor precession frequency of the neutrons within $\vec{B}$ and $\vec{E}$ fields. The signature of an EDM is a frequency shift proportional to any change in the applied electric field.

The innovative feature of this experiment was the use of a cohabiting atomic-mercury magnetometer [1001]. Spinpolarized $\mathrm{Hg}$ atoms shared the same volume as the neutrons, and the measurement of their precession frequency provided a continuous high-resolution monitoring of the magnetic field drift: prior to this, such drift entirely dominated the tiny $\vec{E}$-field induced frequency changes that were sought.

Systematics Analysis of the data revealed a new source of systematic error, which, as the problem of $B$-field drift had been virtually eliminated, became potentially the dominant error. Its origins lay in a geometric-phase (GP) effect [1002] — an unfortunate collusion between any small applied axial $\vec{B}$-field gradient and the component of $\vec{B}$ induced in the particle's rest frame by the Lorentz transformation of the electric field. This GP effect induced a frequency shift proportional to $\vec{E}$, and hence a false EDM signal. In fact, the $\mathrm{Hg}$ magnetometer itself was some 50 times more susceptible to this effect than were the neutrons, so the introduction of the magnetometer brought the GP systematic with it. 
This effect was overcome by careful measurement of the neutron-to- $\mathrm{Hg}$ frequency ratios for both polarities of magnetic field, in order to determine the point nominally corresponding to zero applied axial $B$-field gradient, as well as by a series of auxiliary measurements to pin down small corrections due to local dipole [1003] and quadrupole fields (as well as the Earth's rotation). The final result therefore remained statistically limited.

The experiment is now complete and, as will be discussed below, the equipment will be used for further studies by another collaboration based largely at the PSI.

Still another collaboration, led by the PNPI in Russia, is developing a new room-temperature nEDM apparatus, which they plan to run at ILL. It is also intended to reach a sensitivity of $\sim 10^{-27} \mathrm{ecm}$, to be achieved in part by the use of multiple back-to-back measurement chambers with opposing electric fields to cancel some systematic errors.

Cryogenic experiments overview It has been known for several decades [1004] that $8.9 \AA$ neutrons incident on superfluid ${ }^{4} \mathrm{He}$ at $0.5 \mathrm{~K}$ will down-scatter, transferring their energy and momentum to the helium and becoming ultracold neutrons (UCN) in the process. This so-called super-thermal UCN source provides a much higher flux than is available simply from the low energy tail of the Maxwell distribution. In addition, the immersion of the apparatus in a bath of liquid helium should allow for the provision of stronger electric fields than could be sustained in vacuo. The other two variables that contribute to the figure of merit for this experiment, namely the polarization and the NMR coherence time, should also be improved: the incident cold neutron beam can be very highly polarized, and the polarization remains intact during the down-scattering process; and the improved uniformity of magnetic field attainable with superconducting shields and coil will reduce depolarization during storage, while losses from up-scattering will be much reduced due to the cryogenic temperatures of the walls of the neutron storage vessels.

ILL CryoEDM experiment status The majority of the apparatus for the cryoEDM experiment has been installed at ILL, and testing is underway. UCN production via this superthermal mechanism has been demonstrated [1005], and the solid-state UCN detectors developed by the collaboration have also been shown to work well [1006]. At the time of writing, there are still some hardware problems to be resolved, in particular with components in and around the Ramsey measurement chamber. A high precision scan of the magnetic field was carried out in 2007, and measurements were made of the neutron polarization. An initial HV system will be installed in spring 2008. By the end of 2008, the system is expected to have a statistical sensitivity of $\sim 10^{-27} e \mathrm{~cm}$.
Future plans In order to achieve optimum sensitivity, a number of improvements will need to be made:

- The superconducting magnetic shielding requires additional protective "end caps" to shield fully the ends of the superconducting solenoid.

- The current measurement chamber only has two cells: one with HV applied, and one at ground as a control. It is planned to upgrade to a four-cell chamber, with the HV applied to the central electrode, in order to be able to carry out simultaneous measurements with electric fields in opposite directions. As well as canceling several potential systematic errors, this will reduce the statistical uncertainty by doubling the number of neutrons counted.

- The ILL is preparing a new beam line with six times the currently available intensity of $8.9 \AA$ A neutrons, and wishes to transfer the experiment to that beam line in 2009. Funding for these improvements is expected to be contingent on successful running of the existing apparatus.

A sensitivity of $\sim 2 \times 10^{-28} e \mathrm{~cm}$ should be achievable within two to three years of running at the new beam line.

\subsubsection{PSI}

The present best limit for the neutron electric dipole moment (EDM), $\left|d_{n}\right|<2.9 \times 10^{-26} e \mathrm{~cm}$ [869], was obtained by the Sussex/Rutherford/ILL Collaboration from measurements at the ILL source for ultracold neutrons [1007], as discussed in the previous section. The experiment is at this point statistically limited and also facing systematic challenges not far away $[869,1002,1003]$. In order to make further progress, both, statistical sensitivity and control of systematics, have to be improved. Gaining in statistics requires new sources for ultracold neutrons (UCN). These can be integrated into the experiment as for the new cryogenic EDM searches, delivering UCN in superfluid helium, or a multipurpose UCN source, delivering UCN in vacuum. This high intensity UCN source is presently under construction at the Paul Scherrer Institut in Villigen, Switzerland [1008]. It is expected to become operational towards the end of 2008 and to deliver UCN densities of more than $1000 \mathrm{~cm}^{-3}$ to typical experiments, i.e. almost two orders of magnitude more than presently available.

The in-vacuum technique will be pushed to its limits, delivering first results in about 4 years. The following steps are planned by a sizable international collaboration [1009]:

- While the new UCN source is under construction the collaboration operates and improves the apparatus of the former Sussex/RAL/ILL Collaboration at ILL Grenoble. In order to better control the systematic issues, the magnetic field and its gradients will be monitored and stabilized using an array of laser optically pumped Cs-magnetometers 
[1010, 1011]. An order of magnitude improvement compared to todays field fluctuations over the typical measurement times of $100-1000 \mathrm{~s}$ is certainly feasible. It is also necessary to improve the sensitivity of the $\mathrm{Hg}$ comagnetometer [1012]. Other improvements of the system are with regard to UCN polarization and detection as well as upgrading the data acquisition system. The hardware efforts are accompanied by a full simulation of the system.

- It is planned to move the apparatus from ILL to PSI towards the end of 2008 in order to be ready for data taking for about two years, 2009 and 2010. In addition to the improvements of phase I, an external magnetic field stabilization system and a temperature stabilization are envisaged. Furthermore, work on developing a second comagnetometer using a hyper-polarized noble gas species is ongoing and might further improve the systematics control. In case of a successful development, also the replacement of the Hg system together with an increase of the electric field strength may become possible. In any case, a factor of 5 gain in sensitivity is expected from the higher UCN intensity, corresponding to a limit of about $5-6 \times 10^{-27} e \mathrm{~cm}$ in case the EDM is not found. In parallel to the described activities, the design of a new experimental apparatus will start in 2007. After a major design effort in 2008, set-up of the new apparatus will start in 2009.

- The new experiment will be an optimized version of the room-temperature in-vacuum approach. Another order of magnitude gain in sensitivity will be obtained by a considerable increase of the statistics due to a larger experimental volume $(\times \sqrt{5})$, a better adaption to the UCN source $(\times \sqrt{2})$, longer running time $(x \sqrt{3})$ and by an improvement of the electric field strength $(\times 2)$. Completion of the new experimental apparatus is anticipated for end of 2010, and data taking planned for 2011-2014.

The features of the experiment include

- continued use of the successful Ramsey-technique with $\mathrm{UCN}$ in vacuum and the apparatus at room-temperature,

- increased sensitivity due to much larger UCN statistics at the new PSI source, larger experimental volume, better polarization product and possibly larger electric field strength,

- application of a double neutron chamber system,

- improved magnetic field control and stabilization with multiple laser optically pumped Cs-magnetometers, and

- an improved co-magnetometry system.

As another very strong source for UCN is currently under construction at the FRMII in Munich, in the long run and for the optimum conditions for the experiment, the collaboration will have the opportunity to choose between PSI and FRMII.

\subsubsection{SNS}

A sizable US Collaboration [1013] is planning to develop a cryogenic experiment, following an early concept by Golub and Lamoreaux [1014]. It will be based at the SNS 1.4 MW spallation source at Oak Ridge. A fundamental neutron physics beam line is under construction, which will include a double monochromator to select $8.9 \AA$ A neutrons for UCN production in liquid helium.

In this experiment, spin-polarized ${ }^{3} \mathrm{He}$ will be used both as a magnetometer and as a neutron detector. The precession of the ${ }^{3} \mathrm{He}$ can, in principle, be detected with SQUID magnetometers. Meanwhile, the cross section for the absorption reaction $n+{ }^{3} \mathrm{He} \rightarrow p+{ }^{3} \mathrm{H}+764 \mathrm{keV}$ is negligible for a total spin $J=1$, but very large ( $\sim 5 \mathrm{Mb})$ for $J=0$. In consequence, a scintillation signal from this reaction will be detected with a beat frequency corresponding to the difference between the Larmor precession frequencies of the neutrons and the ${ }^{3} \mathrm{He}$.

An application for funding to construct this experiment is currently under review. Extensive tests are underway to study, for example, the electric fields attainable in liquid helium, the ${ }^{3} \mathrm{He}$ spin relaxation time and the diffusion of ${ }^{3} \mathrm{He}$ in ${ }^{4} \mathrm{He}$. If construction goes according to plan, commissioning will be in approximately 2013, with results following probably four or five years later. The ultimate sensitivity will be below $10^{-28} e \mathrm{~cm}$.

\subsection{Deuteron EDM}

A new concept of investigating the EDM of bare nuclei in magnetic storage rings has been developed by the storage ring EDM Collaboration (SREC) over the past several years. The latest version of the methods analyzed turns out to be very sensitive for light (bare) nuclei and promises the best EDM experiment for $\theta_{\mathrm{QCD}}$, quark and quark-color EDMs.

The search for hadronic EDMs has been dominated by the search for a neutron EDM and nuclear Schiff moments in heavy diamagnetic atoms, such as ${ }^{199} \mathrm{Hg}$. The latter depend on nuclear theory to relate the measured Schiff moment to the underlying $\mathrm{CP}$ violating interaction.

The sensitive 'traditional' EDM experiments are, so far, all performed on electrically neutral systems, such as the neutron, atoms, or molecules. A strong electric field is imposed, together with a weak magnetic field, and using NMR techniques, a change of the Larmor precession frequency is looked for. The application of strong electric fields precludes a straightforward use of this technique on charged particles. These particles would accelerate out of the setup, leaving little time to make an accurate measurement.

Attempt to search for an EDM on simple nuclear systems, such as the proton or deuteron, when part of an atom, are severely hindered by shielding. This so-called Schiffscreening precludes an external electric field to penetrate 
to the nucleus. Due to rearrangement of the atomic electrons, the net effect of the electric field on the nucleus is essentially zero. Known loop-holes include relativistic effects, non-electric components in the binding of the electrons, and an extended size of the nucleus. None of these loopholes are sufficiently strong to allow a sensitive measurement on a light atom. For hydrogen atoms, the atomic EDM resulting from a nuclear EDM is down by some seven orders of magnitude.

Nevertheless, light nuclei, and the deuteron in particular, are attractive to search for hadronic EDMs because of their relatively simple structure. Moreover, a novel experimental technique, using the motional electric field experienced by a relativistic particle when traversing a magnetic field, make it possible to directly search for EDM on charged systems, such as the (bare) deuteron.

\subsubsection{Theoretical considerations}

The deuteron is the simplest nucleus. It consists of a weakly bound proton and neutron in a predominantly ${ }^{3} \mathrm{~S}_{1}$ state, with a small admixture of the D-state. From a theoretical point of view, the deuteron is especially attractive, because it is the simplest system in which the P-odd, T-odd nucleonnucleon (NN) interaction contributes to an EDM. Moreover, the deuteron properties are well understood, so reliable and precise calculations are possible.

In [1015], a framework is presented that could serve as a starting point for the microscopic calculation of complex systems. The most general form of the interaction, based only on symmetry considerations, contains ten P-and T-odd meson-nucleon coupling constants for the lightest pseudoscalar and vector mesons $(\pi, \rho, \eta$ and $\omega)$.

This P-odd, T-odd interaction induces a $P$-wave admixture to the deuteron wave function. It is this admixture that leads to an EDM. Since the proton and neutron that make up the deuteron may also have an EDM, a disentanglement of one- and two-body contributions,

$d_{\mathcal{D}} \simeq d_{\mathcal{D}}^{(1)}+d_{\mathcal{D}}^{(2)}$

to the EDM is necessary to uncover the underlying structure of the P-odd T-odd physics.

The two-body component is predominantly due to the polarization effect, and shows little model dependence for all leading high-quality potentials. Additional contributions arrive from meson exchange.

The one body contribution is simply the sum of the proton and neutron EDMs. The nucleon EDM has a wide variety of sources, as already discussed for the neutron. There exists no good model to describe the non-perturbative dynamics of bound quarks. A commonly used method is to evaluate hadronic loop diagrams, containing mesonic and baryonic degrees of freedom. Within the framework presented in [1015], the EDMs for the proton, neutron and deuteron are found (reproducing only the pion dependence),

$d_{p}=-0.05 \bar{g}_{\pi}^{(0)}+0.03 \bar{g}_{\pi}^{(1)}+0.14 \bar{g}_{\pi}^{(2)}+\cdots$,

$d_{n}=+0.14 \bar{g}_{\pi}^{(0)}-0.14 \bar{g}_{\pi}^{(2)}+\cdots$,

$d_{\mathcal{D}}=+0.09 \bar{g}_{\pi}^{(0)}+0.23 \bar{g}_{\pi}^{(1)}+\cdots$.

These dependences clearly show the complementarity of these three particles.

The magnitudes of the coupling constants can be calculated for several viable sources of $\mathrm{CP}$ violation. In the standard model, there is room for $\mathrm{CP}$ violation via the so-called $\bar{\theta}$ parameter. In the case of the nucleons, one has the relation

$d_{n} \simeq-d_{p} \simeq 3 \times 10^{-16} \bar{\theta} e \mathrm{~cm}$,

which yields the severe constraint $\bar{\theta}<1 \times 10^{-10}$. For the deuteron, one finds

$d_{\mathcal{D}} \simeq-10^{-16} \bar{\theta} e \mathrm{~cm}$.

At the level of $d_{\mathcal{D}} \simeq 10^{-29} e \mathrm{~cm}$, one probes $\bar{\theta}$ at the level of $10^{-13}$. Since $\bar{\theta}$ contributes differently to the neutron and the deuteron, it is clear that both experiments are complementary. Indeed. the prediction

$d_{\mathcal{D}} / d_{n}=-1 / 3$

provides a beautiful check as to whether $\bar{\theta}$ is the source of the observed EDMs, should both be measured. In fact, measurement of the EDMs of the proton, deuteron and ${ }^{3} \mathrm{He}$ would allow to verify if they satisfy the relation

$d_{\mathcal{D}}: d_{p}: d_{3 \mathrm{He}} \simeq 1: 3:-3$

Here, it was assumed that ${ }^{3} \mathrm{He}$ has properties very similar to the neutron, which provides most of the spin.

Generic supersymmetric models contain a plethora of new particles, which may be discovered at LHC, and new $\mathrm{CP}$-violating phases. Following the work by Lebedev et al. [1016] and the review by Pospelov and Ritz [742], we find that SUSY loops give rise to ordinary quark EDMs, $d_{q}$, as well as quark-color EDMs, $\tilde{d}_{q}$. For the neutron and deuteron one finds (with the color EDM part divided in isoscalar and isovector parts)

$$
\begin{aligned}
d_{n} \simeq & 1.4\left(d_{d}-0.25 d_{u}\right)+0.83 e\left(\tilde{d}_{d}+\tilde{d}_{u}\right) \\
& +0.27 e\left(\tilde{d}_{d}-\tilde{d}_{u}\right) \\
d_{\mathcal{D}} \simeq & \left(d_{d}+d_{u}\right)-0.2 e\left(\tilde{d}_{d}+\tilde{d}_{u}\right)+6 e\left(\tilde{d}_{d}-\tilde{d}_{u}\right),
\end{aligned}
$$

and similar relations for e.g. the mercury EDM. The isovector part is limited to $\left|e c\left(\tilde{d}_{d}-\tilde{d}_{u}\right)\right|<2 \times 10^{-26} e \mathrm{~cm}$ by the present limit on the ${ }^{199} \mathrm{Hg}$ atom. The experimental bound 
on the neutron suggests that $\left|e\left(\tilde{d}_{d}+\tilde{d}_{u}\right)\right|<4 \times 10^{-26} e \mathrm{~cm}$, assuming the isoscalar contribution to be dominant. Also in this case, the deuteron and neutron show complementarity. This is in particular in their sensitivity to the isovector contribution, which is 20 times larger for the deuteron.

The large sensitivity to new physics (see e.g.[1016]) and the relative simplicity of calculating the nuclear wave function, make it clear that small nuclei hold great discovery potential and should therefore be vigorously pursued.

\subsubsection{Experimental approach}

All sensitive EDM searches are performed on neutral systems, which are (essentially) at rest. The only exception is the proposed use of molecular ions $\left(\mathrm{HfF}^{+}\right.$and $\mathrm{ThF}^{+}$) [1017], but also for this experiment, the motion of the molecules is not crucial.

In the recent past, several novel techniques have been proposed to use the motional electric field sensed by a particle moving through a magnetic field at relativistic velocities. The evolution of the spin orientation for a spin- $1 / 2$ particle in an electromagnetic field $(\vec{E}, \vec{B})$ is described by the socalled Thomas or BMT equation [1018]. The spin precession vector $\vec{\Omega}$, relative to the momentum of the particle, is given by [1019]

$$
\begin{aligned}
\vec{\Omega}= & \frac{e}{m}\left[a \vec{B}+\left(a-\frac{1}{\gamma^{2}-1}\right) \vec{\beta} \times \vec{E}\right. \\
& \left.+\frac{\eta}{2}\left(\vec{E}+\vec{\beta} \times \vec{B}-\frac{\gamma}{\gamma+1} \vec{\beta}(\vec{\beta} \cdot \vec{E})\right)\right]
\end{aligned}
$$

with $\vec{\mu}=2(1+a)(e / m) \vec{S}$ and $\vec{d}=\eta / 2(e / m) \vec{S}$. It was assumed that $\vec{\beta} \cdot \vec{B}=0$. The first two terms between brackets will be referred to as $\omega_{a}$, whereas the last one will be referred to as $\omega_{\eta}$.

For fast particles, the electric field in the rest frame of the particle is dominated by $\vec{\beta} \times \vec{B}$. For commonplace storage rings, this field can exceed the size of a static electric field made in the laboratory by more than an order of magnitude, thus giving the storage ring method a distinct advantage.

In a homogeneous magnetic field, $\vec{\omega}_{a} \propto \vec{B}$ and $\vec{\omega}_{\eta} \propto$ $\vec{\beta} \times \vec{B}$ are orthogonal, leading to a small tilt in the precession plane and an second order increase in the precession frequency. Although this was used to set a limit on the muon EDM [188, 1020], it does not allow for a sensitive search.

The application of a radially oriented electric field $E_{r}$ to slow down $\omega_{a}$ and thus to increase the tilt, was proposed in [1021]. For a field strength

$$
E_{r}=\frac{a \beta}{1-(1+a) \beta^{2}} B_{z}
$$

the spin of an originally longitudinally polarized beam remains aligned with the momentum at all times. In this case $\vec{\beta} \cdot \vec{E}=0$, and thus

$\vec{\Omega}=\frac{e}{m} \frac{\eta}{2}(\vec{E}+\vec{\beta} \times \vec{B})$.

The EDM thus manifests itself as a precession of the spin around the motional electric field $\vec{E}^{*}=\gamma[\vec{E}+\vec{\beta} \times \vec{B}]$, i.e. as a growing vertical polarization component parallel to $\vec{B}$. This approach can be used for all particles with a small magnetic anomaly, so that the necessary electric field strength remains feasible. Concept experiments, employing this technique, have been worked out for the muon [1022-1024] and the deuteron [1025]. Other candidate particles have been identified as well (see e.g. [1026]).

A third, most sensitive approach is reminiscent of the magnetic resonance technique introduced by Rabi [1027]. The spin is allowed to precess under the influence of a dipole magnetic field. In the original application, an oscillating magnetic field oriented perpendicular to the driving field is applied. By scanning the oscillation frequency, a resonance will be observed when the frequency of the oscillating field matches the spin precession frequency.

In this application, the oscillating magnetic field are replaced by an oscillating electric field [1028]. When at resonance, the electric field coherently interacts with the electric dipole moment. As a consequence, the polarization component along the magnetic field oscillates in the case of a sizable EDM. In practice, only the onset of the first oscillation cycle will be visible in the form of a slow growth of the vertical polarization, proportional to the EDM.

The oscillating electric field is obtained by modulating the velocity of the deuterons circulating in a magnetic field, setting up a so-called synchrotron oscillation. For a time dependent velocity $\beta(t)=\beta_{0}+\delta \beta(t)$ generated by an oscillating longitudinal electric field $E_{\mathrm{RF}}(t)$ and a constant magnetic field $B$, the spin evolution follows from

$$
\begin{aligned}
\vec{\Omega}= & \frac{e}{m}\left[\left\{a B+\frac{\eta}{2} \vec{\beta}_{0} \times \vec{B}\right\}\right. \\
& \left.+\frac{\eta}{2}\left\{\delta \vec{\beta}(t) \times \vec{B}-\frac{\beta^{2} \gamma}{\gamma+1} \vec{E}_{\mathrm{RF}}(t)\right\}\right] \\
\equiv & \vec{\Omega}_{0}+\delta \vec{\Omega}(t) .
\end{aligned}
$$

The first term yields spin precession about $\vec{\Omega}_{0}$, without affecting the polarization parallel to it. For $\delta \beta(t)=$ $\delta \beta \cos (\omega t+\psi)$, and $B \delta \beta \gg \beta^{2} \gamma /(\gamma+1) E_{\mathrm{RF}}$, the parallel polarization component is given by

$$
\frac{d P_{\|}}{d t} \simeq \frac{e}{m} P_{\circ} \eta \delta \beta B \cos (\Delta \omega t+\Delta \phi),
$$

with $\Delta \omega \equiv \Omega_{0}-\omega$ and $\Delta \phi \equiv \phi-\psi$. The beam is assumed to have a longitudinal polarization $P_{0}$ at injection time. For $\Delta \omega=0$ the vertical polarization will grow continuously at 
a rate proportional to the EDM. Maximum sensitivity is obtained for $\Delta \phi=0$ or $\pi$, whereas for $\Delta \phi=\pi / 2$ or $3 \pi / 2$ there is no sensitivity to the EDM. The latter will prove useful in controlling systematic errors. At the same time, the radial polarization component is given by

$P_{\perp} \simeq P_{0} \sin \left(\Omega_{0} t+\phi\right)$.

This polarization component can be incorporated in a feedback cycle, to phase-lock the velocity modulation to the spin precession, i.e. to guarantee $\Delta \omega=0$ and $\Delta \phi$ constant. In addition, observation of $\Omega_{0}$ allows to measure or stabilize the magnetic field.

From (9.23) and (9.24), the main design criteria are easily derived, several of which are common to all other EDM experiments. They include

- high initial polarization $P_{0}$;

- large field strength $E^{\text {eff }} \propto(\delta \beta B)$;

- close control over the resonance conditions $\Delta \omega$ and phase $\Delta \phi$;

- long spin coherence time $P_{\circ}(t)$;

- long synchrotron coherence time $\delta \beta(t)$;

- sensitive method for independent observation of $P_{\|}$ and $P_{\perp}$.

The parameters of the current concept deuteron EDM ring are presented in Table 26. Coherent synchrotron oscillation can be obtained by a set of two RF cavities, one operating at a harmonic of the revolution frequency to bring the beam close to the spin-synchrotron resonance, and a second operating at the resonance frequency to create a forced oscillation.

The statistical reach of the experiment is determined by the number of particles used to determine the polarization,

Table 26 Parameters of the concept deuteron EDM storage ring.

\begin{tabular}{lll}
\hline Parameter & Symbol & Design value \\
\hline Deuteron momentum & $p_{\mathcal{D}}$ & $1500 \mathrm{MeV} / c$ \\
Magnetic field strength & $B$ & $2 \mathrm{~T}$ \\
Bending radius & $\rho$ & $2.5 \mathrm{~m}$ \\
Length of each straight section & $l$ & $5 \mathrm{~m}$ \\
Orbit length & $L$ & $26 \mathrm{~m}$ \\
Momentum compaction & $\alpha_{p}$ & 1 \\
Cyclotron period & $t_{c}$ & $137 \mathrm{~ns}$ \\
Spin precession period & $t_{s}$ & $660 \mathrm{~ns}$ \\
Spin coherence time & $\tau_{s}$ & $1000 \mathrm{~s}$ \\
Motional electric field & $E^{*} / \gamma$ & $375 \mathrm{MV} / \mathrm{m}$ \\
Synchrotron amplitude & $\delta \beta / \beta$ & $1 \%$ \\
Synchrotron harmonic & $h$ & 40 \\
Particles per fill & $N$ & $10^{12}$ \\
Initial polarization & $P_{\circ}$ & 0.9 \\
EDM precession rate @ $d=10^{-26} e \mathrm{~cm}$ & $\omega_{\eta}$ & $1 \mu \mathrm{rad} / \mathrm{s}$ \\
\hline
\end{tabular}

as well as the analyzing power of the polarimeter. The most efficient way to probe the deuteron polarization at the energy considered is by nuclear scattering. To obtain high efficiency, conventional techniques, in which a target is inserted into the beam are unsuitable. Instead, slow extraction of the beam onto a thick analyzer target is necessary. Slow extraction could be realized by exciting a weak beam resonance, or alternatively, by Coulomb scattering off a thin gas jet. The thickness of the analyzer target is optimized to yield maximum efficiency, which may reach the percent level.

The EDM will create a left-right asymmetry in the scattered particle rate, whose initial rate of growth is proportional to the EDM. False signals from, e.g., oscillating radial magnetic fields in the ring will be mitigated by varying the lattice parameters. This will change the systematic error amplitude, while leaving the EDM signal unchanged. Various features of the ring design and bunches with opposite EDM signals will be used to reduce the impact of other systematic effects.

The expected very high observability of most of the field imperfections in the experiment comes from the combination of gross amplification of the original perturbations in the control bunches, and observation and correction of the amplified parasitic growth of the vertical polarization component. This growth is many orders of magnitude more sensitive to ring imperfections than any other beam parameter. Preliminary studies shows no unmanageable sources of systematic errors at the level of the expected statistical uncertainty of $10^{-29} e \mathrm{~cm}$.

There is currently great interest in EDM experiments because of their potential to find new physics complementary to and even reaching beyond that which can be found at future accelerators (LHC and beyond). The new approach described here would be the most sensitive experiment for the measurement of several possible sources of EDMs in nucleons and nuclei for the foreseeable future, if systematic uncertainties can be controlled.

\subsection{EDM of deformed nuclei: ${ }^{225} \mathrm{Ra}$}

In the nuclear sector, the strongest EDM limits have been set by cell measurements which restrict the EDM of ${ }^{199} \mathrm{Hg}$ to $<2.1 \times 10^{-28} e \mathrm{~cm}$. A promising avenue for extending these searches is to take advantage of the large enhancements in the atomic EDM predicted for octupole deformed nuclei. One such case is ${ }^{225} \mathrm{Ra}$, which is predicted to be two to three orders of magnitude more sensitive to T-violating interactions than ${ }^{199} \mathrm{Hg}$. The next generation EDM search around laser-cooled and trapped ${ }^{225} \mathrm{Ra}$ is being developed by the Argon group. They have demonstrated transverse cooling, Zeeman slowing, and capturing of ${ }^{225} \mathrm{Ra}$ and ${ }^{226} \mathrm{Ra}$ atoms in a magneto-optical trap (MOT). They have measured many of the transition frequencies, life-times, hyperfine splittings 
and isotope shifts of the critical transitions. This new development should enable them to launch a new generation of nuclear EDM searches. The combination of optical trapping and the use of octupole deformed nuclei should extend the reach of a new EDM search by two orders of magnitude. A non-zero EDM in diamagnetic atoms is expected to be most sensitive to a chromo-electric induced EDM effect.

Radium-225 is an especially good case for the search of the EDM because it has a relatively long life-time $\left(t_{1 / 2}=\right.$ $14.9 \mathrm{~d}$ ), has spin $1 / 2$ which eliminates systematic effects due to electric quadrupole coupling, is available in relatively large quantities from the decay of the long-lived ${ }^{229} \mathrm{Th}$ $\left(t_{1 / 2}=7300 \mathrm{yr}\right)$, and has a well-established octupole nature. The octupole deformation enhances parity doubling of the energy levels. For example, the sensitivity to T-odd, P-odd effects in ${ }^{225} \mathrm{Ra}$ is expected to be a factor of approximately 400 larger than in ${ }^{199} \mathrm{Hg}$, which has been used by previous searches to set the lowest limit $\left(<2 \times 10^{-28} e \mathrm{~cm}\right)$ so far on the atomic EDM. The 14.9-day half-life for ${ }^{225} \mathrm{Ra}$ is sufficiently long that measurements can be performed and sys- tematics can be checked without resorting to an acceleratorbased experiment. Nevertheless, if a ${ }^{225} \mathrm{Ra}$ beam facility were available for this experiment, approximately a hundred times more atoms could be produced which could have the impact of improving the sensitivity by yet another order of magnitude.

Laser cooling and trapping of ${ }^{225} \mathrm{Ra}$ atoms was developed in preparation of an EDM search. The laser trap allows one to collect and store the radioactive ${ }^{225} \mathrm{Ra}$ atoms that are otherwise too rare to be used for the search with conventional atomic-beam or vapor-cell type methods. Moreover, an EDM measurement on atoms in a laser trap would benefit from the advantages of high electric field, long coherence time, and a negligible so-called " $v \times E$ " systematic effect.

The Argon group has demonstrated a magneto optical trap (MOT) of Ra atoms by using the $7 s^{2}{ }^{1} S_{0} \rightarrow 7 s 7 p$ ${ }^{3} P_{1}$ transition as the primary trapping transition, and $7 s 6 d$ ${ }^{3} D_{1} \rightarrow 7 s 7 p{ }^{1} P_{1}$ as the re-pump transition (see Fig. 68). They used a Ti:Sapphire ring laser system to generate the $714 \mathrm{~nm}$ light to excite the $7 s^{2}{ }^{1} S_{0} \rightarrow 7 s 7 p^{3} P_{1}$ transition.
Fig. 68 Atomic level structure of radium-225 indicating the cycling transition at $714 \mathrm{~nm}$ and the re-pump transition at $1428 \mathrm{~nm}$. The values in boxes indicate the relative transition probabilities

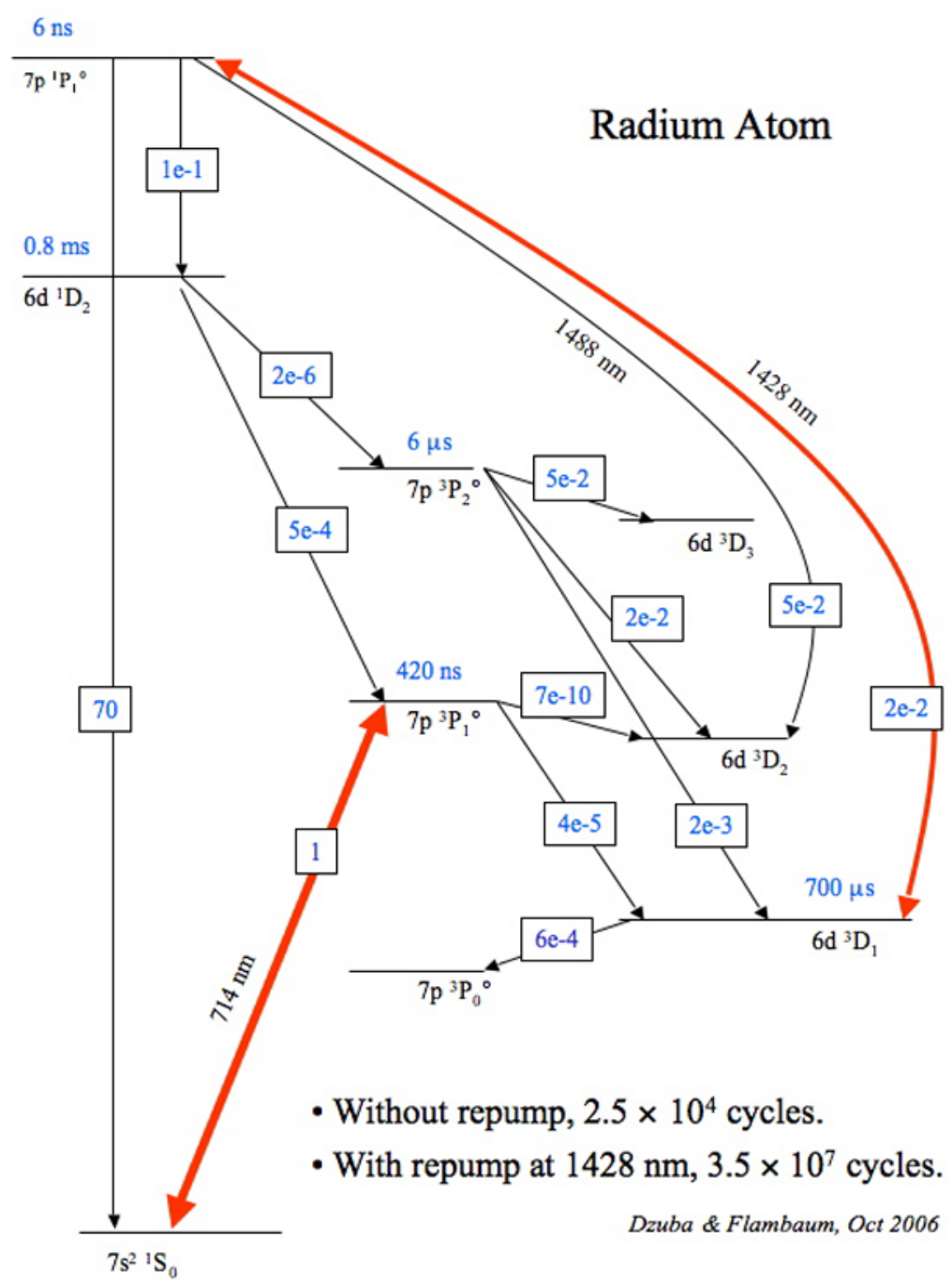


The primary leak channel from this two-level quasi-cycling system is the decay from $7 s 7 p^{3} P_{1}$ to $7 s 6 d^{3} D_{1}$, from which the atoms were pumped back to the ground-level via the $7 s 6 d^{3} D_{1} \rightarrow 7 s 7 p^{1} P_{1}$ transition followed by a spontaneous decay from $7 s 7 p{ }^{1} P_{1}$ back to the ground-level. The re-pump was induced by laser light at $1428.6 \mathrm{~nm}$ generated by a diode laser. This re-pump transition can be excited for an average of 1400 times before the atom leaks to other metastable levels. Therefore, with the re-pump in place, an atom can cycle for an average of $3.5 \times 10^{7}$ times and stay in the MOT for at least $30 \mathrm{~s}$ before it leaks to dark levels. Here the MOT is used only to capture the atoms; the trapped atoms would then be transferred to an optical dipole trap for storage and measurement. They plan to achieve a life-time of $300 \mathrm{~s}$ in the dipole trap.

The ultimate goal of the present series of measurements is to provide a measurement that is comparable in sensitivity to the atomic EDM experiment for ${ }^{199} \mathrm{Hg}$. Because of the enhancement from the octupole deformation of ${ }^{225} \mathrm{Ra}$, the measurement would then be more than two orders of magnitude more sensitive to T-violating effects in the nucleus than that of the ${ }^{199} \mathrm{Hg}$ experiment. The immediate goal over the next two years is to provide an initial atomic EDM limit of $\sim 1 \times 10^{-26} e \mathrm{~cm}$. Thereafter, the plan is to improve the experiment until the ultimate goal is achieved.

\subsection{Electrons bound in atoms and molecules}

\subsubsection{Theoretical aspects}

We discuss here permanent EDMs of diatomic molecules induced by the EDM of the electron and by P- and T-odd $e-N$ neutral currents. In heavy molecules the effective electric field $E_{\text {eff }}$ on unpaired electron(s) is many orders of magnitude higher than the external laboratory field required to polarize the molecule. As a result, the EDM of such molecules is strongly enhanced. The exact value of the enhancement factor is very sensitive to relativistic effects and to electronic correlations. In recent years several methods to calculate $E_{\text {eff }}$ were suggested and reliable results were obtained for a number of molecules.

The study of a non-relativistic electron in a stationary state immediately leads to the zero energy shift $\delta \varepsilon$ for an atom in the external field $\boldsymbol{E}_{0}$ induced by the electron EDM $\boldsymbol{d}_{e}=d_{e} \boldsymbol{\sigma}$. Indeed, the average acceleration $\langle\boldsymbol{a}\rangle=0$, so the average force $-e\langle\boldsymbol{E}\rangle=0$. Therefore, $\delta \varepsilon=-\boldsymbol{d}_{e} \cdot\langle\boldsymbol{E}\rangle=0$. This statement is known as Schiff theorem. In the relativistic case, the position-dependence of the Lorentz contraction of the electron EDM leads instead to a net overall atomic EDM [1029]. Even though $\langle\mathbf{E}\rangle=0$, it still can be (and indeed is) the case that $\left\langle\mathbf{d}_{e} \cdot \mathbf{E}\right\rangle \neq 0$, if $\mathbf{d}_{e}$ is not spatially uniform. Taking account of the fact that the length-contracted value of $\mathbf{d}_{e}$ is NOT spatially uniform for an electron inside the Coulomb field of an atom exactly reproduces the form of the enhancement factor.

Reliable calculations of atomic energy shifts are easier with the relativistic EDM Hamiltonian for the Dirac electron, which automatically turns to zero in the non-relativistic approximation [1030]:

$H_{d}=2 d_{e}\left(\begin{array}{cc}0 & 0 \\ 0 & \sigma\end{array}\right) \cdot \boldsymbol{E} \cong 2 d_{e}\left(\begin{array}{cc}0 & 0 \\ 0 & \sigma\end{array}\right) \cdot \boldsymbol{E}_{\mathrm{int}}$.

This Hamiltonian is singular at the origin and we neglected the external field $E_{0}$. Using (9.25) it is straightforward to show that the induced EDM of the heavy atom $d_{\text {at }}$ is of the order of $10 \alpha^{2} Z^{3} d_{e}$, where $Z$ is the number of protons in the nucleus. If $Z \sim 10^{2}$ the atomic enhancement factor $k_{\text {at }} \equiv d_{\text {at }} / d_{e} \sim 10^{3}$. This estimate holds for atoms with an unpaired electron with $j=\frac{1}{2}$. For higher angular momentum $j$ the centrifugal barrier strongly suppresses $d_{\text {at }}$.

Atomic EDM can be also induced by a scalar P, T-odd $e-N$ neutral current [1030]:

$H_{S}=i \frac{G \alpha}{2^{1 / 2}} Z k_{S} \gamma_{0} \gamma_{5} n(r)$,

where $G$ is Fermi constant, $\gamma_{i}$ are Dirac matrices, $n(r)$ is the nuclear density normalized to unity, and $Z k_{S}=Z k_{S, p}+$ $N k_{S, n}$ is the dimensionless coupling constant for a nucleus with $Z$ protons and $N$ neutrons. Atomic EDMs induced by the interactions (9.25), (9.26) are obviously sensitive to relativistic corrections to the wave function. Numerical calculations also show their sensitivity to correlation effects. For example, the Dirac-Fock calculation for Tl gives $d_{\mathrm{Tl}}=-1910 d_{e}$ while the final answer within all order manybody perturbation theory is $d_{\mathrm{Tl}}=-585 d_{e}$ (see Ref. [1030] for details). Note that the present limit on the electron EDM follows from the experiment with $\mathrm{Tl}$ [186].

The internal electric field in a polar molecule, $E_{\mathrm{mol}} \sim$ $\frac{e}{R_{o}^{2}} \sim 10^{9} \mathrm{~V} / \mathrm{cm}$, is $4-5$ orders of magnitude larger than the typical laboratory field in an atomic EDM experiment. This field is directed along the molecular axis and is averaged to zero by the rotation of the molecule. The molecular axis can be polarized in the direction of the external electric field $\boldsymbol{E}_{0}$. One usually needs the field $E_{0} \sim 10^{4} \mathrm{~V} / \mathrm{cm}$ to fully polarize the heavy diatomic molecule. The corresponding molecular enhancement factor is $k_{\text {mol }} \sim k_{\text {at }} \times \frac{E_{\text {mol }}}{E_{0}} \sim 10^{4} k_{\text {at }}$.

For closed-shell molecules all electrons are coupled and the net EDM is zero. Therefore one needs a molecule with at least one unpaired electron. Such molecules have nonzero projection $\Omega$ of electronic angular momentum on the molecular axis. Again, as in the case of atoms, for the molecules with one unpaired electron the largest enhancement corresponds to $\Omega=\frac{1}{2}$. The centrifugal barrier leads to strong suppression of the factor $k_{\mathrm{mol}}$ for higher values of $\Omega$. On 
Table 27 Calculated values of parameters $E_{\text {eff }}$ and $W_{S}$ from (9.27) for diatomic molecules. The question marks reflect the uncertainty in the knowledge of the ground state

\begin{tabular}{lllccc}
\hline Molecule & State & $\Omega$ & $E_{\text {eff }}\left(10^{9} \mathrm{~V} / \mathrm{cm}\right)$ & $W_{S}(\mathrm{kHz})$ & $-12 \pm 1$ \\
\hline $\mathrm{BaF}$ & ground & $1 / 2$ & $-7.5 \pm 0.8$ & $-44 \pm 5$ & {$[1031,1032]$} \\
$\mathrm{YbF}$ & ground & $1 / 2$ & $-25 \pm 3$ & $-190 \pm 30$ & -144 \\
$\mathrm{HgF}$ & ground & $1 / 2$ & $-100 \pm 15$ & +55 & {$[1032,1033]$} \\
$\mathrm{HgH}$ & ground & $1 / 2$ & -79 & {$[1034]$} \\
$\mathrm{PbF}$ & ground & $1 / 2$ & +29 & {$[1034]$} \\
$\mathrm{PbO}$ & metastable & 1 & -26 & {$[1035]$} \\
$\mathrm{HI}$ & ground & $3 / 2$ & -4 & {$[1036]$} \\
$\mathrm{PtH}^{+}$ & ground (?) & 3 & 20 & {$[1037]$} \\
$\mathrm{HfF}^{+}$ & metastable (?) & 1 & 24 & {$[1038]$} \\
\hline
\end{tabular}

the other hand, such molecules can be polarized in a much weaker external field.

For strong external field $E_{0}$ the factor $k_{\text {mol }}$ depends on $E_{0}$ and it is more practical to define an effective electric field on the electron $E_{\text {eff }}$ so, that the P, T-odd energy shift for a fully polarized molecule is equal to:

$\delta \varepsilon_{P, T}=E_{\mathrm{eff}} d_{e}+\frac{1}{2} W_{S} k_{S}$,

where two terms correspond to interactions (9.25) and (9.26). Calculated values of $E_{\text {eff }}$ and $W_{S}$ for a number of molecules are listed in Table 27.

An EDM experiment is currently going on with $\mathrm{YbF}$ molecules. This molecule has a ground state with $\Omega=\frac{1}{2}$. The P, T-odd parameters (9.27) were calculated with different methods by several groups, and estimates of the systematic uncertainty are available. Several other molecules and molecular ions have been suggested for the search for electron $\mathrm{EDM}$ including $\mathrm{PbO}, \mathrm{PbF}, \mathrm{HgH}$, and $\mathrm{PtH}^{+} . \mathrm{PbF}$ and $\mathrm{HgH}$ have $\Omega=\frac{1}{2}$ and calculations are similar to the $\mathrm{YbF}$ case. The ground state of $\mathrm{PbO}$ has closed shells and the experiment is done on the metastable state with two unpaired electrons and $\Omega=1$. Here electronic correlations are much stronger and calculations are more difficult.

Finally, molecular ions like $\mathrm{PtH}^{+}$are less studied and even their ground states are not known exactly. It is anticipated that such ions can be trapped and a long coherence time for the EDM experiment can be achieved. Recently the first estimates of the effective field for $\mathrm{PtH}^{+}$and several other molecular ions were reported [1037]. These estimates are based on non-relativistic molecular calculations. Proper relativistic molecular calculations for these ions may be extremely challenging.

\subsubsection{Experimental aspects}

Over a dozen different experiments searching for the electron electric dipole moment that are under way or planned will be reviewed here. At present the experimental upper limit on $d_{e}$ is [186]: $\left|d_{e}\right| \leq 1.6 \times 10^{-27} e \mathrm{~cm}$, where $e$ is the unit of electronic charge.

Most of this work is being done in small groups on university campuses. These experiments employ a wide range of technologies and conceptual approaches. Many of the latest generation of experiments promise two or more orders of magnitude improvement in statistical sensitivity, and most have means to suppress systematic errors well beyond those obtained in the previous generation.

To detect $d_{e}$, most experiments rely on the energy shift $\Delta E=-\boldsymbol{d}_{e} \cdot \overrightarrow{\mathbf{E}}$ upon application of $\overrightarrow{\mathbf{E}}$ to an electron. Until recently, most experimental searches for $d_{e}$ used gasphase paramagnetic atoms or molecules and employed the standard methods of atomic, molecular, and optical physics (laser and rf spectroscopy, optical pumping, atomic and molecular beams or vapor cells, etc.) in order to directly measure the energy shift $\Delta E$. Recently, another class of experiments has been actively pursued, in which paramagnetic atoms bound in a solid are studied. Here the principles are rather different than for the gas-phase experiments, and techniques are more similar to those used in condensed matter physics (magnetization and electric polarization of macroscopic samples, cryogenic methods, etc.). We discuss these two classes of experiments separately.

\subsubsection{A simple model experiment using gas-phase atoms} or molecules Experimental searches for $d_{e}$ using gasphase atoms or molecules share many broad features. Each consists of a state selector, where the initial spin state of the system is prepared; an interaction interval in which the system evolves for a time $\tau$ in an electric field $\overrightarrow{\mathbf{E}}$ (and often a magnetic field $\boldsymbol{B} \| \overrightarrow{\mathbf{E}}$ as well); and a detector to determine the final state of the spin. To understand the essential features, we consider a simple model that is readily adapted to describe most realistic experimental conditions. In this model, an "atom" of spin $1 / 2$ with enhancement factor $R$, containing an unpaired electron with spin 
magnetic moment $\mu$ and $\operatorname{EDM} d_{e}$. The spin is initially prepared to lie along $\hat{x}$, i.e., is in the eigenstate $\left|\chi_{+}^{x}\right\rangle$ of spin along $\hat{x}:\left|\psi_{0}\right\rangle=\left|\chi_{+}^{x}\right\rangle \equiv \frac{1}{\sqrt{2}}\left({ }_{1}^{1}\right)$. During the interaction interval the spin precesses about $\overrightarrow{\mathbf{E}}=\mathcal{E} \hat{z}$ and $\boldsymbol{B}=B \hat{z}$, in the $x y$ plane, by angle $2 \phi=-\left(d_{e} R \mathcal{E}+\mu B\right) \tau / \hbar$. At time $\tau$ the quantum state has then evolved to $|\psi\rangle=\frac{1}{\sqrt{2}}\left(\begin{array}{c}e^{-i \phi} \\ e^{i \phi}\end{array}\right)$. Finally, the detector measures the probability that the resulting spin state lies along $\hat{y}$. This is determined by the overlap of the wave function $|\psi\rangle$ with $\left|\chi_{+}^{y}\right\rangle \equiv \frac{1}{\sqrt{2}}\left(\begin{array}{l}1 \\ 1\end{array}\right)$. Hence the signal $S$ from $N$ detected atoms observed in time $\tau$ is $S=N\left|\left\langle\chi_{+}^{y} \mid \psi\right\rangle\right|^{2}=N \cos ^{2} \phi$.

The angle $\phi$ is the sum of a large term $\phi_{1}=-\mu B \tau /(2 \hbar)$ and an extremely small term $\phi_{2}=-d_{e} R \mathcal{E} \tau /(2 \hbar)$. To isolate $\phi_{2}$ one observes $S$ for $\overrightarrow{\mathbf{E}}$ and $\boldsymbol{B}$ both parallel and antiparallel. Reversing $\overrightarrow{\mathbf{E}} \cdot \boldsymbol{B}$ changes the relative sign of $\phi_{1}$ and $\phi_{2}$ and thus changes $S$; the largest change in $S$ occurs by choosing $B$ such that $\phi_{1}= \pm \pi / 4$. With this choice, we have $S_{ \pm} \equiv S\left(\overrightarrow{\mathbf{E}} \cdot \boldsymbol{B}_{<}^{>0}\right)=\frac{N}{2}\left(1 \pm 2 \phi_{2}\right)$. The minimum uncertainty in determination of the phase $\phi_{2}$ in time $\tau$, due to shot noise, is $\delta \phi_{2}=\sqrt{1 / N}$. If the experiment is repeated $T / \tau$ times for a total time of observation $T$, the statistical uncertainty in $d_{e}$ is $\delta d_{e}=\sqrt{1 / N_{0}} \sqrt{1 / T \tau}\left|\hbar / \mathcal{E}_{\text {eff }}\right|$, where we used $R \mathcal{E}=\mathcal{E}_{\text {eff. }}$ In practice, other "technical" noise sources can significantly increase this uncertainty, particularly fluctuations in the magnetic field. Hence, careful magnetic shielding is required in all EDM experiments.

9.5.2.2 Systematic errors The EDM is revealed by a term in the signal proportional to a $\mathrm{P}, \mathrm{T}$-odd pseudoscalar such as $\overrightarrow{\mathbf{E}} \cdot \boldsymbol{B}$. False terms of the same apparent form can appear even without $\mathrm{P}, \mathrm{T}$ violation through a variety of experimental imperfections. The most dangerous effects appear when $\boldsymbol{B}$ depends on the sign of $\overrightarrow{\mathbf{E}}$, which can occur in several ways. For example, leakage currents flowing through insulators separating the electric field electrodes can generate an undesired magnetic field $\boldsymbol{B}_{L}$. Also, if the atoms or molecules have a non-zero velocity $\boldsymbol{v}$, a motional magnetic field $\boldsymbol{B}_{\text {mot }}=\frac{1}{c} \overrightarrow{\mathbf{E}} \times \boldsymbol{v}$ exists in addition to the applied magnetic field $\boldsymbol{B}$; along with various other imperfections in the system, this effect can lead to systematic errors. A related systematic effect involves geometric phases, which appear if the direction of the quantization axis (often determined by $\boldsymbol{B}_{\text {total }}=\boldsymbol{B}+\boldsymbol{B}_{\text {mot }}$ ) varies between the state selector and the analyzer [1030].

A variety of approaches are employed to deal with these and other systematics. Aside from leakage currents, most systematics depend on a combination of two or more imperfections in the experiment (i.e. misaligned or stray fields); these can be isolated by deliberately enhancing one imperfection and looking for a change in the EDM signal. Some experiments utilize, in addition to the atoms of interest, additional species as so-called "co-magnetometers". These co-magnetometer species (e.g., paramagnetic atoms with low $R$ ) are chosen to have negligible or small enhancement factors, but retain sensitivity to magnetic systematics such as those mentioned above.

In paramagnetic molecule experiments, issues with systematic effects are somewhat different. Here the ratio $\mathcal{E}_{\text {eff }} / \mathcal{E}_{\text {ext }}$ is enhanced, and relative sensitivity to magnetic systematics is correspondingly reduced. The $\overrightarrow{\mathbf{E}} \times \boldsymbol{v}$ effect is effectively eliminated by the large tensor Stark effect [1039] typically found in molecular states. The saturation of the molecular polarization $|P|$ (and hence $\mathcal{E}_{\text {eff }}$ ) leads to a wellunderstood non-linear dependence of the EDM signal on $\mathcal{E}_{\text {ext }}$ that can discriminate against certain systematics. Conversely, the extreme electric polarizability leads to a variety of new effects, such as a dependence of the magnetic moment $\mu$ on $\mathcal{E}_{\text {ext }}$, and geometric phase induced systematic errors related to variations in the direction of $\overrightarrow{\mathbf{E}}_{\text {ext }}$.

\subsubsection{Experiments with gas-phase atoms and molecules}

- The Berkeley thallium atomic beam experiment

This experiment gives the best current limit on $d_{e}$. In its final version [186], two pairs of vertical counter-propagating atomic beams, each consisting of $\mathrm{Tl}\left(Z=81, R_{\mathrm{Tl}}=-585\right.$ [1040]) and $\mathrm{Na}\left(Z=11, R_{\mathrm{Na}}=0.32\right)$, were employed (see Fig. 69). Spin alignment and rotation of the $6^{2} P_{1 / 2}(F=1)$ state of $\mathrm{Tl}$ and the $3^{2} S_{1 / 2}(F=2, F=1)$ states of Na were accomplished, respectively, by laser optical pumping and by atomic beam magnetic resonance with separated oscillating rf fields of the Ramsey type. Detection was achieved via alignment-sensitive laser induced fluorescence. In the interaction region, with length $\approx 1$ meter, the side-by-side atomic beams were exposed to nominally identical $\boldsymbol{B}$ fields, but opposite $\overrightarrow{\mathbf{E}}$ fields of $\approx 120 \mathrm{kV} / \mathrm{cm}$. This provided commonmode rejection of magnetic noise and control of some systematic effects. Average thermal velocities corresponded to an interaction time $\tau \approx 2.3 \mathrm{~ms}(1 \mathrm{~ms})$ for $\mathrm{Tl}(\mathrm{Na})$ atoms. Use of counter-propagating atomic beams served to cancel all but a very small remnant of the $\overrightarrow{\mathbf{E}} \times \boldsymbol{v}$ effect. Various auxiliary measurements, including use of $\mathrm{Na}$ as a co-magnetometer, further reduced this remnant and isolated the geometric phase effect. $\mathcal{E}$ and leakage currents were measured using auxiliary measurements based on the observable quadratic Stark effect in Tl. About $5.2 \times 10^{13}$ photo-electrons of signal per up/down beam pair were collected by the fluorescence detectors. The final result is $d_{e}=(6.9 \pm 7.4) \times 10^{-28} e \mathrm{~cm}$, which yields the limit $\left|d_{e}\right| \leq 1.6 \times 10^{-27} e \mathrm{~cm}$ (90\% conf.). - Cesium vapor cell experiments

An experiment to search for $d_{e}$ in a vapor cell of Cs ( $Z=55 ; R_{\mathrm{Cs}}=115$ [1041]) was reported by L. Hunter and co-workers [1042] at Amherst in 1989. The method is being revisited in a present-day search by led by M. Romalis at Princeton [1043]. The Amherst experiment was carried out with two glass cells, one stacked on the other in the $z$ direction. Nominally equal and opposite $\overrightarrow{\mathbf{E}}$ fields 


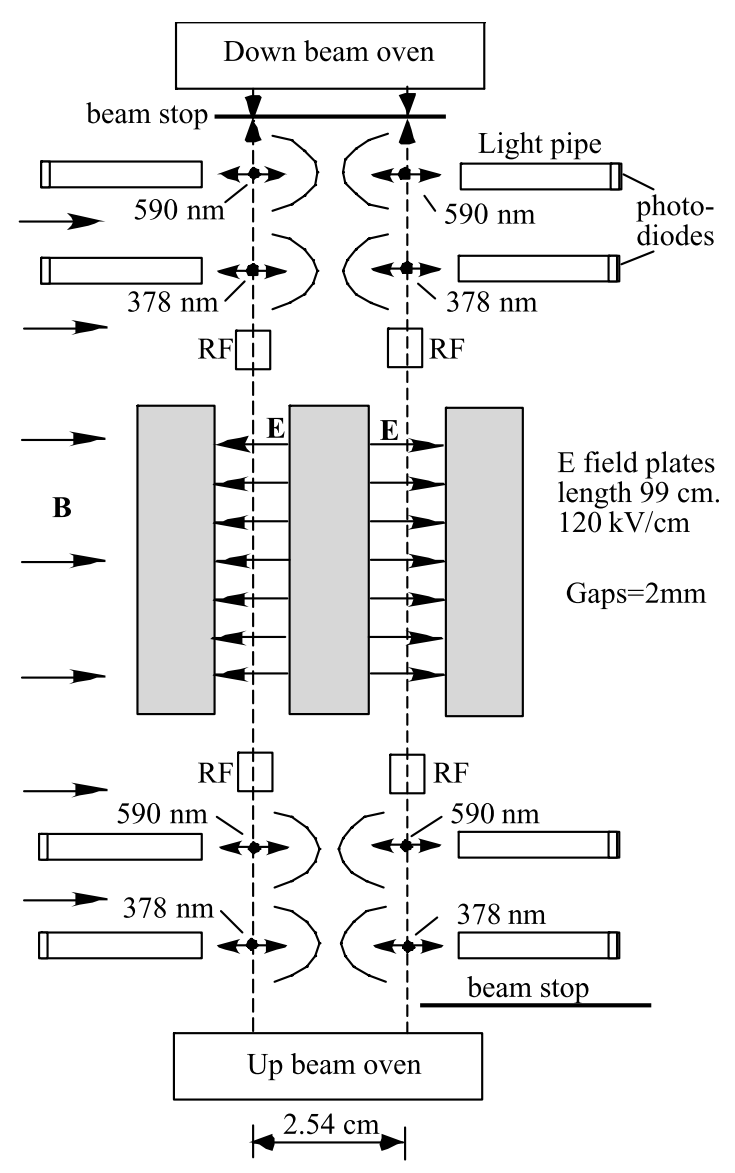

Fig. 69 Schematic diagram of the Berkeley thallium experiment [186], not to scale. Laser beams for state selection and analysis at $590 \mathrm{~nm}$ (for $\mathrm{Na}$ ) and $378 \mathrm{~nm}$ (for $\mathrm{Tl}$ ) are perpendicular to the page, with indicated linear polarizations. The diagram shows the up-going atomic beams active

were applied in the two cells. The cells were filled with Cs, as well as $\mathrm{N}_{2}$ buffer gas to minimize Cs spin relaxation. Circularly polarized laser beams, directed along $x$, were used for spin polarization via optical pumping. Magnetic field components in all three directions were reduced to less than $10^{-7} \mathrm{G}$. Thus precession of the atomic polarization in the $x y$ plane was nominally due to $\overrightarrow{\mathbf{E}}$ alone. The final spin orientation was monitored by a probe laser beam directed along $y$. The effective interaction time was the spin relaxation time $\tau \approx 15 \mathrm{~ms}$. The signals were the intensities of the probe beams transmitted through each cell. A nonzero EDM would have been indicated by a dependence of these signals on the rotational invariant $\boldsymbol{J} \cdot(\boldsymbol{\sigma} \times \overrightarrow{\mathbf{E}}) \tau$, where $\boldsymbol{\sigma}, \boldsymbol{J}$ were the pump and probe circular polarizations, respectively. The most important sources of possible systematic error were leakage currents and imperfect reversal of $\overrightarrow{\mathbf{E}}$. The result was $d_{e}=(-1.5 \pm 5.5 \pm 1.5) \times 10^{-26} e \mathrm{~cm}$.

In the new experiment at Princeton, each cell also contains ${ }^{129} \mathrm{Xe}$ at high pressure. Cs polarization is transferred to the ${ }^{129} \mathrm{Xe}$ nuclei by spin exchange collisions. Under cer- tain conditions this coupling can also give rise to a selfcompensation mechanism, where slow changes in components of magnetic field transverse to the initial polarization axis are nearly canceled by interaction between the alkali electron spin and the noble gas nuclear spin. This leaves only a signal proportional to an anomalous interaction that does not scale with the magnetic moments-for example, interaction of $d_{e}$ with $\mathcal{E}_{\text {eff. }}$ This mechanism (which is understood in some detail [1044, 1045]) has the potential to reduce both the effect of magnetic noise, and some systematic errors.

- Experiments with laser-cooled atoms

Laser-cooled atoms offer significant advantages for electron EDM searches. The low velocities of cold atoms yield long interaction times, and also suppress $\overrightarrow{\mathbf{E}} \times \boldsymbol{v}$ effects. However, these techniques typically yield small numbers of detectable atoms, and magnetic noise must be controlled at unprecedented levels. New systematics due to, e.g., electric forces on atoms and/or perturbations due to trapping fields (see e.g. [1046]) can appear.

Experiments based on atoms trapped in an optical lattice have been proposed by a number of investigators [10471049]. Two such experiments, similar in their design, are currently being developed: one led by D.S. Weiss at Pennsylvania State University and another led by D. Heinzen at the University of Texas. Both plan to use Cs atoms to detect $d_{e}$, along with $\mathrm{Rb}$ atoms $\left(Z=37, R_{\mathrm{Rb}}=25\right)$ as a comagnetometer. The Texas apparatus consists of two side-byside far-off-resonance optical dipole traps, each in a vertical 1-D lattice configuration. These traps are placed in nominally equal and opposite $\overrightarrow{\mathbf{E}}$ fields and a common $\boldsymbol{B}$ field of several $\mathrm{mG}$ parallel to $\overrightarrow{\mathbf{E}}$. To load the atoms into the optical lattice, cold atomic beams from 2D magneto-optical traps exterior to the shields will be captured with optical molasses between the $\overrightarrow{\mathbf{E}}$-field plates. The electric field plates will be constructed from glass coated with a transparent, conductive indium tin oxide layer.

We are aware of two other EDM experiments based on laser-cooled atoms. One employing a slow "fountain", in which Cs atoms are launched upwards and then fall back down due to gravity, has been proposed and developed by $\mathrm{H}$. Gould and co-workers at the Lawrence Berkeley National Laboratory [1050]. Another, using ${ }^{210} \mathrm{Fr}(\tau=3.2 \mathrm{~min}$; $Z=87, R_{\mathrm{Fr}}=1150$ ), has been proposed and is being developed by a group at the Research Center of Nuclear Physics (RCNP), Osaka University, Japan [1051].

- The YbF experiment

E.A. Hinds and co-workers [1052-1054] at Imperial College, London have developed a molecular beam experiment for investigation of $d_{e}$ using YbF. Figure 70 shows the relevant energy level structure of the $X^{2} \Sigma_{1 / 2}^{+}(v=0$, $N=0) \quad J=1 / 2$ ground state of a ${ }^{174} \mathrm{YbF}$ molecule. ${ }^{174} \mathrm{Yb}$ has nuclear spin $I_{\mathrm{Yb}}=0$, while $I_{\mathrm{F}}=1 / 2$; hence the $J=1 / 2$ state has two hyperfine components, $F=1$ and 


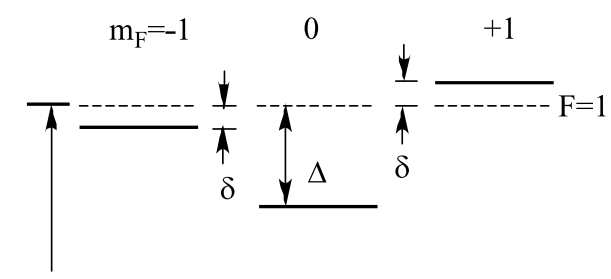

$170 \mathrm{MHz}$

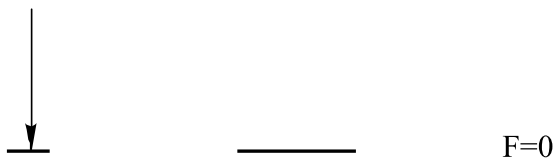

Fig. 70 Schematic diagram, not to scale, of the hyperfine structure of the $X^{2} \Sigma$ electronic state of ${ }^{174} \mathrm{YbF}$ in the lowest vibrational and rotational level. $\Delta$ is the tensor Stark shift. $\delta$ is the shift caused by the combination of the Zeeman effect and the effect of $d_{e}$ in $\overrightarrow{\mathbf{E}}_{\text {eff }}$

$F=0$, separated by $170 \mathrm{MHz}$. An external electric field $\overrightarrow{\mathbf{E}}_{\text {ext }}$ along $\hat{z}$ with magnitude $\varepsilon_{\text {ext }}=8.3 \mathrm{kV} / \mathrm{cm}$ corresponds to $\mathcal{E}_{\text {eff }} \approx 13 \mathrm{GV} / \mathrm{cm}[1033,1052-1054]$, which splits the $F=1, m_{\mathrm{F}}= \pm 1$ levels by $2 d_{e} \varepsilon_{\text {eff. }}$ In this external field, the level $F=1, m_{\mathrm{F}}=0$ is shifted downward relative to $m_{\mathrm{F}}= \pm 1$ by an amount $\Delta=6.7 \mathrm{MHz}$ due to the large tensor Stark shift associated with the molecular electric dipole.

In the experiment, a cold beam of YbF molecules is generated by chemical reactions within a supersonic expansion of $\mathrm{Ar}$ or Xe carrier gas. Laser optical pumping removes all $F=1$ state molecules, leaving only $F=0$ remaining in the beam. Next, a $170 \mathrm{MHz}$ rf magnetic field along $x$ excites molecules from $F=0$ to the coherent superposition $|\psi\rangle=\frac{1}{\sqrt{2}}\left|F=1, m_{\mathrm{F}}=1\right\rangle+\frac{1}{\sqrt{2}}|1,-1\rangle$. While flying through the central interaction region of length $65 \mathrm{~cm}$, the beam is exposed to parallel electric and magnetic fields $( \pm \mathcal{E}, \pm B) \hat{z}(B \sim 0.1 \mathrm{mGauss})$. Next, an rf field drives each $F=1$ molecule back to $F=0$. Because of the phase shift $2 \phi$ developed in the central region, the final population of $F=0$ molecules is proportional to $\cos ^{2} \phi$. These $F=0$ molecules are detected by laser induced fluorescence in the probe region.

The most significant systematic errors in this experiment are expected to arise from variation in the direction and magnitude of $\overrightarrow{\mathbf{E}}$ along the beam axis. If the direction of $\overrightarrow{\mathbf{E}}$ changes in an absolute sense, a geometric phase could be generated, and if $\overrightarrow{\mathbf{E}}$ changes relative to $\boldsymbol{B}$, the magnetic precession phase $\phi_{1}$, proportional to $\overrightarrow{\mathbf{E}}_{\text {ext }} \cdot \boldsymbol{B} /\left|\overrightarrow{\mathbf{E}}_{\text {ext }}\right|$, could be affected. A preliminary result of the $\mathrm{YbF}$ experiment [10521054], published in 2002, is $d_{e}=(-0.2 \pm 3.2) \times 10^{-26} e \mathrm{~cm}$. Many significant improvements have been made since 2002, and it is likely that this experiment will yield a much more precise result in the near future.

\section{- The $\mathrm{PbO}$ experiment}

A search for $d_{e}$ using the metastable $a(1)^{3} \Sigma_{1}$ state of $\mathrm{PbO}$ is being carried out at Yale [1055-1057]. The $a(1)$ state has a relatively long natural life-time: $\tau[a(1)]=82(2) \mu \mathrm{s}$, and can be populated in large numbers using laser excitation in a vapor cell. In this state, the level of total (rotational + electronic) angular momentum $J=1$ contains two closelyspaced " $\Omega$ doublet" states of opposite parity, denoted as $e^{-}$ and $f^{+}$. An external electric field $\overrightarrow{\mathbf{E}}_{\text {ext }}=\mathcal{E}_{\text {ext }} \hat{z}$ mixes $e^{-}$ and $f^{+}$states with the same value of $M$, yielding molecular states with equal but opposite electrical polarization $P$. The degree of polarization $|P| \approx 1$ for $\mathcal{E}_{\text {ext }} \gtrsim 10 \mathrm{~V} / \mathrm{cm}$. When $|P|=1$ the effective molecular field is calculated to be $\varepsilon_{\text {eff }} \cong 26 \mathrm{GV} / \mathrm{cm}$ [1035]. The opposite molecular polarization in the two $\Omega$-doublet levels leads to a sign difference in the EDM-induced energy shift between these two levels. This difference provides an excellent opportunity for effective control of systematic errors, since comparison of the energy shifts in the upper and lower states acts as an "internal co-magnetometer" requiring only minor changes in experimental parameters to monitor.

The Yale experiment is carried out in a cell containing $\mathrm{PbO}$ vapor, consisting of an alumina body supporting top and bottom gold foil electrodes, and flat sapphire windows on all 4 sides. The electric field $\overrightarrow{\mathbf{E}}_{\text {ext }}=\mathcal{E}_{\text {ext }} \hat{z}$ is quite uniform over a large cylindrical volume (diameter $5 \mathrm{~cm}$, height $4 \mathrm{~cm}$ ), and is chosen in the range $30-90 \mathrm{~V} / \mathrm{cm}$. The magnetic field $B_{z}$ is chosen in the range $50-200 \mathrm{mG}$. The cell is enclosed in an oven mounted in a vacuum chamber. At the operating temperature $700 \mathrm{C}$, the $\mathrm{PbO}$ density is $n_{\mathrm{PbO}} \approx 4 \times 10^{13} \mathrm{~cm}^{-3}$.

A state with simultaneously well-defined spin and electrical polarization is populated as follows. A pulsed laser beam with $z$ linear polarization excites the transition $X[J=$ $\left.0^{+}\right] \rightarrow a(1)\left[J=1^{-}, M=0\right]$. ( $X$ is the electronic ground state of PbO.) Following the laser pulse a Raman transition is driven by two microwave beams. The first, with $x$ linear polarization, excites the upward $28.2 \mathrm{GHz}$ transition $a(1)\left[J=1^{-}, M=0\right] \rightarrow a(1)\left[J=2^{+}, M= \pm 1\right]$. The second, with $z$ linear polarization and detuned to the red or blue with respect to the first by $20-60 \mathrm{MHz}$, drives the downward transition $a(1)\left[J=2^{+}, M= \pm 1\right] \rightarrow a(1)[J=1, M= \pm 1]$. The net result is that about $50 \%$ of the $J=1^{-}, M=0$ molecules are transferred to a coherent superposition of $M= \pm 1$ levels in a single desired $\Omega$-doublet component. The subsequent spin precession (due to $\mathcal{E}$ and $B$ ) is detected by observing the frequency of quantum beats in the fluorescence that accompanies spontaneous decay to the $X$ state. The signature of a non-zero EDM is a term in the quantum beat frequency that is proportional to $\overrightarrow{\mathbf{E}}_{\mathrm{ext}} \cdot \boldsymbol{B}$ and that changes sign when one switches from one $\Omega$-doublet component to the other.

The present experimental configuration is sufficient to yield statistical uncertainty comparable to the present limit on $d_{e}$ in a reasonable integration time of a few weeks. However, large improvements can be made in a next generation of the experiment. In the new scheme, detection will be accomplished via absorption of a resonant microwave probe 
beam tuned to the $28.2 \mathrm{GHz}$ transition described above. With this method, the signal-to-noise ratio is linearly proportional to the path length of the probe beam in the $\mathrm{PbO}$ vapor. In a second generation experiment the cell can be made $\sim 10$ times longer than it is now, and the probe beam can pass through the cell multiple times by using suitable mirrors. Improvements in sensitivity of up to a factor of 3000 over the current generation are envisioned.

- Other molecule experiments

E. Cornell and co-workers at the Joint Institute for Laboratory Astrophysics (Boulder, Colorado) have proposed an experiment [1058] to search for $d_{e}$ in the ${ }^{3} \Delta_{1}$ electronic state of the molecular ion $\mathrm{HfF}^{+}$. The premise is to take advantage of the long spin coherence times typical for trapped ion experiments with atoms, along with the large effective electric field acting on $d_{e}$ in a molecule. Preliminary calculations [1036] suggest that the ${ }^{3} \Delta_{1}$ state is a low-lying metastable state with very small $\Omega$-doublet splittings; as in $\mathrm{PbO}$, this state could thus be polarized by small external electric fields $(\lesssim 10 \mathrm{~V} / \mathrm{cm})$ to yield $\mathcal{E}_{\text {eff }} \approx 18 \mathrm{GV} / \mathrm{cm}$ [1037]. To search for $d_{e}$, electron-spin-resonance spectroscopy, using the Ramsey method, is to be performed in the presence of rotating electric and magnetic fields. The electric field polarizes the ions and its rotation prevents them from being accelerated out of the trap. As in $\mathrm{PbO}$, use of both upper and lower $\Omega$-doublet components will yield opposite signs of the EDM signal, but nearly identical signals due to systematic effects. However, this experiment has the unique disadvantage that it is impossible to reverse the electric field: in the laboratory frame it must always point inward toward the trap center.

N. Shafer-Ray and co-workers at Oklahoma University have proposed an experiment to search for $d_{e}$ in the ground ${ }^{2} \Pi_{1 / 2}$ electronic state of $\mathrm{PbF}[1059,1060]$. The proposed scheme is similar to the $\mathrm{YbF}$ experiment, and the value of $\mathcal{E}_{\text {eff }}$ is also approximately the same as for YbF. The primary advantage of $\mathrm{PbF}$ is that its electric field-dependent magnetic moment should vanish when a suitable, large external electric field $\varepsilon_{0} \approx 67 \mathrm{kV} / \mathrm{cm}$ is applied [1059, 1060]. This could dramatically reduce magnetic field-related systematic errors.

9.5.2.4 Experiments with solid-state samples Recently, S. Lamoreaux [1061] revived an old idea of F. Shapiro [1062] to search for $d_{e}$ by applying an electric field $\overrightarrow{\mathbf{E}}_{\text {ext }}$ to a solid sample with unpaired electron spins. If $d_{e} \neq 0$, at sufficiently low temperature the sample can acquire significant spin-polarization and thus a detectable magnetization along the axis of $\overrightarrow{\mathbf{E}}_{\text {ext }}$. Lamoreaux pointed out that use of modern magnetometric techniques and materials (such as $\mathrm{Gd}_{3} \mathrm{Ga}_{5} \mathrm{O}_{12}$ : gadolinum gallium garnet, or $\mathrm{GGG}$ ) could yield impressive sensitivity to $d_{e}$. GGG has a number of attractive properties. Its resistivity is so high $\left(>10^{16} \mathrm{Ohm}\right.$ $\mathrm{cm}$ for $T<77 \mathrm{~K}$ ) that it can support large applied electric fields $\left(\overrightarrow{\mathbf{E}}_{\mathrm{ext}} \approx 10 \mathrm{kV} / \mathrm{cm}\right)$ with very small leakage currents. Moreover, the ion of interest in GGG, $\mathrm{Gd}^{3+}(Z=64)$ has a non-negligible enhancement factor [1063]. A complementary experiment is being done by L. Hunter and co-workers [1064] at Amherst College. Here, a strong external magnetic field is applied to the ferrimagnetic solid $\mathrm{Gd}_{3-x} \mathrm{Y}_{x} \mathrm{Fe}_{5} \mathrm{O}_{12}$ (gadolinium yttrium iron garnet, or GdYIG), thus causing substantial polarization of the $\mathrm{Gd}^{3+}$ electron spins. If $d_{e} \neq 0$, this results in electric charge polarization of the sample, and thus a voltage developed across the sample that reverses with applied magnetic field.

The basic theoretical considerations that must be taken into account to estimate the expected signals [1065] in these solid-state experiments include the same types of calculations needed for free atoms. In addition, however, it is necessary to construct models for the modification of atomic electron orbitals in the solid material, as well as the response of the material to the EDM-induced perturbation of the heavy paramagnetic atom. The results of the calculations are as follows. When all Gd spins are polarized in the GdIG sample, the resulting macroscopic electric field across the sample is $\mathcal{E}=0.7 \times 10^{-10}\left(d_{e} / 10^{-27} e \mathrm{~cm}\right) \mathrm{V} / \mathrm{cm}$. A similar calculation can be used to determine the degree of spin polarization of GGG upon application of an external electric field [1061]. An externally applied electric field of $10 \mathrm{kV} / \mathrm{cm}$ yields an effective electric field $\mathcal{E}^{*}=-\Delta E / d_{e}=3.6 \times 10^{5} \mathrm{~V} / \mathrm{cm}$ acting on the EDM ([1065]; see also [1066]). The resulting magnetization $M$ of the sample is simply related to its magnetic susceptibility $\chi: M=\chi d \mathcal{E}^{*} / \mu_{a}$, where $\mu_{a}$ is the magnetic moment of a $\mathrm{Gd}^{3+}$ ion. Using the standard expression for $\chi(T)$ in a paramagnetic sample, one finds $M \approx 8 n_{\mathrm{Gd}}\left(d_{e} \varepsilon^{*}\right) /\left(k_{B} T\right)$. Here $k_{B}$ is the Boltzmann constant and $T$ is the sample temperature. This yields a magnetic flux $\Phi=4 \pi M S$ over an area $S$ of an infinite flat sheet. In a recent development [1066], Lamoreaux has pointed out that this type of electrically induced spin polarization can be amplified in a system that is super-paramagnetic, so that its magnetic susceptibility $\chi$ is extremely large. It appears that GdIG (GdYIG with $x=0$ ) has this property at sufficiently low magnetic field. If so, the sensitivity of a magnetization measurement in GdIG at $T=4 \mathrm{~K}$ could be similar to that of GGG at much lower temperatures, greatly simplifying the required experimental techniques.

- The Indiana GGG experiment

C.Y. Liu of Indiana University has devised a prototype experiment $[1067,1068]$ in which two GGG disks, $4 \mathrm{~cm}$ in diameter and of thickness $\approx 1 \mathrm{~cm}$, are sandwiched between three planar electrodes. High voltages are applied so that the electric fields in the top and bottom samples are in the same direction. If $d_{e} \neq 0$, a magnetic field similar to a dipole field should be generated, and this is to be detected by a flux pickup coil located in the central ground plane. The latter is designed as a planar gradiometer with 3 concentric loops, arranged to sum up the returning flux and to 
reject common-mode magnetic fluctuations. As the electric field polarization is modulated, the gradiometer detects the changing flux and feeds it to a SQUID sensor. The entire assembly is immersed in a liquid helium bath.

The EDM sensitivity of the prototype experiment is estimated to be $\delta d_{e} \approx 4 \times 10^{-26} e \mathrm{~cm}$. Although this falls short of the ultimate desired sensitivity of $10^{-30} e \mathrm{~cm}$, the prototype experiment is useful as a learning tool for solving some basic technical problems. At Indiana, a second-generation experiment is also being planned, which will operate at much lower temperatures $(\approx 10-15 \mathrm{mK})$, and will employ lower-noise SQUID magnetometers. However, questions remain as to the nature of the magnetic susceptibility $\chi$ of GGG at such low temperatures.

Some thought has gone into possible systematic effects in this system. Although crystals with inversion symmetry such as GGG and GdIG should not exhibit a linear magnetoelectric effect [1069], crystal defects and substitutional impurities can spoil this ideal. Furthermore a quadratic magnetoelectric effect does exist, and to avoid systematic errors arising from it, good control of electric field reversal is required.

\section{- The Amherst GdYIG experiment}

GdYIG is ferrimagnetic, and both $\mathrm{Gd}^{3+}$ ions and Fe lattices contribute to its magnetization $M$. Their contributions are generally of opposite sign, but at moderately low temperatures $T$ the Fe component is roughly constant while the $\mathrm{Gd}$ component changes rapidly with $T$. There exists a "compensation" temperature $T_{C}$ where the $\mathrm{Gd}$ and $\mathrm{Fe}$ magnetizations cancel each other, and the net magnetization $M$ vanishes. For $T>T_{C}\left(<T_{C}\right), M$ is dominated by $\mathrm{Fe}(\mathrm{Gd})$. The $\mathrm{Gd}$ contribution to $M$ can be reduced by replacing some $\mathrm{Gd}^{3+}$ ions with non-magnetic $\mathrm{Y}^{3+}$. With $x$ the average number of $\mathrm{Y}$ ions per unit cell, (so that 3-x is the average number of $\mathrm{Gd}$ ions per unit cell), the compensation temperature becomes $T_{C}=[290-115(3-x)] \mathrm{K}$. This dependence of $T_{C}$ on $x$ is exploited in the Amherst GdYIG experiment. A toroidal sample is employed, consisting of two half-toroids, each in the shape of the letter C. One "C" has $x=1.35$ with a corresponding $T_{C}=103 \mathrm{~K}$. The other " $\mathrm{C}$ " has $x=1.8$ with a corresponding $T_{C}=154 \mathrm{~K}$. These are joined together with copper foil electrodes at the interface. At $T=127 \mathrm{~K}$, the magnetizations of the 2 " $\mathrm{C}$ 's" are identical, but their $\mathrm{Gd}$ magnetizations are nominally opposite. When a magnetic field $H$ is applied to the sample with a toroidal current coil, all Gd spins are nominally oriented toward the same copper electrode. Thus EDM signals from $C_{1}$ and $C_{2}$ add constructively. However below $103 \mathrm{~K}$ (above $154 \mathrm{~K}$ ) the $\mathrm{Gd}$ magnetization is parallel (antiparallel) to $M$ in both C's, which results in cancellation of one EDM signal by the other. Data are acquired by observing the voltage difference $A(B)$ between the two foil electrodes for positive (negative) polarity of the applied magnetic field $H$. An EDM should be re- vealed by the appearance of an asymmetry $d=A-B$ that has a specific temperature dependence, as described above.

A large spurious effect has been seen that mimics an EDM signal when $T<180 \mathrm{~K}$, but which deviates grossly from expectations for $T>180 \mathrm{~K}$. This effect, which is associated with a component of magnetization that does not reverse with $H$, has so far frustrated efforts to realize the full potential of the GdYIG experiment. The best limit that has been achieved so far is [1064]: $d_{e}<5 \times 10^{-24} e \mathrm{~cm}$.

\subsection{Muon EDM}

The best direct upper limits for an electric dipole moment (EDM) of the muon come from the experiments measuring the muon anomalous magnetic moment $(g-2)$. The CERN experiment obtained $1.1 \times 10^{-18} e \mathrm{~cm}(95 \%$ C.L.) [1020] and the preliminary limit from Brookhaven is $2.8 \times$

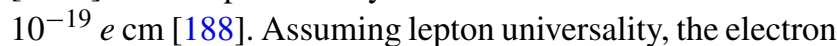
EDM limit of $d_{e}<2.2 \times 10^{-27} e \mathrm{~cm}$ [186] can be scaled by the electron to muon mass ratio, in order to obtain an indirect limit of $d_{\mu}<5 \times 10^{-25} e \mathrm{~cm}$. However, viable models exist in which the simple linear mass scaling does not apply and the value for the muon EDM could be pushed up to values in the $10^{-22} \mathrm{e} \mathrm{cm}$ region (see, e.g., [846, 10701072]). In order for experimental searches to become sufficiently sensitive, dedicated efforts are needed. Several years ago, a letter of intent for a dedicated experiment at JPARC [1073] was presented, proposing a new sensitive "frozen spin" method [1021, 1022]: The anomalous magnetic moment precession of the muon spin in a storage ring can be compensated by the application of a radial electric field, thus freezing the spin; a potential electric dipole moment would lead to a rotation of the spin out of the orbital plane and thus an observable up-down asymmetry which increases with time. The projected sensitivity of the proposed experiment $\left(0.5 \mathrm{GeV} / \mathrm{c}\right.$ muon momentum, $7 \mathrm{~m}$ ring radius) is $10^{-24}$ $10^{-25} \mathrm{e} \mathrm{cm}$. Recently it has been pointed out that there is no immediate advantage from working at high muon momenta and a sensitive approach with a very compact setup (125 MeV/c muon momentum, $0.42 \mathrm{~m}$ ring radius) was outlined [1024]. Already at an existing beam line, such as the $\mu \mathrm{E} 1$ beam at PSI, a measurement with a sensitivity of better than $d_{\mu} \sim 5 \times 10^{-23} e \mathrm{~cm}$ within one year of data taking appears feasible. The estimates for the sensitivity assume an operation in a "one-muon-per-time" mode and the experiment would appear to be statistically limited. With an improved muon accumulation and injection scheme, the sensitivity could be further increased [1074]. Thus the compact storage ring approach at an existing facility could bring the proof of principle for the frozen spin technique and cover the next 3-4 orders of magnitude in experimental sensitivity to a possible muon EDM. 


\subsection{Muon $g-2$}

In his famous 1928 paper [1075-1077] Dirac pointed out that the interaction of an electron with external electric and magnetic fields may have two extra terms where "the two extra terms

$$
\frac{e h}{c}(\sigma, \mathbf{H})+i \frac{e h}{c} \rho_{1}(\sigma, \mathbf{E}),
$$

... when divided by the factor $2 m$ can be regarded as the additional potential energy of the electron due to its new degree of freedom." These terms represent the magnetic dipole (Dirac) moment and electric dipole moment interactions with the external fields.

In modern notation, for the magnetic dipole moment of the muon we have:

$\bar{u}_{\mu}\left[e F_{1}\left(q^{2}\right) \gamma_{\beta}+\frac{i e}{2 m_{\mu}} F_{2}\left(q^{2}\right) \sigma_{\beta \delta} q^{\delta}\right] u_{\mu}$,

where $F_{1}(0)=1$, and $F_{2}(0)=a_{\mu}$.

The magnetic dipole moment of a charged lepton can differ from its Dirac value $(g=2)$ for several reasons. Recall that the proton's $g$-value is $5.6\left(a_{p}=1.79\right)$, a manifestation of its quark-gluon internal structure. On the other hand, the leptons appear to have no internal structure, and the magnetic dipole moments are thought to deviate from 2 through radiative corrections, i.e. resulting from virtual particles that couple to the lepton. We should emphasize that these radiative corrections need not be limited to the standard model particles. While the current experimental uncertainty of $\pm 0.5 \mathrm{ppm}$ on the muon anomaly is 770 times larger than that on the electron anomaly [1078], the former is far more sensitive to the effects of high mass scales. In the lowest order diagram where mass effects appear, the contribution of heavy virtual particles with mass $M$ scales as $\left(m_{\text {lepton }} / M\right)^{2}$, giving the muon a factor of $\left(m_{\mu} / m_{e}\right)^{2} \simeq 43000$ increase in sensitivity over the electron.

\subsubsection{The standard model value of the anomalous magnetic moment}

The standard model value of a lepton's anomalous magnetic moment (the anomaly)

$a_{\ell} \equiv \frac{\left(g_{s}-2\right)}{2}$ has contributions from three different sets of radiative processes: quantum electrodynamics (QED) - with loops containing leptons $(e, \mu, \tau)$ and photons; hadronic-with hadrons in vacuum polarization loops; and weak-with loops involving the bosons $W, Z$, and Higgs:

$a_{\ell}^{\mathrm{SM}}=a_{\ell}^{\mathrm{QED}}+a_{\ell}^{\text {hadronic }}+a_{\ell}^{\text {weak }}$.

The QED contribution has been calculated up to the leading five-loop corrections [1079]. The dominant "Schwinger term" $[1080,1081] a^{(2)}=\alpha / 2 \pi$, is shown diagrammatically in Fig. 71(a). Examples of the hadronic and weak contributions are given in Fig. 71(b)-(d).

The hadronic contribution cannot be calculated directly from QCD, since the energy scale $\left(m_{\mu} c^{2}\right)$ is very low, although Blum has performed a proof of principle calculation on the lattice [1082-1084]. Fortunately, dispersion theory gives a relationship between the vacuum polarization loop and the cross section for $e^{+} e^{-} \rightarrow$ hadrons,

$a_{\mu}(\mathrm{Had} ; 1)=\left(\frac{\alpha m_{\mu}}{3 \pi}\right)^{2} \int_{4 m_{\pi}^{2}}^{\infty} \frac{d s}{s^{2}} K(s) R(s)$,

where

$R \equiv \sigma_{\text {tot }}\left(e^{+} e^{-} \rightarrow\right.$ hadrons $) / \sigma_{\text {tot }}\left(e^{+} e^{-} \rightarrow \mu^{+} \mu^{-}\right)$

and experimental data are used as input $[1085,1086]$.

The standard model value of the muon anomaly has recently been reviewed [1085], and the latest values of the contributions are given in Table 28. The sum of these contributions, adding experimental and theoretical errors in quadrature, gives

$a_{\mu}^{\mathrm{SM}(06)}=116591785(61) \times 10^{-11}$,

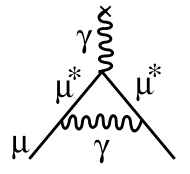

(a)

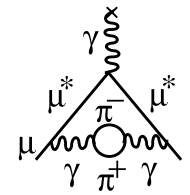

(b)

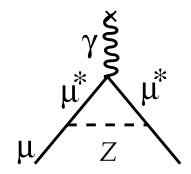

(c)

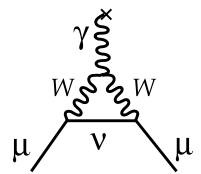

(d)
Fig. 71 The Feynman graphs for: (a) lowest order QED (Schwinger) term; (b) lowest order hadronic correction; (c) and (d) lowest order electroweak terms. The * emphasizes that in the loop the muon is off-shell. With the known limits on the Higgs mass, the contribution from the single Higgs loop is negligible
Table 28 Standard model contributions to the muon anomalous magnetic dipole moment, $a_{\mu}$. All values are taken from Ref. [1085]

\begin{tabular}{lll}
\hline QED & $116584718.09 \pm 0.14_{5 \text { loops }} \pm 0.08_{\alpha} \pm 0.04_{\text {masses }}$ & $\times 10^{-11}$ \\
Hadronic (lowest order) & $a_{\mu}[\mathrm{HVP}(06)]=6901 \pm 42_{\mathrm{exp}} \pm 19_{\mathrm{rad}} \pm 7 \mathrm{QCD}$ & $\times 10^{-11}$ \\
Hadronic (higher order) & $a_{\mu}[\mathrm{HVPh} .0]=.-97.9 \pm 0.9 \exp \pm 0.3_{\mathrm{rad}}$ & $\times 10^{-11}$ \\
Hadronic (light-by-light) & $a_{\mu}[$ HLLS $]=110 \pm 40$ & $\times 10^{-11}$ \\
Electroweak & $a_{\mu}[E W]=154 \pm 2 \pm 1$ & $\times 10^{-11}$ \\
\hline
\end{tabular}


which should be compared with the experimental world average [187]

$a_{\mu}^{\exp }=116592080(63) \times 10^{-11}$.

One finds $\Delta a_{\mu}=295(88) \times 10^{-11}$, a $3.4 \sigma$ difference. It is clear that both the theoretical and the experimental uncertainty should be reduced to clarify whether there is a true discrepancy or a statistical fluctuation. We shall discuss potential improvements to the experiment below.

\subsubsection{Measurement of the magnetic dipole moment}

The measured value of the muon anomaly has a $0.46 \mathrm{ppm}$ statistical uncertainty and a $0.28 \mathrm{ppm}$ systematic uncertainty, which are combined in quadrature to obtain the total error of $0.54 \mathrm{ppm}$. To significantly improve the measured value, both errors must be reduced. We first discuss the experimental technique, and then the systematic errors.

In all but the first experiments by Garwin et al. [1087] the measurement of the magnetic anomaly made use of the spin rotation in a magnetic field relative to the momentum rotation:

$\vec{\omega}_{S}=-\frac{q g \vec{B}}{2 m}-\frac{q \vec{B}}{\gamma m}(1-\gamma)$,

$\vec{\omega}_{C}=-\frac{q \vec{B}}{m \gamma}$,

$\vec{\omega}_{a} \equiv \vec{\omega}_{S}-\vec{\omega}_{C}=-\left(\frac{g-2}{2}\right) \frac{q \vec{B}}{m}=-a_{\mu} \frac{q \vec{B}}{m}$.

A series of three beautiful experiments at CERN culminated in a $7.3 \mathrm{ppm}$ measure of $a_{\mu}$ [1088]. In the third CERN experiment, a new technique was developed based on the observation that electrostatic quadrupoles could be used for vertical focusing. With the velocity transverse to the magnetic field $(\vec{\beta} \cdot \vec{B}=0)$, the spin precession formula becomes

$\vec{\omega}_{a}=-\frac{q}{m}\left[a_{\mu} \vec{B}-\left(a_{\mu}-\frac{1}{\gamma^{2}-1}\right) \frac{\vec{\beta} \times \vec{E}}{c}\right]$.

For $\gamma_{\text {magic }}=29.3(p=3.09 \mathrm{GeV} / c)$, the second term vanishes so $\omega_{a}$ becomes independent of the electric field and the precise knowledge of the muon momentum. Also knowledge of the muon trajectories to determine the average magnetic field becomes less critical, which reduces the uncertainty in $B$.

This technique was used also in experiment E821 at the Brookhaven National Laboratory Alternating Gradient Synchrotron (AGS) [187, 1085]. The AGS proton beam is used to produce a beam of pions that decay to muons in an $80 \mathrm{~m}$ pion decay channel. Muons with $p_{\text {magic }}$ are brought into the storage ring and stored using a fast muon kicker. Calorimeters, placed on the inner radius of the storage ring measure both the energy and arrival time of the decay electrons. Since the highest energy electrons are emitted antiparallel to the muon spin the rate of high energy electrons is modulated by the spin precession frequency:

$N\left(t, E_{\mathrm{th}}\right)=N_{0}\left(E_{\mathrm{th}}\right) e^{-t / \gamma \tau}\left[1+A\left(E_{\mathrm{th}}\right) \cos \left(\omega_{a} t+\phi\left(E_{\mathrm{th}}\right)\right)\right]$.

The time spectrum for electrons with $E>E_{\text {th }}=1.8 \mathrm{GeV}$ is shown in [187] Fig. 72. The value of $\omega_{a}$ is obtained from these data using the five parameter function (9.37) as a starting point, but many additional small effects must be taken into account [187, 1085].

The magnetic field is measured with nuclear magnetic resonance (NMR) probes, and tied through calibration to the
Fig. 72 The time spectrum of $10^{9}$ positrons with energy greater than $1.8 \mathrm{GeV}$ from the Y2000 run. The endpoint energy is $3.1 \mathrm{GeV}$. The time interval for each of the diagonal "wiggles" is given on the right

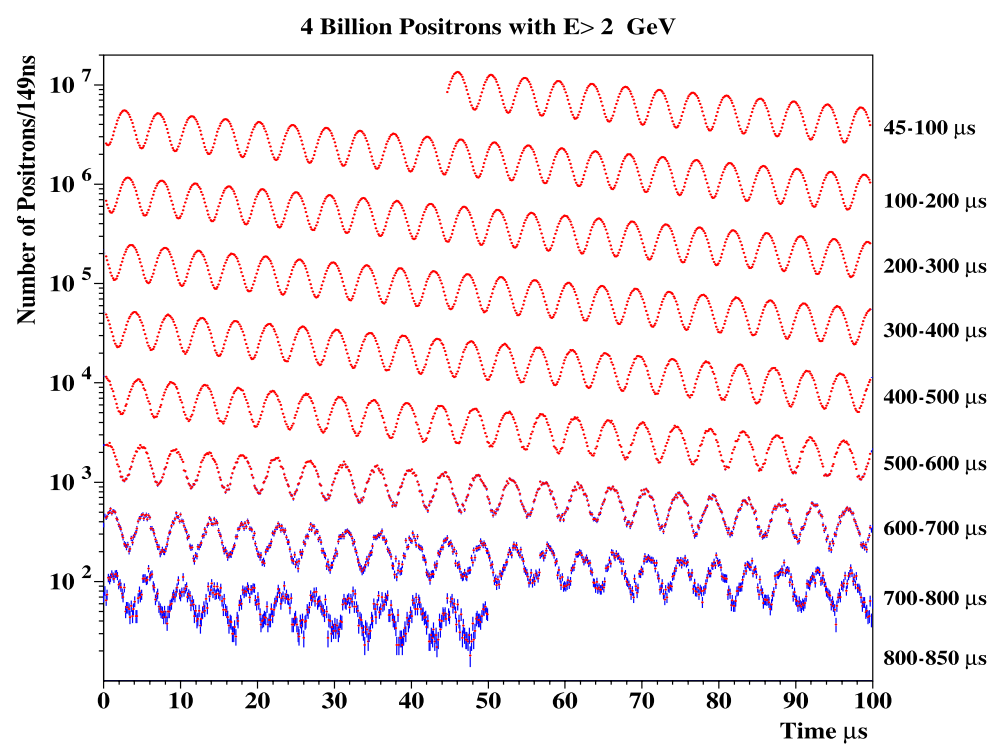


Larmor frequency of the free proton [187]. The anomaly is determined from

$a_{\mu}=\frac{\tilde{\omega}_{a} / \omega_{p}}{\lambda-\tilde{\omega}_{a} / \omega_{p}}=\frac{\mathcal{R}}{\lambda-\mathcal{R}}$,

where the tilde on $\tilde{\omega}_{a}$ indicates that the measured muon precession frequency has been adjusted for any necessary (small) corrections, such as the pitch and radial electric field corrections [1085], and $\lambda=\mu_{\mu} / \mu_{p}$ is the ratio of the muon to proton magnetic moments.

\subsubsection{An improved $g-2$ experiment}

One of the major features of an upgraded experiment would be a substantially increased flux of muons into the storage ring. The BNL beam [187] took forward muons from pion decays, and selected muons $1.7 \%$ below the pion momentum. With this scheme, approximately half of the injected beam consisted of pions. An upgraded experiment would need to quadruple the quadrupoles in the pion decay channel, to increase the beam line acceptance. To decrease the hadron flash at injection one would need to go further away from the pion momentum. Alternatively one could increase the pion momentum to $5.32 \mathrm{GeV} / c$ so that backward decays would produce muons at the magic momentum. Then the pion flash would be completely eliminated, which would significantly reduce the systematic error from gain instabilities.

The inflector magnet that permits the beam to enter the storage ring undeflected would need to be replaced, since the present model loses half of the beam through multiple scattering in material across the beam channel. The fast muon kicker would also need to be improved. With the significant increase in beam, the detectors would have to be segmented, new readout electronics would be needed, and a better measure of lost muons would also be needed.

To reduce the magnetic field systematic errors, significant effort will be needed to improve on the tracking of the field with time, and the calibration procedure used to tie the NMR frequency in the probes to the free proton Larmor frequency [187].

While there are technical issues to be resolved, the present technique-magic $\gamma$, electrostatic focusing, uniform magnetic field — could be pushed to below $0.1 \mathrm{ppm}$. To go further would probably require a new technique. One possibility discussed by Farley [1089] would be to use muons at much higher energy, say $15 \mathrm{GeV}$, which would increase the number of precessions that can be observed. The storage ring would consist of a small number of discrete magnets with uniform field and edge focusing and the field averaged over the orbit would be independent of orbit radius (particle momentum). The averaged field could be calibrated by injecting polarized protons and observing the proton $g-2$ precession.
Acknowledgements The authors of this report are grateful to all the additional workshop participants who contributed with their presentations during the Working Group meetings: A. Baldini, W. Bertl, G. Colangelo, F. Farley, L. Fiorini, G. Gabrielse, J.R. Guest, A. Hoecker, J. Hosek, P. Iaydijev, Y. Kuno, S. Lavignac, D. Leone, A. Luccio, J. Miller, W. Morse, H. Nishiguchi, Y. Orlov, E. Paoloni, A. Pilaftsis, W. Porod, N. Ramsey, S. Redin, W. Rodejohann, M.-A. SanchisLozano, N. Shafer-Ray, A. Soni, A. Strumia, G. Venanzoni, T. Yamashita, Z. Was, H. Wilschut.

This work was supported in part by the Marie Curie research training network "HEPTOOLS" (MRTN-CT-2006-035505).

\section{References}

1. T. Akesson et al., Eur. Phys. J. C 51, 421 (2007). arXiv:hep-ph/0609216

2. A. Blondel et al. (eds.), ECFA/CERN studies of a European neutrino factory complex. CERN-2004-002, ECFA-04230, April 2004

3. V. Barger et al., arXiv:0705.4396 [hep-ph]

4. R.N. Mohapatra et al., Rep. Prog. Phys. 70, 1757 (2007). arXiv:hep-ph/0510213

5. C.D. Froggatt, H.B. Nielsen, Nucl. Phys. B 147, 277 (1979)

6. H. Georgi, C. Jarlskog, Phys. Lett. B 86, 297 (1979)

7. J.R. Ellis, M.K. Gaillard, Phys. Lett. B 88, 315 (1979)

8. G. Anderson, S. Raby, S. Dimopoulos, L.J. Hall, G.D. Starkman, Phys. Rev. D 49, 3660 (1994). arXiv:hep-ph/9308333

9. N. Irges, S. Lavignac, P. Ramond, Phys. Rev. D 58, 035003 (1998). arXiv:hep-ph/9802334

10. J.K. Elwood, N. Irges, P. Ramond, Phys. Rev. Lett. 81, 5064 (1998). arXiv:hep-ph/9807228

11. M. Leurer, Y. Nir, N. Seiberg, Nucl. Phys. B 398, 319 (1993). arXiv:hep-ph/9212278

12. M. Dine, R.G. Leigh, A. Kagan, Phys. Rev. D 48, 4269 (1993). arXiv:hep-ph/9304299

13. Y. Nir, N. Seiberg, Phys. Lett. B 309, 337 (1993). arXiv:hep-ph/9304307

14. M. Leurer, Y. Nir, N. Seiberg, Nucl. Phys. B 420, 468 (1994). arXiv:hep-ph/9310320

15. D.B. Kaplan, M. Schmaltz, Phys. Rev. D 49, 3741 (1994). arXiv:hep-ph/9311281

16. A. Pomarol, D. Tommasini, Nucl. Phys. B 466, 3 (1996) arXiv:hep-ph/9507462

17. C.D. Carone, L.J. Hall, H. Murayama, Phys. Rev. D 53, 6282 (1996). arXiv:hep-ph/9512399

18. R. Barbieri, G.R. Dvali, L.J. Hall, Phys. Lett. B 377, 76 (1996). arXiv:hep-ph/9512388

19. E. Dudas, C. Grojean, S. Pokorski, C.A. Savoy, Nucl. Phys. B 481, 85 (1996). arXiv:hep-ph/9606383

20. R. Barbieri, L.J. Hall, S. Raby, A. Romanino, Nucl. Phys. B 493, 3 (1997). arXiv:hep-ph/9610449

21. P. Binetruy, S. Lavignac, P. Ramond, Nucl. Phys. B 477, 353 (1996). arXiv:hep-ph/9601243

22. P. Binetruy, S. Lavignac, S.T. Petcov, P. Ramond, Nucl. Phys. B 496, 3 (1997). arXiv:hep-ph/9610481

23. Y. Nir, R. Rattazzi, Phys. Lett. B 382, 363 (1996). arXiv:hep-ph/9603233

24. R. Barbieri, L.J. Hall, A. Romanino, Nucl. Phys. Proc. Suppl. A 52, 141 (1997)

25. C.D. Carone, L.J. Hall, Phys. Rev. D 56, 4198 (1997). arXiv:hep-ph/9702430

26. R. Barbieri, L.J. Hall, A. Romanino, Phys. Lett. B 401, 47 (1997). arXiv:hep-ph/9702315

27. R. Barbieri, L. Giusti, L.J. Hall, A. Romanino, Nucl. Phys. B 550, 32 (1999). arXiv:hep-ph/9812239 
28. L.J. Hall, N. Weiner, Phys. Rev. D 60, 033005 (1999). arXiv:hep-ph/9811299

29. R. Barbieri, L.J. Hall, A. Romanino, Nucl. Phys. B 551, 93 (1999). arXiv:hep-ph/9812384

30. A. Aranda, C.D. Carone, R.F. Lebed, Phys. Lett. B 474, 170 (2000). arXiv:hep-ph/9910392

31. R. Barbieri, P. Creminelli, A. Romanino, Nucl. Phys. B 559, 17 (1999). arXiv:hep-ph/9903460

32. Z. Berezhiani, A. Rossi, Nucl. Phys. B 594, 113 (2001). arXiv:hep-ph/0003084

33. M.C. Chen, K.T. Mahanthappa, Phys. Rev. D 62, 113007 (2000). arXiv:hep-ph/0005292

34. A. Aranda, C.D. Carone, R.F. Lebed, Phys. Rev. D 62, 016009 (2000). arXiv:hep-ph/0002044

35. A. Aranda, C.D. Carone, P. Meade, Phys. Rev. D 65, 013011 (2002). arXiv:hep-ph/0109120

36. E. Ma, G. Rajasekaran, Phys. Rev. D 64, 113012 (2001). arXiv:hep-ph/0106291

37. S.F. King, G.G. Ross, Phys. Lett. B 520, 243 (2001). arXiv:hep-ph/0108112

38. S. Lavignac, I. Masina, C.A. Savoy, Phys. Lett. B 520, 269 (2001). arXiv:hep-ph/0106245

39. R.G. Roberts, A. Romanino, G.G. Ross, L. Velasco-Sevilla, Nucl. Phys. B 615, 358 (2001). arXiv:hep-ph/0104088

40. J.L. Chkareuli, C.D. Froggatt, H.B. Nielsen, Nucl. Phys. B 626, 307 (2002). arXiv:hep-ph/0109156

41. K.S. Babu, E. Ma, J.W.F. Valle, Phys. Lett. B 552, 207 (2003). arXiv:hep-ph/0206292

42. G.G. Ross, L. Velasco-Sevilla, Nucl. Phys. B 653, 3 (2003). arXiv:hep-ph/0208218

43. Y. Nir, G. Raz, Phys. Rev. D 66, 035007 (2002). arXiv: hep-ph/0206064

44. H.K. Dreiner, H. Murayama, M. Thormeier, Nucl. Phys. B 729, 278 (2005). arXiv:hep-ph/0312012

45. S.F. King, G.G. Ross, Phys. Lett. B 574, 239 (2003). arXiv:hep-ph/0307190

46. G.G. Ross, L. Velasco-Sevilla, O. Vives, Nucl. Phys. B 692, 50 (2004). arXiv:hep-ph/0401064

47. W. Grimus, A.S. Joshipura, S. Kaneko, L. Lavoura, M. Tanimoto, J. High Energy Phys. 0407, 078 (2004). arXiv:hep-ph/0407112

48. G.L. Kane, S.F. King, I.N.R. Peddie, L. Velasco-Sevilla, J. High Energy Phys. 0508, 083 (2005). arXiv:hep-ph/0504038

49. Z. Berezhiani, F. Nesti, J. High Energy Phys. 0603, 041 (2006). arXiv:hep-ph/0510011

50. G. Altarelli, F. Feruglio, Nucl. Phys. B 741, 215 (2006). arXiv:hep-ph/0512103

51. O. Vives, arXiv:hep-ph/0504079

52. S. Abel, D. Bailin, S. Khalil, O. Lebedev, Phys. Lett. B 504, 241 (2001). arXiv:hep-ph/0012145

53. J.L. Diaz-Cruz, J. Ferrandis, Phys. Rev. D 72, 035003 (2005). arXiv:hep-ph/0504094

54. P.H. Chankowski, K. Kowalska, S. Lavignac, S. Pokorski, Phys. Rev. D 71, 055004 (2005). arXiv:hep-ph/0501071

55. W. Grimus, L. Lavoura, J. High Energy Phys. 0508, 013 (2005). arXiv:hep-ph/0504153

56. I. de Medeiros Varzielas, G.G. Ross, Nucl. Phys. B 733, 31 (2006). arXiv:hep-ph/0507176

57. I. de Medeiros Varzielas, S.F. King, G.G. Ross, arXiv:hep-ph/ 0607045

58. I. Masina, C.A. Savoy, Nucl. Phys. B 755, 1 (2006). arXiv: hep-ph/0603101

59. I. Masina, C.A. Savoy, Phys. Lett. B 642, 472 (2006). arXiv:hep-ph/0606097

60. S.F. King, M. Malinsky, Phys. Lett. B 645, 351 (2007). arXiv:hep-ph/0610250
61. C. Hagedorn, M. Lindner, R.N. Mohapatra, J. High Energy Phys. 0606, 042 (2006). arXiv:hep-ph/0602244

62. T. Appelquist, Y. Bai, M. Piai, Phys. Lett. B 637, 245 (2006). arXiv:hep-ph/0603104

63. L. Ferretti, S.F. King, A. Romanino, J. High Energy Phys. 0611, 078 (2006). arXiv:hep-ph/0609047

64. F. Feruglio, C. Hagedorn, Y. Lin, L. Merlo, arXiv:hep-ph/ 0702194

65. E. Ma, arXiv:hep-ph/0409075

66. E. Ma, Phys. Rev. D 43, 2761 (1991)

67. N.G. Deshpande, M. Gupta, P.B. Pal, Phys. Rev. D 45, 953 (1992)

68. M. Frigerio, S. Kaneko, E. Ma, M. Tanimoto, Phys. Rev. D 71, 011901 (2005). arXiv:hep-ph/0409187

69. E. Ma, Mod. Phys. Lett. A 17, 2361 (2002). arXiv:hep-ph/ 0211393

70. C. Luhn, S. Nasri, P. Ramond, arXiv:hep-th/0701188

71. E. Ma, Mod. Phys. Lett. A 21, 1917 (2006). arXiv:hep-ph/ 0607056

72. W. Grimus, L. Lavoura, Phys. Lett. B 572, 189 (2003). arXiv: hep-ph/0305046

73. E. Ma, arXiv:hep-ph/0612022

74. Y. Koide, Lett. Nuovo Cimento 34, 201 (1982)

75. E. Ma, arXiv:hep-ph/0701016

76. Y. Yamaguchi, Phys. Lett. 9, 281 (1964)

77. J. Kubo, A. Mondragon, M. Mondragon, E. RodriguezJauregui, Prog. Theor. Phys. 109, 795 (2003) [Erratum: Prog. Theor. Phys. 114, 287 (2005)]. arXiv:hep-ph/0302196

78. J. Kubo, H. Okada, F. Sakamaki, Phys. Rev. D 70, 036007 (2004). arXiv:hep-ph/0402089

79. S.L. Chen, M. Frigerio, E. Ma, Phys. Rev. D 70, 073008 (2004) [Erratum: Phys. Rev. D 70, 079905 (2004)]. arXiv:hep-ph/0404084

80. L. Lavoura, E. Ma, Mod. Phys. Lett. A 20, 1217 (2005). arXiv:hep-ph/0502181

81. T. Teshima, Phys. Rev. D 73, 045019 (2006). arXiv:hep-ph/ 0509094

82. Y. Koide, Phys. Rev. D 73, 057901 (2006). arXiv:hep-ph/ 0509214

83. R.N. Mohapatra, S. Nasri, H.B. Yu, Phys. Lett. B 639, 318 (2006). arXiv:hep-ph/0605020

84. S. Morisi, M. Picariello, Int. J. Theor. Phys. 45, 1267 (2006). arXiv:hep-ph/0505113

85. S. Kaneko, H. Sawanaka, T. Shingai, M. Tanimoto, K. Yoshioka, Prog. Theor. Phys. 117, 161 (2007). arXiv: hep-ph/0609220

86. F. Feruglio, Y. Lin, arXiv:0712.1528 [hep-ph]

87. E. Ma, arXiv:hep-ph/0612013

88. O. Felix, A. Mondragon, M. Mondragon, E. Peinado, arXiv: hep-ph/0610061

89. P.F. Harrison, D.H. Perkins, W.G. Scott, Phys. Lett. B 530, 167 (2002). arXiv:hep-ph/0202074

90. X.G. He, A. Zee, Phys. Lett. B 560, 87 (2003). arXiv:hep-ph/ 0301092

91. N. Cabibbo, Phys. Lett. B 72, 333 (1978)

92. L. Wolfenstein, Phys. Rev. D 18, 958 (1978)

93. E. Ma, Phys. Rev. D 70, 031901 (2004). arXiv:hep-ph/0404199

94. G. Altarelli, F. Feruglio, Nucl. Phys. B 720, 64 (2005). arXiv:hep-ph/0504165

95. K.S. Babu, X.G. He, arXiv:hep-ph/0507217

96. E. Ma, Phys. Rev. D 72, 037301 (2005). arXiv:hep-ph/0505209

97. A. Zee, Phys. Lett. B 630, 58 (2005). arXiv:hep-ph/0508278

98. E. Ma, Phys. Rev. D 73, 057304 (2006). arXiv:hep-ph/0511133

99. X.G. He, Y.Y. Keum, R.R. Volkas, J. High Energy Phys. 0604, 039 (2006). arXiv:hep-ph/0601001

100. B. Adhikary, B. Brahmachari, A. Ghosal, E. Ma, M.K. Parida, Phys. Lett. B 638, 345 (2006). arXiv:hep-ph/0603059 
101. B. Adhikary, A. Ghosal, Phys. Rev. D 75, 073020 (2007). arXiv:hep-ph/0609193

102. F. Yin, Phys. Rev. D 75, 073010 (2007). arXiv:0704.3827 [hep$\mathrm{ph}]$

103. G. Altarelli, F. Feruglio, Y. Lin, Nucl. Phys. B 775, 31 (2007). arXiv:hep-ph/0610165

104. X.G. He, Nucl. Phys. Proc. Suppl. 168, 350 (2007). arXiv: hep-ph/0612080

105. E. Ma, Mod. Phys. Lett. A 17, 289 (2002). arXiv:hep-ph/ 0201225

106. E. Ma, Mod. Phys. Lett. A 17, 627 (2002). arXiv:hep-ph/ 0203238

107. K.S. Babu, T. Kobayashi, J. Kubo, Phys. Rev. D 67, 075018 (2003). arXiv:hep-ph/0212350

108. S.L. Chen, M. Frigerio, E. Ma, Nucl. Phys. B 724, 423 (2005). arXiv:hep-ph/0504181

109. M. Hirsch, A. Villanova del Moral, J.W.F. Valle, E. Ma, Phys. Rev. D 72, 091301 (2005) [Erratum: Phys. Rev. D 72, 119904 (2005)]. arXiv:hep-ph/0507148

110. E. Ma, Mod. Phys. Lett. A 20, 1953 (2005). arXiv:hep-ph/ 0502024

111. E. Ma, Mod. Phys. Lett. A 20, 2601 (2005). arXiv:hep-ph/ 0508099

112. E. Ma, Mod. Phys. Lett. A 20, 2767 (2005). arXiv:hep-ph/ 0506036

113. E. Ma, H. Sawanaka, M. Tanimoto, Phys. Lett. B 641, 301 (2006). arXiv:hep-ph/0606103

114. E. Ma, Mod. Phys. Lett. A 21, 2931 (2006). arXiv:hep-ph/ 0607190

115. E. Ma, Mod. Phys. Lett. A 22, 101 (2007). arXiv:hep-ph/ 0610342

116. L. Lavoura, H. Kuhbock, Mod. Phys. Lett. A 22, 181 (2007). arXiv:hep-ph/0610050

117. I. de Medeiros Varzielas, S.F. King, G.G. Ross, Phys. Lett. B 644, 153 (2007). arXiv:hep-ph/0512313

118. S. Morisi, M. Picariello, E. Torrente-Lujan, Phys. Rev. D 75, 075015 (2007). arXiv:hep-ph/0702034

119. Y. Koide, arXiv:hep-ph/0701018

120. M. Hirsch, A.S. Joshipura, S. Kaneko, J.W.F. Valle, arXiv:hep-ph/0703046

121. P.D. Carr, P.H. Frampton, arXiv:hep-ph/0701034

122. M.C. Chen, K.T. Mahanthappa, arXiv:0705.0714 [hep-ph]

123. P.H. Frampton, T.W. Kephart, arXiv:0706.1186 [hep-ph]

124. T. Kobayashi, H.P. Nilles, F. Ploger, S. Raby, M. Ratz, Nucl. Phys. B 768, 135 (2007). arXiv:hep-ph/0611020

125. E. Ma, Fizika B 14, 35 (2005). arXiv:hep-ph/0409288

126. C. Hagedorn, M. Lindner, F. Plentinger, Phys. Rev. D 74, 025007 (2006). arXiv:hep-ph/0604265

127. Y. Kajiyama, J. Kubo, H. Okada, Phys. Rev. D 75, 033001 (2007). arXiv:hep-ph/0610072

128. P.H. Frampton, T.W. Kephart, Phys. Rev. D 51, 1 (1995). arXiv:hep-ph/9409324

129. K.S. Babu, J. Kubo, Phys. Rev. D 71, 056006 (2005). arXiv:hep-ph/0411226

130. J. Kubo, Phys. Lett. B 622, 303 (2005). arXiv:hep-ph/0506043

131. S.L. Chen, E. Ma, Phys. Lett. B 620, 151 (2005). arXiv:hep-ph/0505064

132. E. Ma, Phys. Lett. B 632, 352 (2006). arXiv:hep-ph/0508231

133. Y. Cai, H.B. Yu, Phys. Rev. D 74, 115005 (2006). arXiv:hep-ph/ 0608022

134. H. Zhang, arXiv:hep-ph/0612214

135. Y. Koide, arXiv:0705.2275 [hep-ph]

136. M. Schmaltz, Phys. Rev. D 52, 1643 (1995). arXiv:hep-ph/ 9411383

137. W. Grimus, L. Lavoura, J. High Energy Phys. 0601, 018 (2006). arXiv:hep-ph/0509239
138. W. Grimus, L. Lavoura, arXiv:hep-ph/0611149

139. S.M. Barr, Phys. Rev. D 65, 096012 (2002). arXiv:hep-ph/ 0106241

140. S.F. King, Phys. Lett. B 439, 350 (1998). arXiv:hep-ph/ 9806440

141. S.F. King, Nucl. Phys. B 562, 57 (1999). arXiv:hep-ph/ 9904210

142. S.F. King, Nucl. Phys. B 576, 85 (2000). arXiv:hep-ph/ 9912492

143. S.F. King, J. High Energy Phys. 0209, 011 (2002). arXiv:hep-ph/0204360

144. F. Borzumati, A. Masiero, Phys. Rev. Lett. 57, 961 (1986)

145. L.J. Hall, V.A. Kostelecky, S. Raby, Nucl. Phys. B 267, 415 (1986)

146. S. Ferrara, L. Girardello, F. Palumbo, Phys. Rev. D 20, 403 (1979)

147. P. Fayet, Phys. Lett. B 69, 489 (1977)

148. E. Witten, Nucl. Phys. B 188, 513 (1981)

149. S. Dimopoulos, H. Georgi, Nucl. Phys. B 193, 150 (1981)

150. S. Weinberg, Phys. Rev. D 26, 287 (1982)

151. L. Alvarez-Gaume, M. Claudson, M.B. Wise, Nucl. Phys. B 207, 96 (1982)

152. G.F. Giudice, R. Rattazzi, Phys. Rep. 322, 419 (1999). arXiv:hep-ph/9801271

153. E. Cremmer, S. Ferrara, L. Girardello, A. Van Proeyen, Nucl. Phys. B 212, 413 (1983)

154. S.K. Soni, H.A. Weldon, Phys. Lett. B 126, 215 (1983)

155. V.S. Kaplunovsky, J. Louis, Phys. Lett. B 306, 269 (1993). arXiv:hep-th/9303040

156. A. Brignole, L.E. Ibanez, C. Munoz, Nucl. Phys. B 422, 125 (1994) [Erratum: Nucl. Phys. B 436, 747 (1995)]. arXiv:hep-ph/9308271

157. P.H. Chankowski, O. Lebedev, S. Pokorski, Nucl. Phys. B 717, 190 (2005). arXiv:hep-ph/0502076

158. S.P. Martin, arXiv:hep-ph/9709356

159. M. Shapkin, Nucl. Phys. Proc. Suppl. 169, 363 (2007)

160. G.G. Ross, O. Vives, Phys. Rev. D 67, 095013 (2003). arXiv:hep-ph/0211279

161. S.F. King, I.N.R. Peddie, G.G. Ross, L. Velasco-Sevilla, O. Vives, J. High Energy Phys. 0507, 049 (2005). arXiv:hep-ph/0407012

162. S. Antusch, S.F. King, M. Malinsky, arXiv:0708.1282 [hep-ph]

163. I. Masina, C.A. Savoy, Nucl. Phys. B 661, 365 (2003). arXiv:hep-ph/0211283

164. A. Masiero, S.K. Vempati, O. Vives, Nucl. Phys. B 649, 189 (2003). arXiv:hep-ph/0209303

165. M. Ciuchini, A. Masiero, P. Paradisi, L. Silvestrini, S.K. Vempati, O. Vives, arXiv:hep-ph/0702144

166. K. Hagiwara, S. Matsumoto, D. Haidt, C.S. Kim, Z. Phys C 64, 559 (1994) [Erratum: Z. Phys. C 68, 352 (1995)]. arXiv:hep-ph/9409380

167. W. Buchmuller, D. Wyler, Nucl. Phys. B 268, 621 (1986)

168. Z. Berezhiani, A. Rossi, Phys. Lett. B 535, 207 (2002). arXiv:hep-ph/0111137

169. A. Brignole, A. Rossi, Nucl. Phys. B 701, 3 (2004). arXiv: hep-ph/0404211

170. W.M. Yao et al. (Particle Data Group), J. Phys. G 33, 1 (2006)

171. M.S. Bilenky, A. Santamaria, Nucl. Phys. B 420, 47 (1994). arXiv:hep-ph/9310302

172. L. Lavoura, Eur. Phys. J. C 29, 191 (2003). arXiv:hep-ph/ 0302221

173. T. Inami, C.S. Lim, Prog. Theor. Phys. 65, 297 (1981) [Erratum: Prog. Theor. Phys. 65, 1772 (1981)]

174. A. Brignole, A. Rossi, Phys. Lett. B 566, 217 (2003). arXiv:hep-ph/0304081

175. P. Paradisi, J. High Energy Phys. 0608, 047 (2006). arXiv:hep-ph/0601100 
176. F. Jegerlehner, arXiv:hep-ph/0703125

177. G.A. Gonzalez-Sprinberg, A. Santamaria, J. Vidal, Nucl. Phys. B 582, 3 (2000). arXiv:hep-ph/0002203

178. J. Hisano, T. Moroi, K. Tobe, M. Yamaguchi, Phys. Rev. D 53, 2442 (1996). arXiv:hep-ph/9510309

179. A. Czarnecki, E. Jankowski, Phys. Rev. D 65, 113004 (2002). arXiv:hep-ph/0106237

180. M.L. Brooks et al. (MEGA Collaboration), Phys. Rev. Lett. 83, 1521 (1999). arXiv:hep-ex/9905013

181. K. Hayasaka et al. (Belle Collaboration), arXiv:0705.0650 [hep-ex]

182. B. Aubert et al. (BaBar Collaboration), Phys. Rev. Lett. 96, 041801 (2006). arXiv:hep-ex/0508012

183. S. Banerjee, arXiv:hep-ex/0702017

184. M. Raidal, A. Santamaria, Phys. Lett. B 421, 250 (1998). arXiv:hep-ph/9710389

185. K. Huitu, J. Maalampi, M. Raidal, A. Santamaria, Phys. Lett. B 430, 355 (1998). arXiv:hep-ph/9712249

186. B.C. Regan, E.D. Commins, C.J. Schmidt, D. DeMille, Phys. Rev. Lett. 88, 071805 (2002)

187. G.W. Bennett et al. (Muon g-2 Collaboration), Phys. Rev. D 73, 072003 (2006). arXiv:hep-ex/0602035

188. R. McNabb (Muon g-2 Collaboration), arXiv:hep-ex/ 0407008

189. J. Abdallah et al. (DELPHI Collaboration), Eur. Phys. J. C 35, 159 (2004). arXiv:hep-ex/0406010

190. K. Inami et al.(Belle Collaboration), Phys. Lett. B 551, 16 (2003). arXiv:hep-ex/0210066

191. B. Aubert et al. (BaBar Collaboration), Phys. Rev. Lett. 95, 041802 (2005). arXiv:hep-ex/0502032

192. S. Davidson, C. Pena-Garay, N. Rius, A. Santamaria, J. High Energy Phys. 0303, 011 (2003). arXiv:hep-ph/0302093

193. M. Acciarri et al. (L3 Collaboration), Phys. Lett. B 489, 81 (2000). arXiv:hep-ex/0005028

194. D. Bourilkov, Phys. Rev. D 64, 071701 (2001). arXiv:hep-ph/ 0104165

195. G. Abbiendi et al. (OPAL Collaboration), Eur. Phys. J. C 33, 173 (2004). arXiv:hep-ex/0309053

196. A. Pich, J.P. Silva, Phys. Rev. D 52, 4006 (1995). arXiv: hep-ph/9505327

197. S. Bergmann, Y. Grossman, D.M. Pierce, Phys. Rev. D 61, 053005 (2000). arXiv:hep-ph/9909390

198. M.C. Gonzalez-Garcia, J.W.F. Valle, Mod. Phys. Lett. A 7, 477 (1992)

199. A. De Gouvea, G.F. Giudice, A. Strumia, K. Tobe, Nucl. Phys. B 623, 395 (2002). arXiv:hep-ph/0107156

200. Z. Berezhiani, R.S. Raghavan, A. Rossi, Nucl. Phys. B 638, 62 (2002). arXiv:hep-ph/0111138

201. S. Davidson, D.C. Bailey, B.A. Campbell, Z. Phys. C 61, 613 (1994). arXiv:hep-ph/9309310

202. M. Herz, arXiv:hep-ph/0301079

203. S.L. Glashow, J. Iliopoulos, L. Maiani, Phys. Rev. D 2, 1285 (1970)

204. N. Cabibbo, Phys. Rev. Lett. 10, 531 (1963)

205. M. Kobayashi, T. Maskawa, Prog. Theor. Phys. 49, 652 (1973)

206. L. Wolfenstein, Phys. Rev. Lett. 51, 1945 (1983)

207. M. Battaglia et al., hep-ph/0304132

208. B. Pontecorvo, Sov. Phys. JETP 7, 172 (1958) [Zh. Eksp. Teor. Fiz. 34, 247 (1957)]

209. Z. Maki, M. Nakagawa, S. Sakata, Prog. Theor. Phys. 28, 870 (1962)

210. M. Maltoni, T. Schwetz, M.A. Tortola, J.W.F. Valle, New J. Phys. 6, 122 (2004). arXiv:hep-ph/0405172

211. G.L. Fogli, E. Lisi, A. Marrone, A. Palazzo, Prog. Part. Nucl. Phys. 57, 742 (2006). arXiv:hep-ph/0506083
212. S.T. Petcov, Sov. J. Nucl. Phys. 25, 340 (1977) [Yad. Fiz. 25, 641 (1977); Errata: Yad. Fiz. 25, 698 (1977), Yad. Fiz. 25, 1336 (1977)]

213. S. Weinberg, Phys. Rev. Lett. 43, 1566 (1979)

214. S.M. Bilenky, J. Hosek, S.T. Petcov, Phys. Lett. B 94, 495 (1980)

215. J. Schechter, J.W.F. Valle, Phys. Rev. D 22, 2227 (1980)

216. P. Minkowski, Phys. Lett. B 67, 421 (1977)

217. T. Yanagida, in Proceedings of the Workshop on the Baryon Number of the Universe and Unified Theories, Tsukuba, Japan, 13-14 February 1979

218. M. Gell-Mann, P. Ramond, R. Slansky, in Supergravity, ed. by P. van Nieuwenhuizen, D.Z. Freedman (North Holland, Amsterdam, 1979)

219. S.L. Glashow, NATO Adv. Study Inst. Ser. B Phys. 59, 687 (1979)

220. R.N. Mohapatra, G. Senjanovic, Phys. Rev. Lett. 44, 912 (1980)

221. M. Magg, C. Wetterich, Phys. Lett. B 94, 61 (1980)

222. G. Lazarides, Q. Shafi, C. Wetterich, Nucl. Phys. B 181, 287 (1981)

223. R.N. Mohapatra, G. Senjanovic, Phys. Rev. D 23, 165 (1981)

224. G.B. Gelmini, M. Roncadelli, Phys. Lett. B 99, 411 (1981)

225. E. Ma, Phys. Rev. Lett. 81, 1171 (1998). arXiv:hep-ph/9805219

226. R. Foot, H. Lew, X.G. He, G.C. Joshi, Z. Phys. C 44, 441 (1989)

227. W. Buchmuller, K. Hamaguchi, O. Lebedev, S. RamosSanchez, M. Ratz, Phys. Rev. Lett. 99, 021601 (2007). arXiv:hep-ph/0703078

228. J.R. Ellis, O. Lebedev, arXiv:0707.3419 [hep-ph]

229. J.R. Ellis, J. Hisano, S. Lola, M. Raidal, Nucl. Phys. B 621, 208 (2002). arXiv:hep-ph/0109125

230. S. Pascoli, S.T. Petcov, W. Rodejohann, Phys. Rev. D 68 , 093007 (2003). arXiv:hep-ph/0302054

231. J.A. Casas, A. Ibarra, F. Jimenez-Alburquerque, arXiv:hep-ph/ 0612289

232. J.A. Casas, A. Ibarra, Nucl. Phys. B 618, 171 (2001). arXiv: hep-ph/0103065

233. S. Lavignac, I. Masina, C.A. Savoy, Nucl. Phys. B 633, 139 (2002). arXiv:hep-ph/0202086

234. P.H. Frampton, S.L. Glashow, T. Yanagida, Phys. Lett. B 548, 119 (2002). arXiv:hep-ph/0208157

235. M. Raidal, A. Strumia, Phys. Lett. B 553, 72 (2003). arXiv:hep-ph/0210021

236. A. Ibarra, G.G. Ross, Phys. Lett. B 575, 279 (2003). arXiv:hep-ph/0307051

237. A. Ibarra, G.G. Ross, Phys. Lett. B 591, 285 (2004). arXiv:hep-ph/0312138

238. P.H. Chankowski, J.R. Ellis, S. Pokorski, M. Raidal, K. Turzynski, Nucl. Phys. B 690, 279 (2004)

239. S.T. Petcov, W. Rodejohann, T. Shindou, Y. Takanishi, Nucl. Phys. B 739, 208 (2006). arXiv:hep-ph/0510404

240. T. Endoh, T. Morozumi, T. Onogi, A. Purwanto, Phys. Rev. D 64, 013006 (2001) [Erratum: Phys. Rev. D 64, 059904 (2001)]. arXiv:hep-ph/0012345

241. S. Davidson, A. Ibarra, J. High Energy Phys. 0109, 013 (2001). arXiv:hep-ph/0104076

242. J.R. Ellis, J. Hisano, M. Raidal, Y. Shimizu, Phys. Rev. D 66 , 115013 (2002). arXiv:hep-ph/0206110

243. A. Ibarra, J. High Energy Phys. 0601, 064 (2006). arXiv:hep-ph/0511136

244. E. Ma, U. Sarkar, Phys. Rev. Lett. 80, 5716 (1998). arXiv:hep-ph/9802445

245. K. Huitu, J. Maalampi, M. Raidal, Nucl. Phys. B 420, 449 (1994). arXiv:hep-ph/9312235

246. K. Huitu, J. Maalampi, M. Raidal, Phys. Lett. B 328, 60 (1994). arXiv:hep-ph/9402219

247. M. Raidal, P.M. Zerwas, Eur. Phys. J. C 8, 479 (1999). arXiv:hep-ph/9811443 
248. T. Hambye, E. Ma, U. Sarkar, Nucl. Phys. B 602, 23 (2001). arXiv:hep-ph/0011192

249. A. Rossi, Phys. Rev. D 66, 075003 (2002). arXiv:hep-ph/ 0207006

250. K.S. Babu, C.N. Leung, J.T. Pantaleone, Phys. Lett. B 319, 191 (1993)

251. P.H. Chankowski, Z. Płuciennik, Phys. Lett. B 316, 312 (1993)

252. S. Antusch, M. Drees, J. Kersten, M. Lindner, M. Ratz, Phys. Lett. B 519, 238 (2001)

253. S. Antusch, M. Drees, J. Kersten, M. Lindner, M. Ratz, Phys. Lett. B 525, 130 (2002)

254. S. Antusch, J. Kersten, M. Lindner, M. Ratz, Nucl. Phys. B 674, 401 (2003)

255. P.H. Chankowski, W. Krolikowski, S. Pokorski, Phys. Lett. B 473, 109 (2000)

256. J.A. Casas, J.R. Espinosa, A. Ibarra, I. Navarro, Nucl. Phys. B 573, $652(2000)$

257. S. Antusch, J. Kersten, M. Lindner, M. Ratz, Phys. Lett. B 538, 87 (2002)

258. S. Antusch, J. Kersten, M. Lindner, M. Ratz, M.A. Schmidt, J. High Energy Phys. 0503, 024 (2005)

259. J.W. Mei, Phys. Rev. D 71, 073012 (2005)

260. S. Antusch, M. Ratz, J. High Energy Phys. 0207, 059 (2002)

261. N. Haba, N. Okamura, M. Sugiura, Prog. Theor. Phys. 103, 367 (2000)

262. N. Haba, Y. Matsui, N. Okamura, M. Sugiura, Eur. Phys. J. C 10, 677 (1999)

263. N. Haba, N. Okamura, Eur. Phys. J. C 14, 347 (2000)

264. J.R. Ellis, S. Lola, Phys. Lett. B 458, 310 (1999)

265. J.A. Casas, J.R. Espinosa, A. Ibarra, I. Navarro, Nucl. Phys. B 556, 3 (1999)

266. J.A. Casas, J.R. Espinosa, A. Ibarra, I. Navarro, Nucl. Phys. B 569, $82(2000)$

267. T. Miura, T. Shindou, E. Takasugi, Phys. Rev. D 66, 093002 (2002)

268. N. Haba, Y. Matsui, N. Okamura, Eur. Phys. J. C 17, 513 (2000)

269. S. Antusch, J. Kersten, M. Lindner, M. Ratz, Phys. Lett. B 544, 1 (2002)

270. T. Miura, T. Shindou, E. Takasugi, Phys. Rev. D 68, 093009 (2003)

271. S. Kanemura, K. Matsuda, T. Ota, T. Shindou, E. Takasugi, K. Tsumura, Phys. Rev. D 72, 093004 (2005)

272. T. Shindou, E. Takasugi, Phys. Rev. D 70, 013005 (2004)

273. S.T. Petcov, S. Profumo, Y. Takanishi, C.E. Yaguna, Nucl. Phys. B 676, 453 (2004)

274. J.R. Ellis, A. Hektor, M. Kadastik, K. Kannike, M. Raidal, Phys. Lett. B 631, 32 (2005). arXiv:hep-ph/0506122

275. S.T. Petcov, T. Shindou, Y. Takanishi, Nucl. Phys. B 738, 219 (2006)

276. H.B. Nielsen, Y. Takanishi, Nucl. Phys. B 636, 305 (2002)

277. R. Gonzalez Felipe, F.R. Joaquim, B.M. Nobre, Phys. Rev. D 70, 085009 (2004)

278. G.C. Branco, R. Gonzalez Felipe, F.R. Joaquim, B.M. Nobre, Phys. Lett. B 633, 336 (2006)

279. J.W. Mei, Z.Z. Xing, Phys. Rev. D 70, 053002 (2004)

280. S.T. Petcov, A.Y. Smirnov, Phys. Lett. B 322, 109 (1994). arXiv:hep-ph/9311204

281. M. Raidal, Phys. Rev. Lett. 93, 161801 (2004). arXiv:hep-ph/ 0404046

282. H. Minakata, A.Y. Smirnov, Phys. Rev. D 70, 073009 (2004). arXiv:hep-ph/0405088

283. Y. Kajiyama, M. Raidal, A. Strumia, arXiv:0705.4559 [hep-ph]

284. Euclid, Elements, Book 6, Definition 3. (A straight line is said to have been cut in extreme and mean ratio when, as the whole line is to the greater segment, so is the greater to the less)

285. Q. Duret, B. Machet, arXiv:0705.1237 [hep-ph]
286. B.C. Chauhan, M. Picariello, J. Pulido, E. Torrente-Lujan, arXiv:hep-ph/0605032

287. M. Picariello, arXiv:hep-ph/0611189

288. F. Caravaglios, S. Morisi, arXiv:hep-ph/0611078

289. C.L. Bennett et al. (WMAP Collaboration), Astrophys. J. Suppl. 148, 1 (2003). arXiv:astro-ph/0302207

290. M. Fukugita, T. Yanagida, Phys. Lett. B 174, 45 (1986)

291. V.A. Kuzmin, V.A. Rubakov, M.E. Shaposhnikov, Phys. Lett. B 155, 36 (1985)

292. J. Liu, G. Segre, Phys. Rev. D 48, 4609 (1993). arXiv: hep-ph/9304241

293. M. Flanz, E.A. Paschos, U. Sarkar, Phys. Lett. B 345, 248 (1995) [Erratum: Phys. Lett. B 382, 447 (1996)]. arXiv:hep-ph/ 9411366

294. L. Covi, E. Roulet, F. Vissani, Phys. Lett. B 384, 169 (1996). arXiv:hep-ph/9605319

295. R. Barbieri, P. Creminelli, A. Strumia, N. Tetradis, Nucl. Phys. B 575, 61 (2000). arXiv:hep-ph/9911315

296. A. Abada, S. Davidson, F.X. Josse-Michaux, M. Losada, A. Riotto, J. Cosmol. Astropart. Phys. 0604, 004 (2006). arXiv:hep-ph/0601083

297. E. Nardi, Y. Nir, E. Roulet, J. Racker, J. High Energy Phys. 0601, 164 (2006). arXiv:hep-ph/0601084

298. A. Abada, S. Davidson, A. Ibarra, F.X. Josse-Michaux, M. Losada, A. Riotto, J. High Energy Phys. 0609, 010 (2006). arXiv:hep-ph/0605281

299. T. Fujihara, S. Kaneko, S. Kang, D. Kimura, T. Morozumi, M. Tanimoto, Phys. Rev. D 72, 016006 (2005). arXiv: hep-ph/0505076

300. S. Pascoli, S.T. Petcov, A. Riotto, arXiv:hep-ph/0609125

301. G.C. Branco, A.J. Buras, S. Jager, S. Uhlig, A. Weiler, arXiv:hep-ph/0609067

302. G.C. Branco, R. Gonzalez Felipe, F.R. Joaquim, arXiv:hep-ph/ 0609297

303. S. Uhlig, arXiv:hep-ph/0612262

304. M. Plumacher, Z. Phys. C 74, 549 (1997). arXiv:hep-ph/ 9604229

305. G. Engelhard, Y. Grossman, E. Nardi, Y. Nir, arXiv:hep-ph/ 0612187

306. S. Davidson, A. Ibarra, Phys. Lett. B 535, 25 (2002). arXiv: hep-ph/0202239

307. K. Hamaguchi, H. Murayama, T. Yanagida, Phys. Rev. D 65, 043512 (2002). arXiv:hep-ph/0109030

308. W. Buchmuller, P. Di Bari, M. Plumacher, Nucl. Phys. B 643, 367 (2002). arXiv:hep-ph/0205349

309. G.F. Giudice, A. Notari, M. Raidal, A. Riotto, A. Strumia, Nucl. Phys. B 685, 89 (2004). arXiv:hep-ph/0310123

310. S. Blanchet, P. Di Bari, arXiv:hep-ph/0607330

311. S. Antusch, A.M. Teixeira, arXiv:hep-ph/0611232

312. J.R. Ellis, J.E. Kim, D.V. Nanopoulos, Phys. Lett. B 145, 181 (1984)

313. J.R. Ellis, D.V. Nanopoulos, S. Sarkar, Nucl. Phys. B 259, 175 (1985)

314. J.R. Ellis, D.V. Nanopoulos, K.A. Olive, S.J. Rey, Astropart. Phys. 4, 371 (1996). arXiv:hep-ph/9505438

315. T. Moroi, arXiv:hep-ph/9503210

316. M. Raidal, A. Strumia, K. Turzynski, Phys. Lett. B 609, 351 (2005) [Erratum: Phys. Lett. B 632, 752 (2006)]. arXiv:hep-ph/ 0408015

317. S. Blanchet, P. Di Bari, G.G. Raffelt, arXiv:hep-ph/0611337

318. W. Buchmuller, P. Di Bari, M. Plumacher, Phys. Lett. B 547, 128 (2002). arXiv:hep-ph/0209301

319. S. Davidson, arXiv:hep-ph/0304120

320. E.W. Kolb, M.S. Turner, Front. Phys. 69, 1 (1990)

321. W. Fischler, G.F. Giudice, R.G. Leigh, S. Paban, Phys. Lett. B 258, 45 (1991) 
322. W. Buchmuller, T. Yanagida, Phys. Lett. B 302, 240 (1993)

323. W. Buchmuller, P. Di Bari, M. Plumacher, Nucl. Phys. B 665, 445 (2003). arXiv:hep-ph/0302092

324. W. Buchmuller, P. Di Bari, M. Plumacher, Ann. Phys. 315, 305 (2005). arXiv:hep-ph/0401240

325. W. Buchmuller, P. Di Bari, M. Plumacher, New J. Phys. 6, 105 (2004). arXiv:hep-ph/0406014

326. T. Hambye, Y. Lin, A. Notari, M. Papucci, A. Strumia, Nucl. Phys. B 695, 169 (2004). arXiv:hep-ph/0312203

327. WMAP Collaboration, D.N. Spergel et al., arXiv:astro-ph/ 0603449

328. G.C. Branco, T. Morozumi, B.M. Nobre, M.N. Rebelo, Nucl. Phys. B 617, 475 (2001). arXiv:hep-ph/0107164

329. J.R. Ellis, M. Raidal, Nucl. Phys. B 643, 229 (2002). arXiv:hep-ph/0206174

330. S. Davidson, J. Garayoa, F. Palorini, N. Rius, arXiv:0705.1503 [hep-ph]

331. S. Antusch, S.F. King, A. Riotto, J. Cosmol. Astropart. Phys. 0611, 011 (2006). arXiv:hep-ph/0609038

332. A. Pilaftsis, Phys. Rev. D 56, 5431 (1997). arXiv:hep-ph/ 9707235

333. A. Pilaftsis, Nucl. Phys. B 504, 61 (1997). arXiv:hep-ph/ 9702393

334. J.R. Ellis, M. Raidal, T. Yanagida, Phys. Lett. B 546, 228 (2002). arXiv:hep-ph/0206300

335. A. Pilaftsis, T.E.J. Underwood, Nucl. Phys. B 692, 303 (2004). arXiv:hep-ph/0309342

336. F. del Aguila, J.A. Aguilar-Saavedra, R. Pittau, J. Phys. Conf. Ser. 53, 506 (2006). arXiv:hep-ph/0606198

337. A. Pilaftsis, T.E.J. Underwood, Phys. Rev. D 72, 113001 (2005). arXiv:hep-ph/0506107

338. W. Buchmuller, C. Greub, Nucl. Phys. B 381, 109 (1992)

339. J. Gluza, Phys. Rev. D 66, 010001 (2002). arXiv:hep-ph/ 0201002

340. T. Hambye, G. Senjanovic, Phys. Lett. B 582, 73 (2004). arXiv:hep-ph/0307237

341. T. Hambye, M. Raidal, A. Strumia, Phys. Lett. B 632, 667 (2006). arXiv:hep-ph/0510008

342. S. Antusch, S.F. King, Phys. Lett. B 597, 199 (2004). arXiv:hep-ph/0405093

343. S. Antusch, Phys. Rev. D 76, 023512 (2007). arXiv:0704.1591 [hep-ph]

344. Y. Grossman, T. Kashti, Y. Nir, E. Roulet, Phys. Rev. Lett. 91, 251801 (2003). arXiv:hep-ph/0307081

345. G. D'Ambrosio, G.F. Giudice, M. Raidal, Phys. Lett. B 575, 75 (2003). arXiv:hep-ph/0308031

346. G. D'Ambrosio, T. Hambye, A. Hektor, M. Raidal, A. Rossi, Phys. Lett. B 604, 199 (2004). arXiv:hep-ph/0407312

347. H. Murayama, H. Suzuki, T. Yanagida, J. Yokoyama, Phys. Rev. Lett. 70, 1912 (1993)

348. H. Murayama, T. Yanagida, Phys. Lett. B 322, 349 (1994). arXiv:hep-ph/9310297

349. J.R. Ellis, M. Raidal, T. Yanagida, Phys. Lett. B 581, 9 (2004). arXiv:hep-ph/0303242

350. S. Antusch, M. Bastero-Gil, S.F. King, Q. Shafi, Phys. Rev. D 71, 083519 (2005). arXiv:hep-ph/0411298

351. K. Dick, M. Lindner, M. Ratz, D. Wright, Phys. Rev. Lett. 84 , 4039 (2000). arXiv:hep-ph/9907562

352. D. Atwood, S. Bar-Shalom, A. Soni, Phys. Lett. B 635, 112 (2006). arXiv:hep-ph/0502234

353. T. Asaka, K. Hamaguchi, M. Kawasaki, T. Yanagida, Phys. Lett. B 464, 12 (1999). arXiv:hep-ph/9906366

354. G.F. Giudice, M. Peloso, A. Riotto, I. Tkachev, J. High Energy Phys. 9908, 014 (1999). arXiv:hep-ph/9905242

355. M. Endo, F. Takahashi, T.T. Yanagida, Phys. Rev. D 74, 123523 (2006). arXiv:hep-ph/0611055
356. J.S. Schwinger, Ann. Phys. 2, 407 (1957)

357. H. Georgi, S.L. Glashow, Phys. Rev. Lett. 32, 438 (1974)

358. P.A.M. Dirac, Phys. Rev. 74, 817 (1948)

359. G. 't Hooft, Nucl. Phys. B 79, 276 (1974)

360. A.M. Polyakov, JETP Lett. 20, 194 (1974) [Pisma Zh. Eksp. Teor. Fiz. 20, 430 (1974)]

361. J.C. Pati, A. Salam, Phys. Rev. D 10, 275 (1974)

362. F. Wilczek, A. Zee, Phys. Rev. Lett. 43, 1571 (1979)

363. K. Kobayashi et al. (Super-Kamiokande Collaboration), Phys. Rev. D 72, 052007 (2005). arXiv:hep-ex/0502026

364. A.J. Buras, J.R. Ellis, M.K. Gaillard, D.V. Nanopoulos, Nucl. Phys. B 135, 66 (1978)

365. S. Dimopoulos, S. Raby, F. Wilczek, Phys. Rev. D 24, 1681 (1981)

366. L.E. Ibanez, G.G. Ross, Phys. Lett. B 105, 439 (1981)

367. M.B. Einhorn, D.R.T. Jones, Nucl. Phys. B 196, 475 (1982)

368. W.J. Marciano, G. Senjanovic, Phys. Rev. D 25, 3092 (1982)

369. K. Inoue, A. Kakuto, H. Komatsu, S. Takeshita, Prog. Theor. Phys. 68, 927 (1982) [Erratum: Prog. Theor. Phys. 70, 330 (1983)]

370. L. Alvarez-Gaume, J. Polchinski, M.B. Wise, Nucl. Phys. B 221, 495 (1983)

371. N. Sakai, T. Yanagida, Nucl. Phys. B 197, 533 (1982)

372. J. Hisano, H. Murayama, T. Yanagida, Nucl. Phys. B 402, 46 (1993). arXiv:hep-ph/9207279

373. V. Lucas, S. Raby, Phys. Rev. D 55, 6986 (1997). arXiv: hep-ph/9610293

374. T. Goto, T. Nihei, Phys. Rev. D 59, 115009 (1999). arXiv: hep-ph/9808255

375. H. Murayama, A. Pierce, Phys. Rev. D 65, 055009 (2002). arXiv:hep-ph/0108104

376. B. Bajc, P. Fileviez Perez, G. Senjanovic, Phys. Rev. D 66 , 075005 (2002). arXiv:hep-ph/0204311

377. B. Bajc, P. Fileviez Perez, G. Senjanovic, arXiv:hep-ph/ 0210374

378. D. Emmanuel-Costa, S. Wiesenfeldt, Nucl. Phys. B 661, 62 (2003). arXiv:hep-ph/0302272

379. I. Dorsner, P.F. Perez, Nucl. Phys. B 723, 53 (2005). arXiv:hep-ph/0504276

380. B. Bajc, G. Senjanovic, arXiv:hep-ph/0612029

381. R.J. Wilkes, arXiv:hep-ex/0507097

382. K. Cheung, C.W. Chiang, Phys. Rev. D 71, 095003 (2005). arXiv:hep-ph/0501265

383. W.Y. Keung, G. Senjanovic, Phys. Rev. Lett. 50, 1427 (1983)

384. R.N. Mohapatra, J.C. Pati, Phys. Rev. D 11, 2558 (1975)

385. G. Senjanovic, R.N. Mohapatra, Phys. Rev. D 12, 1502 (1975)

386. G. Senjanovic, Nucl. Phys. B 153, 334 (1979)

387. R.N. Mohapatra, Phys. Rev. D 34, 3457 (1986)

388. A. Font, L.E. Ibanez, F. Quevedo, Phys. Lett. B 228, 79 (1989)

389. S.P. Martin, Phys. Rev. D 46, 2769 (1992). arXiv:hep-ph/ 9207218

390. C.S. Aulakh, K. Benakli, G. Senjanovic, Phys. Rev. Lett. 79, 2188 (1997). arXiv:hep-ph/9703434

391. C.S. Aulakh, A. Melfo, G. Senjanovic, Phys. Rev. D 57, 4174 (1998). arXiv:hep-ph/9707256

392. C.S. Aulakh, A. Melfo, A. Rasin, G. Senjanovic, Phys. Lett. B 459, 557 (1999). arXiv:hep-ph/9902409

393. N.G. Deshpande, E. Keith, P.B. Pal, Phys. Rev. D 46, 2261 (1993)

394. N.G. Deshpande, E. Keith, P.B. Pal, Phys. Rev. D 47, 2892 (1993). arXiv:hep-ph/9211232

395. R.N. Mohapatra, B. Sakita, Phys. Rev. D 21, 1062 (1980)

396. F. Wilczek, A. Zee, Phys. Rev. D 25, 553 (1982)

397. R. Slansky, Phys. Rep. 79, 1 (1981)

398. P. Nath, R.M. Syed, Nucl. Phys. B 618, 138 (2001). arXiv:hep-th/0109116 
399. C.S. Aulakh, A. Girdhar, Int. J. Mod. Phys. A 20, 865 (2005). arXiv:hep-ph/0204097

400. C.H. Albright, S.M. Barr, Phys. Rev. Lett. 85, 244 (2000). arXiv:hep-ph/0002155

401. K.S. Babu, J.C. Pati, F. Wilczek, Nucl. Phys. B 566, 33 (2000). arXiv:hep-ph/9812538

402. T. Blazek, S. Raby, K. Tobe, Phys. Rev. D 60, 113001 (1999). arXiv:hep-ph/9903340

403. E. Witten, Phys. Lett. B 91, 81 (1980)

404. B. Bajc, G. Senjanovic, Phys. Lett. B 610, 80 (2005). arXiv:hep-ph/0411193

405. B. Bajc, G. Senjanovic, Phys. Rev. Lett. 95, 261804 (2005). arXiv:hep-ph/0507169

406. K.S. Babu, R.N. Mohapatra, Phys. Rev. Lett. 70, 2845 (1993). arXiv:hep-ph/9209215

407. H. Fusaoka, Y. Koide, Phys. Rev. D 57, 3986 (1998). arXiv:hep-ph/9712201

408. C.R. Das, M.K. Parida, Eur. Phys. J. C 20, 121 (2001). arXiv:hep-ph/0010004

409. K. Matsuda, Y. Koide, T. Fukuyama, Phys. Rev. D 64, 053015 (2001). arXiv:hep-ph/0010026

410. K. Matsuda, Y. Koide, T. Fukuyama, H. Nishiura, Phys. Rev. D 65, 033008 (2002) [Erratum: Phys. Rev. D 65, 079904 (2002)]. arXiv:hep-ph/0108202

411. T. Fukuyama, N. Okada, J. High Energy Phys. 0211, 011 (2002). arXiv:hep-ph/0205066

412. H.S. Goh, R.N. Mohapatra, S. Nasri, Phys. Rev. D 70, 075022 (2004). arXiv:hep-ph/0408139

413. B. Bajc, G. Senjanović, F. Vissani, arXiv:hep-ph/0110310

414. B. Bajc, G. Senjanović, F. Vissani, Phys. Rev. Lett. 90, 051802 (2003). arXiv:hep-ph/0210207

415. B. Bajc, G. Senjanović, F. Vissani, Phys. Rev. D 70, 093002 (2004). arXiv:hep-ph/0402140

416. B. Brahmachari, R.N. Mohapatra, Phys. Rev. D 58, 015001 (1998). arXiv:hep-ph/9710371

417. H.S. Goh, R.N. Mohapatra, S.P. Ng, Phys. Lett. B 570, 215 (2003). arXiv:hep-ph/0303055

418. H.S. Goh, R.N. Mohapatra, S.P. Ng, Phys. Rev. D 68, 115008 (2003). arXiv:hep-ph/0308197

419. B. Dutta, Y. Mimura, R.N. Mohapatra, Phys. Rev. D 69, 115014 (2004). arXiv:hep-ph/0402113

420. S. Bertolini, M. Malinsky, Phys. Rev. D 72, 055021 (2005). arXiv:hep-ph/0504241

421. K.S. Babu, C. Macesanu, Phys. Rev. D 72, 115003 (2005). arXiv:hep-ph/0505200

422. T.E. Clark, T.K. Kuo, N. Nakagawa, Phys. Lett. B 115, 26 (1982)

423. C.S. Aulakh, R.N. Mohapatra, Phys. Rev. D 28, 217 (1983)

424. D.G. Lee, R.N. Mohapatra, Phys. Rev. D 51, 1353 (1995). arXiv:hep-ph/9406328

425. C.S. Aulakh, B. Bajc, A. Melfo, G. Senjanović, F. Vissani, Phys. Lett. B 588, 196 (2004). arXiv:hep-ph/0306242

426. C.S. Aulakh, arXiv:hep-ph/0506291

427. B. Bajc, A. Melfo, G. Senjanović, F. Vissani, Phys. Lett. B 634, 272 (2006). arXiv:hep-ph/0511352

428. C.S. Aulakh, S.K. Garg, arXiv:hep-ph/0512224

429. S. Bertolini, T. Schwetz, M. Malinsky, Phys. Rev. D 73, 115012 (2006). arXiv:hep-ph/0605006

430. L.J. Hall, R. Rattazzi, U. Sarid, Phys. Rev. D 50, 7048 (1994). arXiv:hep-ph/9306309

431. X.G. He, S. Meljanac, Phys. Rev. D 41, 1620 (1990)

432. D.G. Lee, Phys. Rev. D 49, 1417 (1994)

433. B. Bajc, A. Melfo, G. Senjanović, F. Vissani, Phys. Rev. D 70, 035007 (2004). arXiv:hep-ph/0402122

434. C.S. Aulakh, A. Girdhar, Nucl. Phys. B 711, 275 (2005). arXiv:hep-ph/0405074
435. T. Fukuyama, A. Ilakovac, T. Kikuchi, S. Meljanac, N. Okada, Eur. Phys. J. C 42, 191 (2005). arXiv:hep-ph/0401213

436. T. Fukuyama, A. Ilakovac, T. Kikuchi, S. Meljanac, N. Okada, J. Math. Phys. 46, 033505 (2005). arXiv:hep-ph/0405300

437. T. Fukuyama, A. Ilakovac, T. Kikuchi, S. Meljanac, N. Okada, Phys. Rev. D 72, 051701 (2005). arXiv:hep-ph/0412348

438. C.S. Aulakh, arXiv:hep-ph/0501025

439. C.S. Aulakh, Phys. Rev. D 72, 051702 (2005)

440. D.G. Lee, R.N. Mohapatra, Phys. Lett. B 324, 376 (1994). arXiv:hep-ph/9310371

441. K.S. Babu, I. Gogoladze, Z. Tavartkiladze, arXiv:hep-ph/ 0612315

442. H.S. Goh, R.N. Mohapatra, S. Nasri, S.P. Ng, Phys. Lett. B 587, 105 (2004). arXiv:hep-ph/0311330

443. T. Fukuyama, A. Ilakovac, T. Kikuchi, S. Meljanac, N. Okada, J. High Energy Phys. 0409, 052 (2004). arXiv:hep-ph/0406068

444. S. Bertolini, M. Frigerio, M. Malinsky, Phys. Rev. D 70, 095002 (2004). arXiv:hep-ph/0406117

445. W.M. Yang, Z.G. Wang, Nucl. Phys. B 707, 87 (2005). arXiv:hep-ph/0406221

446. B. Dutta, Y. Mimura, R.N. Mohapatra, Phys. Lett. B 603, 35 (2004). arXiv:hep-ph/0406262

447. B. Dutta, Y. Mimura, R.N. Mohapatra, Phys. Rev. Lett. 94, 091804 (2005). arXiv:hep-ph/0412105

448. B. Dutta, Y. Mimura, R.N. Mohapatra, Phys. Rev. D 72, 075009 (2005). arXiv:hep-ph/0507319

449. W. Grimus, H. Kuhbock, arXiv:hep-ph/0607197

450. W. Grimus, H. Kuhbock, arXiv:hep-ph/0612132

451. C.S. Aulakh, arXiv:hep-ph/0602132

452. L. Lavoura, H. Kuhbock, W. Grimus, arXiv:hep-ph/0603259

453. C.S. Aulakh, arXiv:hep-ph/0607252

454. C.S. Aulakh, S.K. Garg, arXiv:hep-ph/0612021

455. B. Bajc, A. Melfo, G. Senjanović, F. Vissani, Phys. Rev. D 73, 055001 (2006). arXiv:hep-ph/0510139

456. F. Vissani, A.Y. Smirnov, Phys. Lett. B 341, 173 (1994). arXiv:hep-ph/9405399

457. N. Arkani-Hamed, S. Dimopoulos, J. High Energy Phys. 0506, 073 (2005). arXiv:hep-th/0405159

458. G.F. Giudice, A. Romanino, Nucl. Phys. B 699, 65 (2004) [Erratum: Nucl. Phys. B 706, 65 (2005)]. arXiv:hep-ph/0406088

459. B. Bajc, AIP Conf. Proc. 805, 326 (2006). arXiv:hep-ph/ 0602166

460. A. Arvanitaki, C. Davis, P.W. Graham, A. Pierce, J.G. Wacker, Phys. Rev. D 72, 075011 (2005). arXiv:hep-ph/0504210

461. K. Yoshioka, Mod. Phys. Lett. A 15, 29 (2000). arXiv: hep-ph/9904433

462. M. Bando, T. Kobayashi, T. Noguchi, K. Yoshioka, Phys. Rev. D 63, 113017 (2001). arXiv:hep-ph/0008120

463. A. Neronov, Phys. Rev. D 65, 044004 (2002). arXiv:gr-qc/ 0106092

464. N. Arkani-Hamed, L.J. Hall, D.R. Smith, N. Weiner, Phys. Rev. D 61, 116003 (2000). arXiv:hep-ph/9909326

465. K.R. Dienes, E. Dudas, T. Gherghetta, Phys. Lett. B 436, 55 (1998). arXiv:hep-ph/9803466

466. K.R. Dienes, E. Dudas, T. Gherghetta, Nucl. Phys. B 537, 47 (1999). arXiv:hep-ph/9806292

467. N. Arkani-Hamed, M. Schmaltz, Phys. Rev. D 61, 033005 (2000). arXiv:hep-ph/9903417

468. T. Gherghetta, A. Pomarol, Nucl. Phys. B 586, 141 (2000). arXiv:hep-ph/0003129

469. N. Arkani-Hamed, S. Dimopoulos, G.R. Dvali, Phys. Lett. B 429, 263 (1998). arXiv:hep-ph/9803315

470. I. Antoniadis, N. Arkani-Hamed, S. Dimopoulos, G.R. Dvali, Phys. Lett. B 436, 257 (1998). arXiv:hep-ph/9804398

471. N. Arkani-Hamed, S. Dimopoulos, G.R. Dvali, Phys. Rev. D 59, 086004 (1999). arXiv:hep-ph/9807344 
472. G. Barenboim, G.C. Branco, A. de Gouvea, M.N. Rebelo, Phys. Rev. D 64, 073005 (2001). arXiv:hep-ph/0104312

473. T. Appelquist, H.C. Cheng, B.A. Dobrescu, Phys. Rev. D 64, 035002 (2001). arXiv:hep-ph/0012100

474. M.V. Libanov, S.V. Troitsky, Nucl. Phys. B 599, 319 (2001). arXiv:hep-ph/0011095

475. J.M. Frere, M.V. Libanov, S.V. Troitsky, Phys. Lett. B 512, 169 (2001). arXiv:hep-ph/0012306

476. J.M. Frere, M.V. Libanov, S.V. Troitsky, J. High Energy Phys. 0111, 025 (2001). arXiv:hep-ph/0110045

477. M.V. Libanov, E.Y. Nougaev, J. High Energy Phys. 0204, 055 (2002). arXiv:hep-ph/0201162

478. G.R. Dvali, M.A. Shifman, Phys. Lett. B 475, 295 (2000). arXiv:hep-ph/0001072

479. P.Q. Hung, Phys. Rev. D 67, 095011 (2003). arXiv:hep-ph/ 0210131

480. D.E. Kaplan, T.M.P. Tait, J. High Energy Phys. 0006, 020 (2000). arXiv:hep-ph/0004200

481. D.E. Kaplan, T.M.P. Tait, J. High Energy Phys. 0111, 051 (2001). arXiv:hep-ph/0110126

482. M. Kakizaki, M. Yamaguchi, Prog. Theor. Phys. 107, 433 (2002). arXiv:hep-ph/0104103

483. C.H. Chang, W.F. Chang, J.N. Ng, Phys. Lett. B 558, 92 (2003). arXiv:hep-ph/0301271

484. S. Nussinov, R. Shrock, Phys. Lett. B 526, 137 (2002). arXiv:hep-ph/0101340

485. R. Jackiw, C. Rebbi, Phys. Rev. D 13, 3398 (1976)

486. E.A. Mirabelli, M. Schmaltz, Phys. Rev. D 61, 113011 (2000). arXiv:hep-ph/9912265

487. G.C. Branco, A. de Gouvea, M.N. Rebelo, Phys. Lett. B 506, 115 (2001). arXiv:hep-ph/0012289

488. P.Q. Hung, M. Seco, Nucl. Phys. B 653, 123 (2003). arXiv:hep-ph/0111013

489. J.M. Frere, G. Moreau, E. Nezri, Phys. Rev. D 69, 033003 (2004). arXiv:hep-ph/0309218

490. H.V. Klapdor-Kleingrothaus, U. Sarkar, Phys. Lett. B 541, 332 (2002). arXiv:hep-ph/0201226

491. M. Gogberashvili, Int. J. Mod. Phys. D 11, 1635 (2002). arXiv:hep-ph/9812296

492. L. Randall, R. Sundrum, Phys. Rev. Lett. 83, 3370 (1999). arXiv:hep-ph/9905221

493. Y. Grossman, M. Neubert, Phys. Lett. B 474, 361 (2000). arXiv:hep-ph/9912408

494. H. Davoudiasl, J.L. Hewett, T.G. Rizzo, Phys. Lett. B 473, 43 (2000). arXiv:hep-ph/9911262

495. A. Pomarol, Phys. Lett. B 486, 153 (2000). arXiv:hep-ph/ 9911294

496. S. Chang, J. Hisano, H. Nakano, N. Okada, M. Yamaguchi, Phys. Rev. D 62, 084025 (2000). arXiv:hep-ph/9912498

497. B. Bajc, G. Gabadadze, Phys. Lett. B 474, 282 (2000). arXiv:hep-th/9912232

498. A. Pomarol, Phys. Rev. Lett. 85, 4004 (2000). arXiv:hep-ph/ 0005293

499. L. Randall, M.D. Schwartz, J. High Energy Phys. 0111, 003 (2001). arXiv:hep-th/0108114

500. L. Randall, M.D. Schwartz, Phys. Rev. Lett. 88, 081801 (2002). arXiv:hep-th/0108115

501. W.D. Goldberger, I.Z. Rothstein, Phys. Rev. D 68, 125011 (2003). arXiv:hep-th/0208060

502. K.W. Choi, I.W. Kim, Phys. Rev. D 67, 045005 (2003). arXiv:hep-th/0208071

503. K. Agashe, A. Delgado, R. Sundrum, Ann. Phys. 304, 145 (2003). arXiv:hep-ph/0212028

504. K. Agashe, G. Servant, Phys. Rev. Lett. 93, 231805 (2004). arXiv:hep-ph/0403143

505. K. Agashe, G. Servant, J. Cosmol. Astropart. Phys. 0502, 002 (2005). arXiv:hep-ph/0411254
506. S.J. Huber, Q. Shafi, Phys. Lett. B 498, 256 (2001). arXiv: hep-ph/0010195

507. S.J. Huber, Nucl. Phys. B 666, 269 (2003). arXiv:hep-ph/ 0303183

508. S. Chang, C.S. Kim, M. Yamaguchi, Phys. Rev. D 73, 033002 (2006). arXiv:hep-ph/0511099

509. S.J. Huber, Q. Shafi, Phys. Lett. B 544, 295 (2002). arXiv: hep-ph/0205327

510. S.J. Huber, Q. Shafi, Phys. Lett. B 583, 293 (2004). arXiv: hep-ph/0309252

511. S.J. Huber, Q. Shafi, Phys. Lett. B 512, 365 (2001). arXiv:hep-ph/0104293

512. G. Moreau, J.I. Silva-Marcos, J. High Energy Phys. 0601, 048 (2006). arXiv:hep-ph/0507145

513. G. Moreau, J.I. Silva-Marcos, J. High Energy Phys. 0603, 090 (2006). arXiv:hep-ph/0602155

514. A. Ilakovac, A. Pilaftsis, Nucl. Phys. B 437, 491 (1995). arXiv: hep-ph/9403398

515. C.S. Kim, J.D. Kim, J.h. Song, Phys. Rev. D 67, 015001 (2003). arXiv:hep-ph/0204002

516. F. del Aguila, M. Perez-Victoria, J. Santiago, Phys. Lett. B 492, 98 (2000). arXiv:hep-ph/0007160

517. A. Delgado, A. Pomarol, M. Quiros, J. High Energy Phys. 0001, 030 (2000). arXiv:hep-ph/9911252

518. T.G. Rizzo, J.D. Wells, Phys. Rev. D 61, 016007 (2000). arXiv:hep-ph/9906234

519. I. Antoniadis, K. Benakli, M. Quiros, Phys. Lett. B 460, 176 (1999). arXiv:hep-ph/9905311

520. P. Nath, Y. Yamada, M. Yamaguchi, Phys. Lett. B 466, 100 (1999). arXiv:hep-ph/9905415

521. T.G. Rizzo, Phys. Rev. D 61, 055005 (2000). arXiv:hep-ph/ 9909232

522. G. Burdman, Phys. Lett. B 590, 86 (2004). arXiv:hep-ph/ 0310144

523. K. Agashe, G. Perez, A. Soni, Phys. Rev. Lett. 93, 201804 (2004). arXiv:hep-ph/0406101

524. K. Agashe, G. Perez, A. Soni, Phys. Rev. D 71, 016002 (2005). arXiv:hep-ph/0408134

525. K. Agashe, A.E. Blechman, F. Petriello, Phys. Rev. D 74, 053011 (2006). arXiv:hep-ph/0606021

526. K. Agashe, G. Perez, A. Soni, arXiv:hep-ph/0606293

527. F. del Aguila, M. Perez-Victoria, J. Santiago, J. High Energy Phys. 0302, 051 (2003). arXiv:hep-th/0302023

528. M. Carena, T.M.P. Tait, C.E.M. Wagner, Acta Phys. Pol. B 33, 2355 (2002). arXiv:hep-ph/0207056

529. M. Carena, E. Ponton, T.M.P. Tait, C.E.M. Wagner, Phys. Rev. D 67, 096006 (2003). arXiv:hep-ph/0212307

530. M. Carena, A. Delgado, E. Ponton, T.M.P. Tait, C.E.M. Wagner, Phys. Rev. D 68, 035010 (2003). arXiv:hep-ph/0305188

531. M. Carena, A. Delgado, E. Ponton, T.M.P. Tait, C.E.M. Wagner, Phys. Rev. D 71, 015010 (2005). arXiv:hep-ph/0410344

532. K. Agashe, A. Delgado, M.J. May, R. Sundrum, J. High Energy Phys. 0308, 050 (2003). arXiv:hep-ph/0308036

533. K. Agashe, R. Contino, L. Da Rold, A. Pomarol, Phys. Lett. B 641, 62 (2006). arXiv:hep-ph/0605341

534. A. Djouadi, G. Moreau, F. Richard, arXiv:hep-ph/0610173

535. R.S. Chivukula, H. Georgi, Phys. Lett. B 188, 99 (1987)

536. L.J. Hall, L. Randall, Phys. Rev. Lett. 65, 2939 (1990)

537. G. D'Ambrosio, G.F. Giudice, G. Isidori, A. Strumia, Nucl. Phys. B 645, 155 (2002). hep-ph/0207036

538. A.V. Manohar, M.B. Wise, Phys. Rev. D 74, 035009 (2006). hep-ph/0606172

539. B. Grinstein, V. Cirigliano, G. Isidori, M.B. Wise, Nucl. Phys. B 763, 35 (2007). hep-ph/0608123

540. A.J. Buras, P. Gambino, M. Gorbahn, S. Jager, L. Silvestrini, Phys. Lett. B 500, 161 (2001). hep-ph/0007085 
541. M. Bona et al. (UTfit Collaboration), J. High Energy Phys. 0603, 080 (2006). hep-ph/0509219

542. V. Cirigliano, B. Grinstein, G. Isidori, M.B. Wise, Nucl. Phys. B 728, 121 (2005). hep-ph/0507001

543. V. Cirigliano, B. Grinstein, Nucl. Phys. B 752, 18 (2006). hep-ph/0601111

544. S. Davidson, F. Palorini, Phys. Lett. B 642, 72 (2006). hep-ph/0607329

545. S. Pascoli, S.T. Petcov, C.E. Yaguna, Phys. Lett. B 564, 241 (2003). hep-ph/0301095

546. V. Cirigliano, G. Isidori, V. Porretti, Nucl. Phys. B 763, 228 (2007). hep-ph/0608123

547. S. Blanchet, P. Di Bari, J. Cosmol. Astropart. Phys. 0606, 023 (2006). hep-ph/0603107

548. M. Grassi (MEG Collaboration), Nucl. Phys. Proc. Suppl. 149, 369 (2005)

549. W. Grimus, M.N. Rebelo, Phys. Rep. 281, 239 (1997). arXiv:hep-ph/9506272

550. F. Englert, R. Brout, Phys. Rev. Lett. 13, 321 (1964)

551. G.S. Guralnik, C.R. Hagen, T.W.B. Kibble, Phys. Rev. Lett. 13, 585 (1964)

552. P.W. Higgs, Phys. Lett. 12, 132 (1964)

553. P.W. Higgs, Phys. Rev. 145, 1156 (1966)

554. T.D. Lee, Phys. Rev. D 8, 1226 (1973)

555. W. Bernreuther, O. Nachtmann, Eur. Phys. J. C 9, 319 (1999). arXiv:hep-ph/9812259

556. G.C. Branco, L. Lavoura, J.P. Silva, CP Violation (Clarendon, Oxford, 1999)

557. G.C. Branco, M.N. Rebelo, J.I. Silva-Marcos, Phys. Lett. B 614, 187 (2005). arXiv:hep-ph/0502118

558. J. Bernabeu, G.C. Branco, M. Gronau, Phys. Lett. B 169, 243 (1986)

559. L. Lavoura, J.P. Silva, Phys. Rev. D 50, 4619 (1994). arXiv:hep-ph/9404276

560. F.J. Botella, J.P. Silva, Phys. Rev. D 51, 3870 (1995). arXiv:hep-ph/9411288

561. S. Davidson, H.E. Haber, Phys. Rev. D 72, 035004 (2005) [Erratum: Phys. Rev. D 72, 099902 (2005)]. arXiv:hep-ph/0504050

562. J.F. Gunion, H.E. Haber, Phys. Rev. D 72, 095002 (2005). arXiv:hep-ph/0506227

563. I.P. Ivanov, Phys. Lett. B 632, 360 (2006). arXiv: hep-ph/0507132

564. H.E. Haber, D. O’Neil, Phys. Rev. D 74, 015018 (2006). arXiv:hep-ph/0602242

565. G.C. Branco, J.M. Gerard, W. Grimus, Phys. Lett. B 136, 383 (1984)

566. S.L. Glashow, S. Weinberg, Phys. Rev. D 15, 1958 (1977)

567. E.A. Paschos, Phys. Rev. D 15, 1966 (1977)

568. G.C. Branco, M.N. Rebelo, Phys. Lett. B 160, 117 (1985)

569. L. Lavoura, Phys. Rev. D 50, 7089 (1994). arXiv:hep-ph/ 9405307

570. I.F. Ginzburg, M. Krawczyk, Phys. Rev. D 72, 115013 (2005). arXiv:hep-ph/0408011

571. J.F. Gunion, H.E. Haber, Phys. Rev. D 67, 075019 (2003). arXiv:hep-ph/0207010

572. S. Weinberg, Phys. Rev. Lett. 37, 657 (1976)

573. G.C. Branco, Phys. Rev. Lett. 44, 504 (1980)

574. G.C. Branco, Phys. Rev. D 22, 2901 (1980)

575. G.C. Branco, A.J. Buras, J.M. Gerard, Nucl. Phys. B 259, 306 (1985)

576. L. Bento, G.C. Branco, P.A. Parada, Phys. Lett. B 267, 95 (1991)

577. A.E. Nelson, Phys. Lett. B 136, 387 (1984)

578. A.E. Nelson, Phys. Lett. B 143, 165 (1984)

579. S.M. Barr, Phys. Rev. Lett. 53, 329 (1984)

580. G.C. Branco, P.A. Parada, M.N. Rebelo, arXiv:hep-ph/0307119
581. Y. Achiman, Phys. Lett. B 599, 75 (2004). arXiv:hep-ph/ 0403309

582. M.B. Gavela, P. Hernandez, J. Orloff, O. Pene, C. Quimbay, Nucl. Phys. B 430, 382 (1994). arXiv:hep-ph/9406289

583. P. Huet, E. Sather, Phys. Rev. D 51, 379 (1995). arXiv:hep-ph/ 9404302

584. G.W. Anderson, L.J. Hall, Phys. Rev. D 45, 2685 (1992)

585. W. Buchmuller, Z. Fodor, T. Helbig, D. Walliser, Ann. Phys. 234, 260 (1994). arXiv:hep-ph/9303251

586. K. Kajantie, M. Laine, K. Rummukainen, M.E. Shaposhnikov, Nucl. Phys. B 466, 189 (1996). arXiv:hep-lat/9510020

587. L. Fromme, S.J. Huber, M. Seniuch, J. High Energy Phys. 0611 , 038 (2006). arXiv:hep-ph/0605242

588. E. Accomando et al., arXiv:hep-ph/0608079

589. L. Brucher, R. Santos, Eur. Phys. J. C 12, 87 (2000). arXiv:hep-ph/9907434

590. C. Delaere (LEP Collaboration), PoS HEP2005, 331 (2006)

591. A.G. Akeroyd, M.A. Diaz, F.J. Pacheco, Phys. Rev. D 70, 075002 (2004). arXiv:hep-ph/0312231

592. N.G. Deshpande, E. Ma, Phys. Rev. D 18, 2574 (1978)

593. Q.H. Cao, E. Ma, G. Rajasekaran, arXiv:0708.2939 [hep-ph]

594. R. Barbieri, L.J. Hall, V.S. Rychkov, Phys. Rev. D 74, 015007 (2006). arXiv:hep-ph/0603188

595. I.F. Ginzburg, M. Krawczyk, P. Osland, Nucl. Instrum. Methods A 472, 149 (2001). arXiv:hep-ph/0101229

596. T.P. Cheng, M. Sher, Phys. Rev. D 35, 3484 (1987)

597. G.C. Branco, W. Grimus, L. Lavoura, Phys. Lett. B 380, 119 (1996). arXiv:hep-ph/9601383

598. A.K. Das, C. Kao, Phys. Lett. B 372, 106 (1996). arXiv: hep-ph/9511329

599. E. Lunghi, A. Soni, arXiv:0707.0212 [hep-ph]

600. T.P. Cheng, L.F. Li, Phys. Rev. Lett. 38, 381 (1977)

601. S.M. Bilenky, S.T. Petcov, B. Pontecorvo, Phys. Lett. B 67, 309 (1977)

602. J.D. Bjorken, S. Weinberg, Phys. Rev. Lett. 38, 622 (1977)

603. G.C. Branco, Phys. Lett. B 68, 455 (1977)

604. D. Besson et al. (CLEO Collaboration), Phys. Rev. Lett. 98, 052002 (2007). arXiv:hep-ex/0607019

605. R.N. Mohapatra, J.W.F. Valle, Phys. Rev. D 34, 1642 (1986)

606. S. Nandi, U. Sarkar, Phys. Rev. Lett. 56, 564 (1986)

607. P.F. Harrison, D.H. Perkins, W.G. Scott, Phys. Lett. B 458, 79 (1999). arXiv:hep-ph/9904297

608. A. Pilaftsis, Z. Phys. C 55, 275 (1992). arXiv:hep-ph/9901206

609. N. Arkani-Hamed, L.J. Hall, H. Murayama, D.R. Smith, N. Weiner, Phys. Rev. D 64, 115011 (2001). arXiv:hep-ph/ 0006312

610. F. Borzumati, Phys. Rev. D 64, 053005 (2001). arXiv:hep-ph/ 0007018

611. S. Bergmann, A. Kagan, Nucl. Phys. B 538, 368 (1999). arXiv:hep-ph/9803305

612. A. Ioannisian, A. Pilaftsis, Phys. Rev. D 62, 066001 (2000). arXiv:hep-ph/9907522

613. D.A. Dicus, D.D. Karatas, P. Roy, Phys. Rev. D 44, 2033 (1991)

614. A. Datta, M. Guchait, A. Pilaftsis, Phys. Rev. D 50, 3195 (1994). arXiv:hep-ph/9311257

615. F.M.L. Almeida, Y.D.A. Coutinho, J.A. Martins Simoes, M.A.B. do Vale, Phys. Rev. D 62, 075004 (2000). arXiv:hep-ph/0002024

616. O. Panella, M. Cannoni, C. Carimalo, Y.N. Srivastava, Phys. Rev. D 65, 035005 (2002). arXiv:hep-ph/0107308

617. T. Han, B. Zhang, Phys. Rev. Lett. 97, 171804 (2006). arXiv:hep-ph/0604064

618. S. Bray, J.S. Lee, A. Pilaftsis, arXiv:hep-ph/0702294

619. F. del Aguila, J.A. Aguilar-Saavedra, R. Pittau, arXiv:hep-ph/ 0703261

620. N. Arkani-Hamed, A.G. Cohen, H. Georgi, Phys. Rev. Lett. 86, 4757 (2001). arXiv:hep-th/0104005 
621. H.C. Cheng, C.T. Hill, S. Pokorski, J. Wang, Phys. Rev. D 64, 065007 (2001). arXiv:hep-th/0104179

622. N. Arkani-Hamed, A.G. Cohen, H. Georgi, Phys. Lett. B 513, 232 (2001). arXiv:hep-ph/0105239

623. M. Schmaltz, D. Tucker-Smith, Annu. Rev. Nucl. Part. Sci. 55, 229 (2005). arXiv:hep-ph/0502182

624. M. Perelstein, Prog. Part. Nucl. Phys. 58, 247 (2007). arXiv:hep-ph/0512128

625. N. Arkani-Hamed, A.G. Cohen, E. Katz, A.E. Nelson, J. High Energy Phys. 0207, 034 (2002). arXiv:hep-ph/0206021

626. S. Chang, J. High Energy Phys. 0312, 057 (2003). arXiv:hep-ph/0306034

627. H.C. Cheng, I. Low, J. High Energy Phys. 0309, 051 (2003). arXiv:hep-ph/0308199

628. H.C. Cheng, I. Low, J. High Energy Phys. 0408, 061 (2004). arXiv:hep-ph/0405243

629. J. Hubisz, P. Meade, A. Noble, M. Perelstein, J. High Energy Phys. 0601, 135 (2006). arXiv:hep-ph/0506042

630. J. Hubisz, P. Meade, Phys. Rev. D 71, 035016 (2005). arXiv:hep-ph/0411264

631. I. Low, J. High Energy Phys. 0410, 067 (2004). arXiv: hep-ph/0409025

632. J. Hubisz, S.J. Lee, G. Paz, J. High Energy Phys. 0606, 041 (2006). arXiv:hep-ph/0512169

633. M. Blanke, A.J. Buras, A. Poschenrieder, S. Recksiegel, C. Tarantino, S. Uhlig, A. Weiler, Phys. Lett. B 646, 253 (2007). arXiv:hep-ph/0609284

634. S. Yamada, Nucl. Phys. Proc. Suppl. 144, 185 (2005)

635. http://meg.web.psi.ch/

636. S.C. Park, J.h. Song, arXiv:hep-ph/0306112

637. R. Casalbuoni, A. Deandrea, M. Oertel, J. High Energy Phys. 0402, 032 (2004). arXiv:hep-ph/0311038

638. M. Blanke, A.J. Buras, A. Poschenrieder, S. Recksiegel, C. Tarantino, S. Uhlig, A. Weiler, J. High Energy Phys. 0701, 066 (2007). arXiv:hep-ph/0610298

639. M. Blanke, A.J. Buras, B. Duling, A. Poschenrieder, C. Tarantino, arXiv:hep-ph/0702136

640. P.Q. Hung, Phys. Lett. B 659, 585 (2008). arXiv:0711.0733 [hep-ph]

641. M. Blanke, A.J. Buras, A. Poschenrieder, C. Tarantino, S. Uhlig, A. Weiler, J. High Energy Phys. 0612, 003 (2006). arXiv:hep-ph/0605214

642. A.J. Buras, arXiv:hep-ph/9806471

643. S.R. Choudhury, A.S. Cornell, A. Deandrea, N. Gaur, A. Goyal, Phys. Rev. D 75, 055011 (2007). arXiv:hep-ph/0612327

644. B. Aubert et al. (BaBar Collaboration), Phys. Rev. Lett. 92, 121801 (2004). arXiv:hep-ex/0312027

645. Y. Yusa et al. (Belle Collaboration), Phys. Lett. B 589, 103 (2004). arXiv:hep-ex/0403039

646. Y. Enari et al.(Belle Collaboration), Phys. Lett. B 622, 218 (2005). arXiv:hep-ex/0503041

647. K. Abe et al. (Belle Collaboration), arXiv:hep-ex/0609049

648. B. Aubert et al.(BaBar Collaboration), Phys. Rev. Lett. 98, 061803 (2007). arXiv:hep-ex/0610067

649. E. Arganda, M.J. Herrero, Phys. Rev. D 73, 055003 (2006). arXiv:hep-ph/0510405

650. P. Paradisi, J. High Energy Phys. 0602, 050 (2006). arXiv:hep-ph/0508054

651. Y. Kuno, Y. Okada, Phys. Rev. Lett. 77, 434 (1996). arXiv:hep-ph/9604296

652. Y. Farzan, arXiv:hep-ph/0701106

653. A. Czarnecki, W.J. Marciano, A. Vainshtein, Phys. Rev. D 67, 073006 (2003) [Erratum: Phys. Rev. D 73, 119901 (2006)]. arXiv:hep-ph/0212229

654. J. Lee, arXiv:hep-ph/0504136

655. T. Han, H.E. Logan, B. Mukhopadhyaya, R. Srikanth, Phys. Rev. D 72, 053007 (2005). arXiv:hep-ph/0505260
656. F. del Aguila, M. Masip, J.L. Padilla, Phys. Lett. B 627, 131 (2005). arXiv:hep-ph/0506063

657. S.R. Choudhury, N. Gaur, A. Goyal, Phys. Rev. D 72, 097702 (2005). arXiv:hep-ph/0508146

658. A. Abada, G. Bhattacharyya, M. Losada, Phys. Rev. D 73, 033006 (2006). arXiv:hep-ph/0511275

659. G.F. Giudice, C. Grojean, A. Pomarol, R. Rattazzi, arXiv: hep-ph/0703164

660. D. Acosta et al. (CDF Collaboration), Phys. Rev. Lett. 93, 221802 (2004). arXiv:hep-ex/0406073

661. V.M. Abazov et al. (D0 Collaboration), Phys. Rev. Lett. 93, 141801 (2004). arXiv:hep-ex/0404015

662. K. Huitu, J. Maalampi, A. Pietila, M. Raidal, Nucl. Phys. B 487, 27 (1997). arXiv:hep-ph/9606311

663. C.X. Yue, S. Zhao, arXiv:hep-ph/0701017

664. E. Ma, M. Raidal, U. Sarkar, Phys. Rev. Lett. 85, 3769 (2000). arXiv:hep-ph/0006046

665. E. Ma, M. Raidal, U. Sarkar, Nucl. Phys. B 615, 313 (2001). arXiv:hep-ph/0012101

666. E. Ma, U. Sarkar, Phys. Lett. B 638, 356 (2006). arXiv: hep-ph/0602116

667. N. Sahu, U. Sarkar, arXiv:hep-ph/0701062

668. G. Marandella, C. Schappacher, A. Strumia, Phys. Rev. D 72, 035014 (2005). arXiv:hep-ph/0502096

669. T. Han, H.E. Logan, B. McElrath, L.T. Wang, Phys. Rev. D 67, 095004 (2003). arXiv:hep-ph/0301040

670. T. Han, H.E. Logan, L.T. Wang, J. High Energy Phys. 0601, 099 (2006). arXiv:hep-ph/0506313

671. G. Azuelos et al., Eur. Phys. J. C 39S2, 13 (2005). arXiv: hep-ph/0402037

672. J.F. Gunion, C. Loomis, K.T. Pitts, in Proceedings of 1996 DPF/DPB Summer Study on New Directions for High-Energy Physics (Snowmass 96), Snowmass, CO, 25 June-12 July 1996, p. LTH096. arXiv:hep-ph/9610237

673. J.F. Gunion, J. Grifols, A. Mendez, B. Kayser, F.I. Olness, Phys. Rev. D 40, 1546 (1989)

674. M. Muhlleitner, M. Spira, Phys. Rev. D 68, 117701 (2003). arXiv:hep-ph/0305288

675. A.G. Akeroyd, M. Aoki, Phys. Rev. D 72, 035011 (2005). arXiv:hep-ph/0506176

676. T. Rommerskirchen, T. Hebbeker, J. Phys. G 33, N47 (2007)

677. A. Hektor, M. Kadastik, M. Muntel, M. Raidal, L. Rebane, arXiv:0705.1495 [hep-ph]

678. T. Han, B. Mukhopadhyaya, Z. Si, K. Wang, arXiv:0706.0441 [hep-ph]

679. F. Gabbiani, A. Masiero, Nucl. Phys. B 322, 235 (1989)

680. F. Gabbiani, E. Gabrielli, A. Masiero, L. Silvestrini, Nucl. Phys. B 477, 321 (1996). arXiv:hep-ph/9604387

681. P. Paradisi, J. High Energy Phys. 0510, 006 (2005). arXiv:hep-ph/0505046

682. R. Barbieri, L.J. Hall, Phys. Lett. B 338, 212 (1994). arXiv:hep-ph/9408406

683. R. Barbieri, L.J. Hall, A. Strumia, Nucl. Phys. B 445, 219 (1995). arXiv:hep-ph/9501334

684. I. Masina, C.A. Savoy, Phys. Rev. D 71, 093003 (2005). arXiv:hep-ph/0501166

685. B.C. Allanach et al., in Proceedings of the APS/DPF/DPB Summer Study on the Future of Particle Physics (Snowmass 2001), ed. by N. Graf, Snowmass, CO, 30 June-21 July 2001, p. P125. arXiv:hep-ph/0202233

686. S. Antusch, E. Arganda, M.J. Herrero, A.M. Teixeira, J. High Energy Phys. 0611, 090 (2006). arXiv:hep-ph/0607263

687. F. Deppisch, H. Päs, A. Redelbach, R. Rückl, Phys. Rev. D 73, 033004 (2006). arXiv:hep-ph/0511062

688. L. Calibbi, A. Faccia, A. Masiero, S.K. Vempati, arXiv:hep-ph/ 0610241 
689. M. Maltoni, T. Schwetz, M.A. Tortola, J.W.F. Valle, Phys. Rev. D 68, 113010 (2003). arXiv:hep-ph/0309130

690. S. Eidelman et al. (PDG Collaboration), Phys. Lett. B 592, 1 (2004). arXiv:hep-ph/0310053

691. S.T. Petcov, T. Shindou, Phys. Rev. D 74, 073006 (2006). arXiv:hep-ph/0605151

692. G. Weiglein et al. (LHC/LC Study Group), Phys. Rep. 426, 47 (2006). arXiv:hep-ph/0410364

693. F. Deppisch, H. Päs, A. Redelbach, R. Rückl, Y. Shimizu, arXiv:hep-ph/0206122

694. A.G. Akeroyd et al. (SuperKEKB Physics Working Group), arXiv:hep-ex/0406071

695. F. Deppisch, H. Päs, A. Redelbach, R. Rückl, Y. Shimizu, Phys. Rev. D 69, 054014 (2004). arXiv:hep-ph/0310053

696. K. Agashe, M. Graesser, Phys. Rev. D 61, 075008 (2000). arXiv:hep-ph/9904422

697. Yu.M. Andreev, S.I. Bityukov, N.V. Krasnikov, A.N. Toropin, arXiv:hep-ph/0608176

698. A. Bartl, K. Hidaka, K. Hohenwarter-Sodek, T. Kernreiter, W. Majerotto, W. Porod, Eur. Phys. J. C 46, 783 (2006). arXiv:hep-ph/0510074

699. W. Porod, Comput. Phys. Commun. 153, 275 (2003). arXiv:hep-ph/0301101

700. U. Bellgardt et al. (SINDRUM Collaboration), Nucl. Phys. B 299, 1 (1988)

701. S. Ritt (MEGA Collaboration), on the web page http://meg.web. psi.ch/docs/talks/s_ritt/mar06_novosibirsk/ritt.ppt

702. T. Iijima, talk given at the 6th Workshop on a Higher Luminosity B Factory, KEK, Tsukuba, Japan, November 2004

703. J. Aysto et al., arXiv:hep-ph/0109217

704. The PRIME Working Group, Search for the $\mu-e$ Conversion Process at an Ultimate sensitivity of the order of $10^{18}$ with PRISM, unpublished

705. LOI to J-PARC 50-GeV PS, LOI-25, http://psux1.kek.jp/jhf-np/ LOIlist/LOIlist.html

706. Y. Kuno, Nucl. Phys. Proc. Suppl. 149, 376 (2005)

707. A. Masiero, S.K. Vempati, O. Vives, New J. Phys. 6, 202 (2004). arXiv:hep-ph/0407325

708. E. Ables et al. (MINOS Collaboration), Fermilab-proposal0875

709. G.S. Tzanakos (MINOS Collaboration), AIP Conf. Proc. 721, 179 (2004)

710. M. Komatsu, P. Migliozzi, F. Terranova, J. Phys. G 29, 443 (2003). arXiv:hep-ph/0210043

711. P. Migliozzi, F. Terranova, Phys. Lett. B 563, 73 (2003). arXiv:hep-ph/0302274

712. P. Huber, J. Kopp, M. Lindner, M. Rolinec, W. Winter, J. High Energy Phys. 0605, 072 (2006). arXiv:hep-ph/0601266

713. Y. Itow et al., arXiv:hep-ex/0106019

714. A. Blondel, A. Cervera-Villanueva, A. Donini, P. Huber, M. Mezzetto, P. Strolin, arXiv:hep-ph/0606111

715. P. Huber, M. Lindner, M. Rolinec, W. Winter, arXiv:hep-ph/ 0606119

716. J. Burguet-Castell, D. Casper, E. Couce, J.J. GomezCadenas, P. Hernandez, Nucl. Phys. B 725, 306 (2005). arXiv:hep-ph/0503021

717. J.E. Campagne, M. Maltoni, M. Mezzetto, T. Schwetz, arXiv: hep-ph/0603172

718. T. Blazek, S.F. King, Nucl. Phys. B 662, 359 (2003). arXiv: hep-ph/0211368

719. S.F. King, J. High Energy Phys. 0508, 105 (2005) arXiv:hep-ph/0506297

720. S. Antusch, S.F. King, Phys. Lett. B 631, 42 (2005). arXiv:hep-ph/0508044

721. S. Antusch, S.F. King, arXiv:0709.0666 [hep-ph]

722. P.H. Chankowski, S. Pokorski, Int. J. Mod. Phys. A 17, 575 (2002). arXiv:hep-ph/0110249
723. G. Altarelli, F. Feruglio, Phys. Rep. 320, 295 (1999)

724. A.Y. Smirnov, Phys. Rev. D 48, 3264 (1993). arXiv:hep-ph/ 9304205

725. J. Pradler, F.D. Steffen, Phys. Rev. D 75, 023509 (2007). arXiv:hep-ph/0608344

726. S. Davidson, J. High Energy Phys. 0303, 037 (2003). arXiv:hep-ph/0302075

727. P.H. Chankowski, K. Turzynski, Phys. Lett. B 570, 198 (2003). arXiv:hep-ph/0306059

728. A. Pilaftsis, Int. J. Mod. Phys. A 14, 1811 (1999). arXiv: hep-ph/9812256

729. S.F. King, Rep. Prog. Phys. 67, 107 (2004). arXiv:hep-ph/ 0310204

730. F.R. Joaquim, I. Masina, A. Riotto, arXiv:hep-ph/0701270

731. J.R. Ellis, K.A. Olive, Y. Santoso, V.C. Spanos, Phys. Lett. B 565, 176 (2003). arXiv:hep-ph/0303043

732. T. Blazek, S.F. King, Phys. Lett. B 518, 109 (2001) arXiv:hep-ph/0105005

733. F. Cuypers, S. Davidson, Eur. Phys. J. C 2, 503 (1998). arXiv:hep-ph/9609487

734. E.J. Chun, K.Y. Lee, S.C. Park, Phys. Lett. B 566, 142 (2003). arXiv:hep-ph/0304069

735. A. Strumia, F. Vissani, arXiv:hep-ph/0606054

736. F.R. Joaquim, A. Rossi, arXiv:hep-ph/0607298

737. T. Moroi, Phys. Lett. B 493, 366 (2000)

738. J. Hisano, Y. Shimizu, Phys. Lett. B 565, 183 (2003)

739. M. Ciuchini, A. Masiero, L. Silvestrini, S.K. Vempati, O. Vives, Phys. Rev. Lett. 92, 071801 (2004)

740. S. Dimopoulos, L.J. Hall, Phys. Lett. B 344, 185 (1995)

741. J. Hisano, M. Kakizaki, M. Nagai, Y. Shimizu, Phys. Lett. B 604, 216 (2004)

742. M. Pospelov, A. Ritz, Ann. Phys. 318, 119 (2005)

743. V.M. Khatsimovsky, I.B. Khriplovich, A.R. Zhitnitsky, Z. Phys. C 36, 455 (1987)

744. A.R. Zhitnitsky, Phys. Rev. D 55, 3006 (1997)

745. F.R. Joaquim, A. Rossi, arXiv:hep-ph/0604083

746. M. Dine, W. Fischler, M. Srednicki, Nucl. Phys. B 189, 575 (1981)

747. S. Dimopoulos, S. Raby, Nucl. Phys. B 192, 353 (1981)

748. C.R. Nappi, B.A. Ovrut, Phys. Lett. B 113, 175 (1982)

749. S. Dimopoulos, S. Raby, Nucl. Phys. B 219, 479 (1983)

750. M. Dine, A.E. Nelson, Phys. Rev. D 48, 1277 (1993). arXiv:hep-ph/9303230

751. M. Dine, A.E. Nelson, Y. Shirman, Phys. Rev. D 51, 1362 (1995). arXiv:hep-ph/9408384

752. M.A. Giorgi et al. (SuperB Group,), INFN Roadmap Report, March 2006

753. Y. Mori et al., (PRISM/PRIME Working Group), LOI at J-PARC 50-GeV PS, LOI-25. http://psux1.kek.jp/ jhf-np/LOIlist/LOIlist.html

754. M. Cvetic, I. Papadimitriou, G. Shiu, Nucl. Phys. B 659, 193 (2003) [Erratum:Nucl. Phys. B 696, 298 (2004)]

755. M. Cvetic, P. Langacker, arXiv:hep-th/0607238

756. R. Barbieri, D.V. Nanopoulos, G. Morchio, F. Strocchi, Phys. Lett. B 90, 91 (1980)

757. P. Langacker, Phys. Rep. 72, 185 (1981)

758. D. Chang, A. Masiero, H. Murayama, Phys. Rev. D 67, 075013 (2003). arXiv:hep-ph/0205111

759. T. Moroi, J. High Energy Phys. 0003, 019 (2000). arXiv:hep-ph/0002208

760. N. Akama, Y. Kiyo, S. Komine, T. Moroi, Phys. Rev. D 64, 095012 (2001). arXiv:hep-ph/0104263

761. J. Sato, K. Tobe, T. Yanagida, Phys. Lett. B 498, 189 (2001). arXiv:hep-ph/0010348

762. M. Apollonio et al.(CHOOZ Collaboration), Phys. Lett. B 466, 415 (1999). arXiv:hep-ex/9907037 
763. L. Calibbi, A. Faccia, A. Masiero, S.K. Vempati, Phys. Rev. D 74, 116002 (2006). arXiv:hep-ph/0605139

764. K. Abe et al. (Belle Collaboration), Phys. Rev. Lett. 92, 171802 (2004). arXiv:hep-ex/0310029

765. A.J. Buras, P.H. Chankowski, J. Rosiek, L. Slawianowska, Nucl. Phys. B 659, 3 (2003). arXiv:hep-ph/0210145

766. G. Isidori, P. Paradisi, Phys. Lett. B 639, 499 (2006). arXiv:hep-ph/0605012

767. E. Lunghi, W. Porod, O. Vives, Phys. Rev. D 74, 075003 (2006). arXiv:hep-ph/0605177

768. J. Foster, K.i. Okumura, L. Roszkowski, Phys. Lett. B 641, 452 (2006). arXiv:hep-ph/0604121

769. G.R. Farrar, P. Fayet, Phys. Lett. B 76, 575 (1978)

770. L.J. Hall, M. Suzuki, Nucl. Phys. B 231, 419 (1984)

771. R. Barbier et al., Phys. Rep. 420, 1 (2005). arXiv:hep-ph/ 0406039

772. D. Comelli, A. Masiero, M. Pietroni, A. Riotto, Phys. Lett. B 324, 397 (1994). arXiv:hep-ph/9310374

773. R. Kuchimanchi, R.N. Mohapatra, Phys. Rev. D 48, 4352 (1993). arXiv:hep-ph/9306290

774. K. Huitu, J. Maalampi, Phys. Lett. B 344, 217 (1995). arXiv:hep-ph/9410342

775. R. Kitano, K.y. Oda, Phys. Rev. D 61, 113001 (2000). arXiv:hep-ph/9911327

776. M. Frank, K. Huitu, Phys. Rev. D 64, 095015 (2001). arXiv:hep-ph/0106004

777. J.R. Ellis, G. Gelmini, C. Jarlskog, G.G. Ross, J.W.F. Valle, Phys. Lett. B 150, 142 (1985)

778. S. Davidson, M. Losada, Phys. Rev. D 65, 075025 (2002). arXiv:hep-ph/0010325

779. K.S. Babu, R.N. Mohapatra, Phys. Rev. Lett. 64, 1705 (1990)

780. M. Hirsch, M.A. Diaz, W. Porod, J.C. Romao, J.W.F. Valle, Phys. Rev. D 62, 113008 (2000) [Erratum: Phys. Rev. D 65, 119901 (2002)]. arXiv:hep-ph/0004115

781. M.A. Diaz, C. Mora, A.R. Zerwekh, Eur. Phys. J. C 44, 277 (2005). arXiv:hep-ph/0410285

782. A. Deandrea, J. Welzel, M. Oertel, J. High Energy Phys. 0410, 038 (2004). arXiv:hep-ph/0407216

783. W. Porod, M. Hirsch, J. Romao, J.W.F. Valle, Phys. Rev. D 63, 115004 (2001). arXiv:hep-ph/0011248

784. A. Heister et al.(ALEPH Collaboration), Eur. Phys. J. C 31, 1 (2003). arXiv:hep-ex/0210014

785. P. Abreu et al.(DELPHI Collaboration), Phys. Lett. B 500, 22 (2001). arXiv:hep-ex/0103015

786. P. Achard et al. (L3 Collaboration), Phys. Lett. B 524, 65 (2002). arXiv:hep-ex/0110057

787. B. Abbott et al. (D0 Collaboration), Phys. Rev. D 62, 071701 (2000). arXiv:hep-ex/0005034

788. S. Aid et al. (H1 Collaboration), Z. Phys. C 71, 211 (1996). arXiv:hep-ex/9604006

789. C. Adloff et al. (H1 Collaboration), Eur. Phys. J. C 20, 639 (2001). arXiv:hep-ex/0102050

790. W. Beenakker, R. Hopker, M. Spira, P.M. Zerwas, Phys. Rev. Lett. 74, 2905 (1995). arXiv:hep-ph/9412272

791. J. Kalinowski, R. Ruckl, H. Spiesberger, P.M. Zerwas, Phys. Lett. B 414, 297 (1997). arXiv:hep-ph/9708272

792. G. Moreau, E. Perez, G. Polesello, Nucl. Phys. B 604, 3 (2001). arXiv:hep-ph/0003012

793. G. Moreau, M. Chemtob, F. Deliot, C. Royon, E. Perez, Phys. Lett. B 475, 184 (2000). arXiv:hep-ph/9910341

794. S. Dimopoulos, L.J. Hall, Phys. Lett. B 207, 210 (1988)

795. H.K. Dreiner, P. Richardson, M.H. Seymour, Phys. Rev. D 63, 055008 (2001). arXiv:hep-ph/0007228

796. F. Deliot, G. Moreau, C. Royon, Eur. Phys. J. C 19, 155 (2001). arXiv:hep-ph/0007288

797. M. Chaichian, A. Datta, K. Huitu, S. Roy, Z.h. Yu, Phys. Lett. B 594, 355 (2004). arXiv:hep-ph/0311327
798. S. Dimopoulos, R. Esmailzadeh, L.J. Hall, G.D. Starkman, Phys. Rev. D 41, 2099 (1990)

799. B. Allanach et al. (R Parity Working Group Collaboration), arXiv:hep-ph/9906224

800. E.L. Berger, B.W. Harris, Z. Sullivan, Phys. Rev. Lett. 83, 4472 (1999). arXiv:hep-ph/9903549

801. M. Chaichian, K. Huitu, Z.H. Yu, Phys. Lett. B 490, 87 (2000). arXiv:hep-ph/0007220

802. E.D. Richter-Was, L. Poggioli, ATLAS note ATLAS-PHYS-98131 (1998)

803. W.W. Armstrong et al., ATLAS technical proposal CERNLHCC-94-43, 1994

804. A. Belyaev, M.H. Genest, C. Leroy, R.R. Mehdiyev, J. High Energy Phys. 0409, 012 (2004). arXiv:hep-ph/0401065

805. K.S. Babu, C.F. Kolda, Phys. Rev. Lett. 84, 228 (2000). arXiv:hep-ph/9909476

806. K.S. Babu, C. Kolda, Phys. Rev. Lett. 89, 241802 (2002). arXiv:hep-ph/0206310

807. A. Dedes, J.R. Ellis, M. Raidal, Phys. Lett. B 549, 159 (2002). arXiv:hep-ph/0209207

808. D. Chang, W.S. Hou, W.Y. Keung, Phys. Rev. D 48, 217 (1993). arXiv:hep-ph/9302267

809. M. Sher, Y. Yuan, Phys. Rev. D 44, 1461 (1991)

810. M. Sher, Phys. Rev. D 66, 057301 (2002). arXiv:hep-ph/ 0207136

811. R. Kitano, M. Koike, S. Komine, Y. Okada, Phys. Lett. B 575, 300 (2003). arXiv:hep-ph/0308021

812. S. Kanemura, Y. Kuno, M. Kuze, T. Ota, Phys. Lett. B 607, 165 (2005). arXiv:hep-ph/0410044

813. A. Broncano, M.B. Gavela, E. Jenkins, Phys. Lett. B 552, 177 (2003) [Erratum: Phys. Lett. B 636, 330 (2006)]. arXiv:hep-ph/ 0210271

814. M.C. Gonzalez-Garcia, J.W.F. Valle, Phys. Lett. B 216, 360 (1989)

815. S. Antusch, C. Biggio, E. Fernandez-Martinez, M.B. Gavela, J. Lopez-Pavon, J. High Energy Phys. 0610, 084 (2006). arXiv:hep-ph/0607020

816. M. Apollonio et al., Eur. Phys. J. C 27, 331 (2003). hep-ex/ 0301017

817. T. Araki et al. (KamLAND Collaboration), Phys. Rev. Lett. 94, 081801 (2005). hep-ex/0406035

818. B. Aharmim et al. (SNO Collaboration), Phys. Rev. C 72 , 055502 (2005). nucl-ex/0502021

819. M.H. Ahn et al. (K2K Collaboration), Phys. Rev. Lett. 90, 041801 (2003). hep-ex/0212007

820. Y. Ashie et al. (Super-Kamiokande Collaboration), Phys. Rev. D 71, 112005 (2005). hep-ex/0501064

821. P. Astier et al. (NOMAD Collaboration), Nucl. Phys. B 611, 3 (2001). hep-ex/0106102

822. K. Eitel (KARMEN Collaboration), Nucl. Phys. Proc. Suppl. 91, 191 (2001). hep-ex/0008002

823. Y. Declais et al., Nucl. Phys. B 434, 503 (1995)

824. D.A. Petyt (MINOS Collaboration) First MINOS results from the NuMI beam. http://www-numi.fnal.gov/

825. M.C. Gonzalez-Garcia, Phys. Scr. T 121, 72 (2005). hep-ph/0410030

826. V. Barger, S. Geer, K. Whisnant, New J. Phys. 6, 135 (2004). arXiv:hep-ph/0407140

827. D.S. Ayres et al. (NOvA Collaboration), hep-ex/0503053

828. J.J. Gomez-Cadenas et al. (CERN Working Group on Super Beams Collaboration), hep-ph/0105297

829. J.E. Campagne, A. Cazes, Eur. Phys. J. C 45, 643 (2006). hep-ex/0411062

830. P. Zucchelli, Phys. Lett. B 532, 166 (2002)

831. S. Geer, Phys. Rev. D 57, 6989 (1998) hep-ph/9712290

832. A. De Rujula, M.B. Gavela, P. Hernandez, Nucl. Phys. B 547, 21 (1999) hep-ph/9811390 
833. W.J. Marciano, A. Sirlin, Phys. Rev. Lett. 71, 3629 (1993)

834. S. Eidelman et al. (Particle Data Group), Phys. Lett. B 592, 1 (2004)

835. L. Fiorini (for the NA48/2 Collaboration), at ICHEP 2005

836. V.D. Barger, G.F. Giudice, T. Han, Phys. Rev. D 40, 2987 (1989)

837. P.H. Chankowski, A. Dabelstein, W. Hollik, W.M. Mosle, S. Pokorski, J. Rosiek, Nucl. Phys. B 417, 101 (1994)

838. J.F. Gunion, H.E. Haber, G.L. Kane, S. Dawson, The Higgs hunter Guide (Addison-Wesley, Reading, 1990)

839. A. Masiero, P. Paradisi, R. Petronzio, Phys. Rev. D 74, 011701 (2006). arXiv:hep-ph/0511289

840. M. Krawczyk, D. Temes, Eur. Phys. J. C 44, 435 (2005). arXiv:hep-ph/0410248

841. P. Krawczyk, S. Pokorski, Phys. Rev. Lett. 60, 182 (1988)

842. J.R. Ellis, J. Hisano, M. Raidal, Y. Shimizu, Phys. Lett. B 528, 86 (2002). arXiv:hep-ph/0111324

843. I. Masina, Nucl. Phys. B 671, 432 (2003). arXiv:hep-ph/ 0304299

844. Y. Farzan, M.E. Peskin, Phys. Rev. D 70, 095001 (2004). arXiv:hep-ph/0405214

845. I. Masina, C. Savoy, Phys. Lett. B 579, 99 (2004). arXiv: hep-ph/0309067

846. K.S. Babu, B. Dutta, R.N. Mohapatra, Phys. Rev. Lett. 85, 5064 (2000). arXiv:hep-ph/0006329

847. S. Abel, O. Lebedev, J. High Energy Phys. 0601, 133 (2006)

848. T. Ibrahim, P. Nath, Phys. Rev. D 58, 111301 (1998) [Erratum: Phys. Rev. D 60, 099902 (1999)]. arXiv:hep-ph/9807501

849. J. Hisano, Y. Shimizu, Phys. Rev. D 70, 093001 (2004). arXiv:hep-ph/0406091

850. M. Brhlik, L.L. Everett, G.L. Kane, J.D. Lykken, Phys. Rev. Lett. 83, 2124 (1999). arXiv:hep-ph/9905215

851. S. Abel, S. Khalil, O. Lebedev, Phys. Rev. Lett. 86, 5850 (2001). arXiv:hep-ph/0103031

852. J.R. Ellis, S. Ferrara, D.V. Nanopoulos, Phys. Lett. B 114, 231 (1982)

853. S. Abel, S. Khalil, O. Lebedev, Nucl. Phys. B 606, 151 (2001). hep-ph/0103320

854. D.A. Demir, O. Lebedev, K.A. Olive, M. Pospelov, A. Ritz, Nucl. Phys. B 680, 339 (2004). hep-ph/0311314

855. K.A. Olive, M. Pospelov, A. Ritz, Y. Santoso, Phys. Rev. D 72, 075001 (2005). hep-ph/0506106

856. N. Arkani-Hamed, S. Dimopoulos, G.F. Giudice, A. Romanino, Nucl. Phys. B 709, 3 (2005). hep-ph/0409232

857. G.F. Giudice, A. Romanino, Phys. Lett. B 634, 307 (2006). hep-ph/0510197

858. D. Chang, W.-F. Chang, W.-Y. Keung, Phys. Rev. D 71, 076006 (2005). hep-ph/0503055

859. A.D. Linde, Phys. Lett. B 129, 177 (1983)

860. S. Weinberg, Phys. Rev. Lett. 59, 2607 (1987)

861. V. Agrawal, S.M. Barr, J.F. Donoghue, D. Seckel, Phys. Rev. D 57, 5480 (1998). hep-ph/9707380

862. R. Bousso, J. Polchinski, J. High Energy Phys. 06, 006 (2000). hep-th/0004134

863. N. Arkani-Hamed, S. Dimopoulos, S. Kachru, hep-th/0501082

864. R. Arnowitt, J.L. Lopez, D.V. Nanopoulos, Phys. Rev. D 42, 2423 (1990)

865. G. Degrassi, E. Franco, S. Marchetti, L. Silvestrini, J. High Energy Phys. 11, 044 (2005). hep-ph/0510137

866. M. Pospelov, A. Ritz, Phys. Rev. Lett. 83, 2526 (1999). hep-ph/9904483

867. M. Pospelov, A. Ritz, Phys. Rev. D 63, 073015 (2001). hep-ph/0010037

868. A. Arvanitaki, C. Davis, P.W. Graham, J.G. Wacker, Phys. Rev. D 70, 117703 (2004). hep-ph/0406034

869. C.A. Baker et al., Phys. Rev. Lett. 97, 131801 (2006). hep-ex/0602020
870. O. Lebedev, W. Loinaz, T. Takeuchi, Phys. Rev. D 61, 115005 (2000)

871. M.J. Ramsey-Musolf, Phys. Rev. D 62, 056009 (2000). arXiv:hep-ph/0004062

872. O. Lebedev, W. Loinaz, T. Takeuchi, Phys. Rev. D 62, 055014 (2000). arXiv:hep-ph/0002106

873. J.H. Park, J. High Energy Phys. 0610, 077 (2006)

874. M.J. Ramsey-Musolf, S. Su, arXiv:hep-ph/0612057

875. M.A. Sanchis-Lozano, Contributed paper to the Workshop on B-Factories and New Measurements, KEK, 13-14 September 2006. arXiv:hep-ph/0610046

876. B.A. Campbell, D.W. Maybury, Nucl. Phys B 709, 419 (2005)

877. W. Loinaz, N. Okamura, S. Rayyan, T. Takeuchi, L.C.R. Wijewardhana, Phys. Rev. D 70, 113004 (2004)

878. M. Finkemeier, Phys. Lett. B 387, 391 (1996). arXiv:hep-ph/ 9505434

879. G. Czapek et al., Phys. Rev. Lett. 70, 17 (1993)

880. D.I. Britton et al., Phys. Rev. Lett. 68, 3000 (1992)

881. A.Y. Smirnov, R. Zukanovich Funchal, Phys. Rev. D 74, 013001 (2006). arXiv:hep-ph/0603009

882. D. Pocanic, A. van der Schaaf (PEN Collaboration), PSI experiment R-05-01

883. M.A. Bychkov, Ph.D. thesis, University of Virginia (2005), unpublished. http://pibeta.phys.virginia.edu/docs/publications/ max_thesis/thesis.pdf

884. B.A. VanDevender, Ph.D. thesis, University of Virginia, 2005, unpublished. http://pibeta.phys.virginia.edu/docs/ publications/brent_diss.pdf

885. D. Bryman and T. Numao (PIENU Collaboration), TRIUMF experiment 1072

886. NA48/2 Collaboration, Report CERN/SPSC/2000-003, 16 January 2000

887. L. Fiorini, $\mathrm{PhD}$ thesis, Scuola Normale Superiore, Pisa, Italy, 2005, unpublished. Available from http://lifiorini.home.cern.ch/lfiorini/

888. L. Fiorini (for the NA48/2 Collaboration), in Proc. HEP 2005 Europhysics Conference, Lisbon, 21-27 July 2005, PoS (HEP 2005) 288

889. W.J. Marciano, A. Sirlin, Phys. Rev. Lett. 61, 1815 (1988)

890. J.Z. Bai et al.(BES Collaboration), Phys. Rev. D 53, 20 (1996)

891. V.V. Anashin et al. (KEDR Collaboration), Nucl. Phys. Proc. Suppl. 169, 125 (2007). arXiv:hep-ex/0611046

892. A. Lusiani (BaBar Collaboration), Nucl. Phys. Proc. Suppl. 144, 105 (2005)

893. K. Abe et al. (Belle Collaboration), arXiv:hep-ex/0608046

894. R. Decker, M. Finkemeier, Phys. Lett. B 334, 199 (1994)

895. W. Buchmüller, R.D. Peccei, T. Yanagida, Annu. Rev. Nucl. Part. Sci. 55, 311 (2005)

896. W. Bernreuther, private communication

897. L. Michel, Proc. Phys. Soc. A, 63, 514 (1950)

898. W. Fetscher, H.-J. Gerber, K.F. Johnson, Phys. Lett. B 173, 102 (1986)

899. P. Langacker, Commun. Nucl. Part. Phys. 19 (1989)

900. A. Aktas et al. (H1 Collaboration), Phys. Lett. B 634, 173 (2006). arXiv:hep-ex/0512060

901. W. Fetscher, H.-J. Gerber, Eur. Phys. J. C 15, 316 (2000)

902. F. Scheck, Electroweak and Strong Interactions (Springer, Berlin, 1996)

903. J. Kirkby et al., PSI proposal R-99-06, 1999

904. A. Barczyk et al. (FAST Collaboration), arXiv:0707.3904 [hepex]

905. W. Marciano, Phys. Rev. D 60, 093006 (1999)

906. I.C. Barnett et al., Nucl. Instrum. Methods A 455, 329 (2000)

907. Y.S. Tsai, in Stanford Tau Charm (1989), pp. 0387-0393

908. Y.S. Tsai, Phys. Rev. D 51, 3172 (1995)

909. J.H. Kuhn, E. Mirkes, Z. Phys. C 56, 661 (1992) [Erratum: Z. Phys. C 67, 364 (1995)] 
910. J.H. Kühn, E. Mirkes, Phys. Lett. B 398, 407 (1997)

911. I.I. Bigi, A.I. Sanda, Phys. Lett. B 625, 47 (2005)

912. A. Datta et al., hep-ph/0610162

913. J. Bernabeu et al., Nucl. Phys. B 763, 283 (2007)

914. D. Delepine et al., Phys. Rev. D 74, 056004 (2006)

915. A. Pich, Int. J. Mod. Phys. A 21, 5652 (2006)

916. S. Anderson et al. (CLEO Collaboration), Phys. Rev. Lett. 81, 3823 (1998)

917. J.J. Sakurai, Phys. Rev. 109, 980 (1958)

918. A.R. Zhitnitkii, Sov. J. Nucl. Phys. 31, 529 (1980)

919. V.P. Efrosinin, I.B. Khriplovich, G.G. Kirilin, Yu.G. Kudenko, Phys. Lett. B 493, 293 (2000). arXiv:hep-ph/0008199

920. I.I. Bigi, A.I. Sanda, CP Violation (Cambridge University Press, Cambridge, 2000)

921. A.I. Sanda, Phys. Rev. D 23, 2647 (1981)

922. N.G. Deshpande, Phys. Rev. D 23, 2654 (1981)

923. H.Y. Cheng, Phys. Rev. D 26, 143 (1982)

924. H.Y. Cheng, Phys. Rev. D 34, 1397 (1986)

925. I.I.Y. Bigi, A.I. Sanda, Phys. Rev. Lett. 58, 1604 (1987)

926. M. Leurer, Phys. Rev. Lett. 62, 1967 (1989)

927. H.Y. Cheng, Phys. Rev. D 42, 2329 (1990)

928. G. Belanger, C.Q. Geng, Phys. Rev. D 44, 2789 (1991)

929. R. Garisto, G.L. Kane, Phys. Rev. D 44, 2038 (1991)

930. M. Fabbrichesi, F. Vissani, Phys. Rev. D 55, 5334 (1997). arXiv:hep-ph/9611237

931. G.H. Wu, J.N. Ng, Phys. Lett. B 392, 93 (1997). arXiv: hep-ph/9609314

932. W.F. Chang, J.N. Ng, arXiv:hep-ph/0512334

933. J.A. Macdonald et al., Nucl. Instrum. Methods Phys. Res. Sect. A 506, 60 (2003)

934. M. Abe et al., Phys. Rev. D 73, 072005 (2006)

935. J-PARC experiment proposal P06. http://j-parc.jp/NuclPart/ Proposal_0606_e.html

936. W. Bernreuther, U. Low, J.P. Ma, O. Nachtmann, Z. Phys. C 41, 143 (1988)

937. M. Skalsey, J. Van House, Phys. Rev. Lett. 67, 1993 (1991)

938. M. Felcini, Int. J. Mod. Phys. A 19, 3853 (2004). arXiv:hep-ex/0404041

939. O. Jinnouchi, S. Asai, T. Kobayashi, Phys. Lett. B 572, 117 (2003). arXiv:hep-ex/0308030

940. M. Ahmed et al. (MEGA Collaboration), Phys. Rev. D 65, 112002 (2002). arXiv:hep-ex/0111030

941. L. Willmann et al., Phys. Rev. Lett. 82, 49 (1999). arXiv:hep-ex/9807011

942. P. Wintz, in ICHEP 98, Vancouver, Canada, 23-29 July 1998

943. J. Kaulard et al. (SINDRUM II Collaboration), Phys. Lett. B 422, 334 (1998)

944. W. Honecker et al. (SINDRUM II Collaboration), Phys. Rev. Lett. 76, 200 (1996)

945. W. Bertl et al. (SINDRUM II Collaboration), Eur. Phys. J. C 47, 337 (2006)

946. T. Mori et al., PSI proposal R-99-5, May 1999, available as UTICEPP 00-02

947. A. Baldini et al., Research Proposal to INFN, Septembet 2002. http://meg.web.psi.ch/docs/

948. S. Mihara et al., Cryogenics 44, 223 (2004)

949. W. Bertl et al. (SINDRUM Collaboration), Nucl. Phys. B 260, 1 (1985)

950. A. Czarnecki, W.J. Marciano, K. Melnikov, AIP Conf. Proc. 549, 938 (2002)

951. T.S. Kosmas, I.E. Lagaris, J. Phys. G 28, 2907 (2002)

952. R. Kitano, M. Koike, Y. Okada, Phys. Rev. D 66, 096002 (2002). arXiv:hep-ph/0203110

953. T.S. Kosmas, J.D. Vergados, O. Civitarese, A. Faessler, Nucl. Phys. A 570, 637 (1994)

954. R.M. Dzhilkibaev, V.M. Lobashev, Sov. J. Nucl. Phys. 49, 384 (1989) [Yad. Fiz. 49, 622 (1989)]
955. M. Bachman et al., MECO, BNL proposal E 940, 1997

956. Letter of Intent to J-PARC, L24: The PRISM Project, a Muon Source of the World-Highest Brightness by Phase Rotation, 2003

957. Letter of Intent to J-PARC, L26: Request for a Pulsed Proton Beam Facility at J-PARC

958. G. Cvetic, C. Dib, C.S. Kim, J.D. Kim, Phys. Rev. D 66, 034008 (2002) [Erratum: Phys. Rev. D 68 (2003) 059901]. arXiv:hep-ph/0202212

959. J.R. Ellis, M.E. Gomez, G.K. Leontaris, S. Lola, D.V. Nanopoulos, Eur. Phys. J. C 14, 319 (2000). arXiv:hep-ph/9911459

960. T. Fukuyama, T. Kikuchi, N. Okada, Phys. Rev. D 68, 033012 (2003). arXiv:hep-ph/0304190

961. C.X. Yuex, Y.M. Zhang, L.J. Liu, Phys. Lett. B 547, 252 (2002). arXiv:hep-ph/0209291

962. A. Ilakovac, Phys. Rev. D 62, 036010 (2000). arXiv:hep-ph/ 9910213

963. W.J. Li, Y.D. Yang, X.D. Zhang, Phys. Rev. D 73, 073005 (2006). arXiv:hep-ph/0511273

964. D. Black, T. Han, H.J. He, M. Sher, Phys. Rev. D 66, 053002 (2002). arXiv:hep-ph/0206056

965. R.D. Cousins, V.L. Highland, Nucl. Instrum, Methods Phys. Res. Sect. A 320, 331 (1992)

966. R. Barlow, Comput. Phys. Commun. 149, 97 (2002)

967. K. Abe et al. (Belle Collaboration), arXiv:0708.3272 [hep-ex]

968. Y. Miyazaki et al. (Belle Collaboration), Phys. Lett. B 648, 341 (2007). arXiv:hep-ex/0703009

969. N.G. Unel, arXiv:hep-ex/0505030

970. M. Della Negra et al., CMS Physics Technical Design Report, vol. 1,2006

971. T. Speer et al., CMS Note 2006/032, 2006

972. E. James et al., CMS Note 2006/010, 2006

973. R. Santinelli, M. Biasini, CMS Note 2002/037, 2002

974. R. Santinelli, Nucl. Phys. Proc. Suppl. 123, 234 (2003)

975. M.C. Chang et al. (Belle Collaboration), Phys. Rev. D 68, 111101 (2003). arXiv:hep-ex/0309069

976. F. Abe et al. (CDF Collaboration), Phys. Rev. Lett. 81, 5742 (1998)

977. D. Ambrose et al. (BNL Collaboration), Phys. Rev. Lett. 81, 5734 (1998). arXiv:hep-ex/9811038

978. W. Bonivento, N. Serra, LHCb Note 2007-028, 2007

979. S.N. Gninenko, M.M. Kirsanov, N.V. Krasnikov, V.A. Matveev, P. Nedelec, D. Sillou, M. Sher, CERN-SPSC-2004-016

980. M. Sher, I. Turan, Phys. Rev. D 69, 017302 (2004). arXiv: hep-ph/0309183

981. D.E. Groom et al. (Particle Data Group), Eur. Phys. J. C 15, 1 (2000)

982. B. Aubert et al. (BaBar Collaboration), Phys. Rev. Lett. 95, 191801 (2005). arXiv:hep-ex/0506066

983. Y. Yusa et al. (Belle Collaboration), Phys. Lett. B 640, 138 (2006). arXiv:hep-ex/0603036

984. G. Ingelman, A. Edin, J. Rathsman, Comput. Phys. Commun. 101, 108 (1997). arXiv:hep-ph/9605286

985. M.V. Romalis, W.C. Griffith, E.N. Fortson, Phys. Rev. Lett. 86, 2505 (2001). arXiv:hep-ex/0012001

986. R.D. Peccei, H.R. Quinn, Phys. Rev. Lett. 38, 1440 (1977)

987. J.S.M. Ginges, V.V. Flambaum, Phys. Rep. 397, 63 (2004). arXiv:physics/0309054

988. I.B. Khriplovich, S.K. Lamoreaux, CP Violation Without Strangeness: Electric Dipole Moments of Particles, Atoms, and Molecules (Springer, Berlin, 1997)

989. R.J. Crewther, P. Di Vecchia, G. Veneziano, E. Witten, Phys. Lett. B 88, 123 (1979) [Erratum: Phys. Lett. B 91, 487 (1980)]

990. K. Kawarabayashi, N. Ohta, Prog. Theor. Phys. 66, 1789 (1981)

991. D. Chang, W.Y. Keung, A. Pilaftsis, Phys. Rev. Lett. 82, 900 (1999) 
992. O. Lebedev, M. Pospelov, Phys. Rev. Lett. 89, 101801 (2002). arXiv:hep-ph/0204359

993. M. Pospelov, A. Ritz, Y. Santoso, Phys. Rev. Lett. 96, 091801 (2006). arXiv:hep-ph/0510254

994. M. Pospelov, A. Ritz, Y. Santoso, Phys. Rev. D 74, 075006 (2006). arXiv:hep-ph/0608269

995. C. Grojean, G. Servant, J.D. Wells, Phys. Rev. D 71, 036001 (2005). arXiv:hep-ph/0407019

996. D. Bodeker, L. Fromme, S.J. Huber, M. Seniuch, J. High Energy Phys. 0502, 026 (2005). arXiv:hep-ph/0412366

997. S.J. Huber, M. Pospelov, A. Ritz, Phys. Rev. D 75, 036006 (2007). arXiv:hep-ph/0610003

998. P.G. Harris et al., Phys. Rev. Lett. 82, 904 (1999)

999. K.F. Smith et al., Phys. Lett. B 234, 191 (1990)

1000. I.S. Altarev et al., Phys. At. Nucl. 59, 1152 (1996) [Yad. Fiz. 59(N7) 1204 (1996)]

1001. K. Green et al., Nucl. Instrum. Methods A 404, 381 (1998)

1002. J.M. Pendlebury et al., Phys. Rev. A 70, 032102 (2004)

1003. P.G. Harris, J.M. Pendlebury, Phys. Rev. A 73, 014101 (2006)

1004. R. Golub, J.M. Pendlebury, Phys. Lett. A 62, 337 (1977)

1005. C.A. Baker et al., Phys. Lett. A 308-1, 67 (2003)

1006. C.A. Baker et al., Nucl. Instrum. Methods A 501, 517 (2003)

1007. A. Steyerl et al., Phys. Lett. A 116, 347 (1986)

1008. http://ucn.web.psi.ch

1009. http://nedm.web.psi.ch

1010. S. Groeger et al., J. Res. NIST 110, 179 (2005)

1011. S. Groeger et al., Appl. Phys. B 80, 645 (2005)

1012. K. Green et al., Nucl. Instrum. Methods A 404, 381 (1998)

1013. http://p25ext.lanl.gov/edm/edm.html

1014. R. Golub, K. Lamoreaux, Phys. Rep. 237, 1 (1994)

1015. C.P. Liu, R.G.E. Timmermans, Phys. Rev. C 70, 055501 (2004). arXiv:nucl-th/0408060

1016. O. Lebedev, K.A. Olive, M. Pospelov, A. Ritz, Phys. Rev. D 70, 016003 (2004). arXiv:hep-ph/0402023

1017. R. Stutz, E. Cornell, Bull. Am. Soc. Phys. 89, 76 (2004)

1018. J.D. Jackson, Classical Electrodynamics, Wiley, New York (1975)

1019. A.J. Silenko, arXiv:hep-ph/0604095

1020. J. Bailey et al. (CERN Muon Storage Ring Collaboration), J. Phys. G 4, 345 (1978)

1021. F.J.M. Farley et al., Phys. Rev. Lett. 93, 052001 (2004). arXiv:hep-ex/0307006

1022. Y.K. Semertzidis et al., arXiv:hep-ph/0012087

1023. J.P. Miller et al. (EDM Collaboration), AIP Conf. Proc. 698, 196 (2004)

1024. A. Adelmann, K. Kirch, arXiv:hep-ex/0606034

1025. Y.K. Semertzidis et al. (EDM Collaboration), AIP Conf. Proc. 698200 (2004). arXiv:hep-ex/0308063

1026. I.B. Khriplovich, Phys. Lett. B 444, 98 (1998). arXiv: hep-ph/9809336

1027. I.I. Rabi, J.R. Zacharias, S. Millman, P. Kusch, Phys. Rev. 53, $318(1938)$

1028. Y.F. Orlov, W.M. Morse, Y.K. Semertzidis, Phys. Rev. Lett. 96, 214802 (2006). arXiv:hep-ex/0605022

1029. E.D. Commins, J.D. Jackson, D.P. DeMille, Am. J. Phys. 75, 532 (2007)

1030. E.D. Commins, Am. J. Phys. 59, 1077 (1991)

1031. M.G. Kozlov, A.V. Titov, N.S. Mosyagin, P.V. Souchko, Phys. Rev. A 56, R3326 (1997)

1032. M.K. Nayak, R.K. Chaudhuri, B.P. Das, Phys. Rev. A 75, 022510 (2007)

1033. N. Mosyagin, M. Kozlov, A. Titov, J. Phys. B 31, L763 (1998)

1034. M.G. Kozlov, L. Labzowski, J. Phys. B 28, 1933 (1995)

1035. A.N. Petrov et al., Phys. Rev. A 72, 022505 (2005)

1036. T.A. Isaev et al., Phys. Rev. Lett. 95, 163004 (2005)

1037. E.R. Meyer, J.L. Bohn, M.P. Deskevitch, Phys. Rev. A 73, 062108 (2006)
1038. A.N. Petrov, N.S. Mosyagin, T.A. Isaev, A.V. Titov, Phys. Rev. A 76, 030501(R) (2007)

1039. M.A. Player, P.G.H. Sandars, J. Phys. B 3, 1620 (1970)

1040. Z.W. Liu, H.P. Kelly, Phys. Rev. A 45, R4210 (1992)

1041. W.R. Johnson, D.S. Guo, M. Idrees, J. Sapirstein, Phys. Rev. A 34, 1043 (1986)

1042. S.A. Murthy, D. Krause, Z.L. Li, L. Hunter, Phys. Rev. Lett. 63, 965 (1989)

1043. M.V. Romalis, private communication

1044. T.W. Kornack, M.V. Romalis, Phys. Rev. Lett. 89, 253002 (2002)

1045. T.W. Kornack, R.K. Ghosh, M.V. Romalis, Phys. Rev. Lett. 95, 230801 (2005)

1046. M.V. Romalis, E.N. Fortson, Phys. Rev. A 59, 4547 (1999)

1047. C. Chin, V. Leiber, V. Vuletic, A.J. Kerman, S. Chu, Phys. Rev. A 63, 033401 (2001)

1048. D. Heinzen, private communication

1049. D.S. Weiss, F. Fang, J. Chen, Bull. Am, Phys. Soc. APR03 J1.008 (2003)

1050. J. Amini, H. Gould, arxiv.org/abs/physics/0602011 (2006)

1051. Y. Sakemi, private communication

1052. J.J. Hudson, B.E. Sauer, M.R. Tarbutt, E.A. Hinds, Phys. Rev. Lett. 89, 023003 (2002)

1053. M.R. Tarbutt et al., J. Phys. B 35, 5013 (2002)

1054. B.E. Sauer, J. Wang, E. Hinds, J. Chem. Phys. 105, 7412 (1996)

1055. D. DeMille et al., Phys. Rev. A 61, 052507 (2000)

1056. L.R. Hunter et al., Phys. Rev. A 65, 030501(R) (2002)

1057. D. Kawall, F. Bay, S. Bickman, Y. Jiang, D. DeMille, Phys. Rev. Lett. 92, 133007 (2004)

1058. E. Cornell, co-workers, private communication

1059. N.E. Shafer-Ray, Phys. Rev. A 73, 34102 (2006);

1060. N.E. Shafer-Ray, private communication

1061. S.K. Lamoreaux, Phys. Rev. A 66, 022109 (2002)

1062. F.L. Shapiro, Sov. Phys. Usp. 11, 345 (1968)

1063. V.A. Dzuba, O.P. Sushkov, W.R. Johnson, U.I. Safronova, Phys. Rev. A 66, 032105 (2002)

1064. B.J. Heidenreich et al., Phys. Rev. Lett. 95, 253004 (2005)

1065. T.N. Mukhamedjanov, V.A. Dzuba, O.P. Sushkov, Phys. Rev. A 68, 042103 (2003)

1066. S. Lamoreaux, arXiv:physics/0701198 (2007)

1067. C.-Y. Liu, S.K. Lamoreaux, Mod. Phys. A 19, 1235 (2004)

1068. C.-Y. Liu, private communication

1069. M. Mercier, Magnetism 6, 77 (1974)

1070. J.L. Feng, K.T. Matchev, Y. Shadmi, Nucl. Phys. B 613, 366 (2001). arXiv:hep-ph/0107182

1071. A. Romanino, A. Strumia, Nucl. Phys. B 622, 73 (2002). arXiv:hep-ph/0108275

1072. A. Bartl, W. Majerotto, W. Porod, D. Wyler, Phys. Rev. D 68, 053005 (2003). arXiv:hep-ph/0306050

1073. M. Aoki et al., J-Parc Letter of Intent, 2003

1074. A. Adelmann, K. Kirch, C.J.G. Onderwater, T. Schietinger, A. Streun, to be published

1075. P.A.M. Dirac, Proc. R. Soc. (London) A 117, 610 (1928),

1076. P.A.M. Dirac, Proc. R. Soc. (London) A 118, 351 (1928).

1077. P.A.M. Dirac, The Principles of Quantum Mechanics, 4th edn. (Oxford University Press, London, 1958). Eq. 9.28 uses Dirac's original notation

1078. B.C. Odom, D. Hanneke, B. D’Urso, G. Gabrielse, Phys. Rev. Lett. 97, 030801 (2006) [Erratum: Phys. Rev. Lett. 99, 039902 (2007)]

1079. T. Kinoshita, M. Nio, Phys. Rev. D 73, 053007 (2006). arXiv:hep-ph/0512330

1080. J. Schwinger, Phys. Rev. 73 416L (1948)

1081. J. Schwinger, Phys. Rev. 76, 790 (1949). The former paper contains a misprint in the expression for $a_{e}$ that is corrected in the longer paper 
1082. T. Blum, Phys. Rev. Lett. 91, 052001 (2003)

1083. C. Aubin, T. Blum, PoS LAT2005:089 (2005). hep-lat/ 0509064,

1084. M. Hayakawa et al., PoS LAT2005:353 (2005). hep-lat/ 0509016

1085. J.P. Miller, E. de Rafael, B.L. Roberts, Rep. Prog. Phys. 70, 795 (2007). arXiv:hep-ph/0703049

1086. It has been proposed that the hadronic contributions could also be determined from hadronic $\tau$ decay, plus the conserved vector current hypothesis, but this prescriptions seems to have internal consistency issues which are still under study. See Ref. [1082] and references therein

1087. R.L. Garwin, L.M. Lederman, M. Weinrich, Phys. Rev. 105, 1415 (1957)

1088. J. Bailey et al. (CERN-Mainz-Daresbury Collaboration), Nucl. Phys. B 150, 1 (1979)

1089. F.J.M. Farley, Nucl. Instrum. Methods A 523, 251 (2004). arXiv:hep-ex/0307024 